\title{
An Introduction to Contemporary Population Geographies
}

\begin{abstract}
An Introduction to Contemporary Population Geographies provides a foundation to the incredibly diverse, topical and interesting field of twenty-first-century Population Geography. It establishes the substantive concerns of the subdiscipline, acknowledges the sheer diversity of its approaches, key concepts and theories and engages with the resulting major areas of academic debate that stem from this richness.

Written in an accessible style and assuming little prior knowledge of topics covered, yet drawing on a wide range of diverse academic literature, the book's particular originality comes from its extended definition of Population Geography that locates it firmly within the multiple geographies of the life course. Consequently, issues such as childhood and adulthood, family dynamics, ageing, everyday mobilities, morbidity and differential ability assume a prominent place alongside the classic Population Geography triumvirate of births, migrations and deaths. This broader framing of Population Geography allows the book to address more holistically aspects of lives across space often provided little attention in current textbooks. Particular note is given to how these lives are shaped though hybrid social, biological and individual arenas of differential life course experience. By engaging with traditional quantitative perspectives and newer qualitative insights, the authors engage students from the quantitative macro scale of population to the micro individual scale.

Aimed at higher-level undergraduate and graduate students, this introductory text provides a well-developed pedagogy, including "real world" illustrations of theory, concepts and issues.
\end{abstract}

Holly R. Barcus is a Professor and Chair in Geography at Macalester College, St. Paul, Minnesota, USA.

Keith Halfacree is a Reader in Human Geography at Swansea University, Swansea, UK. 
| 7215 INTRO CONTEMP GEOGRAPHIES-Acg_246x189 mm 23/05/2017 $\$ 7: 35$ Page ii

\section{ST PROOFS: NOT FOR DISTRIBUTION}




\section{An Introduction to Contemporary Population Geographies}

\section{Lives Across Space}

Holly Barcus and Keith Halfacree 
First published 2018

by Routledge

2 Park Square, Milton Park, Abingdon, Oxon OX14 4RN

and by Routledge

711 Third Avenue, New York, NY 10017

Routledge is an imprint of the Taylor \& Francis Group, an informa business

(c) 2018 Holly Barcus and Keith Halfacree

The right of Holly Barcus and Keith Halfacree to be identified as authors of this work has been asserted by them in accordance with sections 77 and 78 of the Copyright, Designs and Patents Act 1988.

All rights reserved. No part of this book may be reprinted or reproduced or utilised in any form or by any electronic, mechanical, or other means, now known or hereafter invented, including photocopying and recording, or in any information storage or retrieval system, without permission in writing from the publishers.

Trademark notice: Product or corporate names may be trademarks or registered trademarks, and are used only for identification and explanation without intent to infringe.

British Library Cataloguing in Publication Data

A catalogue record for this book is available from the British Library

Library of Congress Cataloging in Publication Data

[CIP data]

ISBN: 978-0-415-56994-1 (hbk)

ISBN: 978-0-415-56995-8 (pbk)

ISBN: 978-0-203-85584-3 (ebk)

Typeset in Akzidenz Grotesk and Eurostile

by Florence Production Ltd, Stoodleigh, Devon, UK 


\section{CONTENTS}

List of figures

List of tables

List of boxes

Preface

Chapter 1 Viewing populations spatially: Population Geography as lives across space

\section{Chapter $3 \quad$ Global spatial distributions of population}

4.1 Introduction: the production of children 000

4.2 Modeling geographies of fertility 000

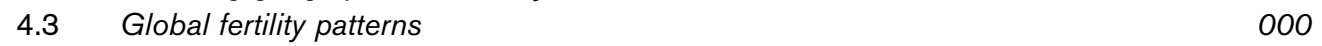

4.4 The "value" of a child 000

4.5 Managing timing and spacing of births 000

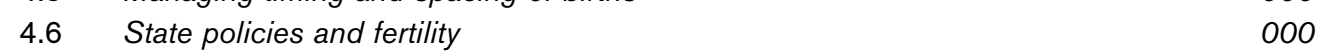

4.7 "New" fertilities $\quad 000$

4.8 Conclusion: fertility's multiple entanglements 000

$\begin{array}{llr}\text { Chapter } 5 & \text { Placing human migration } & \mathbf{0 0 0}\end{array}$

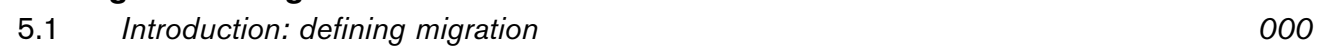

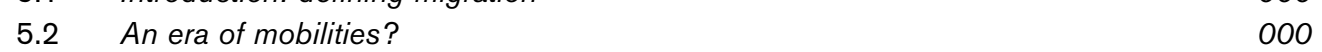

5.3 Migration as natural expression 000 
vi CONTENTS

$\begin{array}{lll}5.4 & \text { Migration as societal expression } & 000\end{array}$

5.5 Migration as individual expression 000

5.6 Migration as life course expression 000

5.7 Conclusion: broadening understanding of human migration 000

$\begin{array}{lll}\text { Chapter } 6 & \text { From everyday to residential mobilities } & 000\end{array}$

6.1 Introduction: mobilities to migrations 000

6.2 Everyday mobilities 000

$\begin{array}{lll}6.3 & \text { Residential mobility } & 000\end{array}$

6.4 Conclusion: from mobilities to migrations 000

$\begin{array}{llr}\text { Chapter } 7 & \text { Employment migrations } & \mathbf{0 0 0}\end{array}$

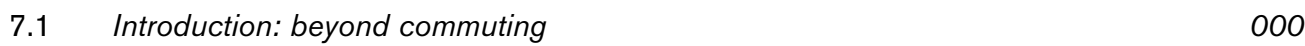

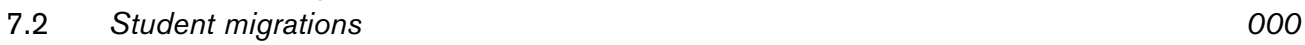

7.3 General employment migrations 000

$\begin{array}{lll}7.4 & \text { Specialist employment migrations } & 000\end{array}$

7.5 Employment migrations as family and community practices 000

$\begin{array}{ll}7.6 \text { Conclusion } & 000\end{array}$

$\begin{array}{llr}\text { Chapter } 8 & \text { Lifestyle migrations } & \mathbf{0 0 0}\end{array}$

8.1 Introduction: migration for the project of the self 000

8.2 The lure of the city 000

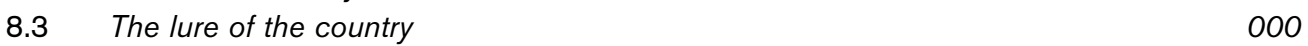

8.4 Lifestyle retirement migrations 000

8.5 Conclusion: relational lifestyle migrations 000

$\begin{array}{llr}\text { Chapter } 9 & \text { Forced migrations } & \mathbf{0 0 0}\end{array}$

9.1 Introduction: the importance of labels 000

9.2 Recognizing forced migration and mobilities 000

9.3 Forced mobilities 000

9.4 Refugees, internally displaced persons (IDPs) and asylum-seekers 000

9.5 Conclusion: looking beyond victimhood 000

$\begin{array}{llr}\text { Chapter } 10 & \text { Ageing and mortality } & \mathbf{0 0 0}\end{array}$

10.1 Introduction: the ageing body and the end of a life course 000

10.2 Elderly lives 000

10.3 Mortality: measurement and global trends and patterns 000

10.4 Modeling mortality geographies 000

10.5 Differentiating mortality: causes of death 000

10.6 Conclusion: mortality, resources and access in an ageing world 000

$\begin{array}{lll}\text { Chapter } 11 & \text { Twenty-first-century lives across space } & 000\end{array}$

11.1. Twenty-first-century perspectives 000

11.2 Twenty-first-century challenges 000

$\begin{array}{lll}11.3 & \text { Twenty-first-century populations } & 000\end{array}$

11.4 Conclusion: twenty-first-century population geography 000

$\begin{array}{lr}\text { Bibliography } & 000\end{array}$

$\begin{array}{lr}\text { Index } & 000\end{array}$ 


\section{FIGURES}

Global population growth

Trewartha's central place for Population within Geography

How the Wales Coastal Path at Swansea expresses four kinds of space

Representations of home and place in the real estate window

A simplified diagram of an individual life course

World population growth through history

World population distribution 2015

Population pyramid for Russia in 1950

Population pyramids for China and Afghanistan in 2010

World's largest cities: 2007 and 2025

Gentrification

The Statue of Liberty

The late nineteenth-century growth of London's railway lines

Suburbia-housing developments near Markham, Ontario, Canada, 2005

The Demographic Transition Model

Global distribution of fertility rates by country, 2010

Youthful (male) protestors in Tahrir Square, Cairo, January 2011

Margaret Higgins Sanger, 1879-1966

Births to unmarried mothers, North and West Europe, 1960-2009

Potential consequences of infertility for women in the Global South

The costs of everyday mobility

The mobility continuum

Representation of the Mobility Transition Model

Two behavioral models of migration: a. General model of decision on moving house simplified from and based on Roseman (1971); b. Rubicon model of the migration process simplified from and based on Kley (2010)

Instructions for Immigrant Sense of Belonging Walking Project

Commuters at Paddington Station, England

A typology of visiting friends and relatives travel

Overlapping emphases within residential mobility explanation

Diversity of population by housing tenure across Europe, 2013

Buying a first home is a major financial commitment

The growing sectarian divide in Baghdad, Iraq, 2003-2007

The model migration schedule

Entering university education, selected OECD countries, 2000 and 2010

Student migrations in a differentiated and differentiating educational and labor market context

Birthplace of US Undocumented Population, 2005 (Millions)

The restructuring US meat industry's labor migration consequences

Eastern Europeans resident in EU15, 1997-2009 
7.7 The escalator region concept

7.8 Highly skilled emigrants to OECD countries, \% of total, 1990 and 2000

7.9 Countries where UK medical doctors trained

7.10 Classifying highly-skilled migrants

7.11 Top 10 Global South recipients of remittances, by amount (a) and \%GDP (b)

7.12 The Urtehagen Mosque: part of the transnational landscape of Oslo, Norway

8.1 Model for the development of "alternative" urban districts

8.2 London's Docklands in the 1980s, just before gentrification

8.3 Resisting gentrification displacement

8.4 Graphical representation of counterurbanization

8.5 Counterurban household strategies in the Czech Republic

8.6 Sea Change coastal towns in New South Wales, Australia

8.7 Model of rural counter-urban populations

8.8 Goals within back-to-the-land

8.9 A recreational vehicle (RV): Class A motorhome with slide-out extended floors

8.10 Necessary conditions for undertaking lifestyle migration

9.1 Refugee camp: Lesvos Island, Greece

9.2 Human trafficking in East Asia

9.3 Contexts of modern slavery

9.4 Number of refugees by country or territory of asylum, 2010

9.5 Number of IDPs by country, 2010

9.6 Number of asylum-seekers by origin country, 2010

9.7 Number of asylum-seekers by destination country, 2010

9.8 A conceptual framework defining the core domains of integration

10.1 Active retirees

10.2 Global distribution of crude death rates, 2010

10.3 Age adjusted death rates globally, 2012

10.4 Life expectancy at birth by world region, 1950-2010

10.5 Deaths in the US (2013) and South Africa (2011), percentage total by age group

10.6 Infant mortality rates globally, 2014

10.7 Stages of the epidemiological transition model

10.8 Screenshot of Gapminder graph for rates of tuberculosis deaths globally

10.9 Differentiating causes of mortality

10.10 Global and regional distribution of deaths by group, 2000 and 2012

10.11 Global tuberculosis deaths, 2014

10.12 HIV/AIDS prevalence in Africa, 2007-2008

10.13 Interrelationships between health, migration and deprivation

10.14 Global regional breakdown of deaths by cancer, 2012 (\% total deaths)

11.1 Gini coefficient of OECD income inequality 2012

11.2 World petroleum consumption by region 1980-2012

11.3 Some claimed benefits and risks from genetically modified crops 


\section{TABLES}

1.1 Comparative infant mortality rates

1.2 Some key research poles within Population Geography 1950s-1970s

$1.3 \quad$ Migration research undertaken within different research traditions

1.4 Population Geography in the 1980s-1990s

1.5 The perceived neglect of social theory and its potential for Population Geography c.1995

1.6 Reviews of Population Geography published in Progress in Human Geography, 2000-2015

1.7 The expanding scope of Population Geography

2.1 The "classic" family life-cycle

$2.2 \quad$ Key life transitions

2.3 Basic principles of the life course perspective

2.4 The US class structure today

2.5 Class as relation

2.6 Environmental barriers impeding urban wheelchair mobility (in order of significance)

2.7 Ethnicity in New Zealand Statistics

2.8 Contrasting sex and gender: two expressions

3.1 Three place classification codes used by US Economic Research Service

3.2 Regional and global populations, 2014 and 2050 (projected): urban versus rural

3.3 Global "slum" populations

4.1 Calculating and comparing fertility rates, Norway 2012

4.2 First and second Demographic Transitions in western Europe: demographic and societal characteristics

4.3 Intermediate fertility variables and proximate determinants of fertility

4.4 European and Asian countries with continuing TFR below 1.5

4.5 Central tenets of preference theory

4.6 Percentage of women married or in sexual union, generally aged 15-49 years, using contraception

$5.1 \quad$ Three expressions of migration

5.2 Measuring migration

5.3 Diverse theoretical approaches to studying migration

5.4 How different academic disciplines have studied migration

5.5 Ravenstein's "Laws of Migration"

5.6 Selection from autobiographies of three emigrants from Hong Kong to Canada

5.7 Life course transitions associated with migration

6.1 "Mobility revolution" in the Global South

6.2 The changing British commuter through the twentieth century

6.3 Six weeks' trips in two German cities

6.4 Intensifying homophobia in Africa: the case of Uganda

6.5 Distance moved by living arrangement and status for Dutch movers

6.6 School League Tables: Secondary Schools on Devon-Somerset Border, England, 2014 


\section{$x$ TABLES}

7.1 Migrating to work in New Zealand

7.2 Characteristics of China's "rural laborers" according to migrant status, 2006

7.3 Profiles of smuggled male Pakistani migrants in London, UK

7.4 Immigrants in Spain 2000-2011 in low-skill occupation groups (\%)

7.5 Migration backgrounds of international academics working in universities in North West England 2011

7.6 Top 10 countries exporting highly skilled people to OECD, 2000

7.7 Migrants' perceived impacts of FIFO, Queensland, Australia, 2009

7.8 Types of return migration

7.9 Remittance flows to Global South, 2011 estimates

8.1 Contrasting representations of city and country

8.2 Ownership of rural micro-businesses by migrant status, NE England 2007 survey

8.3 Key attributes of intentional communities

8.4 Elements of "radical rural" representations

8.5 Origins of flights to Alicante, 3 July 2014

8.6 Reasons for retiring to Torrevieja, Spain

9.1 UNHCR definitions of displaced persons

9.2 Terms associated with environmental migrants and migration

9.3 Four kinds of mixed migrations

9.4 Book-length accounts of human trafficking and modern slavery

9.5 Forms of modern slavery

9.6 Five requirements for ideal refugee and IDP return

10.1 Residential options for adults needing regular assistance

10.2 Selected gerontic dependency ratios by country, 2013

10.3 Mortality rates, Norway 2013

10.4 Odds ratios for mortality by key mortality covariates

10.5 World Health Organization classification of deaths

10.6 Globally prominent infectious diseases

10.7 Major modes of HIV transmission

11.1 The UN's five priority areas for future population and development

11.2 Progressing the Millennium Development Goals

11.3 Areas of enquiry for population geographers relative to health and climate change

11.4 UN estimates of world and regional populations, 2015 and 2050

11.5 Top 10 countries by population size, 2015 and 2050

11.6 The UN's paths to sustainability

11.7 Top 10 countries by percentage population aged 80+ years, 2015 and 2050 


\section{BOXES}

1.1 Some key -isms underpinning the diverse approaches to Population Geography

$2.1 \quad$ The complex transition to adulthood

2.2 Media representations of the older aged population

2.3 Social stratification within the caste system

2.4 Superman, Fascism and challenging the disabled body

2.5 Race, genetics and ill health

3.1 Agricultural population in prehistory

3.2 The population census

3.3 Revolutionary Road

3.4 Rural

4.1 The post-1945 "Baby Boom"

4.2 The costs of children

4.3 Homebirth in the United States

4.4 Fertility policies in Romania

4.5 A Brave New World of fertility

5.1 Changing expressions of migration and mobility from Ireland to the US and UK

5.2 The legacy of "push" and "pull" factors in migration scholarship

5.3 From cross-sectional to longitudinal migration data

5.4 Migration in twentieth-century love letters between Italy and Canada

5.5 Momentous mundane mobility moments

6.1 Relationship mobilities of internet dating

6.2 The yellow school bus

6.3 Campaigning for children's free-range

6.4 Mobility consequences of housing busts

6.5 Ethnic segregation in the UK

6.6 Chinese suburbia and gated communities

7.1 Landscapes of studentification

7.2 The migrant "flood" that never was

7.3 International relocations of financial workers

7.4: $\quad$ Building Qatar's World Cup stadia

7.5 Skype, social media and transnationalism

7.6 The complexities of migrations and mobilities: the case of Mongolian Kazakhs

8.1 The rural as destination for LGBT migrations

8.2 The city and protest mobilization

8.3 Rurality: from representational to more-than-representational

8.4 Balance quickly restored: cabin life in Norway

8.5 Countercultural Wales

8.6 Authenticity-through-place in amenity migrations

9.1 Uncertain places of children within forced migration 
xii BOXES

9.2 Frontex and European Union border security

9.3 Refugee modes of transit: use of boats

9.4 Population displacement by the Three Gorges Dam in China

9.5 Ethnic cleansing: the dark side of democracy?

10.1 Disability: social and embodied (im)mobilities

10.2 Images of retirement and the perpetuation of ageism

10.3 Early epidemiological mappings

10.4 Vaccination and mortality reduction

10.5 Mortiferous environments

11.1 Population projections 


\section{PREFACE}

Georges Perec's short book The Art and Craft of Approaching Your Head of Department to Submit a Request for a Raise, originally published in 1968, begins with a flow-chart, designed by Jacques Perriaud. This complex set of questions, yes or no answers, and consequent outcomes is a veritable maze of detail. The book itself, written in one continuous sentence, manages somehow to address all of this detail, albeit with much repetition and reiteration. And yet, although this was another of Perec's remarkable literary achievements, one has the feeling at the end of it that there are numerous other issues that could have been brought into this story of how one might attempt to procure a pay rise. But, plainly, everything cannot be covered in one book.

The present book makes the same claim, in this case regarding Population Geography rather than obtaining a raise. We hope it is less repetitive than Perec's text but that it still manages to reveal both the scope of and interlinkages so central to our take on the subject. We also hope that this account is also presented in line with one of Perec's repeated assertions, namely that "we must do our best to keep things simple," whilst not losing our sense of the complex reality of lives lived across space. 
7215 INTRO CONTEMP GEOGRAPHIES-Acg_246x189 mm 23/05/2017 $\$ 7: 35$ Page xiv

\section{ST PROOFS: NOT FOR DISTRIBUTION}




\section{CHAPTER 1}

\section{Viewing populations spatially: Population Geography as lives across space}

\subsection{INTRODUCTION: LIVES ACROSS SPACE}

We are born, we live, and then we die. ${ }^{1}$ And our lives are always emplaced, always have a "where." From the cradle, through life, to the final resting place, life is lived across space. Population Geography is the study of such experiences and this book seeks to draw out some key features of the diverse lives lived across the spaces of the world today.

The aim of this chapter is to work towards this conception of Population Geography as lives across space, setting the scene for the rest of the book. It is structured as follows. Section 1.2 asserts the importance of population and of examining it through a spatial or geographical lens by engaging with some stark facts about the state of the world today. Second, the history of Population Geography as a sub-discipline is briefly narrated in Section 1.3, concentrating on the post-1950s period when it became recognized as a key component of Human Geography. A conclusion of this section, also made by many other academic commentators, is how Population Geography needs to reach out beyond the discipline of Demography, with which it was initially strongly linked, if it is to (re)assert itself within the established "social science" (Johnston 2009a) of Human Geography. This call for a broader intellectual base will be built upon in Chapter 2's development of a life course framing for Population Geography but is set-up here in Section 1.4 's call for a more fully contextualized or relational sub-discipline.

\subsection{OF POPULATIONS AND POPULATION GEOGRAPHY}

\subsubsection{Big numbers and big issues ...}

It is not difficult to make a case for writing another book on the human population. On the one hand, all of us are part of that population and we like to know about ourselves! However, on the other hand, it is also because it is hard to accept that our human population-homo sapiens-is simply another component within the immense diversity of animate groups populating planet Earth. Humans, in short, have evolved to such a degree that what we do collectively, whether on an everyday basis or more extraordinarily, often has global impacts, whether good or bad. Sometimes these impacts are immediate but often, as with global warming $\left(11.2 .3^{2}\right)$, they are long-term. And, of course, there are so many of us humans to bring about these impacts, with numbers rising daily, that humanity is very definitely a global force that cannot be denied.

According to the best estimates of the United Nations (UN), the year 1999 witnessed the birth of the six billionth co-resident of the earth, whilst 2011 saw much press and popular coverage (e.g. Kunzig 2011) given to population projections that predicted this figure had risen to seven billion. As Figure 1.1 demonstrates starkly, the global human population appears to be on an almost inexorable upward trajectory, its total of around three billion in 1950 now seeming very modest compared to the seven billion of 60 years later (UN Population Division 2011).

A number of implications of this seemingly huge global human population immediately call attention. First are those concerning immediate survival: questions of basic needs for food, water, shelter, space. The ghost of the English scholar Thomas Malthus inevitably makes an appearance here. At the turn of the nineteenth century-when England's population was a mere 8.3 million (by 2001 it was 49.1 million) (Jefferies 2005)-Malthus (1798) put forward his first Essay on the Principle of Population. This hypothesized that whilst population tended to increase geometrically 


\section{VIEWING POPULATIONS SPATIALLY}

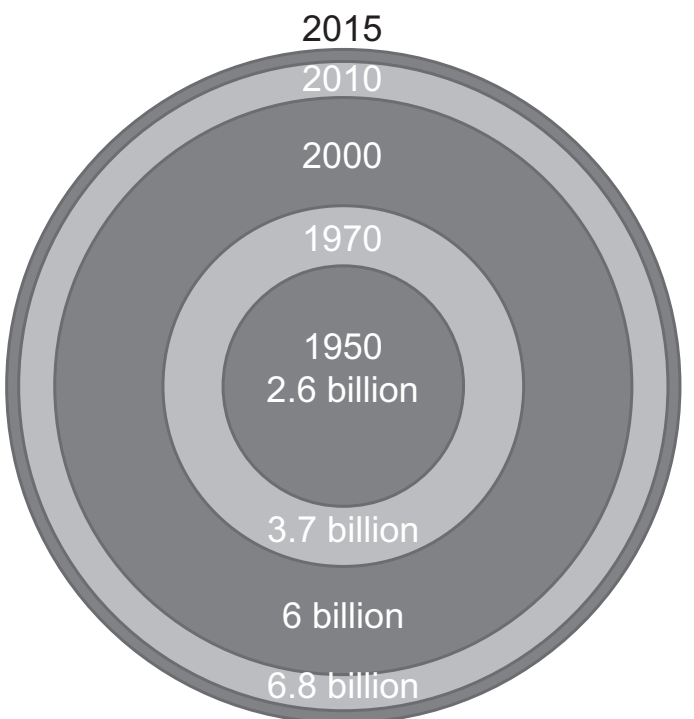

7.3 billion

Figure 1.1 Global population growth.

Data source: US Bureau of Census International Program. Data downloaded on 5 April 2016.

$(1,2,4,8,16 \ldots)$ food supplies could only manage arithmetical increase $(1,2,3,4,5 \ldots)$, leading to the specter of immediate famine and mortality if restraints on population growth were not put in place. This issue, and the reasoning and debates accompanying it, are returned to later (11.3.1), but it has not gone away. For example, it partly underpins the often near apocalyptic press coverage that was given to Earth's 7 billion plus future (e.g. Daily Mail 2011; Dean 2011; Dorling 2013).

Second, there are some less immediate but by no means less significant implications raised by the huge and still growing global population. These are expressed within arguments about the extent to which the Earth has the overall ability to support or sustain its human population. Can Earth's carrying capacity-the idea that the planet can only sustain a limited number of people (e.g. Ehrlich 1968)-cope with 7 billion, let alone the 9 billion some predict for 2045 (Kunzig 2011) or possible 10 billion by 2100 (Dorling 2013)? This is not just an issue of food supply, or even provision of other basic needs (water, shelter, space), but must take into consideration issues such as pollution produced (and its link to phenomena from ill health to global warming), the extent to which humanity relies on largely non-renewable resources to underpin lifestyles, and how humanity's expansion has often been at the expense of the Earth's other living species. The carrying capacity issue (11.3.1) is also, fundamentally and unavoidably, a question of politics and of global and local power relations. Any discussion must also consider the distribution of food and other aspects of basic needs within a society. The Earth that may be seen to have a carrying capacity is an economic, social, cultural and political Earth as well as a physical entity. As eminent Geographer David Harvey (1996: 147) forcefully put it, "scarcity is socially produced" at least as much as it somehow resides in the laws of the natural world. Population matters, then, are never solely matters of nature, making them still more important and interesting to study.

By now, it should be clear to the reader that consideration of global numbers of people alone suggests immediately why the human population merits specific and detailed attention by students, researchers and policymakers. There is a clear prima facie case for the discipline of Demography: "the scientific study of the size, composition, and distribution of human populations and their changes resulting from fertility, mortality, and migration" (Poston and Bouvier 2010: 3). Births, deaths and human migrations between locations across the globe certainly require measurement, presentation and discussion, and future trends predicted. But whilst Demography also involves itself with the causes of the patterns, trends and magnitudes it identifies-"the factors that affect these components" (Poston and Bouvier 2010: 3)-a more dedicated emphasis on their contextualized spatial expression has become the focus of Population Geography. 


\subsection{2 . . across real places}

Population Geography can be defined initially as the study of "the geographical organization of population and how and why this matters to society" (Bailey 2005: 1). An immediate illustration of how such a spatial lens is both significant and important comes, once again, via some simple demographic facts about the state of the world today (some developed more fully in Chapter 3). Consider infant mortality rates (10.3.1) in a small selection of countries in Asia and Europe, shown in Table 1.1. First, even across these ten countries there is a considerable range of values. This is true even within the same continent, such as the contrast between Romania and Norway in Europe. Second, although there is a general trend towards declining infant mortality, countries such as Mongolia and India retain extremely high rates. Furthermore, many countries affected by civil strife, such as Afghanistan, Congo or Iraq, are understandably unable to provide data. Thus, Table 1.1 is biased in favor of countries experiencing relative political stability. Third, the table also shows infant mortality rates tending to be higher in rural as compared to urban areas, although this gap tends to reduce with overall decline and some countries, such as Armenia, have higher urban rates. In short, therefore, it is clear that there is a strong geography of infant mortality worldwide.

More generally, as world population continues to expand, experiences of individuals across space remain extraordinarily diverse and in some cases are even diverging. This statement may seem initially surprising, given that academic and other writers have for decades suggested how a range of global forces, especially those linked to the economy, are making the world's human geography increasingly similar. The concept of globalization, defined as "the widening, deepening and speeding up of worldwide interconnectedness in all aspects of contemporary social life, from the cultural to the criminal, the financial to the spiritual" (Held et al. 1999: 2), suggests, at least on the (distant?) horizon, "a single world society, global society" (Albrow 1990: 9) or even the long-anticipated utopian "global village" (McLuhan 1962). Such a convergence may be expected to embrace demography. Indeed, this is as a more or less explicit assumption in key concepts such as demographic transition (3.3) and mobility transition (5.3.1). However, in spite of global processes encouraging convergence, Human Geographers such as Massey (2005), Yeung (2003) and Taylor et al. (2001), plus numerous other social scientists (e.g. Held et al. 1999) and even business commentators (e.g. Kay 2001), have emphasized how seemingly global processes explored in their spatial context exhibit sustained and even increased diversity. Globalization's spatial expressions operate at a range of different scales and it is not solely "an essential global force that homogenizes national and local differences" (Yeung 2003: 105).

Returning more directly to Population Geography, within this framework of continued diversity, experiences of birth, childhood, adolescence, and adulthood, for example, are all to a greater or lesser extent conditioned by moreor-less specific circumstances. On the one hand, these circumstances are a product of each individual's geographic location. Crudely put, for example, a baby born in the Global South can expect a quite different childhood and life prospects from a baby born in the Global North: most children's lives in St Paul, Minnesota are very differently configured from those in Saint-Paul, Réunion. On the other hand, though, the lives of all children born in either St Paul or Saint-Paul are not the same. Family circumstances, not least expressed by class or relative wealth, play a critical role. Taken together, what can be termed a child's life expectations are related to the cultural, social and environmental circumstances that meet (Massey 2005) not only within the child's individual home but also within their city, region, country and so on. And finally but critically, these circumstances are, to greater or lesser degrees, everchanging through a life, through their embodiment in a life course (Chapter 2). In short, life experiences are always relational in respect of spaces and individual lives.

Understanding and explaining the population geographies of births, deaths and population movement requires taking into account a wide array of economic, social, cultural, political and environmental processes. These are reflected in, for example, resource conflict, everyday living environments, differences in health and well-being, levels of poverty and inequality, and relationships formed both within and between peoples and countries. These processes both shape the population characteristics of any particular society and its individual members, and are in turn shaped by them. Thus, studying Population Geography requires core demographic indicators such as births, deaths and migrations not to be thought of in isolation but examined through their entanglements with the full panoply of diverse processes that construct everyday life. This is, of course, no mean feat but reflects both the challenge and potential sense of achievement to be had in explaining what the present authors are calling lives across space. Next, though, an historical account of Population Geography up to the present day is outlined. This both establishes a fuller academic context for the rest of the book and demonstrates how an expanded notion of Population Geography is congruent with recent critical reflection within the sub-discipline.

\section{ST PROOFS: NOT FOR DISTRIBUTION}


4 VIEWING POPULATIONS SPATIALLY

Table 1.1 Comparative infant mortality rates

Infant mortality rate

$2002 \quad 2006$

ASIA

Armenia

Total

Urban

14.0

13.9

Urban
Rural

14.416 .3

India

Total

13.1

9.7

Urban

63.0

57.0

Urban

40.0

39.0

Rural

69.0

62.0

Israel

Total

5.4

4.0

Urban

5.3

4.0

Rural

$-$

Japan

Total

3.0

2.6

Urban

3.0

2.6

Rural

3.1

2.6

Mongolia

Total

29.6

19.1

Urban

Rural

33.4

18.3

EUROPE

$\begin{array}{llll}\text { Belarus } & \text { Total } & 7.8 & 6.1 \\ & \text { Urban } & 7.0 & 5.7 \\ \text { Rural } & 10.2 & 7.0 \\ \text { Hungary } & \text { Total } & 7.2 & 5.7 \\ & \text { Urban } & 6.9 & 5.3 \\ \text { Rural } & 7.6 & 6.6 \\ \text { Netherlands } & \text { Total } & 5.0 & 4.4 \\ & \text { Urban } & 5.0 & 4.2 \\ \text { Rural } & 5.0 & 4.8 \\ \text { Norway } & \text { Total } & 3.5 & 3.2 \\ & \text { Urban } & - & - \\ \text { Romania } & \text { Rural } & - & - \\ & \text { Total } & 17.3 & 13.9 \\ & \text { Urban } & 14.5 & 11.2 \\ & \text { Rural } & 19.8 & 17.1\end{array}$

Source: United Nations Demographic Yearbook 2006 at:

http://unstats.un.org/unsd/demographic/sconcerns/mortality/mort2.htm\#DYB

\subsection{A SHORT HISTORY OF POPULATION GEOGRAPHY}

\subsubsection{The emergence of Population Geography}

"[F]ields and sub-disciplines make and remake themselves" (Bailey 2005: 9) but, paraphrasing Karl Marx, they do not make themselves under self-selected circumstances. So far in this chapter, the fundamental idea of "population" has been largely unexamined. All of us will have a fairly taken-for-granted understanding of what the word means. However, when investigated further and how it came to be used is drawn out, specifically by government and academia, interesting insights emerge into how people have been represented. Such representation leads to particular ways of understanding people in society. 
Drawing on the work of the late French social theorist (and proposed Population Geographer) Michel Foucault (Legg 2005; Philo 2005), Curtis (2002) examined how representation and study of what he termed the "social body" saw the term "populousness" increasingly displaced by a newer concept of "population" from around the eighteenth century. This is not just of terminological interest. Populousness was an idea whose meaning was set within the strongly stratified and hierarchically ordered society of pre-capitalism. For example, feudalism was built on a rigid hierarchical structure from the monarch at the top through lords to vassals at the social base. Populousness was rooted in a "sense that units of government (kingdoms, empires, countries, parishes, cities) contain greater or lesser numbers of entitieshearths, soldiers or souls, for instance-distributed across different orders or classes" (Curtis 2002: 508). In other words, populousness regarded society primarily in terms of orders or classes, whether represented by people or their artifacts, such as the hearth, rather than in terms of the individual.

In contrast to populousness, population as a concept stressed a more abstract notion of the individual. It presented "practical equivalences among subjects, objects or events... [It] depends upon the notion of a common abstract essence ... of so many undifferentiated atoms distributed through abstract space and time" (Curtis 2002: 508). Such an atomistic re-imagination of people was facilitated by "destruction of the status differences of the ancien régime" (Curtis 2002: 529), notably following the French Revolution of 1789. People became, in short, both individual and, as entities, equal. Population, as "primarily a statistical artifact" (Curtis 2002: 509) totaling up these equal units was soon expressed both in a country's inhabitants experiencing "common subordination to sovereign political authority" (Curtis 2002: 509)-formal political equality, expressed in equal voting rights, for example-and from a demographer's point of view in the rise of "nominal census enumerations" (Curtis 2002: 530)-Norway 1801-, England 1841-, Belgium 1846-, USA 1850- (Box 3.2)-formal demographic equality. Population was atomized and discrete but equal individuals consequently went on to become the predominant way people were represented within Population Geography.

Population Geography lagged by some considerable period this emergence of the modern concept of population. It is a relatively young sub-discipline within the broader field of Human Geography. Specifically, it did not clearly emerge in a recognizable and distinct form until the mid-1950s and, as with all of Geography's sub-disciplines, has been in fairly constant change and evolution ever since. This rest of this section of the chapter will seek to give the reader a brief sketch of this emergence and evolution, drawing in particular on Adrian Bailey's (2005) Making Population Geography, although much the same broad story is told, more briefly, in most textbooks.

Perhaps the key trajectory mapped by Bailey is how Population Geography has, over time, concerned itself with an increasingly "enlarged" (Bailey 2005: 8) notion of its principal subject matter-population. Building upon an initial focus on the spatial manifestation of the core elements of Demography (births, deaths, migrations), it now encompasses issues of geopolitics, economic growth and development, individual and family livelihoods, consumption issues generally, social structures and cultural forms and transformations. In short, "population geography [has] shifted its topical emphases to reflect key issues in society, rather than to follow the dictates of a demographic framework" (Bailey 2005: 192). The present book falls in line with Bailey (also Bailey 2010) in promoting this notion of Population Geography "beyond Demography." Yet, it also aims to return to something of the more integrated sense of the subdiscipline presented within its intellectual emergence in the 1950s but then subsequently rather lost.

\subsubsection{Early twentieth century}

There was scholarship that can be seen as Population Geography prior to the 1950s, notably in the half century when Human Geography established itself as a significant academic discipline (Johnston and Sidaway 2004). In these early years, in line with predominant regional and empirical paradigms (predominant ways Human Geography was practiced and understood), Population Geography was extremely descriptive. It concerned itself primarily with measuring, mapping and monitoring areal differentiation of population or how various measurable aspects of populations varied spatially and in relation to other geographical phenomena. Epitomized by Richard Hartshorne's (1939) influential text, The Nature of Geography, interest was given both to the extent to which regions differed from one another and to the extent they were similar (Agnew 1989). Within Population Geography a strong national scale emphasis was evident and differences between dominant world powers and the rest of the world used to reinforce colonial and imperial ambitions and statements of superiority (Bailey 2005). 


\section{VIEWING POPULATIONS SPATIALLY}

Population Geography prior to the 1950s, therefore, was largely subsumed within a descriptive Human Geography mainstream. Within such an imaginary, space was seen in absolute terms, simply there to be measured and calculated. It could be presented either as comprising a largely inert container or environment, within which the objects of interest to Human Geographers such as people or cities fitted, or as a more active determinant force, whereby physical features of this "container," such as climate, determined its more social characteristics (environmental determinism). Human Geography, and by default Population Geography, was from this perspective not seen as very "alive." In summary, Jones (1990: 2) was not being excessively critical when he concluded that prior to the 1950s population was "relegated to consideration in the more sterile forms of Regional Geography as part of a place-work-people chain" where the physical environment begat the economy which begat population in a fairly unexamined manner.

\subsubsection{0s-1970s}

As with its practice in the early part of the twentieth century, Bailey (2005; also Jones 1990) argued that the rise of Population Geography as a distinctive academic sub-discipline in the 1950s, expressed through key figures and influential texts, reflected developments within both Human Geography and society generally. With respect to the latter, population issues featured heavily in: post-World War II national projects of economic and social reconstruction; promoting geopolitical stability, economic prosperity and social cohesion; and often pushed progressive social and cultural agendas. For example, reducing infant mortality as a pressing issue for democratic societies of equals could be associated with all these aforementioned topics. However, whilst states "became absorbed by population matters" (Bailey 2005: 58), these challenges were usually not framed in practice in objectively neutral ways but were always constituted under the shadows cast by their societies. In particular, there were the post-1945 East versus West/Communist versus non-Communist geopolitical world reorganizations, struggles and tensions.

Human Geography generally was similarly evolving within global and national contexts (Johnston and Sidaway 2004). Within the positive haze of a post-war utopian faith in reason, science and progress, it sought to reposition itself away from both the unambitious descriptive emphasis expressed by areal differentiation and, in the wake of Nazi Germany's blood-and-soil rhetoric of societies and cultures rooted in their physical environments (Bramwell 1985), very firmly away from environmental determinism. It retained an absolute sense of space, however.

Embracing ideas, concepts and approaches adopted and adapted from what were seen as politically untainted and intellectually progressive sciences, notably Physics, Human Geography experienced from the late 1950s a "quantitative revolution" (Burton 1963). It sought to become an objective modern spatial science. In line with generally integrationist aims within academia at the time, it was especially inspired by positivist philosophies (Box 1.1) and sought out order and the general. In short: "[a] central goal ... was to verify and falsify empirical observations and construct generalizable laws that informed complex models and theories with widespread (i.e. universal) applicability" (Bailey 2005: 60).

What, then, of Population Geography? First, the quantitative revolution was both congruent with and reinforced the interpretation of population as comprising discrete atomized objects (1.3.1). Group/class and more subjective concerns were largely overlooked in favor of a formal demographic equality for each person-unit, an abstraction little different from the areal differentiation years. Second, support for Population Geography came from governmental concerns with population matters. There was a desire for knowledge obtained through science that could be put to supposedly rational use for the benefit of all, although the global geopolitical shadow noted earlier remained. Third, however, Population Geography also had some difficulties emerging as a sub-discipline. Although Demography was widely acknowledged as an important body of knowledge-and fuelled Population Geography's own quantitative revolution (Findlay and Graham 1991)-it did not provide the equivalent strong and broad disciplinary leaping off point that Sociology, Economics and Anthropology provided for Social, Economic and Urban Geography, respectively (Zelinsky 1966).

Nonetheless, Population Geography emerged as a vibrant component of Human Geography in the 1950s. This was heralded, in particular, by the 1953 Presidential speech to the annual conference of the Association of American Geographers by Glen Trewartha (for broader roots than this speech, see Kosiński 1984). Trewartha (1953: 97) asserted Population Geography as "the pivotal element in Geography, and the one around which all the others are oriented." It comprised one component of the essential trinity of Geography that, for him, was Population, Cultural Earth and Physical Earth. Yet, unlike the increasing specialization that accompanied the quantitative revolution's

\section{ST PROOFS: NOT FOR DISTRIBUTION}




\section{BOX 1.1 KEY -ISMS UNDERPINNING THE DIVERSE APPROACHES TO POPULATION GEOGRAPHY}

Numerous conceptual approaches have been brought to the study of Population Geography over its relatively short existence as a distinctive sub-discipline. Some sense of this diversity is described in the present chapter and will become clearer throughout the book. Underpinning these approaches is an equally bewildering range of philosophical frameworks, often termed the -isms. Some of these, ordered by their appearance in the chapter, are now given extremely short definitions:

- Empiricism: knowledge primarily coming from experiences of the senses

- Positivism: prioritizes knowledge obtained via repeatable, verifiable observations

- Behavioralism: emphasizes cognitive processes underlying individual decision making

- Marxism: focuses on the material, especially economic, relations between humans

- Structuralism: understanding surface patterns of human behavior requires knowledge of underlying causal structures

- Humanism: accords primary attention to thinking, acting, feeling individuals

- Postmodernism: emphasizes the relativism of reality and expresses considerable skepticism towards grand claims to knowledge and truth

- Post-structuralism: focuses on openness of meanings, ambivalence of texts and multiple production of identities

For further details on these terms, consult the wide range of books now available on Geographical thought!

scientization of Human Geography, Trewartha advocated Population Geography practiced within a unified geographical approach or an emphasis on "oneness" (Trewartha 1953: 85). This is a "lost" perspective returned to in Section 1.4. Indeed, whilst Trewartha's intervention may have been very politically useful for Population Geography, the subdiscipline by the 1980s was more a product of developments that took place within 1960s Human Geography than being rooted in his holistic 1950s expression (Jones 1990).

Table 1.2 gives an overview of some of the main areas of Population Geography that subsequently developed during the 1950s-1970s. This was a buoyant time for the discipline as it moved to develop a more scientificallysounding Spatial Demography. Population Geography moved away from Demography's focus on "the intrinsic nature and universal attributes of populations and [the] temporal dimension" (Jones 1990:3) to concentrate on more or less scientifically outlining "demographic facts in their present environmental context" (Beaujeu-Garnier 1966: 3, our emphasis). Although still absolute, space was at last being brought more fully out of the shadows, assuming an equal significance to demographic facts. Thus, as in much of Human Geography (Johnston and Sidaway 2004), a positivistinfluenced approach, heralded at the time by Zelinsky (1966), increasingly triumphed at the expense of more empiricist descriptive and synthetic local studies.

Within positivist Population Geography, a deductive approach, seeking to test carefully a priori theorized hypotheses or propositions put forward to explain some phenomenon, increasingly overshadowed more inductive work that sought generalizations from pre-gathered facts. Again, this suggested greater academic rigor and organization. Thus, Bailey $(2005: 103,105)$ concluded that by the end of the 1970s "population geography had come of age as a sub-discipline with a recognizable focus upon the spatial context of demographic phenomena.... [This] meant theoretical convergence around a positivist search for demographic order in spatial economic landscapes." Population Geography was an essential piece of the positivist Human Geography jigsaw.

Looking further at Table 1.2, whilst empiricism and positivism predominated, investigations concerned with understanding or explanation also began to take on board more explicitly societal context (structuralist) or experiential (humanist) approaches (Box 1.1). These will be considered more fully in the next section but both finally challenged the atomistic interpretation of population as well as the absolute or fixed status of space. The reader should also note the presence in Table 1.2 of numerous core Population Geography concepts, all revisited within subsequent chapters of the present book. 


\section{VIEWING POPULATIONS SPATIALLY}

Table 1.2 Some key research poles within Population Geography 1950s-1970s

\begin{tabular}{llll}
\hline Research pole & Indicative topics & Approaches & Views of population \\
\hline Describing demographic structures & Concentration & Empiricist & Aggregate \\
& Segregation & Positivist & \\
& Density & & \\
Flows & Balancing equation & Empiricist & Aggregate \\
Describing demographic change & Spatial demography & Positivist & \\
& Transition theory & Positivist & Aggregate \\
Explaining demographic change & Diffusion & Structuralist & Individual \\
& Systems theory & & \\
& Migration & Empiricist & Aggregate \\
Lived worlds and cultural systems & Culture & Humanist & Individual \\
& Environment & & \\
\hline
\end{tabular}

Source: based on Bailey (2005: Table 3.3).

\subsubsection{0s-1990s}

Almost as soon as the quantitative revolution had begun to generate a significant body of new Human Geography scholarship, a backlash began. In particular, upwelling politically radical perspectives developing within many societies across the Global North from around 1968 soon had counterparts within academia (Blunt and Wills 2000; Watts 2001). The philosophical and political standpoints of these perspectives led to different emphases and foci within scholarship, generating different results and conclusions. Almost all, either explicitly or more implicitly, rendered increasingly problematic any supposed objectivity about knowledge or the knowledge acquisition process. Thus, the global geopolitical shadow that had long hung over geographical scholarship, for example, was finally given central attention. Context and reflexivity, in short, became paramount.

British geographer David Smith (1984: 129) colorfully expressed the radicals' dilemma within a series of recollections on this turbulent period. It was one in which students of Geography "saw the heroes of the quantitative revolution content to rotate their principal components ${ }^{3}$ while the city ghettos burned." In short, radicals became increasingly discontented with the relevance, usefulness and embodied values of much of the work inspired by the quantitative revolution, whatever its scientific validity. An ethically directed realignment was demanded of Human Geography, the political direction of which is expressed well in a later commentary from the best known radical geographer to emerge from the 1960s, David Harvey (1984: 7):

Geography is ... far too important to be left to generals, politicians, and corporate chiefs... The selling of ourselves and the geography we make to the corporation is to participate directly in making their kind of geography, a human landscape riven with social inequality and seething geopolitical tensions.

Responding to critiques such as these, Human Geography scholarship increasingly moved through the 1970s away from abstracted and objectified value-free conceptions of both the research process and what was researched. This initiated the flowering of what is now a great diversity of post-positivist ways of thinking (Johnston and Sidaway 2004; Peet 1998). Particular attention was given to the development of scholarship in two broad rival directions. Both rejected any absolute, fixed and therefore distanced notion of space and instead acknowledged the spatial as intimately entangled with the human.

First, and strongly underpinned by Marxism (Box 1.1), a quasi-scientific emphasis was retained within scholarship that primarily saw Human Geography through a structuralist lens. In particular, the geography of our cities, regions and countries was seen as reflecting, more or less directly, the underlying social and political structures that societies were thought to be made up of and built upon. In particular, existing as we do within capitalist societies, space was produced by capitalism (Smith 1984). Geography expressed the inequalities and tensions intrinsic to such societies. 
It was the radicals' task to tease out these connections, with the ultimate aim of changing the world for the better (Blunt and Wills 2000).

The second broad direction taken in response to what were seen as political and academic failings of positivist influenced work moved away from a scientific perspective. Rejecting the supposed objectivity of both positivism and structuralism, this work focused on the feeling, emotional, living individual, with all their/our diverse and often inconsistent traits and qualities (Ley and Samuels 1978). Analysis by group-whether defined by such established and taken-for-granted ways as "national populations" or by more structurally defined groups such as Marxian classeswas displaced by a sense that such categorization dehumanized the subject of study: living people. The space of the individual was the key, often irrespective of its relationship with more objective spatial reality, a concept itself rendered increasingly problematic. Work developed in this humanist (Box 1.1) tradition, utilizing in-depth, interpersonal and empathetic techniques to present the qualitative side of people and their experiences-values, feelings, emotions, understandings.

The influence of such radical Human Geography currents on Population Geography was not as immediate as in some sub-disciplines. For example, in Urban Geography, Harvey's (1973) Social Justice in the City, rooted in Marx, and Edward Relph's (1976) Place and Placelessness, rooted in humanism, made near immediate intellectual impacts. The former traced Harvey's path from feeling that ultimately attaining social justice in the city was centrally about achieving "efficiency," facilitated by the kinds of positivist methods he had outlined a few years earlier in Explanation in Geography (Harvey 1969), to a realization that urban injustice was not a flaw in the system but an in-built characteristic. It required more than simply developing better models if it were to be eliminated (Peet 1998: 75-7). For example, Harvey (1973: 137) argued that whilst there was plenty of theory describing the structure and evolution of inner-city ghettos, if "[o]ur objective is to eliminate ghettos ... the only valid policy with respect to this objective is to eliminate the conditions which give rise to the truth of the theory." Relph (1976: i), in contrast, sought "to elucidate the diversity and intensity of our experiences of place," proceeding to argue for the importance of sense of place to human identity and everyday life but also how this was being increasingly undermined by the placeless character of modern design (and the urban processes outlined by Harvey). For Relph, an "authentic" place had an inevitably personal or subjective element that should neither be ignored nor conceptually eliminated, either through the rational models of positivism or a reduction to Marxian class issues.

There was some early response within Population Geography to these radical critiques and the scholarship they advocated. For example, attempts were made to bring individuals into the picture from the late 1960s through behavioral approaches. These sought to model people as having a range of possible priorities underpinning their decision-making behavior (Cox and Golledge 1969, 1981) relating to both societal context and individual traits. Behavioral approaches were most influential on studies of migration (5.3.3), which was typically presented as a sequential series of decisions that when cognitively resolved in the person's head ultimately brought about relocation (e.g. Clark 1986). However, behavioralism was criticized considerably for not breaking conclusively with positivism and even came to be regarded by White and Jackson (1995) as one of the causes of Population Geography's alleged academic backwater status by the 1990s.

More structural perspectives, in contrast, eventually went on to have considerable influence on Population Geography. These typically Marxian interpretations reinforced the linkages between Population Geography and the economy that both behaviorism and humanistic perspectives queried. From such a perspective, the demographic triumvirate of births, deaths and migration had to be contextualized firmly within the priorities and expressions of capitalist society. Thus, migration was seen from this point of view largely as responding to the dynamic labor demands of an ever-changing capitalist economy (Boyle et al. 1998: 68-70), at both intra- and inter-national scales (Chapter 7). Additionally, from a Marxian perspective, governments' interest in population was seen in critical terms, the state itself seen as a crucial component of the overall capitalist infrastructure rather than some kind of independent and neutral societal referee. Consequently, critical attention was given to government policies concerned with fertility and migration, in particular, and how these ultimately benefitted the capitalist classes.

Humanistic perspectives developed somewhat more slowly within Population Geography. Although they feature within the "lived worlds and cultural systems" research pole of Table 1.2, prior to the 1980s, even in its second edition, a textbook such as Jones (1990) largely overlooked this approach. However, there were notable pioneering engagements, such as Murray Chapman's village studies of population circulation in Melanesia from the early 1960s (Bedford 1999). This was facilitated through Chapman keeping a highly detailed "mobility register" of all movements

\section{ST PROOFS: NOT FOR DISTRIBUTION}




\section{VIEWING POPULATIONS SPATIALLY}

into and out of the village of Duidui in Guadalcanal for 24 hours or more, for example (Chapman 1987).

Unlike positivism's emphasis on the formal equality of the individual person or Marxism's placing of individuals into unequally resourced groups (classes), a key feature pioneered by humanistic Population Geography was explicit acknowledgement of differences between people on a more individual basis. Emphasis on diversity was boosted significantly from the 1980s as a further school of thought, postmodernism (Box 1.1), became increasingly influential. Postmodernism questioned all of the predominant theoretical perspectives previously noted-empiricism, positivism, Marxism, structuralism, even humanism-through expressing "scepticism towards the grand claims and grand theory of the modern era, and their privileged vantage point, stressing in its place an openness to a range of voices" (Ley 1994: 466). For Geography, it implied production of multiple spaces-and of diverse population geographies rather than any singular Population Geography.

From postmodernism, the challenge became investigation and expression of these often highly diverse geographies, indicating "a shift towards more differentiated understandings of population processes" (Graham 2000: 262), such as fertility behaviors (Sporton 1999). Foregrounding complexity, the research material used to investigate a specific topic was often obtained through utilizing a range of eften-contrasting methods: multiple methods research (McKendrick 1999). Table 1.3 contrasts a postmodern approach to migration research with approaches informed by positivism and humanism. Note the importance given to positionality, a concept that stresses how a person's relative location within a specific social expression (age, class, ethnicity, gender) influences their understanding of the world. Postmodern research requires strong sensitivity to such positionality, both as it relates to research subjects and the researcher.

Given all of these diverse strands, by the 1990s it is perhaps unsurprising that Bailey (2005) characterized Population Geography as becoming increasingly fragmented into a series of specialist topics and niches. Cutting edge postmodernists, in-depth humanists, revolutionary Marxists, and plenty of scholars retaining faith in the scientific promises of positivism, could make very uncomfortable bedfellows. All were Population Geographers but they barely communicated in the same language or concerned themselves with the same priorities, even if studying the same topic (Table 1.3). Similar arguments can been made for Human Geography as a whole (Johnston and Sidaway 2004).

Within this fragmented Population Geography sub-discipline, the balance also changed in terms of which elements of population were most studied. In short, migration became increasingly prominent, even predominant (Boyle 2002, 2003, 2004). This reflected, in part, the retreat, on the one hand, from "Domesday-like concerns over population growth" (Bailey 2005: 107; 11.3.1) and the growth, on the other hand, of state and popular concerns with international migration flows (Chapter 9). Diverse but entangled flows of refugees, asylum seekers and economic migrants heralded an "age of migration" (Castles et al. 2014).

Bailey (2005) again provides something of a summary of the immense variety of work undertaken within Population Geography in the 1980s and 1990s. However unlike his map of the 1950s-1970s, this time he emphasized (Table 1.4) different strands of Population Geography in terms of whether they:

- $\quad$ Reflected continuity with previous work (commonplace);

- Brought new perspectives to bear on established traditions, such as adding a humanistic dimension to topics such as migration that had until then been largely addressed quantitatively:

- Took a more explicitly critical perspective on scholarship to date, such as feminist influenced work that placed migration's often highly gendered character center-stage.

Table 1.4 reinforces how diversity became a core feature of Population Geography during this period. Although Table 1.2 was not fully comprehensive in its coverage of the earlier period, note the greater number of topics cited in Table 1.4, not least the rise of diverse migration related strands. Also highly significant is how Bailey was unable (or unwilling) to confine some topics, such as gendered aspects of migration, to the demographic triumvirate of births, deaths and migrations. Such evidence signifies the beginning of a sense of Population Geography that goes beyondbut still includes-this triumvirate. In summary, by 2000, Population Geography was increasingly diverse and hard to pin down and delineate as a distinctive sub-discipline. In postmodern spirit, it broke out from former sub-disciplinary fetters.

Leaving Population Geography at this point might suggest another period in which the sub-discipline thrived. Indeed, although hinting at some of the concerns raised shortly, whilst Jones (1990: 6) felt Population Geography

\section{ST PROOFS: NOT FOR DISTRIBUTION}


VIEWING POPULATIONS SPATIALLY 11

Table 1.3 Migration research undertaken within different research traditions

\begin{tabular}{|c|c|c|c|c|c|c|}
\hline \multirow{2}{*}{$\begin{array}{l}\text { Research } \\
\text { tradition }\end{array}$} & \multirow[t]{2}{*}{ Objectives } & \multirow{2}{*}{$\begin{array}{l}\text { Migration } \\
\text { example }\end{array}$} & \multicolumn{4}{|c|}{ METHODS AND APPLICATION } \\
\hline & & & $\begin{array}{l}\text { Modeling/ } \\
\text { survey }\end{array}$ & Interview & Fieldwork & Secondary \\
\hline Positivist & $\begin{array}{l}\text { Establish } \\
\text { empirical } \\
\text { regularities } \\
\text { assumed to be } \\
\text { of general } \\
\text { significance }\end{array}$ & $\begin{array}{l}\text { Patterns, } \\
\text { reasons for and } \\
\text { outcomes of } \\
\text { counter- } \\
\text { urbanization }\end{array}$ & $\begin{array}{l}\text { Mathematical } \\
\text { modeling of } \\
\text { census data to } \\
\text { provide national } \\
\text { overview }\end{array}$ & $\begin{array}{l}\text { Analysis of } \\
\text { structured } \\
\text { interview } \\
\text { focusing on } \\
\text { reasons for } \\
\text { migrating }\end{array}$ & $\begin{array}{l}\text { Survey of urban } \\
\text { fringe to identify } \\
\text { suitable sites for } \\
\text { "migrant" } \\
\text { residential } \\
\text { developments }\end{array}$ & $\begin{array}{l}\text { Comparative } \\
\text { systematic } \\
\text { analysis of } \\
\text { migrant and } \\
\text { non-migrant } \\
\text { children's } \\
\text { writings about } \\
\text { their homes }\end{array}$ \\
\hline Humanist & $\begin{array}{l}\text { Valorize human } \\
\text { experience and } \\
\text { seek to } \\
\text { understand the } \\
\text { meaning, value } \\
\text { and human } \\
\text { significance of } \\
\text { events }\end{array}$ & $\begin{array}{l}\text { "Return migration" } \\
\text { of American } \\
\text { Jewish families } \\
\text { to Israel }\end{array}$ & $\begin{array}{l}\text { Detailed social } \\
\text { attitudes } \\
\text { questionnaire to } \\
\text { establish and } \\
\text { understand the } \\
\text { personal context } \\
\text { of this migration }\end{array}$ & $\begin{array}{l}\text { In-depth interview } \\
\text { to explore the } \\
\text { meaning of the } \\
\text { migration } \\
\text { experience to } \\
\text { each individual } \\
\text { migrant }\end{array}$ & $\begin{array}{l}\text { Ethnographic, } \\
\text { participant } \\
\text { observation to } \\
\text { share the } \\
\text { emotions, } \\
\text { experiences and } \\
\text { significance of } \\
\text { this particular } \\
\text { migration }\end{array}$ & $\begin{array}{l}\text { Analysis of } \\
\text { accounts of } \\
\text { return migration } \\
\text { to situate their } \\
\text { personal } \\
\text { significance } \\
\text { within the wider } \\
\text { socio-political } \\
\text { context }\end{array}$ \\
\hline $\begin{array}{l}\text { Post- } \\
\text { modernist }\end{array}$ & $\begin{array}{l}\text { Establish that the } \\
\text { multiple } \\
\text { positioning of the } \\
\text { author (or reader) } \\
\text { influences the } \\
\text { production (or } \\
\text { interpretation) of } \\
\text { the story told }\end{array}$ & $\begin{array}{l}\text { Migration of "elite" } \\
\text { women from the } \\
\text { Global South }\end{array}$ & $\begin{array}{l}\text { Log-linear } \\
\text { modeling of } \\
\text { migration data to } \\
\text { estimate the } \\
\text { significance of } \\
\text { different } \\
\text { "positions" on the } \\
\text { propensity to } \\
\text { migrate }\end{array}$ & $\begin{array}{l}\text { In-depth interviews } \\
\text { to "unpack" } \\
\text { women's } \\
\text { rationalizations } \\
\text { of their } \\
\text { migrations }\end{array}$ & $\begin{array}{l}\text { Unobtrusive } \\
\text { access to } \\
\text { migrants' } \\
\text { experiences via } \\
\text { a service function } \\
\text { (e.g. labor market } \\
\text { consultant, } \\
\text { removal } \\
\text { contractor) }\end{array}$ & $\begin{array}{l}\text { Unpack } \\
\text { autobiographies, } \\
\text { personal journals } \\
\text { and letters of } \\
\text { migrants that } \\
\text { discuss their } \\
\text { migration } \\
\text { experiences }\end{array}$ \\
\hline
\end{tabular}

Source: adapted and simplified from McKendrick (1999: 45), Table 1.

Table 1.4 Population Geography in the 1980s-1990s

\begin{tabular}{lll}
$\begin{array}{l}\text { Position relative to "established" Population } \\
\text { Continuity }\end{array}$ & $\begin{array}{l}\text { Geography } \\
\text { Pluralist }\end{array}$ & Critical \\
\hline Migration differentials & Skilled international migration & Geopolitics of mobility \\
Migration systems & Gendered migration & Migrancy and sedentarism \\
Regional migration & Family migration & Gendering, racialization and sexualization \\
Random utility theory & Life course & of migration \\
Second demographic transition & Social capital & Politics of reproduction \\
Health inequalities & Environmental justice & Politics of aging \\
Replacement migration & Social context of age & \\
Remittances & Immigrant labor markets & \\
Population and environment & & \\
\hline
\end{tabular}

Source: simplified from Bailey (2005: Table 4.1). 


\section{VIEWING POPULATIONS SPATIALLY}

needed to "shed ... its traditional unambitious concern for pattern distribution and inferential interpretation" and engage with a broader range of perspectives, he also noted more positively:

- $\quad$ Sustaining the trend from describing patterns to examining causal processes;

- $\quad$ Emergence of more sophisticated analyses, often appropriated from Demography (e.g. Woods 1979, 1982);

- The value to Population Geography of developments in Geographical Information Systems (GIS) and emergence of complex datasets-a "data feast" (Champion 1992: 224)-especially at smaller geographic scales.

The influence of the popularization of versatile, powerful and affordable personal computers, associated software and comprehensive desktop mapping and analysis packages also cannot be understated. Nevertheless, by the early 1990s, some Population Geographers begged to differ from this buoyant impression and perceived the state of the subdiscipline very differently.

\subsubsection{Population Geography by the early twenty-first century}

Whilst Jones (1990) was sanguine about the clear links Population Geography had retained with Demography, others were more circumspect. In a sustained, no-holds-barred critique, Scottish Population Geographers Allan Findlay and Elspeth Graham (1991) argued that the sub-discipline was in crisis. It had stagnated after it had ceased to follow the evolution of Human Geography that had characterized its development in the 1960s and 1970s. In particular, Population Geography had been reluctant to shed a still prominent positivist skin. Consequently, through the 1980s "population geography entered ... a period of separate development" (Findlay and Graham 1991: 153), strengthening links with Demography but marginalizing and isolating itself from a Human Geography that had plotted very different courses (Peet 1998).

As subsequent related interventions made clear, Findlay and Graham were not alone in their concerns. However, the problem with Population Geography by the 1990s was less its links with Demography-which, it must be noted, had not stagnated conceptually or theoretically (Ogden 2000; Greenhalgh 1996)-than failure to embrace or even to engage substantively with many of the debates enlivening Human Geography over this period. Bailey's (2005) "continuity" category (Table 1.4) was both too big and too complacent. Thus, within migration scholarship, Halfacree and Boyle (1993) advocated more engagement with social theory in order to progress ideas, understanding and explanations. More broadly, in the first volume of the International Journal of Population Geography (re-named Population, Space and Place in 20044), White and Jackson (1995) reiterated Population Geography's separatism from other Human Geography sub-disciplines and its relative neglect of social theory. Empirical, quantitative, statistical analyses of abundant data-Champion's (1992) data feast-had to date kept most of those calling themselves population geographers happy, but was this (still) enough? Specifically, White and Jackson (1995) considered the neglect of social theory stemming from several factors. These are listed in Table 1.5 and some returned to below. They called for a re-theorized Population Geography, some dimensions of which Table 1.5 also sketches.

Five years after White and Jackson's intervention, Graham (2000) was still asking the question "What kind of theory for what kind of population geography?" She emphasized the postmodernist importance of drawing out difference and diversity. The following year, she again intervened, with Boyle, after a conference that sought to promote re-theorizations of Population Geography, to argue that the sub-discipline was still marginal(ized) within Human Geography. Reflecting on papers published to date within the International Journal of Population Geography, Graham and Boyle (2001) characterized the Population Geography mainstream as: methodologically conservative, neglecting theory, and dominated by migration studies.

What, then, of Population Geography in the first two decades of the new millennium in the wake of these critiques? To give some flavor of this, one can draw on reviews of the sub-discipline published in the leading journal Progress in Human Geography since 2000. Table 1.6 lists these articles and gives the focus of each review. Looking in detail, they show, first, just how dominant migration has become within the sub-discipline (Graham 2004). Indeed, two reviews by Boyle $(2003,2004)$ were written explicitly to counter any narrowing of Population Geography to the study of migration and to emphasize the sustained and even increased importance of acknowledging geographies of fertility and mortality. The latter was taken up more fully by Tyner $(2013,2015 a, 2015 b)$ in all three of his reviews, where, from a strongly Marxian position, examination and explanation of the fate of the world's most vulnerable populations 
Table 1.5 Perceived neglect of social theory and its potential for Population Geography c.1995

Causes of the neglect of social theory in Population Geography:

- Over-emphasis on "events"

- Preoccupation with data

- Acceptance of data constraints, such as established categories

- Attachment to essentialist ideas, such as neglecting to consider how categories are constructed

- Following theoretical cul-de-sacs, such as behavioralism

Considerations for a re-theorized Population Geography:

- Researchers' positionality: how who we are impinges upon our research

- Challenging established and supposedly "objective" categories

- Use of more intensive, ethnographic methods

- Acknowledging wider political, economic and social context

- Seeing population "events" as placed within the biographical context of people's lives

Source: White and Jackson (1995).

Table 1.6 Reviews of Population Geography published in Progress in Human Geography, 2000-2015

\begin{tabular}{ll}
\hline Author & Focus of review \\
\hline Ogden (2000) & Weaving demography into society, economy and culture \\
Boyle (2002) & Transnational women on the move \\
Boyle (2003) & Does geography matter in fertility research? \\
Boyle (2004) & Migration and inequalities in mortality and morbidity \\
Hugo (2006) & Population geography from the Southern Hemisphere \\
Hugo (2007) & Population geography from the Southern Hemisphere \\
Bailey (2009) & Lifecourse matters \\
Bailey (2010) & Gender and the migration-development nexus \\
Bailey (2011) & Population geographies and climate change \\
Tyner (2013) & Surplus populations \\
Tyner (2015a) & Mortality, premature death, and the ordering of life \\
Tyner (2015b) & Precarity, dead peasants, and truncated life \\
\hline
\end{tabular}

Note: reviews not published every year.

was advocated strongly. Second, all the reviews are in tune with the 1990s concern over the future of the sub-discipline, expressed from Ogden (2000) to Tyner (2013) as a need to engage more fully with other branches of Geography and allied disciplines, where relevant scholarship on population issues is widely noted. Third, where such engagement has occurred, the reviews note the results in the presence of major bodies of often interlinked work. These include: transnational migration, where people "actively maintain simultaneous, multi-stranded social relations linking their place of origin and destination" (Boyle 2002: 533); gender dimensions of migration, such as how gender-sensitive analysis expands understanding of the "migration-development nexus" (Bailey 2010); the importance of local cultures and traditions in shaping population characteristics, embracing Hugo's $(2006,2007)$ concern of the relative lack of Population Geography influence on censuses and their continued use of outdated urban-rural classifications (Champion and Hugo 2004) within Asia; foregrounding flows (of people and objects), instability and change over stasis, fixity and stability, as people strive to live mobile lives through and across space; and how a change in terminology from premature death to truncated life both directs attention to the scale of the body and helps confront the inequalities of life that exist (Tyner 2015b: 11, 4). All are themes picked up explicitly in the present book.

So, overall, what is the state of Population Geography today? What seems clear is that there remains a very lively range of scholarship being conducted within the boundaries of a fairly clearly defined sub-discipline, both broad and deep. This was something even the critiques noted above consistently recognized. Substantial activity is reflected by such groups as the Population Specialty Group of the Association of American Geographers, the Population Geography Research Group (PGRG) of the UK's Royal Geographical Society with the Institute of British Geographers, 


\section{VIEWING POPULATIONS SPATIALLY}

and the Population Geography Commission of the International Geographical Union. However, it is perhaps the boundaries implied by and to some extent also inscribed and reproduced by these same groups that paradoxically represent a problem as well as a strength.

Appreciation of the crucial role of boundaries requires introducing one final theoretical perspective, or -ism, influential within Human Geography since the 1980s but having had less impact within Population Geography. This is post-structuralism (Box 1.1). One issue this approach draws explicit attention to is construction of categories and boundaries, since language is seen as constituting, reproducing and sometimes transforming everyday realities rather than simply reflecting these realities. Within Population Geography, a post-structuralist perspective has been used, for example, to interrogate what is meant by counterurbanization (8.3.2) and specifically how dominant interpretations of this phenomenon can be unduly limiting (Halfacree 2001, 2008, 2012a).

Informed by a post-structuralist sensibility, it appears that whilst the memberships of all of the defined Population Geography groups undertake diverse, interesting and original scholarship within all the areas (and more) suggested in Table 1.4, much informed strongly by social theory, these groups' intellectual boundaries must not be too rigorously enforced. Specifically, there is increasingly geographical scholarship than can at the very least be seen as closely affiliated to Population Geography but, for whatever reason, often fails to fall under any of its sub-disciplinary shadow (see also note 3 above) (e.g. Silvey 2004 on the place of "critical migration studies"). Once again, the result is that Population Geography can come across as unduly intellectually constricted, conservative and constrained. There seems, in conclusion, to be a prima facie case to support calls for Population Geography to at least (re)consider its twentyfirst-century identity and the scope of its sub-disciplinary competence and interest. This is part of what the present book aims to do. Such an aim will be developed more fully in the next chapter but requires, first, an expanded representation of Population Geography as explicitly relational.

\subsection{TOWARDS RELATIONAL POPULATION GEOGRAPHIES}

\subsubsection{An expanded notion of population and population geography}

The last section began with discussion of how a particular concept of population emerged from the eighteenth century and was strongly rooted in the liberal idea of formally equal but atomized and discrete individuals within any overarching idea of society. Such a perspective was then shown to permeate many of the rich seams of Population Geography that emerged from the 1950s, since it fitted very well with the philosophical underpinnings of empiricism and positivism that proved so influential and profitable for the sub-discipline in this period. However, there were also problems in adopting such an abstracted perspective on the individual person. These came through more generally via the critical strands within Human Geography that emphasized in particular either the socio-economic structure that individuals' lives are always entangled with, or the personality, subjectivity and individuality-the lives themselvesof the human "atoms." Population Geography was seen to embrace these strands to varying degrees, whilst still holding on to positivism and empiricism more firmly than did Human Geography overall.

Putting the story somewhat differently, Population Geography has seemingly been very reluctant to discard the eighteenth-century concept of population (1.3.1). From this perspective, debates on the present condition and hoped for future of the sub-discipline that developed through the 1990s can be seen as reflecting not just fears about resistance to social theory or of over-dependence on Demography. Instead, they also express anxiety about Population Geography letting go of a fixed, essential, abstract characterization of population. However, with both postmodern and post-structural strands challenging population with their iconoclastic critique of fixed categories, positions and lasting truths, the writing seems increasingly on the wall for the concept.

The alternative advocated here is not a return to the pre-eighteenth-century concept of populousness. This presents an equally essentialist, conservative and fixed sense of the person-this time confined to stratified groups or classes. Instead, the present authors suggest a notion of population that transcends both the fixed individual (population) and the fixed group (populousness). It foregrounds more fluid roles for both socio-economic and bioenvironmental context and individual personal character and agency in shaping a life. Population needs to center both postmodernism's emphasis on diversity and post-structuralism's critical sensitivity to the importance of categorization. A postmodern influence supports continued research on established Population Geography topics but studied more

\section{ST PROOFS: NOT FOR DISTRIBUTION}


closely and with sharper sensitivity to the complexities of Sameness and Otherness ${ }^{5}$ contained therein (Philo 1992), whilst post-structuralism destabilizes the stability or fixity of any category and thus promotes recognizing people through their lives as being frequently in motion between categories. Together, these twin emphases call for a new imagination that encompasses both recognizing people existing together within categories of the Same but also seeing people simultaneously distributed into an indeterminate variety of Others. Both geography and history are implicated strongly within this dynamic condition. For example, it varies across the globe but also appears to be increasing and accelerating within an "era of mobilities" (5.2). Population Geography thus breaks away from a focus on the fixed, Same, point of population (or populousness) (Curtis 2002) to encompass the fluid, Other, surface of populations.

The call, in short, is for a thoroughly relational Population Geography. This presents who and what people are in relation, both to one another-understood to encompass a range of dimensions of positionality and scales-and to the spaces with which they are entangled and the times they live through. These spaces and times are also seen relationally as never fixed but always being produced and reproduced, always being (re)written. People are always individuals and people are always grouped but these conditions are far from static or stable. People-you and I-and what we do, and how we do it, and why we do it, must always be understood in context, and "context" is itself never wholly static.

The present book advocates this broader sense of Population Geography, informed strongly by social theory, diverging still further from spatial Demography to express the historically grounded lives of populations as experienced across and through "real" spaces and places. However, whilst following this call for an "enlarged" (Bailey 2005: 164) sub-discipline, "a broader definition of [our] field" (Findlay and Graham 1991: 161), it must also be appreciated that there is a danger such an eclectic and referential Population Geography could become largely indistinguishable from Human Geography overall. It also requires some kind of anchoring or integrating concept to sustain a relatively distinctive focus. This, Chapter 2 will argue, can be achieved through following people through their lives.

\subsubsection{Trewartha revisited}

Before concluding, it is worth returning briefly to the advocacy of Population Geography made by Glen Trewartha in 1953. As argued in Section 1.3.3, although his speech provided strong impetus to the growth of the sub-discipline, the actual form Population Geography took differed quite markedly under the influence of the quantitative revolution to that he promoted.

Illustrated in Figure 1.2, Trewartha positioned Population as at least equal (actually, dominant; Trewartha 1953: 96) to Cultural Earth and Physical Earth in his organization of Geography. He also advocated an integrated approach

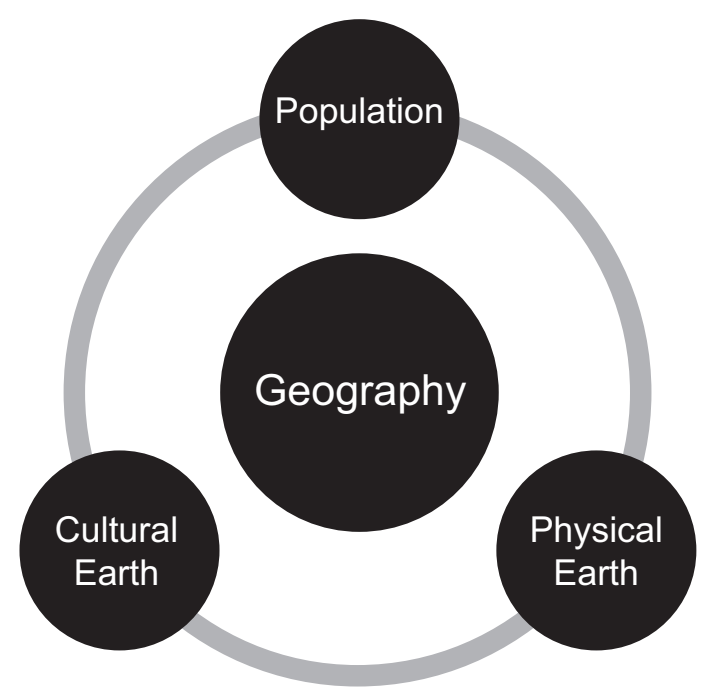

Figure 1.2 Trewartha's central place for Population within Geography.

Source: developed from Trewartha 1953: 81. 


\section{VIEWING POPULATIONS SPATIALLY}

to the study of Geography overall. Both issues merit reconsideration after the call for a more relational Population Geography. First, although wary of trying to separate population, culture and the physical environment, even diagrammatically, ${ }^{6}$ one may cautiously support Trewatha's recognition of according population issues a primary significance (Silvey 2004). This conclusion should be clearer after the association of Population Geography with the life course developed in Chapter 2. Second, although again throwing up often considerable methodological and philosophical challenges, a more relational sense of Population Geography also chimes with Trewartha's advocacy of an integrated approach to (human) Geography, including a need to move beyond treating people "in terms of numbers almost exclusively" (Trewartha 1953: 96). This extends to noting the continued importance of people's relationships with the physical environment.

Properly and fully recognizing the fundamental relationality of Population Geography necessitates recognizing the vast range of issues potentially implicated in the ultimate "production" of any population phenomenon. Births, deaths and migrations can be seen, at least initially, as relatively straightforward demographic facts but beyond this initial impression, and especially when causality is considered, cultural and physical dimensions soon become engaged (e.g. Halfacree 2004a). Together, then, Geography is, as Trewartha suggested, always a combination of population, culture and the physical sphere and within this, therefore, population indeed "move[s] from being a demographically defined object that exist[s] in space to a socially constituted process that help[s] to make space" (Bailey 2005: 192). Trewartha is thus vindicated.

\subsection{CONCLUSION: LIVES ACROSS SPACE}

An expanded field of Population Geography and a critical return to Trewartha encompasses a wider range of perspectives and issues than the sub-discipline has conventionally been seen to cover these past 60 years. Bailey (2005) once again gave a good sense of this breadth through a table (Table 1.7) that burrows both downwards, from the (highlighted) demographic core of fertility, mortality and migration to other linked life course issues (Chapter 2), and laterally, to indicate the huge range of places, practices and experiences, as well as the languages or discourses that accompany them. There is not space to explain all of these further here but many will come to inform the remaining content of this book.

We are born, we live, and then we die. The chapter thus ends as it began but hopefully the reader now has a better appreciation of how and why that simple summation of a life has provided the stimulus for the ever-changing,

Table 1.7 The expanding scope of Population Geography

\begin{tabular}{lll}
\hline Demographic events and concepts & $\begin{array}{l}\text { Indicative population acts, } \\
\text { performances and institutions }\end{array}$ & Discourses \\
\hline Migration & Residence, home & Migrancy \\
& Sedentarism \\
Fertility & Pregnancy, parenting & Nomadism \\
Mortality and morbidity & Disease & Nationalism and transnationalism \\
& & Ageism \\
Age grades & Childhood, youth, adolescence, & Ableism \\
& adulthood, old age, retired & Familism \\
& & Healthy bodied \\
Marriage and divorce & Dating, partnering, widowhood & Sexualization \\
& & Individualism \\
Positionality & Gender, class, sexuality, race, ethnicity, & Secularization \\
& nationality, religion, family & Racialization \\
\end{tabular}

Source: modified from Bailey (2005: Table 4.2). 
often contested but always relevant and important sub-discipline that is Population Geography. It may remain in a fundamental sense the geographical organization of population and how and why this matters to society (Bailey 2005: 1) but the proposed re-specifying of the sub-discipline through the influences of, in particular, postmodernism and post-structuralism, and through the relational framing device of everyday lives emphasizes that these stories of lives across space to be told in the rest of this book are ones well worth reading about, contemplating, and even debating in greater depth. These Population Geography stories are the stuff of all of our lives.

\section{NOTES}

1 Depiction inspired by the 1986 Smiths' song Cemetry (sic.) Gates, from The Queen is Dead (WEA 4509-91896-2).

2 This notation refers the reader to Section 11.2.3 within Chapter 11 and its style is adopted throughout the book.

3 Principal components analysis is a statistical technique for analyzing and synthesizing multiple variables. It is used by Smith to epitomize the predominant academic style of the time.

4 This change of name was itself informative of a growing sense that much Population Geography was being undertaken outside of the sub-discipline. Population, Space and Place's inaugural editorial spoke of how one reason for a change of name that removed Population Geography' from the title was "to attract articles from those working in any discipline who are interested in population issues and how they vary across space and place" (Boyle et al. 2004: 1), aiming especially to include more qualitative work.

5 Otherness, usually capitalized, represents "the quality of difference which [an] Other possesses" (Cloke et al. 2005: 608) and is the opposite of Sameness or the quality of similarity which an Other possesses. Postmodernism emphasizes appreciating both Others and Otherness in their own terms, whilst acknowledging their relational existence.

6 For example, the cultural turn in Human Geography has increased awareness of how culture infuses all of life, including senses of the physical environment (Crang 2000). For a Trewarthian take on the population-environment nexus, see Conway (2004). 


\section{CHAPTER 2}

\section{Population Geographies of the life course}

\section{$2.1 \quad$ INTRODUCTION}

Chapter 1's review of the history of Population Geography advocated scholarship continuing the trajectory that has focused increasingly on emplacing the key demographic expressions of births, deaths and migration within the diverse currents of everyday life. Demographic practices must be placed within broader contextual understandings and explanations of lives across space. This call for a more fully relational Population Geography, besides providing rounded understanding and explanation, may also help to re-unite Population Geography into a more coherent sub-discipline, reinforcing its position within Human Geography as a whole.

To begin to achieve these aims, this chapter begins to develop a life course framing for Population Geography. First, Section 2.2 introduces a life course perspective, extended from conventional life course concerns to stress how individual lives are continuously and inextricably entangled with those of others. This is followed by Section 2.3, introducing nine arenas through which life courses are collectively shaped within everyday life. Such arenas comprise the relational contexts whose specific demographic significance will be interrogated in subsequent chapters.

\subsection{LIVES INDIVIDUAL AND COMMON}

\subsubsection{Life course: from life-cycle to life transitions}

Life course denotes the longevity of a specific human being-from birth to death-as marked by the "sequence of socially defined events and roles that the individual enacts over time" (Gielle and Elder 1998: 22). Resolutely relational, life courses collectively express "age-graded patterns ... embedded in social institutions and history ... [since individual] lives are influenced by an ever-changing historical and biographical context" (Elder et al. 2003: 4, 7). A life course is thus first of all individual, expressing an individual's life experiences. However, it is also fundamentally social, in that these individual lives always exist in context, as inherently relational. It is, thirdly, also centrally concerned with change, as individuals live their life. As Hockey and James (2003: 5) express it, the life course is "a way of envisaging the passage of a lifetime less as a mechanical turning of a wheel and more as the unpredictable flow of a river." All rivers flow downhill, from source to sea, from birth to death, but the routes taken are infinitely variable.

The focus on change through life not resembling "the mechanical turning of a wheel" emphasizes that individuals do not necessarily move through a highly and clearly defined and delineated series of changes and life stages from birth to death. Yet, such an assumption underpins the influential life-cycle concept, pioneered by Glick's (1947) use of United States Census data to define seven stages in the development of the "average" American family (Table 2.1). The implication from such a model was not only how subsequent cohorts-individuals born within the same time period-would experience these same cycles and stages but that these stages directly fed into equally distinctive sets of consumption practices and demographic behaviors.

Whilst the life-cycle model has been considerably refined since Glick's outline, it has increasingly been seen as problematic (Boyle et al. 1998). First, there is its inadequately developed sense of time. The model, classically expressed, presents a very singular, linear idea of time from birth until death; with life passing through a series of sequential stages. Whilst time from birth to death is linear if measured in years, minutes and seconds, it is not only 
Table 2.1 The "classic" family life-cycle

\begin{tabular}{lll}
\hline Phase & Begins & Ends \\
\hline I. Formation & Marriage & Birth of first child \\
II. Extension & Birth of first child & Birth of last child \\
III. Completed extension & Birth of last child & First child leaves home \\
IV. Contraction & First child leaves home & Last child leaves home \\
V. Completed contraction & Last child leaves home & First spouse dies \\
Vl. Dissolution & First spouse dies & Death of survivor \\
\hline
\end{tabular}

Source: Hohn (1987), after Glick (1947).

this. Instead, people live in or through multiple, overlapping times: linear time from birth to death, cyclical time of the seasons, linear experiential time of a specific career, cyclical time of a typical day, and so on (2.3.2.1). Such fuller sense of times not only emphasizes the complexity of any individual's life at any given moment but leads to a key realization that an individual may not pass through a sequence of stages anything like as neatly as life-cycle models suggest. A life course can jump between and even repeat stages (e.g. through inter-personal relationships changing or new families being formed with other partners) and the stages are themselves internally differentiated.

Second, the life-cycle model downplays the role of both socio-cultural context and individual or family agency. People come to be seen as "determined" by their stage in life, neglecting myriad relational decisions everyone constantly makes within life every day. Associated with this concern over an excessive and narrow determinism are concerns that the life-cycle model is at least implicitly normative (Hohn 1987). Thus, Uhlenberg (1974) pithily observed how to have followed the "preferred" life-cycle, a woman should (sic.) by the age of fifty have married, remained within this marriage, and borne at least one child. In addition, any normative life-cycle is also at best historically and geographically specific, notably perhaps being a fair composite of "family circumstances of white urban middle-class Americans in the 1950s and 1960s" (Murphy 1987: 36) but increasingly anachronistic within present-day complex family lives within both Global North and South. The supposed, if highly elusive, "golden age of the family" (Hall 1995), in which people married, did not divorce and reserved sex for marriage, is certainly now no more. Moreover, it is not just marriage and family demands-vital as they are-that shape Population Geographies of the life course, as Section 2.3 will emphasize.

Having made these critical observations, however, the reason the life-cycle concept has been discussed so much is because its sense of relatively distinctive stages occurring within a life course remains highly pertinent. Whilst lives may poorly map any simple model of the type shown in Table 2.1 , they still do usually express a reasonably discernible structure, characterized by periods of both change and relative stability. Totally randomly lived or disordered lives are both rare and frequently problematic for all concerned (G. Evans et al. 2005). It is with reference to this more fuzzily defined idea of structured lives that the life transitions concept has been developed, which is suited to the nondeterminacy of the life course approach.

Life transitions are rooted in the principle that "rather than following fixed and predictable life stages, we live dynamic and varied lifecourses which have, themselves, different situated meanings" (Hopkins and Pain 2007: 290). They occur "across the life course rather than at predefined, age-related points in life" (Hörschelmann 2011: 379). Lives, in short, follow, and embody (Teather 1999), diverse pathways, defined both by circumstances and by the individual and those around them. Within these pathways, some life transitions demonstrate "biographical ruptures and discontinuities" (Hörschelmann 2011:378)-becoming a wage-earner, getting married, becoming a parent. Useful here is the "fateful moment" (Giddens 1991: 142), when "[o]ne's protective cocoon of comfortable everyday experiences is challenged by being forced to choose one future path or another" (Worth 2011: 406). Fateful moments challenge one's ontological security, yet can also express "periods of reskilling and empowerment" (Giddens 1991: 142). Other life transitions, in contrast, are more transitionally experienced, as an individual becomes (Worth 2009, 2011), more-or-less conclusively, a child, an adolescent, an adult, a senior (Box 2.1). Taking both types together, Table 2.2 lists some key life transitions, many of which are explicitly demographic.

One may go farther than appreciating the complexities of all transitions to question the very idea of an either/or existence between, for example, childhood and adulthood (Evans 2008; Jeffrey 2010). Supposed children often

\section{ST PROOFS: NOT FOR DISTRIBUTION}


20 POPULATION GEOGRAPHIES OF THE LIFE COURSE

Table 2.2 Key life transitions (N.B. The reader is invited to fill in the blank cells and add more if necessary.)

\begin{tabular}{|c|c|c|c|}
\hline Birth & Cohabitation & Moving house & Moving away from home \\
\hline Bereavement & Divorce & & $\begin{array}{c}\text { Retirement from paid } \\
\text { employment }\end{array}$ \\
\hline Having a child & Becoming terminally ill & Leaving school & Leaving a job \\
\hline $\begin{array}{c}\text { Becoming independently } \\
\text { mobile }\end{array}$ & Starting new job & Marriage & Puberty and adolescence \\
\hline
\end{tabular}

express "adult" behavior (e.g. child street traders; Bromley and Mackie 2008, 2009), whilst adults can adopt quasichildlike behavior (e.g. terms such as "kidulthood" or "adultescence" suggest a blurring of "youth" and "adult" roles; Heath 2004). Even more clearly defined fateful moment transitions may also retain ambiguity. For example, when one becomes a parent one's behavior and attitudes may both reflect this change (e.g. behaving in a more responsible and caring manner) and at other times and/or in other places be much less parental (e.g. behaving irresponsibly through dangerous leisure activities).

In sum, life transitions are of core existential significance but are often not "clean processes." They are messily "emergent" (Del Casino 2009: 212) and present not always conclusive or irreversible becomings within the life course. Thus, becoming a parent is likely to be a very key event within a person's life course but, whilst a birth may trigger a transition to "motherhood" or "fatherhood" on one level, the details of what it is to exist within this new state are there to be worked out, often messily, as any parents will readily attest! For Population Geographers, therefore, demographic life transitions alone do not define lives. They are far from the end of the matter when it comes to constructing everyday Population Geographies from a life course perspective.

\subsubsection{An extended life course perspective}

It is a truism reaffirmed by the birth of every child: no two lives are the same and each one of us is unique among the billions of people who have ever lived and who will ever live... And yet it is also a truism that the human condition brings with it a set of seemingly universal experiences.

(Shanahan and Macmillan 2008: 3)

In terms of bringing greater life course sensitivity to Population Geography, an overall aim is to understand demographic transition behaviors and practices in the fullest possible context of the lives being led by the persons concerned. It is to be undertaken in the spirit of a biographical approach (Halfacree and Boyle 1993; Halfacree 2004a), which emphasizes three core issues. First, it acknowledges how all demographic transitions are entangled within an everyday life that is inevitably multi-faceted and messy. A person's demographic behavior, no matter how seemingly rationally calculated, has a history, geography and socio-biological dimensions (2.3). Second, and even though the currents feeding into a transition may be poorly recognized, any such change is likely to involve multiple reasons, not least when attempting to explain all of its dimensions. Third, an explicitly contextual and relational reading reinforces the idea that demographic transitions are always shaded in their expressions, performances, meanings and understandings by some degree of shared cultural colors (Fielding 1992a; Halfacree and Merriman 2015). Overall, a biographical perspective "bring[s] to the fore how individuals negotiate and understand social structures, expectations and shifts in relation to the circumstances of their own lives, needs and desires" (Hörschelmann 2011: 381).

Such multi-relational biographical sensitivity lies at the heart of life course scholarship (Mortimer and Shanahan 2003). Summarized by Shanahan and Macmillan (2008), this body of work emerged in the US from a series of studies 


\section{BOX 2.1 THE COMPLEX TRANSITION TO ADULTHOOD}

Illustrating how life transitions may not be as simple, immediate or one-directional as one might at first imagine, consider the transition to adulthood. Clearly, one could simply define and understand "young people" as aged between birth and an upper limit, after which they are deemed to be "adults." However, there is immediately potential disagreement with where this cut-off point is set. Instead, there are proxy measures that can be used to define "adulthood" by age. These include: age at which one can marry, buy and/or drink alcohol, vote in elections or drive a car.

Defining "adulthood" by age

\begin{tabular}{|c|c|c|c|c|}
\hline \multirow[t]{2}{*}{ Country } & \multicolumn{4}{|l|}{ Age one can: } \\
\hline & Marry (male/female) & Drink alcohol & Vote in elections & Drive a car independently \\
\hline Argentina & $18^{1}$ & 18 & 18 & 18 \\
\hline Australia & $18^{2}$ & 18 & 181 & $15-18$ \\
\hline Brazil & $18^{3}$ & 18 & 16 & 18 \\
\hline Canada & $18^{4}$ & $18 / 19$ & $18-19$ & $17^{12}$ \\
\hline China & $22 / 20$ & No limit & 18 & 18 \\
\hline Ethiopia & 18 & 18 & 18 & 18 \\
\hline France & 18 & 18 & 18 & 18 \\
\hline Iran & $18 / 16$ & Illegal & 18 & 18 \\
\hline Japan & $20^{5}$ & 20 & 20 & 18 \\
\hline Kenya & 16 & 18 & 18 & 18 \\
\hline New Zealand & $18^{6}$ & 18 & 18 & 16 \\
\hline Sweden & $18^{7}$ & 18 & 18 & 18 \\
\hline Tunisia & $20 / 17^{8}$ & 18 & 20 & 18 \\
\hline United Kingdom & $16-18^{9}$ & 18 & 18 & 17 \\
\hline United States & $15-19^{10}$ & 21 & 18 & $16-21$ \\
\hline Venezuela & $18^{11}$ & 18 & 18 & 18 \\
\hline
\end{tabular}

Notes: 1. Younger with judicial consent in exceptional cases. 2. 16 with permission from a court and both parents. 3. 17 or younger with parental and other consent. 4.16 with parental consent, 14 with judicial consent. 5. 18/16 with parental consent. 6. 16 with parental consent. 7. Younger with permission from county administrative board. 8. Younger with judicial special permission and with clear interest for both spouses. 9. Varies between component countries; 15-16 with parental consent, again varying geographically. 10. Varies by state but mostly 18; mostly 15-16 with parental consent but in Mississippi no minimum with parental and judicial consent. 11.16/14 with parental consent. 12. Complex provincial variations.

All vary considerably between each other, between (even within) countries, and by gender with respect to marriage age. The plethora of footnotes suggests the complexity of the issue. Nevertheless, 18 years is the clear modal value within the table and is widely accepted internationally as the age of majority or the legal threshold of adulthood. Cross-cutting this, however, is how the age range 16-25 years is frequently used to define "youth," sometimes seen as a transitional phase between childhood and adulthood.

Yet, making the transition to adulthood is generally seen as involving much more than simply attaining a particular age. Consequently, whilst an 18th or 21 st birthday party might be a key threshold for many lives-and signifies receipt of a whole host of legal rights and responsibilities in most countries-when a person wakes next day their lives are usually not that altered! Considerably more complexity is involved in the life transition to adulthood, involving issues of competence, psychology and agency, and is expressed in diverse pathways.

Attention can be given to more performative or qualitative considerations in making the transition to adulthood. This focuses attention on what a particular person is both able to do and actually does, how they act in life, and how they are expected to behave. For example, the predominant account from the Global North is of childhood "characterised by play, frivolity, freedom, innocence, dependency and a lack of responsibility, [whereas adulthood expresses] ... work, seriousness, independence and responsibility" (Evans 2008: 1663). How an individual expresses himself or herself in these terms, then, may define whether or not they are deemed "adult." Additionally, attaining adulthood is not solely an individual process but involves the young person in relations with immediate family, family circumstances, local community, wider society, and so on.

(Sources: Evans 2008; Shanahan 2000; Skelton 2002: Valentine 2003; Weller 2006; Young et al. 2008) 
22 POPULATION GEOGRAPHIES OF THE LIFE COURSE

by Sociologist Glen Elder in the 1970s, notably Children of the Great Depression (Elder 1974; also Elder 1985, 1994; Hareven 2000). Elder presented the Great Depression of the 1930s as a key event that all individuals in the United States experienced and which subsequently shaped their lives and attitudes and, ultimately, the long term life paths they followed. It was a cohort marking experience. However, whilst each person had common or shared experience of the Great Depression they also expressed a degree of uniqueness within individual experiences. The Depression did not impact on each life in precisely the same manner: individual experiences were both Same and Other (1.4.1).

Today, the Great Depression is largely historical memory. Instead, 9/11 (September 11th 2001) in the US or $7 / 7$ (July 7th 2005) in the UK might be proposed as equivalent discrete cohort-shaping events or, as with the Depression, more extended periods, such as the culturally transformative "1960s" (McWilliams 2000). Indeed, any (sub-)period of history, plus specific events within it, provide both group and personal significance to those who experience them. In all cases, each person experiencing these events or times is to a greater or lesser extent part of a collective experience shared with others and yet also has a personal response to it.

It was from such appreciation of the relational impact of major historical events and times as being both collective and unique that life course scholarship developed. It proposed that while each individual person lives a unique life filled with different experiences from travel, relationships, education, work, leisure and so on, these same individuals are not atomized units but express and are constituted in part through diverse and differentiated links with others in their cohort or generation through some degree of shared experience. Re-stated again, a person's life course is both driven by agency but also always sensitive to historical context.

Table 2.3 lists six basic principles that a life course approach prioritizes (also Elder et al. 2003: 10-14). All six should be seen as fundamentally intertwined. The first principle can be linked immediately to life transitions and expresses how an "age-graded sequence of roles, opportunities, constraints, and events ... shape the biography from birth to death" (Shanahan and Macmillan 2008: 40, emphasis removed). This emphasis on the map of the individual's life focuses on the timing of transitions within it. When in one's life one has a child or moves house, for example, matters. Second, and related to the first principle, there is the core theme of the biographical approach of how past biography is relevant to and works to modify behavior. Migration or child-bearing experience, for example, will be influenced by past migration and child-bearing experiences. Third, central to the life course is ability of individuals to "make a difference" to their lives; a humanistic emphasis (Chapter 1).

Nonetheless, a life course perspective does not over-state individuals' "freedom" to construct their demographic lives. Whilst "[i]ndividuals choose the paths they follow, ... choices are always constrained by the opportunities structured by social institutions and culture" (Elder et al. 2003: 8). Table 2.3's remaining three principles build on this sense of a life course constrained. Fourth, agency is recognized as being greater in practice-in the "real world"-in some circumstances than in others. Fateful moments, such as birth of a child or marriage, may strongly "encourage" migration, for example. Yet, recognizing such situational imperatives must also incorporate realization of what is required for any demographic outcome. Thus, actual ability to move depends greatly on having sufficient resources, which in turn is influenced by present or past employment, for example. Fifth, agency is also related to an individual's networked connections, links and ties, which can again either magnify or dampen it. The institution of the family is of vital significance. For example, becoming emotionally and materially settled within a family may encourage child-bearing but it may also discourage the upheaval of migration. More broadly, social capital, benefits one gets from social

Table 2.3 Basic principles of the life course perspective

\begin{tabular}{ll}
\hline $\begin{array}{l}\text { 1. Life stage } \\
\text { 2. Accentuation }\end{array}$ & $\begin{array}{l}\text { Meaning and consequences of events depend in part on when they occur in a life. } \\
\text { 3. Agency }\end{array}$ \\
$\begin{array}{ll}\text { 4. Situational imperatives } & \text { The more demanding a situation, the more behavior is constrained to meet role and other } \\
\text { expectations and requirements. }\end{array}$ \\
$\begin{array}{ll}\text { 5. Linked lives } & \text { Life course development depends on the person's network of interpersonal relationships, including } \\
\text { immediate and extended family members, mentors and close friends. }\end{array}$ \\
6. Time and space & A life course takes on different patterns in different historical times and geographic places.
\end{tabular}

Source: modified from Shanahan and Macmillan (2008: 55, 57-58). 
connections, is of direct relevance here (Putnam 2000). Finally, considerable attention is given within a life course perspective to the role of geography and history in shaping demographic behaviors and how they are expressed. Both where an individual lives and when s/he lives and molds a life course is critical, as the importance Elder attributed to cohort demonstrated.

From Table 2.3's six principles, the importance of age, agency, geography and history to shaping a life course is clear. However, from the "social institutions" (Elder et al. 2003) also central to this constitution, it is imperative to draw out a host of other arenas within which a population (1.3.1) is differentiated. The results work to intersect with, reinforce, counteract and entangle an individual's life choices (Crockett 2002; Hockey and James 2003; Hunt 2005). They express such factors as social class or status, age, gender, culture and beliefs, biomedical condition, and bodily abilities, and are developed shortly in Section 2.3. Thus, pursuing life course informed Population Geography must consider: historical period in which an individual lives (1500s, early 1900s, mid-2000s); relative position in society (wealthy, poor, in-between); present or previous job(s) (pay, authority, health and safety); cultural norms within the community (religious beliefs, gender roles); the political system in which the community is situated (authoritarian, liberal, socialist, clerical, neo-liberal); and biomedical conditions (healthy, fertile, "disabled," strong).

In conclusion, adopting an extended life course perspective within scholarship, with this added emphasis on arenas of population differentiation, requires ranging very widely "to describe the structures and sequences of events and transitions through an individual's life" (Bailey 2009: 407) as they relate to key demographic topics. Fundamentally, it involves recognizing lives as individual and common; transcending the population versus populousness divide (1.4.1). Notably, whilst invoking "biography" and the potential of individual agency, it is equally important to avoid granting the individual life course any "spurious sense of oneness" (Thrift 2007: 7; Hörschelmann 2011). Thus, whilst a life course perspective has been credited with revolutionizing Demography "by focusing attention away from the behaviors of aggregate populations to the consideration of the demographic behaviors of individuals" (Hogan and Goldscheider 2003: 690), these individuals do still come together collectively in different life course arenas, expressing group defined Population Geographies.

Finally, the present authors are not alone in suggesting the value of adopting a life course framing for Population Geography. Bailey (2009: 407) suggested that work brought together under the umbrella term "lifecourse geographies" provided "new knowledge on topics that include: mobility, work, housing, childhood, changing families and social networks, age, generation, disability, health and well-being inequalities, and vulnerability." Within this approach, biography provides a means of integrating (changing) lives, geography and history, since biographies "help relate trajectories (or 'careers', including residential location, mobility, work, incarceration) to transitions (such as the demographic triumvirate of birth, death, and migration events, and nest-leaving, partnering, separating, retirement) and the spaces and times they flow through" (Bailey 2009: 408). Relationality is paramount, with experiences through the life course constituting lives both individual and common, and demographic behavior expressing "a complex articulation between the accumulation of life experience and resources, contingency, and inequality and participation" (Bailey 2009: 412).

\subsection{ARENAS OF DIFFERENTIAL LIFE COURSE EXPERIENCE}

\subsubsection{Differentiating experiences across the life course}

Each phase of life, including its timing and defining features, is a social construct, as are the transitions among the phases, and perhaps even people's subjective understanding of their lives.

(Shanahan and Macmillan 2008: 174)

Promoting Population Geography through a biographical life course perspective foregrounds and promotes an inherently holistic sensitivity, emphasizing the fundamental interconnected and relational status of the demographic phenomenon under scrutiny. However, this openness runs the danger that scholarship gets bogged down in seeking to express and investigate the almost infinite aspects of a person's life that may feed into demographic outcomes. For an individual this is hard enough but at the aggregate level it is clearly unworkable! To deflect this problem, attention is focused within subsequent chapters on key arenas most pertinent for structuring the particular demographic 


\section{POPULATION GEOGRAPHIES OF THE LIFE COURSE}

experience of interest. These arenas will now be identified and introduced. They both frame an individual's practical competence, or ability to get on in the world, and their subsequent progress. Relative position within each provides constraints or privileges that result in individuals becoming disempowered or empowered, reducing or enhancing their agency, respectively. Together, these arenas comprise the socially differentiated landscapes of everyday life across which individual lives are relationally lived out.

Nine arenas of differential experience are introduced (also Crockett 2002; Hockey and James 2003; Hunt 2005). Following the traditional importance life course perspectives accord history, this comes first, age receiving special attention. History is followed by its entangled co-axis of geography. These two macro-contextual arenas are then joined (alphabetically) by six bio-social arenas: class and status; health and differential ability; race and ethnicity; religion and belief; sex and gender; and sexuality. Discussion ends with consideration of the final key arena: expression of human agency or individuality. Each life ultimately "speaks on its own terms" (Shanahan and Macmillan 2008: 236), even within a potentially wide range of structured group biographies.

The astute reader may be surprised to see culture absent from the previous list. Following the so-called "cultural turn" (Crang 2000), culture-being "what humans do" (Anderson 2010: 3)-is so infused within all aspects of the life course that it is ubiquitous within all nine arenas. It cannot be usefully abstracted. Indeed, a key theme running through this Section is how all life course arenas are never more than partly "natural." Instead, they are to a greater or lesser extent "produced" (Smith 1984), with culture (and economics; focused on specifically in terms of class but of ubiquitous significance) featuring prominently within these productions. Calling for "a more integrated vision of social scientific explanation," US Sociologists Pachuki and Breiger (2010: 219) concluded their extensive literature review by emphasizing how "[s]ocial relations are culturally constituted, and shared cultural meanings also shape social structure." This is endorsed.

\subsubsection{History}

\subsubsection{Families of time}

The importance of the place in history that an individual life course exists featured prominently within Elder's (1974) original development of the life course perspective. However, it is important not to see history and time (or geography and space, below) as a singular absolute variable. The single term time, trying to capture "the eel-like dimension of our existence, slippery and ungraspable" (Turner 2012: 1217), instead "summarizes a multitude of unfoldings, all making their way into the world at different rates" (Thrift 2004a: 873). Time and temporality, the condition of being set in time, ranges from the philosophical enigma of finitude and infinity to something regarded simply as "a banal and evident feature of day-to-day life" (Giddens 1984: 34-5; Shove et al. 2009). Time features in many guises within everyday life and the resulting contrasting temporalities have varying consequences with respect to framing and forming the life course and demographic behaviors.

Four families of time are distinguished here (also Giddens 1984; Thrift 2009). First, a supra-individual sense of history inscribes the times lived in. Such historical periods, eras or epochs are marked by the major events of world order and disorder-from formation and dissolution of states, empires and "world orders," to wars and revolutions. These shape popular understanding of History. Historical landmarks partly defining population cohorts, such as Elder's (1974) Great Depression, are included here. This "big history" expresses many outcomes with immediate relevance to and impact upon demographic behavior: inventions (e.g. the contraceptive pill); socio-political policies, institutions and legislation relating to births and reproductive rights (e.g. regarding abortion and birth control), deaths (e.g. judicial executions) and migration (e.g. travel restrictions); specific high-impact events (e.g. wars or famines); and general levels of health and well-being (e.g. levels of malnutrition, epidemic diseases). Once again, this notes the importance of cohort within life course scholarship.

Second, there are times operating at much smaller scales. These express the rhythms central to the reproduction of everyday life (Lefebvre 2004). They include the seemingly endless regularities and repetitions inscribed by clock time (Griffiths 2000) but also much less explicitly articulated or represented times of reaction/response, intuition and creativity that express the largely taken-for-granted working of everyday life (Thrift 2007). Illustrating the latter with reference to speech, think of the temporality of the "pregnant pause," the excuse spoken too quickly to be convincing, 
or the avoidance of an answer through loquaciousness! Expressions of these times of everyday life pertinent to demographic behavior include expressions of emotions and their affect on others (Pile 2010) and times of work and leisure. For example, engaging in "excessive" labor may not only be bad for one's health and happiness, thus potentially raising the specter of morbidity and mortality, but may also suppress fertility (both biomedically and more sociologicallylibido and opportunity). However, it may increase migration as the exhausted worker seeks better quality of life elsewhere.

Third, sitting between these two temporal scales are intermediate times, notably the "irreversible" longevity of the individual. So much of who and what individuals are-character, personality, tastes, attitudes, outlook on life-is shaped by birth context and upbringing experiences from infant to adulthood (Clausen 1991a, 1991b). Especially important are the "impressionable years" (Alwin and McCammon 2003: 34) when emerging adults (Arnett 2000) appear especially receptive to ongoing socio-cultural changes. Clearly influenced by and to an extent embodily integrating the bigger and smaller scale times just introduced, an individual's longevity-life course-has its own fatalistic logic. Within what Roman Emperor Marcus Aurelius termed the "incessant flux" of life, and notwithstanding all that buffets and redirects it, biological times for having children arise and wither, and a time for dying inevitably looms larger by the year.

A fourth family of time, suggested throughout the categories so far addressed as it overlaps them all, is time represented or imagined. Time is widely expressed within culture-whether as "history," "clock time" or the "life of a person"-emphasizing how it should be seen as much more than a natural phenomenon. For the individual, for example, "age represents not only a point in the life span and a historical marker ... but also a subjective understanding about the temporal nature of life" (Elder et al. 2003: 10). Rather than see time as "shaped by external 'forces'[,] ... temporalities are themselves continually reproduced, enacted and transformed" (Shove et al. 2009: 4). This endless (re)shaping involves not just technological innovations, such as accurate clocks (Glennie and Thrift 2009), but embodied, emotional and often impassioned disputes over what times (should) mean and be associated with. Within such disputes, different representations of time contest, as in the gradual imposition of a now taken-for-granted "clock time" (Glennie and Thrift 2009; Thompson 1967).

How time is seen or represented, and the temporalities that result from struggles to assert rival representations, combine with biological times to impact on demography. There is, in summary, as Ecclesiastes 3:2 puts it in the Bible, "a time to be born and a time to die, a time to plant and a time to uproot." Mediated through cultural representations there are, in other words, times for fertility (e.g. in stable sexual union), migration (e.g. when out of work) and, arguably, dying (e.g. beyond a certain period on a life-support machine). However, as encultured representations, such associations are always subject to disputation (Cresswell 1996). This is clear from considering the life course divided by age.

\subsubsection{Age}

Within the time of longevity, age can, not unproblematically, be broken down into categories such as childhood/youth, adulthood/middle age, and older age (Box 2.1), each with implications for what Settersten (2003) termed the rhythm of the life course. Starting with childhood, researchers now reject any notion of a "universal child," since across the world-whether between or within countries-there remain "vastly different understandings of what it is to be young" (Ruddick 2003: 357). Such understandings have changed historically and continue to change, linked once again to broader cultural and socio-political currents within society. However defined nonetheless, childhood expresses a relatively distinct arena of differential demographic experience.

The distinctiveness of childhood comes from at least two perspectives. First, there is biological "capability," related to age, which is particularly connected to inability to have children and enhanced susceptibility to disease and physical harm. However, whilst biology is a critical force defining a young person, especially when a baby or very young, it has arguably been overshadowed throughout human history by the roles played by extra-biological forces shaping young lives across space. This is true for demographic experiences. "Childhood," "youth," "teenager" and related age-based terms for young people are strongly socio-culturally constructed. Such constructs carry with them rules, norms and expectations concerning demographic behavior and competence. For example, questions may arise over issues such as: adequate responsibility to have children of their own; whether they should have a say within family migration 


\section{POPULATION GEOGRAPHIES OF THE LIFE COURSE}

decision-making; or whether sick young people should relinquish responsibility for managing their morbidity. Featuring prominently within such debates is likely to be adultism, adults' power over children (Flasher 1978). Away from arguably more positive associations with adult responsibility and care, adultism can result in discrimination and prejudice against young people and their potential agency (Matthews and Limb 1999).

Attaining adulthood (Box 2.1) usually assumes much more responsibility for demographic behavior. Both decisions - whether or not to have children, when and where to move, how to deal with illness-and subsequent actionshaving and raising a family, moving house, caring for sick relatives-are now supposedly largely down to the individual, at least within liberal democracies. The "helpless" child is comprehensively replaced by the "responsible adult." Nonetheless, as Del Casino (2009: 236) argues, scholars have been somewhat "blind to ... [a] normative adultcentredness." Being adult is actually something that merits scrutiny in its own right, since it is also thoroughly constructed by and contested within the socio-cultural sphere. Individual adult agency can easily be over-stated.

Adult agency, including that associated with demographic behaviors, is highly constrained from numerous directions. Being an adult within any society has attached to it much cultural baggage and expectation. Transitioning from childhood or youth does not necessarily transition one from the cultural imagination. It may take much learning and performative work to be(come) the culturally normative "responsible adult" society expects and demands (Mezirow 2000). This includes learning and embodying appropriate fertility, migration and mortality/morbidity behaviors (Settersten 2003).

Focus on adult demographic performances also draws attention to the importance of the home and, in particular, the family or household institutional setting (Blunt and Dowling 2006). Many life course transitions are associated with the household (Table 2.2), including forming lasting sexual unions-expressed through co-habitation or marriage. This brings about often significant changes in lifestyle-more than just cooking for two!-that feed into demographic outcomes such as deciding to have children or moving to a bigger home. Equally, breakdown of sexual unionsseparation and divorce-have demographic outcomes, commonly migration for at least one partner but also increased mortality through suicide (Ide et al. 2010), for example. Overall, adult household arrangements impact substantially on demographic behavior.

Whilst older people remain adults and frequently retain mid-life responsibilities, priorities and concerns, attaining seniority also typically represents a key life course transition. Again, both biology (age based capability) and society (socio-culturally based capability) converge in structuring older people's ability to engage with their immediate environments. First, ageing typically signifies and is signified by heightened and reconfigured bodily prominence. Whilst lack of energy and fatigue in older age should not be assumed and often indicate serious health problems (Avlund 2010; Cheng et al. 2008), it is a truism that as people get older their bodies age. Parts may be replaced-artificial limbs, transplants, medical devices such as heart pacemakers-and technological aids, such as scooters, facilitate mobility but the overall effects of ageing see parts of the body gradually wear out. Consequently, ageing is associated with slowing down, loss of bodily resilience and, therefore, increasing energy impairment. Demographically, this feeds into loss of fertility, reduced or increasingly selective migration (notably to access care), further morbidity and, ultimately, mortality.

Moving away from the physicality of bodily ageing, the substantial role of social and cultural processes in shaping older lives across space must again be noted. Cultural expectations and attitudes are encapsulated in terms such as "pensioner," "OAP," "geriatric," "golden-ager," "old fogey," "old-timer," "oldster" or "senior," the sheer number indicating cultural resonance (Box 2.2). Prevalent in many societies is ageism, "culturally prescribed sets of norms and prejudices against people and behaviour at various stages in the life course" (Mowl et al. 2000: 190) (thus not just affecting older people). For older people, ageism typically results in prejudice, through, for example, misrepresentation of needs, priorities and capacities, including those relating to demographic issues.

There is a final way age becomes a critical aspect of Population Geography, which operates at the macro-scale, beyond the individual. This refers to how the population structure of a defined area, typically a country, broken down by age, will have implications for births, deaths and migrations. This issue is discussed in the context of population pyramids in Chapter 3. In brief, if a country has a relatively large number of young adults then overall fertility level (births) is likely to be high, just as if a country has a very aged population then mortality (and morbidity) is likely to be high. In other words, at the macro-scale, a population's age structure has biologically built-in demographic implications. How individuals behave within such a structural context remains, of course, more relational and individual. 


\section{BOX 2.2 MEDIA REPRESENTATIONS OF THE OLDER AGED POPULATION}

In 2008, the Irish government proposed to revoke a key element of the Health Act 2001 which had granted all people aged 70 years and over automatic entitlement to a medical card. It was proposed that entitlement should be restricted to those able to determine their need for such a social benefit through a means test. Unsurprisingly, this controversy led to much debate in the media on the lives of older people.

Within newspaper coverage, older people were regularly collectively classified, with "chronological age ... reified as a facet of personhood ... that establishes a discourse of us/them" (Fealy et al. 2012: 99). Numerous terms sought to express this assumed chronologically-grouped identity: older folk, senior citizens, pensioners, over-70s, retired, grannies and granddads, little old ladies, old and vulnerable, ageing parents. However, within this process of representation, five broader identity types were recognized:

- Victims: vulnerable citizens who had been made "hapless and undeserving victims of a cruel act perpetrated by an uncaring and mean-spirited Government" (Fealy et al. 2012: 91);

- Frail, infirm and vulnerable: an especially vulnerable group, eternally at risk of illness and infirmity, and terrified of their immediate future;

- $\quad$ Radicalised citizens on the march: stories typically deployed romantic and military metaphors, with "grey panthers" armed with Zimmer frames besieging the Irish Parliament;

- Deserving old: a group who have more than paid their dues to society and deserve sympathy; and

- Undeserving old: an arrogant and pampered group, whose unreasonable demands hold back Irish society.

Overall, there was "a latent ageism in the texts in the way that older people were conferred with a uniform identity of implied dependency" (Fealy et al. 2012: 98), whether or not this dependency was seen as deserved. In other words, the combination of reified chronological identity and its association with neediness "construct[ed] older people as unproductive/dependent and other/marginal" (Fealy et al. 2012: 100). Thus, there was no representational space for older Irish residents to be healthy, self-reliant, autonomous or, most especially, individual.

(Source: Fealy et al. 2012)

\subsubsection{Geography}

A similar case to that made for the importance of history and time for understanding demographic behaviors within the life course can be made for geography and space. Experience across the life course is also differentiated according to "where" one is. Space and/or place matter.

In the simple terms, all demographic life course behaviors of births, deaths and migrations take place somewhere. However, beyond this immediate case for Population Geography attention must be given to conceptual distinctions within geography/space that, as with history/time, emphasize multiple co-existence. Thus, Thrift (2009) started his account of space, "the fundamental stuff of geography" (p.85), with a request to:

abandon the idea of any pre-existing space in which things are passively embedded, like flies trapped in a web of co-ordinates-the so-called absolute view of space-for an idea of space as undergoing continual construction as the result of the agency of things encountering each other in more or less organized circulations.

In other words, the idea of any singular Geography is immediately rejected. Instead, Thrift advocated a relational view of space, simplified into four kinds or families (Figure 2.1).

First, "spaces of empirical construction" are the "fabric of our daily lives" (Thrift 2009: 86). Such spaces-"houses, cars, mobiles, knives and forks, offices, bicycles, computers, clothes and dryers, cinemas, trains, televisions, garden paths" (ibid.)-may be so mundane as often to be largely overlooked by "serious" scholarship. Yet, they represent remarkable and complex achievements. Certainly, they do not just exist. Moreover, one can extend and expand the 


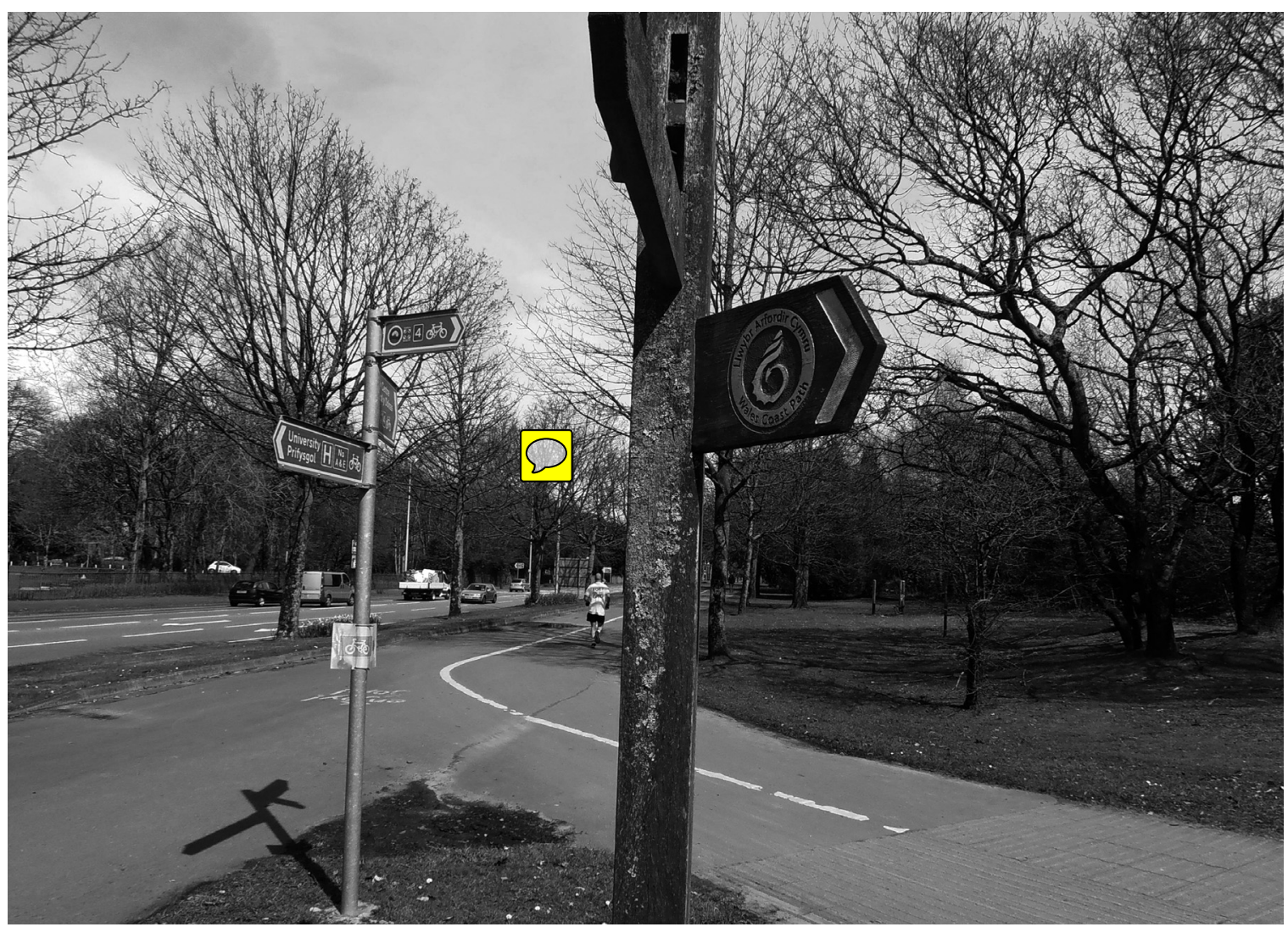

Figure 2.1 How the Wales Coastal Path at Swansea expresses four kinds of space.

Source: categories of space after Thrift (2009); see also www.walescoastpath.gov.uk.

scope of these spaces to include larger scale everyday fabrics, equally largely taken for granted. These are the scaled places (Marston 2000) that embody and express spatially the "social relations of empowerment and disempowerment" (Swyngedouw 1997: 169) introduced in the next seven arenas. They include locality, region, state, geopolitical constructions such as Global North and South, even Earth as a whole. Demographically, these are the spaces within which we conceive, give birth, take ill, die, and migrate. Their material configurations, themselves partly (re)produced through these same behaviors, are thus of great relevance. For example, how a house is configured-size, number of bedrooms, presence of garden, adequacy of heating, rooms on one level or two-can have immediate consequences for fertility (e.g. enough space), migration (e.g. desire to stay or leave) and mortality/morbidity (e.g. healthiness), just as the forms housing take also express in part the demands of these same demographic considerations (e.g. central heating, private bedrooms).

Second, the fluidity of geography is not just in the production, reproduction and transformation of empirical spaces. Thrift (2009: 88) next outlined "flow spaces" referencing the "pathways which bind often quite unalike things together." Developed further in Chapter 5, various mobilities (Cresswell 2006) increasingly bind the everyday world more-orless together. Think of globalization (Sparke 2009) integrating the world through trade, political structures, cultures, and so on. Pathways are absolutely central to the practices (e.g. facilitating through modern transport networks) and potentials (e.g. presenting potential destinations to potential migrants) of migration but also impact on births (e.g. facilitating pre-natal, natal and post-natal care) and deaths (e.g. facilitating spread of epidemic disease or life-saving medicines).

Third, Thrift called attention to place. This core geographical concept (Cresswell 2004) seeks to express "particular rhythms of being that confirm and naturalize the existence of certain spaces" (Thrift 2009: 92). As such, place particularly engages with how spaces become embodied; are sensed and felt; affect a person's mind and body; and

\section{ST PROOFS: NOT FOR DISTRIBUTION}


become a part of the fabric of everyday taken-for-granted life. Place works to integrate geography, including scaling daily life (Marston 2000), and shape the overall demographic expressions of the life courses that live through them. Being "in place" (Cresswell 1996) almost always has demographic dimensions in terms of norms and rules of behavior, for example. Describing and explaining the (em)placing of Demography is thus another way of expressing the core of Population Geography (Chapter 1).

Within the idea of place must also be noted the existence and importance of the physical and non-human biological world. Such a world of, broadly speaking, Physical Geography and Biosciences has been largely absent from discussions of space thus far. Experiencing the bio-physical world (just as experiencing other people) can impact on people and their behavior. On the one hand, affect broadly expresses the feelings, emotions and actions brought about through bodily engagement with worldly materiality (Anderson 2014; Blackman and Venn 2010). Its "sense of push in the world" (Thrift 2004b: 64) can entangle itself within the shaping of demographic behaviors. For example, Halfacree and Rivera (2012) suggested the affective role of "rurality" or the spatialities of the countryside in shaping the experience of migration to rural areas. On the other hand, the bio-physical world is also effective, albeit often indirectly, on demography. Climate and weather, soil type and quality, proximity to water, presence of certain other types of living things, and so on, whilst rarely directly bringing about demographic outcomes in the refuted style of environmental determinism (1.3.2), may be causally implicated in such outcomes. For example, high levels of background radiation in rocks such as granite have been associated with raised levels of childhood cancers (Knox et al. 1988), or substantial migration outflows might be expected to result from severe environmental catastrophe (Black et al. 2011).

Fourth, Thrift (2009) drew attention to the importance of images of space or their representations. This strongly cultural realm of geography is so important (as it was for history) "because it is so often through [representations] that we register the spaces around us and imagine how they might turn up in the future" (Thrift 2009: 90). Images

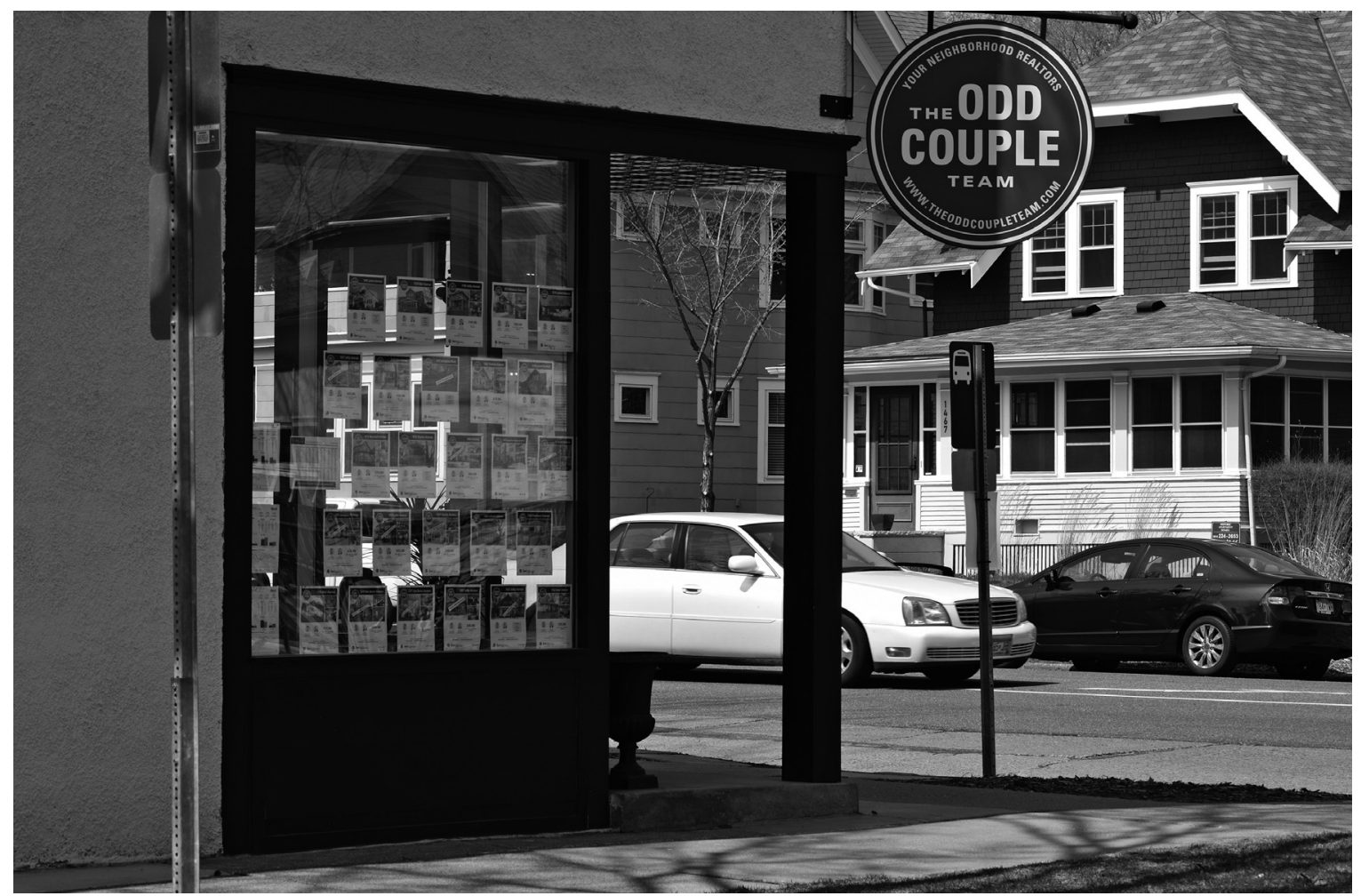

Figure 2.2 Representations of home and place in the real estate window. (In the real estate agent's window, representations of houses and their accompanying text promote residential relocation to "better" properties and neighborhoods. Photo taken in Saint Paul, Minnesota.)

Source: authors. 


\section{POPULATION GEOGRAPHIES OF THE LIFE COURSE}

of space and place are ubiquitous within migration behavior, deterring people from moving to places with poor place images whilst promoting lifestyle migration (O'Reilly and Benson 2009) to places with positive images, for example. They are utilized in marketing both homes and neighborhoods (Figure 2.2). However, representations of space also impact on fertility decisions through, for example, contributing to a psychological "feel good" or "feel bad" factor, and even on deaths, through health providers failing to "see" needs within seemingly beautiful and healthy places (Watkins and Jacoby 2007), for example. For a "healthy neighborhood," place image or reputation is vitally important, since "[h]ow areas are perceived, by their residents, by service or amenity planners and providers, by banks and investors, may influence the infrastructure of the area, the self-esteem and morale of the residents, and who moves in and out" (Macintyre et al. 2002: 131).

There has been much commonality in the discussions of the role within the life course and key demographic transitions of geography and history. Together, as internally differentiated as they have been shown to be, they present macro-contextual arenas for Population Geographies of the life course. Indeed, in the broader Geography literature, both have sometimes been combined as time-space or space-time. Usually, Schatzki (2009: 36) argues, this retains both components as independent "phenomena that are not intrinsically related" but is useful as it highlights "contingent connections" between time and space. Simply put demographically, it may link the when of a birth (e.g. 1965) with a where (e.g. London), enabling us to appreciate, for example, that this birth took place at a time-space of relative national (British) prosperity and a comprehensive operative National Health Service. However, Schatzki (2009: 39) also proposes another expression of time-space, "activity time[-]space," that is "a feature of an individual human life" and expresses how life is lived. Whilst unable to develop this concept here, all demographic behaviors "require" their own time-space to manifest themselves. Intrusion into this domain, not least via technological innovations, will be seen to be impacting on the very definition of both life and death in Chapters 4 and 10, respectively, for example. Time and space can rarely be meaningfully disconnected when talking about life as lived.

\subsubsection{Class and status}

Introducing arenas of differential demographic experience within the life course now turns to key bio-social stratifications. Together, these constitute an individual's positionality, those "[f]acets of the self - institutional privilege, for example, as well as aspects of social identity-[which] are articulated as 'positions' in a multidimensional geography of power relations" (Rose 1997: 308). In other words, a person's various social locations (e.g. middle class, disabled, Hispanic, man, homosexual) lead into differentiated links to resources of all kinds (material, cultural, social, experiential). Given the importance of such access, positionality significantly shapes a life course and demographic transitions within it. Age is a further key aspect of positionality, already introduced.

From Marxists to "free market" advocates, the economy has long been acknowledged as providing a key-often the key-way people are socially subdivided. It is thus unsurprising that class as a key aspect of positionality has long been prioritized, including by Population Geographers (Chapter 1). However, the exact meaning of class-let alone its contemporary significance-is immensely debated, both within academia (e.g. Wright 2009) and without (e.g. Arnot 2009). It can simply be defined as the principal social expression of "systems of stratification derived from social relations of property and work" (Pratt 2000: 85). In other words, it is generally seen at base as originating in the waged workplace, yet also meshing with other arenas of stratification. Sociologist Erik Olin Wright suggested the hierarchical picture of the US class structure shown in Table 2.4. This hierarchy is cross-cut by an interaction between race and class in which the working poor and marginalized disproportionately comprise racial minorities.

Gough (2001: 20-22; following Wood 1995) distinguishes class as personal attribute from class as relation. In the former, a class position or identity is given to an individual or a household according to one or more variables. These variables include "income, skill, social status, security of employment, type of knowledge or cultural competences possessed..., accent, consumption pattern" (Gough 2001: 20). The number of ways class can be defined from them is nearly infinite. In contrast, class as relation argues that class only comes about through a person's or household's relations to others. The variables Gough noted above, including status, express the consequences of these relations, they are the results of class not its defining bases. David Harvey's Marxist understanding of the place and role of class within capitalism exemplifies this approach (Table 2.5).

However class is understood and defined, its core importance in this book comes from its link to uneven distributions of the "occupational reward" (Hunt 2005: 41) of resources between people. These resources comprise 
Table 2.4 The US class structure today

\begin{tabular}{|c|c|}
\hline $\begin{array}{l}\text { 1. Extremely rich capitalist } \\
\text { class and corporate } \\
\text { managerial class }\end{array}$ & $\begin{array}{l}\text { Extraordinarily high consumption and relatively weak constraints on their exercise of } \\
\text { economic power. }\end{array}$ \\
\hline 3. Established working class & $\begin{array}{l}\text { Previously unionized with standards of living and security similar to the middle class but now largely } \\
\text { lacking protections. }\end{array}$ \\
\hline $\begin{array}{l}\text { 4. Poor and precarious } \\
\text { working class }\end{array}$ & $\begin{array}{l}\text { Low wages and insecure employment, subject to unconstrained job competition in the labor } \\
\text { market and with minimal state protection. }\end{array}$ \\
\hline $\begin{array}{l}\text { 5. More marginalized and } \\
\text { impoverished "class" }\end{array}$ & $\begin{array}{l}\text { Without skills and education needed for above poverty line jobs and with living conditions making } \\
\text { it extremely difficult to acquire them. }\end{array}$ \\
\hline
\end{tabular}

Based on Wright (2009: 114).

Table 2.5 Class as relation

David Harvey (2001: 79) expresses both how class is an essential defining relation of capitalist society and how the "logic" of that relation, in turn, drives the fundamental reproduction of society:

The two themes [accumulation and class struggle] are integral to each other and have to be regarded as different sides of the same coin ... The class character of capitalist society means the domination of labor by capital. Put more concretely, a class of capitalists is in command of the work process and organizes that process for the purpose of producing profit. The laborer, on the other hand, has command only over his or her labor power which must be sold as a commodity on the market. The domination arises because the laborer must yield the capitalist a profit in return for a living wage. All of this is terribly simplistic, of course, and actual class relations and an actual system of production (comprising production, services, necessary costs of circulation, distribution and exchange, and so on) are much more complicated. The essential Marxian insight, however, is that profit arises out of the domination of labor by capital but that the capitalists as a class must, if they are to reproduce themselves, expand the basis of profit. We thus arrive at a conception of a society founded on the principle of 'accumulation for accumulation's sake, production for production's sake.'

not just money, or even exist just in material terms, but also embrace more explicitly cultural expressions: knowledge; taste, style and authority (cultural capital); status and power; and general "awareness" (Bourdieu 1984). Differential access to this diverse panoply of resources, all somewhat connected to class, promotes differentiated demographic behaviors across the life course and between individuals. Such a general conclusion is true even if alternative expressions of occupational stratification within society substitute for class, such as the South Asian caste system (Box 2.3), other measures of status, or even just occupational category (frequently used in population censuses).

\subsubsection{Health and differential ability}

With clear links to fertility, births, morbidity and deaths, and more recently acknowledged significant associations with migration (e.g. Boyle and Norman 2010), issues of health have long been central to Population Geography, although Health and Medical Geography is a well-established sub-discipline in its own right (Brown et al. 2010; Gatrell and Elliott 2015). More recently, geographical focus on the differentially enabled body has expanded its scope further (Butler and Parr 1999; Chouinard et al. 2010), an area with direct implications for life course demographics.

It is useful to begin any introduction of health and differential ability by observing how all of us are Other (Chapter 1) when judged against some fully enabled mythical "superhuman" (Box 2.4). In other words, "[w]e are all 'disabled' in some way or another," as a researcher stated to Castrodale and Crooks (2010: 94). However, a central theme within research on disability, in particular, is the need to acknowledge, challenge, overcome and move beyond an underlying assumption of the "normal" lack of any disability. This assumption is termed ableism, a set of "ideas, practices, institutions and social relations that presume ablebodiedness, and by so doing ... construct persons with disabilities as marginalized, oppressed, and largely invisible 'others'" (Chouinard 1997: 380).

\section{ST PROOFS: NOT FOR DISTRIBUTION}




\section{BOX 2.3 SOCIAL STRATIFICATION WITHIN THE CASTE SYSTEM}

Class is not the only way social stratification is expressed globally. Within India in particular, but extending more broadly into parts of South Asia, there endures what is known as the caste system. This comprises numerous groups expressing diverse styles of life. Caste is highly relational, acquiring "different shadings in the context of village, locality, region, and nation" (Galanter 1984: 7) but is understood overall as "a system of social organization in which society comprises ranked hereditary kinship groups associated with a division labor and organized into a unified and integrated whole" (ibid.).

At the broad aggregate level, four classes of varnas can be identified within the caste system:

- Brahmins: priests, scholars;

- Kshatriyas: rulers, soldiers;

- Vaishyas: merchants, agriculturalists; and

- Sudras: menial workers.

All four groups are highly internally differentiated, not least by geography, and form thousands of sub-castes (jatis). Additionally, there is a further aggregation that expresses the bottom tier in the system:

- Dalits (also known as Untouchables, Scheduled Castes or Harijans).

Whilst terminologically problematic and hard to relate precisely to the Hindu-rooted caste system, "untouchability" has long had numerous negative life course consequences for those it encompasses. These are summarized by Galanter (1984: 15) as "a catalog of ... disabilities":

- Denied or restricted access to public facilities, holy places and services;

- Exclusion from profitable and prestigious forms of employment;

- $\quad$ Required deference to higher castes;

- $\quad$ Liability for unremunerated menial labor for higher castes;

- Residential segregation;

- Restrictions in everyday lifestyles, especially in using items of comfort or luxury; and

- Restricted movement, especially near higher castes.

Finally, caste "still insinuates itself into social, economic, and political fabric of the country" (Bidner and Eswaran 2015: 142), notwithstanding India's considerable recent economic development and political mobilization of Dalits and their supporters. And it has notable demographic consequences.

(Sources: Bidner and Eswaran 2015; Galanter 1984)

Ableism draws immediate attention to the cultural framing of differential abilities. Indeed, a key theme in health and disability scholarship is tension between the relative importance of and priority given to the biological or biomedical as compared to the social. Within Medical Geography, the rise of post-positivist approaches (Chapter 1) saw a shift away from a biomedical focus on, for example, geo-epidemiological mapping of the incidence of diseases and linking this to corresponding distributions of their determinants (e.g. Krieger 2003; Milo and Kahana 2010; Rican and Salem 2010). Instead, health was understood more as constituted through social and cultural environments (Kearns and Moon 2002; Parr 2002). Furthermore, the resulting Health Geography (Kearns 1993) articulates "definitions of health that go beyond the biomedical perspective of an absence of disease, ... framing ... health issues within criticalsocial-theoretical frameworks, [often using] qualitative methodology and mixed methods, and ... [attending] to the meaning of place" (Rosenberg 2011: 109).

The biological-social tension is even more central within disability scholarship. Indeed, within the emerging subdiscipline of Geographies of Disability in the 1990s, a dominant theme was of the social model of disability displacing a biomedical model (Barnes and Mercer 2003). The latter focused on impairment of the physical body, portrayed as "individual medical tragedy" (Shakespeare 1993: 255). Resultant "abnormal" and "failing" bodies were regarded as physically and, through extension, socially inferior, yet deserving sympathy and help, often through medical 


\section{BOX 2.4 SUPERMAN, FASCISM AND CHALLENGING THE DISABLED BODY}

Long before naming a well-known comic character, the concept of the Superman came from German philosopher Friedrich Nietzsche (1844-1900). The Übermensch was proposed as a liberatory goal for humanity to set itself in his novel Thus Spoke Zarathustra (1883). With this concept, Nietzsche sought to provoke humanity to excel and transcend what he saw as unnecessary constraining existing limits on everyday living.

With a focus on the bio-physical, desire for a physique closer to such an imagined Superman has long been entangled with Far Right politics. On the one hand, there was the avowed interest of political parties-notably Mussolini's Italian Fascists and Hitler's Nazis in Germany-in the years between the World Wars to attain an assumed racial superiority. This was to be done, in part, through cultivating the perfect body. For example, Nazi Germany's Strength through Joy initiative had a strong bodily fitness element. On the other hand, most populations defined as inferior to the potential race of Supermen subsequently experienced attempted genocide in the Holocaust. One may thus understandably wish to reject striving to overcome perceived bodily dis-ability as potentially Fascist.

However, physical body "improvement" was not just the concern of the inter-war Far Right. The contemporary situation in Britain illustrates this. On the one hand, a Fascist tendency to fetishize the Superman was apparent. The Superman, a glossy magazine, was launched in 1930 with a belief expounded by its editor "that Man must go forward, must climb the ladder of Evolution, must transform himself into a being loftier in mind, more powerful and vastly more fit in both his physical health and strength" (quoted in Zweiniger-Bargielowska 2010: 603). Two years later, Oswald Mosley, founder of the British Union of Fascists, "envisioned ... the creation of a British new Fascist man whose perfect physique was to be showcased in physical training displays" (ibid.). On the other hand, desire for physical bodily improvement extended far beyond the political Far Right, and had a much longer pedigree. A "physical culture movement" was stimulated by various experiences and concerns: popular rise of sport and outdoor leisure; official recognition of the unfitness of many soldiers in both the Boer War (1899-1902) and World War I (1914-1918); popular magazines promoting fitness, such as Health and Strength; Britain's relatively poor performance in the 1936 Berlin Olympics; and a general desire for a fitter nation.

This evidence from Britain in the inter-war years suggests that although seeking to become Superman can promote extremely dis-abling narratives and consequences, that at the very least stigmatize and discriminate against those regarded as failing this ideal, there may nonetheless also be negative life consequences from simply remaining as one is. Promoting a fitter nation in 1930s Britain was a reasonable and positive ambition, and remains thus today. Nietzsche's Superman imagined lives becoming more fully realized; it was not essentially a device for social discrimination.

(Source: Zweiniger-Bargielowska 2010)

interventions-from medication to invasive techniques (e.g. cochlea implants for deafness) to technical aids (e.g. wheelchairs or prosthetic limbs) (Oliver 1998). In contrast, the body, medicine and technology take a back seat within the social model. Attention switches to how society creates conditions which dis-able people. For example, the social model stresses how the modern city dis-ables people with particular impairments (Hahn 1986; Imrie 1996), illustrated in Table 2.6. Only relatively recently has concern not to "concede ... the body to medicine" (Hughes and Paterson 1997: 326) renewed appreciation of how "people are disabled by society and by their bodies" (Shakespeare $2006:$ 56, emphasis added), allowing biology back (Hansen and Philo 2007).

Dis-ability, through the biomedical sources of either ill health or bodily impairment but always set within (typically ableist) socio-cultural context, inevitably produces populations with bodies of differential ability, "a continuum of experiences, rather than polar opposites" (Worth 2008: 307). This, in turn, is expressed in how they live their lives across space, including consequent differentiated demographic behaviors. Biologically, for example, health problems may reduce fertility or enhance morbidity and mortality. Additionally, expressions of dis-ability, not least through association with reduced incomes but also in terms of how lack of everyday mobility may act as a more general brake on movement, both physical and socio-economic, may also suppress migration. Furthermore, through cultural norms 
34 POPULATION GEOGRAPHIES OF THE LIFE COURSE

Table 2.6 Environmental barriers impeding urban wheelchair mobility (in order of significance)

\begin{tabular}{ll}
\hline 1. High curbs and/or lack of dropped curbs & 1. People on sidewalks \\
2. Steep gradients or ramps & 2. Accessing shops \\
3. Uneven paving slabs & 3. Lack of dropped curbs \\
4. Rough or cobbled surfaces & 4. Uneven surfaces \\
5. Slippery surfaces & 5. Dropped curbs on roads not adjacent \\
6. Narrow sidewalks & 6. High curbs \\
7. Street furniture poorly placed & 7. Steps without adjacent ramp \\
8. Congested sidewalks & 8. Busy roads \\
9. Steps without adjacent ramp & 9. Narrow sidewalks \\
10. Dropped curbs on roads not adjacent & \\
11. Difficult camber on sidewalks & (1-3 considered a "major obstacle" by over $50 \%$ \\
12. Deep gutters along roadside & of respondents) \\
13. Busy roads & Top five demands of wheelchair users: \\
14. Lack of resting places on slopes and ramps & 1. More dropped curbs \\
15. Handrails not provided on ramps & 2. Improve quality of sidewalks \\
16. Insufficient designated road-crossings & 3. Improve overall access \\
17. Drains near dropped curbs & 4. Better policing of disabled parking bays \\
18. Cars parking adjacent to dropped curbs & 5. More disabled parking bays \\
19. Raised manhole covers at road-crossings & \\
20. Poor pathway maintenance: dog fouling, litter &
\end{tabular}

(Source: adapted from Matthews and Vujakovic 1995: 107)

(Source: adapted from Bromley et al. 2007: 234, 238)

and legislative measures, a dis-abled individual may be "discouraged" from parenting, whilst a society's ableism may even enhance morbidity and mortality through the consequences of abuse, bullying and discrimination.

\subsubsection{Race and ethnicity}

The previous two Sections demonstrated how even relatively specific arenas of differential life course experience are themselves internally differentiated (disability/ill health/impairment, for example). This is even truer in this Section, which embraces two terms in popular understanding often seen as equivalent but which are markedly different. Nonetheless, they are not quite as different as scholarship can too simply imply, since both have the twin faces of the biological and social that characterize all the positional categories being introduced.

Race is a classifier of individuals emphasizing biological difference and/or physical difference in appearance, notably skin color. In contrast, ethnicity is regarded as more definitively socio-cultural and expresses cultural identitydefined by the group itself and/or by non-group members of the wider society-rooted in the sense of a "people" defined by birth (Barke and Fuller 2001; Lewis and Phoenix 2004). However, whilst these distinctions at first sight appear relatively clear, this is not the case, especially when seen in terms of life experiences.

First, making an easy association between race and biology runs the danger of over-stating the everyday significance of one (occasionally more) relatively arbitrary aspect of a person's composition. This runs against, for example, the more multiply relational individual expressed within the life course concept. Race, suggestive of clearly bounded and defined categories, also implies a degree of abstraction from the "real world," which comprises constant flows of people and genetic intermixing (see Chapter 5 on mobilities). Second, it is both equally hard and problematic to define ethnicity in a clear-cut way, for similar reasons (Table 2.7). New Zealand Statistics recognizes ethnicity as a key social indicator that population surveys need to gather. It also recognizes how complex a concept it can be, reflected in its classification methodology,

Whatever the status of their more "objective" reality, race and ethnicity are united in being culturally constructed categories, widely articulated within the everyday life of almost every society today, with far from neutral or innocent outcomes (Gilroy 2000; Hunt 2005). Both are themselves relationally expressed. Their existence, characteristics and importance within everyday life for an individual is only understandable through reference to that individual's encultured relations to others. In summary: 
Table 2.7 Ethnicity in New Zealand Statistics

\begin{tabular}{|c|c|c|}
\hline Assumptions & Hierarchy & Results \\
\hline $\begin{array}{l}\text { - Ethnicity is a measure of cultural affiliation } \\
\text { - } \text { Ethnicity is self-defined } \\
\text { - } \text { can changication with a particular ethnic group } \\
\text { - Declaration of ethnicity can change with } \\
\text { specific context } \\
\text { - People often identify with more than one } \\
\text { ethnic group }\end{array}$ & $\begin{array}{l}\text { - Classification of ethnicity utilizes } \\
\text { four levels } \\
\text { - Going from Level } 4 \text { to Level } 1 \text {, the } \\
\text { ethnic groups get progressively broader } \\
\text { - Number of categories: Level } 1=7 \text {, } \\
\text { Level } 2=27 \text {, Level } 3=42 \text {, Level } 4=239 \\
\text { - Each level also has residual categories }\end{array}$ & $\begin{array}{l}\text { - Ethnicity detail recorded } \\
\text { depends on the survey and its } \\
\text { purpose } \\
\text { - Up to three or six responses } \\
\text { to ethnicity are usually } \\
\text { recorded per person } \\
\text { - Where more ethnicities than } \\
\text { three or six, respectively, are } \\
\text { given, these are reduced to } \\
\text { the maximum using a random } \\
\text { number method }\end{array}$ \\
\hline
\end{tabular}

\section{EXAMPLE}

- In response to the question "Which ethnic group do you belong to?" an individual self-defines as French, Niuean, Cambodian, Vietnamese, English, Algerian and New Zealander

- These seven identities are classified according to their Level 1 category:

- French \& English-European; Niuean-Pacific Peoples; Cambodian \& Vietnamese-Asian; Algerian-Middle Eastern/Latin American/African; New Zealander-Other Ethnicity

- To reduce the seven categories to six, one member of the Level 1 categories with two ethnicities cited (European \& Asian) is eliminated using a random number selection

- Cambodian is "lost" in this way

- The individual now assumes French, Niuean, Vietnamese, English, Algerian, New Zealander ethnicity

Source: developed from New Zealand Statistics (no date).

"[e]thnicity" and "race" are about the processes of marking differences between people on the basis of assumptions about human physical or cultural variations and the meanings of these variations.... [I]ndividuals and groups are racialized or ethnicized.

(Lewis and Phoenix 2004: 124, emphasis added).

Crucial are practices of racism that typically elide race and ethnicity to define a group of people as somehow inferior. Such marking of difference initiates a series of negative consequences for members of that group. The scope and depth of racism varies considerably but without doubt remains a key feature of discrimination and prejudice within modern societies.

In terms of shaping demographic aspects of the life course, race and ethnicity come through in numerous ways. First, racism, long studied by Geographers for associated spatial outcomes-such as ethnic segregation (e.g. Johnston et al. 2007; Simpson and Finney 2009)-clearly interacts with geographies of fertility, migration and mortality. From the consequences of poorer access to health care by certain ethnic groups, to ethnicity-based migration policies, to racist murders, the demographic relevance of racism is unfortunately inescapable. Second, however, recognizing the importance of group and individual agency, relative racial and ethnic positioning and identity construction can also (re)produce cultural differentiations which, in turn, express demographic outcomes irreducible to racism. Ethnic groups frequently demonstrate relatively distinctive practices of fertility, migration and even mortality. Third, returning to the biological, genetic make-up ("race") may sometimes be more directly linked to particular demographic expressions, notably causes of morbidity. However, one must be cautious in making such links as race may prove a poor proxy for genetics (Box 2.5). 


\section{BOX 2.5 RACE, GENETICS AND ILL HEALTH}

Racial categorization has long had an important if controversial place within medical scholarship and practice. Apart from ignorant or explicitly racist expressions, research seemingly revealed some regular associations between racial categories and the likelihood of experiencing particular diseases. Such associations have been consolidated with the rise to prominence of genetics, where specific genes, for which race may be seen as a proxy, are linked with specific diseases. However, it is vital to observe this medical evidence through an additional social lens, demonstrated by the classic case of the alleged "black" disease of sickle cell anemia.

Sickle cell anemia originates from a genetic modification to a haemoglobin protein that, somewhat ironically, prospered through natural selection by conferring immunity to falciparum malaria. Consequently, those with the sickle cell trait historically correlated strongly geographically with places where this form of malaria flourished. The majority of Africa was notable here, thereby linking the disease with black Africans and promoting the "black" disease label.

However, the fuller story of sickle cell anemia and race is much more complex. On the one hand, prevalence just within sub-Saharan Africa, and even within small areas, ranges between 10 percent and 40 percent of the population. In Gambia alone, prevalence amongst the Mandika is 4 percent, amongst the Wolof 14 percent and amongst the Fula nearly 30 percent. Such considerable socio-spatial variability queries any racial homogeneity. On the other hand, away from Africa, India has some of the highest global rates of sickle cell anemia, some of its peoples having prevalence of around 33 percent. This further questions the disease's racial correlation. In short, more is needed to understand the prevalence of sickle cell anemia than race.

More is also needed to understand ill health generally than just genetics. Whilst medical research may legitimately suggest connections between genetics and specific diseases, using race as the former's proxy seems increasingly inadequate. First, self-identified racial groups often demonstrate considerable genetic variability and any assumption of genetic homogeneity must be rejected. Second, actual diseases do not develop in abstraction but in the context of individual relational life courses, with such things as living conditions having a role to play as well as genetics. Third, within medical practice, if a disease is too simply associated with a racial group, there is a tendency to neglect the detailed life history of the patient. In summary, "[o]nce race is presumed, the ways in which multiple genetic inheritances interact with the environment within that individual seem to disappear" (Braun et al. 2007: 1426). Race, even where racism seems little prevalent, can obstruct effective medical practice and thus negatively impact on the life courses of the sick.

(Source: Braun et al. 2007)

\subsubsection{Religion and belief}

Deeply held beliefs can strongly influence identity and behavior, as any study of adherents to the major religions demonstrates (e.g. Aitchison et al. 2007; Hopkins et al. 2012). A resurgent Geographies of Religion (Kong 1990, 2001, 2010) immediately suggests that rather than existing as "important epiphenomena" (Yorgason and della Dora 2009: 635) within an increasingly secular world, religiosity-how religious a person is-may be the key marker of identity. Scholarship now pays increasing attention to post-secularization, a concept expressing the significance of religion for different population groups at different spatial scales within the world today (Kong 2010). Moreover, post-secularization may also be seen less as representing a backlash against an increasingly secular world and more as a belated acknowledgement-informed by postmodern sensitivity to difference and diversity (1.2.4)-of "an abiding spirituality that has persisted in the face of modernity" (Kong 2010: 765; Keddie 2003).

One reason for the prominence of religion within identity construction comes from the "energy" it gets from its interrelationship with other arenas introduced in this section. This includes the energy it gets from "spiritual" places (e.g. Holloway 2003a, 2003b, 2006). Linking with age, Gardner and Shukur (1994) noted how young Muslim British Bengalis increasingly defined their identities in terms of religion, as opposed to parental country of origin, class, and so on. Whilst first generation immigrants were equally "religious" in abstract terms (i.e. strongly Muslim), this was expressed differently, as more entangled with the culture of Bangladesh, for example (Ahmed 2013). Another

\section{ST PROOFS: NOT FOR DISTRIBUTION}


expression, strongly shaded by experiences of racism, is how young, second generation Indian Americans have used Hindu identity to contest marginalization (Kurien 2005). Castells (2010) termed this "resistance identity."

Notwithstanding a global rise in religious fundamentalisms (Castells 2010; Keddie 1998), with their absolute demands on daily living practices, to understand the potential importance of religion and belief within the life course, two important qualifications should be noted. First, there is a need for a broad perspective on religion, belief and spirituality that neither over-emphasizes the major religions, at the expense of other belief systems, nor the importance of formal religious sites, such as the Mosque, Church, Temple or Synagogue, over more informal locations (Kong 2001, 2010). Spiritual belief, in other words, can be relatively ubiquitous. Second, and reinforcing the last point, religion and belief are strongly relational. In short, "[r]eligion, like class and race, must be a matter for historical and place-specific analysis rather than taken as a priori theory" (Kong 2001: 226; Proctor 2006). McGuire's (2008) concept of "lived religion" (also Ammerman 2007) seems useful. This distinguishes "actual experience of religious persons from the prescribed religion of institutionally defined beliefs and practices." Whilst the latter focuses on "entire, single package[s] of beliefs and practices," lived religion acknowledges "how individuals attend to matters of the religious or the spiritual ... at a particular time and context ... in their own lives" (McGuire 2008: 25, 24, 19).

Religion and belief mold demographic aspects of the life course in numerous ways. Most obviously, belief systems usually have much to say about fertility and sexual behavior, from frequent condemnation of sexual activity outside of marriage to strong prohibitions on abortion. Attitudes towards morbidity and mortality are also significant, leading to contrasting outcomes via such things as acceptance or not of blood transfusions or organ donations. Even migration can be seen to be both enabled and constrained by religious discourses and practices (Freeman 2005), not least when understood in relation to ethnicity and gender.

\subsubsection{Sex and gender}

The life course arena of sex and gender again strongly expresses and entangles the biological and social. As with race and ethnicity, one can recognize a trajectory that goes from understanding the terms as relatively autonomous, expressing a biological/social divide, to both being seen as having bio-social aspects (Fausto-Sterling 2005).

To the general public, sex and gender tend to be treated as largely equivalent, fundamentally conjoined. Moreover, both are split into two "opposed" categories: woman versus man, boy versus girl, female versus male. In contrast, official bodies and social science recognize fundamental differences between the terms (Table 2.8). First, sex has been defined as primarily biological: "the characteristics you are born with as male or female; a different set of genitals and, for females, the ability to carry children, give birth and breastfeed" (Pain 2001: 120). In contrast, the social took the upper hand with gender. Understood less conclusively than sex as "an ongoing accomplishment" (Dozier 2005: 298), gender expressed "socially constructed ... aspects of being male or female" (Pain 2001: 120). As pioneering feminist Simone de Beauvoir (1949: 525) put it, "one is not born but becomes a woman." This performative perspective has shaped feminist scholarship strongly.

Both sex and gender clearly focus attention strongly on the body but such a focus reveals that any clear separation of the terms soon becomes problematic and oversimplified. Both have become "elusive ... mercurial" (Peake 2010: 55), expressed by bodies "simultaneously composed of genes, hormones, cells, and organs ... and of culture and

Table 2.8 Contrasting sex and gender: two expressions

\begin{tabular}{ll}
\hline World Health Organization & American Psychological Association \\
\hline Gender refers to the socially constructed characteristics of & Sex refers to a person's biological status and is typically \\
women and men such as norms, roles and relationships of & categorized as male, female, or intersex ... [I]ndicators of \\
and between groups of women and men. It varies from & biological sex ... include sex chromosomes, gonads, internal \\
society to society and can be changed. While most people & reproductive organs, and external genitalia. \\
are born either male or female [sex], they are taught & Gender refers to the attitudes, feelings, and behaviors that a \\
appropriate norms and behaviors. & given culture associates with a person's biological sex.
\end{tabular}

Both articulations of gender emphasize a link to socially-defined "appropriate" and "inappropriate" behaviors, and how nonconformity can provoke discrimination.

Source: APA (2011); WHO (2015). 


\section{POPULATION GEOGRAPHIES OF THE LIFE COURSE}

history" (Fausto-Sterling 2005: 1495). Complication has been reinforced by work with trans individuals (transsexual and/or transgender) "who live as members of the opposite sex to which they were born or as people who live between the categories male/female or man/woman" (Browne 2007: 116). Trans people fail to conform to any simple binary within either sex or gender, and may shift between categories (e.g. Dozier 2005; Lim and Browne 2009; Rubin 2003) through surgery and other biomedical procedures, for example.

Taking sex, a check-list of bodily characteristics could define "men" and "women," as with using years lived to define age (Box 2.1). However, "biological sex is a complex constellation of chromosomes, hormones, genitalia, and reproductive organs" (Dozier 2005: 298) that does not always neatly coalesce. With respect to sex-reassigned children, for example, where the sex initially attributed to a young person is subsequently changed, a social perspective on the individual's "appropriate" sex has been used to override a confusing biological situation (Fausto-Sterling 2000). This idea of social determination of sex has been taken still further, to argue that "gender is the knowledge that establishes meanings for bodily differences" (Scott 1988: 2). Such perspectives lead Dozier (2005: 298) to conclude that "sex is both a physical attribute and socially constructed."

Turning to gender, presenting it as completely socially constructed would, of course, immediately go against its popular correlation with sex. However, whilst much feminist scholarship has sought to promote separation (FaustoSterling 2005), it is hard to shake off all bio-genetic elements. This is particularly the case if "the social" within the social construction of gender is regarded as heterogeneous. This mixture includes, and probably prioritizes, the cultural (perhaps entangled with the economic; as in some accounts of patriarchy, introduced next) but can also include the biological sphere, making gender truly a co-construction. Nonetheless, the present authors reject biologically deterministic explanations for gender differences in (demographic) behavior.

Within the social construction of gender, a key role has been accorded to patriarchy. This can be defined, following Walby (1989: 214; also Walby 1990, 2003), as "a system of social structures and practices in which men dominate, oppress and exploit women." For Walby, patriarchy comprises six elements: gendered exploitation within the family; gendered exploitation within paid work; a gender-biased state; male violence; sexuality; and a patriarchal culture reinforcing the other elements. The net result is, simply put, diverse manifestations of sexism. This refers to prejudice or discrimination based on a person's sex, that negatively impacts on women (trans people also experience forms of sexism). Whilst patriarchy is deeply rooted historically and geographically (e.g. Bennett 2006), Walby regards the paid workplace and the domestic sphere as its key sites today. This emphasizes again the contextual relationality of gender construction and interconnection between gender and class.

Both patriarchy and gender, within the household/family and beyond, have clear demographic expressions. Male priorities and domination have been strongly linked to expressions of fertility behavior, migration and, although less clear overall, mortality and morbidity. Sex, too, very obviously takes a leading role with respect to fertility (only women give birth!). It is also expressed through differential mortality and morbidity rates, although, as with race (Box 2.5), one must be wary of making simplistic genetic explanations.

\subsubsection{Sexuality}

The final arena of differential demographic life course experiences that places people into relatively distinct population groupings is equally as elusive as sex and gender. Indeed, it is frequently considered together with these categories (e.g. Hockey and James 2003; Peake 2010). Sexuality is defined as the capacity for sexual feelings and the subsequent orientations and preferences expressing this capacity (sexual orientation). Typically, a three-fold distinction is made, based on the sex-defined group a person is sexually attracted to: heterosexuality, persons of the opposite sex; homosexuality, persons of the same sex; bisexuality, persons of either sex. However, unsurprisingly perhaps given the problems of defining sex, both sexuality as existing on a continuum rather than in discrete categories (APA 2011) and the presence of other forms of human sexual attraction have been recognized. Pansexuality expresses attraction toward all people, oblivious to biological sex or gender identity, whilst generally considered morally deviant forms, such as zoophilia (attraction to animals) and pedophilia (attraction to children), also exist.

Understanding sexuality has, once again, increasingly seen social-cultural accounts displace bio-genetic explanations. Whilst sexuality is generally accepted as emerging through a life course, notably associated with life transitions of adolescence and adulthood (Table 2.2), there is considerable debate as to the extent it is established in the womb, as a baby develops, or is contextually shaped through life. A key proponent of the latter has been

\section{ST PROOFS: NOT FOR DISTRIBUTION}


Philosopher Judith Butler (1990,1993). Through her emphasis on life as performance, Butler regards both sexuality and gender as social identities only achieved, written on the body, and attaining a taken-for-granted "normal" state through forms of contextualized embodied repetitive actions and practices.

Whether or not one accepts Butler's ideas, attention to sexuality as performed immediately draws attention to heteronormativity within everyday life across most of the world. This refers to how "heterosexuality is the norm through which all other sexual identities must be understood" (Del Casino 2009: 203). Resisting such "compulsory heterosexuality" (Rich 1980) can be challenging. For example, young people's experiences of schooling typically reinforce numerous heterosexual norms within formal classes, physical educational activities and the culture of the playground (Del Casino 2009). Associated with heteronormativity and reinforcing it is homophobia, or prejudice and antipathy towards homosexual people.

Sexuality is now widely acknowledged, within both life course (e.g. Hockey and James 2003; Hutchison 2010) and Geography scholarship (e.g. Browne et al. 2007; Oswin 2013), as of core importance within everyday life. Resisting both heteronormativity and homophobia, non-heterosexual experiences have frequently been brought to the fore, expressing difference. In terms of demographic behavior, sexuality is expressed in contrasting practices of fertility, as in how same-sex couples obtain and raise children, and in distinctive forms of migration, not least in "queer"-"a shorthand, umbrella term for all lesbians, bisexuals, gay men and transgendered [LGBT] people" (Brown et al. 2007: 12)relocation to more tolerant or homonormative residential environments. In terms of mortality and morbidity, relationships between sexuality and different sexually transmitted diseases merit attention.

\subsubsection{Individuality}

After introducing macro-contextual and bio-social arenas shaping differential demographic experiences across the life course, there remains the question of where the individual as individual fits into all of this (Dienstbier and Crockett 2002). An initial sense of this place comes from a widely repeated quotation from Karl Marx, expressed in his 1852 extended essay which examined the historical role of the individual. This is presented next to a not dissimilar quote from life course researchers Michael Shanahan and Glen Elder:

For Marx (2010/1852: 5):

"Men (sic.) make their own history, but they do not make it as they please; they do not make it under self-selected circumstances, but under circumstances existing already, given and transmitted from the past."
For Shanahan and Elder (2002: 176):

"People can shape their lives, but they do so within socially structured boundaries, as reflected in historically-changing opportunities and limitations."

In other words, central to a life course perspective (also Elder et al. 2003) is the proposition that while people "create" their lives, this activity does not spring from wholly independent and original human agents. Instead, people construct their life courses more circumstantially or relationally. Such relationality expresses the "weight of history," where this history is itself a metaphorical onion of many layers. "History" is also geographical and bears the marks not just of "great events" but also, at the very least, the bio-social categories introduced above.

In exerting human agency across the life course, the practical realm of "planning, reflexivity, and perseverance" (Shanahan and Elder 2002: 173) or the ability to think through options and then enact decisions should not be overlooked. The experience of having a child, for example, may be very different when carefully planned and organized than when more accidental and improvised. Or the forms of migration predominant across the Global North, for example, being usually highly intentional and heavily invested in, differ considerably from the often immediate and lifesignificant upheavals of migrating as a refugee. Some sense of this differentiation of individual experiences can be captured by an individual's overall demographic competence (after Clausen 1991a, 1991b) or capability to perform demographic actions. This ability both to assess options and potentials and to act on them is, first, strongly related to resources. Second, and related to the latter through, for example, psychological resources of knowing who and where one is within the bigger positional scheme of things, demographic competence is further related to belief in ability to make a difference, or self-efficacy (Bandura 1997; Gecas 2003). Gaining demographic competence is complex,

\section{ST PROOFS: NOT FOR DISTRIBUTION}




\section{POPULATION GEOGRAPHIES OF THE LIFE COURSE}

incorporating "genes, parental [and other] care and guidance, ... physical developmental experience, the social matrix in which we find ourselves, and large social and cultural processes" (Clausen 1991b: 5; Gecas 2003).

To leave things at the structured situation of demographic competence would still rather lose the individual as individual. Production of history is not just a matter of competence but involves a degree of individuality and personality (Moser 2008). The individual is not just constructed by society (Alwin and McCammon 2003). All the biosocial categories noted above may be associated with certain demographic behaviors but congruence is never 100 percent. There are always anomalies and outliers. Sometimes these may reflect biomedical conditions, such as ability to have children, but frequently they will express individual or family decisions unrelated to positionality, except in the sense that "[a]gency and choice imply an empowered and embodied individual" (Hockey and James 2003: 139); hence the significance of acquiring diverse resources.

More individualistic expressions of demographic behavior within the life course cover various strands. First, there are expressions that may be explained through reference to the unconscious and other psychological concepts (Callard 2003; Pile 1996) or "subliminal processes" (Crockett 2002: 23). Second, there are expressions brought about by social and extra-social affects (Anderson 2014; Blackman and Venn 2010), felt and consequently embodied emotionally but often poorly expressed in language (Pile 2010). Third, there is more deliberate expression of demographic difference. This includes "political" agency articulating how "[p]eople have a million ways of saying No . .. [through] scream[s] of non-identity" (Holloway 2002: 205, 151) expressing (demographic) dissidence. Such behavior may extend beyond individual acts to become "a movement of refusal-and-other-creation" (Holloway 2010: 6). Fourth, there is demographic expression of individuality contained within "simple" human variety and its variation of likes and dislikes, priorities and persuasions. This is an "excess" that makes each of us who we are. Chiming with Marx's quote, Clausen (1991a: 805) observed that "societies and their cultures provide rough scripts and casts of characters whose interactions tend to shape individual lives" but ultimately there are still individual demographic lives. Lives, as the chapter has stressed, are common and individual, individual and common.

\subsection{CONCLUSION: REPRESENTING LIVES ACROSS SPACE}

This chapter has introduced key elements and principles of a modified life course perspective through which the essential relationality of individual lives can assume a more prominent place within Population Geography. This was labeled an extended life course perspective to reflect not just Elder's (1974) focus on cohort but all arenas-macrocontextual, bio-social, individual-through which life courses are constituted. Centrally, while all lives are individual, they are also common, relatively distinct population groups congealing from the arenas.

It is important to add here that the nine arenas of life course differentiation do not simply operate independently. Whilst something of their respective constitutive significance has been suggested in Section 2.3, the complex consequences of their entanglements with each other through a life course and its specific demographic expressions must be noted. This is notwithstanding that specific arenas may (often) assume overwhelming predominance (Walby et al. 2012) in particular situations.

Useful for understanding better this sense of contextual relationality is the concept of intersectionality. Developed within feminist scholarship, this signifies:

complex, irreducible, varied, and variable effects which ensue when multiple axis of differentiation-economic, political, cultural, psychic, subjective and experiential-intersect in historically specific contexts. The concept emphasizes that different dimensions of social life cannot be separated out into discrete and pure strands.

(Brah and Phoenix 2004: 76, emphasis added)

In other words, interrogating a specific demographic expression, such as a birth, must neither "explain" it too narrowly-simply reflecting parental class position, for example-nor assume the role played by class in explanation will necessarily be the same if the parents are of the same class but differ in other dimensions, such as ethnicity. Whilst the former qualification is generally appreciated, the latter presents considerable research challenges. For example, as if defining gender and sex was not hard enough, intersectionality emphasizes how "'race', social class and sexuality differentiated women's experiences has [additionally] disrupted notions of a homogeneous category 


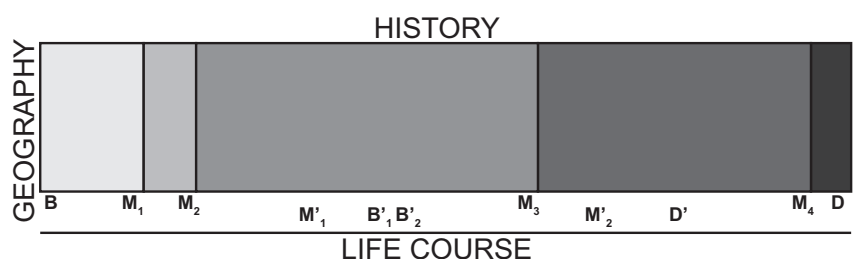

Figure 2.3 A simplified diagram of an individual life course.

"woman'" (Brah and Phoenix 2004: 82). Intersectionality focuses attention on how, in the "mutual shaping" of an experience, "systems and social relations change each other at the point of intersection but do not become something totally different" (Walby et al. 2012: 235). Arenas operate according to their own logic but when they intersect the result may not simply be the sum of each.

Adopting an extended life course perspective not only foregrounds the relationality of lives lived across space but allows Population Geography more fully to reconnect with other aspects of the spatiality of everyday life overall. Whether or not the sub-discipline should or could even ultimately be re-cast as Life Course Geography remains a moot point. Certainly, such a significant overhaul would demote substantially the key demographic elements of births, deaths and migrations that have long served as the basis of the sub-discipline and whose priority the scholarly division of labor may well wish to retain.

Leaving the potential future of Population Geography and to conclude this chapter, Figure 2.3 presents an indicative simple graphical representation of a life across space. It depicts a life, from birth (B) to death (D), along the intermediate time scale (2.3.2.1) of individual longevity. Across this period, the person lived in five different locations, each new place attained by a migration $\left(M_{1}-M_{4}\right)$ and shaded differently in the figure. The individual also did not live all their adult life on their own. After leaving the family home, s/he was joined by a partner $\left(\mathrm{M}_{1}{ }_{1}\right)$. They went on to have two children $\left(B_{1}^{\prime}, B_{2}^{\prime}\right)$ but later separated, with the partner moving away $\left(M_{2}^{\prime}\right)$ before dying soon afterwards ( $\left.D^{\prime}\right)$. Towards the end of the individual's life, s/he also moved $\left(M_{4}\right)$, possibly into sheltered housing.

Clearly, diagrams similar to Figure 2.3 could be constructed for "real" individuals, although needing to be much more complex and messy ${ }^{1}$. Whilst mapping an individual life course may prove to be extremely challenging, real world convergences of demographic outcomes in more regular expressions enables Population Geographers to map characteristics of populations, not least their spatial distributions. Some of these are now introduced and explored globally in Chapter 3.

\section{NOTE}

1 Nancy Worth's $(2009,2011)$ life maps methodology could also be used to demonstrate this messiness. She has used it to investigate young visually-impaired lives, not least through allowing them to highlight key life transitions, including those associated with demography. 


\section{Global spatial distributions of population}

\subsection{INTRODUCTION: SNAPSHOTS OF PEOPLE IN THE WORLD}

Chapter 2 argued that geography is one of the key arenas through which individual lives across space are constructed. However, those same individual lives considered in aggregate also express a series of recognizable geographies. This empirical perspective of spatial distribution (1.3)-in this case of the global population-is this chapter's focus. The results of such an exercise are always snapshots that temporarily freeze process. Nevertheless, these pictures present challenges taken up by subsequent chapters, namely to explain the patterns through engagement with those temporarily hidden processes. Furthermore, whilst observed spatial distributions may not determine demographic behavior, they do provide vital contextual elements of the geographical arena of life course experience within which such behavior occurs (2.3.3). In summary, one recognizes the importance of the following questions long posed by Population Geographers:

- Where are the people?

- Why do they live there?

- What types of spaces are these habitations?

By sketching some answers to these fundamental questions, the chapter establishes an overall empirical framework that engages themes addressed throughout the book. Section 3.2 provides a snapshot of the spatial distribution of the Earth's present population, plus a brief history of how humanity got there. It introduces basic concepts of population composition and growth, and the demographic implications in-built within different population structures from the past for future growth. Section 3.3 then divides the populated world into urban and rural places. It introduces key patterns and processes perpetuating these settlement types. Qualifying the preceding empirical snapshots, Section 3.4 concludes by emphasizing the inherent dynamism within the ever-evolving placement of people on the planet.

\subsection{THE POPULATED WORLD: A GLOBAL DEMOGRAPHIC PERSPECTIVE}

\subsubsection{Global population growth, population density and the Ecumene}

October 312011 was symbolically attributed by the United Nations as the day Earth's population reached seven billion (UN 2011). Only a dozen years earlier, in 1999, this population had attained 6 billion: in less than 13 years, humanity had added 1 billion people to the planet! An estimate for mid-2015-relentlessly rising on the Population Research Bureau's World Population Clock-was 7.328 billion (PRB 2015a). Elsewhere, there is the widely reported United Nations 10.1 billion projection for 2050 (Dorling 2013), returned to in Chapter 11.

In spite of this recent dramatic global population growth, illustrated earlier in Figure 1.1, it took all of human history until 1850 to reach the first billion humans worldwide (Poston and Bouvier 2010). In other words, it is over just the last 150 years that the global human population has, fairly consistently, grown so rapidly. In the twentieth century alone, world population increased by 50 percent between 1900 and 1950 but by 200 percent between 1950 and 
2000 (Hirschman 2005). All-in-all, the numerical presence of humanity on Earth has pushed itself aggressively forward for scholarly and popular scrutiny in recent decades as never before: big numbers and big issues (1.2.1).

Figure 3.1 presents a long-term simplified picture of global population growth. It depicts, first, a J-curve of accelerating exponential growth, before anticipating a leveling off of population through declining growth rates, eventually producing the S-curve of logistic growth (Woods 1982). The final steady state for such a curve would be a relatively stable global population. Again, Chapter 11 revisits this issue.

Reflecting briefly on the history of global population, the dominant theme is slow growth (Biraben 2003; Hirschman 2005). Prehistoric hunter-gatherer societies had both high fertility and mortality and while mortality declined over time for agricultural societies, largely because of increased nutrition and food security (Box 3.1), population growth remained quite low. Beginning around 1600, however, global population began to expand more consistently, albeit frequently violently disrupted by population crashes linked to such things as plague epidemics. After reaching 1 billion (around 1850), further improvements in food security and advances in medicine (antibiotics and immunizations, in particular) facilitated longer life expectancies and lowered mortality. Population growth rapidly accelerated. Consequently, global population reached 2 billion in 1930, 3 billion in 1959, and 6 billion in 1999. Put differently, the time it took world population to double-doubling time-declined between 1850 and 1999 from 130 years (1-2 billion) to just 40 years (3-6 billion). With global population growth rates recently estimated at around 1.2 percent per year, doubling time rose to about 58 years (Poston and Bouvier 2010: 275) and continues to grow.

Discussion so far has only considered the global birds' eye view of population growth. Where human populations are growing (or declining) and why are further key questions posed by Geographers to qualify this overall picture. Simply put, today, at a national scale, some countries are growing rapidly, such as China and India, while others, such as Russia and Japan, are facing possible population decline. Refining this view, urban areas are growing notably faster than rural areas, and particular neighborhoods within urban areas are growing strongly while others lose residents. Global figures and perspectives are thus important but such changing of the scale at which population growth, decline, and distribution are assessed greatly changes their implications for local areas. How scale is produced and utilized

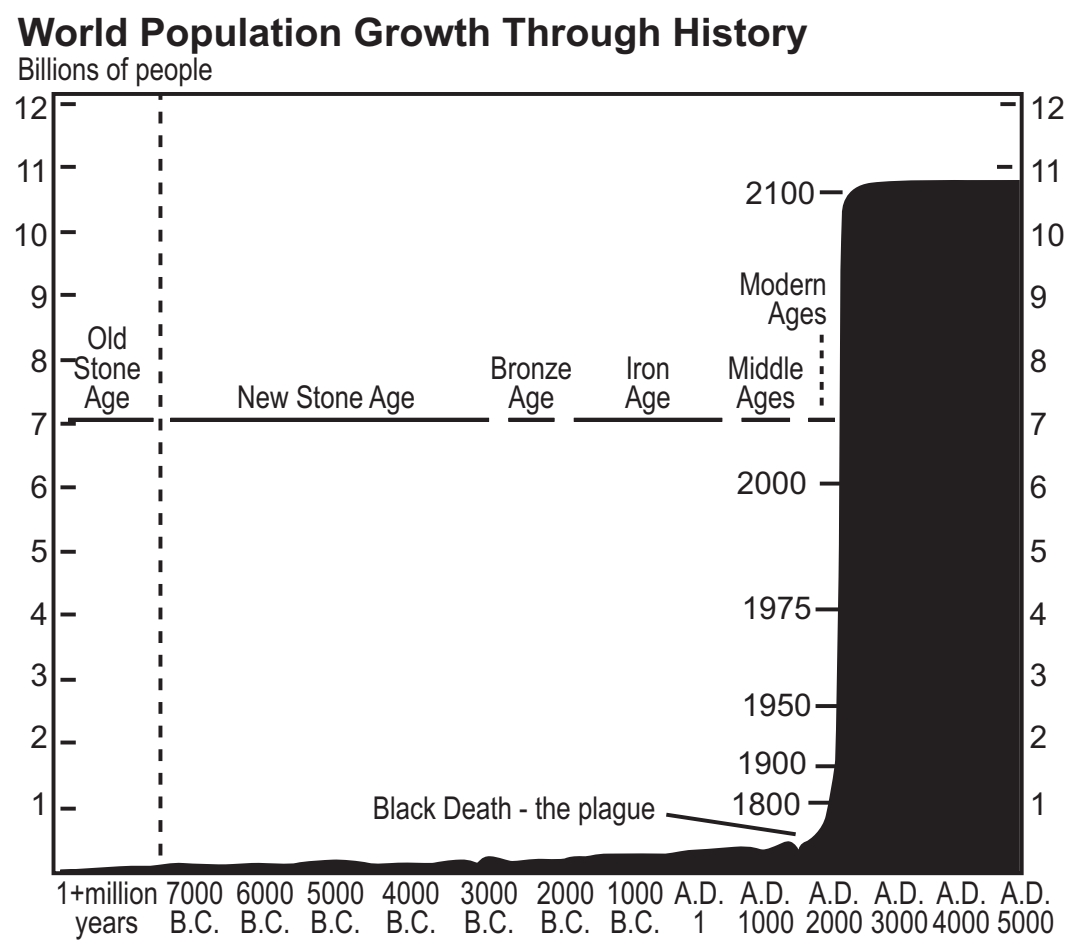

Figure 3.1 World population growth through history.

Source: McFalls (2001: 33). 


\section{BOX 3.1 AGRICULTURE AND POPULATION IN PREHISTORY}

Centrally but complexly entwined with global scale growth of the human population in the Prehistoric period was the development of agriculture. As a distinctive set of practices-contrasting with previous hunter-gathering or pastoral transhumance (seasonal movement of people with livestock) societies-agriculture emerged in the Fertile Crescent of west Asia (from the Persian Gulf through parts of Iraq, Syria, Lebanon, Jordan and Israel to Egypt) around 11,000-12,000 years ago. It signified the beginning of the Neolithic period, which lasted until the Bronze Age around 5,000 years ago. Within the Neolithic, and reflecting how the ancient world was substantially less interconnected than today, subsequent developments in agriculture occurred in various locations largely independently: central China, West Africa, New Guinea Highlands, Mesoamerica, central Andes and eastern North America. In Europe, agriculture was not developed so independently but was imported via migrants from the Middle East.

Agricultural evolution began with the cultivation of wild cereals (e.g. einkorn, wheat, millet and spelt). This promoted a largely sedentary place-based way of living that can ultimately be seen leading to the development of the first cities. Agricultural development also saw domestication of animals-dogs, sheep and goats, and later cattle and pigs. Food supply generally became more stable and certain.

Focusing on Europe as an example, population growth at first sight seems clearly to be linked to this development of sedentary agricultural society. At the start of the Neolithic period, Europe's entire human population has been estimated at around just 200,000. By around 7,000 years ago this had risen to just 400,000 but by the start of the Bronze Age it had attained 2 million.

However, to see any definitive causal link between agricultural development and population growth is oversimplistic. First, the increase in Europe's population was not solely or even largely due to increased births and/or reduced infant mortality (both of which might be associated with improved regular food supplies from agriculture). Instead, as already suggested, growth significantly involved in-migration as Europe's climate improved, such as from modern Turkey to Greece. Second, societal change during the Neolithic period did not just comprise development of agriculture but also involved many other technological innovations, from manufacturing to transportation, all of which may be seen to have greater or lesser population change consequences. Third, research has recently suggested that rather than a one-way relationship of sedentary agriculture stimulating population growth, the reverse may also have been true. Specifically, genetic evidence indicates major population growth may have occurred in Africa, Europe and the Americas prior to the development of agriculture. In other words, increased population may have driven the development of agriculture, turning the latter from supplementary to primary food source. Fourth, whilst overall population grew during the Neolithic period, this was very uneven. Overall, still relatively low levels of technology and food security, plus considerable poverty and frequent occurrence of disease epidemics constrained substantially both population growth and socio-economic development. In summary, population growth must again be understood in full relational context rather than reduced to the result of any simple cause and effect occurrence.

(Sources: Bellwood 2004; Biraben 2003; Gould 2015; Zheng et al. 2012)

(Marston 2000) thus becomes a pertinent issue for Population Geographers. Just thinking about all of these dimensions immediately indicates the potential complexity contained within the sub-discipline even at just the spatial distribution level.

Attention now turns to considering population distribution, how populations are arranged across the globe. They can be concentrated, clustered in a particular place, or dispersed across a large geographic area. One way to measure such distribution is by calculating population density (Poston and Bouvier 2010: 304). Density is simply the frequency by which something occurs across space, and population density is the number of persons per square kilometer, square mile, or other areal measure.

Consider the residents of the US state of Minnesota. It had a population of 5.3 million in 2010, distributed across $128,199.5$ square kilometers. This gives a population density of 5.3 million divided by $128,199.5$ or 41.3 persons per square kilometer (US Bureau of Census 2010). However, also consider the population density of defined groups within 
the overall Minnesota population. For example, for this quite agricultural state, one might look at the number of farmers per unit of area of farmland. Or, one could calculate the physiological or nutritional density, which is the number of persons per unit of arable land (Poston and Bouvier 2010: 305). Each of these measures relates something different about the population of Minnesota and are usually compared to the same measures calculated elsewhere.

Population density, however, fails to tell us where within Minnesota the population is located. As it turns out, 2.8 million of the 5.3 million (53 percent) lived in just seven of the state's 87 counties in 2010 (MetCouncil 2012). In other words, the population was highly clustered, concentrated in a relatively small geographic space. Such clustering can be measured using various levels of statistical sophistication (Woods 1982). These enable quantification and description of the spatial distribution of people in diverse ways.

Briefly observing the distribution of the Minnesota population highlights how, even at quite small geographic scales, population growth and distribution are frequently spatially highly uneven. This is true for virtually any areal unit assessed: Earth, continent, country, state, district. Even with more than 70 percent of the planet's surface area covered in water bodies, the remaining land areas can be divided into areas that are more or less habitable, and more or less inhabited.

The Ecumene is the portion of the Earth's surface occupied by permanent human settlement. Within it, several areas of high population density are apparent, shown in Figure 3.2. These include South Asia, primarily India; East Asia, primarily China; Southeast Asia; Western Africa; and Western Europe. High density areas also include megalopolises (chains of near-continuous metropolitan areas) on the east coast of the US, areas around Lake Victoria in eastern Africa, and the Sub-Saharan area of central Ethiopia. At the global scale, one can also generalize about populations being largely concentrated in particular physiographic environments, such as areas in close proximity to oceans.

A variety of factors have historically influenced population distribution at a sub-national scale. These include proximity to resources, historical distribution of lands and land tenure issues, transportation systems, distribution of

\section{Distribution of World Population 2015}

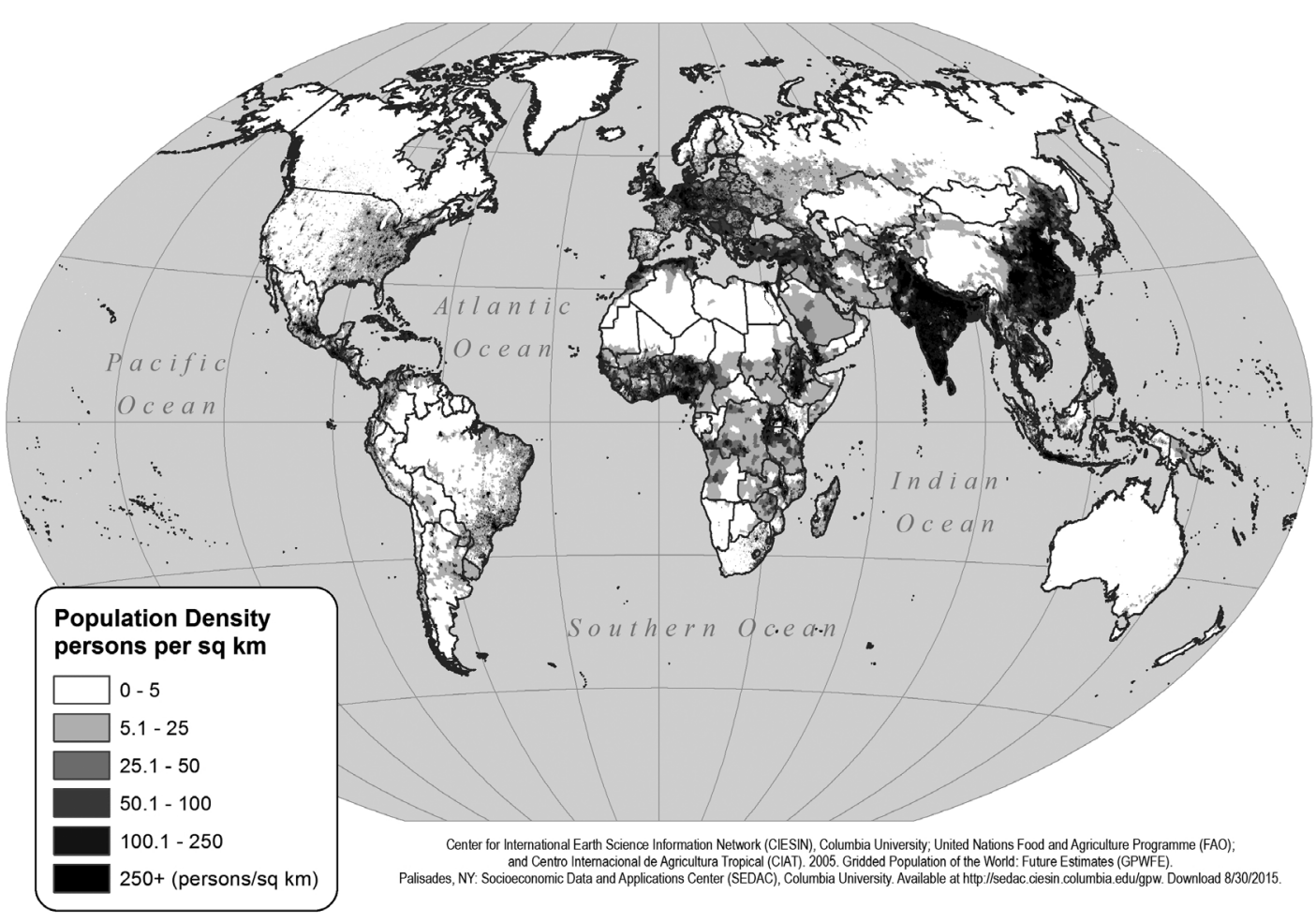

Figure 3.2 World population distribution 2015.

Data Source: Center for International Earth Science Information Network (CIESIN), Columbia University; United Nations Food and Agriculture Programme (FAO); Centro Internacional de Agricultura Tropical (CIAT) 2005; SEDAC. 
amenities and individual preferences. They are inextricably entangled with the arenas of life course construction outlined in Section 2.3. In South America, for example, the population is not evenly distributed but clustered along the coastlines and a few interior cities, leaving large areas of low population density. Knowing the extensiveness of the Amazon rainforest and the difficulty of living in the Andes mountains, for example, gives immediate physical geographic clues to these spatial patterns, even if these factors alone are unable to explain the full picture.

Fortunately, Geographers' methods to measure population density and distribution have evolved as rapidly as the populations being measured. Censuses (Box 3.2), population registers and surveys that count the number of people who reside in a particular area help production of density measures. Advanced technologies, such as satellite images and aerial photography, can further be employed for population estimation. For example, Rindfuss et al. (2002) utilized Geographic Information Systems and remote sensing to link data about changes in village practices and landscape change in Nang Rong, Thailand. Similarly, Fox (2002) utilized aerial photography and socioeconomic data to assess the multiple dimensions of land change, including the implications of land tenure policies, on land use practices in several villages in northeastern Cambodia. These technologies are increasingly accessible-think of images available via Google Earth-and provide yet another way to visualize population patterns.

\section{BOX 3.2 THE POPULATION CENSUS}

A census is "[a]n enumeration ... to provide needed data for state purposes" (Johnston 2009b: 74) and a population census is a count specifically targeting people. The population census has a long history, stretching back to Egypt 3,000 years ago and other ancient civilizations. Of note was its use in Roman times, with the word census coming from the Latin censere, "to estimate." The census played a crucial role in determining taxes and administering the Roman Empire. It was usually carried out every 5 years and provided a record of citizens and their property.

Censuses are found today worldwide, as the United Nations' Demographic Yearbook records, split between de facto enumerations, recording individuals where they are found on the census day, and de jure enumerations, recording individuals where they usually reside. Mostly, the world's censuses focus on measuring numbers and selected characteristics of people and/or housing. There are also more specialized versions that enumerate agriculture, industry, retailing, traffic, and so on. A 10 year period is typical for census enumeration, cost and complexity working against more frequent counts.

The US Constitution requires a census every decade in order to apportion the number of members of the House of Representatives for several states. This example is indicative of how census data tend to be used today. With taxation now usually deducted through the workplace, their data have become less associated with this role. Instead, they are used for such things as apportioning state funding that comes from this taxation and investigating issues pertinent to social policy. In general, "the primary role of the census is to collect factual information to inform public policy" (Johnston 2009: 74).

As indicated, administration of a census is a major task even for small countries, let alone huge states such as China or India. This is because they target as large a proportion of the population as possible, at least officially. Key aspects of the process include, first, division of the country into small manageable areas, known by terms such as "collectors' districts" or "enumeration districts." Second, these areas are overseen by a highly trained administrator and his/her team responsible for gathering in the forms on which census data are collected. Data are usually entered on these forms by residents themselves but in some cases, such as due to illiteracy, the census team may do it. Then begins the huge tasks of analyzing the information gathered, producing summaries and reports, and making allowance for people missed. For these, computers have become an invaluable tool, and websites are now the main way census data are accessed by interested parties.

Whilst the census is now globally institutionalized and its data widely used by government and researchers, it is a far from uncontroversial data gathering tool. Besides its tendency to put people into "boxes" or abstract "populations" (1.3.1), often more clearly defined (e.g. ethnicity, occupational classes) than they exist within the multiple positions of the life course, and the difficulty of defining even such seemingly straightforward facts as place of residence (problems: students living away from home; refugees; people on vacation, with more than

\section{ST PROOFS: NOT FOR DISTRIBUTION}


one home, without a fixed address), it is how the data collected may be used that provokes most concern. From breaching the privacy of the home that data collection involves, to subsequent deployment of the "facts" collected, ethical issues abound. Reflecting such concerns, access to and presentation of census data typically requires a tough ethical audit. For example, in terms of the geographical areas for which data are released, where very few people fit a specific category in a certain area (e.g. people born in Africa in remote rural Ireland), data are usually not released for that area to avoid possible recognition of individuals.

Further reflecting ethical concerns but also due to the high costs and a feeling that requisite data are available by other means, recent years have seen some countries cancelling regular population censuses. The UK census of 2011 may be its last, in spite of Dugmore et al. (2011: 620) arguing that "no other resource provides an equivalent demographic snapshot of the population, taken at a single point in time, by a consistent method, across all areas in the UK." In Germany, the Constitutional Court stopped the census in 1980 and 1983 , and the country's last census was in 1987. Since then, a sample of the population has been surveyed rather than attempting to engage everyone. Such an alternative requires complex further statistical work to ensure conclusions drawn from the sample are representative of the whole population. The Netherlands and Scandinavian countries also rely on population registers rather than censuses. Registers require residents to notify their municipality where they live and provide details of partners, children and migrations. As such, they are not immune from concerns about privacy and civil liberties. Indeed, any census replacement brings strongly into the light just how much information about each person is obtained and retained by the state and, increasingly, the private sector. Lives lived across space are certainly not unrecorded lives but how they are and should be recorded present tough political and ethical challenges.

(Sources: Dugmore et al. 2011; Holdsworth et al. 2013: 41-46; Johnston 2009; Statewatch 2012; UNStats 2015)

\subsubsection{Population pyramids, migration and the demographic equation}

Although climate and resource accessibility, particularly water and fertile soils, historically played important roles in determining where populations concentrate, numerous other factors facilitate or favor contemporary population growth and distribution. Many relate to the arenas of life course experience (2.3) whose influences on Population Geography are discussed in subsequent chapters. Of immediate demographic consequence are two "internal" influencing factors of the population: age and sex structure. These contribute to population change through age distribution and sex ratios, respectively. They are given graphical expression in population pyramids (Poston and Bouvier 2010: 240-245; Holdsworth et al. 2013: Chapter 4). These bar graphs or histograms depict separately but back-to-back the number of men and women in discrete (generally 5- or 10-year increments) and ordered age categories. A pyramid's exact shape has major implications for subsequent growth or decline in a population, as well as bearing the marks of historic events that have major differential impacts on a population, such as wars and periods of economic prosperity. Population pyramids, in other words, present "a kind of social memory" (Vassin 1996: n.p.).

Consider Russia's population pyramid for 1950, shown in Figure 3.3. Here, the notably small bar for adult males strongly expresses the immense losses experienced during World War II that differentially impacted on younger men (Demeny 2003). Now compare recent population pyramids for China and Afghanistan, in Figure 3.4. These reveal distinct demographic experiences. China's state-sponsored efforts to restrict population growth (4.6.3) served to check the country's relatively young and growing population, pinching the pyramid at the base (reduced fertility). In contrast, Afghanistan's pyramid expresses a comparatively young and rapidly growing population of large families, indicated by the wide base and narrow peak.

Yet more critically, population pyramids not only express history but also suggest future population trends through demographic momentum (Holdsworth et al. 2013: 66). This is due to the macro or structural effects of age's association with demographic behaviors. For example, relatively young populations with at least moderately high birth rates, such as in Afghanistan, will likely experience population growth faster than populations with smaller cohorts of young people and correspondingly larger numbers of older people. This, quite simply, reflects the relatively higher number of women of childbearing age in the former. Such a fast growth rate expresses high natural increase (excess of births over deaths). 


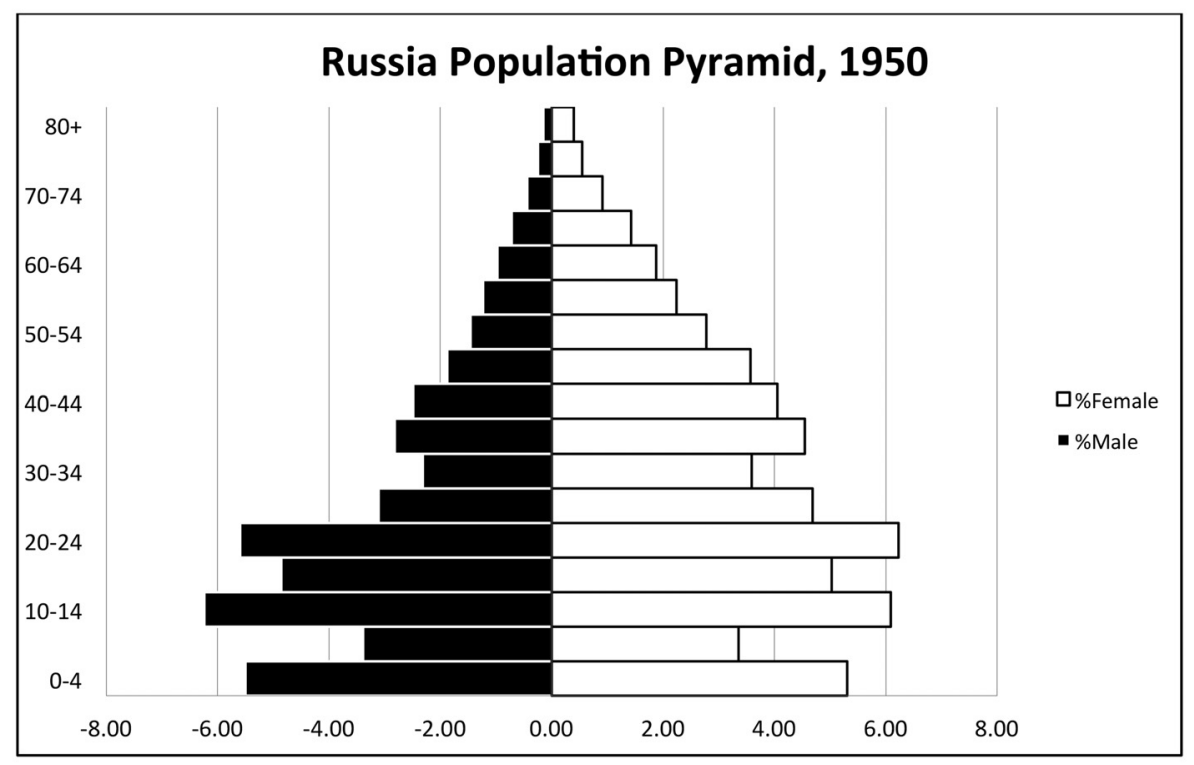

Figure 3.3 Population pyramid for Russia in 1950.

Source: UN (2015).

And, within a country, if some regions have higher natural rates of increase than others, population growth and increases in density will occur more rapidly in these areas. Population Geographies thus take shape, subsequently feeding into socio-spatial issues such as, for example, how an ageing dependent population (10.2.3.5) is to be supported by the rest of the population (Holdsworth et al. 2013: Chapter 4).

Whilst population pyramids can reveal demographic pasts and have considerable structural implications for the demographic futures of a specified geographic area, any account of the spatial population distribution must also take into account migration's role shaping its geography (Boyle et al. 1998; Castles et al. 2014; Samers 2010). Migration redistributes humans from one area to another, as Section 3.3 and Chapters 5-9 abundantly illustrate. Associated with migration, urbanization continues to relocate a greater and greater proportion of the global population into cities, occurring particularly fast in the Global South. Additionally, international migration redistributes people between countries, to the extent that by the early 1990s it was estimated that 1 in 40 of the global population (nearly 120 million people) resided outside their country of birth (Bailey 2005: 108).

As the only ways the size of a population can change, demographers have brought together mathematically natural increase (or decrease) and migration. As Poston and Bouvier (2010: 5) expressed it: "There are only two ways of entering a population-being born or moving into it. There are also two, and only two, ways of leaving a populationdying or moving out of it." This overall situation is represented by the demographic equation:

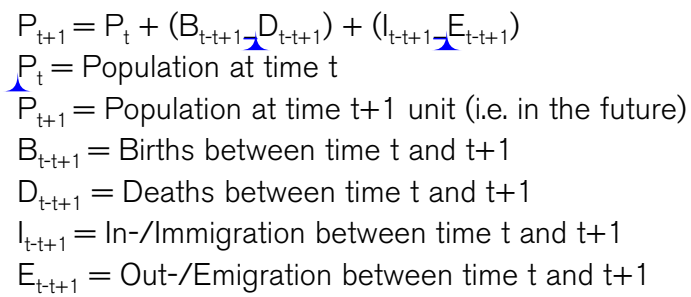

Subsequent chapters will consider the constitution of all four of the variables $(B, D, I, E)$ that together continuously but variably add to or subtract from a population. Next though, this chapter delves into the socio-demographic processes shaping sub-national population distributions. 

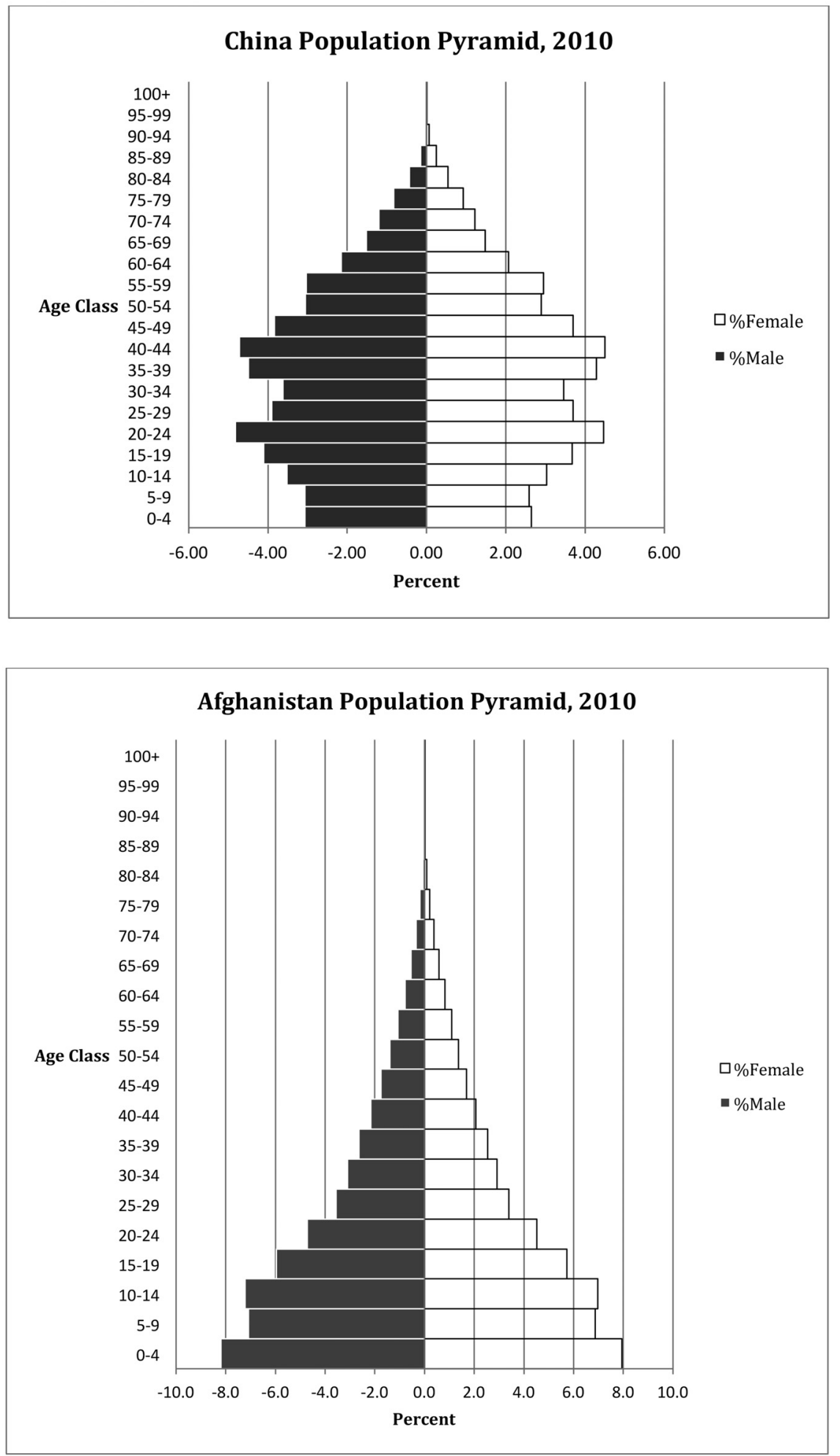

Figure 3.4 Population pyramids for China and Afghanistan in 2010.

Source: United States Census Bureau (2010). 


\subsection{THE ECUMENE: URBAN, INTRA-URBAN, RURAL SPACES}

\subsubsection{The populated world}

The section introduces the more detailed spatial distributions of population across the Ecumene. It focuses, first, on the ongoing rapid growth of urban areas; second, on four processes working to differentiate these urban areas both demographically and in broader social ways; and third, by paying attention to the changing demographics of remoter, rural locations. Throughout, numerous processes and experiences impacting and expressing births, migrations and deaths are noted, all returned to in more detail in subsequent chapters.

Throughout this section, precise definitions of "urban," "rural," "suburban," and so on remain largely unconsidered. Producing such definitions remains a lively, contested and seemingly endless task both within and beyond Population Geography (e.g. Champion and Hugo 2004; National Academy of Sciences 2015). Within national or transnational statistics agencies, for example, there are typically numerous different classification schemes. Table 3.1 gives an example from the US of three complementary or competing classification codes used by the US Department of Agriculture's Economic Research Service. These classifications utilize such things as settlement size, distance from large settlements, and dominant commuting patterns to define the extent an area is urban(ized).

Utilizing Population Research Bureau (2015b) definitions both of urban and rural, and of regional and economic development country groupings, Table 3.2 presents the spatial distribution of the population across the globe in further detail. Setting the context for the rest of this section, it indicates the global predominance of urban populations and their projected future growth, the continued numerical significance of the rural population, and the considerable variation in both these observations by region and developmental state.

\subsubsection{Urban areas: densest and fastest growing places on earth}

As recently as 1950, only just over a quarter (29 percent) of humanity lived in an urban area (United Nations 2010). The situation is now very different, as both Table 3.2 and Figure 3.5 demonstrate. In 2008, the United Nations announced that the world had reached "an invisible but momentous milestone" of having more than half its population, 3.3 billion people, living in urban areas. By 2030, this population is expected to reach almost five billion (UNFPA 2007a). In other words, contemporary populations within the Ecumene are increasingly urban, with ever greater numbers crowding into a growing and increasingly dense but still limited number of major centers (Knox and Pinch 2009).

This urbanization, the transition from a predominantly distributed, rural settlement pattern to a densely populated urban settlement pattern, is not, of course, a novel geo-demographic trend. Notwithstanding early cities, modern times have seen two main urbanization waves. The first took place across the Global North, notably in Europe and North America, between 1750 and 1950. Consequently, having attained highly urbanized societies, the North is now seeing only small increases in its proportion of population residing in urban areas. The second urbanization wave, ongoing since 1950, is occurring across the Global South (UNFPA 2007a). Table 3.2 thus shows the Less and Least Developed categories accounting for the vast majority of ongoing growth, with Asia and Africa the most rapidly urbanizing regions. The net consequence of this South-North variation in urban growth today is movement towards global convergence of urbanization levels.

There are other notable geographical contrasts within urbanization besides distinctions between Global North and South. For example, mega-cities (Yeung 2009)-those with 10 million or more population, such as Buenos Aires, Calcutta, and Seoul-may be very high profile but are increasingly not the major recipients of urban growth. Instead, smaller cities are capturing the bulk of such expansion. These urban areas are expected to continue to grow rapidly and it is estimated they will house half of near future global urban residents. In contrast, mega-cities' growth will slow substantially (UNFPA 2007a).

Although extremely interesting, it is nonetheless insufficient simply to know where most urban growth is occurring. Whilst both waves of urbanization have facilitated socio-economic development of urban industrial societies, which makes the 2008 urbanization milestone vitally important from this economic development perspective alone, this is not evenly experienced by urban residents. Thus, today's rapidly growing urban areas of the Global South offer diverse opportunities and yet also present many challenges for everyday living (Knox and Pinch 2009). Consequently, the composition of urban populations, from both demographic and social perspectives, is a vital consideration. Such 
Table 3.1 Three place classification codes used by US Economic Research Service

\begin{tabular}{|c|c|c|c|}
\hline Classification & Scale & Overview & Categories \\
\hline $\begin{array}{l}\text { Rural-Urban } \\
\text { Continuum (Beale) } \\
\text { Codes }\end{array}$ & Counties & $\begin{array}{l}9 \text { categories: } \\
3 \text { Metro population } \\
6 \text { Non-metro urban population } \\
\text { and adjacency to Metro area }\end{array}$ & $\begin{array}{l}\text { 1. Metro area } 1 \text { million+ } \\
\text { 2. Metro area } 0.25-1 \text { million } \\
\text { 3. Metro area under } 0.25 \text { million } \\
\text { 4. Urban population } 20,000+\text {, adjacent to Metro } \\
\text { 5. Urban population } 20,000+\text {, not adjacent to Metro } \\
\text { 6. Urban population } 2,500-19,999 \text {, adjacent to Metro } \\
\text { 7. Urban population } 2,500-19,999 \text {, not adjacent to Metro } \\
\text { 8. Under } 2,500 \text { population, adjacent to Metro } \\
\text { 9. Under } 2,500 \text { population, not adjacent to Metro }\end{array}$ \\
\hline $\begin{array}{l}\text { Urban Influence } \\
\text { Codes (UICs) }\end{array}$ & Counties & $\begin{array}{l}12 \text { categories: } \\
2 \text { Metro population } \\
9 \text { Non-metro size of largest city, } \\
\text { adjacency to Metro areas by size } \\
\text { of Metro areas, Micropolitan status }\end{array}$ & $\begin{array}{l}\text { 1. Metro area } 1 \text { million+ } \\
\text { 2. Metro area under } 1 \text { million } \\
\text { 3. Micro (Micropolitan) area adjacent to } 1 \text { million+ Metro } \\
\text { 4. Noncore adjacent to } 1 \text { million+ Metro } \\
\text { 5. Micro area adjacent to under } 1 \text { million Metro } \\
\text { 6. Noncore adjacent to under } 1 \text { million Metro, with town } \\
2,500+ \\
\text { 7. Noncore adjacent to under } 1 \text { million Metro, without } \\
\text { town } 2,500+ \\
\text { 8. Micro area not adjacent to Metro } \\
\text { 9. Noncore adjacent to Micro, with town } 2,500+ \\
\text { 10. Noncore adjacent to Micro, without town } 2,500+ \\
\text { 11. Noncore not adjacent to Metro or Micro, with town } \\
2,500+ \\
\text { 12. Noncore not adjacent to Metro or Micro, without town } \\
2,500+\end{array}$ \\
\hline
\end{tabular}

Source: Cromartie 2015; www.ers.usda.gov

composition, notably as represented through Section 2.3's bio-social arenas, shapes strongly the urban life course experiences of individuals, families and larger population sub-groups. For example, general advantages of urban residence, including access to greater public services, health care, and education-all highly pertinent for demographic behavior-are not equally attainable in practice. In particular, populations living in poverty have significantly lower ability to access these urban features than wealthier residents.

Drilling deeper into the impoverished urban population, it is women, children and the elderly who are especially vulnerable. Reflecting patriarchy (2.3.8), women and girls often face greater dangers from crime and violence, while potentially having greater access to education and employment compared to those in rural areas. Although urban 
GLOBAL SPATIAL DISTRIBUTIONS OF POPULATION

Table 3.2 Regional and global populations, 2014 and 2050 (projected): urban versus rural

\begin{tabular}{lllll}
\hline Region & \multicolumn{3}{l}{ Population } & \\
\cline { 2 - 5 } & $\begin{array}{l}\text { Urban 2014 } \\
(\%)\end{array}$ & $\begin{array}{l}\text { Rural 2014 } \\
(\%)\end{array}$ & $\begin{array}{l}\text { Urban 2050 } \\
\text { (projected \%) }\end{array}$ & $\begin{array}{l}\text { Urban Change } \\
\text { 2014-50 (\%) }\end{array}$ \\
\hline More developed & 78 & 22 & 85 & +7 \\
Less developed & 48 & 52 & 63 & +15 \\
Least developed & 31 & 69 & 50 & +19 \\
Northern America & 82 & 18 & 87 & +5 \\
Latin America \& Caribbean & 80 & 20 & 86 & +6 \\
Europe & 73 & 27 & 82 & +9 \\
Oceania & 71 & 29 & 74 & +3 \\
Asia & 48 & 62 & 64 & +16 \\
Africa & 40 & $46(3,329,000)$ & 66 & +16 \\
TOTAL & $54(3,909,000)$ & & & +12 \\
\hline
\end{tabular}

Source: adapted from PRB (2015b: 3).

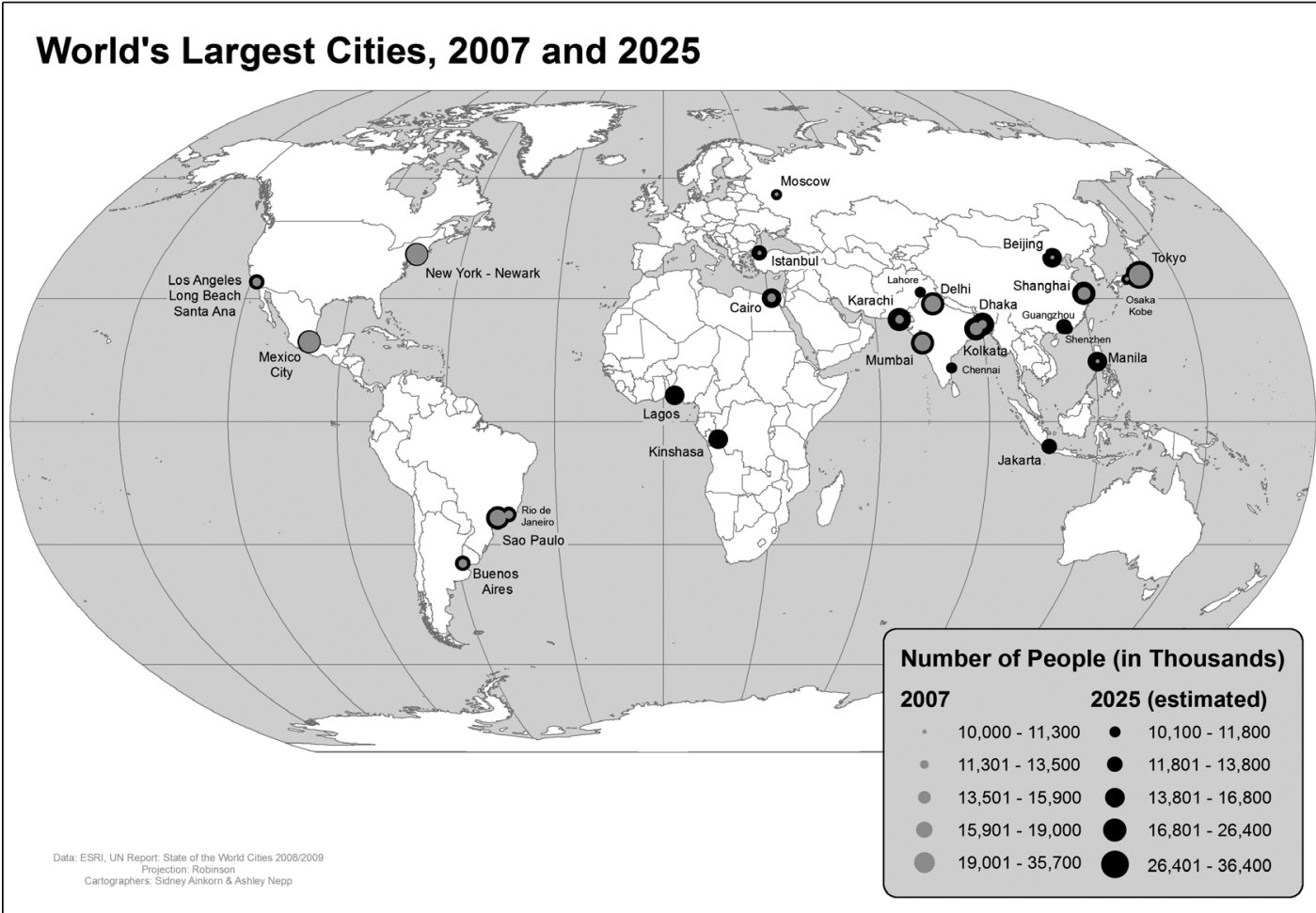

Figure 3.5 World's largest cities: 2007 and 2025.

Source: ESRI, UN Report: State of the World Cities 2008/2009, Projection: Robinson.

residence increases the likelihood that girls attend school, they are still the most likely to be taken out of school early in order to perform household chores, for marriage, or because of an unexpected pregnancy. And yet, urban residence also increases the likelihood of women participating in the labor force, which may in turn increase status within a family and facilitate positive changes in gender roles and decision-making power. Such can be the ambiguity of the urban experience (UNFPA 2007a).

Moving from the South to countries of the Global North that passed through the first major wave of urbanization, the clear majority of people now reside in urban areas and, as Table 3.2 suggests, further growth is likely to be small.

\section{ST PROOFS: NOT FOR DISTRIBUTION}


On the one hand, these agglomerations consequently face some quite different issues to rapidly growing cities of the South. Challenges include provision of mass transportation, suburbanization, social segmentation, and absorbing new international immigrant populations. Additionally, unlike emerging urban areas seeking to install infrastructure, ageing urban areas struggle to update decaying provision. Nonetheless, on the other hand, the bio-social arenas also strongly shape and differentiate residents' life course experiences, often markedly.

Urban lives in the transition economies of eastern Europe and the former Soviet Union (USSR) are yet further differentiated. Since the post-1989 collapse of previously dominant centralized control and planning, "many of the positive aspects ... preserved and accrued during communism, including compact form, socially integrated urban populations, ample public parks and other public spaces and well-developed mass transit systems, were perhaps permanently lost" (Hirt and Stanilov 2005: 3). Subsequent variations in exposure to and engagement with the global economy, as well as different and evolving forms of governance, have diversely affected their populations' well-being. Particularly hard hit have been settlements that were designated for specific industrial purposes. These struggle to obtain global investments and are largely dependent upon government, European Union and international financial support (Hirt and Stanilov 2005). However, almost all "transitional" urban places have seen significant increases in poverty and socio-spatial stratification.

In summary, the Ecumene may be becoming increasingly urban but such recognition of a global convergence in levels of urbanization must never assume equivalent convergence of life course experiences and contexts, or of the Population Geographies inscribed through these. Even at the individual city scale people are differentiated by income, class, ethnicity, and so on (Hubbard 2006). Cities are extremely socially variegated (Amin and Thrift 2002), especially the larger ones (Frey 2002). Building these segregated or more generally differentiated urban social geographies are, in particular, four general migration processes. These are introduced next.

\subsubsection{Urban areas: diversifying migration processes}

\subsubsection{Gentrification}

Gentrification (Figure 3.6) can be defined as the upgrading and renovation of older housing and other buildings in what have become generally impoverished residential neighborhoods (Jones 2012: 186). Such physical change usually goes hand-in-hand with social changes which contribute to restructuring the overall socio-demographic profile of the areas involved (Hammel 2009). Gentrification typically expresses a process of population turnover within urban neighborhoods characterized by a change in both the residents and structures of the neighborhood. Demographically, this usually involves in-migration of the relatively wealthy and the resultant displacement and out-migration of lowerincome residents (Smith 1996). Gentrification is closely tied to the life course, with "typical" gentrifiers singles or childless dual-income couples in their mid-thirties, highly educated and working in professional occupations (Rérat et al. 2010). Gay householders have also been linked to this process (Lauria and Knopp 1985).

A variant of gentrification, new build gentrification, has many of the same characteristics but is associated with development of areas not presently utilized for residential housing. Consequently, it is linked with more indirect social displacement (Davidson and Lees 2010). From a study of new build gentrification in Shanghai, China, He (2010: 346) argued that gentrification in general is a "global urban strategy" extending beyond simple reinvestment in housing stock to encompass "the class remake of urban/rural landscapes," usually supported by the state.

\subsubsection{Immigrant neighborhoods}

Historically, cities have long been attractive gateway destinations to immigrant populations (Price and Benton-Short 2008) (Figure 3.7). These populations often first settle in neighborhoods in which earlier migrants have created a relatively distinct community (Frey and Johnson 1998). Such communities help new arrivals adjust to their new country, providing familiar forms of support and ethnic context, from shops to churches. Consequently, Brama (2008) described a process by which immigration to Sweden led to increasingly segregated neighborhoods, reinforced both through net births over deaths within the existing immigrant population and through newcomers finding these neighborhoods a "comfortable" point of entry into Sweden. However, the ultimate situation is often more complex than

\section{ST PROOFS: NOT FOR DISTRIBUTION}




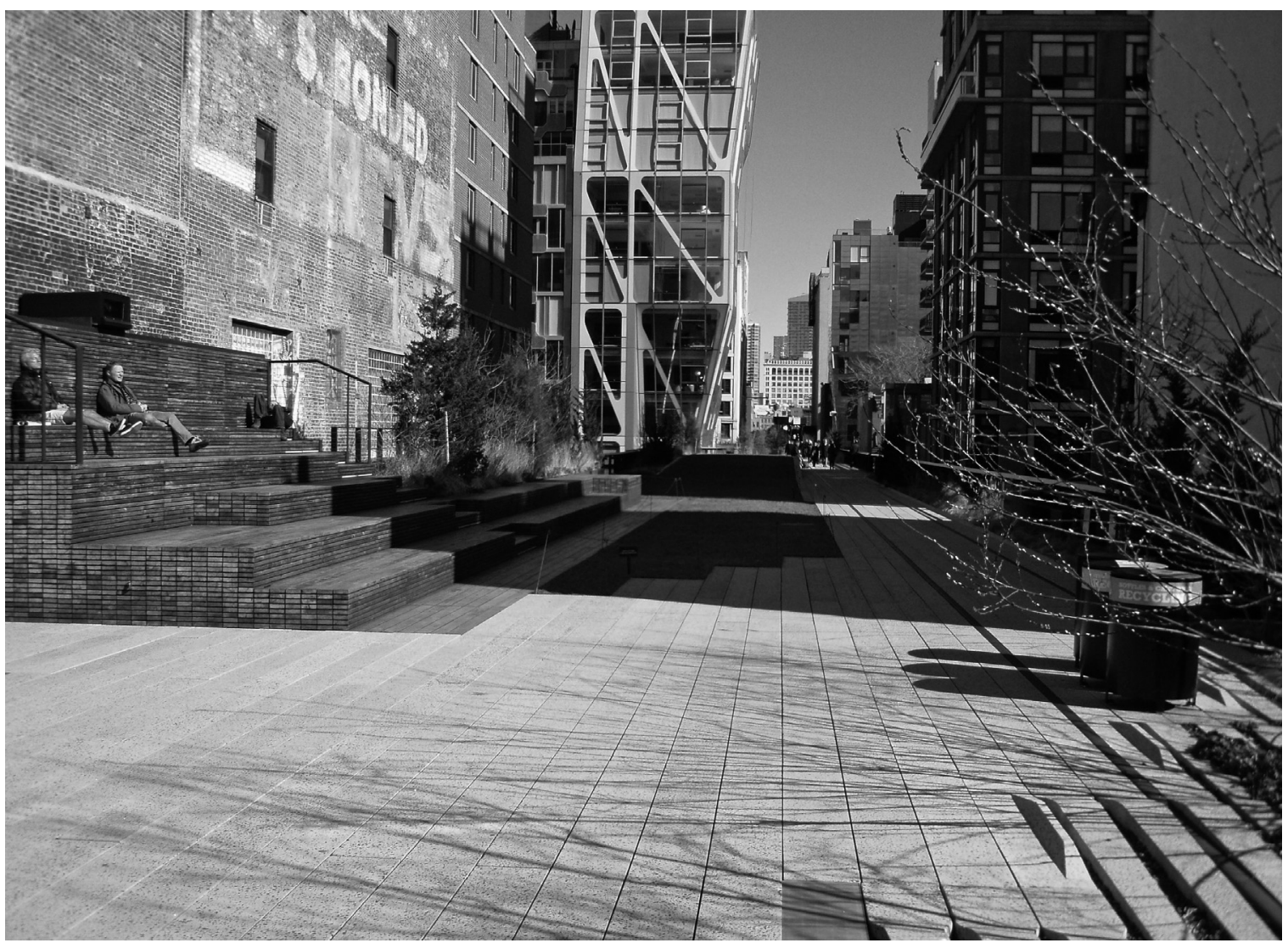

Figure 3.6 Gentrification.

Source: Dan Trudeau, High Line in New York City.

simply immigrant neighborhoods growing demographically. Thus, Newbold (1999) argued that many immigrants settle initially in such neighborhoods but, as they gain resources, subsequently relocate further afield.

Ethnic minority neighborhoods sometimes evolve into ethnic enclaves (Wilson and Martin 1982) or, more accurately according to Logan et al. (2002), immigrant enclaves. Even for wealthier residents who could live elsewhere, the neighborhood may remain the preferred residential choice for the various everyday life course resources and social capital (Putnam 2000) it can supply. Logan et al. (2002) further postulated that suburban immigrant enclaves can offer equivalent benefits, albeit associated with higher social and economic status. A further elaboration is Zelinsky and Lee's (1998) concept of heterolocalism, developed to express how many US minority communities express neither full assimilation nor relatively isolated cultural and ethnic islands. Instead, they adopt a dispersed pattern of residential location at the metropolitan scale, whilst retaining strong ethnic identity. This can be facilitated by key spaces of empirical construction (2.3.3) such as the Shikharbandhi Jain Deraser temple in the north London suburb of Potters Bar for an otherwise dispersed Jain population (Dwyer et al. 2013).

\subsubsection{Suburbanization}

Expressively pushing the edges of cities outward, suburbanization (Mace 2009) refers to the spatial growth of areas dominated by residential development on the fringes of major cities. Across the Global North, suburbanization processes building at the end of the nineteenth century were fueled, in particular, by transport innovations. Growth of the railways facilitated commuting to employment in city centers from origins that would otherwise have been seen as too distant. An excellent example is London, with its railway lines radiating from centrally located termini (Figure 3.8) driving

\section{ST PROOFS: NOT FOR DISTRIBUTION}




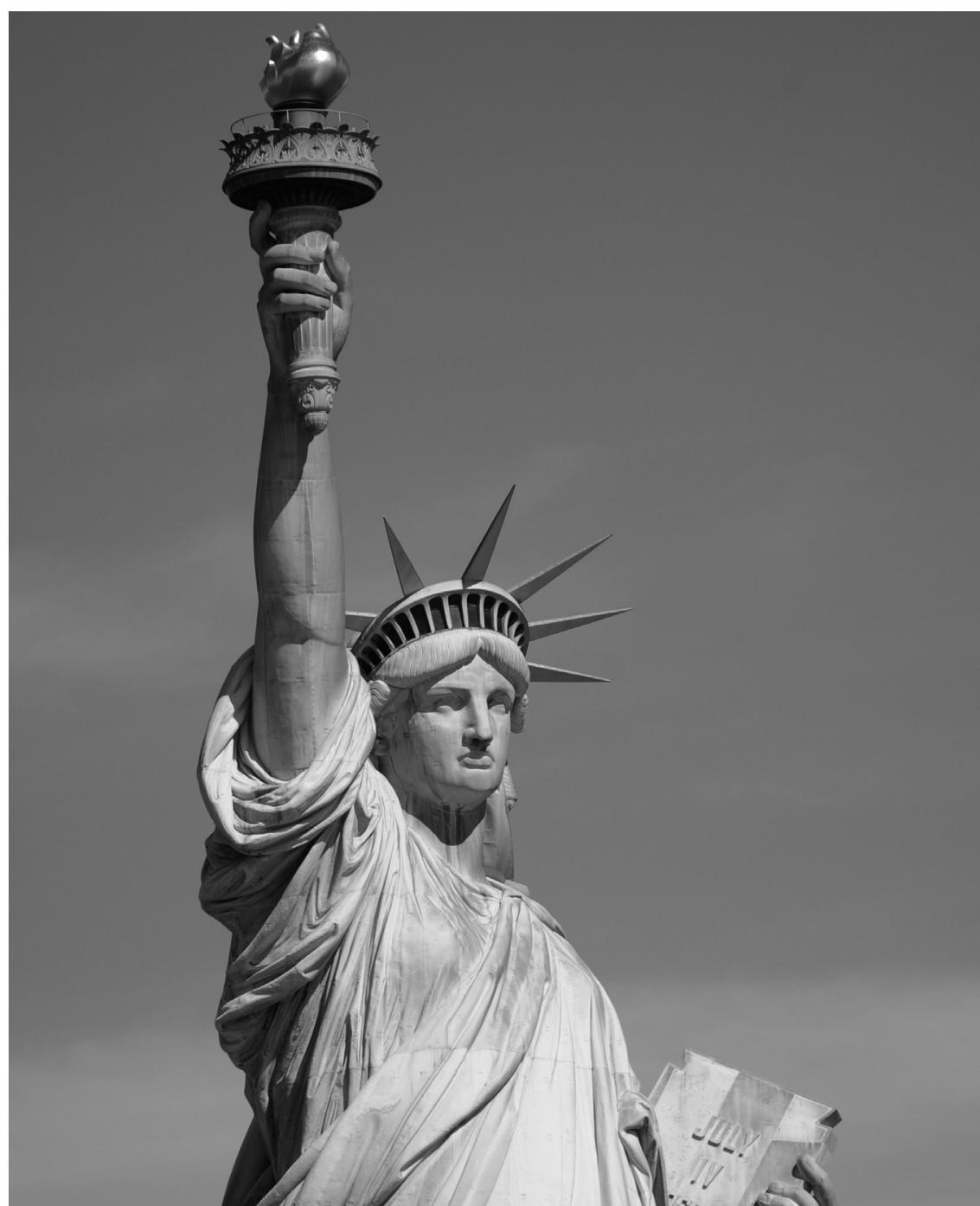

Figure 3.7 The Statue of Liberty.

Source: Dan Trudeau.

development of residential suburbs (Thompson 1982). As Porter (1994: 234) put it, "the railways made outer suburbs possible."

Most important for US suburbanization was the astonishing rise of automobile ownership, which similarly facilitated transportation between city centers and peripheries that offered greater access to more spacious housing and property ownership (Clapson 2003). Creating and building these US suburbs began in the 1920s but exploded after World War II, when returning service personnel began to settle in communities in close proximity to urban centers. One of the most famous new suburbs was William Levitt's Levittown on Long Island, New York. Begun in 1947, it became the first and one of the largest mass-produced suburbs and a symbol of postwar suburbia (Kelly 1993).

Besides transportation, several other key factors facilitated movement into the suburbs. These included new incentives for homeownership, such as Federal Housing Authority insured loans in the US, which made homeownership accessible to a broader range of families; lower cost housing; and cultural factors. An example of the latter with clear demographic implications was cultivation of the normative ideal of the "classic" suburban household of stay-at-home wife homemaker and commuting husband "breadwinner" (England 1993) (Box 3.3).

\section{ST PROOFS: NOT FOR DISTRIBUTION}



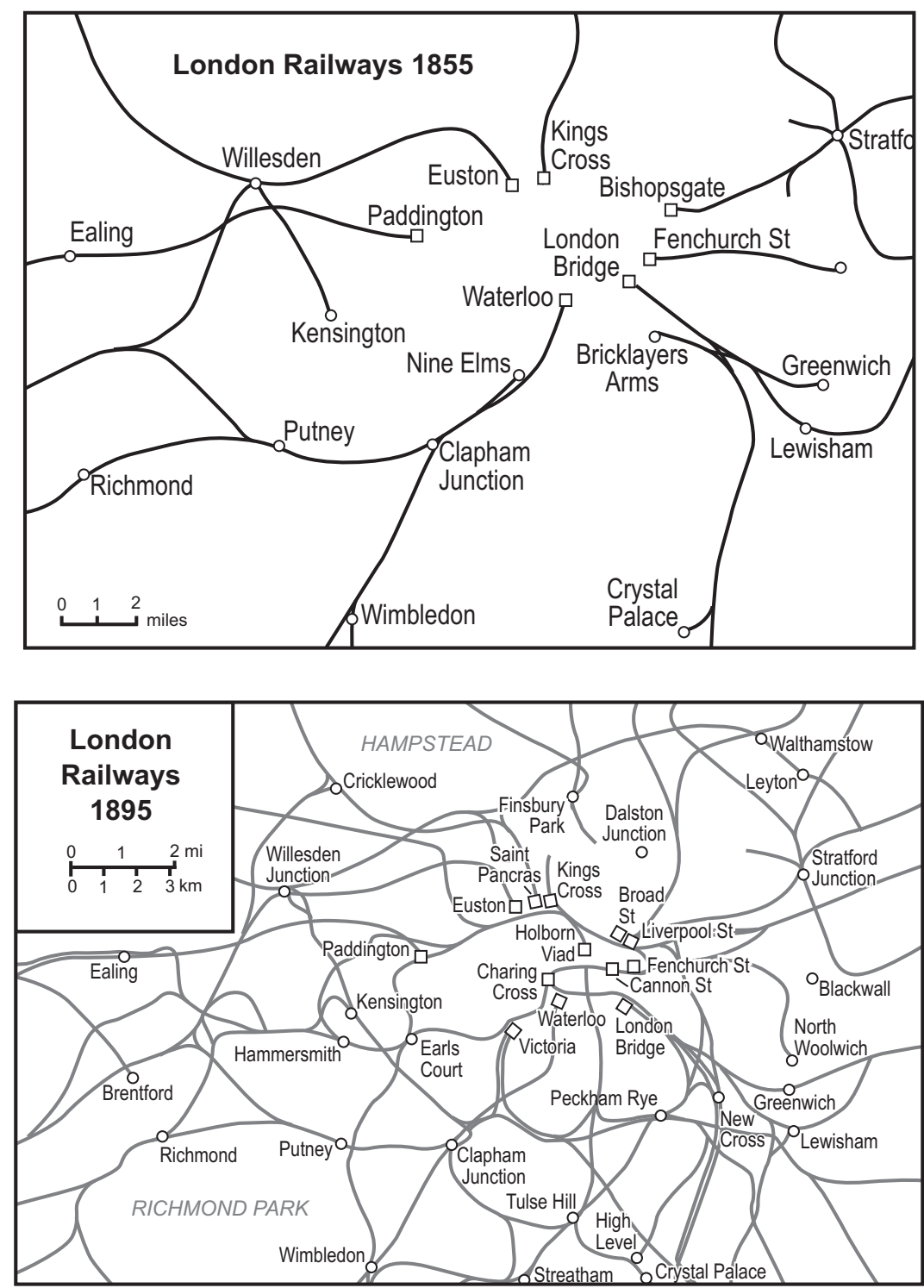

Figure 3.8 Late nineteenth-century growth of London's railway lines. (Two maps-1855 and 1895).

Source: Redrawn and simplified from Porter (1994: 230).

Suburbs are generally characterized by single-family homes built at low densities, each unit including its own small piece of land (Figure 3.9). Accompanying their evolution, as suggested, was a branding or stereotyping of residents of suburbia. Besides clear gender divides, suburbs have been commonly thought of as the domain of white, middle-class families (Dwyer et al. 2013). Yet, today they are increasingly home to a wide range of ethnic and other minority populations. As noted, immigrants may initially settle in gateway cities but eventually leave for suburban neighborhoods. Moves may be undertaken as families gain socio-economic status and seek higher quality housing. They may also occur as lower income households seek lower cost housing outside of an urban area (Tammaru and Kontuly 2011), not least on account of city center gentrification.

Indeed, as with gentrification, suburbanization is no longer exclusive to the Global North. Returning to Shanghai, the city has grown from demand for new housing and availability of inexpensive land on the city's periphery. The 1982 


\section{BOX 3.3 REVOLUTIONARY ROAD}

Suburbia and suburban lives feature strongly within US culture, not least as the site of various forms of repression and frustration. Writer Ernest Hemingway, reflecting on his upbringing in the Protestant upper middle-class suburb of Oak Park, allegedly dismissed it as a place of "wide lawns and narrow minds" (Vejdovsky and Hemingway 2011), albeit being only ten miles from central Chicago. Similarly, Malvina Reynolds's 1962 song Little Boxes attacked what she saw as the bourgeois values embodied in "Little boxes all the same" and their equally uniform residents.

Typically, the implicit or even explicit target within such critical perspectives is one of suburbia's key imagined cultural aspects: a normative ideal of the stay-at-home wife homemaker and the commuting husband "breadwinner." This was expressed in the award-winning 2008 film (directed by Sam Mendes, starring Leonardo Di Caprio and Kate Winslet) Revolutionary Road, based on a 1961 novel by Richard Yates.

Revolutionary Road narrates a tale of Frank and April Wheeler. In 1955, they move to a suburban home in Revolutionary Road, Connecticut, to raise their children. While on the surface the Wheelers appear a contented if stereotypical suburban couple-he commuting to a white-collar job in the city, she staying home as mother and housewife-Frank hates his boring job and April dreams of becoming an actor. Both also dream of moving to Paris, France, with its romantic appeal. Although they subsequently plan to relocate there, this never happens. Instead, Frank has a promotion and an affair and April becomes pregnant again. Not wishing to have another child, April wants to have an abortion but Frank furiously objects. April attempts the procedure herself, with fatal consequences. Frank then relocates back to the city and a new idealistic couple move into the suburban house, blissfully ignorant of the emotional traumas that occurred there.

Revolutionary Road, in summary, articulates the cultural and life course ambiguity or duplicity of the suburbs. On the one hand, they offer clear material comforts, notably good housing and a solid base from which to develop a (male) career and (for the wife) to raise a family. In other words, they can provide a stable geographical base for life course consolidation. On the other hand, suburban life demands culturally a lot from those buying into it, against which challenges from within the life course arenas of gender and individuality can prompt rebellion.

census revealed that for the first time more than 50 percent of the city's population was resident in an "outskirts" area. This percentage had reached 65 percent by 2008 (He 2010).

Thinking of suburbanization and gentrification together suggests something of the complexity of urban evolution. As urban populations continue to expand, their population (re)distribution becomes ever more spatially complex. In a study of 158 urban agglomerations in Europe, Kabisch and Haase (2011) found any simple linear progression within urban growth not sustained. Rather, they recognized processes of re-urbanization (gentrification) and suburbanization together producing complex urban developmental forms.

\subsubsection{Informal settlements}

Rapid urbanization, particularly in the Global South, results in a further process of urban growth: informal "squatter" or "slum" settlements (Harris 2009). These are estimated to house "one out of every three city dwellers, a billion people, a sixth of the world's population" (UNFPA 2007a: 16). They are characterized by poor quality housing, lack of access to clean water, and constant exposure to sewage and infectious and parasitic diseases endemic to areas with poor sanitation (Davis 2006). Clearly, such factors can be linked to mobility and mortality, yet the United Nations's The Challenge of the Slums anticipated their number of residents to rise to 2 billion by the 2030s (UNHSP 2013). Political activist Mike Davis (2004: 11) described this report as "the first truly global audit of urban poverty," finally acknowledging how, according to Owusu et al. (2008: 180), "[m]uch of the 21 st century urban world squats in squalor, surrounded by pollution, excrement and decay."

The size of these especially vulnerable "surplus" populations (Tyner 2013) varies by region. Highest concentrations are found in urban areas of Sub-Saharan Africa and South-Central Asia (Table 3.3), although presence in Eastern European transition states must be recognized (Tsenkova 2010). For example, Vassilev (2004: 42) described 


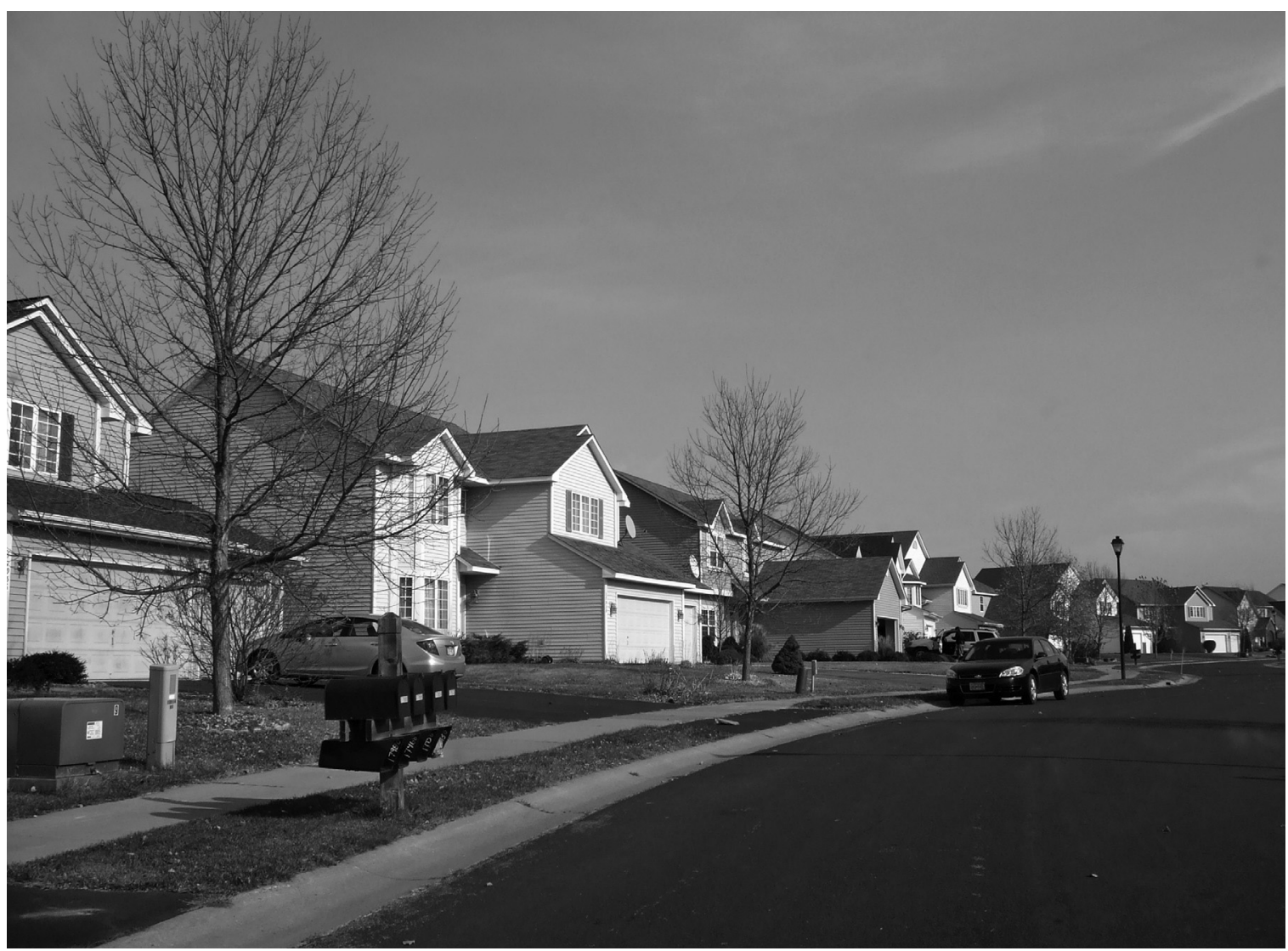

Figure 3.9 Suburbia.

Source: Charlotte Fagan, Oak Grove, a suburban subdivision in Eden Prairie, Minnesota USA in July 2011.

Table 3.3 Global "slum" populations

\begin{tabular}{ll}
\hline Region & "Slum" population 2001 \\
\hline \% urban population & \\
Sub-Saharan Africa & $71.9 \%$ \\
South-Central Asia & $58.0 \%$ \\
Eastern Asia & $36.4 \%$ \\
Western Asia & $33.1 \%$ \\
Latin America \& Caribbean & $31.9 \%$ \\
Northern Africa & $28.2 \%$ \\
Southeast Asia & $28.0 \%$ \\
Oceania & $24.1 \%$ \\
\hline
\end{tabular}

Source: UN 2003: xxv.

Bulgaria's Fakulteta district in Sofia as "a slum in which about 35,000 Roma [Gypsy people] live in squalor, penury, and hunger." Understandably, residents may be prompted to seek better lives through labor migration abroad, as discussed in Chapter 7 (Marinov 2015).

Today's slum settlements of the South have historical antecedents in the "classic" Northern slums of the nineteenth century that caught the attention of reformers and political radicals. For example, here is Friedrich Engels's account of a district within Manchester, England, in 1847:

\section{ST PROOFS: NOT FOR DISTRIBUTION}


the most horrible spot ... lies ... immediately south west of Oxford Road, and is known as Little Ireland.... The race that lives in these ruinous cottages, behind broken windows, mended with oilskin, sprung doors, and rotten door-posts, or in dark, wet cellars, in measureless filth and stench ... must really have reached the lowest stage of humanity.

Such "slums of despair" (Eckstein 1990: 165) have mostly relocated today to the outer city. Doubly marginalized areas (socially and spatially) of the urban environment, they are characterized by unstable land tenure, poor quality housing, lack of sanitation and clean water, and limited access to basic services, such as garbage collection. Living within such a blighted spatial life course context clearly has major demographic implications, not least likelihood of premature death (Tyner 2013).

Nonetheless, broad-brush condemnations, politically useful in conveying the overall scandal of slum settlement, fail to dissect critically the day-to-day lived experiences of residents. As Owusu et al. (2008: 180) observed:

Slums are universally assumed to be the worst places for people to live in, and it is often taken for granted that the livelihood situations of slum communities are also uniform and homogenous. So pervasive is the latter idea that most studies examining the livelihood situations of slum communities do not compare the socio-economic and cultural differences within such communities.

Such differences can be considerable. Slums such as the favelas of Brazilian cities, for example, have developed distinctive social micro-geographies, even emerging as tourist attractions (Freire-Medeiros 2009).

Critically dissecting the "slum" still further, Agnew (2010) challenged the idea of homogenous spaces of marginality in a comparison within the Global North between impoverished neighborhoods of Chicago and Paris. He argued that not only are the processes creating these environments politically and geographically specific, resident populations are substantively differentiated between marginalized areas as well as within these areas.

Finally, the UN has drawn attention to another relevant urban statistic here: the Global South's youth bulge. With predictions that 60 percent of urban dwellers will be under the age of 18 by 2030 (UN 2016), a large proportion will likely live in informal urban settlements. Consequently, the drive to "regularize" slums through granting a degree of tenurial security, providing some basic infrastructure and services, and generally moving away from seeing residents as unlawful "squatters"-as in the community-engaged Baan Mankong program in Thailand (Boonyabancha 2009)seems especially imperative.

\subsubsection{Rural areas: sparsely populated but demographically dynamic}

Globally, both the size and, more unambiguously, the proportion of the population residing in rural areas-however defined (Box 3.4)-continues to shrink. Table 3.2's regional scale presentation clearly showed this. Nonetheless, this population still comprises a substantial number of people, estimated at more than 3.3 billion in 2014. It remains in the majority in Asia and Africa. The rural population is also still expected to comprise one third of the global total in 2050. Moreover, the experiences, livelihoods and challenges faced by rural peoples are possibly even more varied than those of their urban counterparts. Local histories, geographies, cultures and traditions play a large role in determining gender roles, household economics, and access to basic services, all of which feed into demographic life course behavior. As with urbanization, one can discuss these geographies through the Global North-South dichotomy, although considerable variation again exists within these categories.

Exploring the geographies of rural populations in the Global North requires some understanding of contemporary rural issues (Woods 2005, 2011). The historical dichotomy between urban and rural places, largely based on economic structure and livelihoods - with rural places predominantly associated with agriculture and extractive industries and urban places linked with manufacturing and services - has evolved significantly over the past 50 years. From the rural perspective, both technological developments and the evolution of global markets and economies have profoundly changed these economies (McDonagh et al. 2016) and, by extension, the populations residing within contemporary rural communities. 


\section{BOX 3.4 RURAL}

What do people mean when they talk about rural? Engaging with this seemingly simple question has long been a scholarly preoccupation with one of the present authors for at least two reasons. First, as Michael Woods (2005: 15) put it, "'Rural' is one of those curious words which everyone thinks they know what it means, but which is actually very difficult to define precisely." Second, engaging with the definition of rural requires engagement with a long tradition within social science scholarship that has seen "rural" as a significant geographical concept repeatedly and frequently written off. Since at least the mid nineteenth century, with the rise of industrial and urban society within the Global North, the rural has appeared as a concept out of time-an anachronism-and on the road to extinction. Modern times, it seems, has no place for rural. And yet, in spite of such valedictions, it simply does not go away. As Sarah Whatmore (1993: 605) effectively expressed it over two decades ago: "For a subject repeatedly dismissed as a figment of our analytical imagination ..., the rural world has an unruly and intractable popular significance."

How, then, must rural be understood today? First, just as all urban areas are not somehow "the same," so too are rural places fundamentally diverse. This diversity is not simply Global North versus South but reflects often considerable geographical variability within even just one country. Second, with the word rural rooted in the Latin noun rus meaning "open area," and with "distinction between 'urban' and 'rural', between the city and the country, [being] one of the oldest and most pervasive of geographical binaries" (Woods 2011: 3), it is clear that rural tries to express the world beyond or outside the built-up urban. Third, it is also apparent that the ruralor associated terms, such as countryside or country-is popularly understood. For many people, it represents an idea very dear to their hearts, influencing where they live, where they identify with culturally, where they look for recreation, and/or where they see "nature" residing. This is the rural's "intractable popular significance."

Diversity, openness, everyday recognition and significance-all point to the complexity of pinning down the rural. This challenge is further expressed in this sequence of questions contained within the introduction to a popular Rural Geography textbook:

Clear your mind and think of the word 'rural'. What image do you see? Maybe you see the rolling green downland of southern England, or the wide open spaces of the American prairie? Perhaps it's the golden woodlands of the New England fall, or the forests of Scandinavia? The Rocky Mountains or the sun-baked outback of Australia? Are there any people in your rural picture? If so, what are they doing? Are they working? Or maybe they are tourists? What age are they? What colour are they? Are they men or women? Rich or poor? Do you see any buildings in your rural scene? Perhaps a quaint thatched cottage, or a white-washed farmstead? Maybe a ranch, or a simple log cabin? Or do you see a run-down dilapidated home, barely fit for human habitation; or an estate of modern, identikit, housing? Is there any evidence of economic activity? Farming, probably, but then do you see a farmyard of free-range animals, as the children's storybooks would have us believe, or do you see battery hen sheds, or endless fields of industrially produced corn? Maybe you see quarrying or mining or forestry? But what about factories, or hi-tech laboratories or office complexes? Are there any shops, or banks, or schools-or have they been converted into holiday homes? Are there any roads or traffic in your image? Is there any crime, or sign of police on patrol? Do you see any problems of ill-health, or alcoholism, or drug abuse? Who owns the land you are picturing? Who has access to it?

Do you still have a clear picture of what 'rural' means to you, or are you beginning to think that defining the rural is more complicated than you thought?

(Woods 2005: 3) 
US economic restructuring of rural areas throughout the later twentieth century, including shifts away from resource extraction, mechanization of agriculture, increasing scale of corporate farms, growth (and decline) of tourism, and increased recognition of the amenity and lifestyle valuations of rural places has created a very heterogeneous landscape of population change (Kirschner et al. 2006: 53). It expressed often complex combinations of out- and inmigration processes.

Out-migration resulted from economic decline in remoter areas, especially of working-age adults, causing general depopulation and rapid ageing of the remaining population. Moreover, when youth migrate away from these rural places, they take not only themselves but also their potential children. Rural depopulation, however, is unevenly distributed. While some regions, such as the Great Plains and Corn Belt, have witnessed significant population loss and ageing, others, such as the West, now have to engage with issues associated with population growth (Jobes 2000).

Rural population growth in the US reflects the migration-driven resurgence during the 1970s and early 1980s that became labeled a "rural renaissance" (e.g. Frey 1987; Morrison and Wheeler 1976) after decades of population decline. However, rather than signaling any comprehensive rural population revival, the renaissance was short-lived (Frey 1990), especially for communities traditionally rooted in agriculture or extractive industries. They soon began losing population again (or never stopped declining). Nonetheless, experience of a rural "population turnaround" (Fuguitt 1985) away from urbanization and towards counterurbanization (Berry 1976) has become highly significant in many other locations.

Indeed, across much of the Global North-albeit beginning at different times, being highly geographically uneven, and fluctuating considerably both historically and spatially-the decades since the 1960s have seen migration scholars widely acknowledging counterurbanization (e.g. Boyle and Halfacree 1998a; Champion 1989). Woods (2005: 75), for example, argued that it was "a product of the economic restructuring of both urban and rural societies, combined with societal and technological changes that mean that people are more mobile physically and socially than in previous generations."

Counterurbanization is intimately entangled with another important trend influencing rural population growth and social restructuring: amenity migration (Gosnell and Abrams 2011). This is itself a sub-set of lifestyle migration, defined as "relocation of people ... searching for a better way of life" (Benson and O'Reilly 2009a: 608). It especially impacts on rural communities possessing what are perceived as desirable geographic and climate features. Desirability can be largely aesthetic or more practical, not least linked to recreational activities. Destinations favored are areas with mountains, large bodies of water or other outdoor recreation features, such as hiking and ski trails or natural areas. Amenity migration may be permanent-linking clearly to counterurbanization-or more temporary or seasonal. The latter is reflected in, for example, large numbers of second homes in Scandinavian countries, with around 40 percent of the Norwegian population accessing an estimated 420,000 properties (Overvåg 2009).

Amenity communities, including those selected through international migration flows such as North Americans relocating to Ecuador (Hayes 2014), often struggle to incorporate the influx of affluent, second-home or permanent residents. Newcomers value the aesthetic qualities of a particular landscape but may also contribute to significant increases in the cost and availability of local housing, potentially displacing long-term residents: rural gentrification (Phillips 1993). Ironically, while a driving force behind much counterurban amenity migration is the perception of rural places as idyllic, remote, pastoral and peaceful, clashes between their consumption and the productive demands placed on them may lead to local controversies and conflicts (Frisvoll 2012; Gosnell and Abrams 2011; Woods 2005).

Counterurbanization is not just an expression of amenity migration, however, but also reflects more "economic" life course requirements (Halfacree 2008). For example, it expresses the ongoing restructuring of US agriculture and the movement of large-scale animal and vegetable processing plants out of urban areas and into rural communities, such as the Great Plains. Indeed, to promote economic growth, many small communities actively seek out agribusiness processing plants. Unfortunately, such businesses only offer predominantly low-skill, low-pay work largely undesirable to community residents. Rapid in-migration of Asian and Latino workers therefore responds to fill these positions, resulting in the gradual ethnic restructuring of the local area (Barcus and Simmons 2013). With these workers also often culturally and socially distinct from the existing population, the result may be significant community disharmony. In-migrants, nonetheless, increase the rural population and contribute to continued economic development through local spending and entrepreneurialism, helping offset population decline. Similarly, in the UK and elsewhere, workers may be "imported" annually for planting, harvesting and other agricultural tasks, becoming at least temporarily part of the rural population (Halfacree 2008).

\section{ST PROOFS: NOT FOR DISTRIBUTION}




\section{GLOBAL SPATIAL DISTRIBUTIONS OF POPULATION}

Turning now to the Global South, rural out-migration is more unambiguously predominant. This is largely related to lack of local employment or livelihood opportunities, combined with a perception that migration elsewhere, often to a nearby urban area, will be advantageous, socially and economically. Of course, as in the North, one can question just how distinct rural and urban places are, especially given mobility's role in linking the two. As Rigg (1997: 153) observed: "[t]he notion that there are distinct and separate worlds, where agrarian change is fundamentally linked to agrarian processes, and urban change to urban and industrial processes is ... deeply flawed as more and more people, and with greater frequency, cross the 'divide' between the two."

Whilst rural environs and people are dynamic and continuously changing within the Global South, national and regional scale statistics paint a relatively distinctive rural picture. Rural people generally have fewer resources, lower levels of education and access to it, poorer health care and fewer non-agriculturally based employment options. They often comprise another vulnerable surplus population (Tyner 2013). Poverty levels are significantly higher and livelihoods still more precarious than for urban residents.

Within spatial representations (2.3.3), the "rural" in the "developing world" is still largely tied to images of agriculture (e.g. rice paddies in Southeast Asia, subsistence farming in South America, nomadic herders in Sub-Saharan Africa). This is despite a strong ongoing shift away from livelihoods exclusively dependent upon agriculture, especially among the young (Rigg 2006). Indeed, Rigg (1997: 174-178) earlier identified this tendency within several "threads of change" across rural Southeast Asia: growth of cash crop farming, increasing mechanization of production, bigger inputs of chemical fertilizers and wage labor, raising of livestock for sale, wage labor generally, off-farm local employment such as seasonal work or factory jobs, and employment migration to regional centers or larger cities. All these threads, whether directly or indirectly, have profound demographic consequences.

Transportation and communication technologies are also fundamentally transforming Southern rural communities. And, as economic changes take place, access to these technologies is increasing. Hence, Rigg (1997: 166) also noted how "the pick-up truck made the transformation from 'status symbol' to 'necessity' in the space of just five years" in Chachoengsao province in Central Thailand. Similarly, in the five year period 2004 to 2009, proliferation of cell phone technology and internet access in Bayan-Ulgii, Mongolia, meant individuals and households were not only better connected to each other within the province but could communicate with Mongolian emigrants in Kazakhstan daily via text messages. This supplanted weekly or monthly phone calls or letters (Werner and Barcus 2009).

Finally, the rural household economy in the Global South is also greatly affected by growing involvement of women in non-household employment, even if this is often moderated or suppressed by numerous barriers. The latter include the social acceptability of women working outside the household and cultural and religious norms preventing them seeking employment. Nevertheless, other contemporary cultural changes facilitate greater involvement in nonhousehold work. These include "declining fertility, delayed marriage, greater mobility and rising female education" (Rigg 1997: 185), demonstrating the intimate entanglement of demographic behavior, gender and culture.

\subsection{CONCLUSION: DYNAMISM OF LIVES ACROSS SPACE}

This chapter has provided a fairly straightforward introductory overview of the spatial distribution of the human population across the Ecumene. It presented summative snapshots of the multitude of ever-dynamic lives across space that express themselves through the life course and which are subject to detailed examination in the rest of the book. Focusing first on the global scale, the chapter introduced some basic concepts of population composition and growth, and placed the current global population in historical perspective. Each section noted the danger of over-generalizing and over-simplifying a spatial complexity which itself can be seen as inevitable given its rootedness in the multitude of life courses present.

A key conclusion to take from the chapter is relatively simple but also extremely profound. This is how dynamism has been a central feature of the spatial distributions of populations in the past, remains absolutely prominent in the present, and is highly likely to persist in the future. Given the complexity of issues involved in how demographic behaviors are relationally constructed, proposed in Chapter 2, this overarching theme of dynamism within Population Geography should perhaps be expected. Thus, the simple demographic equation may summarize population size and change, and maps and tables present neatly these spatial distributions, but the detailed specification and explanation 
of its components is highly situationally complex. Consequently, Population Geographies both differ and fluctuate considerably across the globe.

Within this heightened sensitivity to demographic diversity and change, however, emerge clear specific foci. For example, meriting special attention are places rapidly gaining population, notably urbanized areas. And one must acknowledge how, as populations continue to redistribute through migration in particular, new forms of urban expansion are taking shape. Processes and patterns of gentrification and suburbanization, for example, are no longer found exclusively in the Global North but their expressions across the South may be both quantitatively and qualitatively different to what Population Geographers have conventionally recognized. The issue of global population, it is clear, involves much more than just concerns about its absolute size, raised at the start of the chapter. Its geography, as expressed by spatial distribution, is the result of complex historical and contemporary processes, guided both by the economy and socio-cultural and political contexts, but also by decisions made by individuals and families as they seek to adapt practically every day to living across space. This overall strong sense of complexity, dynamism and change hopefully encourages the reader to investigate life course demographics in greater detail. Such a task begins, as a life course perspective anticipates, by examining geographies of fertility and births. 


\section{CHAPTER 4}

\section{Fertility and births}

\subsection{INTRODUCTION: THE PRODUCTION OF CHILDREN}

\subsubsection{Fertility geographies}

Every day, around 400,000 children are born (PRB 2014)-over 16,000 per hour! Each of these births is both usually a very intimate experience and a statistical demographic fact with implications for local communities, entire countries, even the world. Each marks the start of an individual life course, the beginning of a life across space. It might represent a long-awaited blessing, celebrated by the mother and her family, or a desperate strain on family resources or for a woman lacking support of a partner, close friends and/or family. Yet, no matter how received, a birth is clearly a major occurrence for all involved, and one typically subsequently celebrated each year as a birthday!

Births are also the living expression of what is known as fertility or "actual production of children" (Poston and Bouvier 2010: 39). Patterns of fertility and what shape them are one of the three core areas, beside mortality and migration, Population Geographers traditionally study. Whilst fertility geographies are probably least well represented of the three (Boyle 2003), they have still been examined at various socio-spatial scales: woman, family, community, nation, global humanity. Geographers are interested in how births are both experienced and have consequences on all these scales.

After introducing how Demographers measure fertility, this chapter presents a few key aspects of fertility geographies. It stresses fertility's always relational connection to the other elements of the demographic equation and the multiple arenas of life course constitution (2.3). Section 4.2 starts by summarizing two key ways fertility has been modeled at the aggregate scale. The empirical correlates of such models in the present day are then introduced in Section 4.3's overview of a global fertility divergence. The children produced then come to the fore in Section 4.4's account of the "value" of a child, before Section 4.5 engages with individual and family practices managing timing and spacing of births. Also central to shaping fertility geographies are various state policies, some explicitly demographic, introduced in Section 4.6. Lastly, before the conclusion, Section 4.7 extends the traditional scope of fertility geographies through a broader perspective on conception, birth and parenthood today.

\subsubsection{Measuring production of children}

Fertility refers to the number of children born to an individual or a defined population, often a country. To begin to articulate a process with such far-reaching implications and dimensions, Demographers have devised a range of statistics-based definitions (Holdsworth et al. 2013: 10, 72-76; Poston and Bouvier 2010: 40-52). Three are particularly useful, but increase in both sophistication and data demands, respectively.

Crude Birth Rate (CBR) expresses the total number of births occurring in a specified population over a specific period of time. It is easy to calculate and may have direct practical implications (e.g. numbers of new mouths to feed). For a given year:

$$
\left.\mathrm{CBR}=\text { - Total Number of Live Births }-{ }^{*}{ }^{*}\right] 1000
$$

Note that only live births are used to define CBR and that the rate uses the estimated mid-year area population as denominator. Accurate statistics are clearly required for both measures. The calculated value is then multiplied by 
1000 to give manageable figures because the numerator size (births) is likely to be much smaller than the denominator (population) as no group produce that many children! CBR thus expresses the total annual birth rate per 1000 people.

Whilst CBR is useful and easily calculable, there is a major weakness in using total (mid-year) population as a denominator. Much of this population is unable to bear children (but see 4.7): all males, many females. Here, it is useful to distinguish between fertility and fecundity (Poston and Bouvier 2010: 53-56). While fertility expresses number of children born, fecundity refers to a woman's physiological ability to have a child. Biologically, women cannot conceive before first menses (menstruation; typically in early teens) or after menopause (typically some time in the mid-late 40s). Consequently, the childbearing population is usually considered as women aged between 15 and 49 years. However, fecundity is also a property of a woman's overall health. This is never itself simply a matter of biology but also influenced by both socio-economic factors-such as ability to access a healthy environment, adequate food or medical support-and institutional factors-such as the health service supply system.

Infecundity occurs when a woman cannot physiologically have a child. It may be permanent or temporary. It is caused by a range of factors, including sickness, poor nutrition or contraceptive use. For example, breastfeeding women are temporarily infecund following childbirth (postpartum amenorrhea). Again, infecundity can be associated with particular socio-economic and institutional contexts.

By taking into consideration the ability to have children, Demographers define what is known as the at risk population. This is much smaller than total mid-year population and is used for a second fertility measure, General Fertility Rate (GFR). GFR expresses the number of births per 1,000 women within a standardized childbearing age range (ages 15-49).

Whilst GFR demonstrates awareness of the at risk population, it is also not without drawbacks. In particular, at risk population is quite bluntly defined, since rates of fecundity vary across a woman's lifetime, as noted above. Instead therefore, if adequate data are available, Age Specific Fertility Rates (AFSRs) for age-defined sub-groups can be calculated. AFSRs are usually calculated for 5-year cohorts: 15-19, 20-24, 25-29, 30-34, 35-39, 40-44, 45-49 years.

An AFSR breakdown enables a third fertility measure to be calculated. Total Fertility Rate (TFR) is calculated by adding together all the AFSRs and multiplying by five to account for the 5-year groupings. It expresses the average total number of children a woman would have over her lifetime if she followed AFSR expectations. TFR is widely used to compare fertility rates of populations and evaluate trends for different countries or communities. It is the default fertility measure in the rest of this chapter. Geographical variation in TFRs reflects the multitude of economic, social, political, and cultural systems and norms that exist in different places, many of which are discussed below.

To bring the three fertility measures together, Table 4.1 calculates them for Norway in 2012. Note how the rates differ considerably. They reveal that Norway had around 12 live births per 1000 population (CBR), 52 live births per 1000 women of principal child-bearing ages (GFR), and that on average these same women would be expected to give birth to just under two children (TFR). Notice also the variation in ASFRs between age groupings, the key years for bearing children being between 25 and 34 .

Table 4.1 Calculating and comparing fertility rates, Norway 2012

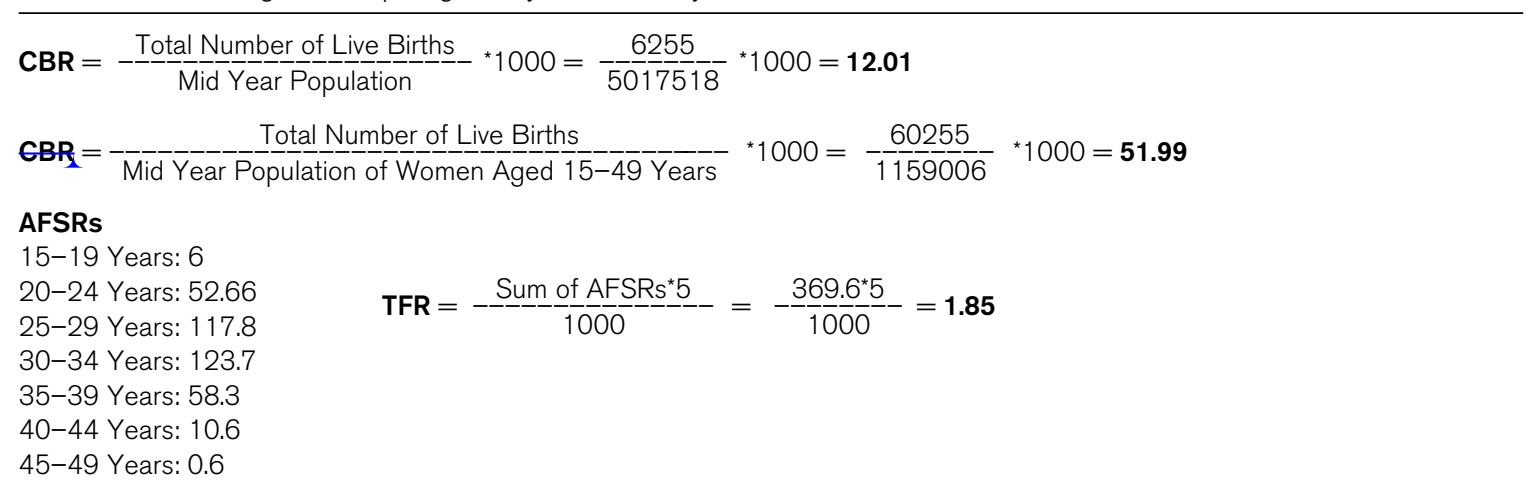

Source: calculated from http://www.ssb.no (accessed September 2013). 


\subsection{MODELING FERTILITY GEOGRAPHIES}

\subsubsection{Aggregate perspectives}

Before presenting some general global TFR patterns, it is useful to note how Population Geographers have developed ways to summarize or model these aggregate fertility geographies. This task is vital not least because fertility represents new human beings, new people who will need feeding, housing, educating, finding work, and so on.

Two key perspectives are considered below. First, the idea of fertility transition is developed out of the broader Demographic Transition Model (TTM) Here, associations between fertility and mortality, and their links in turn to changing socio-economic contexts, are emphasized. Second, a range of key bio-social variables regarded as having immediate or proximate impacts on fecundity and thus, ultimately, on fertility rates are outlined. Again, the relative importance of these variables is seen as strongly relationally shaped through the operations of specific societies.

\subsubsection{Fertility transition}

As was detailed in Chapter 3, population growth worldwide continues. Yet, there is an overall trend towards fertility decline. Global population continues to expand through population momentum (3.2.2) but within TFR a global, albeit uneven, transition from high to low rates is apparent. To begin to understand this trend in historical context, Demographers proposed the "enduring concept" (Holdsworth et al. 2013: 14) of the Demographic Transition Model $(\mathrm{DTM})$

The DTM seeks to connect population growth via interconnected fertility and mortality variables with social and economic change-"development"-across geographies. The model positions a country within a model of generalized demographic evolutionary stages, based on the historical experience of western European countries. Initially developed in the mid-twentieth century, Kirk (1996) saw the model occupying a place in modern Demography where some viewed it as a keystone while others saw it as "non-theory." Numerous scholars nonetheless continue to utilize it to help explain or at least describe the transition of both Global North and South countries from levels of high to low population growth (Reher 2004.)

There are four stages to the DTM, illustrated in Figure 4.1. These are configured by relatively stable or changing fertility and/or mortality rates (Poston and Bouvier 2010: 271-273; Holdsworth et al. 2013: Chapter 2). Stage One is a "low growth" pre-industrial or pre-transitional stage, where high rates of births and deaths (although both are likely to fluctuate substantially) cumulatively result in low levels of population growth. This stage was common prior to the Neolithic Agricultural Revolution (Box 3.1), with population groups largely hunter-gatherers. Although fertility rates were high, birth control measures being largely non-existent, high mortality rates from disease, accidents and malnutrition resulted in short life expectancies.

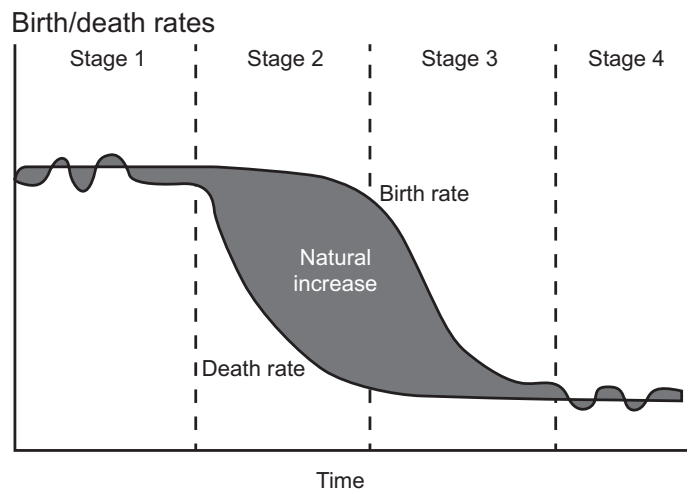

Figure 4.1 The Demographic Transition Model.

Source: Redrawn from McFalls (2001: 34). 
Stage Two is a "high growth" transitional stage. Mortality begins to decline but fertility rates remain high, leading to an excess of births over deaths and subsequently "intense" (Poston and Bouvier 2010: 272) rates of population growth. Of key significance was the Industrial Revolution, roughly covering the century from 1750, where huge developments in industrial technology (e.g. steam engines and mass production) were mirrored by more efficient and productive agriculture. This allowed both greater food production and increased wealth in rapidly growing urban areas that eventually saw improvements in sanitation and public health, leading to a decline in disease and mortality. However, fertility rates lagged and remained high, as children represented valuable assets economically (4.4.1) and there was minimal birth control.

In Stage Three, "decreasing growth", fertility rates start to fall. Although births still exceed deaths, rates of population growth begin to decline. Living and working conditions continue to improve and mortality rates keep falling. Families also begin to have fewer children, not least because costs relative to benefits increase (e.g. having children educated), and family size declines.

Stage Four has both fertility and mortality rates and overall population growth low, within countries that are conventionally termed "more developed." Improvements in medical technologies and sanitation continue to decrease mortality and the increasing costs associated with raising and educating children mean most families explicitly choose to have relatively few offspring. Birth control rises in prominence.

Stage Four is also known as expressing "incipient decline", which has led scholars to propose a Stage Five (Poston and Bouvier 2010: 273). Here, fertility rates drop below mortality rates and population growth is negative, leading to overall population decline. This is a stage countries such as Germany, Japan and Russia are entering (4.3.3).

The DTM has proved very influential within Demography and Population Geography, and for understanding countries' population experiences generally (Holdsworth et al. 2013: Chapter 2). However, it is not without critics. Jones (1990: 20) noted a quarter of a century ago how "patterns of demographic transition ... can be recognized clearly enough in most developed countries, but the way in which they are derived from, and interact with, the social and economic changes which constitute development... is a more complex matter." In particular, while the DTM is useful for expressing broad empirical trends, it is criticized for being overly focused on the experiences of western Europe. Simply to assume applicability elsewhere is an example of what Taylor (1993: 9) called the error of developmentalism, the "futuristic speculation" that all countries follow the same path of "development" rather than recognizing the more relationally defined paths actually taken. Critics have queried the applicability of a model rooted in the European experience to countries currently undergoing demographic transition. Moreover, the DTM is a macro-scale generalization even for western Europe, where studies have shown fertility decline, for example, extremely complex in terms of timing, geography and underlying causes (Coale and Watkins 1986). Fertility within a life course is complexly configured.

Rather than reject it, other scholars have sought to add to the traditional DTM. For example, Lesthaeghe (1995) and Van de Kaa (1987) proposed a "second demographic transition" to expand the model's scope to engage with economic, cultural and gender issues. The result is summarized in Table 4.2. Lesthaeghe (1995) later identified three distinct phases in western Europe linked with particular time periods (1955-1970, 1970-1985, 1985-) and has gone on to develop his model still further (Lesthaeghe 2014).

Key characteristics of the second demographic transition are decline of fertility below replacement level (4.2.1) and linking demographic changes, especially fertility and sexual union formation, with behavioral changes. Sabotka et al. (2003: 252, emphases removed) evocatively summarized Van de Kaa's stages as "1) from the golden age of marriage to the dawn of cohabitation; 2) from an era of the king-child with parents to that of the king-pair with a child; 3) from preventative contraception to self-fulfilling conception; and 4) towards pluralistic families and households." Human agency is being brought to the fore. However, these changes also have economic links, specifically tied to growth of a service economy and expansion of a welfare state. Exemplifying the model in a study of Romania, Russia, Bulgaria and Hungary, Hoem et al. (2009) found evidence of a second demographic transition, particularly changes in union formation practices, in each country (also Sabotka et al. 2003 on the Czech Republic).

Returning to the fertility transition from a state of high to low fertility rates between Stages Three and Four of the original DTM, Demographer Ansley Coale (1973) proposed three primary preconditions:

- Acceptance of calculated choice as a valid element in marital fertility;

- $\quad$ Perception of advantages from reduced fertility; and

- Knowledge and mastery of effective techniques of control (especially acceptance of and access to contraception). 
68 FERTILITY AND BIRTHS

Table 4.2 First and second Demographic Transitions in western Europe: demographic and societal characteristics

\begin{tabular}{|c|c|}
\hline First demographic transition & Second demographic transition \\
\hline $\begin{array}{l}\text { Marriage } \\
\text { - Rise in proportions married, decline in age at first marriage } \\
\text { - Low or reduced cohabitation } \\
\text { - Low divorce } \\
\text { - High remarriage }\end{array}$ & $\begin{array}{l}\text { - Fall in proportions married, rise in age at first marriage } \\
\text { - Rise in cohabitation (pre- and post-marital) } \\
\text { - Rise in divorce, earlier divorce } \\
\text { - Decline of remarriage following both divorce and widowhood }\end{array}$ \\
\hline $\begin{array}{l}\text { Fertility } \\
\text { - Decline in marital fertility via reductions at older ages, } \\
\text { lowering mean ages of first parenthood } \\
\text { - Deficient contraception, parity failures } \\
\text { Declining illegitimate fertility } \\
\text { - Low definitive childlessness among married couples }\end{array}$ & $\begin{array}{l}\text { - Further decline in fertility via postponement, increasing mean } \\
\text { age of first parenthood, structural sub-replacement fertility } \\
\text { - Efficient contraception (exceptions in specific social groups) } \\
\text { - Rising extra-marital fertility, parenthood within cohabitation } \\
\text { - Rising definitive childlessness in unions }\end{array}$ \\
\hline $\begin{array}{l}\text { Societal background } \\
\text { - Preoccupation with basic material needs: income, work } \\
\text { conditions, housing, health, schooling, social security; } \\
\text { solidarity prime value } \\
\text { - Rising memberships of political, civic and community } \\
\text { oriented networks; strengthening social cohesion } \\
\text { - Strong normative regulation by State and Churches; first } \\
\text { secularization wave; political and social "pillarization" } \\
\text {-egregated gender roles, familistic policies, } \\
\text { "embourgeoisement", promotion of breadwinner family model } \\
\text { Ordered life course transitions, prudent marriage and } \\
\text { dominance of one single family model }\end{array}$ & $\begin{array}{l}\text { - Rise of "higher order" needs: individual autonomy, } \\
\text { self-actualisation, expressive work and socialization values, } \\
\text { grass-roots democracy, recognition; tolerance prime value } \\
\text { - Disengagement from civic and community oriented networks, } \\
\text { social capital shifts to expressive and affective types; } \\
\text { weakening social cohesion } \\
\text { - Retreat of the state, second secularization wave, sexual } \\
\text { revolution, refusal of authority, political "depillarisation" } \\
\text { - Rising symmetry in gender roles, female economic autonomy } \\
\text { - Flexible life course organization, multiple lifestyles, open } \\
\text { future }\end{array}$ \\
\hline
\end{tabular}

Source: Lesthaeghe, R. (2010: 5).

These preconditions suggest that families must both desire fewer children and have access to the knowledge and technologies (such as contraception) to control fertility before rates at a community scale decline. In short, there is considerable individual and household control of the fertility process, a perspective developed further later in the chapter.

Focus on fertility transition within the DTM is one way to model, examine and arguably explain the forces and considerations that both bring about and accompany a shift from high to low fertility rates. However, to reiterate, it also attracts the same criticism as that leveled at the overall DTM: based on the experiences of western European countries, developmentalism, and neglectful of spatial and temporal variations increasingly evident in contemporary experiences. One can thus turn to a second perspective on modeling fertility geographies, which focuses more on individual (family) decision-making processes but starts with fecundity.

\subsubsection{Fecundity and the proximate determinants of fertility}

Demographers have been extremely influential in drawing attention to the importance of fecundity, the ability of a woman to have children, in debates about fertility determination. Fecundity has been associated closely with a series of intermediate fertility variables. Davis and Blake (1956) identified eleven, listed in Table 4.3. These were seen to influence a society's fertility levels by affecting both fecundity directly and through formation of sexual unions and birth control practices, both of which have fecundity implications. Such variables are present in all societies but their individual importance varies considerably.

In the 1970s, Bongaarts (1978) simplified things further. He determined that four primary variables within those listed in Table 4.3 explained nearly all fertility level variation across populations. These are the proximate determinants of fertility: proportion of women married (nuptiality) or in a sexual union; percentage of women using contraception; proportion of women infecund; and societal level of induced abortion (Holdsworth et al. 2013: 87-89; Poston and Bouvier 2010: 52-56). All are considered later in the chapter and feature prominently when statistically modeling fertility behavior. A simple model (Singh et al. 1985: 113; Holdsworth et al. 2013: 89) is: 
Table 4.3 Intermediate fertility variables and proximate determinants of fertility

\begin{tabular}{|c|c|}
\hline Factor type, affecting: & Variables affecting fertility \\
\hline Exposure to intercourse & $\begin{array}{l}\text { - Age of entry into sexual unions** } \\
\text { - Permanent celibacy: proportion of women never entering sexual unions } \\
\text { - Amount of reproductive period spent after or between unions: } \\
\text { a. Unions broken by divorce, separation or desertion } \\
\text { b. Unions broken by death of husband }\end{array}$ \\
\hline Exposure to conception & $\begin{array}{l}\text { - Fecundity or infecundity from involuntary causes }{ }^{* *} \\
\text { - Use or nonuse of contraception** } \\
\text { a. Mechanical or chemical means } \\
\text { b. Other means } \\
\text { - Fecundity or infecundity from voluntary causes (sterilization, subincision, medical treatment) }\end{array}$ \\
\hline
\end{tabular}

Source: summarized and adapted from Bongaarts (1978); Davis and Blake (1956).

** Proximate determinants of fertility.

$\left.\mathrm{F}=\mathrm{C}_{m}{ }^{[*}\right] \mathrm{C}_{c}\left[{ }^{*}\right] \mathrm{C}_{a}\left[{ }^{*}\right] \mathrm{C}_{i}\left[{ }^{*}\right] \varnothing$

$\mathrm{F}=$ Actual level of fertility predicted, a function of:

$\mathrm{C}_{m}=$ Index of nuptiality/marriage: $1=$ all reproductive age women married, $0=$ none married

$\mathrm{C}_{c}=$ Index of contraception: $1=$ no effective contraception, $0=$ all reproductive age women using efficient contraception

$\mathrm{C}_{\mathrm{a}}=$ Index of abortion: $1=$ no induced abortion, $\mathrm{O}=$ all foetuses aborted

$\mathrm{C}_{i}=$ Index of post-partum infecundability: $1=$ no women infecund for any period, $0=$ infinite infecundity

$\emptyset=$ Total fertility possible

F varies between zero and the total hypothetical fertility possible ( $\emptyset$ ). Thus, if all women of reproductive age are married, use no contraception, have no induced abortions and are not infecund for any time after a birth, predicted fertility is $\left.1\left[^{*}\right] 1\left[^{*}\right] 1\left[^{*}\right] 1{ }^{*}\right] \varnothing$, or the maximum value of $\emptyset$. In contrast, if no women of reproductive age are married, all use effective contraception, all abort foetuses somehow still formed and are all infecund, predicted fertility is $0\left[^{*}\right] 0\left[{ }^{*}\right] 0$ ${ }^{[*} 0\left[{ }^{*}\right] \emptyset$, or zero. Of course, it only takes one of these indices to be zero for predicted fertility to fall to zero.

Applications of Bongaarts's model, usually in variations of the basic expression given above, have proved insightful. Singh et al. (1985) applied a version to data from 29 countries across the world. They found reduced post-partum infecundability (the index becomes closer to 1), which increases fertility, typically counterbalanced by decrease in nuptiality and increase in contraceptive use (both indices becoming closer to zero.) However, they also concluded that the "extent to which observed variations in sub-national fertility can be attributed solely to concomitant variations in the [main] proximate determinants ... [is] limited" (Singh et al. 1985: 132.) Clearly, one must step away from proximate determinants to engage with broader cultural, social and economic factors. This will be done after indicating some spatial variations of TFR across the world today.

\subsection{GLOBAL FERTILITY PATTERNS}

\subsubsection{Fertility divergence}

Globally, public discourse on fertility is largely dominated by two primary but opposing trends: high (often increasing) and low (often decreasing) birth rates. These trends, reflecting different relative positions of countries in the DTM, 


\section{FERTILITY AND BIRTHS}

display a clear geography, illustrated in Figure 4.2. Countries with the highest TFR are generally located in the Global South, heavily concentrated in sub-Saharan Africa (PRB 2010.) In contrast, countries with low TFRs, specifically at or below the replacement level of 2.1-the fertility rate required to produce enough babies to replace a cohort of adults when they die-are predominantly within the Global North. Note however, some countries usually positioned within the South, such as Brazil and Chile, have transitioned from high to low fertility levels.

Overall level of fertility has major implications for a country. High TFR prompts increasing demand for economic expansion to meet the employment needs of growing youth populations, while also foregrounding issues of resource availability and environmental change. In contrast, low and especially below replacement level TFR countries struggle to accommodate ageing populations and the prospect of slower economic and demographic growth. These two broad groups will be considered in more detail, although inevitably oversimplifying a more complex global demographic picture.

\subsubsection{High fertility countries}

Figure 4.2 showed highest fertility countries clustered in sub-Saharan Africa, with moderate fertility rates in South America and Asia. The latter contain many countries experiencing a fertility transition away from high TFR. The cases of a few African countries, utilizing 2012 data (PRB 2012), will be used to express something of the experiences of the high fertility group.

Sub-Saharan countries, such as the Democratic Republic of Congo (TFR =6.3), Tanzania (5.4), Uganda (6.2) and Zambia (6.3), have some of the world's highest fertility rates. Yet such rates should not be regarded as inherently problematic and certainly not by resident populations. For example, a report from the Population Reference Bureau showed both men and women in the Democratic Republic of Congo desiring very high numbers of children: 6.8 and 8.2, respectively (PRB 2012: 3.) Both figures were above the country's TFR of 6.3, suggesting the latter would rise

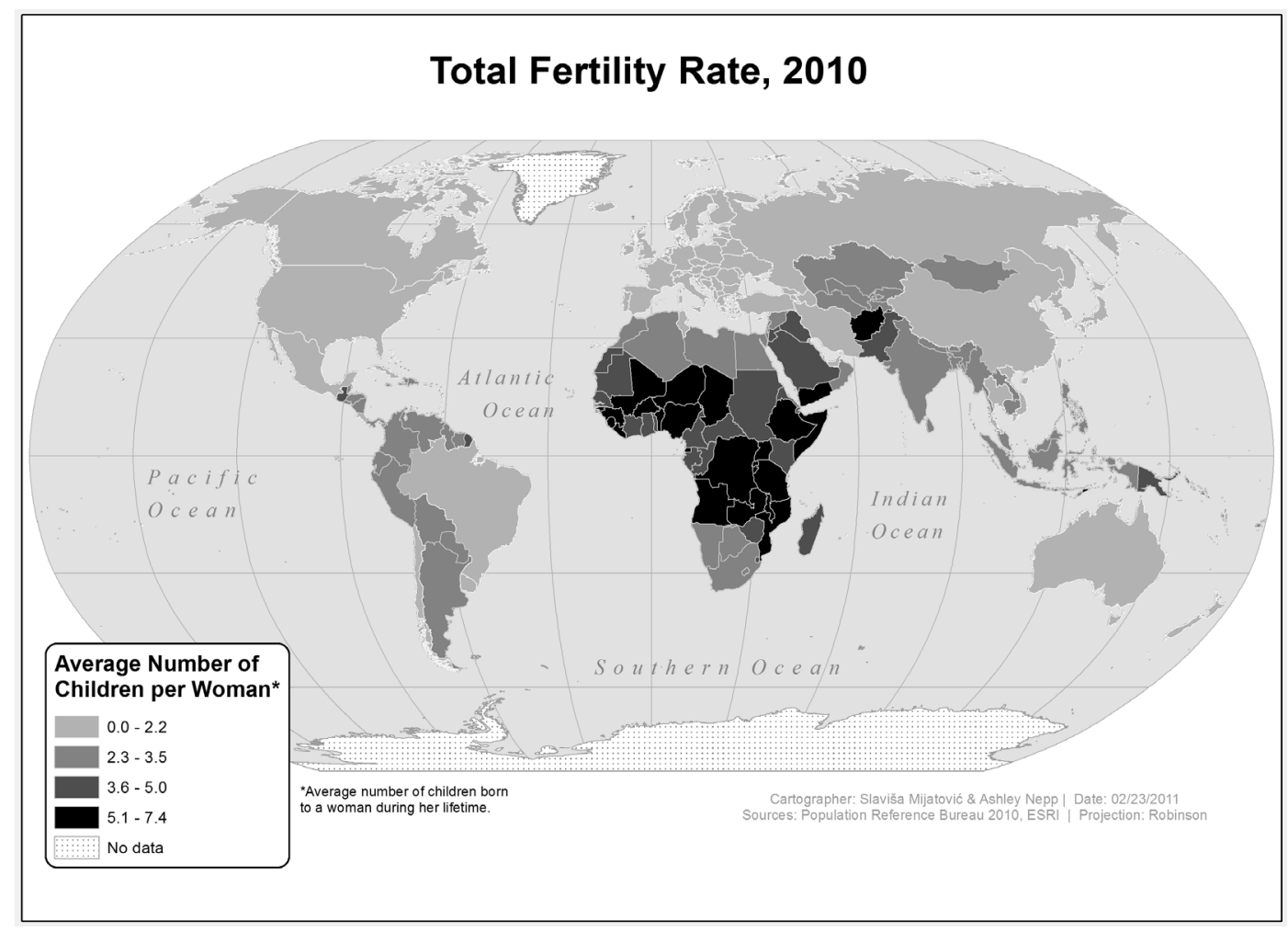

Figure 4.2 Global distribution of fertility rates by country, 2010.

Data source: Population Reference Bureau (2010). 
still higher if desires were met. Furthermore, whilst high fertility figures typically indicate fast growing populations, they must not be judged against the global replacement tevel fertility level of 2.1. For any individual country in 2012, replacement level could be up to 3.4 (Sierra Leone), due to higher levels of mortality (Espenshade et al. 2003.) Reiterating Section 4.2.2, fertility must always be analyzed in the context of mortality (and migration) to get the more complete picture of why it is at a particular level and the significance of that level.

Geographical clusters of high fertility, such as in sub-Saharan Africa, tend to be observed from global and national vantage points. However, countries also express tremendous variation in fertility rates among different sub-groups within the population and between geographic regions. Understanding these is often more complex than simply correlating with economic development, as simple reference to the DTM might suggest. Briefly consider fertility rates

\section{BOX 4.1 THE POST-1945 "BABY BOOM"}

When one thinks of high fertility countries today, the Global South features most strongly in the geographical imagination. However, it is not long ago that the North expressed a burst of relatively high fertility. Recognition of such events illustrates how general patterns of fertility (and mortality) modeled by the DTM may be punctuated by localized disruptions, the consequences of which work to shape life course cohorts born during these periods.

Many factors encourage people to have children. Understandably, the end of a major war and a sense of living in a period of general economic optimism is likely to work in the former manner! This occurred across much of the Global North in the years immediately following the end of World War II in 1945. The period has become known as the "baby boom" years, producing a life course cohort labeled the "baby boom generation."

Although the precise period is debated, the baby boom is usually dated from around 1947 until the mid 1960s. Young adults in general through this period chose to have considerably more children than their parents' generation. US TFR peaked at more than 3.7 per woman, resulting in 30 million people added to the population in the 1950s compared to 19 million in the 1940s. Such a fertility boost resulted in a distinct bulge in the population pyramids of countries affected.

The baby boom generation's ageing and consequent moving up the population pyramid had a number of socio-demographic consequences, reiterating the importance of cohort recognition within life course studies. First, the large numbers involved have strained provision of services such as education, where lack of both space and teachers led to overcrowding. Second, it also meant that when "boomers" entered job and housing markets, there was, at least initially, not enough to go around. Third, today, as the generation reaches old age, a key challenge is provision of adequate support.

From the mid 1960s, fertility in baby boom countries declined and there emerged the "baby bust" cohort, also labeled Generation X. Following the baby boom, this cohort was presented with both benefits and problems. On the one hand, supply had to some extent caught up with boomers' demands and there were now far more schools, university places, jobs, houses, and so on available, many of which the now fewer numbers of young people had greater choice over. Yet, on the other hand, with the baby boom generation becoming elderly, a smaller working age cohort increasingly has to support them in their retirement through taxes, direct financial support or hands-on care.

Finally, the baby boom generation is often associated with relatively distinctive cultural experiences and identities. Whilst one must not take this distinction too far and be wary of simply seeing the large size of this group as somehow the cause, boomers grew up in times of often dramatic social change. In spite of their numbers, and with memories of the 1930s Great Depression still relatively fresh, they were a relatively wealthy group, often subsidized by the state for education and housing. At least initially, they had high expectations of the world becoming a better place. And, as deeply socially divisive and traumatic concerns, such as the Vietnam War and global environmental crises, quickly dampened the latter, baby boomers were at the forefront of countercultural challenges in the 1960s and 1970s. As boomer Jim Morrison of the Doors sang in 1968, rather optimistically from the counterculture's point of view: "They got the guns but we got the numbers; gonna win, yeah, we're taking over."

(Sources: Goffman and Joy 2005; Poston and Bouvier 2010: 8-10, 290-291) 


\section{FERTILITY AND BIRTHS}

within India, a country with a moderate and declining national fertility rate. While overall TFR halved from 5.2 in the early 1970 s to about 2.6 in 2008, these aggregate numbers masked tremendous differences between Indian states (PRB 2012). In the 1970s, rates varied from 3.9 in Tamil Nadu to 6.7 in Uttar Pradesh, and in 2008 ranged from 1.7 in Tamil Nadu and Kerala in the south to 3.9 in Bihar in the north. These geographical differences illustrate how important local disparities in development, education and cultural preferences are for determining fertility decisions. For example, while social development, such as education and literacy levels of women, religious reform, land reform and provision of public healthcare, contributed to declining fertility for all social groups in Kerala from the nineteenth century, Tamil Nadu's low value is related to more recent economic and social changes. Levels and types of economic development, as well as social factors such as education, literacy, caste status, income, declines in infant mortality, and family planning programs each play a role in fertility reduction, exerting varied levels of importance for segments of the population in different areas (Dyson et al. 2005; JSK 2012; Sinding 2007).

Ultimately, sustained high fertility rates are associated with high levels of population growth (Box 4.1). Such growth has numerous societal and environmental implications. In particular, high fertility prompts concerns about declining resource availability as populations expand, noted in Chapter 3 and revisited in Section 4.5.2.1 and Chapter 11. This is clearly of global significance. There are also other reasons why the international community now concerns itself with high birth rates. One concern centers on the consequences of so-called youth bulge cohorts.

The youth bulge refers to the demographic shape of populations in which a sizable portion of persons are concentrated into younger age groups, particularly those just entering reproductive years. This concentration, towards the base of the population pyramid, means that absolute number of births is likely to remain high for many years, even if fertility rates are declining, simply because so many people are in or entering their childbearing years. Demographic

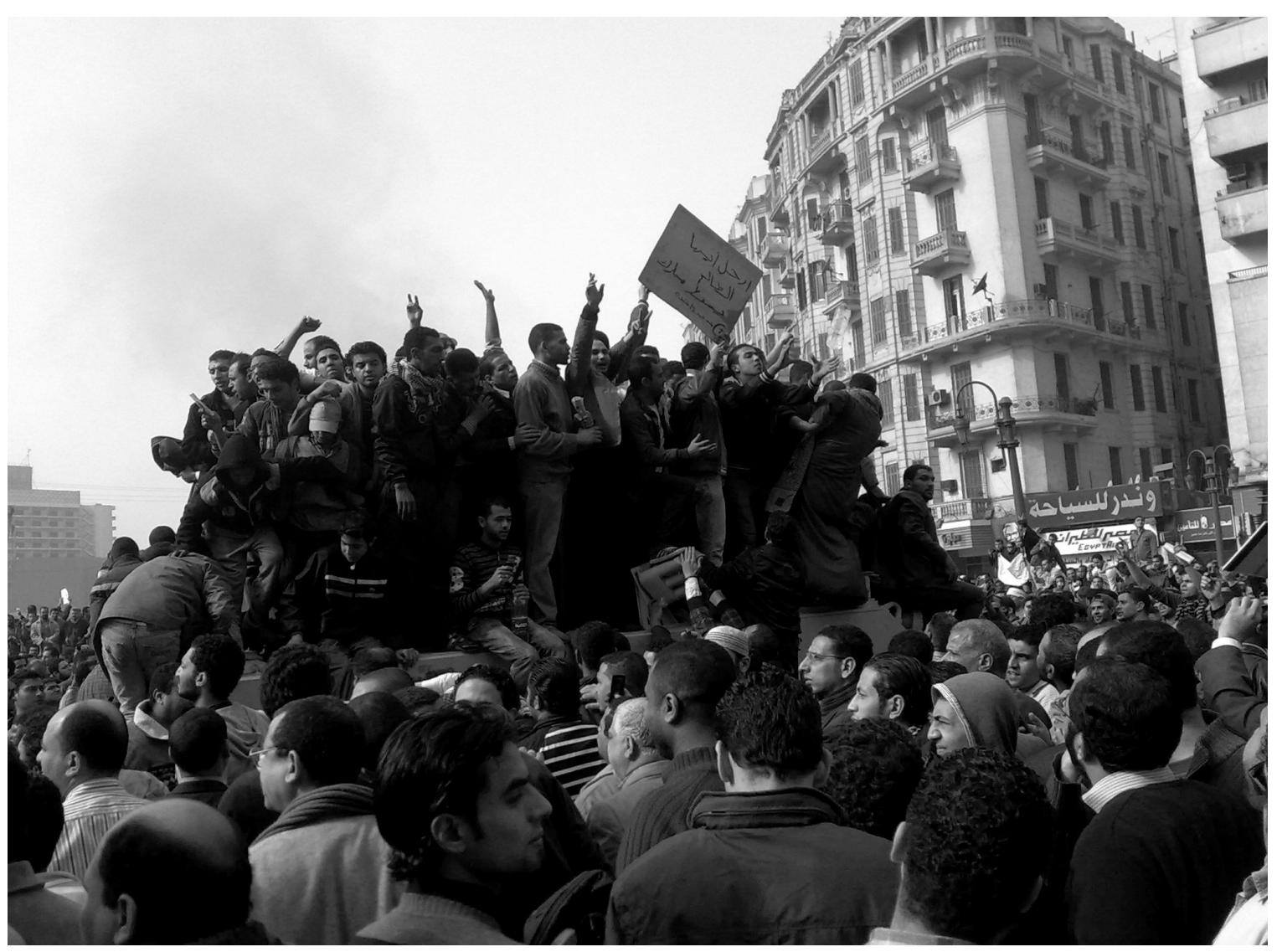

Figure 4.3 Youthful (male) protestors in Tahrir Square, Cairo, January 2011.

Source: Ramy Raoof. 
momentum (3.2.2), in other words, remains strong, reflecting how population age structure has implications for population growth regardless of fertility rate.

The youth bulge also has implications for employment and political stability, and via these for migration (Urdal 2012). Many countries with a youth bulge already struggle to generate enough employment opportunities for their populations. As members of the bulge come of age, this situation worsens. Without employment, youth are unable to earn a living, afford suitable and safe housing, and/or access adequate food resources. Demographically, their quest for employment pushes many out of rural regions and into expanding urban centers (7.3.3.1), further exacerbating poverty, unemployment and the poor living conditions already present (Davis 2006). Such circumstances sometimes further prompt searches for employment and life opportunities at increasingly distant global cities, fueling international migration movements (7.3.3.2). Finally, having many young adults lacking basic requirements for a decent twentyfirst-century life can provoke social upheaval and political instability.

Egypt well illustrates the consequences of having a distinctive youth bulge (Figure 4.3), with nearly half of the population under the age of 25 years (Roudi-Fahimi et al. 2011). Whilst experiencing considerable problems with unemployment, housing and so on, young adults have been important drivers of social change. They played a leading role in the 2011 protests that ultimately led to the fall of President Hosni Mubarak (Roudi-Fahimi et al. 2011). Indeed, members of the youth bulge formed a key element within all the "Arab Spring" uprisings of that year, even if certainly not solely responsible for changes that occurred (Hoffman and Jamal 2012).

\subsubsection{Low fertility countries}

While many countries grapple with high fertility rates, there is growing concern elsewhere about rates dropping below replacement level. From Figure 4.2, these countries are predominantly, although not exclusively, located in the Global North. For example, China had a 2012 TFR of 1.5, the US 2.0, and Sweden 1.9 (PRB 2012.) Yet, each of these countries' low fertility originated in quite different circumstances. China's low value reflected the impact of a one-child state policy (4.6.3) and Sweden's changes in age structure and family-size preference (it had even actively sought to increase childbearing; King 2001). For the US, there was a shift from an average of seven children per woman in the early 1800s to four by the early 1900s and, after a brief upsurge following World War II, a decline to 2.1 (global replacement level) by 2009 (PRB Staff 2004).

Table 4.4 lists many more countries with low TFRs. In most, fertility declines "coincided with trends toward delayed marriage, more divorce, and an increase in the percentage of women going to college and working outside the home" (PRB Staff 2004: 7). These associations reinforce fertility's positive association with being in a stable sexual union

Table 4.4 European and Asian countries with continuing TFR below 1.5

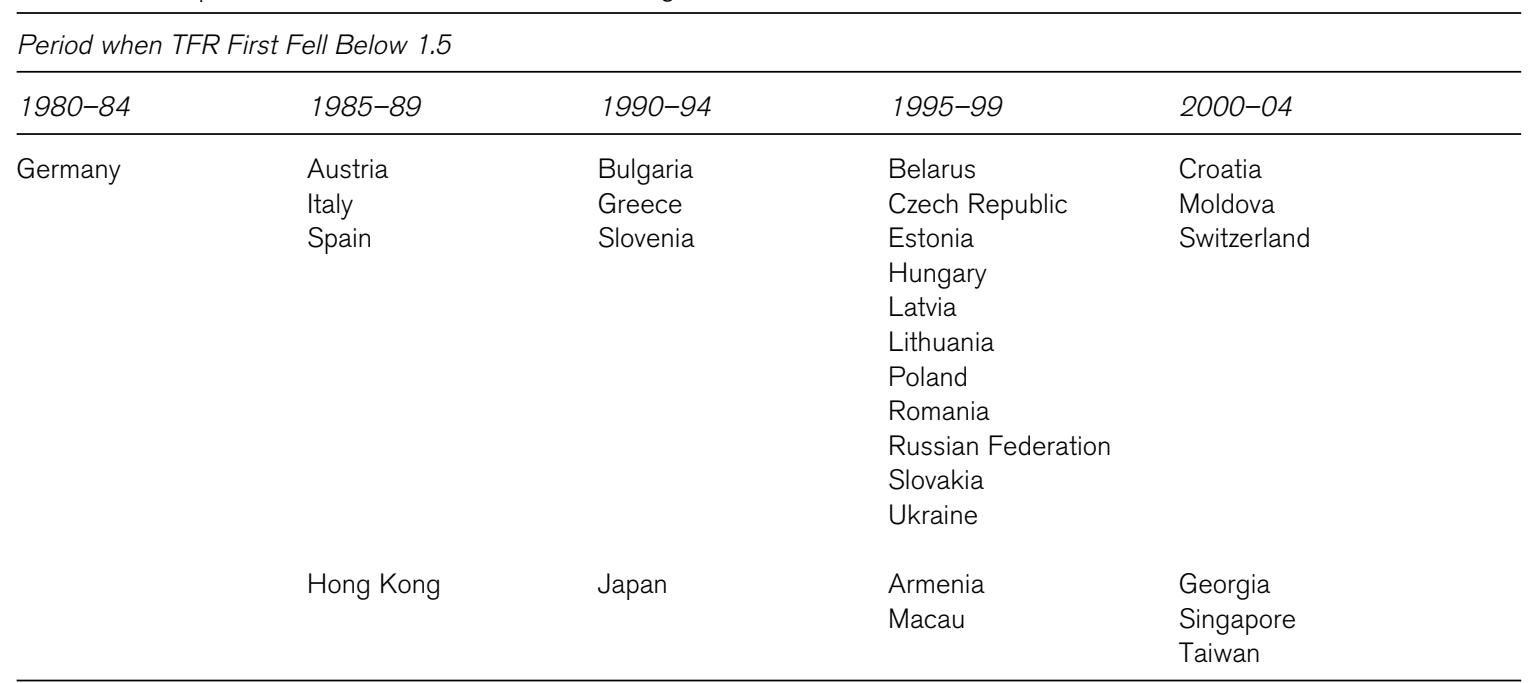




\section{FERTILITY AND BIRTHS}

(notably marriage) and women's non-engagement with paid work. The latter illustrates that the importance of fecundity to fertility expands beyond intermediate fertility variables by linking ability to have children to employment practices, in particular.

What, then, of the societal consequences of low fertility rates? Concerns were first voiced in the 1930s in the context of ethically controversial eugenicist attempts to "improve" the supposed genetic composition of a population. Such improvement was to be achieved through genetically "strong" sub-populations being encouraged to have children, whilst the genetically "weak" were discouraged. It was epitomized by Nazi Germany's selective promotion of births from Aryan parents and brutal repression of other sub-groups, such as the disabled (Bock 1983; also Box 2.4).

Renewed concern today over low fertility and countries entering Stage Five of the DTM is fortunately less underpinned by explicit eugenicist or racist discourses. What Caldwell and Schindlmayr (2003) summarize as a "fertility crisis" for many European and some Asian countries is regarded as primarily a matter of the consequences that stem from population ageing. Economically, low fertility sees reduced cohorts of young people entering the workforce. The result is a declining proportion of the population contributing through taxes to the social service and health budgets needed to support, in contrast, a growing cohort of older dependent adults. Furthermore, fewer workers and a dampening of consumption-both working age people and the elderly have less surplus income to spend-potentially leads to lower productivity and growth for the whole economy.

In contrast and reflecting critiques of the dependency argument (10.2.3.5), Morgan (2003: 600) did not see low fertility as a crisis. Instead, he depicted it as

the kind of problem we want to have ... the result of solving a bigger, more threatening social problem: the crisis of continued population growth. [It] can be addressed through public policy and institutional adjustments ... [and] befalls ... countries that, by and large, have the resources to respond.

He argued that problems could potentially be offset by increasing immigration, raising average retirement age or other public policy management. Indeed, such debates are being engaged with in some countries (4.6.2). Anderson and Hussey (2000) concurred, arguing that the US, for example, is well-positioned relative to comparison countries but must pay particular attention to formulating public policies mitigating the costs of low fertility, such as those engaging with the costs of long-term health care and pharmaceuticals. The World Bank has also raised concerns about the sustainability of public pensions systems (World Bank 1994). Additionally relevant here are the relative costs (and benefits) of having children, considered next.

\subsection{THE "VALUE" OF A CHILD}

\subsubsection{Economic value}

What is the "value" of a child? This question may appear shocking even to ask, as people are generally brought up to regard children as "priceless," outside monetary calculus. Yet, if one temporarily suspends this moral reluctance to value living people, a number of important issues come to light. First, children do clearly cost or, put another way, require supply of a range of resources if they are to survive and prosper. They need food, drink and shelter as basics for survival; education and socialization if they are to thrive; and love and emotional interaction if they are to belong. All of these, either directly or indirectly, can be put into monetary terms-albeit imperfectly in many cases. Children also provide benefits, which can be similarly monetized. They can provide love and emotional sustenance; family labor within the household, such as cooking, caring, collecting fuel and cleaning; and, whether through child labor or later as adults through remittances, monetary support to family, not least aged parents. In other words, a baby or child embodies a composite of different values. How these values are defined, selected and evaluated is something that is strongly relational. It is also something that researchers, not least Economists, have tried to express and link with fertility behavior.

A classic approach engaging with the economic value of a child as it impacts on fertility behavior is the intergenerational wealth flow theory of fertility associated with Demographer John Caldwell (1982). Usually considered in conjunction with the DTM, attention is paid to whether it is parents or the resulting new person who are the likely 
net beneficiaries of a birth. Where net wealth flow is towards parents-from children to adults-it is "rational" for the family to have as many children as possible, each fortifying family wealth. In contrast, where costs of having children rise and inter-generational wealth flows in reverse, in favor of the child, it becomes rational to have few or even no children. Costs can rise as a result, for example, of laws compelling a child to attend school or regulating work both inside and outside the home. They can fall as a result, for example, of improved social child welfare provisions.

An important implication of inter-generational wealth flow theory is that it is simplistic and often incorrect to regard populations with high fertilities as somehow "irrational." Family life course context can encourage, even almost necessitate, high fertility for its survival. Nonetheless, there are problems with the theory (e.g. Cleland and Wilson 1987; Kaplan 1994).

First, there is the underlying assumption of rational behavior by families with respect to responding to intergenerational wealth flows. On the one hand, it assumes both perfect and accurate information for decisions to be made. This is highly improbable. On the other hand, it assumes that rationality within (fertility) behavior predominates. As all who have children will attest, deciding to become a parent is rarely expressed in such "objective" ways! The demographics of a life course are much more complex in their relational construction.

Second, there are practical problems with the theory. Cleland and Wilson (1987: 27) observed how "conscious exercise of birth control within marriage" was largely absent in "traditional societies," implying that relatively high birth rates were inevitable. They also noted how empirical data do not support the theory. Indeed, these tend to suggest children are probably always a net family cost, with "net intergenerational wealth flow ... downward in all societies" (Kaplan 1994: 785). Certainly, costs are widely noted today across the Global North (Box 4.2). However, a perspective focusing on the relative costs and benefits of children at different places within a life course is useful here. Young children may be a net cost, yet are financially bearable for relatively young and healthy parents. When the latter age, now-adult offspring become of net economic benefit through provision of support. Children, from this perspective, are an investment.

Third, greater attention must be paid to valuation of a child other than just "wealth." Thus, perhaps it is better to see what changes between high and low fertility societies as more "ideational" (Cleland and Wilson 1987): "what matters ... is a shift in family morality that encourages parents to be aspirational for their children" (Holdsworth et al. 2013: 24), requiring investment in them (costs). Even relatively homogeneous societies today exhibit considerable variations in fertility behavior, related to religious belief or more secular cultural values, norms and expectations, often mediated by birth control technologies. Attention thus switches from economic to cultural evaluation of a child.

\subsubsection{Cultural value}

There was, in fact, always a strong cultural dimension within inter-generational wealth flow theory. First, Caldwell (1982) only saw it operating properly where the family unit was the center of economic calculations. Second, historically, the practice of having several children in pre-fertility transition societies would have become very firmly culturally embedded. There would thus be substantial lag before families likely acknowledged wealth flows changing direction and had fewer children. Third, Caldwell et al. (1992) also recognized how such socio-cultural demographic factors as urbanization and contraceptive use reduced fertility.

Within the cultural framing of fertility decision-making within a life course, the religious arena (2.3.7) can play a major role. For example, in a study of high fertility within sub-Saharan Africa, Caldwell and Caldwell (1987) suggested religious beliefs intertwined with and reinforced a societal structure whereby high fertility was rewarded. In consequence, fertility decline was again likely to lag any reversal of wealth flows in favor of the child. Generally, the major world religions are pro-natalist-encouraging fertility-but more differentiated and inconsistent concerning use of birth control and contraception.

Different cultural post-natal practices also impact on fertility behavior and consequences. For example, breastfeeding a baby suppresses a woman's fecundity and can provide reliable contraception for up to nine months (McNeilly 2002). Postpartum sexual abstinence, a delay in resuming sexual relations following birth, also reduces fertility. Thus, the Yoruba and Benin peoples of Nigeria regard a woman's body following birth as needing time to recover. There is also a belief that a man's ejaculate would contaminate her breast milk (Sule-Odu et al. 2008). Sule-Odu and colleagues went on to show that whilst 80 percent of Yoruba mothers abstained from sex for up to 2 months, only 6 percent were still doing so after 6 months.

\section{ST PROOFS: NOT FOR DISTRIBUTION}




\section{BOX 4.2 THE COSTS OF CHILDREN}

The cost of raising children is a popular topic of media interest but is also of concern from welfare and equality perspectives. Consider these two examples from the UK and US. First, the UK: "At a time when many families are finding it hard to make ends meet, how much does it cost to bring up a child?" (Hirsch et al. 2012: 7):

\begin{tabular}{lll}
\hline Cost of a child in 2012 & \multicolumn{2}{c}{ Minimum additional cost of a child } \\
\cline { 2 - 3 } & Couple & Lone parent \\
\hline Basic cost* over 18 years & $£ 79,742$ & $£ 88,330$ \\
Cost over 18 years, including childcare & $£ 142,680$ & $£ 155,015$ \\
Percentage of basic cost covered by child benefit & $20 \%$ & $18 \%$ \\
Percentage of basic cost covered by child benefit plus maximum child tax credit & $87 \%$ & $78 \%$ \\
\hline
\end{tabular}

* Net of rent, childcare and council tax

To the US: "estimates of expenditures on children from birth through age 17 since 1960. These estimates may be used in developing state child support guidelines and foster care payments, as well as in family education programs" (Mark 2012: 1):

\section{Categories of household expenses}

Housing: shelter, utilities, house furnishings and equipment

Food: food and non-alcoholic beverages

Transportation: vehicle loans, down-payments, gasoline and motor oil, maintenance and repairs, insurance, public transportation

Clothing: children's apparel, footwear and clothing services (dry cleaning, alterations, repair)

Health care: medical and dental services not covered by insurance (prescription drugs, health insurance premiums not paid by employer or other organization)

Child care and education: day care and supplies, baby-sitting, school tuition

Miscellaneous: personal care items, entertainment, reading materials

Taking these expenses into account, the costs of raising two children were estimated as:

\begin{tabular}{lcc}
\hline Age of children (years) & \multicolumn{2}{c}{ Estimated annual expenditure, 2011(\$) } \\
\hline & Couple $^{1}$ & Lone parent ${ }^{2}$ \\
\hline $2 \& 16$ & 26,690 & 16,660 \\
$5 \& 16$ & 26,710 & 17,510 \\
$8 \& 16$ & 26,610 & 17,350 \\
$11 \& 16$ & 27,430 & 17,930 \\
$14 \& 16$ & 28,140 & 18,340 \\
$15 \& 16$ & 28,640 & 18,080
\end{tabular}

1 Before-tax income $\$ 59,410-\$ 102,870$

2 Before-tax income less than $\$ 59,410$ 
Finally, cultural and economic converge in a more psychological evaluation of whether to have a child, expressing macro-contextual life course arenas of history (2.3.2) and geography (2.3.3). Box 4.1 described the "baby boom" across much of the Global North following World War II, driven in part by a sense of optimism for the way society in general was developing. In contrast, during wars and other crises (famines, natural disasters, economic collapse) fertility tends to decline. In part biological (e.g. inadequate food for women to become fecund or take a baby to full-term or birth), decline also expresses individual or family reasoning not to bring life into the world at that perilous moment. Thus, survey data from Ethiopia showed how upheavals of war, political instability and famine in the 1970s and 1980s impacted negatively on conception rates (Lindstrom and Berhanu 1999). In another example, economic recession, with rising job insecurity and unemployment especially experienced by young adults, was a downward influence on fertility, albeit often overshadowed by other influencing factors (Sobotka et al. 2011). Furthermore, it was "bettereducated women in particular [who] perceive childbearing to be an especially risky strategy during recession and are likely to react by postponing their childbearing plans" (Sobotka et al. 2011: 291). These issues of managing timing and spacing of births are now considered in detail.

\subsection{MANAGING TIMING AND SPACING OF BIRTHS}

\subsubsection{Preferentially managing fertility}

\subsubsection{Going beyond age and health}

The management of fertility is one of the most important functions of adulthood.

(Germaine Greer 1984: 40)

The age and health of a woman (and her partner) are clearly of critical importance in shaping fecundity and fertility across a life course. This is represented, for example, through ASFRs (4.1.2). From a biological perspective, the younger a woman is when she begins having children, the more children she can have over her lifetime. Hence the fertility significance, for example, of the recent rise in the number of child brides in remote parts of Nigeria (Mark 2013). Although illegal under Nigerian law, marriage of girls, in particular, is encouraged by older customs such as the tradition that "as soon as a girl is of age [starts menstruating], she should be married" (local father, quoted in Mark 2013: 21), and by recent imposition of strict Islamic Sharia law in some parts of the country.

While there is a theoretical maximum ( $\varnothing)$ number of children a woman can bear, in addition to the risks to the mother's life associated with pregnancy and birth, numerous social and cultural mediating norms mean no woman ever bears this many children. Indeed, the number is usually shaped strongly by such norms. They include, for example, postponing childbearing until at least aged 16-18 years in many cultures or as late as aged 30 years or more in countries where women's engagement with waged labor is advanced. Norms also create social pressures to have families of particular sizes, neither "too big" nor "too small." Across the Global North, the normative-if widely satirized and increasingly unrepresentative-nuclear family of mother, father and "2.4 children" expresses this well (Duckworth 2002).

Compare the relative roles played by biology and culture in Uganda and Germany. In Uganda, 48 percent of the population is aged 15-49 years, with an additional 49 percent entering reproductive ages within the next 15 years (PRB 2009). In 2009, TFR was 6.7, the population expanding rapidly. Even if fertility rates declined, the population would continue to grow substantially through demographic momentum because of large numbers of emerging young adults of childbearing age. In contrast, although Germany has a larger proportion of the population of childbearing age (66 percent), social and cultural norms have reduced the fertility rate to about 1.3 children per woman. Combined with a small number of young people reaching childbearing ages within the next 15 years (14 percent), the probability of significant population growth is thus low (PRB 2009).

In summary, the age a woman conceives her first child, the number of children she eventually has, and the spacing between births are only partially biologically determined. Fertility behavior is always more broadly relationally constructed, culture featuring prominently. Aggregate statistics, collected worldwide on the total number of births in a population at national and more local scales, reflect countless individual choices, always set within both constraints 


\section{FERTILITY AND BIRTHS}

and opportunities, from a woman's status in society and her personal preferences for having children, to community norms of acceptable fertility practice, including what might be legal or illegal. All this relationality results in marked differentials in fertility behavior and outcomes, some examined in Section 4.5.3. First, however, the importance of appreciating the life lived by the individual woman will be exemplified through reference to preference theory.

\subsubsection{Preference theory}

The trend toward lower fertility across the Global North has been tied to increases in women's education, later marriages, pursuit of careers, desire to remain in the workforce, as well as to increasing costs of raising children. All relate closely to historical and geographical macro-scale life course contexts. There is also a suggestion of a rising desire to remain without children. Increasing use of the term "childfree" in place of "childless" for women without children suggests a subtle shift in how such individuals are viewed. Indeed, childfree is now a term actively embraced (Agrillo and Nelini 2008). This growing minority often associate child rearing with a loss of freedom, reduced career prospects and financial burdens (BBC 2006). A small but growing movement even argues that people with children unfairly receive greater benefits from governments. However, one must not get carried away. Using data for 25 countries from the United Nations Family and Fertility Survey in the mid-1990s, Hakim (2006) found levels of being voluntarily childfree in childbearing ages (20-39 years) generally below 10 percent, with the exception of Belgium at 14 percent.

Nevertheless, at least across the Global North, the twenty-first century is becoming characterized by increasingly divergent childbearing preferences and behaviors. This has been conceptualized through preference theory, a microscale or individual-level approach to understanding fertility decisions. Particularly developed by British Sociologist Catherine Hakim $(2000,2003,2004)$, it is concerned with individual decisions by women rather than aggregate generalizations. It portrays fertility choices as based on a complex set of social and individual factors-attitudes, values, life goals-converging within preferences or favored attitudes towards a particular family size and lifestyle. Understanding these preferences can help predict employment and fertility patterns (Hakim 2003.)

Hakim laid out four basic premises, presented in Table 4.5. She argued strongly against any assumption that women can be treated as "homogeneous" at a specific historical period and geographical location. Such treatment, as in the traditional DTM, was seen as a primary reason fertility behavior is so difficult to predict. Instead, Hakim identified three distinct and sometimes conflicting groups:

- Women with a lifestyle centered on the home, who often have several children;

- Women with a paid work-centered lifestyle, who often remain childless; and

- $\quad$ Adaptive women, who combine paid work, key household roles and raising children.

The groups express different fertility behaviors associated with work and lifestyle preferences. Importantly, Hakim also argued that these orientations are not based on socio-economic status or other traditional childbearing "variables" (noted above) but rather on the self-defined goals and priorities of individual women.

Whilst drawing attention to the undoubted importance of lifestyle choice and associated "strategic life planning" (Giddens 1991: 85) for fertility practice, preference theory has still attracted criticisms. First, it should be reiterated that it primarily addresses women's decisions in the Global North, its propositions assuming that the country of residence has experienced fertility transition. Second, women rarely have the level of unrestrained agency to act on preferences that the theory implies, even within the North. Numerous constraints can impinge on ability to realize preferences (McRae 2003). One may be actually having children (fecundity), thereby making (in)fertility more cause than effect of lifestyle.

A test of preference theory using comparative data from the 2004-2005 European Social Survey tested the links between individual level preferences and both fertility outcomes and intentions (Vitali et al. 2009; also Rabušic and Manea 2008). Results confirmed an association between work-family lifestyle preferences and realized fertility outcomes in several countries. However, they did not show a relationship between lifestyle preferences and fertility intentions, except in Britain and Denmark. Preference theory failed to link preferences to fertility decision-making. The key role of constraints is reflected in the conclusions that "heterogeneity in lifestyle preferences is mirrored differently in different societies and in different welfare state regimes" (Vitali et al. 2009: 435) and how it is often that "lifestyle preferences are more influenced by actual fertility than vice versa" (Vitali et al. 2009: 436). 
Table 4.5 Central tenets of preference theory

\begin{tabular}{ll}
\hline Premise & Description \\
\hline 1 & Five separate historical changes in society and the labor market that started in the late twentieth century produce \\
qualitatively different and new scenario of options and opportunities for women: \\
1) Contraceptive revolution; \\
2) Equal opportunities revolution; \\
3) Expansion of white-collar occupations, more attractive to women than most blue-collar occupations; \\
4) Creation of jobs for secondary earners, who do not want to give priority to paid work at the expense of other life \\
interests; \\
5) Increasing importance of attitudes, values and personal preferences in the lifestyle choices of affluent modern \\
societies. \\
Women are heterogeneous in their preferences and priorities vis-à-vis conflict between family and employment. \\
Heterogeneity of women's preferences and priorities creates conflicting interests between groups of women. \\
Women's heterogeneity is the main cause of their variable responses to social engineering policies in the new \\
scenario of modern societies.
\end{tabular}

Source: summarized from Hakim (2003: 356).

One thus again returns to the importance of the globally and socially differentiated relational contextual arenas within which individuals, women and their families live their lives and within which they decide to bear, or not to bear, children (2.3). Such contexts also change more-or-less continuously over the life of a woman and her family, as they progress through their life course. Their significance emerges at different scales-from within individual-level decisionmaking to expressing more collective responses to social and community cultural norms. In short, across the globe, myriad influences shape individual fertility decisions. For example, the decision to have children may be textured by questions about how best to conceive, whether a surrogate mother should be used (4.7.2), the choice of where to give birth (Box 4.3), and choices about leaving the workforce or enrolling children in day care. All become potential topics to negotiate. For others, lack of access to contraception, health insurance, and affordable day care may create barriers for childbearing and/or child rearing. Attention now turns to the first of these, a vital agent in shaping fertility geographies.

\subsubsection{BIRTH CONTROL, CONTRACEPTION AND ABORTION}

Attempts to prevent the production of children as a consequence of engaging in sexual intercourse are as old as recorded human history (Poston and Bouvier 2010: 72-74). However, the modern family planning movement finds significant beginnings with the early twentieth-century efforts of Margaret Sanger in the US (Figure 4.4) and Marie Stopes in the UK (Hall 1977). Sanger worked tirelessly from 1914 to provide women with information about contraception and legalize distribution of contraceptives to married couples (Engelman 2011). She opened a Brooklyn clinic in New York in response to what she perceived as frequent adverse effects of childbirth, miscarriages and abortions. It was the first family planning clinic in the US and, needless to say, proved highly controversial.

Whilst in the first half of the twentieth century, following Sanger and Stopes, the family planning movement focused on the needs of married couples, demands to broaden this focus soon grew. Rapidly extending across the Global North and now near global, there evolved a contraceptive revolution. By the 1960s, birth control options, such the Pill and Intrauterine Device (IUD), were approved by regulatory bodies such as the US Food and Drug Administration (CDC 1999). These options coincided with a second wave of feminism (Stansell 2010), one of whose defining features was women's increasing demand to exert greater control over their sexual behavior, childbearing and family size decisions (Greer 1970). More women were seeking higher education and expanding their career goals, further increasing their desire to control fertility.

Globally, contraceptive availability and use today involves numerous techniques. Table 4.6 shows that nearly two thirds of women use some form, mostly "modern" methods, notably sterilization. Looking more closely, contraception across the Global North favors the use of male condoms and the Pill, whereas sterilization and IUDs are more predominant in the South. There are marked variations within these groups of countries, too. Table 4.6 also shows a slight increase in contraceptive use since 2002. 


\section{BOX 4.3 HOMEBIRTH IN THE UNITED STATES}

Over the course of the twentieth century childbirth in the US moved out of the home and into the hospital. This reflects both "push" and "pull" factors. In terms of the former, the rise of the medical profession coincided with a decline in midwifery. Prior to the twentieth century, midwives were self- or apprentice-trained women who attended births largely in the birthing woman's home. They lost their "professional edge" as predominantly white middle class men practicing medicine increasingly placed childbirth under their jurisdiction. However, the rise of the hospital as the normative birth site also reflected the pull of considerable attractions to expectant mothers. Hospital birth came to represent the safest option for birthing women, as well as a symbol of financial security and affluence. New pharmacological options for pain relief available within them were also very attractive to overcome "a great deal of fear and trepidation about the potential pain (and danger) of childbirth" (Beckett 2005: 253).

Notwithstanding this historical backdrop, some women still consider a planned homebirth. There is fierce debate within local communities, as well as on the national level, about their relative safety as compared to birth in hospital. Moreover, since planned homebirth represents a tiny minority of births, discerning comparative safety is challenging. Statistics for 2005 showed 37,402 infants born out of hospitals in the US, representing just 0.9 percent of over 4 million births. Over two-thirds of those took place at home.

For the average US citizen, homebirth is an unfamiliar concept. It may conjure up an image of women giving birth in dangerous conditions in another time or place. In reality, planned US homebirths are typically attended by trained midwives. Recent studies demonstrated neonatal and maternal mortality rates for low-risk homebirths similar to low-risk hospital births. Nonetheless, this does not necessarily make homebirth appealing for low-risk pregnant women. For those who do choose one, reasons are varied and complex. Crucial is overall awareness of the option. In addition, some women actively identify reasons to avoid hospital, such as fear of medical intervention (particularly Cesarean section, also epidurals and episiotomies) and a belief that the hospital atmosphere is less conducive to "natural" birth than the home.

Research shows US women who plan homebirths more likely to be white, older, married, wealthier, and more educated than those giving birth in hospitals. This suggests homebirth is most accessible to relatively privileged members of society. One major contributor to this association is reluctance of insurance companies to provide coverage for out-of-hospital births. Families seeking certified professional midwives typically pay out of pocket for related expenses. Thus, lower-income women may not find homebirth financially feasible, even if interested. Only nine states allow reimbursement to direct entry midwives through Medicaid. Out-of-hospital midwifery is not even legal or regulated in all states and women pursuing homebirth must use informal networks to locate providers, who run great personal legal risk in their work. Therefore, financial and legal barriers shape the demographic trend of who pursues homebirth.

In conclusion, birth is simultaneously a highly individual and universal experience. Women across the US and around the world have dramatically different access to quality care, let alone the opportunity to make choices about their experiences. While learning about the choices of a relatively privileged group of women to have homebirths is useful, it is important to situate within a backdrop of inequality. Further research is necessary to understand more fully and critique the influence of social mechanisms on perceptions of birth, risk, choice, and place.

(Sources: Beckett 2005; Boucher et al. 2009; Cassidy 2006; Emple 2010; Johnson and Daviss 2005; McDorman et al. 2011; NARM 2006) 
FERTILITY AND BIRTHS

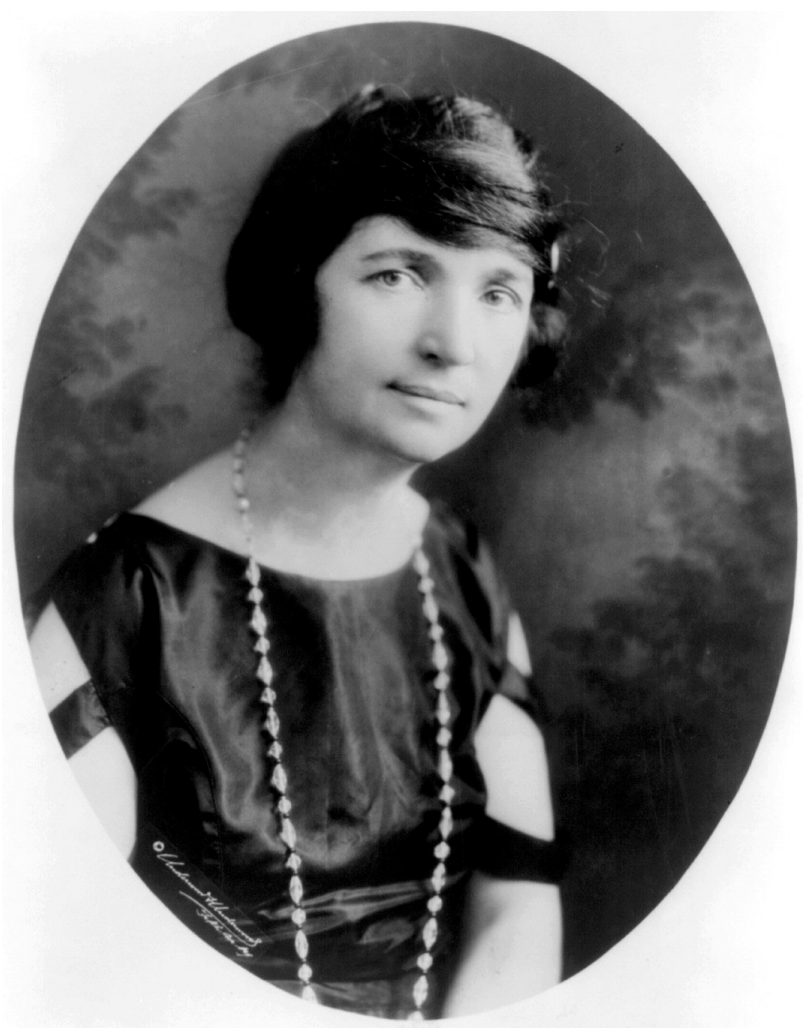

Figure 4.4 Margaret Higgins Sanger, 1879-1966.

Source: Underwood \& Underwood, Library of Congress Prints and Photographs division, reproduction number LC-USZ62-29808.

Table 4.6 Percentage of women married or in sexual union, generally aged 15-49 years, using contraception

\begin{tabular}{lllllc}
\hline Location & Any method & $\begin{array}{l}\text { Any "modern" } \\
\text { method }\end{array}$ & Pill & \multicolumn{1}{l}{$\begin{array}{l}\text { IUD } \\
\text { Sterilization (Male \& Female) }\end{array}$} \\
\hline World (2008) & $\mathbf{6 3}$ & $\mathbf{5 7}$ & $\mathbf{8}$ & $\mathbf{1 4}$ & $\mathbf{6}$ \\
World (2002) & 61 & 55 & 7 & 15 & 5 \\
Global North (2008) & 71 & 61 & 18 & 6 & 20 \\
Global North (2002) & 68 & 58 & 14 & 9 & 15 \\
Global South (2008) & 62 & 56 & 7 & 15 & 4 \\
Global South (2002) & 60 & 54 & 6 & 15 & 3 \\
N. Europe (2002) & 82 & 77 & 33 & 11 & 28 \\
North America (2008) & 74 & 69 & 18 & 2 & 12 \\
Latin America \& Caribbean (2008) & 72 & 63 & 13 & 7 & 5 \\
Oceania (2008) & 72 & 64 & 22 & 1 & 17 \\
Asia (2008) & 67 & 61 & 6 & 18 & 6 \\
S. Europe (2008) & 62 & 44 & 10 & 5 & 18 \\
Africa (2008) & 30 & 25 & 8 & 5 & 2
\end{tabular}

Source: PRB $(2002,2008)$. 


\section{FERTILITY AND BIRTHS}

Contraception/birth control and family planning is a major concern of Demography (Poston and Bouvier 2010: Chapter 4), not least because it is a vitally important explanation of fertility decline and also associated with other social changes, especially benefiting women. For example, use of contraception substantially accounted for dramatic decreases in fertility in Thailand, Columbia, Jamaica and Mexico (CDC 1999). In impoverished areas of Nepal, where girls as young as 12 years still marry, even though child marriage is illegal, contraceptive availability has given these young women much greater control over their fertility behavior (Williams 2012.) Use of contraception is not culturally taboo and "family planning has rocked and continues to rock this society-the changes will not stop at smaller family sizes and lower infant mortality and higher female literacy and better maternal health" (Williams 2012: 35).

Universal access to contraceptives is still far from realized, of course. In Afghanistan (e.g. Mojumdar 2012) in 2008 only 8.5 percent of women were using modern methods (PRB 2008). Nevertheless, as seen in Nepal, increasing availability and knowledge is beginning to shift control of fertility to women. For Hakim (2003: 350-351), consequently, "women's views, perspectives, and goals become the key to understanding current and future changes in women's position in society and ... patterns of fertility" (4.5.1.2).

It must be recognized, however, that throughout its existence, use and promotion of contraception has provoked considerable controversy (Engelman 2011), in particular from certain strict moral religious viewpoints. For example, there are ongoing often heated debates on the (non-) place of contraception (and certainly of abortion) within the Roman Catholic faith community (e.g. Catholics Against Contraception 2012). Sanger's and Stopes's legacy remains a lively area of ethical and political debate (e.g. Planned Parenthood 2012), even though by 2012 the UN had declared access to family planning a human right (UNFPA 2012a).

By the 1990s, the family planning movement had begun to broaden into concerns about reproductive health generally (CDC 1999). Here, the international community, including the UN and Non Governmental Organizations (NGOs), plays a vital role in disseminating information about and sponsoring programs that facilitate access to contraceptives and family planning resources. This is often underpinned by a strong health and welfare discourse. A major milestone was the 1994 Cairo International Conference on Population and Development (UN 1995), which expanded debate about population growth from just fertility rates to the need to integrate socio-economic development with demographic goals. At the conclusion of the conference, 179 countries adopted a 20 year Program of Action, recognizing women's empowerment, gender equity and reproductive health rights as key intertwined aspects of population and development programs (UN 1995).

It is against this key-backdrop of the family planning movement and contraceptive revolution that three key dimensions of fertility differentiation within the relational arenas of the life course will now be examined. This focus on the influence of arenas that promote such differentials across and within societies moves away from a focus on statistical correlations-such as contraceptive use or age of marriage with fertility rates-to engage more fully with the potential mother's (and father's) place or position within and consequent experiences of society generally as their life course plays out.

\subsubsection{Fertility differentials}

\subsubsection{Class and status}

Class and status are critical differentiating variables within fertility behavior. They influence behavior fairly directly, as through association with having sufficient income to afford a child (4.4.1). However, they also influence in more indirect ways, notably through their strong correlation with levels of education, a key fertility determinant.

Education levels, notably those of women, are closely linked to childbearing practices and preferences (Holdsworth et al. 2013: 23-24). In general, as levels rise for women, particularly beyond the primary stage, fertility rates decline (Bongaarts 2003). The longer a woman is enrolled in education, be it elementary, high school, or post-secondary, the less likely she is to begin having children. Remaining in school also delays marriage and this proximate determinant's (4.2.3) frequent initiation of childbearing, resulting in fewer reproductive years and likely fewer children.

Engaging with the broader issues of reproductive and general health, greater education of women is also associated with healthier families. Women able to remain in school will have higher literacy levels, allowing access to a greater variety of information, including material about contraception, vaccinations and other important health resources for herself and her children. Education also facilitates diffusion of new ideas and technologies, again notably 
the proximate determinant of contraception. In a 2003 study of 57 poorer countries, Bongaarts (2003) thus concluded that the educational levels of women represented a key predictor of overall fertility, whilst Gould (2015) noted how in Kenya that same year over half of women with at least secondary education used contraception, compared to only 8 percent lacking such education.

Geographically, however, the relationship between fertility and education levels is more complex. In the Global South, studies suggest that fertility often declines more rapidly where schooling is widespread or primary school enrollment is nearly universal (e.g. Osili and Long 2008). However, fertility behaviors can also be affected by exposure to mass media, such as radio, television and advertisements. In Brazil, India, Ethiopia, Mexico and Tanzania, for example, mass media campaigns and entertainment broadcast media, such as radio and television soap operas have played important roles in changing reproductive behavior (e.g. Singhal et al. 2003; Singhal and Rogers 1989). Finally, education has other positive effects on child health, as mothers with higher levels of education are more likely to have higher status in their communities, which puts them in a position to seek pre-natal care and be better advocates for their children's education, immunization, and health care.

Education is often linked to opportunities in the workplace. As a woman's education increases, so too do her options for employment outside of the home. By spending longer in school and then beginning a career, women across the Global North, in particular, are increasingly delaying the birth of the first child. Family sizes also tend to be smaller as the costs of raising a child increase for such "working women."

Although traditional explanations for the relationship between education and fertility reflect making a choice between taking up employment opportunities (higher education) or having children (lower education), other social changes and factors make this relationship much less direct. These include availability of childcare, quality of employment opportunities, and attitudes towards mothers in the workplace. Thus, variations across the Global North reflect differences in government policies, including those affecting parental leave and availability of childcare services. Thus, greater state provision of services in northern European countries, such as Norway and Sweden (Duvander et al. 2010), may partially explain why they have higher fertility levels than other countries, such as Italy, where dominant religious beliefs (Catholicism) might anticipate higher fertility levels than actually seen. Indeed, research in Austria concluded that increases in parental leave resulted in increases in fertility, whilst leaving the mother's paid work career relatively unaffected (Lalive and Zweimüller 2009).

\subsubsection{Race and ethnicity}

Racial or ethnic minority status within broader society may also be linked with fertility behavior. Across the Global North, members of minority groups commonly have higher fertility levels than the majority population. Both age profileswith minorities often more concentrated into the main child-bearing years-and class factors-with minorities typically more concentrated into the lower social strata-are important here. However, higher fertility may also stem from different religious or cultural backgrounds. Immigrants frequently bring with them the fertility and childbearing norms of their home countries, which might be higher (or lower) than those of their host country. However, over successive generations, reproductive behavior tends towards mirroring that of the adopted country. For example, during the recent period of multiculturalism in Australia, Abbasi-Shavazi and McDonald (2000) found that most immigrants adjusted fertility levels to correspond with those of more established Australians, except for Italian and Greek immigrants, who were more likely to maintain prior cultural norms.

Ethnically expressed fertility differences within a country's population are not limited to new immigrant minority groups. Native-born minority groups also tend to have higher fertility levels. This more explicitly tends to reflect lower socieeconomic status.Fertility rates for Hispanic and non-Hispanic Black populations in the US, for example, are higher than for Whites (PRB 2007). While this gap has decreased over time, a key reason for these differences is the overall concentration of Hispanics and Blacks into lower socioeconomic status groups. This relates again, in part, to education: Yang and Morgan (2003) found higher fertility among African Americans limited to those with lower levels of education.

\subsubsection{Gender}

Clearly shown throughout this chapter, gender roles and a woman's status, both within her family and broader society, strongly influence decisions about fertility and family size (Holdsworth et al. 2013: 162-167). "High status" within a

\section{ST PROOFS: NOT FOR DISTRIBUTION}




\section{FERTILITY AND BIRTHS}

family-or more independently-better allows a woman to negotiate timing of childbirth and number of children she bears. Such a status may be related to economic power from waged labor, for example, or from a more culturally defined position of relative equality to men. Both issues will shortly be considered. However, also of relevance are further entwined employment and fertility issues, notably "when is the best time to take a break from employment to have children; whether to return to work when children are young; whether to work full or part-time" (Holdsworth et al. 2013: 162). These issues apply to both women and men but awareness of gender as a social construction producing differential experience (2.3.8) means that, historically, they have been seen as of particular relevance to women.

Engaging with paid work outside the home can accord women greater gender role status and potential power within fertility decision-making, on account of the wages or salaries received. On the one hand, paid work can enable a woman financially to afford a child. On the other hand, waged labor may discourage production of children, as potential loss of income through leaving the paid workforce for at least a short time may be too high a price to pay. How serious this loss will be depends not only on the size of the earned income but also:

- How childcare is to be undertaken-by parent(s), relatives or friends, the state, commercial companies?

- How affordable paid childcare is-can the mother (or father) afford to return to work?

- How much work is "family friendly"-how well can (a) parent(s) balance the demands of paid work and children?

All such issues vary considerably globally and are returned to in the context of the role of the state in influencing fertility in Section 4.6. In summary, female employment is associated in very complex ways with either increasing or decreasing fertility (Bernhardt 1993). The latter is usually emphasized but it is not the only direction the relationship can take.

In a society where patriarchy, men's domination of women, is strong, women's employment may initially be expected to be low. This will be due to factors such as an assumption of running the home as women's sole responsibility, general lack of recognition and/or valuation of women's skills and abilities in a paid work context, and workplace sex discrimination (Walby 1986). In contrast, within less patriarchal societies the cultural authority or status of women may at least approach parity with that of men. Consequently, a woman's relational ability through gender role status to engage with paid work, to control that engagement-and thus also its association with fertility-and to control fertility behavior more independently of work should be enhanced.

In practice, the sheer complexity of the fertility decision-making process makes it extremely difficult to link conclusively levels of patriarchy-however defined-within a country or population with explicit expressions of fertility, such as TFR. The extent to which patriarchy is undermined can, however, be associated with indirect influences on fertility. First, it can be linked with the development of policies that seek to facilitate the smooth running of the production of children (4.6). Second, in societies where women have more access to media or education, as already suggested, they also tend to be more knowledgeable about reproductive health and contraceptive use. This may allow them more direct control over childbearing decisions. Third, another dimension of encultured gender norms of relevance here is preference for sons in some societies (e.g. Kulkarni 2007 on India). This may encourage a couple to continue to have children until at least one son is born, regardless of desired family size. Hence, undermining this aspect of gender inequality will also likely have fertility consequences. In sum, women's relative equality with men does appear to lead to greater equality in control of fertility behavior and practices.

Emphasis on the importance of gender, combined with the intertwined roles played by the other socio-cultural arenas of differential life course experience (2.3), iterate how understanding lives lived by women in particular is as vitally important for explaining overall fertility geographies as more biological population measures, such as age or fecundity. Socio-economic and cultural factors strongly mediate and in the twenty-first century loom ever larger over Bongaarts's proximate determinants (4.2.3). Thus, while working in Mongolia, one of the present authors witnessed the changing perceptions of women's roles and responsibilities at home for nomadic pastoralists, as these families gained greater access to television and internet. Expectations for young Mongolians related to consumer expenditures, employment and gender roles were changing rapidly to reflect the examples they saw on shows. All had fertility implications.

Finally in terms of differential life course experiences, the role of the government or state policies in influencing fertility behavior has been noted several times in this chapter. Such policies that directly or more indirectly target fertility will now be introduced, located within the macro-contextual arenas of history and geography.

\section{ST PROOFS: NOT FOR DISTRIBUTION}




\subsection{STATE POLICIES AND FERTILITY}

\subsubsection{Returning to history and geography}

Chapter 2 placed History and Geography, often intimately entwined, as macro-contexts of the life course. Models such as the DTM or the various differentiations of fertility discussed above are all more-or-less historically and geographically specific and should be recognized this way. However, this section focuses on how such specificity is expressed through particular state political contexts framing fertility and fertility decision-making by individuals and families. These contexts present a variety of national laws, policies and practices which work, whether intentionally or not, to shape fertility. Such influences are diverse but can be very considerable.

The section focuses on two main families of historically- and geographically-specific policies: those that strive to promote fertility and those that try to reduce it (Poston and Bouvier 2010: 343-351; PRB Staff 2004; Morgan 2003). Both reflect the fact that while individual women and families ultimately control fertility, the state can play a pivotal role in providing or prohibiting access to reproductive health, family planning and other resources (such as childcare) associated with fertility behavior. These influences of the state and their policies can directly target fertility or more indirectly shape the broader relational contexts of fertility geographies.

\subsubsection{Pro-natalist policies and contexts}

Pro-natalist policies seek to incentivize increased fertility. They have a long historical pedigree and have been adopted by numerous countries, notwithstanding the civil liberties issues they can raise. While no single policy appears most effective, such policies do appear to influence fertility rates.

Within Europe, some countries (notably Germany, France, Sweden, Italy and Spain) had pro-natalist policies in place as early as the 1930s (King 2001) but by the turn of the present century around 88 countries provided incentives for women to have children (PRB Staff 2004). In general, besides explicit exhortation and propaganda, pro-natalism is usually expressed in policies embracing welfare issues. It comes through, for example, in schemes that facilitate women leaving the paid labor force, grant mothers significant maternity pay, or provide substantial support for childcaring mothers (Holdsworth et al. 2013: 165). For example, the French government worked to increase birth rates through such measures as the 1939 Family Code, provision of social and family benefits such as birth premiums, loans to young married couples, and housing subsidies (King 2001). In 2009, France had a TFR of 2.0, higher than any other country in western Europe, although still below replacement level (PRB 2009).

Many policies and the socio-cultural environments they support work more indirectly in promoting fertility than explicit pro-natalist policies. Typically, pro-natalism becomes entangled with equal-opportunities designs (Poston and Bouvier 2010: 350), as will be noted for same-sex couples legally parenting children (4.7.3). For example, acknowledging factors linked to fertility reviewed in this chapter, governments may seek to ensure greater equality within the workplace and a reduction of the burden of family-work responsibilities by creating more flexible working hours, child care assistance, tax incentives, family allowances or low cost housing loans (PRB Staff 2004). Such policies can make having a child more economically feasible; highly relevant when noting the high average "cost" of raising a child (Box 4.2). In support of this, an Austrian study found increases in parental leave increased fertility (Lalive and Zweimüller 2009).

Pro-natalist policies may also be motivated by concerns about dependency consequences of an ageing society of low fertility. As noted in Section 4.3.3, governments may respond through facilitating immigration. Since much of the world's population is still young, working-age immigrants from the Global South, in particular, can potentially provide both needed workers for countries with declining populations as well as increased fertility rates on account of their demographic concentration within the child-bearing years. Such policies, however, are likely to be extremely politically sensitive (Chapters 7 and 9).

\subsubsection{Anti-natalist policies and contexts}

In contrast to policies that seek either (selectively) to boost a country's populations or promote equal and positive fertility experiences within the life course arenas of gender, class, sexuality and beyond, countries may enact

\section{ST PROOFS: NOT FOR DISTRIBUTION}




\section{FERTILITY AND BIRTHS}

anti-natalist policies designed to reduce fertility. In fact, some countries have alternated between pro- and anti-natalist policies (Box 4.4).

The most well known-for many, infamous-anti-natalist attempt to reduce fertility has been China's "one child" policy (Poston and Bouvier 2010: 348-349; Greenhalgh 2008). Breakdown of a campaign to promote birth control in the 1950s saw China's TFR shoot up to over 6.0 by the early 1960 s. Official concern over this high figure saw the wan-xi-shao program launched in 1971: later marriages (wan), longer intervals between children ( $x i$ ), fewer children (shao). Yet, prompted by continued worries at high population growth rates, wan-xi-shao was superseded in 1979 by

\section{BOX 4.4 FERTILITY POLICIES IN ROMANIA}

Romania has seen both anti- and, most especially, pro-natalist population policies applied strongly during different periods between 1945 and 1989. The most controversial form of birth control-abortion-featured strongly within these policies. Although the country returned to democracy in 1989, the legacy of this period remains.

By the mid 1950s, Romanian CBR had fallen to around 23 births per 1000 people, from around 40 at the turn of the century, not least due to widespread use of abortion, although illegal. In response to this reality, the government legalized abortion in 1957 to enable women to have more control over their fertility. Contraception, on the other hand, was discouraged, not least because contraceptives were not widely available. The result was Romanian abortion policy becoming one of the most liberal in Europe and abortion becoming still more significant in preventing unwanted fertility. CBR fell to around 14 by 1966 .

By the mid 1960s, however, the new Romanian government under Nicolae Ceauşescu became concerned about this low birth rate. Under Decree 770, the country switched to a strict pro-natalist policy. In 1966 abortion became restricted to tightly defined categories, including where the woman's life was in danger if she carried on with the pregnancy, where she had been raped, if there was a strong risk of congenital malformation, and where the woman was over 45 years of age or already had four or more living children. To enforce this dramatic shift in population policy, society became still more strictly controlled. Contraceptives that had been available disappeared and women were forced to be monitored monthly by a gynecologist, with detected pregnancies closely monitored until birth. Secret police closely observed operations in hospitals. Young women's sex education focused on becoming a "heroic" mother who gives her beloved homeland many children.

There was a huge baby boom. CBR trebled from 13 per 1000 in December 1966 to 40 by September 1967. Thousands of nursery schools had to be built, and the generation born in 1967 and 1968 was the largest in Romanian history. The late 1960s was a high point for Romanian fertility, however, and it subsequently declined. In the 1970s, as economic pressures on families grew, people sought ways around the law. Richer women obtained contraceptives illegally or bribed doctors to give them a diagnosis allowing an abortion. Among less educated and poorer women, such options were unavailable and many unwanted pregnancies occurred. The award-winning 2007 Romanian film 4 Months, 3 Weeks and 2 Days (Director: Cristian Mungiu) tells the story of a pregnant student looking for an illegal abortion. Such attempts to abort frequently led to infections, sterility or even death, and mortality for pregnant women became the highest of Europe. Furthermore, many children who were born subsequently became malnourished due to poverty. They often ended up in state care, where conditions were typically grim, with considerable abuse and high rates of child mortality. Their fate attracted worldwide media attention when the realities of life in Romanian orphanages emerged after the 1989 revolution that deposed Ceauşescu.

A few years earlier, in 1985, another decree had further restricted contraception and abortion but fertility still did not rise significantly. During the revolution, such was their significance that both abortion and contraception were legalized and Romania now retains the highest induced abortion rates in Europe. This reflects a continued legacy of limited sex education and information on contraception, mistrust and misinformation on modern methods of birth control, and continued unevenness of contraceptive supply. Fertility remains very low. Even by 2008 TFR was just 1.3, below replacement level, reflecting the economically parlous state of the country. It was still estimated at this low level in 2012, firmly locating Romania within Section 4.3.3's low fertility countries.

(Sources: Balkan Insight 2012; Poston and Bouvier 2010: 346; PRB 2012; Serbanescu et al. 1995) 
the One Child Campaign. This combined incentives for a family's first child with increasing financial sanctions for each subsequent birth. The latter has been the main cause of much ethical disquiet, not least due to coercion not to have any more children by local officials, reported as frequent. This has included forced abortions and sterilizations (Jian 2013). Notwithstanding such concerns, and noting there are many exceptions to the one child rule outside of urban areas, China's policy has been seen as contributing to rapidly and dramatically decreased fertility rates, from 5.5 in 1970 to 2.3 in 1990 to 1.6 in 2011 (Shen 1998; UN 2015). So successful has it been that by 2015 there was talk of replacing the one-child policy, already somewhat eased, with a two-child policy. This was to counter the "demographic time-bomb" of a rapidly ageing population and, supposedly, also in response to Chinese public opinion (Phillips 2015).

Beyond explicit anti-natalist policies, other nominally non-demographic state initiatives may reduce fertility. As with pro-natalist outcomes, these again typically involve the demands of an equality-seeking agenda. For example, it was noted in Section 4.4.1 how legislation protecting children from exploitation and requiring them to attend school may increase their relative "cost," thereby discouraging fertility. From a gender perspective especially, access to family planning resources through state-sponsored programs is critical. Besides contraceptive provision, such programs typically involve media resources and propaganda that promote small families, women's rights and reproductive health. Thailand is a good example of a country in which the state has taken an active role in promoting small families. Between 1969 and 1979 fertility rates dropped dramatically (from 4.3 in 1975 to 2.2 in 1987), contraceptive prevalence increased, and family size preferences dropped from an average of six to seven children to only two (Knodel et al. 1984; Pritchett 1994). Paralleling these changes in preferences and contraceptive use, the government instituted an active national planning program which introduced modern contraceptive methods to rural Thais (Knodel et al. 1984). Combining increasing economic and social development and an effective family planning program facilitated rapid change in fertility preferences and behaviors. However, not all family planning programs work. For success, a program must be tailored to the particular circumstances of a specific country (Hemachudah and Rosenfield 1975).

While many countries across the Global South have witnessed a strategic dip in TFR since the mid 1960s, pockets of high fertility remain. Partly in response to this, the mid-1990s marked a turning point at which family planning programs began to re-focus. As already noted, the 1994 Cairo conference brought reproductive health care issues to the forefront of international population and development debates. Such issues and concerns underlie many of the fertility decisions made by women and families, clearly including family planning but also encompassing safe motherhood, pre- and post-natal care, adolescent health, HIV/AIDS awareness and complications during pregnancy and childbirth. National and international programs must evolve and expand to embrace these issues. For example, in Cambodia maternity related complications are the leading cause of death for women aged 15-49 years (PRB 2003). Poor access to pre- and ante-natal care, poor access to key nutrients during pregnancy, such as iron, and lack of skilled assistance at delivery all contribute to high mortality rates. Reproductive health is thus a broad and complex challenge for any official body. However, its scope expands further with "new" fertilities.

\section{7 "NEW" FERTILITIES}

\subsubsection{Fertilities beyond the marital bed}

As if getting a handle on fertility geographies was not challenging enough from the multitude of factors and considerations discussed so far, the modern era has made it harder still by throwing up for debate what exactly is meant by "fertility". Fuller engagement with this issue expands the traditional scope of Population Geography. Of course, at one level, fertility remains the number of children born to an individual or defined population-the production of children. But at another less abstract and empirical level, a new range of considerations must be taken on board if Population Geographers are to understand more fully the global fertility experience and its geographies. The scope of fertility has expanded widely.

An initial challenge comes from a need to revisit the close association between fertility and being in a marital relationship traditionally assumed within much scholarship, such as by the proximate determinants of fertility model (4.2.3). Whilst being married remains positively associated with fertility, the converse is much less reliable. Children are increasingly conceived and born outside wedlock, although-of course-such births have always existed. This is illustrated in Figure 4.5, which shows how in European countries such as France and Iceland most babies are now

\section{ST PROOFS: NOT FOR DISTRIBUTION}


88 FERTILITY AND BIRTHS
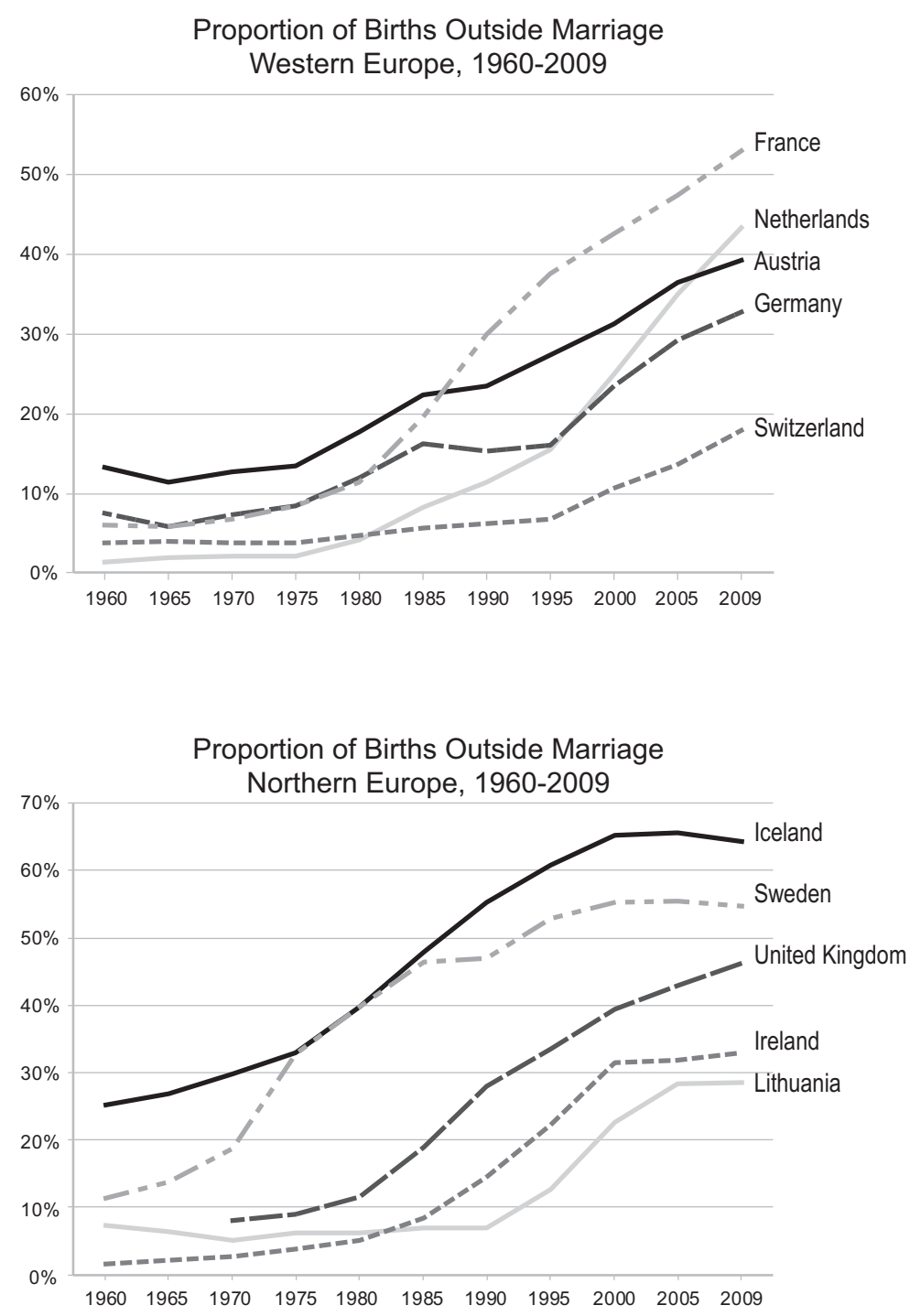

Figure 4.5 Births to unmarried mothers, Western and Northern Europe, 1960-2009.

Source: http://www.prb.org/Publications/Articles/2010/birthsoutsidemarriage.aspx.

born outside marriage. Such trends reflect strongly increases in cohabitation, which one might represent as an informal style of marriage, but there is also a rise of births to women not in settled cohabiting relationships. This is also demonstrated in the figure.

\subsubsection{New fertilities}

Bio-medical and sociological developments have expanded the domain of fertility. This expansion has diverse sociocultural and geographical expressions and comprises what can be termed "new" fertilities.

Fertility was not conventionally possible if a person was infecund or not in a heterosexual relationship, a situation traditionally symbolizing the end of any hope of starting a family. This is no longer the case. Significant bio-medical developments have led the way in tackling problems of infertility through treatments aimed at improving the fecundity, the physiological ability to have a child, of both women and men. However, as with all such bio-medical initiatives, both 
their development and implications are shaped through a socio-cultural lens. Such a lens has generally, if not uncritically, grown increasingly sympathetic to and supportive of the plight of those wanting a child.

In 2010, nearly 50 million couples worldwide experienced infertility (Mascarenhas et al. 2012). This is a huge figure-others put it considerably higher (e.g. Ombelet et al. 2008)-and, although Mascarenhas and colleagues went on to suggest that infertility globally was not increasing, media stories on increasing male rates in the Global North (e.g. Roberts 2005) add to popular recognition of the problem. Infertility is, in fact, a much more serious problem in the Global South, not least due to higher incidence of untreated reproductive tract infections (Inhorn and BirenbaumCarmeli 2008).

Fortunately, much medical research has gone into addressing infertility, resulting in the emergence of a wide range of treatments to overcome the problem (Inhorn and Birenbaum-Carmeli 2008). Moving on from the large-scale development of drugs to tackle infecundity in the US in the 1950s, what are known collectively as Assisted Reproductive Technologies (ARTs) emerged (Greil et al. 2009). The best known, In Vitro Fertilization (IVF), involves sperm and eggs from prospective parents mixed outside of the body. If successful, this produces a fertilized embryo which can be transferred to the future mother's uterus to develop in the conventional manner. The first child via IVF was "test-tube baby" Louise Brown, born in Oldham, England, in 1978. Another ART is Intrauterine Insemination (IUI), which involves sperm from a male partner or donor being injected into a woman's uterus (Inhorn and BirenbaumCarmeli 2008).

A further development that may be placed with ART is surrogate motherhood. Surrogacy involves a third party woman gestating a fetus, possibly her own but usually donated by the prospective parents who pay for the process. As Inhorn and Birenbaum-Carmeli (2008: 182) put it, surrogacy deconstructs the dominant idea of motherhood as fundamentally rooted in an "indissoluble mother-child bond." It also has some interesting geographical expressions which raise difficult ethical questions. For example, India has become a key source for surrogate mothers, attracting prospective parents from all over the world, not least the US. This reflects, according to Rudrappa (2010), three key features: a developed consumer market in surrogacy, low cost and flexible female labor, and intermediating brokerage companies to do the deals. All provoke questions of power and ethics. Generally, it is explicit monetary relations involved in surrogacy, in tune with a general reluctance to "value" children economically (4.4.1), that makes surrogacy controversial and helps explain why it remains strongly legally regulated.

As the Indian surrogacy example suggests, the geography of ARTs globally but also within individual countries closely mirrors the geography of wealth and class, again overlain by cultural considerations. ARTs tend to be expensive, often extremely so. They thus tend to be rationed closely within state health schemes and are only privately available to wealthier families (Inhorn and Birenbaum-Carmeli 2008). Hence, in the US, it is estimated that just one per cent of infertile women utilize ARTs (Spar 2006). In the Global South, any (rare) state support for ARTs can divert resources from more life-and-death medical priorities: vaccinations or diseases such as HIV (Ombelet et al. 2008). Use of ARTs thus illustrates stratified reproduction (Colen 1995): technology-assisted fertility accessible only to elites, whilst the poor-the most likely to be infertile-have little or no access to such an option.

Culture overlays this matter of differential resources when considering the geography of ARTs. There are considerable differences both between and within cultures regarding infertility. Women experiencing infertility, in particular, tend to suffer considerably, not least in the Global South (Figure 4.6). Via patriarchy, they are often blamed for a failure to produce children (Inhorn and Birenbaum-Carmeli 2008). Childlessness may even result in being unable "properly" to attain the life transition to adulthood (Greil et al. 2009). Religious differences are also important. For example, Roman Catholicism tends to denounce ARTs but Jewish pro-natalism, as expressed through the state of Israel, presents ARTs "as a way to reproduce the nation through the birth of Jewish babies" (Inhorn and BirenbaumCarmeli 2008: 184). Consequently, Israel subsidizes IVF and even surrogacy (Greil et al. 2009).

\subsubsection{New parents}

Based on Biology, human "parents" have traditionally been defined as a man and a woman who have "made a baby" through sexual intercourse. Developments such as IVF and surrogate parenthood challenge this representation, as do developments now discussed. Once again, both bio-medical and socio-cultural changes have converged, most strongly within the Global North, to present new understandings of parenthood well beyond the conventional nuclear family. The relational arenas of sexuality (2.3.9) and differential ability (2.3.5) feature strongly.

\section{ST PROOFS: NOT FOR DISTRIBUTION}




\section{FERTILITY AND BIRTHS}

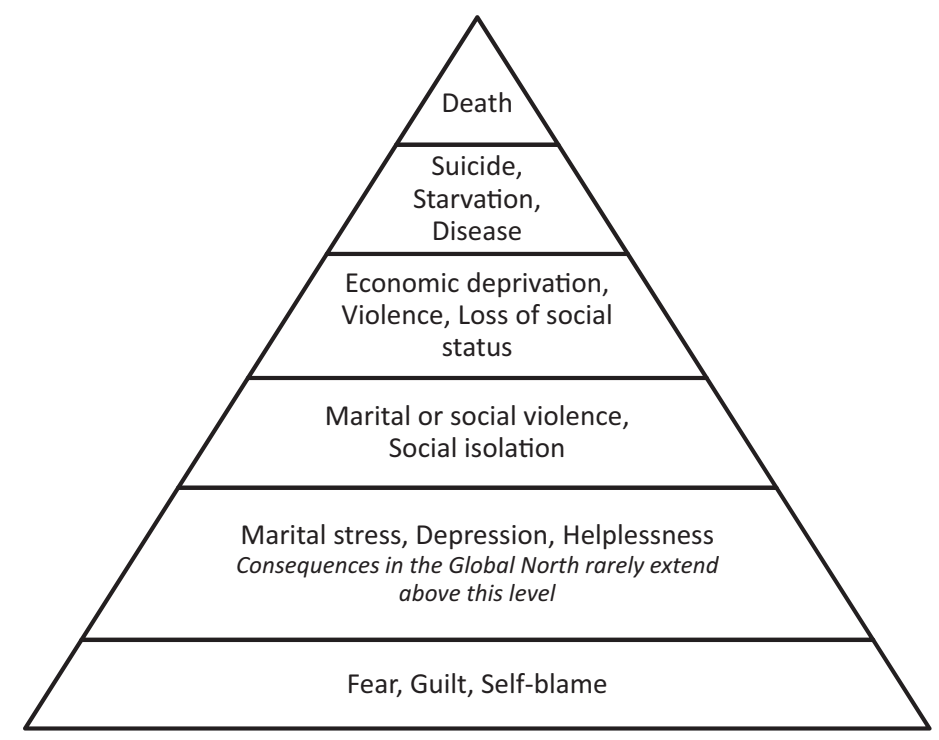

Figure 4.6 Potential consequences of infertility for women in the Global South.

Source: adapted from Ombelet et al. (2008: Figure 1).

An initial and long accepted way in which one may become a parent non-sexually is through adoption of a baby or older child. A famous early adoptee was Moses in the Bible, a Hebrew baby left on the banks of the River Nile to be adopted by an Egyptian mother (Sperry 2008). From the 2000 US census, it was estimated that 2.5 percent of young people under 18 years were adopted (Hutchison 2012). Of course, adoption does not reflect changed fertility but the geography of adoption and its life course consequences for those involved are interesting issues.

There are two main geographical expressions of adoption. First, there is within-country practice. This developed in modern form from the mid-nineteenth century, when specific US legislation was passed, for example (Sperry 2008). Adoptees have tended to be orphans or other offspring who could not be raised by their biological parents, often because of poverty. Second, there is international adoption, with adoptees sourced abroad. This has seen major growth since the mid-twentieth century but with the US by far the main recipient country, accounting for over half of all international adoptions in the early twenty-first century. In contrast, the top three source countries were China, Russia and Guatemala (Selman 2009).

Conventional legislative and cultural norms of adoption have also been extended. Early obsession with "matching" adopted children to new parents by race, religion and ethnicity has increasingly been discontinued. Furthermore, adoption increasingly includes same-sex couples (and trans individuals) as prospective parents (Hutchison 2012). Within all adoption situations, however, numerous key challenges arise, not least concerned with disclosing to the adoptee and working with the fact that s/he has, in simple terms, two families-birth and adopted (Hutchison 2012).

Same-sex couples have also utilized ARTs, such as taking genes from one partner and a donor of the opposite sex to produce a child brought up by the couple as her or his "parents." It was estimated that by 2000 in the US around 20 percent of gay male and 33 percent of lesbian couples were raising children (Hutchison 2012). In the UK, Palmer (2013: 3) noted how the "family landscape is changing" since introduction of quasi-marital "civil partnerships" in 2005 enabled "both people in a same-sex relationship to be the legal parents of a child born to one, conceived by surrogacy or adopted." In short, adoption, ARTs and surrogacy are "unseating traditional notions of heterosexual parenthood" (Inhorn and Birenbaum-Carmeli 2008: 183).

Approaching the idea of "new parents" from yet another angle, particular forms of disability, excluding infecundity, have traditionally deterred parenthood. This barrier is also now much less certain. Indeed, as a barrier it is more sociocultural than bio-medical, reflecting society's perception of the supposed demographic competence (2.3.10) of people with varying disabilities to become parents. Arguably, social attitudes increasingly accept disabled people becoming parents, coming to recognize, for example, that disabled people are sexual beings too (O'Toole 2002). 


\section{BOX 4.5 A BRAVE NEW WORLD OF FERTILITY}

English writer Aldous Huxley's dystopian 1930s novel Brave New World opens in an imagined London 2540AD, when most of humanity is unified under a World State. In this peaceful, stable global society in which goods and resources are plentiful (population limited to two billion), everyone seems happy. Natural fertility has been replaced by children created, "decanted" and raised in Hatcheries and Conditioning Centres. Here, they are divided into five castes designed to fulfill predetermined positions within society. Fetuses chosen to become members of the highest caste are allowed to develop naturally, while those chosen to become members of the lower castes are subjected to in situ chemical interference to cause arrested development in intelligence or physical growth. Moreover, members of these lower castes are not unique but created from a single egg spawning up to 96 children, one ovary producing thousands of children. To further increase lower caste birthrate, all the eggs in the ovary mature simultaneously, allowing the hatchery to get full use. People of these lower castes comprise the majority of the World State. The production of such specialized children bolsters the efficiency and harmony of society, since they are deliberately limited in cognitive and physical abilities, ambitions and desires, rendering them easier to control. In short, Huxley truly painted a terrifying fertility dystopia!

(Source: Huxley 1932)

Finally, all developments covered in this section so far have indicated a broader scope of parenthood and, therefore, might be expected potentially to increase production of children. However, contextualized bio-medical developments do not all point this way. For example, forms of prenatal diagnosis allow tests on fetal tissue for presence of disease genes, whilst genetic screening enables future parents at risk of transmitting a genetic disease to test an embryo created via IVF for presence of disease-associated genetic alterations (Pray 2008). Use of such forms of screening could lead to selective rejection of embryos somehow deemed "problematic." This is clearly another ethically challenging area (Pray 2008). Besides the potential use of abortion-unacceptable to many, particularly those in certain faith groups-criteria used to define an embryo as unwanted are extremely difficult to reach consensus on. They might include such things as having or even being likely to have a terminal illness, having a greater or a lesser disability, or even being of the "wrong" sex. The latter acknowledges the widespread global presence of male preference (Kulkarni 2007), whereby sex-selective abortion of girls, for example, has spread across the Global South and is controversially claimed to account for 160 million "missing girls" and women in Asia alone (Hvistendahl 2011). A frightening specter of "unnatural selection" within fertility controlled dystopias, such as envisaged by writer Aldous Huxley in the 1930s (Box 4.5), soon emerges.

Finally, in contrast to extra-marital fertility, which might be said to destabilize the nuclear family institution, increasing diversity expressed by both new fertilities and new parents can actually work to reinforce the conventional idea of family. Of course, there are many reasons why a couple, or even a single person, might want to bring up a child even when their "natural" fecundity tells them this is not possible (Hutchison 2012). Explicit concern with reproducing the nuclear family is unlikely to be one of them! However, a consequence that flows from resultant developments in fertility is reinforcement of "the common belief that a couple without children cannot possibly be a family unit" (Rudrappa 2010: 260). This suggests potential influence of cultural demographic pressures in shaping life course practices.

\subsection{CONCLUSION: FERTILITY’S MULTIPLE ENTANGLEMENTS}

This chapter discussed both some key expressions and aspects of fertility geographies worldwide and introduced a range of the factors that influence fertility behaviors and decisions. Attention also focused specifically on the implications for both high and low fertility at national and local scales. A range of theories help explain different patterns of fertility decline at scales from the individual to the community to the global. Local contexts, cultures, and economies play critical roles within individual fertility decisions and Population Geographers are uniquely positioned to investigate these and to contribute to associated debates (Boyle 2003). 
92 FERTILITY AND BIRTHS

In summary, fertility demonstrates very clearly indeed the complexity of factors that impinge upon any "Population Geographies" that may be represented singly by numbers, as in this chapter's use of TFR. Fertility behavior is fundamentally entangled with the overall lives across space of both potential parents but especially with that of the mother. It is entangled with Biology, of course, but also with the social, cultural and economic life of the person. It is thus entangled, through the latter, with societal forces and experiences that emerge and are expressed well "beyond" the individual but with which that individual must engage through their life course. And yet finally, as an ultimate expression of human intimate connection, one must never forget fertility-production of children-remains in most cases the ultimate preserve of, and the cause of joyous celebration for, those immediately and intimately involved. 


\section{CHAPTER 5}

\section{Placing human migration}

\subsection{INTRODUCTION: DEFINING MIGRATION}

\subsubsection{What is migration?}

Migration remains the most widely studied and examined element within Population Geography (Boyle 2003, 2004). Moreover, whilst Geographers have played a pivotal role in shaping our contemporary understanding of it, the topic is of interest to numerous academic disciplines, including Demography, Sociology, Political Science, Economics and Anthropology (Brettell and Hollifield 2008a). In this respect, approaching migration as it occurs within the life course has considerable potential for bringing together a scattered body of scholarship often fragmented by "disciplinary partitioning" (Olwig and Sørensen 2002: 7).

But what exactly is migration? Initially put, as in a recent textbook, it is "the movement of people to live in a different place" (Holdsworth et al. 2013: 96) or a "permanent change in residence." It is residential relocation. Or, as expressed in UK and US censuses, a migration is deemed to have occurred when one's "usual address" has changed within the last 1 or 5 years, respectively (ONS 2013; USCB 2013). Simple, then, one might think! However, as Holdsworth et al. (2013: 98) also noted, careful consideration of these definitions immediately raises a host of questions: what precisely is meant by "different place," "live in," "permanent," or "usual address"? Consequently, by the end of the present chapter, "migration" will have been demonstrated to be at least as complex and multi-dimensional a concept as Chapter 4 revealed "fertility" to be.

Starting with the idea of "different place," the type of areal unit(s) involved in a migration is an initial important consideration when defining it specifically. A crucial starting point is whether a political boundary is crossed during a move. For example, an individual could move from one county to another within the same US state or from one state to another. Both moves are conventionally described as intra-national or internal migration, because neither involves leaving the US. In contrast, a move from the US to Canada, crossing an international border, is an international migration. Actual distance moved is unimportant in differentiating these two kinds of migration, just political boundaries.

Individuals moving to a different place, defined at any spatial or political scale, are defined as in-migrants to that place, while those moving out are out-migrants. More specifically, within international migration, movement to a new country is termed immigration, movement out is emigration. In contrast, migration over short distances, such as family relocation from one home to another within the same urban area, is labeled intra-urban or residential mobility. In contrast, movement from one urban area to another expresses inter-urban mobility.

But not all movements within or across defined areal units are considered migrations. Here, the issues of "live in," "permanent" and "usual address" come under the spotlight. Time is an important element in separating migration from mobility or more general movement. In general, migration involves a move seen as signifying an intention to reside at the destination. Working with this distinction, an individual daily commuting from Brighton to London in the UK for work, for example, would not be considered a migrant, as their intention is to return home each day, but as engaging in mobility. Likewise, a German couple on vacation in France would not be considered migrants. Neither would a Norwegian family relocating to their holiday home for the whole summer. All three cases are fairly easy to appreciate. However, what if the commuter stayed in a flat in London during the week, only returning to Brighton at weekends? Or, if the German couple extended their stay indefinitely because they liked France so much? And what if the Norwegian family spent more of their year in the second home than in their first home? In all three cases the divide 


\section{PLACING HUMAN MIGRATION}

between mobility and migration becomes fuzzy. However, it would take the (former) commuter giving up her Brighton property, the couple their German residence, and the Norwegian family redefining their second home as their primary residence before a migration would definitively be seen to have occurred.

Stepping back from these initial reflections, Table 5.1 illustrates what is conventionally meant by migration through three vignettes. Whilst all three refer to people moving to live in different places and to permanent change of residence, in many other respects they are quite different. One can also see them all as relational with respect to their associations with key life course issues: housing, love, employment, safety. Migration, like life course (Chapter 2), has both common and individually differentiated features.

Having gained some idea of what is understood as migration but also some sense of the challenges in delineating precisely the phenomenon, the remainder of the chapter seeks to establish how academics have engaged with or placed migration. Following a short introduction to how migration is conventionally measured, Section 5.2 presents the argument that much of the world is now experiencing an era of mobilities, highlighting but not restricted to residential relocation, challenging any idea of migration as an aberrant human condition. From this heightened sense of everyday movement, Sections 5.3 to 5.6 present four predominant perspectives apparent within scholarship: migration as natural, societal, individual, or life course expression. The latter follows the chosen focus of the present book. Discussion concludes by suggesting that migration can present a key life course "event," acting as metaphorical portal to the development of a range of contrasting lived experiences. Section 5.7's chapter conclusion sets up conceptually the diverse forms of migration and mobility covered in Chapters 6 to 9 .

\subsubsection{Measuring migration}

Table 5.2 defines five principal descriptive measures of migration. All are usually expressed as rates, which facilitates comparison between places and time periods; and as rates per 1000 people, which presents analysts with manageable numbers. They tend to measure migrant flow (moves between defined places over a defined period), as compared to migrant stock (number of migrants in one place at one time) (Holdsworth et al. 2013: 107), unless taken at a

Table 5.1 Three expressions of migration

Intra-urban mobility:

Dave Smith, 20, Brighton, UK

It is approaching Christmas and unemployed Dave has had enough of his Brighton bedsit. It is always colddue to poor heating and drafty windows-and his new neighbor's noisy late night behavior is the last straw. Unfortunately, Dave has no money to buy as much as a onebedroom flat in one of the most expensive cities in England outside London. And government housing policy means there is very little social housing available. However, Dave has struck lucky. A friend puts him in touch with a landlord who has a suitable vacancy. Moreover, it is just two streets away from where he is presently living. Thus, after some exceptionally frugal weeks, he has managed to put together enough money for the deposit required to sign the rental agreement. Hopefully sorted for Christmas...
Intra-national or internal migration: Jean-Luc and Vanessa, mid-20s, Paris, France

Jean-Luc and Vanessa have been seeing each other romantically since they met in their second year at University in Paris. Whilst Vanessa is Parisienne, a resident since birth of the 17th arrondissement, Jean-Luc comes from the small village of Pontours in the Dordogne. However, with both now having graduated, it is time for a major life course decision. After much soul-searching, since he is very close to his family, Jean-Luc decides to make his move to Paris permanent. He is to live with Vanessa. Whilst love is clearly the key factor here, Paris is also deemed to be a suitable location by both Jean-Luc and Vanessa, and by their families, as it is a more likely place for both graduates to make best use of their degrees in employment than Pontours. A new life stage begins ...
International migration:

Luis and Isabella, early 30s, Mexican-US border

Luis and Isabella are poor peasants in the southern Mexican state of Chiapas. Whilst they have survived thus far, and brought Valeria, Antonella and baby Hernando into the world, their life is very hard by any reckoning. Now, however, things have become progressively worse! Somehow, the local powerful landowner in their community has discovered their sympathies for the radical Zapatista movement and Luis and Isabella fear their lives are in danger. Also, the poor soils they till are becoming increasingly infertile. A drastic decision is thus reached that they must try to cross the

Mexican-US border to seek a better life in the latter. We leave all five on the border at the tender mercies of the coyote people-smugglers who will attempt to get them into Arizona tonight... 
Table 5.2 Measuring migration

\begin{tabular}{|c|c|}
\hline Measure & Equation \\
\hline $\begin{array}{l}\text { In-migration rate (IMR): number of migrants arriving } \\
\text { into a specified area over a specified time period (usually } \\
1,5 \text { or } 10 \text { years). }\end{array}$ & IMR $=-\frac{\ln -\text { migrants }}{\text { Population }}\left[{ }^{*}\right] 1000$ \\
\hline $\begin{array}{l}\text { Out-migration rate (OMR): number of migrants leaving } \\
\text { a specified area over a specified time period: }\end{array}$ & OMR $=$ - Out-migrants ${ }_{\text {Population }}\left[{ }^{*}\right] 1000$ \\
\hline $\begin{array}{l}\text { Net migration rate (NMR): the difference between the } \\
\text { number of people arriving and departing (In-migrants } \\
\text { minus Out-migrants) }\end{array}$ & NMR $=\frac{\text { In-migrants }- \text { Out-migrants }}{\text { Population }}\left[{ }^{*}\right] 1000$ \\
\hline $\begin{array}{l}\text { Gross migration rate (GMR): sums the number of } \\
\text { people arriving and departing (In-migrants plus } \\
\text { Out-migrants) }\end{array}$ & $\mathrm{GMR}=\frac{\ln -\underline{m i g r a n t s}+\text { Out-migrants }}{\text { Population }}\left[{ }^{*}\right] 1000$ \\
\hline $\begin{array}{l}\text { Migration effectiveness or efficiency ratio (MER): } \\
\text { expresses how effectively migration is redistributing } \\
\text { population }\end{array}$ & $\mathrm{MER}=\frac{\text { Net migration }}{\text { Gross migration }}\left[{ }^{*}\right] 100$ \\
\hline
\end{tabular}

Sources: Holdsworth et al. (2013: 108); Poston and Bouvier (2010: 171-174).

cross-sectional snapshot in time and space, such as a population census (Box 3.2). Focusing on flow, the measures consequently usually include in-migrants who have subsequently left (no longer present), out-migrants who have returned, and/or multiply enumerated migrants who have moved in or out more than once.

Considering migration effectiveness ratio (MER), if migration for a specified place is dominated by either inmigration or out-migration, MER will have a relatively high value, close to the limits of +100 (all in-migration) and -100 (all out-migration). This demonstrates migration redistributing population spatially, either into or out of the area, respectively. However, if net migration is low in magnitude, and especially if gross migration is nonetheless relatively large, MER will approach zero and migration is merely moving population between areas, with little significant numerical redistributive effect.

All of these statistical measures express migration compared to non-migration or "staying put" (Hjälm 2013). The latter, the mirror image of migration, is of course the everyday state for the vast majority of people the majority of the time. As such, it tends to be somewhat taken-for-granted, a status recently questioned by critical scholars (e.g. Halfacree and Rivera 2012). Critique includes reappraising any normal state of staying put, engaged with next in a section recognizing the ubiquity of migration and mobility generally within everyday life.

\subsection{AN ERA OF MOBILITIES?}

\subsubsection{Sedentarism and migration}

Culturally underpinning most understanding of migration is an assumption of sedentarism. Recognized by numerous authors (e.g. Cresswell 2006; Gustafson 2014; Malkki 1992; Urry 2007), it is expressed in social science's acceptance of "the place-fixated paradigm of the modern age" (Rolshoven 2007: 21). Reinforced by philosophical reflections on place (e.g. Heidegger 1971), sedentarism expresses the idea that being still, bounded and "authentic" through being in-place is a foundational feature of human life. In consequence, mobility is regarded with suspicion. It is at root "inauthentic," inherently disruptive of normal settled states of affairs. It is thus potentially deviant, requiring close sociopolitical scrutiny and control (Cresswell 2006: 55). This attitude comes through in state policies towards immigrants, for example (Chapters 7 and 9).

Halfacree (2012a) argued that sedentarism implicitly frames how migration is expressed within everyday life. For example, within the Global North, "moving house" has much in common with going to see a doctor or dentist and, 


\section{PLACING HUMAN MIGRATION}

importantly, with other forms of mobility, such as commuting or general travel. On the one hand, it has become a relatively mundane practice, to be undertaken as efficiently and painlessly as possible so as to minimize disruption to the normal sedentary condition. To smooth its passage it has become heavily institutionalized in and through facilitating networks encompassing: real estate companies, banks, building societies, letting agencies, mortgage providers, removal companies, builders, decorators, utility companies, and others. In addition, strong socio-cultural norms serve to explain and legitimize a migration, preventing it being classed as "deviant" (Halfacree 1996). These relate to moving for such things as economic betterment, quality of life and amenity, accessibility, retirement, and children's welfare. On the other hand, moving house is still widely recognized as stressful (e.g. Mann and Seager 2007) and disruptive (Fielding 1992a). It may be regarded as threatening the supposed goal of a "natural state" of place-based dwelling (Ingold 1995).

An assumption of sedentarism at first sight has much commonsense plausibility in the context of migration. For those who move, numerous different stressful aspects will probably be experienced. And, in the age of globalization, searching for a settled and defined place in the world seems one of humanity's preoccupations (Lewicka 2011) (1.2.2), exemplified through lifestyle migration (Chapter 8). Nonetheless, to assume "natural" desire for immobility or fixity is a step too far. Not only are there groups for whom mobility and migration are "normal" behavioral expressions shaping their identity, such as many Gypsies (Acton 2010), but to overemphasize the settled state can suggest a person "clotted with rootedness" (Mabey 2005: 1). It neglects considering adequately personal life course transformative aspects of both migration and general mobility. Movement shapes the person no less than a settled state of stillness.

Interesting issues are raised from reflecting critically on the legacy of sedentarism within migration scholarship. First, whilst the idea of "usual address" is central to the predominant understanding of migration, what if someone is unable or unwilling to acknowledge any single such location? How does this undermine Section 5.1.1's representation of migration? Does "home" have to be one place? Think how slippery it is to define spatially-house, village, region, country, and so on (Blunt and Dowling 2006). Certainly, migration scholars promoting ideas such as transnationalism (7.5.6) challenge any simple singular idea of home. Second, how long does one have to live somewhere before acquiring the label "migrant" (Holdsworth et al. 2013: 102)? Weeks, months, a year? And how long before one is no longer a migrant? Third, migration also typically hinges on relocation being "permanent," from one settled state to another. However, as most people move residence many times in their lives, how can anyone ever really declare a move permanent? The migrant may think so but no residential future is ever conclusively pre-defined. Fourth, the sedentarist legacy framing understanding migration is tested still further by recent announcements of an "age of migration." Engagement with this literature and its broader recognition of diverse forms of mobility within everyday life potentially expands the scope of Population Geographers' concerns with movement very considerably. It does this through relegating the prominence of both sedentarism and the equally assumed special geographical status of migration within life course mobilities.

\subsubsection{Age of migration}

The Age of Migration was originally published in 1993 but is now into its fifth edition. In the book, Stephen Castles and Mark Miller portray the present period of human history as fundamentally shaped through international migration flows. Whilst people have moved residentially from place to place throughout humanity's existence (Brettell 2013), with migration in the early "modern" period frequently under-appreciated (Pooley and Turnbull 1998), the magnitude and complexity of population flows today is unparalleled (Box 5.1). For Castles and Miller, such flows comprise a core feature of globalization. They are certainly a key component of the historical and geographical arenas (2.3) of twenty-first century life courses, expressing a strong force shaping individual lives (Fielding 1992a). Migration almost inevitably accompanies the increased global movements of commodities, capitals and culture widely noted. Ever more countries feature prominently as both international migration origins and destinations, migration flows become more sizeable, and there is a heightened everyday presence of diverse motivations for all this relocation (Castles and Miller 1993).

Castles and Miller's strong sense of life courses now playing out within an increasingly mobile world does not just implicate international migration, however. Indeed, whilst a sense of near constant change and transformation has long been recognized as a key feature of both a capitalist economy and life within it (Berman 1983), a number of writers now consider flux, both experiential and metaphorical, increasingly displacing fixity and coming to

\section{ST PROOFS: NOT FOR DISTRIBUTION}




\section{BOX 5.1 CHANGING EXPRESSIONS OF MIGRATION AND MOBILITY FROM IRELAND TO THE US AND UK}

Interesting historical perspectives on the changing place of migration and mobility for Ireland come through in cultural expressions of the Irish abroad. Two are briefly related here, relating to nineteenth-century emigration to the US and late-twentieth century shorter-term migration to the UK, plus a present-day indication of the ubiquity of outward- and inward-movement by air from the perspective of flights from and to Dublin airport in summer $2015_{\text {i }}$

Devastating famines between 1845 and 1852, the cause of a bitter political legacy, provoked huge emigration from Ireland. Amongst destinations, the US featured especially prominently. With such moves, relocation was likely to be permanent, with not even temporary return, due to distance, costs of travel, and so on. Thus, desperate need to escape poverty and misery through emigration became an existentially traumatic experience for all involved. Very tightly bonded families would be broken apart through emigration of family members-often an eldest son or daughter-even if the idea was that remittance of monies subsequently earned would enable other family members to follow.

This major life course experience soon became deeply culturally embedded. One tradition that powerfully expressed it was the American Wake. This usually took place on the night before the person departing left to board their ship and sail away. It became commonplace during the 1840s and 1850s and reflected Catholic reverence for holding a wake for the dead, watching over a body on the night before a burial to prevent evil spirits entering it. Reflecting the importance generally of demographic events in Irish life, the American Wake and its associated traditions expressed:

- Death-departure;

- Burial-boarding the ship;

- $\quad$ Birth-arrival in the US;

- Marriage-letters sent back and forth across the Atlantic.

The Wake itself was widely advertised to the local community, whose members had a moral obligation to attend. At a party, often paid for on credit in the expectation that the migrant would soon be sending money back from the US, guests gave various gifts to the soon to be departed. Finally, in the early hours of the morning after the father danced with the emigrant-probably for the last time ever-a period of lamentation would begin, led by older women, and then at dawn the emigrant would make his or her final farewells.

Joseph O'Connor's short story Mothers Were All the Same centers on a few days in the life of the narrator, Eddie, as he flies from Dublin to London, via Luton Airport, supposedly to look for work around 1991.

On the plane, Eddie notices Catriona, whom he vaguely recognizes and who latter turns out to have met him at a party of a mutual friend in Dublin. After a slightly uneasy entry to the UK, where he "told the customs guy l'd just arrived from Dublin, and I didn't know how long l'd be staying" (p. 17), Eddie and Catriona travel from the airport to London together.

Catriona persuades Eddie to put off going to stay with relatives elsewhere in London and instead check in to her somewhat depressing but cheap hotel in the King's Cross district. This reinforces Eddie's sense of being in a transitional state between his parental home in Dublin and full life course independence, which is seen as dependent on the work he hopes to get: "l'd have to get a job soon. Then my own place" (p. 19). Indeed, even for this relatively short-distance move, the legacy of the Irish Wake is evoked in his mind: "I never knew the folks would be so upset about me going. When I told them first they were delighted. But the morning I left it was a different story. Tears and scribbled addresses and folded-up tenners in the suit pocket. You'd have sworn I was going to the moon, the way they went on" (p. 19).

Eddie and Caitriona go out on the town and soon become lovers. However, Catriona has not told Eddie that she has come to London for an abortion. She mysteriously goes out and has the operation but it is both emotionally and physically traumatic and she returns to Ireland without saying goodbye to Eddie, who moves on to his relatives and his search for work. 


\section{PLACING HUMAN MIGRATION}

O'Connor's story involved both Dublin Airport and the budget airline Ryanair, which began business in 1985. Both are now very big players within air travel mobilities. Indeed, July 2015 saw the airport set a new monthly passenger record of 2.7 million people.

Consideration of the 2015 summer schedule of flights from and to Dublin airport throws up some further impressive facts and statistics, all providing further support to the idea of a contemporary era of mobilities:

- Flights to and from around 164 destinations;

- Around 25 of these destinations were in the UK, demonstrating how flying has displaced sea ferries as the main way Ireland and the UK are linked;

- In mid-August, the three principal London destinations (Heathrow, City, Gatwick) had over 40 flights each way per day listed;

- To around 14 US destinations at same time, New York received around seven flights per day.

In context, Ireland in 2015 still had a population of well under 5 million inhabitants.

(Sources: Fenagh Visitors Centre 2015; O'Connor 1991/2008; Dublin Airport 2015a, 2015b)

predominate within everyday life and consciousness (Halfacree 2012a). Some social scientists thus asset that "mobility" is becoming the spirit of our times-its zeitgeist.

Sociologist John Urry and co-workers, for example, described this broader age of migration (e.g. Hannam et al. 2006; Urry 2007; Sheller and Urry 2006; the journal Mobilities) or era of mobilities (Halfacree 2012a). To get to grips with this mobile age, metaphors of "movement, mobility and contingent ordering" need to replace conventional sociological emphasis on "stasis, structure and social order" (Urry 2000: 18). In other words, sedentarist assumptions must be fundamentally undermined and displaced. A "new mobilities paradigm" may even be required to understand the condition (Sheller and Urry 2006). Within such a paradigm, one might expect enhanced social scientific status for human migration.

It appears that the era of mobilities does have a central place for migration (e.g. Cresswell and Merriman 2011). Quantitatively, this is expressed in increased frequency and diversity of everyday migration experiences, illustrated very simply and directly by Holdsworth et al. $(2013: 97,111)$ through a handful of official statistics:

- 214 million-214,000,000-international migrants worldwide in 2008, 3.1 percent of global population;

- 15.2 million refugees in the world in 2009;

- One-in-ten people in the UK moving house every year;

- Migrants in the US rising from 23 million in 1990 to nearly 43 million by 2010.

Qualitatively, too, mobilities researchers acknowledge heightened migration impacting on the human condition: belonging, community, home, identity, social-cultural expression (Adey 2010; Samers 2010). Such qualitative themes will be pursued throughout Chapters 6-9.

However, acknowledging the increased quantitative and qualitative significance of migration within everyday life, while necessary, is not enough. Migration's own sedentarist underpinnings presenting residential relocation as inherently unsettling and abnormal, must be challenged (Halfacree 2012a). Migration needs to be seen as an everyday component within the general mobile rhythms of lives (Lefebvre 2004). Sometimes this type of movement expresses disjuncture and disruption but it is always also constructive and creative, not least for the production of identity and biography (Fielding 1992a; Halfacree 2014a). Migration should not be seen as an abject experience, to be cast off, forgotten and denied with passage of time, but recognized as a force that, in both positive and negative ways, helps make people who they are.

Through more fully embracing the mobilities paradigm, Population Geographers can shift from presenting residential migration as an essentially unique or distinctive form of movement to recognizing and emphasizing its location within a broad spectrum of mobilities that both expresses and shapes everyday life. Within this spectrum, mundane everyday mobilities, for example, can also be seen having numerous significant impacts on the human condition (Figure 5.1). 


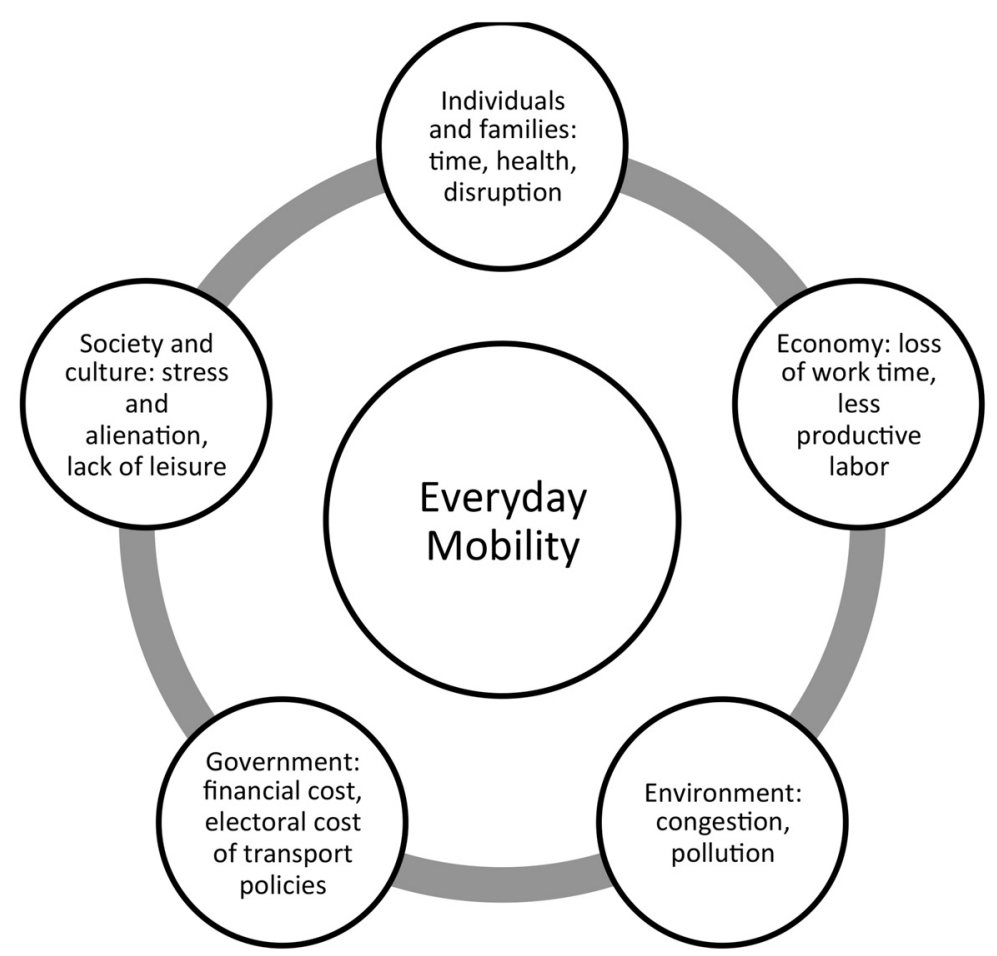

Figure 5.1 The costs of everyday mobility

Source: based on Pooley et al. (2005: Figure 1.2).

\begin{tabular}{|c|l|}
\hline Everyday movement & -Home, Garden \\
\hline Daily short-distance trips & $\begin{array}{l}\text { - School, Work, Shopping, Family, Social activities, Leisure, } \\
\text { Pleasure }\end{array}$ \\
\hline Regular longer trips & -Business, Family, Social, Leisure, Other \\
\hline Cyclical mobility between & $\begin{array}{l}\text { Long-distance weekly commuting, Students, Children between } \\
\text { two homes }\end{array}$ \\
\hline polidayts
\end{tabular}

Figure 5.2 The mobility continuum

Source: based on Pooley et al. (2005: Figure 1.1). 


\section{PLACING HUMAN MIGRATION}

Conceptual re-imagining of the place of migration vis-à-vis both societal significance and relations to other forms of mobility has significant implications for the scope of Population Geography's interest in people "on the move." In particular, it suggests not automatically bracketing out residential forms of migration for specific circumscribed analysis. Instead, acknowledging "the never-straightforward boundary between migration and mobility" (King 2002: 90), migration's connections and parallels with other forms of mobility must be recognized; independent not separate spheres (Sheller and Urry 2006).

Population Geographers have two particular challenges from this discussion. First, they need to give attention to the spectrum of mobilities if the relative place of migration within lives across space in an era of mobilities is to be adequately understood and appreciated. Pooley et al.'s (2005: 2) "mobility continuum," shown in Figure 5.2 (Green and Shuttleworth 2015), stretching from mundane everyday movements to diverse migrations, is useful here. Second, and convenient because Population Geographers will probably wish to retain a focus on migration, they must also become more fully aware of just how important various forms of migration may be becoming for shaping humanity's overall twenty-first century existence beyond acknowledged importance for the individual. How Population Geographers have sought to understand and investigate migration thus acquires added significance and is now introduced.

\subsubsection{Understanding and investigating migration in an era of mobilities}

Having noted migration's and mobility's increased recognition as vital components of everyday life, it is perhaps unsurprising that "there is no shortage of theories to explain why and where people migrate" (Samers 2010: 52). Table 5.3 reproduces a simplified version of Samers's overview of theoretical perspectives on international migration alone, whilst to demonstrate the multidisciplinary investment shown in migration (Brettell and Hollifield 2008a), Table 5.4 summarizes how different academic disciplines have approached the topic.

Such richness and diversity within theory clearly helps to explain migration's leading status within Population Geography (Boyle 2003, 2004). As suggested by the tables, to get a grip on this abundance, authors utilized different classificatory meta-frameworks. For example, Boyle et al. (1998; also Bakewell 2010; Samers 2010) distinguished "determinist," "humanist" and "integrated" approaches. Determinist accounts lay principal stress on factors within the potential migrant's "environment," understood more or less broadly, encouraging relocation (structure; Box 1.1). Humanist accounts, in contrast, focus on the individual as a more controlling, active migration decision maker (agency; Box 1.1). Integrated accounts attempt to bring these two perspectives together and transcend what can be regarded as an unhelpful divide.

The above framework is not adopted exactly in the rest of this chapter. Instead, inspired by the ultimately inextricably entangled areas of nature (Biology) and lives in common and individual presented as underpinning Chapter 2's life course perspective, four "expressions" of migration and mobility are distinguished. Each tends to be associated with more specific theories, many noted in Tables 5.3 and 5.4. Some are noted in the present chapter but elaborated across Chapters 6-9. Whilst there are often fundamental differences between theories, there is not space in this book to address these substantially. Moreover, with Castles $(2007,2010)$, this book does not promote a single theory of migration but favors more contextually specific articulations. It is felt that, with Massey et al., specific migration theories:

cannot be assumed, a priori, to be inherently incompatible ... [but often] reflect different research objectives, focuses, interests, and ways of decomposing an enormously complex subject into analytically manageable parts. (Massey et al. 1993: 433, quoted in Samers 2010: 53; also Massey et al. 1998;

cf. Bakewell 2010)

Finally, within the sections below, mobility more generally is brought into the discussion where possible and where it informs discussion. 
Table 5.3 Diverse theoretical approaches to studying migration

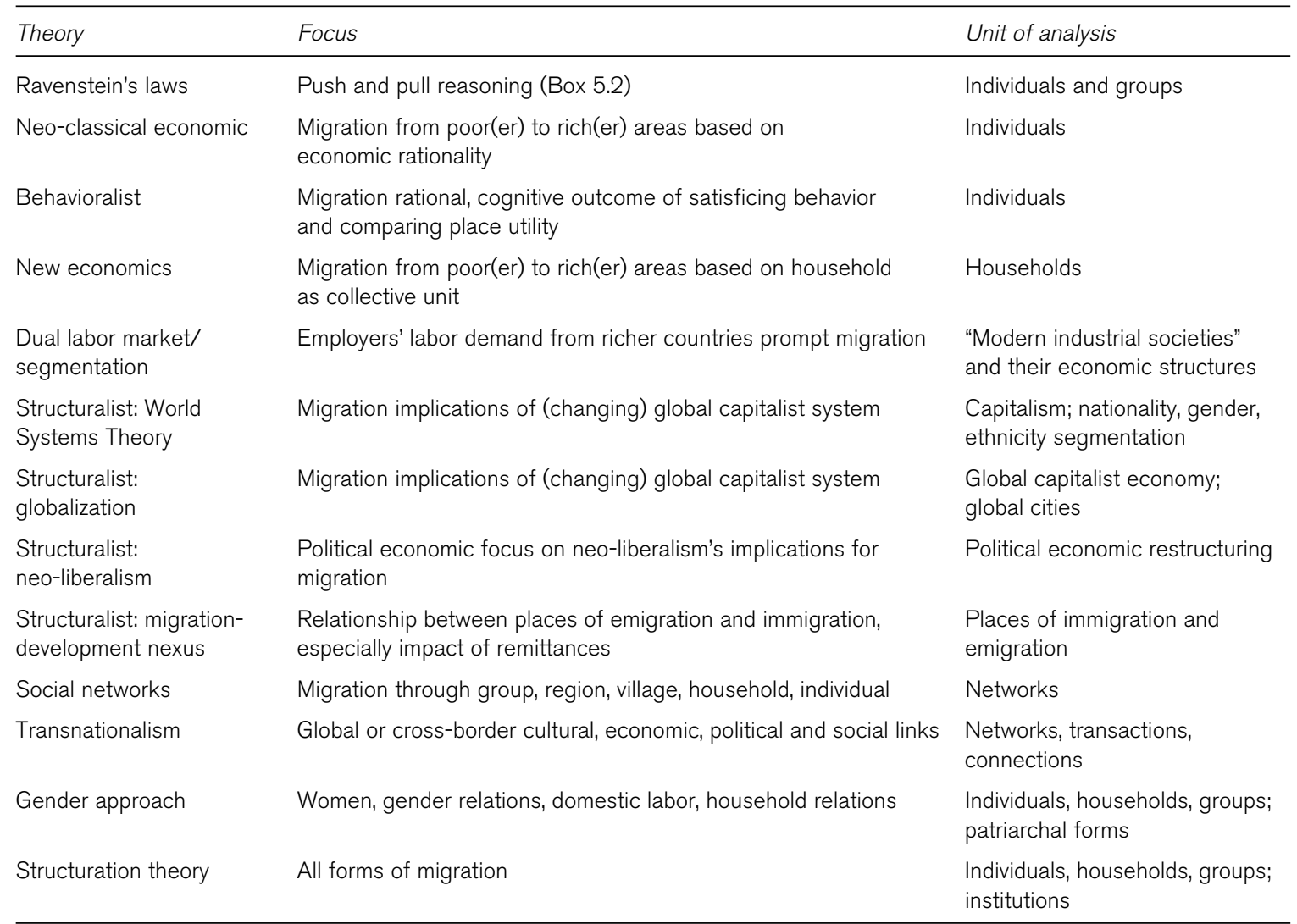

Source: simplified substantially from (Samers 2010: Table 2.1).

Table 5.4 How different academic disciplines have studied migration

\begin{tabular}{|c|c|c|c|c|}
\hline Discipline & Research question & Focus & Main theories & Sample hypothesis \\
\hline Geography & $\begin{array}{l}\text { What explains spatial } \\
\text { patterns of migration? }\end{array}$ & $\begin{array}{l}\text { Individuals, households, } \\
\text { groups }\end{array}$ & $\begin{array}{l}\text { Relational, structural, } \\
\text { transnational }\end{array}$ & $\begin{array}{l}\text { Ethnic incorporation } \\
\text { depends on networks and } \\
\text { residential patterns }\end{array}$ \\
\hline Demography & $\begin{array}{l}\text { How does migration } \\
\text { influence population change? }\end{array}$ & Populations & Economic rationality & $\begin{array}{l}\text { Migration has a significant } \\
\text { impact on population size }\end{array}$ \\
\hline Sociology & $\begin{array}{l}\text { What explains incorporation } \\
\text { and exclusion? }\end{array}$ & $\begin{array}{l}\text { Ethnic groups and } \\
\text { social class }\end{array}$ & $\begin{array}{l}\text { Structural and } \\
\text { institutional }\end{array}$ & $\begin{array}{l}\text { Incorporation varies with } \\
\text { human and social capital }\end{array}$ \\
\hline Economics & $\begin{array}{l}\text { What explains the propensity } \\
\text { to migrate? }\end{array}$ & Individuals & Economic rationality & $\begin{array}{l}\text { Incorporation varies with } \\
\text { human capital }\end{array}$ \\
\hline Law & $\begin{array}{l}\text { How does law influence } \\
\text { migration? }\end{array}$ & $\begin{array}{l}\text { Political and legal } \\
\text { systems }\end{array}$ & $\begin{array}{l}\text { Institutional and } \\
\text { rational }\end{array}$ & $\begin{array}{l}\text { Rights create incentive } \\
\text { to incorporate }\end{array}$ \\
\hline $\begin{array}{l}\text { Political } \\
\text { Science }\end{array}$ & $\begin{array}{l}\text { How do states try to } \\
\text { control migration? }\end{array}$ & $\begin{array}{l}\text { Political and } \\
\text { international systems }\end{array}$ & $\begin{array}{l}\text { Institutional and } \\
\text { rational }\end{array}$ & $\begin{array}{l}\text { Anti- and pro-immigrant } \\
\text { interests influence policy }\end{array}$ \\
\hline
\end{tabular}

Source: Brettell and Hollifield (2008b: 4). 


\subsection{MIGRATION AS NATURAL EXPRESSION}

\subsubsection{Natural laws and models of migration}

An immediate critique of sedentarism is to assert residential relocation as not somehow discordant but "natural" for people to undertake. One should look to (human) nature to explain migration. At one level, this is clearly true. Many forms of movement outlined in Figure 5.2 have been prominent within the human experience throughout history. Humankind, in short, is a mobile species! However, going beyond this ultimately biologically-reductionist perspective, and recognizing at least a degree of distinctiveness to human beings as a species, theories have sought to express "natural laws" of human migration. Such laws fit with empiricist and positivist accounts of human behavior (Chapter 1) that assume it is open to objective description and typically expressing generalizable regularities.

Between 1876 and 1889, a German-born former cartographer for the British War Office named Ernst Georg Ravenstein published around eleven laws of migration, presented in Table 5.5. These express the methodological individualism of the individual as the central "unit of analysis" (Samers 2010: 54-55). Ravenstein derived his laws from nineteenth-century British census data, supplemented in his final paper by data from censuses in North America and mainland Europe. All his laws, excluding the fifth, were more or less accurate accounts of migration across the Global North at the time. Many remain true today and indicate "some persistent patterns and processes" (Samers 2010: 56). Consequently, Ravenstein's laws have had lasting importance for Population Geography (Box 5.2). Nevertheless, they leave the key challenge of explaining why they are or have been true, since they lack explicit theoretical grounding (Woods 1982).

To provide such grounding, and predating the Quantitative Revolution (1.3.3), academics attempted from the 1940s onwards to extend laws of Physics to the human sphere in the form of the gravity model (Boyle et al. 1998: 46-50). Drawing on Newton's Law of Universal Gravitation, which stated that "two bodies in the universe attract each other in proportion to the product of their masses and inversely as the square of the distance between them" (cited in Jones 1990: 189), the basic gravity model for migration was specified as:

$$
\begin{array}{ll}
\hat{M}_{i j}=k^{*} \frac{P_{i}^{*} P_{j}}{d_{i j}^{b}} & \text { Estimated number of migrants moving between } i \text { and } j ; \\
\hat{M}_{i j} & \text { Constant value, depends on specific case; } \\
k & \text { Populations of origin and destination, respectively; } \\
P_{i} \text { and } P_{j} & \text { Distance between origin and destination; } \\
d_{i j} & \text { Distance decay parameter the larger it is, the more significantly distance influences migration. } \\
b &
\end{array}
$$

The gravity model provides estimates of flows between pairs of places. It states that the number of migrants is positively correlated with population size of both origin (potential migrants) and destination (potential "opportunities"). However, the number is inversely (negatively) related to distance between origin and destination. Here, "distance" can also act

Table 5.5 Ravenstein's "Laws of Migration"

1. Most migrants go only a short distance.

2. Migration proceeds step by step.

3. Long distance migrants generally choose to go to main urban centers.

4. Each current of migration produces a compensating counter current.

5. Urban natives are less migratory than those of rural areas.

6. Females are more intra-nationally migratory but males are more inter-nationally migratory.

7. Most migrants are adults: families rarely migrate out of their county of birth.

8. Large towns grow more by migration than by natural increase.

9. Migration rates increase as industries and commerce develop and transport improves

10. The main direction of migration is from rural to urban areas.

11. The major causes of migration are economic.

Source: simplified from Grigg (1977). 


\section{BOX 5.2 THE LEGACY OF "PUSH" AND "PULL" FACTORS IN MIGRATION SCHOLARSHIP}

There is a long pedigree within migration research of identifying so-called "push" and "pull" factors. The classic formulation envisaged push factors at the origin and pull factors at the destination combining to bring about migration. Bogue summarized these factors as follows:

\section{Push factors}

- Decline in a national resource or the prices it commands; decreased demand for a particular product or service; exhaustion of mines, timber or agricultural resources.

- Loss of employment due to incompetence, changing employer's needs, automation or mechanization.

- Discriminatory treatment on the grounds of politics, religion or ethnicity.

- Cultural alienation from a community.

- Poor marriage or employment opportunities.

- Retreat due to natural or humanly-created catastrophe.

\section{Pull factors}

- Improved employment opportunities.

- Superior income-earning opportunities.

- Opportunities for specialised training or education.

- Preferable environment or general living conditions.

- Movement as a result of dependency on someone else who has moved, such as a spouse.

- $\quad$ Novel, rich or varied cultural, intellectual or recreational environment (especially the city for rural populations).

From a present-day perspective or from within specific societal contexts one may wish to modify these factors considerably. Indeed, citing just push and pull factors is now generally considered too simplistic to explain observed migrations. For example, one can recognize both push and pull factors in both origin and destination. In addition, attention must also be paid in particular to "intervening obstacles" (as compared to intervening opportunities; 5.3.1) impeding particular migrations, such as family obligations at the origin, the costs of moving, legal constraints and personal anxiety about migration.

(Sources: Bogue 1969; Gilmartin 2008; Lee 1966; Lewis 1982)

as a proxy for influences on migration besides physical spacing, including knowledge about potential destinations and physical and psychological costs of moving.

The key area of statistical migration modeling operationalizing the gravity model, used widely in research today, is regression analysis. Usually, ordinary least squares (OLS) multiple regression techniques using logarithms are deployed. With the same terminology as the previous equation, the basic formula is:

$$
\ln M_{i j}=a_{0}+\left(a_{1}{ }^{*} \ln P_{i}\right)+\left(a_{2}{ }^{*} \ln P_{j}\right)-\left(b^{*} \ln d_{i j}\right)
$$

The equation replaces $k$ with a range of case specific constants: $a_{0}, a_{1}$ and $a_{2}$ (population parameter estimates) and $b$ (the distance decay parameter estimate). The latter is usually expressed, as here, by a power function (distance variable logged). An alternative is the negative exponential (distance not logged). Fotheringham and O'Kelly (1989) suggested the former for analyzing population migration, the latter for residential mobility.

Many variations to the basic regression model have been devised. These include:

- $\quad$ Adding additional variables relating to socio-economic conditions;

- Using alternative specifications, such as entropy-maximizing models (Wilson 1967, 1970; Senior 1979), models based on the Poisson distribution (Flowerdew and Aitkin 1982; Flowerdew 1991), or models based on populations alone (Simini et al. 2012); 


\section{PLACING HUMAN MIGRATION}

- $\quad$ Playing down the independent importance of distance through emphasizing the role of intervening opportunities (Stouffer 1940), where number of movements is proportional to number of "opportunities" at the destination but inversely proportional to "opportunities" between origin and destination.

All these models are known collectively as spatial interaction models since they focus attention on supposed interdependence between areas (Ullman 1980). They are not only used to study migration. Spatial interaction modeling (SIM) also seeks to explain and predict more general movements across space, including passenger numbers, traffic movement, commuter flows, movements of shoppers, trade flows and telecommunications (Fotheringham and O'Kelly 1989; Guldmann 2004). For example, an intervening opportunities model for shopping would suggest the number of trips between village $A$ and town $B$ would be proportional to the number of shops at $B$ but inversely proportional to the number of shops encountered on the way. Thus, presence of a mall between $A$ and $B$ would reduce flows as shoppers were diverted (e.g. Lorch and Smith 1993).

Substantial developments in Geographical Information Systems (GIS) technologies and applications have been vital in developing SIM. Indeed, discussion of such approaches now tends to be located within GIS-focused text books (e.g. Lloyd 2010, 2011). In addition, emerging data sets from online social networks, such as Facebook (Hofleitner et al. 2013) or Foursquare, whose users give their current location, have considerable potential for the study of mobility in general. An example of the latter (Noulas et al. 2012) reiterates the importance of intervening opportunities to urban mobility.

A further influential attempt to generalize about migration was made by Wilbur Zelinsky in the 1970s and 1980s. Inspired by the Demographic Transition Model's (4.2.2) omission of migration, Zelinsky (1971) sought to parallel the DTM's focus on fertility and mortality with how migration evolved as a society developed. The result was the fivephase Mobility Transition Model (MTM), shown in revised and simplified form in Figure 5.3. The MTM recognized modern society's high migrancy but also posited a general decline in migration in favor of circulation and communications media, suggesting the interdependence of mobilities. It also incorporated some of Ravenstein's laws, such as the prevalence of rural-to-urban migration and the central role of economics in directing migration.

The MTM, characterized by Hirschman (2005: 397) as "a very ambitious essay," retains a prominent place within migration scholarship. It has been effective at describing the evolution of migration when carefully qualified. The latter is necessary because the model has been subjected to much of the same criticism as the DTM: a vague notion of "modernization" as the driving force of change, strong Eurocentric universality, and supposed political naïvety (e.g. Woods 1993; Cadwallader 1993). Boyle et al. (1998: 61) even suggested it "tends towards the trivial when we attempt to understand and explain migration patterns and behaviour in detail." Indeed, beyond the very general, a comprehensive model for migration (change) over time or across space remains elusive.

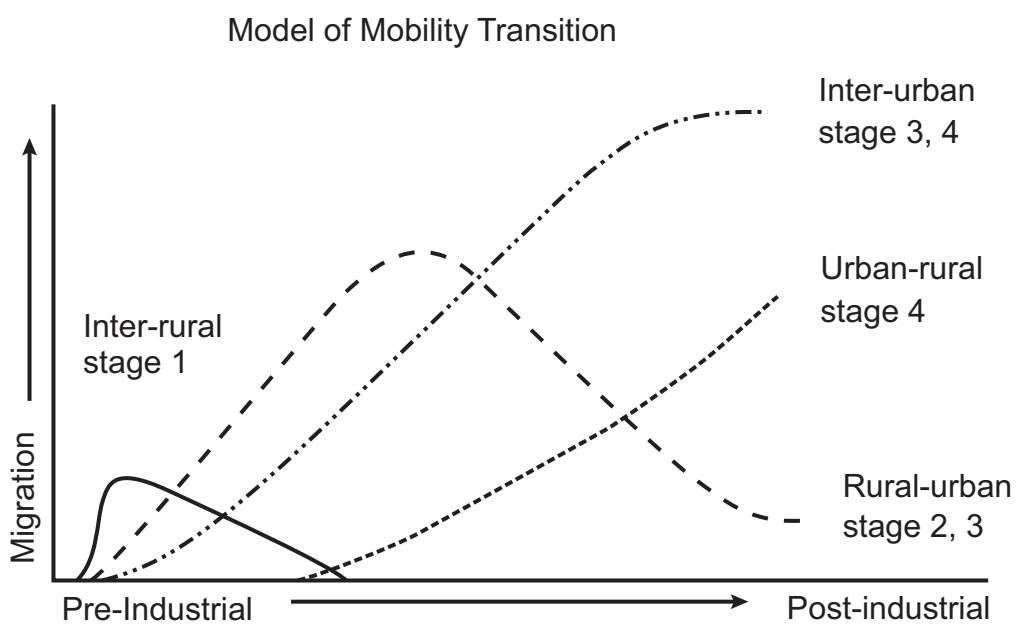

Figure 5.3 Representation of the Mobility Transition Model.

Source: Jones (1990: 208), after Zelinsky (1971). 


\subsubsection{Migration as rational economic behavior}

One of Ravenstein's principal aims was to understand migration's association with labor demand and supply. This came through clearly in his law: "[t]he major causes of migration are economic" (Table 5.5). Zelinsky's MTM also stressed the economic underpinning of migration. This strong association between migration and the economic endures (Halfacree 2004a). It is thus unsurprising that most early migration theories were rooted strongly in neoclassical economics (Greenwood 1975, 1985; Massey 1990), the predominant Economics paradigm, then as today.

A particular emphasis within this scholarship has been on workers responding economically rationally to variations in wages across space. Essentially, workers are seen to react to wage differentials by migrating from countries or regions with low wages to destinations with higher wages. This can be studied and expressed at the "macro" scale, with a focus on the supply of and demand for labor between countries, for example. Alternatively, a more methodologically individualistic "micro" perspective can be deployed (Massey et al. 1998). In both cases, migration expresses "labor reallocation in response to market need" (Ritchey 1976: 364). Moreover, this is likely to be a highly dynamic situation as the result of such (individually) rational relocations would be (socially) irrational over-supply of workers in high wage areas, which then depresses wages and promotes (individually) rational migration elsewhere (e.g. Rogers 1967).

Neoclassical models have become increasingly sophisticated over time (Borjas 1989) and any short introduction risks homogenizing and over-simplifying (Samers 2010: 314). A key development was Todaro's (1969, 1976; Harris and Todaro 1970) inclusion of a measure of the probability of finding employment, prompting wage differentials to be weighted by a proxy of such probability: unemployment rates. A further key extension came with human capital theory (Milne 1991), which takes into account different types of "income." For example, Sjaastad (1962) emphasized a process of weighing-up the costs of a potential migration against its benefits. Potential migrants based migration decisions on "assessment of the anticipated future stream of benefits (both monetary and psychic) as a consequence of migration" (Molho 1986: 398, emphasis removed). "Income" was still maximized but could now cover more than wages (McNabb 1979; Borjas 1989). As a consequence, migration patterns need not be dominated by flows from low to high wage areas. A huge variety of social, environmental and economic factors can drive relocation. The human capital approach, through the idea of investment, also recognizes that the time horizon for expected returns from migration is not necessarily immediate or minimal. Overall, it presents migration as a holistic investment decision, based on long-term as much as short-term considerations.

A further key development of broadly neoclassically rooted migration theory was the new economics of migration (Massey et al. 1993, 1998), particularly the work of Oded Stark (e.g. Stark 1991; Stark and Bloom 1985). This recognizes families and households, rather than individuals, as the primary unit within most migration (Cooke 2008a; Smith 2011), qualifying previous methodological individualism. It also focuses on risk reduction-playing safe-as much as on riskier capital maximization. In sum, migration is guided by family-based risk reduction strategies. The new economics approach has been especially useful interpreting migration originating in the Global South. For example, in a poor country with a weak and unstable economy, very real risk of extreme poverty through job loss can be reduced by relocating a family member abroad, who can then remit money back home (7.5.5).

A principal criticism of the neoclassical approach in general is that it treats information too unproblematically. Potential migrants are implicitly assumed to receive and process accurate and near complete information on wages, amenities, policy benefits, risk, and so on. This is an unrealistic assumption. Thus, researchers such as Stark (1991) tried to incorporate variations in stocks of accurate relevant information into their models. Also highly problematic is an assumption that individuals behave in a rational-(human) capital maximizing-manner. Third, even with perfect information and a rational attitude, migration may still not happen due constraints potential migrants experience. These include family and personal ties to place and, from a global perspective, how not all individuals are free to decide to migrate away from a home country, especially in an era of increasingly restrictive immigration laws and policies (Newbold 2010). All such critiques lead first to theories giving greater attention to the individual decision-making process, before Section 5.4's perspectives emphasize the constraints (and opportunities) presented by any migration's societal setting.

\subsubsection{Migration as decision-making behavior}

Behavioral approaches (Chapter 1) take their cue from Psychology. They focus on the supposed cognitive mechanisms behind individual acts of migration and mobility generally. Such individual perception and processing of information 


\section{PLACING HUMAN MIGRATION}

are ignored by measuring attributes of different places alone when modeling migration flows. Behavioral approaches, therefore, investigate how psychological processes of cognition and decision-making mediate between "environment" and "individual."

The key figure in the development of behavioral approaches to human migration was Julian Wolpert. In a series of papers in the mid-1960s (Wolpert 1964, 1965, 1966), he outlined a number of key concepts. First, there was place utility:

the net composite of utilities which are derived from the individual's integration at some time and space .... [I]t may be expressed as a positive or negative quality, expressing respectively the individual's satisfaction or dissatisfaction with respect to that place.

(Wolpert 1965: 60)

Wolpert argued that individuals move to places awarded higher overall place utility. This has similarities with the human capital approach, except in how utility is expressed. It might be actual characteristics, as neoclassical theory assumed, or highly subjective and/or inaccurate individual perceptions. Consequently, migration may be irrational and sub-optimal, at least to an "objective" external observer (or researcher!).

Wolpert further expressed migration's potential irrationality through the concept of satisficer. People were depicted as satisficing rather than maximizing beings, migration decisions expressing aspirations for satisfaction rather than optimality. Aspirations changed constantly but could be linked to biographical factors, such as previous experiences, and relational considerations, such as behavior of "similar" people (Boyle and Halfacree 1998b; Savage 2010). Also important was quasi-sedentarist concern with the satisfaction derived from settlement in place; acquisition of which was termed cumulative inertia (Huff and Clark 1978).

Wolpert further recognized migrants' limits on knowledgeability and the importance of biography by emphasizing a tendency to relocate to familiar places. Individuals' action space of potential migration destinations predominantly comprised known places, although being dynamic it might expand as information was gathered on more places (Kley 2011). Important were locational ties, such as presence of family or friends, and positive experiences of particular places, all part of what Moon (1995) termed moorings.

Finally, the concept of stress is important in behavioral perspectives. Wolpert assumed a satisficing human would tolerate a degree of discomfort at their current location but only up to a threshold. When stress levels crossed this line, either the threshold was raised as the individual reassessed his or her life or the current residential location became unsatisfactory and migration was desired. Stress was generated by such life course experiences as changed household structure, dwelling characteristics or environment, or by a person's tolerance threshold lowering as aspirations rose. Responding to such stresses through short-distance movement is illustrated widely in Chapter 6 .

Numerous studies have sought to operationalize behavioral perspectives on migration. In a pioneering study of intra-urban relocation within Philadelphia in the US, Rossi (1955) separated the decision-making process into three stages: decision to leave current location, search for new location, and choice from alternative destinations. This became the basis of the influential behavioral stage model that presents migration as a sequential decision-making process. Expressed by Zuiches (1980: 184):

the decision to move is analytically divided, first, into a phase of evaluation of one's current residence, in which a threshold of dissatisfaction may be reached, bringing the household to consider the possibility of a move. The second stage involves the search-and-selection procedures and includes a comparative evaluation of alternative sites. It is at this stage that locational preferences play a role in the selection process and that, finally, a decision to move is made.

Figure 5.4 gives two examples of behavioral stage models. Note how Clark (1986) included the concept of stress, seen as originating both in household needs and expectations and the environment. For Kley (2011: 470), enacting a migration involved crossing a metaphorical Rubicon, triggered by life course experiences "that dissolve bonds at the place of residence and ... scatter daily routines" (also Mulder 1993). Similar stage-style cognitive behavioral models can also be constructed to explore other forms of mobility, such as way-finding behavior, where spatial information is processed and drawn upon (often as cognitive maps) to get a person from origin to destination (Golledge 1999).

\section{ST PROOFS: NOT FOR DISTRIBUTION}




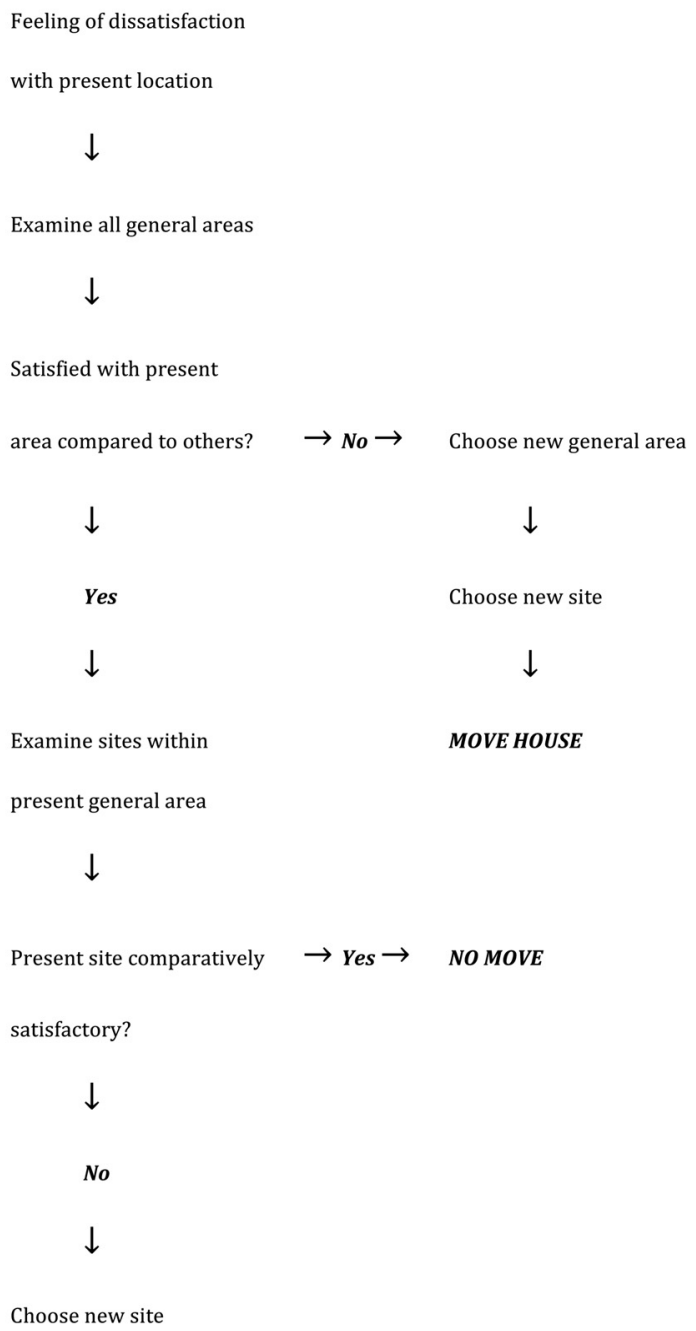

Figure 5.4 Two behavioral models of migration. a. General model of decision on moving house simplified from and based on Roseman (1971). b. Rubicon model of the migration process simplified from and based on Kley (2010).

\section{ST PROOFS: NOT FOR DISTRIBUTION}




\section{PLACING HUMAN MIGRATION}

Whilst the behavioral model begins to recognize individual diversity and how migration may be inspired by considerations well beyond the economic (Halfacree 2004a), expanding through multi-attribute utility theory (MAUT) to regard migration as seeking multiple goals simultaneously (e.g. Lin-Yuan and Kosinski 1994), it has also been strongly critiqued. First, stage models tend to treat migration as a relatively systematic and rational response to the need to overcome stress. In particular, the decision-maker is presented as something of "a cognitive drone ... governed by some 'inner', on-board computer" (Thrift 1986: 87) to make the "right" decision. As a result, models still at least implicitly anticipate an ability to produce generalizations of migration behavior, potentially expressed as empirical laws. Second, the behavioral approach concentrates only on what can be measured and quantified: causes of migration in terms of stress levels, place utility or, as a result of surveys, tables of reasons for moving. Third, behavioral models still naturalize migration. Whilst implicitly critiquing sedentarism, reducing migration to an omnipresent "good thing" that leads to better lives is contextually weak. Other approaches express migration more contextually, as a (political) outcome of society. These are considered next.

\subsection{MIGRATION AS SOCIETAL EXPRESSION}

\subsubsection{Migration in a capitalist society}

From structuralist (Chapter 1) perspectives emphasizing the societal context of behavior, patterns of migration and the processes giving rise to them are anything but "natural." One could no longer "avoid ... the question, 'why does migration occur?" (Woods 1982: 152, emphasis added). In answer, migration patterns and processes are regarded as rooted within the forces and currents shaping society. There is definitive move away from methodological individualism, as "patterns of migration are [regarded] not ... merely the result of the aggregation of individual decisions and actions but the product of objective social and spatial structures" (Goss and Lundquist 1995: 322). Complexity is also noted by recognizing that since any society is historically and geographically specific, so too is migration. Regularities do occur-hence the continued value of Ravenstein's nineteenth-century propositions and the sheer volume of work fitting into Section 5.3-but these are always relational outcomes of behavior set within a specific society.

Marxian perspectives, especially, have been highly influential within migration scholarship (Tables 5.3 and 5.4; Samers 2010: 65-85) and express society's influences very strongly. Starring role is given to the economic sphere, expressed by the mode of production or how specific societies organize productive activities. This importance of economic considerations for migration was made earlier in Section 5.3. However, from the Marxian perspective, this link is not "naturalized" nor taken as read but is expressed within the context of the predominant mode of production throughout the world today: capitalism. To understand migration, primary attention must be given to how the capitalist economy operates and evolves.

A Marxian perspective presents the links between the economic and migration as being more urgent yet contingent, dynamic yet unstable, and more fundamentally political than do naturalistic representations. Marxist Geographer David Harvey (1982: 381) expressed the overall situation thus:

In search of employment and a living wage, the labourer is forced to follow capital wherever it flows. ... Wage differentials ... provide the means to co-ordinate workers' moves to capital's requirements.... The more mobile the labourer, the more easily capital can adopt new labour processes and take advantage of superior locations. The free geographical mobility of labour power appears a necessary condition for the accumulation of capital.

Coverage and explanation within migration scholarship, as a consequence, cannot rest at just modeling migrants relocating to areas with higher wages (and/or other economic benefits) but must also note who migrants are (not least their economic class), why the destination has higher wages than the origin (where places fit within spatial divisions of labor, Massey 1984), and what are the consequences of non-migration (not least in terms of (non) receipt of state support). In short, a Marxian perspective requires a much broader canvas than just abstract economic rationality or personal stress motivated cognitive information processing to understand and explain migration adequately.

Such a more holistic societal perspective-not always Marxian-is especially strong within studies of international migration (Brettell and Hollifield 2008a; Massey et al. 1993; Samers 2010). What emerges clearly from these studies,

\section{ST PROOFS: NOT FOR DISTRIBUTION}


albeit with diverse emphases, is the role global geographies of capitalism play in shaping migration. Four insights demonstrate this.

First, studies rooted in Piore's (1979) influential Birds of Passage presented international labor migration in the context of a dual labor market within the Global North. In these richer nations, a capital-intensive primary economic sector is increasingly distinguished from a labor-intensive secondary sector. Secondary sector jobs are poorly paid, insecure and display little opportunity for advancement. They are unattractive options for existing residents, who largely monopolize primary sector employment. However, they are relatively attractive to poorer residents of the Global South. The latter, through migration, seek to access them.

Second, other studies adapted and fine-tuned Piore's ideas. For example, scholarship highlighted the key role of global cities as prime sites for secondary jobs (e.g. Sassen 1998). Other studies focused on mapping a complex economic geographical picture of employment than just primary and secondary labor markets, not least emphasizing the highly dynamic economic geographies of capitalism, whose ebbs and flows have considerable consequences for human migration (e.g. Smith 1984).

Third, there was in contrast cautioning against getting carried away with this sense of dynamic economic geographies, in spite of recent added mobilities emphasis (5.2.2). Instead, stubborn geographical inertia is recognized within the international division of labor, the global geographical expression of jobs and workers. Such a geography underpins the terms Global North and Global South, for example. The South, in consequence, has been portrayed by Cohen (1987) as providing a Marxian reserve army of labor, a force "called up" by capitalists whenever labor shortages threaten and/or workers in the North demand too high wages.

Fourth, the idea of a structured global capitalist economy was strongly apparent in Immanuel Wallerstein's (1974, 1979, 1983; Taylor 1989) World System Theory. This focused on the global socio-spatial growth of the capitalist economy. Wallerstein considered this economy covered the entire world by around 1900, economic variations between countries subsequently reflecting the uneven spatial character of its internal development (Smith 1984). Migration responded to this unevenness of both employment supply and demand. It has been assisted by developments falling under the heading globalization (1.2.2), notably the transportation and communications technologies also featuring strongly within the mobilities literature. Global migration patterns are also underpinned by ideologies such as neo-liberalism, whereby a drive to reduce wages and cut back substantially on state welfare support can "motivate" out-migration. This was shown by Massey and Capoferro (2006) in Peru, for example. Finally, all perspectives discussed in this section tended to focus on migration from peripheral to core locations but the consolidated idea of a global economy also helps explain other flows. These include international migrations of students and highly skilled expatriate workers (Chapter 7).

Throughout their existence, Marxian and other economic societal readings of migration have been subject to frequent modifications and refinements. Nevertheless, whilst not necessarily accepting fully the radical political message often associated with this perspective, emphasis on migration as primarily a consequence of economic considerations within a capitalist society remains widely recognized (Boyle et al. 1998; Halfacree 2004a; Samers 2010). However, migration's societal contextualization that begins with the economic does not have to end with the imperatives and calculations of and within capitalism alone, as the next section demonstrates.

Discussion thus far has largely been confined to medium and longer distance migration, albeit with links to mobilities. Whilst shorter distance migration-residential mobility (Chapter 6)-is much less associated with employment considerations, the economic, expressed particularly through income, is equally critical for explaining and understanding such moves. The same can be said for broader mobility. Adapting a term used in the mobilities literature, motility or ability to move (Kaufmann 2002)-whether with respect to international labor migration, intra-national lifestyle migration, intra-urban housing-related migration, or the daily commute-is molded strongly by relative position within a specific capitalist society. Migration and mobility are, in short, strongly shaped through the differential life course arena of social class (2.3.4).

\subsubsection{Migration within a more-than-capitalist society}

No society today is solely defined by the capitalist mode of production, even if all societies are largely capitalist and to a degree fundamentally interconnected (Gibson-Graham 1996, 2006). The role society plays in shaping migration and mobility generally must acknowledge the role of the capitalist mode of production and/or the economy more loosely 


\section{PLACING HUMAN MIGRATION}

but this should not lead to interpreting migration only in such terms. Each other arena through which the life course is shaped (2.3) also have implications for migration and mobility.

Considering age first, personal mobility is typically closely linked with this arena, being low before a toddler learns to walk and if infirmities, often in older age, become significant. Migration's strong associations with key life course transitions, considered more fully in Section 5.6.3.1, show broad correlations with age (Mulder 1993). In addition, socially sanctioned ability to relocate-and even to influence a relocation (Bushin 2009)-is strongly determined by acquisition of normative levels of "adult" competence. It is thus entangled with transition to adulthood (Tyrrell and Kraftl 2015). Child-led migration solely encompassing people under 16 years of age, for example, is rarely seen as "legitimate," at least in the Global North.

Second, health and differential ability impacts on migration and mobility in numerous ways (Darlington et al. 2015). As a limiting case, being ill can prevent both, thereby possibly impacting on ability to access health care, for example (Chapter 10). At the other end of the mobility continuum, a key strand within lifestyle migration (Chapter 8 ) involves the search for supposedly healthier places to live. Between these extremes are migration flows such as retirement migration, where both health and lifestyle considerations typically help shape the relocation rationale and outcome.

Third, considerations from within historically and geographically specific economic geographies play a central role in explaining migration differentials by race and ethnicity (Finney et al. 2015). However, this societal arena is directly significant for prejudice and discrimination impacting on migration and mobility. This is reflected, for example, in concentration of ethnic minority groups into certain parts of a city (6.3.4), at the larger scale by distributing and blocking flows of employment migrants and refugees (Chapters 7 and 9) and, in mobility terms, in deterring ethnic minorities from venturing out at night (6.2.5).

Fourth, religion and belief has a structural association with forms of mobility reflecting a duty to undertake pilgrimage to holy sites (Collins-Kreiner 2010). Notable is the requirement for Muslims to undertake the hajj to Mecca in Saudi Arabia. Numbers undertaking hajj now regularly exceed 2 million annually (Collins-Kreiner 2010). On top of this, an Islamic conception of migration is expressed in Islamic law through issues such as the obligations to and of migrants or when emigration should occur (Abu-Sahlieh 1996). Historically, religious persecution and/or perceived need to find a relatively "empty" space to enact beliefs has also underpinned some international migration, not least to the so-called New World (e.g. Portes and Rumbaut 2006). Persecution and prejudice can also again encourage everyday immobilities.

Fifth, sex and, most especially, gender relations shape both migration and mobility very strongly. Yet, prior to the 1980s, although Ravenstein regarded them as migratory as men, women and their identities, spaces of work, tasks, dress and domains were largely ignored in migration studies (Boyle 2002; Pessar and Mahler 2003). Over the last 30 years, however, scholars have increasingly acknowledged the importance of considering gender in migration research (Pessar and Mahler 2003; Mahler and Pessar 2006; Chant and Radeliff 1992) and there are now plenty of examples of well developed gender perspectives (Gilmartin 2008). This allowed Donato et al. (2006: 4) to observe a:

change in perspective [that] reflects two important developments. First, scholars ... have succeeded in bringing female migration out of the shadows .... Indeed, with demographers claiming that, globally, female migration is now virtually equal to that of males, the phrase 'the feminization of migration' is gaining currency. Second, and perhaps more important, many migration scholars now insist that migration itself is a gendered phenomenon that requires more sophisticated theoretical and analytical tools than studies of sex roles and of sex as a dichotomous variable allowed in the past.

Despite a slow start, in other words, Population Geographers are engaging with and contributing to a rich and diverse scholarship on women in migration, analyzing gender dimensions of scale, politics and spatialities of power, as well as unequal geographies of mobility, belonging, exclusion and displacement (Silvey 2006a).

Within this work on gender and migration (e.g. Boyle and Halfacree 1999; Hondagneu-Sotelo and Cranford 2006; Palmary et al. 2010), a paramount structuring role has been given to patriarchy (2.3.8), operating through all its elements (Walby 1989): family, paid work, state, male violence, sexuality, culture. Consider willingness to move. Even when controlling for factors widely noted to influence it, such as occupational status and home ownership, research has found women in the Global North more reluctant migrants than men. For married or cohabiting women, many reasons have been proposed to explain this (Halfacree 1995a). Internal perspectives, which focus on the woman in

\section{ST PROOFS: NOT FOR DISTRIBUTION}


family context, examine issues such as relative net gains in human capital or gender roles. External perspectives, which locate the woman and her family within broader social context, focus on patriarchy more acutely. Differences in willingness and propensity to migrate by sex follow the patriarchal model. For example, the delineation "women's jobs" versus "men's jobs" (Bradley 1989) has resulted in the former being lower paid and less career-orientated, and thus less worth migrating for in economic rationality terms. Furthermore, the patriarchal household accords women less influence than men within the migration decision-making process. Yet, more than just the specifics of either patriarchy or the household, gendering of migration also reflects the more nebulous expression of "migration decision making processes ... shaped by sex-specific family and friendship sources of approval, disapproval, assistance and information" (Boyd 1989: 657).

Everyday mobility is also molded strongly by patriarchal expression. Evidence ranges from studies showing women restricting their spatial and temporal organization and extent of mobility due to fear of physical and sexual assault (e.g. Pain 1997), to mobility consequences of relative lack of time from multiple daily roles and tasks (e.g. Domosh and Seager 2001: Chapter 4), to constraints from transport inaccessibility (e.g. Pooley et al. 2005).

Sixth, sexuality also works to structure migration. On the one hand, despite the rise of the dual location household in the Global North (Green 1997; Smith 2011), long-term sexual relationships and especially marriage remain strongly associated with the migration of one or both partners as a new household is established (6.3.2). This is as true for same-sex couples as heterosexuals but with the former an additional societal driver of migration may be experience of prejudice and discrimination. This can prompt international migration but is more commonly represented by migratory drift towards supposedly more same-sex friendly environments (e.g. Smith and Holt 2005; Weston 1995). As with those experiencing racism, religious persecution or patriarchal threat, homophobia can also impact negatively on everyday mobilities of lesbian, gay and transgender people (6.3.5).

In summary, both migration and mobility generally are shaped more-or-less independently and more-or-less strongly by all the societal life course arenas identified in Section 2.3, all placed, furthermore, within the critical contexts of geography and history. From this, one might even suggest that due to any person's multiple positionalities, migration and mobility should be seen in very individual terms. This is considered next.

\subsection{MIGRATION AS INDIVIDUAL EXPRESSION}

Absent from the societal arenas introduced in Section 5.4 as molding migration and mobility was the role of individuality (2.3.10), a vital dimension within the life course perspective. Whilst, on the one hand, individuality may be seen partly or even largely socially determined, on the other hand, both the present book and migration scholarship accord a role for migration less reductively expressing individuality; a "personal experience" (McHugh 2000: 76). For example, in critique typically leveled at structuralist approaches an argument is made that agency must not be removed from individuals involved in migration. To do so is to render people powerless and impotent to forces of societal determination, which is regarded as essentially anti-human. Resistance to such relegation is even appropriate when considering "forced" migration (King 2002) (Chapter 9).

A humanist stance (Chapter 1) rejects "mechanical perspectives" (Mendoza and Morén-Alegret 2013: 16) and positions people first and foremost as individuals. Any decision on whether or not to migrate and how migration plays out becomes substantially that of the individual. Individuals are active and creative decision-makers, not just cognitive data processors or structural configurations. Their ultimate decisions to migrate or stay put may or may not be rational from any particular perspective. Migration thus cannot be so confidently predicted by measuring characteristics of individuals (such as class) or places (such as wage rates). Instead, beliefs, values, aspirations, emotions and feelings, priorities and obligations must be noted; they represent more than just "noise." Empathetic understanding is prioritized over evaluated explanation.

To gain such understanding, humanist approaches usually involve in-depth study of the persons involved, utilizing intensive qualitative methodologies (1.2.4). Longitudinal data sets, where the migrant is engaged with for an extended period, are also very useful (Box 5.3). Lack of such detailed material, not least due to time and cost constraints, helps explain the relative under-representation of humanistic migration research (Boyle et al. 1998). Consequently, McHugh (2000: 72) called for much fuller valuation of ethnographic studies to further "understanding of migration and circulation systems." 


\section{BOX 5.3 FROM CROSS-SECTIONAL TO LONGITUDINAL MIGRATION DATA}

Most migration data, often obtained from surveys, is cross-sectional. It comes from a temporal cross-section or snapshot of a population at a particular point in time. For example, in the UK census a person is categorized as a "migrant" if their permanent address on the day of the census was different from their permanent address exactly one year earlier. The US census, similarly, uses a 5-year period. However, some migration data sets are more longitudinal, where migrants' characteristics and how they change over a long(er) period of time can be measured. For example, a migrant's employment or social status can be tracked from data taken at a number of time periods. Importantly, from such longitudinal data the migration researcher can examine the before and after of a migration, with respect to employment type for example. Such analysis can thus show whether migration-residential change-tends to be associated with other changes in a person's life. Although this correlation investigation cannot itself demonstrate causality-e.g. migration leading to people getting better jobsit is suggestive of linkages and associations in a way cross-sectional analysis cannot be.

A longitudinal data set can be constructed from a number of cross-sectional data sets if the same individuals can be identified and tracked within the latter. This has been done in the English and Welsh census through its Longitudinal Study (LS). After the 1971 census, an original LS sample of all people born on one of four birth days, comprising around 1 percent of the population at that time, was selected. For each subsequent census members of the sample were removed if they had died or emigrated and new members recruited if they had one of the four birthdays. This resulted in a sample of around half a million people.

For those in the LS sample, their census returns at each census have been linked together so their lives as defined by the variables measured in the census can be tracked. From such data sets a great diversity of research can be carried out. From a migration point of view, one can analyze whether, for example, those who migrated between two or more of the census dates have fared differently, in terms of employment, family structure, health and so on, compared to non-migrants. Clearly, such research requires considerable care and detail but results are now widely reported in the migration literature. For example, using LS data for the 1971-1991 period, Paul Norman and colleagues demonstrated young migrants in England and Wales to be generally healthier than equivalent non-migrants, and migrants moving from more to less deprived areas to be healthier than migrants from less to more deprived areas. Indeed, within more deprived areas, in-migrants tended to be less healthy than non-migrants. Such studies demonstrate clearly the value of constructing longitudinal data sets for understanding the likely consequences of migration more fully.

(Sources: Hattersley and Creeser 1995; Norman et al. 2005)

Nonetheless, there is a substantial and growing body of humanistic-inclined migration scholarship. This has been inspired by general recognition, endorsed here, that:

[m]igration is an inherently complex spatial and social phenomenon ... [and] shedding light on the complexity of the migration process itself (e.g. how many moves over what distances, and to what places over what period of time) as well as the motives for, and personal experiences of, moving involves a commitment to more qualitative methods and techniques, as well as to longitudinal analyses.

(Gibson and Argent 2008: 136)

Growing scholarship also builds on how "quantitative reduction of migration into equations, formulae and prediction caused significant unease in the middle of geography's cultural turn" (Gilmartin 2008: 1839). Details of the response cover seven areas.

First, a migrant history approach has provided detailed individual insights into migration behavior. Typically deploying "biographical narrative interviews" (Apitzsch and Siouti 2007: 7), it traces individuals through their lives. Emphasis is given to uncovering the migrations that occur in a life (e.g. Bartholomew 1991; Chapman 1985; Courgeau 1989; Miles and Crush 1993) and their relationship to such things as sense of place (Pascual-de-Sans 2004). For 
Forrest and Murie (1991: 64), migrant histories avoided the "arid and depersonalized" results of surveys, retaining the coherence of the individual.

An excellent example of migrant history research is Ní Laoire's (2008) life narrative study of late 1980s/ early 1990s emigrants from Ireland who had returned a decade later. Using in-depth interviews in particular, Ní Laoire constructed an oral history of the migrants' lives that gave a clear sense of why and how they moved. The study revealed considerable diversity within a group otherwise united through emigration and subsequent return. Away from such primary data gathering, various secondary information sources are also extremely useful for teasing out the details and individuality of migrant histories, such as personal letters (Box 5.4) and, increasingly, emails and blogs.

Migrant history perspectives can be extended, second, to recognize how migration is a highly cultural experience (Bottomley 1992; Fielding 1992a; Halfacree and Merriman 2015). Most sharply acknowledged within Anthropology (Brettell 2008, 2013; Vertovec 2007), migration accretes meanings for a given social group that cannot be reduced to economic or social factors alone (e.g. King et al. 1995). Thus, Boyle et al. (1998: 207) propsed the concept of cultures of migration, defined as "express[ing] how both the association between migration and key events and experiences in people's lives and the selectivity of any specific migration process are reflected in the relative [cultural] position that migration holds within specific societal groups." Migration appears as an integral part of a "way of life"

\section{BOX 5.4 MIGRATION IN TWENTIETH-CENTURY LOVE LETTERS BETWEEN ITALY AND CANADA}

Canadian Historian; Sonia Cancian; has used the texts of letters between lovers to present a deep qualitative insight into more individual and expressive experiences of international migration. She focused, in particular, on correspondence between Italians who migrated to Canada in the years 1948-1957 and their romantic partners who remained behind in Italy. For Cancian (2011: 92), these letters, filled as they are with expressions of love, passion and longing as their authors sought "to sustain a romantic relationship across geographical distances" also "represent a new window onto the human experience of migration, mobility, and separation."

Both women and men migrated to Canada amongst the couples researched. Some migration occurred through accompanying other family members but the key aim was predominantly to obtain employment. In other words, emigrants in the study were largely-directly or indirectly-international employment migrants (Chapter 7).

The first, and most obvious, theme the letters convey is just how emotionally stressful and hurtful the resultant romantic separation could be. Whilst migrants were generally seeking a better material standard of living through their relocation to Canada, this was often a tough emotional experience to endure. These employment migrants were no happy-go-lucky chancers, principally trying their luck in a new environment. Whilst material benefits might be acquired, this was often at the expense of considerable emotional cost. Giordano Rossini's letter of April 1957 to Ester di Leonardi powerfully articulated this sense of loss from one left behind in Italy: "I saw our star tonight and it seemed to be saying to me: 'Ester sends you many, many kisses and is always thinking of you' to which I answered: 'Dearest little star, bring many, many kisses to my Love ... '" (quoted p. 99).

Second, the letters give strong individual insights into the working and everyday lives of the migrants. The migrant life was often physically hard, as Antonietta Petris confided to Loris Palma in October 1948. Such conditions could change for the better, however, as Antonietta's subsequent letters made clear.

Third, in this pre-mobilities era any promise of eventual reconciliation in place for the lovers could never be guaranteed and the letters also spoke of the long time for reunion to occur. Certainly, relationships that changed from face-to-face bodily intimacy to "a romantic love nurtured on paper" (p. 104) could be difficult to come to terms with. Thus, Dante del Moro, anticipating the imminent arrival in Canada of his wife, Sara, and two children in September 1956, spoke of his desperation whereby "a day feels like a month" (quoted p. 101).

Cancian's material originated in "the last postal age" (p. 105). In contrast, today, letters may no longer express the migrant experience so convincingly. Instead, other sources have taken their place. Many of these are less material than the letter, such as telephone calls, emails and Skype conversations, and to access and analyze them presents a worthy contemporary challenge for migration researchers.

(Sources: Cancian 2011) 


\section{PLACING HUMAN MIGRATION}

garbed in cultural-economic clothes for specific groups (Brettell 2013). For higher status professionals in the Global North, being mobile is often a core requirement of career development (Chapter 7). Or, consider the individuals and families studied by Cohen (2004) in the Mexican state of Oaxaca, for whom migration, whether just to the state capital or further afield to other parts of Mexico or the US, was firmly encultured as a way of making a living. Stark's riskreducing new economics of migration behavior, in other words, is also cultural.

Importantly, one must not assume "culture" within such analyses as all-encompassing or singular, even for a relatively well defined social group; individual insight must not be lost. For Ní Laoire (2000: 329; also Silvey and Lawson 1999) it was often more interesting and insightful "to go behind apparent 'cultures of migration' to tease out the differences and inequalities which are often hidden by them." Finally, an interesting extension of the cultures of migration idea comes from Bushin's (2009) examination of the role children do or do not play within family migration decision making. The extent to which a child is granted agent status depends on such things as culturally inscribed ideas of responsibility, maturity and competence, especially as recognized by family adults.

Third, returning more directly to individuality yet retaining emphasis on culture, postmodern perspectives (1.2.4) have become active within efforts to understand migration. Emphasizing difference, diversity and context and rejecting the sweeping claims of "grand theory" (Graham 1995), postmodernism has much in common with humanism's emphasis on the individual, if often drawing from contrasting philosophies. An example of the resulting work and its recognition of relative, situated knowledges (Smith and King 2012) is use of polyphonic interviewing. In this approach, migrants' voices are recorded with minimal interference from the researcher. Next, the diversity of different responses and their internal inconsistencies are acknowledged and presented instead of being generalized and thereby reduced (Clifford and Marcus 1986; Crang 1992). A partial illustration is Li's (1997) parallel presentation of three migration autobiographies of Hong Kong emigrants, simplified in Table 5.6.

Fourth, much better at gaining more personal or individual insights into migration than studies of the act of relocation is the large body of scholarship that has examined experiences of migrants in their new residential locations. Migrant stock rather than flow is emphasized. Anthropologists, with "central interest ... in the human dimensions ... and the lived experience of being a migrant" (Brettell 2008: 136), have contributed considerably (Vertovec 2007). They are relatively well positioned to cope with the often considerable cultural, social and linguistic demands (Mendoza and Morén-Alegret 2013) such work entails. Specifically, scholarship has focused on immigrant experiences, whilst lives following relocation have been less considered within internal migration research (Halfacree 2014a). Ethnographically rich research has also promoted greater recognition of the place of gender in migration practices (Mahler and Pessar 2006).

Work on immigrants' experiences is numerous and diverse (Brettell and Hollifield 2008a), extending to considerable recent interest in the post-displacement lives of forced migrants, for example (Chapter 9). Generally, researchers engage via such qualitative methods as ethnography, textual analysis, participant observation, focus group discussions and audio-visual methods to gain a deep insight into lives within the immigrant community (Brettell 2013; Mendoza and Morén-Alegret 2013) and how both individual and community identity are being shaped through migration (McHugh 2000; Silvey 2004).

A key theme within research into the immigrant experience is the migrant's sense of belonging and home. Increasingly moving away from a "'sedentarist analytic bias' (Chu 2006: 397) that sees home as a fixed, bounded and enclosed site" (Ralph and Staeheli 2011: 518), scholarship acknowledges complex, multiple configurations of this central spatial site(s) of attachment and belonging (Blunt and Dowling 2006). A cutting edge example, utilizing participatory action research (PAR) and arts practice, is O'Neill and Hubbard's (2010) exploration of the senses of belonging negotiated by asylum seekers, refugees and undocumented migrants across the English East Midlands. A walking event (Figure 5.5) involved new arrivals guiding established residents through the city on an imaginary and real journey, "shar[ing] aspects of their biographies connected to the here and now-Nottingham, Derby, Leicester and Loughborough-to memories of there and then (home)" (O'Neill and Hubbard 2010: 50). Other studies have engaged with how migrants are represented within immigrant host societies (e.g. Chavez 2001, 2008; Finney and Robinson 2008) and the extent to which cities are "immigrant-friendly or immigrant-hostile places" (Brettell 2013: 3). Out of this work emerged the key concept of transnationalism (McEwan 2004; McHugh 2000), a sense of home that crosses national borders (5.6.1, 7.5.6).

A fifth growing area of migration scholarship expressing the humanistic and individual strongly concerns the emotional dimensions of relocation (Brooks and Simpson 2012; Mendoza and Morén-Alegret 2013; Tyrrell and Kraftl

\section{ST PROOFS: NOT FOR DISTRIBUTION}


Table 5.6 Selection from autobiographies of three emigrants from Hong Kong to Canada

\begin{tabular}{lll}
\hline Lo & Siu & Chan \\
\hline - Born and educated in Hong Kong & - Born and educated in Hong Kong up & ・ Born and educated in Hong Kong up \\
up to first degree in Engineering & to tertiary level in Engineering & to MBBS \\
- Worked in Hong Kong 1981-1990 & - Worked in Hong Kong 1985-1990 & ・ Worked in Hong Kong 1980-1984 \\
- Married in Hong Kong & - Emigrated in 1990 & - Married in the USA \\
- Emigrated in 1990 & - Currently resident in Vancouver, & ・ Emigrated to USA in 1984 \\
- Currently resident in Toronto, & director of own company & - Emigrated to Canada in 1989 \\
employed as manager & Single & - Currently resident in Vancouver, \\
- Two school-aged children & & Assistant Professor in teaching hospital \\
& & - Two school-aged children
\end{tabular}

Migration intention in earlier stages

[In secondary school] I thought quite naively that maybe I could go to university abroad. My family would not be able to support me financially. In my secondary school, many pupils ... went abroad to study and I did consider going as well. All the universities I applied to accepted me and I also got a scholarship to go. However, I didn't go eventually and I can't remember why... . . I did not consider going abroad when I was at university. I had identified the issue of 1997 even before it was raised. At the time, the phrase "the future of Hong Kong" was not even mentioned. I said even then that Hong Kong should be given back to the Chinese government.... It was an unfair treaty .... Also because this is mainly Chinese society, the correct way is to return it to China.... I never considered leaving.
There were many people in my secondary school who went to study in Britain because my school had alliances with some high schools [there] ... I didn't consider going myself at the time because I felt that I couldn't earn any money and my family was not upper-middle class .... So I didn't even think about it. I only thought about going when I was finishing my tertiary education.... During the last year at college, I did consider going abroad because my professor suggested that I apply to scholarships provided by my college to go to the UK or USA. But even with a scholarship, I would still need a large sum of money for daily expenses.... I didn't want my family to spend such a sum of money .... and I couldn't find a quick way to earn such a large sum .... So I didn't even apply.
[In my secondary school] I think about 70 or 80 percent of people went abroad to study. They usually returned to Hong Kong after their studies. I wanted to live abroad, not just to study. .. I always wanted to leave. I have an elder brother and ... sister. They were both abroad when I was in secondary school. My elder brother went to university abroad and my elder sister went for secondary school education in Canada. So I always wanted to leave but it turned out that I stayed on ... I applied to go abroad many times, and I almost left but stayed on at the last moment.... My [High School] results were very good .... So the opportunity was there and my dad and mum were in Hong Kong. I was the youngest and my elder brother and sister had both left.

Source: selected and simplified from Li (1997: 77).

2015). Emotions feature strongly within the immigrant experiences just noted but this aspect of migration is of general relevance, not least given the upheaval in everyday life migration can present (Fielding 1992a; Halfacree 2014a). From this, Shubin $(2012,2015)$ demonstrated the emotional significance of spiritual practices for Eastern Europeans in Scotland. Elsewhere, Gorman-Murray (2009) examined emotional embodiment within the migration experiences of gay and lesbian people. Drawing on Australian case study material, he showed how "feelings of comfort and love were emphasised as triggers for displacement and re-placement," before making the more general conclusion that "emotions, desires and intimate attachments [potentially] play a critical - but under-recognised - role in migration processes" for everyone (Gorman-Murray 2009: 454-455).

Sixth, a sense of individuality is very apparent within the mobilities literature, with its strong emphasis on experience, embodiment and encounter. However, critically, and in a bridge to Section 5.6, this is not a solipsistic obsession with a unique and bounded self, a criticism sometimes made of overly humanistic work, but of the individual always "in relation." As Adey (2010: 17) emphasized, mobilities must be understood relationally, personal mobilities always "positioned in relation to something or somebody," from a trip to the shops to a visit to close family. Performance of mobilities, moreover, is both reliant on the presence of "mobility systems" (Urry 2007: 52), involving such things as railway stations or airports, and productive of relatively enduring socio-spatial relations, such as specialized shopping environments or geographically scattered communities (Zelinsky and Lee 1998; Hardwick 2008). 
116 PLACING HUMAN MIGRATION
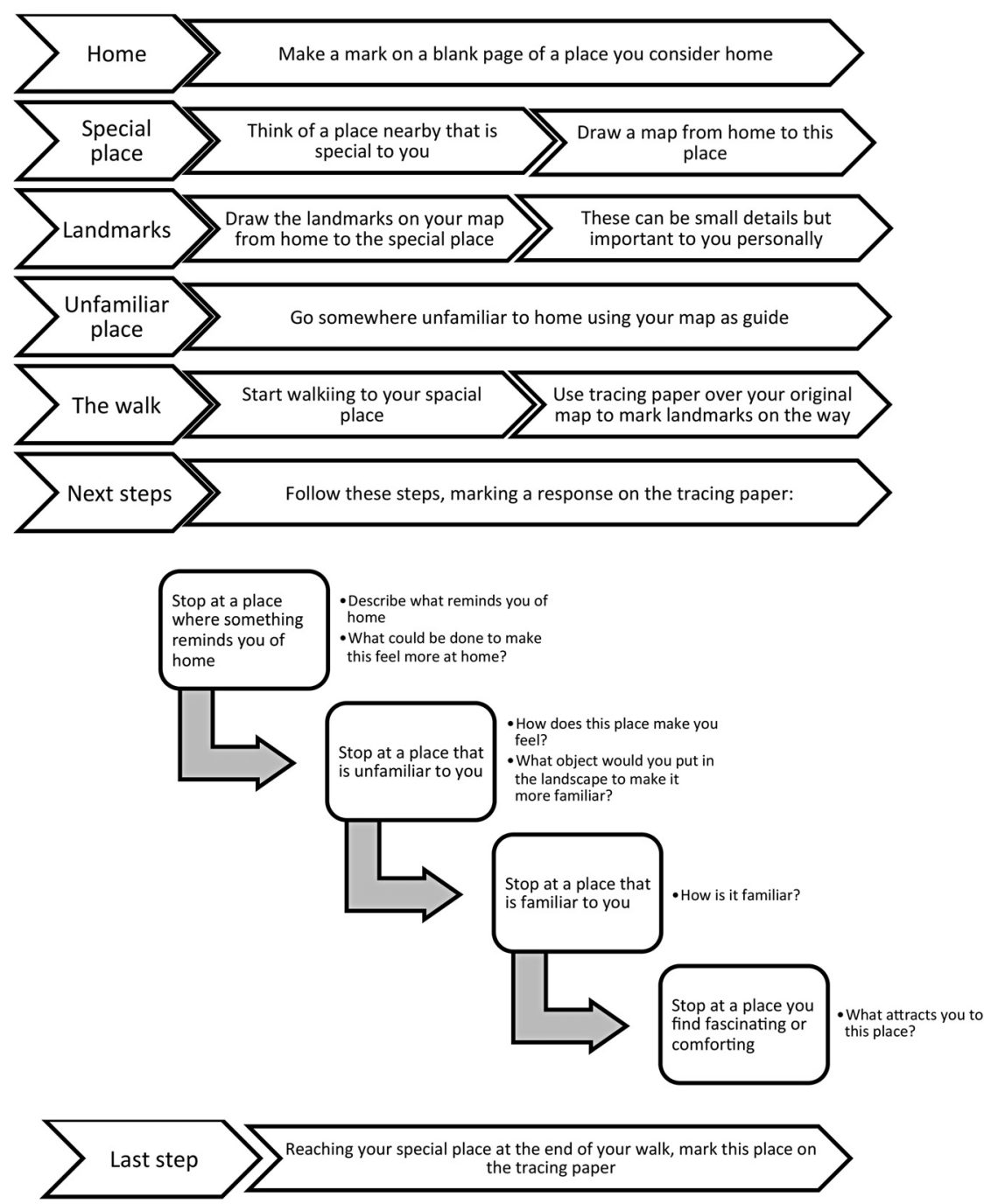

Figure 5.5 Instructions for Immigrant Sense of Belonging Walking Project

Seventh and finally, attention returns to methodology. From a mobility perspective, Cresswell (2012: 647) noted strong "interest in new kinds of methods that are suited to both actual mobility in the world and new theoretical approaches to mobility." These mobile methods enable researchers to move, be or see with research subjects. They include video ethnography and "go-along" interviews (e.g. Büscher et al. 2010; Fincham et al. 2009; Merriman 2012). Spinney (2011), for example, used mobile video techniques, such as head-mounted cameras, to capture cyclists' behavior. Footage was analyzed to examine the often instant decisions occurring within the active negotiation of space, so as to build a detailed ethnography. Besides such innovative methods, more established approaches have adjusted, "[e]thnography, in particular, [having] gone mobile... mov[ing] from a deep engagement with a single site, to analysis of several sites at once (multisite ethnography), to ethnography that moves along with, or besides, the object of research (mobile ethnography)" (Cresswell 2012: 647). Not least through work on mobilities, therefore, both mobility and by extension migration are increasingly seen in much more contextualized and multi-dimensional ways. Finally, and linking migration and broader mobilities, recent scholarship has drawn from the performance literature to suggest the embodied presentations involved in all acts of migration, whether spontaneous and improvised or more choreographed and acted out (Halfacree and Merriman 2015). This takes the perspective on migration deep into the embodied life course. 


\subsection{MIGRATION AS LIFE COURSE EXPRESSION}

\subsubsection{Openness, integration, multi-dimensionality}

Scholarship reviewed in Sections 5.3-5.5 revealed migration to exhibit both a range of regularities and variations in both geographical and social expression. Many research findings have remained relatively consistent and stable over time (Kley 2011), such as the economic impetus behind much migration. They have been relatively immune to contrasting theoretical perspectives if not always so neatly reproduced at the individual level. Accorded naturalistic law-like status by some scholars, others emphasized the causal emplacement of regularities by the structured workings of society. In contrast, other researchers (re)turned to focus on the individual migrant and how s/he lived a life, including migrations, in their own terms. As the life course perspective elaborated in Chapter 2 anticipates, migration-and mobility-has thus been seen as lived both in common and individually.

Building on this duality of the role of context and individual agency but seeking to transcend it through the medium of the life course, this chapter's insights will be brought together through the elaboration and promotion of a biographical understanding of migration. In this model, biologically-rooted processes of human lives, including a degree of rational calculation and an everyday individuality, work themselves out within a more-or-less multiply structured and organized society. Migration-as life generally-is lived relationally across space.

To date, migration research has often not adopted such an holistic perspective. Instead, it has been frequently distinguished and separated through a variety of either/or dichotomies or dualisms (King 2002; White 1980) and "disciplinary parochialisms" (Massey 1990: 4). These include divisions of nature versus society, individual versus society, micro (individual) versus macro (group), and quantitative versus qualitative. One may recognize the imprint of such divisions within this chapter's previous sections. However, "taking sides" within such divides is often not very intellectually worthwhile as it can lead to one-sided and partial perspectives on a multifaceted whole. Advocated instead, after Boyle et al. (1998), is a more integrated "cartography" (King 2002: 89) for migration theory.

A similar call for more integrated and interdisciplinary approaches to interrogating migration have been made elsewhere (e.g. Brettell and Hollifield 2008b; King 2002; Vertovec 2007). Population Geographers need to pay greater attention to migration scholarship undertaken within other disciplines and even within other branches of the discipline (e.g. Skeldon 1995). Acknowledgement can provide ideas to stimulate and extend existing perspectives, as in Halfacree's (2012a) "domestication" of the previously "international" concept of transnationalism. In short, echoing the critical reflection on Population Geography expressed in Chapter 1, all migration scholars need openness to unfamiliar ideas and interpretations to keep their subject intellectually vibrant (Smith and King 2012).

Over the years, attempts have been made to promote a more integrated and open perspective on migration. A strong sense of interconnectedness and of migration as a system (Castles and Miller 1993) has played a vital role here. Research on international migration recognizes the central importance of linkages between sending and receiving countries expressed in migration networks (Hardwick 2008; Kritz et al. 1992; Newbold 2010; Samers 2010). Chapter 7 engages with this theme much further but as an example, there is a well-established labor migration system linking certain Asian countries to the Arab Gulf states. Its significance was starkly exposed during the 1990-1991 Gulf War when there was a return home of 2 million migrant workers within 4 months. This caused much hardship for these migrants and their families (Connell 1992).

Another illustration of migration networks is demonstrated through the causal power of the Polish diaspora in the UK to promote and direct migration (Morawska 2001). This finding supports Massey's (1990) argument, drawing on macroeconomic theory, that international migration networks promote positive feedback mechanisms of cumulative causation. Building links facilitating greater levels of migration in turn reinforces the "reality" of the network, prompting more migration and so on. Thus, employment growth in one country initially stimulating in-migration from an origin country linked to it in some way (perhaps via common language and/or through being a former colony) becomes increasingly sedimented over time.

Networks express much more than just people flows, however. Vitally, they also encompass other mobilities, again reinforcing how migration is only relatively distinctive from these other movements. First, they are social, leading to diverse attempts to maintain links between origin and destination countries (Shinozaki 2004). For example, Gulati's (1993) study of wives "left behind" in Kerala, India, by male international labor migrants demonstrated how the women 


\section{PLACING HUMAN MIGRATION}

kept in touch with their husbands. Ultimately, sustaining these social linkages led to husbands' return migration. Generally, family, friendship and community linkages underpin many contemporary geographical patterns of international migration, extending economic relations between countries through providing information conduits and a "cultural cushion" (Boyle et al. 1998: 77) for new arrivals.

Second, however, international migration networks are very strongly economic. Crucial for Gulati (1993), but now widely acknowledged within international migration studies, was the role of money sent home by those abroad. These monies are typically used to support family and to invest in property, indicating migrants' intention to return. This further demonstrates interconnectedness of diverse expressions of mobility (Adey 2010)-here, people and money. The previously noted concept of transnationalism (McEwan 2004) brings together these ideas of connectivity and multidimensionality excellently. Returned to later (7.5.6), this seeks to capture "processes by which immigrants forge and sustain multi-stranded social relations that link together their societies of origin and settlement" (Basch et al. 1994: 7). Transnationalism expresses how migration may "lie ... at a tangent to both the region of origin and the region of settlement ... constitut[ing] a form of existence in its own right" (Apitzsch and Siouti 2007: 16). To examine this place of migration within a life lived across space, one may consider a biographical approach.

\subsubsection{A biographical approach to migration}

The specific biographical approach to migration introduced here was proposed two decades ago (Halfacree and Boyle 1993; also Halfacree 2004a) but inevitably name-checks a longer and broader tradition (Apitzsch and Siouti 2007). It is an approach whose explicit focus on migration within the context of relational lives lived associates it firmly with the present book's life course perspective.

Within the biographical approach, migrants are firmly positioned as "interpretive subjects of their own mobility. . . [but operating within] webs of socially negotiated meanings. . . constantly negotiated" (Silvey and Lawson 1999: 126, 124-125). This parallels how lives across space are lived generally (Hörschelmann 2011). Migrant interpretation of their mobility is informed by present contexts or circumstances but is also shaped by past experiences and future anticipations. In consequence, migration shifts from being regarded predominantly as a relatively discrete, focused and tightly bounded action-responding to immediate "stress," for example-to being recognized as a much more distributed, indeterminate and entangled process. It is "a process over time" (Kley 2011: 469), "a search, a project, rather than an act" (Benson and O'Reilly (2009a: 610). As a result, besides overcoming Section 5.3-5.5's nature, structure and agency separations, both stock versus flow and process versus product divides (King 2002) are transgressed. The aim is to acknowledge "migratory process ... migration ... not [as] a single [action] ... but a lifelong process" (Castles 2000: 15).

The biographical approach initially emphasized three core issues. First, as indicated, it locates all migrations within everyday life. Whilst migration may not be as "everyday" as other forms of mobility, it is nonetheless something that occurs every day (Halfacree 2014a). For an individual, this emplaces each migration within a life course that is unavoidably multi-faceted, multi-layered and multi-textured. Thus, migration decision-making, no matter how seemingly rational and calculated it appears, has a history, a geography, and socio-cultural dimensions; it is undertaken within the arenas of differential life course experience, relationally composed as well as an individual action (Apitzsch and Siouti 2007). Put slightly differently, migration is always entangled within the multiple existential currents that flow and mingle together to comprise "life" (Thrift 2004b): experiences, sensations, emotions, encounters, affects, memories, reflections, thoughts, hopes, fears, aspirations, anticipation .... Or, still differently, reflecting the importance of sense of place to migration behavior:

How an individual senses a given place and how that sensory experience is expressed are also related to his/her own biography, including previous migration movements but also childhood experiences and dreams, education paths, languages spoken and political engagements

(Mendoza and Morén-Alegret 2013: 7)

In sum, biographical emphasis on the everyday undermines any "exceptional" status being accorded to residential migration as a form of mobility, no matter how significant such a migration can be(come) for an individual. It thus builds on deconstructing the exceptionalism of migration relative to other mobilities begun in Section 5.2. 
Second-and fortunately for overworked migration researchers!-all the multiple existential currents flowing through and eddying within a migration will not be of equivalent significance. Some aspects of life course usually predominate in informing a migration. Nonetheless, the biographical approach argues that multiple currents typically inform a specific migration. It thus concurred with Lawson (1999: 263) that "[w]hile migration is often prompted by economic motivations, the migration literature all too frequently stops there." It is generally necessary to assume multiple reasons underpin, albeit unevenly in prominence, a particular relocation. This becomes clear when one attempts to explain all of the dimensions of an urban-to-rural relocation, where different reasons may underpin selection of house, district, rurality, and so on (Halfacree 1994; Rivera 2004). In this respect, Kley's (2011) Rubicon model (Figure 5.4) usefully extended the dimensions of conventional behavioral models. A biographical approach also agrees with Ní Laoire's (2000) emphasis on the need "to look inside, round, across, under, over and beyond dominant cultures of migration" (Halfacree 2004a: 242). It is also suggestive to note that not every current co-constructing a migration will necessarily be acknowledged. They may not even be capable of being acknowledged as potentially expressible discursive consciousness (Giddens 1984) if originating within the deep unconscious, yet may still help shape the individual character of a migration.

Third, the biographical approach's explicitly contextual emplacing reinforces the importance of recognizing migration as always encultured (Fielding 1992a). This parallels Section 2.3.1's assertion that culture is both deeply infused within and thereby transcends all arenas of differential experience identified. Thus, Section 5.5's concept of cultures of migration-always multiple, always diverse, always underdetermined-expresses well the biographical appearance of migration. It is not that migration somehow maps culture or culture maps migration but that the two mutually, if often rather partially and untidily, co-constitute one another. Widely noted regularities of migration-such as its occurrence following retirement from paid work in the Global North-are always encultured and not just empirical expressions. As Bakewell (2010) asserted, migration theory should not be restricted to "upgrading the formal status of [such] empirical observations" (Arango 2000: 294) but must strive to explain these regularities within encultured everyday existence.

Throughout all this discussion, invocation of "biography" requires one central overall clarification. Crucially, solipsistic, subject-orientated readings of the term are rejected, so as to recognize and emphasize instead the beyondthe-individual structuring of a biography/life course. This should be clear from the emphasis on culture and arenas of life course experience but merits explicit re-statement. As Thrift $(2007: 7,109)$ warned, there is a danger in the notion of biography-expressed in the biographies and autobiographies that fill bookshops, including those of Geographers (Daniels and Nash 2004)-of arriving at a "spurious sense of oneness" from "putting [a] ... life in order through text." Typically, such ordering tells the reader a moral story, "[l]ife history ... plotted as story of self-reflection and self-improvement" (Daniels and Nash 2004: 454; Apitzsch and Siouti 2007). Use and advocacy of the term biography for interpreting migration is shorn of such moral or coherent narrative implications but still aims "to retain a certain minimal humanism ... [and] personal authorship" (Thrift 2007: 13, emphasis removed) that acknowledges "the 'human' in human migration" (McHugh 2000: 72).

Affiliating biography with life course prompts a final additional key aspect of the biographical perspective on migration. This is to reiterate how migration is usually a fundamentally social process. Whilst not unknown, rare are migrations bearing no relation to the existence, feelings and opinions, exhortations or awareness of other people. Here, family or household context merits particular note (Bailey and Boyle 2004; Cooke 2008a). The family or householdincreasingly diverse in its patterning (Smith 2011)-is a further central relational consideration in the playing out of individual migration biographies. Cooke (2008a: 262) even argued that "family migration should move front and centre in discussions regarding migration in general."

Overall, the biographical approach to human migration builds on the idea of all lives as "storied" (Gutting 1996: 483). Population Geographers must "read" these stories to understand and explain migration behaviors. As such, the approach encourages use of "biographical narrative interview[s]" (Apitzsch and Siouti 2007: 5), which "allow the individual to relate how he or she has experienced certain life history processes and his or her own life history" (Apitzsch and Siouti 2007: 8-9). Yet, subsequent examination of these often "spontaneous autobiographical narrative[s]" (Apitzsch and Siouti 2007: 9) must also show awareness of their positioning as entangled within the webs woven by the arenas of life course experience, including the family. Investigating migration stories and the place not only of migration but also mobility generally thus should always extend beyond humanist preoccupation with the individual. 
120 PLACING HUMAN MIGRATION

\subsubsection{Migration and the life course}

\subsubsection{Migration and life course transitions}

All kinds of geographical mobility can, very simply, occur at just about any time across a life course. This is in line with not seeing the life course as rigidly (pre-)defined or irreversible. However, not least when specifying a type of move more exactly-e.g. commuting, employment migration, lifestyle migration-many forms show strong association with relatively well-defined life course stages. More precisely, while forms of mobility often link closely to the duration of life course stages (e.g. school and work commutes, shopping trips), different kinds of migration typically accompany and reinforce key life course transitions (2.2.1) (Geist and McManus 2008; Jasso 2003; Mulder 1993; Tyrrell and Kraftl 2015). Table 5.7 summarizes many of these, if simplified and a Global North perspective.

As Tyrrell and Kraftl (2015) emphasized, not only do all life courses not experience all of Table 5.7's forms of migration but any life course may experience many of them more than once, sometimes repeatedly. What the table clearly demonstrates is just how integral migration is to shaping and patterning an evolving life course. Migration not only signifies a change in the geographical arena in which a life is playing out but can also signify the biographical changes that express a life. This assumes still greater significance when some migrations attain event status.

\subsubsection{The event of migration}

Tony Fielding (1992a), in calling to recognize more fully the cultural dimensions of residential relocation, spoke of migration as an "event." This term can be taken up and used to extend the biographical approach's re-imagining of how migration can work within a life course (Halfacree 2013a, 2014a; Halfacree and Rivera 2012). Specifically, although elusive to pin down (Anderson and Harrison 2010), the event is a key concept within non-representational theory (Thrift 2007). Here it is not just understood as "something that happens" (Fraser 2010: 57), as in commonplace understanding. Instead, what happens "presents a metaphorical gateway or portal" (Halfacree 2014a: 100) to more radical change and consequences. Events provide "new potentialities for being, doing and thinking" (Anderson and Harrison 2010: 19, emphasis removed). In an international migration context, Apitzsch and Siouti (2007:13) present a partial example of this excess to the "intentional action":

Intentional action with the aim of integration in the country of destiny ... will be halted or altered by new experiences, false expectations, the hostility of neighbours and the discovery that strangeness may increase instead of disappearing as time goes by.

Table 5.7 Life course transitions associated with migration

\begin{tabular}{ll}
\hline Life course stage & Transitional migration \\
\hline Infancy (dependent) & Family: employment \\
& Family: quality of life \\
& Family: education \\
& (Higher) education \\
Younger adult & Leaving parental home \\
& Employment: new job, job change, promotion \\
Adult & Response to economic hardship: job loss \\
& Relationship formation: marriage, cohabitation \\
& Relationship dissolution: divorce, separation \\
& At or prior to birth of child: bigger and/or better property \\
& Lifestyle \\
& Retirement lifestyle \\
& Following bereavement \\
& Due to frailty or chronic ill health \\
& For caring responsibilities: recipient or provider (e.g. grandchildren)
\end{tabular}

Sources: inspired by Tyrrell and Kraftl (2015); Warnes (1992). 
With its contextualization within the relational complexity of everyday life and its diverse indeterminacy in terms of temporality and cause, the biographical approach primes migration for receiving event-like status. As event, whilst a residential relocation may usually be explicable and understandable, seeking a goal such as a better job, safety or a pleasanter environment, its life course significance and relevance does not end with this "explanation." Indeed, relocation is only the starting point of migration as event. Thus, a specific relocation may initially be capable of representation as, for example, employment or lifestyle migration, but this categorization can turn out to be initial and provisional rather than definitive and conclusive in its significance to the life course. Subsequent experiences gained through this act of migration may go on to re-write what the migration becomes about within the later life course biography.

Attributing potential event-like status to a migration radically re-imagines how it is to be interpreted and described. The physical act of relocation and the reasons given for it remain of considerable importance but just mark the beginning of detailed analysis of its significance. Such appreciation comes through within studies of the immigrant experience, introduced in Section 5.5. As the biographical approach emphasizes, migration becomes "distributed" into the future as much as it is triggered in the present and rooted in the past (Halfacree and Boyle 1993). An event perspective makes it hard to determine the central "meaning" of any migration and, thus, when it is "over" and capable of final inventory. Migration as expressing permanent relocation becomes further refuted (5.1.1). Eventful migration brings to central attention post-relocation (Benson 2011) lives, issues and experiences. This is illustrated later in the book for counterurban migration (8.3.2).

Integrating an eventful reading of migration within the biographical perspective suggests how a particular migration may be both more ordinary and more extraordinary than conventionally understood. Reinforcing Section 5.6.2, whilst

\section{BOX 5.5 MOMENTOUS MUNDANE MOBILITY MOMENTS}

The idea a migration can be an event that opens up a potentially very different life course future than explicitly "intended" by the action of relocating also has resonance within other mobility forms. A sense of how mobility "choice" can open different futures was illustrated creatively in two feature films from 1998. Neither expresses a complete event situation but in both a simple mobility choice has event-like consequences.

Sliding Doors was a romantic comedy-drama written and directed by Peter Howitt. It sketched out two different futures for its main protagonist, Helen, played by Gwyneth Paltrow, dependent on whether or not she succeeds in catching a London underground train before its (sliding) doors close. In the first scenario she catches the train, arriving home to find her boyfriend has been unfaithful. She begins a relationship with somebody she met on that same underground trip, only to die later after being hit by a car. In the second scenario Helen misses the train. After subsequent injury takes her to hospital, delaying her further, she arrives home after her boyfriend's lover has left. The couple stay together until Helen discovers her boyfriend has made his lover pregnant. Although she injures herself when leaving him, Helen survives in this scenario.
Run Lola Run (Lola Rennt) was written and directed by Tom Tykwer and has three alternate scenarios. These depend on the run that the principal character, Lola, played by Franka Potente, undertakes in her near impossible quest to obtain 100,000 Deutsche Marks for her boyfriend within 20 minutes. This is to prevent him being murdered by the gangster to whom he owes this money. In each scenario Lola encounters a variety of people, whose own fate is seen later in the film to vary depending on Lola's run. In the first scenario, Lola ends up getting accidentally shot; in the second it is her boyfriend who is seriously injured; and in the third both are okay and also recover the original money that was the source of the debt Lola was seeking to replace.

Both films express explicitly the role of chance in shaping everyday life. A sense of very different futures hinging on the starting points of key mobility-related pivots-an underground train or a run-suggests how the significance of an event far exceeds its functional, mundane original purpose.

(Howitt 1998; Tykwer 1998) 
such a migration is every day, it is not everyday (Schillmeier 2011). On the one hand, it is an every day mobility in that almost everyone migrates during their life, most many times. As a form of mobility it is not so clearly distinguishable or as unique as Population Geographers' focus on it has perhaps tended to imply. At very least, one must recognize migration's contextual position within a continuum of everyday mobilities (Figure 5.2). And yet, on the other hand, eventful migration is not everyday, in that both the significance of the relocation and its post-relocation consequential playing out can lead to profound life course consequences. Reiterating however, this is not to suggest that a migration is always eventful. Some (most?) are explicable in fairly simple and bounded terms. The challenge is to distinguish those that exceed everyday status. A similar case can be made for other expressions of mobility: all every day and mostly everyday (Middleton 2011; cf. Edensor 2008) but sometimes eventful (Box 5.5).

\subsection{CONCLUSION: BROADENING UNDERSTANDING OF HUMAN MIGRATION}

This chapter introduced the considerable body of academic scholarship seeking conceptual understanding of human migration. It presented four broad approaches: migration as expressing nature, society, individuality, and life course. There is much overlap between these categories-and other migration scholars have proposed alternative mappingsbut taken together they indicate how human migration, initially seemingly simply defined as expressing a permanent change in usual address, is an extremely complex and multidimensional human practice or set of practices. It was traced from being regarded as a relatively simple, singular, individual action to being seen through a biographical lens as potentially a complex, multiply configured, relational event.

Understandably, there remains much debate over the state of migration theory within Population Geography and beyond. Consequently, Smith and King (2012: 127) argued in a special issue of Population, Space and Place that "establishing robust theoretical frameworks and migration theories that effectively capture the increasing diversity of migration should be a major preoccupation for scholars." They called for the advance of migration theories for a range of scales, "to substantiate the changing social, economic, and political contexts within which different forms of migration are couched and to explore if different theoretical frameworks are required to understand some of the new regimes of migration" (Smith and King 2012: 128, after Castles 2007). Three aspects within this quote exemplify key future challenges for migration theorizing.

First, in terms of scale, one may question the enduring influence of the internal versus international dichotomy (King 2002). Whilst crossing a national boundary-defining a migration as "international"-is likely to be highly for a migration and its experience, this is not necessarily true. As Favell (2008: 270, emphasis added) argued, the "defining" role of state boundaries can be overemphasized, since "the world is not only one of nation-state units." In addition, Olwig and Sørensen (2002: 7) noted how "strong focus on international migration has had the unfortunate result that other kinds of population movement have received less attention." Instead therefore, it is sometimes more useful for migration researchers to examine their subject according to themes cross-cutting both the internal/international and the earlier critiqued (5.2.2) migration/mobility divides. This is pursued to some extent in Chapters 6-9. De-prioritizing a priori these two divides may facilitate development of migration theory. Work on internal migration can learn lessons from international migration's concepts, and vice-versa, and migration and mobilities scholarship can similarly speak more fully to one another.

Second, one can again reiterate Smith and King's (2012) emphasis on the importance of context. This chapter's outlining and advocacy of a biographical life course perspective on migration foregrounds both arenas of everyday life and their institutional forms, notably the family, for shaping migration. Migration, as with all Population Geography, is irredeemably relational. Thus, except in the trivial sense that humans are mobile life forms, naturalized theories of migration are rejected. Chapters 6-9, therefore, also seek to draw out key relational contexts when illustrating their various expressions of migration and mobility.

Third, Smith and King (2012) noted the emergence of "new regimes of migration." Partly expressing this, Section 5.2 proposed an emerging era of mobilities. Mobility, both metaphorical and material, has attained something of a zeitgeist status. Clearly, this poses many challenges to migration theorizing and understanding, arguably promoting its existential significance to the first division for us mobilized humans. Within this, though, any "mobilities paradigm" (Sheller and Urry 2006) must recognize migration's place within a diverse galaxy of mobilities. Whilst residential

\section{ST PROOFS: NOT FOR DISTRIBUTION}


migration presents a fundamentally qualitatively different human experience to an everyday work commute, a "hard" boundary must not be erected between migration and other mobilities. This sensitivity has a number of consequences. In particular, it questions the permanent versus temporary binary conventionally so significant in distinguishing migration (permanent) from other forms of mobility (temporary) (Bell and Ward 2000; King 2002). Restating how any migration is often "temporary" from the perspective of an individual's life course biography, to assume a migration has an implicit goal of permanent settlement is overly sedentarist. Chapters 6-9 thus attempt where possible to transcend this migration/permanent versus mobility/temporary boundary through noting connections between migration and other mobilities.

From these three emphases, this book's four subsequent chapters engaging with migration are organized as follows. Eschewing the usual internal/international divide as a predominant structuring device (e.g. Boyle et al. 1998), they address four themes:

- $\quad$ Chapter 6: emphasizes how everyday mobilities can build into residential mobility;

- Chapter 7: explores how employment considerations underpin a huge range of relocations at all scales, many far from "permanent";

- Chapter 8: illustrates the many migrations underpinned by what may be loosely termed "lifestyle considerations," especially across the Global North;

- Chapter 9: engages with the substantial numbers of migrations globally driven by fear, danger, disaster, and absolute necessity.

The specific types of migration present within the four chapters' broad families are often, as already noted, associated strongly with life course stages or transitions. The chapters also draw out such associations. Finally, overall, migration and other mobilities speak of people's demands to maintain or improve their quality of life, understood very broadly as referring to an individual's general sense of everyday well-being. As such, just as quality of life involves working, living (not least family), playing (leisure) and simply surviving, Chapters 6-9 show migration and mobility expressing ways these issues are engaged with across a life course. 


\section{CHAPTER 6}

\section{From everyday to residential mobilities}

\subsection{INTRODUCTION: MOBILITIES TO MIGRATIONS}

The next four chapters provide detailed illustration of the great diversity of migrations widely undertaken across the world today. These chapters together map a path from everyday mobilities through residential mobility to much less everyday expressions of migration. Most focus is on relatively clear examples of residential relocations rather than mobilities more generally. Nonetheless, given the conceptual background introduced in Chapter 5, migrations are linked to such mobilities. The present chapter, in particular, makes such connections.

Following this introduction, the chapter has two main sections. First, Section 6.2 outlines areas of everyday mobilities and immobilities which express the priority given to key aspects of quality of life within the life course: working, living, playing, surviving. Crucially, these everyday mobilities can morph into forms of migration, reflecting the mobility continuum (Figure 5.2). Whilst some resulting migrations may be relatively long-distance, others will be more localized, expressing intra-urban migration (although it need not be urban) or residential mobility. Second, key areas of such residential mobility are presented in Section 6.3, principally expressing priorities of everyday living related to the needs of a family, for housing, and for a livable neighborhood. Rarely across Chapters 5-9, employment issues take a relative back seat. A short conclusion in Section 6.4 reiterates the importance of placing migration within the mobilities continuum.

\subsection{EVERYDAY MOBILITIES}

\subsubsection{Introducing everyday mobilities}

In line with migration scholarship generally, everyday mobilities covered in this section are all expressions of physical or embodied mobility, where people are in movement. Virtual mobilities, not least those associated with the internet, may also be implicated with these migrations, even just by informing people about potential destinations (Appadurai 1996), a perspective that can extend to linking to other people (Box 6.1). However, there is not space here to cover them. Interestingly, such virtual mobilities can also, somewhat paradoxically, work to reduce both physical mobility and migrations. For example, the rise of teleworking or telecommuting, which involves working from home with computer and other communications technologies (Jackson and van der Wielen 2002), can reduce a need either to commute or to consider more permanent migration to live nearer work. Similarly, distance-learning educational courses, such as massive open online courses (MOOCs) (Daniel 2012), can lessen students' need to attend educational institutions.

The ubiquity of everyday physical mobilities was indicated in the last chapter but their prevalence warrants reiteration. They refer to how "[m]ost people travel every day: to school, to work, for leisure, or for a multitude of other reasons" (Pooley 2013: 1; also Pooley et al. 2005). As such, they are a vital component of the "mundane mobilities" (Binnie et al. 2007) that express the "[c]ollective rhythms and routines of daily life ... made and remade everyday, in micro and detailed ways" (Southerton 2009: 62). Indication of the scale of everyday mobilities in terms of distance moved was derived from research that studied 35 million "location broadcasts" from nearly 1 million users in 34 worldwide cities of the location-based social network app Foursquare during six months of 2010 (Noulas et al. 2012). 


\section{BOX 6.1 RELATIONSHIP MOBILITIES OF INTERNET DATING}

Formation of lasting emotional-sexual relationships between two adults is often closely associated with a range of mobilities. These include travelling to meet on dates, telephoning and other communicative mobilities, and ultimately residential mobility or longer-distance migration involving one or both individuals so that co-habitation can occur. The internet is also now important here.

Institutionalized technologies have long been involved in the development of interpersonal relationships and romantic interactions. Classic examples are seen in the love letter, movie theater, dance/discotheque/nightclub, $\mathrm{bar} /$ pub and, of course, workplace. All allow potential partners to meet, interact and get to know one another. The same is now true for the internet, which started to become a key vehicle for interpersonal interaction in the 1990s, soon bringing together people who had never met. Use of information technology developed from the 1960s, when questionnaires and databases were used to match supposedly compatible individuals. This linking has now burgeoned into a huge online data industry. For example, here is the supposed top 10 internet dating sites in the UK according to http://www.top10bestdatingsites.co.uk/ in April 2014:

\begin{tabular}{|c|c|c|}
\hline Dating site & Users & Descriptions \\
\hline Zoosk & Booming UK user base & Fun and easygoing-online dating your way \\
\hline Match.com & $\begin{array}{l}\text { Over a million singles in the UK looking to go } \\
\text { on dates and start relationships }\end{array}$ & $\begin{array}{l}\text { Great fun for those ready to meet their perfect } \\
\text { match }\end{array}$ \\
\hline eHarmony & Millions of users looking for a lasting relationship & $\begin{array}{l}\text { Personalized dating experience to help compatible } \\
\text { partners find each other }\end{array}$ \\
\hline Elite Singles & $\begin{array}{l}\text { Educated singles over } 30 \text { looking for their } \\
\text { perfect match }\end{array}$ & $\begin{array}{l}\text { Perfect for those who expect more from their } \\
\text { dating site }\end{array}$ \\
\hline Lovestruck & Professionals in the city seeking romance & $\begin{array}{l}\text { For busy people looking for great dates and } \\
\text { excitement }\end{array}$ \\
\hline Christian Mingle & Lively community of devoted Christian singles & For Christians seeking singles with similar values \\
\hline Speed Dater & $\begin{array}{l}\text { Young or busy professionals, looking for real } \\
\text { connections }\end{array}$ & $\begin{array}{l}\text { A great, easy way to get out and meet like-minded } \\
\text { people without pressure of first dates }\end{array}$ \\
\hline DatingDirect.com & Seeking serious relationships & Impressive search criteria and unique features \\
\hline matchaffinity.com & Seeking long term relationships & $\begin{array}{l}\text { In-depth personality testing, combines matching } \\
\text { and searching }\end{array}$ \\
\hline Be Naughty & $\begin{array}{l}\text { Wide UK user base seeking fun, casual } \\
\text { relationships }\end{array}$ & $\begin{array}{l}\text { For fun flirting, dating and casual dating. } \\
\text { Naughty teasing optional }\end{array}$ \\
\hline
\end{tabular}

This table gives a good idea of the range of options available for the online dater.

Who, then, uses internet dating? And what are their detailed motivations? In terms of who, and mirroring everyday use of the internet generally, there remains a bias in favor of the middle classes and younger adults. In terms of motivations, themes that emerge include:

- Companionship: seeking friendship, conversation, social activity and, of course, partnership.

- Comfort: after a life crisis such as serious illness, bereavement, divorce or job loss, the internet can provide a support network.

- Presentation of a "different" self: the anonymity of the internet environment allows the user to present in a more positive or flattering light, perhaps even as pure romantic fantasy.

- $\quad$ Freedom from stereotypical expectations: allowing one's "true" self to be expressed, less encumbered by the normative expectations and commitments of everyday life. (Women online may feel more able to act assertively and men to be more open and emotionally expressive.)

- Adventure: paralleling the gamble of blind dates, online dating can be an emotional-sexual adventure, involving potential danger if physical meeting actually occurs. 


\section{FROM EVERYDAY TO RESIDENTIAL MOBILITIES}

Whilst internet mobilities are undoubtedly here to stay in terms of their use to facilitate the emergence of new partnerships, and thus of residential mobilities, they should be neither over-stated nor regarded as inherently benign. Even across the hyper-mobile Global North, most people still meet partners in "traditional" ways, notably via mutual friends. For example, a 2009 survey of 4,000 US residents found around 30 percent met online, although for homosexual couples this was much higher at 61 percent. Furthermore, there are also downsides to internet dating, revolving around issues of trust, honesty, exploitation and abuse. One must note involvement of sexual predators and maliciously motivated fantasists and self-presenters. Most extreme is internet use by pedophiles to "groom" and ultimately abuse child victims. Notwithstanding such concerns, however, Lawson and Leck (2006: 206) concluded that "need to obtain companionship motivates people to seek out romantic relationships in a variety of ways, and the Internet is merely the latest technological development used by people to assist their romantic goals. [B]enefits appear ... to outweigh the risks."

(Sources: Blue $2010^{* *} A Q^{* *}$; Couch and Liamputtong 2008; Lawson and Leck 2006)

Movements were derived from users of the app indicating presence at a place. These data demonstrated the very high relative likelihood of movements covering short distances.

From the perspective of the Global South, Rigg (2007), whilst not neglecting the ubiquity of mobilities in the past, noted how movement in general has increased rapidly in significance in recent years. This is in part linked to changing geographies of employment (Chapter 7) but also expresses how a key feature of "being modern" is being mobile (Mills 1997). Table 6.1 illustrates some aspects of the associated "mobility revolution" within the South.

Everyday mobilities form a key structuring mechanism of the life course (Edensor 2011). They both underpin daily routines and feed into the key life transitions that can transform these routines (Table 2.2), not least those associated with the core demographic triumvirate of births, deaths and, as shown here, migrations. When disrupted, perhaps due to bad weather, much of everyday life in schools or work places frequently comes to a grinding standstill. Nonetheless, as Pooley (2013) went on to note, everyday mobilities are generally taken for granted.

As with the life course overall, a key feature of everyday mobilities is their relational construction. This can be illustrated by the role of the private car within everyday transportation. Whilst across the Global North this mode of transport strongly predominates, its presence should not be assumed. For example, the concept of the "transport poor"

Table 6.1 "Mobility revolution" in the Global South

\begin{tabular}{ll}
\hline Category & Causal factor \\
\hline Resources \& environment & $\begin{array}{l}\text { Land squeeze in rural areas } \\
\text { Declining land productivity from environmental degradation } \\
\text { Growing inequalities in land access from wealth-concentrating effects of market-led growth }\end{array}$ \\
Economic & Declining terms of trade between farm and non-farm activities \\
& Declining value of agricultural commodities \\
& Rising costs of farm inputs \\
& Increasing availability of non-farm work \\
& Growing wants driven by consumerism \\
Social & Changing norms on mobility acceptability, especially for women \\
& Farming becoming low status occupation \\
Mobility as route to emancipation & Growing wants driven by consumerism \\
Political & Lifting or easing barriers to movement (selective) \\
Bechnological & Cheaper travel \\
Improved communications between places
\end{tabular}

Source: simplified from Rigg (2007: Table 6.2). 
(Wibberley 1978) draws attention both to how many people do not have access to such transport but also to how this lack is skewed statistically towards certain categories: those on low income, the elderly and youth, the disabled, and women. While private cars have also increased markedly in everyday prominence across the Global South, studies such as Howe (2001) qualify this through showing 83 percent of trips made by women in rural Tanzania were on foot. In particular, the life course arenas of class, gender, age, health and disability, geography and history are vital relational contexts shaping everyday mobilities. This will now be demonstrated further, starting with commuting mobilities.

\subsubsection{Commuting}

Chapter 5 noted how migration research is dominated strongly by economic considerations. It is therefore appropriate to begin illustrating everyday mobilities with "the act of travel from one's home to one's place of employment" (Walker 2013: 1). This is usually termed commuting (Gately 2014) (Figure 6.1).

Commuting takes diverse forms, differentiated along at least four key axes: time, distance, mode of travel, and life course arena. First, commuting varies according to average time taken. Thus, whilst walking down the street to work in a shop may hardly be defined as comprising a commute, a daily combined drive, train ride and subway journey of 2 hours, one way, certainly does! However, time is also a vital defining dimension in terms of frequency, distinguishing between commutes undertaken daily, at the start and end of a working week, and over longer periods, such as months.

Second, much of the temporal dimension of commuting is interlinked with space, in terms of commutes being defined by distances covered and geography of the population (Shen 2000; Walker 2013). The general trend has

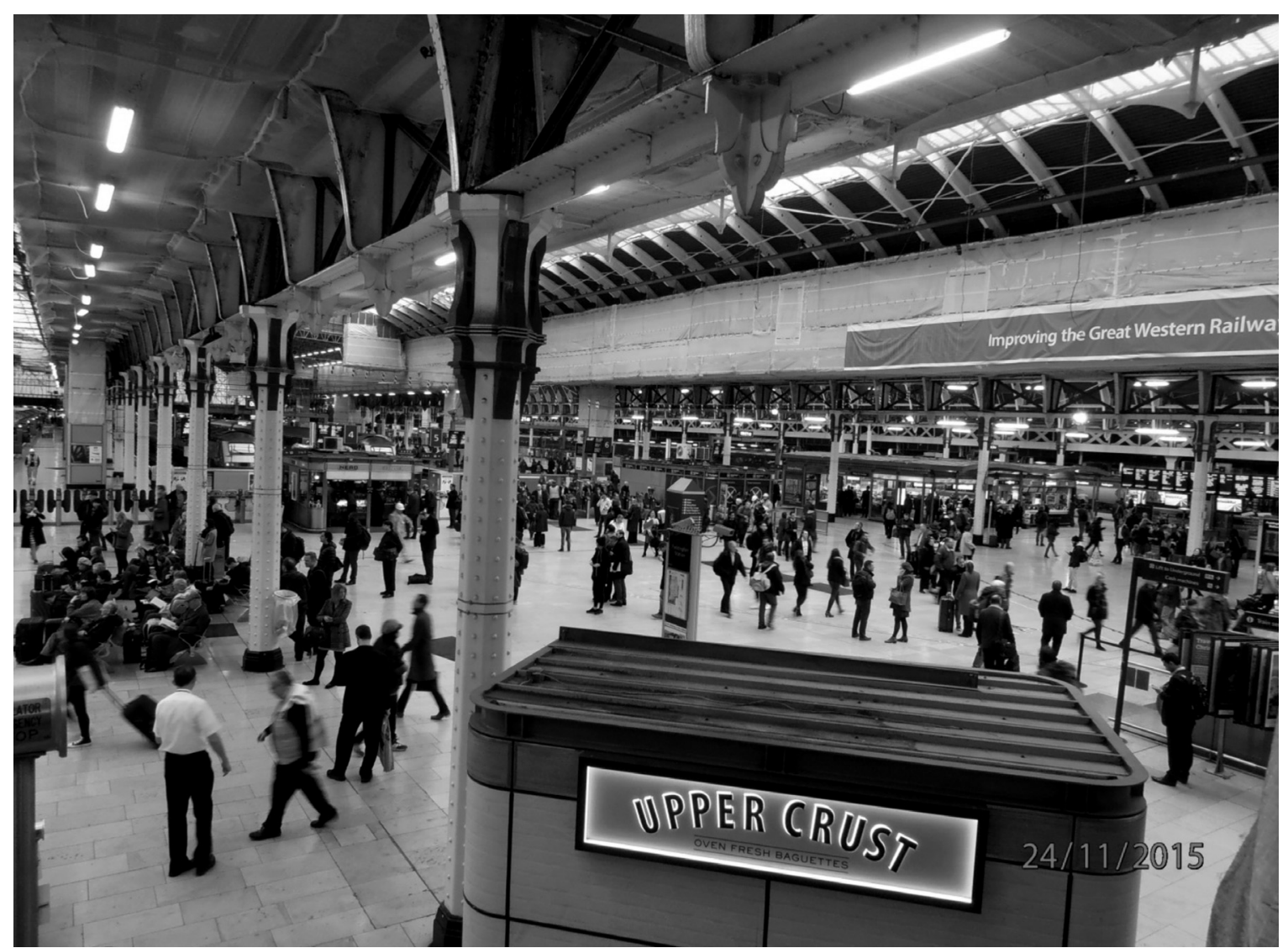

Figure 6.1 Commuters at Paddington Station, England.

Source: author, 


\section{FROM EVERYDAY TO RESIDENTIAL MOBILITIES}

been towards longer distances over time, linked strongly to suburban spread and, more recently in much of the Global North, counterurbanization (Chapter 3). Both trends also imply how jobs remain concentrated in downtown locations.

Third, time and distance of commuting are not always neatly correlated as the mode of travel and the transport infrastructure must also be considered. Commuting by foot or by bicycle, for example, expresses a very different experience than one by car, which in turn is very different from commuting by train, plane, helicopter and so on. Furthermore, it is only through considerable improvements in road and rail infrastructures, most notably, that today's long distance commutes-and the resultant geographies of population-are now practically possible (Baum-Snow 2007). Mobilities, in other words, are strongly dependent upon certain fixities (Urry 2007).

Fourth, commuting varies considerably according to relative position within the bio-social arenas of differential life course experience. Perhaps most notably, commuting is strongly gendered in favor of men. This reflects men's greater involvement with paid work but, from the perspective of accessing paid work that is undertaken, lengthy travel is also skewed in favor of men (Green and Shuttleworth 2015). Community's gendering may thus also be seen to reflect women's greater overall responsibilities for the home and child care within broadly patriarchal family environments (e.g. Hjorthol 2008; Turner and Niemeier 1997). Commuting prevalence and distance are also biased in favor of the healthy, the able-bodied, and people in higher status jobs, whilst displaying complex relationships with ethnicity and race (e.g. Shen 2000).

To illustrate something of the complex changing constitution of commuting, Table 6.2 summarizes empirically relevant changes that have taken place in Britain through the twentieth century. Overall, there was an increase in commuting. Average distances increased four-fold, a rise only partly compensated by increased speed (2.5 times increase), which led to time spent commuting increasing by half. Gender is important, as these trends apply to both men and women but are more marked for men. Table 6.2 also shows how the mode of transport used in commuting changed, from dominance by walking and buses at the start of the century to cars and trains by the end. Most notable was rising use of cars from the 1960s. Finally, although this analysis was from Britain, similar trends were broadly apparent across the Global North (e.g. Santos et al. 2011) and, less clearly, the South (e.g. Rigg 2007; Singh and Shandilya 2012).

Globally, commuting in all its various forms now comprises a commonplace and largely normative part of the everyday life course routines for those of employment age (Gately 2014; Pooley et al. 2005: Chapter 6). This status has been attained across much of the Global South as it is clearly present throughout the North. Yet, it is an experience considered very ambiguously for those involved.

On the one hand, as O'Dell (2009: 96) concluded from his study in Sweden, commuting is an activity whose necessity is generally taken for granted, even to the extent that "most people are actually fairly content with their commutes, and even ... say they would not like to live a life without any commute at all" (challenging any strong sedentarist norm; 5.2.1). One reason for apparent contentment is that time spent commuting should not be regarded

Table 6.2 The changing British commuter through the twentieth century

\begin{tabular}{|c|c|c|c|c|c|c|c|c|c|}
\hline \multirow[t]{2}{*}{ Decade } & \multicolumn{3}{|l|}{ Males } & \multicolumn{3}{|l|}{ Females } & \multicolumn{3}{|l|}{$A / l$} \\
\hline & $\begin{array}{l}\text { Distance } \\
(\mathrm{km})\end{array}$ & $\begin{array}{l}\text { Time } \\
\text { (mins) }\end{array}$ & $\begin{array}{l}\text { Speed } \\
(\mathrm{km} / \mathrm{h})\end{array}$ & $\begin{array}{l}\text { Distance } \\
(\mathrm{km})\end{array}$ & $\begin{array}{l}\text { Time } \\
\text { (mins) }\end{array}$ & $\begin{array}{l}\text { Speed } \\
(\mathrm{km} / \mathrm{h})\end{array}$ & $\begin{array}{l}\text { Distance } \\
(\mathrm{km})\end{array}$ & $\begin{array}{l}\text { Time } \\
\text { (mins) }\end{array}$ & $\begin{array}{l}\text { Speed } \\
(\mathrm{km} / \mathrm{h})\end{array}$ \\
\hline 1900-09 & 4 & 22 & 11 & 3 & 25 & 8 & 4 & 22 & 10 \\
\hline 1910-19 & 6 & 27 & 14 & 5 & 27 & 11 & 6 & 27 & 13 \\
\hline 1920-29 & 7 & 28 & 15 & 6 & 31 & 12 & 7 & 29 & 14 \\
\hline 1930-39 & 7 & 31 & 14 & 7 & 32 & 13 & 7 & 31 & 14 \\
\hline $1940-49$ & 8 & 34 & 15 & 7 & 33 & 13 & 8 & 34 & 14 \\
\hline $1950-59$ & 10 & 34 & 18 & 7 & 34 & 13 & 9 & 34 & 16 \\
\hline 1960-69 & 12 & 35 & 21 & 8 & 32 & 14 & 10 & 34 & 18 \\
\hline 1970-79 & 13 & 35 & 23 & 8 & 29 & 16 & 10 & 32 & 20 \\
\hline 1980-89 & 16 & 37 & 25 & 9 & 29 & 18 & 12 & 33 & 22 \\
\hline 1990-98 & 19 & 39 & 30 & 11 & 31 & 21 & 15 & 35 & 25 \\
\hline
\end{tabular}

Source: Simplified from Pooley et al. (2005: Tables 6.1 and 6.3). 
as "dead time" but, as emphasized within mobilities scholarship (Cresswell 2010; see Jain and Lyons 2008; Jensen 2009), filled with meaning, significance, creativity and activity (Edensor 2011).

On the other hand, however, few people "actually look forward to" (O'Dell 2009: 96) their commute and frequently bemoan the time it takes, the amount it costs, its discomfort, and so on. Thus, "Johanna" in Sweden could write in a letter to her local newspaper:

it's tragic that you have to pay 2000 Swedish Crowns every month to stand two hours a day, and you don't just stand. Usually you have some unknown person's breath working as a fan in your face, and in the morning it's not all that fresh. I promise you.

(quoted in O'Dell 2009: 89)

Still more radically, French revolutionary Raoul Vaneigem (1967/1994: 52) expressed the existential costs of the shattering daily cycle of commuting and its inextricable entanglement with the world of work thus:

What spark of humanity ... can remain alive in a being dragged out of sleep at six every morning, jolted about in suburban trains, deafened by the racket of machinery, ... spun dry by statistical controls, and tossed out at the end of the day into the entrance halls of railway stations. .

In sum, and reflecting the experiential ambiguity within mobilities of all kinds: commuting comprises "a mobile practice that offers a rich variety of pleasures and frustrations" (Edensor 2011: 189).

From experiences such as those of Johanna (also Stutzer and Frey 2008) and Vaneigem, one might expect a need to commute prompting residential migration, not least if the behavioral concept of stress is deployed (5.3.3). However, this response is not always possible. Whilst relocations do at least partly occur as a result of the stresses of commuting, a family life course perspective makes things much more complicated. For example, if children are present the family may not wish them either to leave their current school or to endure lengthy commutes themselves as a result of a change of residence. Similarly, in households with more than one adult engaged in paid work, reducing the commute for one partner through migration may increase it for another. Finally, factors such as high city center house prices can mean living outside the center and commuting may be the only way certain jobs can be undertaken; increased commuting thus becomes a consequence of residential relocation, rather than a prompter of it (e.g. Guardian 2014a). Residential migration to reduce commuting, in short, is highly constrained. Whilst the era of mobilities may suggest increased flexibility in access to jobs across a broader area, for example, this sense of "freedom" can be very misleading from the perspective of lives led within a family context. Indeed, there is a suggestion that mobilities may be being deployed to reduce migration within US society (Cooke 2011) and elsewhere.

Even with high personal mobility, the challenge to access employment without excessive commuting has increasingly been expressed through less conventional or traditional household forms. Life course experiences within such arrangements are, likewise, relatively novel. These forms include families where one adult is away from home for the working week (weekly commute), possibly residing Monday-Thursday nights in a small flat in a distant city. More extreme still are monthly or longer commutes, such as to oil or gas rigs (Collinson 1998), or the "fly-in, fly-out" lifestyle (7.4.4) associated with enormous open cast mines in countries such as Australia (Cheshire 2010). There are also a variety of dual location households (Green et al. 1999), such as "commuter marriages" (Holmes 2004; van der Klis and Mulder 2008) and "living-alone-together" arrangements (Duncan and Phillips 2011; Levin 2004), where couples do not have a single home location. The latter further challenges the normative concept of a single "permanent" home, axiomatic within migration scholarship (5.1.1).

\subsubsection{Trips to education}

Access to education, with weekday daily movement to and from school and later in life to and from college, university or other site of learning and/or training is a second key area of everyday mobilities (Pooley et al. 2005). This expression of mobility has potential employment implications but also picks up strongly on wider quality of life considerations. Whilst accessing a "good" school or university course may help a person gain suitable qualifications to go on to obtain a "good" job (Dorling 2014), it may also make the individual directly experiencing the favorable educational environment 


\section{BOX 6.2 THE YELLOW SCHOOL BUS}

A resonant symbol of the school commute, recognized iconically widely across the Global North, is the US yellow school bus. It epitomizes the US public school system's ubiquitous reach into all of the country, urban and rural. Mostly funded through local taxation, the US's largest mass transit system has grown from taking around a quarter of a million students to school daily in 1920 to around 25 million today in nearly half a million buses. The Los Angeles Unified School District alone ferried around 83,000 children in over 2,000 buses in 2005. The buses themselves, adopting National School Bus Chrome Yellow as their high visibility uniform color in 1939, now seat between 45 and 70 passengers. With well trained drivers, their safety record is exemplary. Furthermore, as early as 1972 van Til heralded the yellow school bus as blending seamlessly into a US that was already an extremely mobile society.

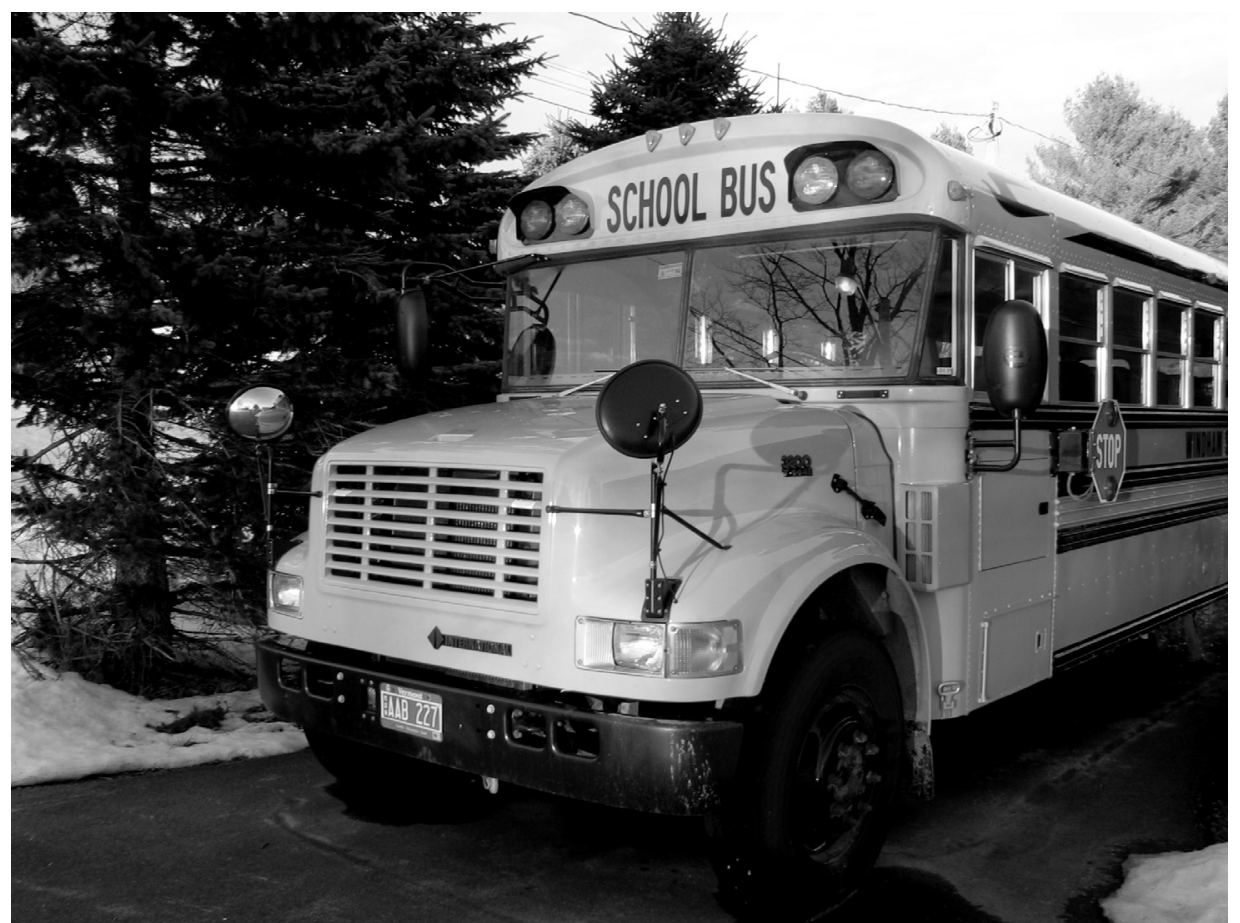

Source: https://commons.wikimedia.org/wiki/File:School_bus_zoom_in_front.jpg (accessed 09/2015)

Besides such positive resonances, however, the yellow school bus also speaks of the costs that have accompanied late-twentieth century US school mobilities. Notably, the buses played a prominent role in the push for greater racial equality and desegregation in the country. Following the 1954 Brown versus Board of Education of Topeka, Kansas Supreme Court decision, which ruled then commonplace racial segregation laws for public schools illegal, the bus switched from taking African-American children in the southern states, in particular, often long distances to poorly funded and equipped "Black" schools to mobilizing their integration within better provisioned and often closer "White" schools. Indeed, the term busing soon became shorthand for these moves towards greater equalization of educational experience by race across the US. However, whilst the educational and equality benefits of busing could be considerable, the experience for the transported children could initially be very frightening as they often experienced racist resistance and threat. This was not just in the south but anywhere busing was initiated. Everyday mobilities can be extremely political.

(Sources: Humphreys 2005; Irons et al. 2014; van Til 1972) 
(Box 6.2), or even the family as a whole, feel they are accessing a "good" life. However, time and cost issues are again of vital consideration. For example, excessive travel to education may prove not only monetarily demanding but also stressful, often also impacting negatively on a young person's home life.

Within studies of travel to school across the Global North, a key focus has been the potential health benefits of active travel to school (ATS), such as walking, cycling and skateboarding (Lubans et al. 2011). This reflects general concern within these same countries about children becoming increasingly spatially constrained within the home (Burdette and Whitaker 2005). Local neighborhoods, such as nearby streets and parks, have often become perceived as supposedly increasingly dangerous for young people: "stranger danger" (Valentine 2001), traffic, gangs and other anti-social presences. This has reduced children's engagement with fresh air, nature and exercise, leading to reduced fitness and consequent actual or potential health problems. Promoting ATS represents at least a partial response to such concerns as it involves significant physical activity-reflected, for example, in higher pedestrian step counts (Murtagh and Murphy 2011)-compared to more passive travel modes, such as being driven by car or taking the bus. Research has suggested positive associations between ATS and better health (Davison et al. 2008; Lubans et al. 2011). Again touching on broader quality of life, the more independent travel implied by ATS may also not only facilitate the life transition to independent mobility (Table 2.2) but promote building of links with other people, places and the natural environment (Ross 2007). The latter are all elements within a life course's "free range" (Box 6.3). Nevertheless, ability to promote such practices depends both on assuring parents of child safety and creating child-friendly routes (e.g. with safe road crossings) (Dalton et al. 2011; Kerr et al. 2006; Timperio et al. 2006).

\subsubsection{Accessing relations, friends and leisure}

This section focuses still more strongly on the quality of life emphasis behind everyday mobility, seen not just as narrowly functional but expressive of broader lifestyle choices (Pooley et al. 2005). Implicit within commuting or travelling to school, quality of life becomes increasingly explicit within mobility that seeks to access relations, friends and leisure activities. First, there is "social" mobility undertaken for the purpose of accessing friends and relations. Whilst strong inter-personal relations, exemplified by marriage, usually-although not always (Coulter et al. 2016; Green et al. 1999)-involve at least one partner migrating, converging life courses spatially (Mulder 2013), being with other relatives or friends typically involves more temporary mobilities. Second, whether with such friends, family or individually, accessing leisure activities in free time also often involves frequent mobility decisions, constraints and choices. All these expressions of mobility are important for promoting social inclusion and resisting social exclusion (Cass et al. 2005).

Any consideration of the mobilities involved in visiting relatives or friends returns to the tendency identified in Chapter 5 of being too eager to distinguish internal from international movements. Much of this kind of mobilityknown as "visiting friends and relatives travel" (VFR) (Moscardo et al. 2000)-involves long-distance movement that may cross international borders. Indeed, the latter is very important within tourism generally (Moscardo et al. 2000). It is demonstrated by Figure 6.2's typology of VFR, which expands the usual definition to include the numerous visits that do not require overnight accommodation and also transcends the domestic (internal)/international divide.

Within the tourism literature, Larsen et al. (2007) argued for the importance of recognizing visits to family and friends, which have been sharply increasing. Although they focused on international flows, their argument is equally valid for everyday visits. All such forms of tourism should not be seen as "marginal" but as highly "necessary to much social life conducted at a distance... [since] tourism often involves connections with ... social relations and the multiple obligations of everyday social life" (Larsen et al. 2007: 245). Larsen and colleagues went on to argue how such obligations "involve very strong normative expectations of presence and attention" and become some of "the top necessities of life" (pp.247-248). Moreover, whilst the era of mobilities' great leap in transportation might facilitate such travel, paradoxically it can make it more challenging. This is because mobilities both enabled wider geographical spread of family and friends (Box 5.1) but also encourage an encultured expectation of visits taking place. As a young male Briton put it:

[Travel] is essential. I don't think we could go on just by making emails and phone calls. It is very necessary for us to go and see friends and family. ... I think it would be emotionally bad for us if we didn't. We need to travel.

(Larsen et al. 2007: 258, emphasis added) 


\section{BOX 6.3 CAMPAIGNING FOR CHILDREN'S FREE-RANGE}

Free-range refers to the "places ... children [are] able to go without permission" (Matthews 1987: 46). A particular young person's free-range will be the product of numerous factors, including "caretaking conventions, age-related capabilities and gender-related expectations, all set within a context of social, cultural and environmental contingencies" (Matthews and Limb 1999: 71). Thus, a study of free-range amongst Paris households revealed it to reflect internal family dynamics and parental experiences (den Besten 2011). It is in part a product of interactions between a young person and her or his carer(s), involving the "negotiation and performance of trustworthiness" (Skelton and Hamed 2011: 217). The idea of children's free-range can also assume political and campaigning momentum.

A good example comes from the UK, where the charity Sustrans (Sustainable Transport) promotes travelling by foot, bike or public transport for everyday journeys, including attending school. Sustrans is concerned about declining levels of walking and cycling to school and elsewhere. They want to make it easier for young people to be active outside. In 2011 they launched the Free Range Kids campaign. This was a response to rising speeds and volumes of traffic on the streets linked to children staying indoors more and being ferried around largely by car when venturing out. According to Sustrans, parents' everyday fear of traffic dangers was around twice as great as concerns about risks from strangers in public places. The campaign's aim is to produce Free Range Kids-young people who "experience freedom from their front door" by having the confidence, opportunities and safety to "travel independently, play outdoors and explore their local community." Building on previous campaigns such as Safe Routes to School, Free Range Kids promotes families walking and cycling, facilitating safe street play, carrying very young children by bike, making journeys to school healthier, using the outdoors as a classroom, and lobbying local and national politicians. It also has competitions for children, such as designing a "Dream Street", and a blog covering a wide range of free range issues.

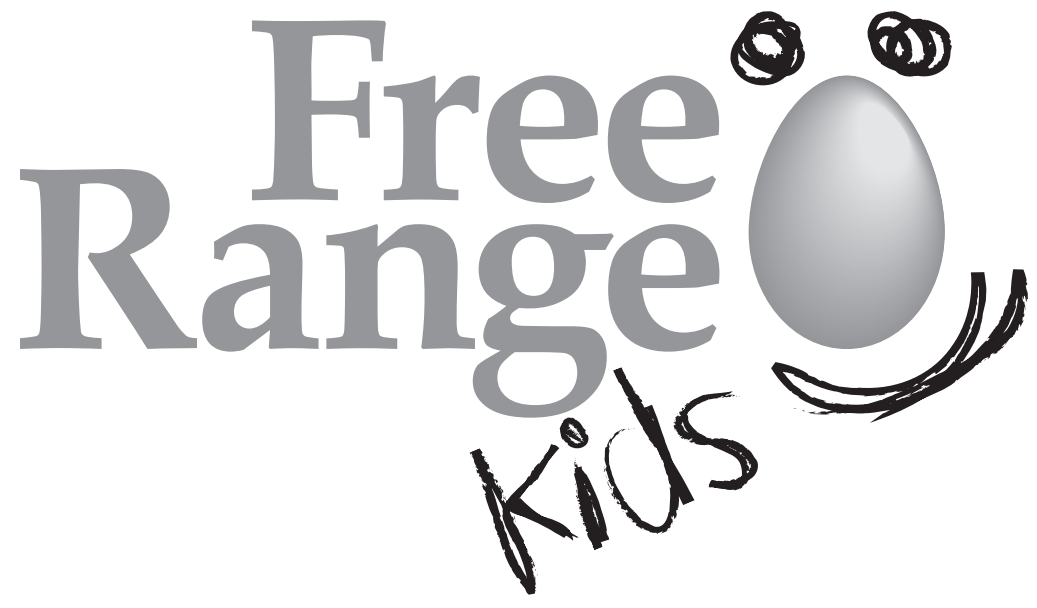

Source: http://blogs.educationscotland.gov.uk/globalcitizenship/files/2011/08/freeer.png, (accessed 09/2015); http://www.sustrans.org.uk/ (accessed 09/2014)

Studies of children's journeys to school in the Global South often focus on health and safety concerns in a more acute way. Mode of transport is more strongly dominated by walking, especially for lower income groups (Gough 2008; Porter et al. 2010). For example, in her study of the poor Kalingalinga area of Lusaka, Zambia, Gough (2008: 247) found walking commutes of up to $6 \mathrm{~km}$. Young people:

left early in the morning, often in the company of friends, and although they sometimes tried to hitch a life they usually made the journey on foot. Few could afford the bus fare and many reported leaving home on an empty stomach.... The pupils made the return journey home also on foot.... [Yet, d]espite the long 
distances, for many of the young people the journey to and from school was a period of freedom from adult control during which social relations with peers were strengthened.

Despite such benefits, risks abounded: dangerous and aggressive drivers, uncontrolled dogs, snakes and attacks by other humans (Porter et al. 2010). The latter risk is especially marked for girls, who also have to contend with "moral" risks, represented by general patriarchal linking of female mobility with sexual impropriety and promiscuity (Porter 2011).

Recognizing the importance of traveling to school can be extended to noting the migratory behavior of university students and other usually younger adults in training. For example, whilst many students commute on a daily basis to university from the parental or their own home, others move to accommodation provided by the university itself-halls of residence-or private landlords near the university for the university term or academic year. Are students migrants, or a form of commuter? In terms of how migration is usually defined (5.1.1), their moves are not "permanent," nor may they see themselves as changing their "usual address." These moves are returned to in Chapter 7.

\begin{tabular}{|c|c|c|c|}
\hline Sector & Scale & Effort & Accommodation \\
\hline \multirow{5}{*}{$\begin{array}{l}\text { Visiting friends } \\
\text { and relatives as } \\
\text { motive }\end{array}$} & Local & Short haul & $\begin{array}{c}\text { None } \\
\text { Friends/relatives } \\
\text { Commercial (e.g. hotel) }\end{array}$ \\
\hline & \multirow[t]{2}{*}{ National } & Short haul & $\begin{array}{c}\text { None } \\
\text { Friends/relatives } \\
\text { Commercial }\end{array}$ \\
\hline & & Long haul & $\begin{array}{l}\text { Friends/relatives } \\
\text { Commercial }\end{array}$ \\
\hline & \multirow[t]{2}{*}{ International } & Short haul & $\begin{array}{c}\text { None } \\
\text { Friends/relatives } \\
\text { Commercial }\end{array}$ \\
\hline & & Long haul & $\begin{array}{l}\text { Friends/relatives } \\
\text { Commercial }\end{array}$ \\
\hline
\end{tabular}

Figure 6.2 A typology of visiting friends and relatives travel.

Source: developed from Moscardo et al. (2000: Figure 1).

Within the Global South, furthermore, in spite of much rural-to-urban population redistribution (3.3.2), for example, sustained significance of "the natal bond that links the migrant and his or her [extended] household" (Rigg 2007: 142) promotes visits to family, emphasizing origin and destination of migration as linked. Prevention of such visits, not least due to poverty, can be a major cause of stress (Gough 2008).

Similar issues regarding the importance of everyday mobilities to engage family and friends can be made about those in pursuit of leisure. Indeed, many of these activities combine family/friends and leisure (Schlich et al. 2004). Leisure is arguably as central to everyday life and the life course worldwide (Williams et al. 2009) as social linkages. As with the latter, whilst it can be pursued at home it frequently involves often not inconsiderable travel. From going for a country walk, to leisure shopping, to eating out in restaurants, to spectating at sports events, to just "hanging out," mobility's presence is ubiquitous. Table 6.3 demonstrates this, giving mean distances, duration and frequency of trips, disaggregated by gender, from a 6-week travel diary supplied by 361 urban German residents in 1999 (Schlich et al. 2004). 52,000 trips for all purposes were recorded, covering 14,500 person days. The table demonstrates the importance of leisure trips but also reveals negligible differences between men and women. Finally, in terms of the mode of transport used to access leisure, an urban study from Switzerland that deployed multivariate cluster analysis of survey results identified four "leisure mobility styles": Sporty, showing affinity with bicycles; Fun and Distraction Seekers, linked to cars; Culture-oriented, multi-modal but critical of cars; and Neighborly home-lovers, associated with cars and public transport (Ohnmacht et al. 2009). 
134 FROM EVERYDAY TO RESIDENTIAL MOBILITIES

Table 6.3 Six weeks' trips in two German cities

\begin{tabular}{lcl}
\hline & Females & Males \\
\hline Mean Distance $(\mathrm{km})$ & & \\
All trips & 8 & 11 \\
Leisure trips & 13 & 14 \\
Mean Duration (mins) & & \\
All trips & 21 & 22 \\
Leisure trips & 27 & 27 \\
Mean Person Trips per Day & & \\
All trips & 3.9 & 3.9 \\
Leisure trips & 0.7 & 0.7 \\
Working trips & 0.6 & 0.7 \\
Household-related trips & 1.0 & 0.8 \\
Back home & 1.7 & 1.6 \\
\hline
\end{tabular}

Source: simplified from Schlich et al. (2004: Table 2).

\subsubsection{Immobility due to fear}

Talk of mobility and of an era of mobilities should not overlook the equal ubiquity of immobility. This may be as much a practice as mobility (Coulter et al. 2016). On the one hand, immobility can be chosen-opting to be still in a mobile world (Bissell and Fuller 2011), such as going on retreat (Conradson 2011) or engaging in quiet contemplation (Maitland 2009). On the other hand, immobility can more or less forcefully be imposed. This may be through lack of resources-notably money and/or time-a relational perspective that qualifies the argument for an era of mobilities from the perspective of the Global South, for example (e.g. Gough 2008; Rigg 2007). Forced immobility can also originate from fear (Pain 2000; Pain and Smith 2008) of the consequences of becoming mobile. For example, there may be fear of "hate crime" (Hall 2013), a crime partly underpinned by prejudice towards a social group. The latter is the focus of this section. It is not only spatially and temporally specific but also defined relationally through key social life course arenas. Discrimination and prejudice, in short, can be major causes of unwanted immobility.

Both direct experience and fear of violence may be related to sexuality (Pain 2000) and this can feed into constraining mobility and promoting immobility. On the one hand, victim surveys "consistently show that the wide spectrum of homophobic violence, from physical assault to harassment and verbal abuse, is an everyday experience for lesbians and gay men" (Moran et al. 2003: 175). On the other hand, fear expresses such violence, being associated with not only homophobic people but also particular places and times (Corteen 2002; Moran et al. 2003). In terms of geography, relatively LGBT-friendly neighborhoods, such as the Castro District of San Franciso or Kemptown in Brighton, England, can provide a degree of security (Storey 2012). Nonetheless, as Storey went on to note, being in such locations can also present targets for homophobic attack. A temporal dimension must also be recognized, with night times and weekends usually perceived as most dangerous, not least due to groups of drunk young men on the streets. As Norman, a gay businessperson in Manchester's Gay Village in England sharply put it:

you only need a couple of straight lads to come down here and have a good time and they start fetching their friends; it's when you get gangs of twelve or fifteen leaving pubs in Salford and it's like, 'Let's go down the Gay Village and kick a fucking queer's head in.' It happens, believe me.

(quoted in Moran et al. 2003: 181).

Reflecting these threats, therefore, gay people might understandably choose to remain in "safe" locations or regulate behavior, including mobilities, very carefully. All expresses (relative) immobility.

As this section was being written, a still more acute case of homophobia-induced likely immobility was in the news. This was from Uganda, outlined in Table 6.4, whereby a law had been proposed to criminalize severely "homosexual acts." Uganda was not alone. Africa, in particular (Harris 2013), had seen a growth in homophobic expressions and legislation associated with a confluence of bitter political power struggles and Christian 
Table 6.4 Intensifying homophobia in Africa: the case of Uganda

Ugandan President Yoweri Museveni signs anti-gay bill

Uganda's leader has signed into law a bill toughening legal penalties for gay people:

- Includes life sentences for gay sex and same-sex marriage

- Museveni had previously agreed to hold it back, pending US scientific advice

- Homosexual acts already illegal in Uganda

- Law allows life imprisonment as penalty for acts of "aggravated homosexuality"

- Criminalizes "promotion" of homosexuality," where activists encourage others to come out

- Lesbians covered for the first time

- Bill originally proposed death penalty for some homosexual acts but removed amid international criticism

- A gay rights Ugandan activist said he was "very scared" about the new bill: "I didn't even go to work today. I'm locked up in the house and I don't know what's going to happen now"

- US President Obama described it as "more than an affront, and a danger to, Uganda's gay community. It will be a step backwards for all Ugandans."

Source : edited from http://www.bbc.co.uk/news/world-africa-26320102 (accessed February 2014).

fundamentalism (Sharlet 2010). This serves further to immobilize LGBT populations; permanently as in the case of Ugandan gay rights activist David Kato, "bludgeoned to death by hammer in broad daylight on his own front porch" (Harris 2013: np).

Immobility due to threat and fear, absolute or more relative, is also constituted through other relational arenas of society. One of the most widely noted links is to gender. On the one hand, as already noted, patriarchal association of female mobility with sexual impropriety and promiscuity makes mobility a "moral" risk (Porter 2011), prompting gossip or worse (Williams et al. 2009). On the other hand, women's fear of physical assault, including sexual attack, often constrains mobility (Meyer and Post 2006; Pain 2000; Valentine 1989). Recognizing both the presence and consequences of such fear should not reinforce any spurious "public-private divide" (Whitzman 2007), as gendered violence is still more commonplace within the home (Pain 1991, 1997). Nonetheless, shocking evidence of the dangers facing women who venture into the "public" realm comes from India. This rapidly mobilizing country has featured frequently on global news bulletins in recent years following a number of gang rapes of women (Martinson 2014). This has seemingly confirmed Delhi's "dubious distinction of being one of most unsafe cities in the world for women" (Viswanath and Mehrotra 2007: 1542).

Absolute and relative immobility across space can additionally be associated with race, age, religion and other relational social arenas. Racial or noted ethnic differences, strongly underpinned by racism, for example, can lead to "no go areas" and lack of mobility between ethnically-badged neighborhoods within cities (Van Kempen and Şule Özüekren 1998; Webster 2003). With age, although evidence is more ambiguous (Pain 2000), greater fear of crime by the elderly can, once again, manifest in fear of going out, especially at night (e.g. Meyer and Post 2006). Overall, immobility is clearly as much relationally constructed as mobility. It is also intersectional (2.4). This is exemplified by the practice of purdah, associated with certain forms of Islam and Hinduism (Williams et al. 2009), whereby women of reproductive age may only move around outside their house when accompanied by a male relative (Bose 2007). Besides the intersection of religion and gender, this restrictive practice has a class dimension, being often only practically possible for wealthier families, where adult males earn sufficient income for women to stay at home (Williams et al. 2009).

\subsection{RESIDENTIAL MOBILITY}

\subsubsection{Introduction: Rossi's legacy}

Everyday mobility, even within the socially selective but heightened motility-ability to move (5.4.1)-implied by any era of mobilities, may be inadequate to access work, education, friends and relatives, or leisure activities. And immobility due to fear may reach intolerable levels-although it is hard to think of an "acceptable" level. And life course changes or transitions occur for the individual or his/her family that make the present accommodation or neighborhood no 


\section{FROM EVERYDAY TO RESIDENTIAL MOBILITIES}

longer adequate for needs or aspirations. In all such cases, "stress" or "shocks" (Rabe and Taylor 2010) cross a critical threshold, the current residential location becomes unsatisfactory and residential relocation becomes increasingly desired (5.3.3).

Whilst migration is often imagined as comprising residential relocations whereby all or most of one's everyday spatial routines and social interactions are substantially reconstituted (e.g. Bartram et al. 2014; Samers 2010), a change of "usual address" can be much more modest, both spatially and experientially. Such small-scale relocations are termed residential mobilities. They "are in general short-distance moves which do not disrupt general patterns of living and are, with few exceptions, within a specific labor and housing market" (Clark and Huang 2003: 323). Although comprising the majority of migrations across the globe, such relocations remain the "poor relation" within migration scholarship (Coulter et al. 2016), which tends to focus on longer-distance moves. But when residential mobilities are the focus, family or household life course considerations-linked lives-immediately spring to the fore.

The pioneering study that placed residential mobility squarely on the migration map, linking it to what was then termed life-cycle stage, was Peter Rossi's (1955) Why Families Move (Boyle et al. 1998: 105-106). This was a US study of four census tracts in Philadelphia, stratified according to socio-economic status and mobility level. Rossi undertook around 200 interviews in each tract, collecting information on frequency and motivations of residential moves. $\mathrm{He}$ argued that such mobility was underpinned by five themes: creation of new households, circulation of existing households, mortality, household dissolution, and work. All five are clearly linked to life course concerns and transitions. However, Rossi downplayed moves stimulated by work, divorce and death of a household member compared to those of families wanting different housing. He summarized his position:

the major function of mobility [is found] to be the process by which families adjust their housing to the housing needs that are generated by the shifts in family composition that accompany life cycle changes.

(Rossi 1955: 9)

Focusing on these moves to adjust housing, Rossi emphasized how amount of space available in existing accommodation was especially important in encouraging household relocation. Space issues were also the most frequently cited characteristics determining choice of destination property. Nonetheless, Rossi also observed how low or declining social status of a neighborhood could be an important additional motive for leaving an area. In part, this reflected parental concern to ensure children were socialized within a preferable social milieu and would go on to interact with potentially "suitable" marital partners. Arrival and perceived needs of children were, therefore, powerful determinants of residential mobility.

Rossi's pioneering ideas went on to underpin much subsequent research on residential mobility. Two overall findings stand out (Boyle et al. 1998). First, there is a need to acknowledge a strong empirical link between residential mobility and life course changes related to the family (Clark and Dieleman 1996; Mulder 2007) but qualified relationally by the economic, demographic, social and political conditions of a particular time and place (Warnes 1992: 177-178). Residential mobility is, once again, highly relational (Coulter et al. 2016). Second, whilst residential mobility can be motivated-"forced"-by immediate experiences of employment change, bereavement, domestic violence, hate crime, eviction and so on, more "voluntary" desires for greater or lesser residential characteristics numerically predominate. This particularly marks the cultural relationality of such mobility, reflecting norms, aspirations, inter-subjective engagements, and so on.

Bringing both emphases together, subsequent sub-sections focus on key prompts of residential mobility. It should be emphasized how there is no clear divide between such mobilities and more comprehensive socio-spatial routinedisrupting migrations (Chapters 7-9). In both, biographical life course experiences underpin but rarely definitively determine the relocation. For residential mobility, such experiences are expressed predominantly through the three overlapping areas shown in Figure 6.3: family, housing, neighborhood. Put differently, Rabe and Taylor (2010: 533) presented "maximum level of satisfaction that can be obtained from housing consumption" $\left(\mathrm{h}^{*}\right)$ as:

$$
h^{*}=h\left(D_{t} H_{t} N_{t} u_{t}\right)
$$

Loosely interpreted, in this equation housing satisfaction $(h)$, has elements of demographic $(D)$, housing $(H)$ and neighborhood $(N)$ expression, plus more subjective tastes $(u)$, all specified at time $t$.

\section{ST PROOFS: NOT FOR DISTRIBUTION}




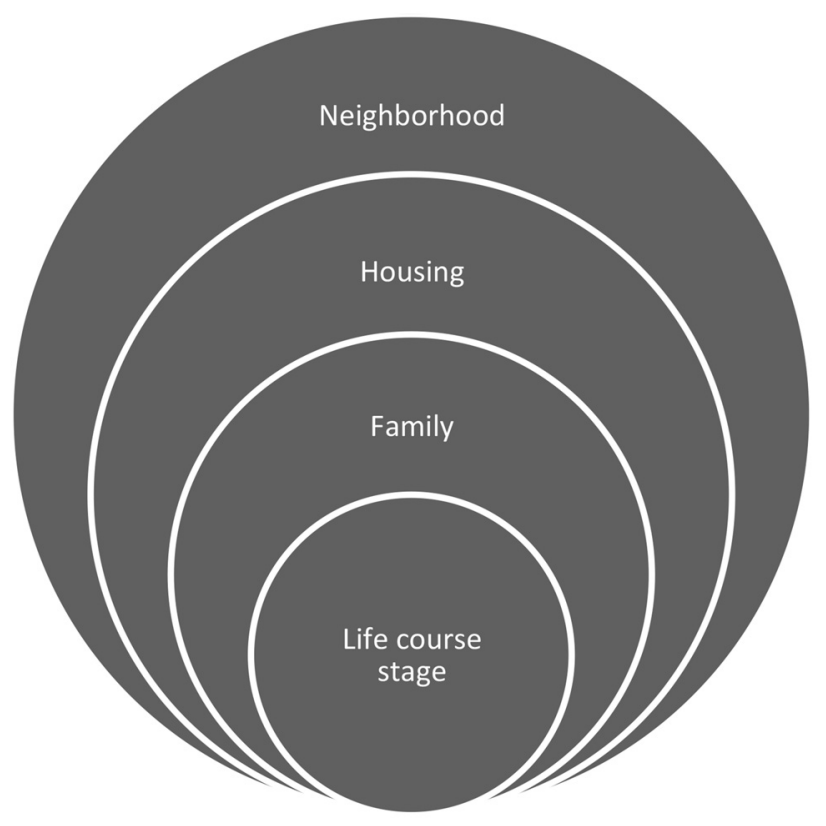

Figure 6.3 Overlapping emphases within residential mobility explanation.

Notably absent from Figure 6.3 and under-stated within Rabe and Taylor's equation are employment issues. Whilst economic matters, notably (family) income, underpin most strongly all the residential mobilities covered below (e.g. determining what type of housing is affordable), relocation for the purposes of employment generally tends towards "migration." This is Chapter 7's focus. An exception is residential relocation into housing supplied with a job, known as tied accommodation, but such opportunities are relatively rare and can also involve longer-distance movement (7.5.3).

\subsubsection{Family matters}

Residential mobility can be presented, first, as comprising a "family matter," as the structure and content of the family or household unit is frequently at its center. It is associated with family formation, expansion, evolution, contraction and break-up. A further sense of this comes from now well-noted links between stages and especially transitions in the life course and migration generally (5.6.3.1; Table 5.7). This, in turn, is noted by age being the most consistent factor associated with likelihood of moving (Long 1988; Nam 1994; Shryock 1964). For example, Long (1988) illustrated the age-mobility profile for four time periods between 1935 and 1980 with US interstate mobility rates. For all periods, the 20 to 29 year age group had the highest rate of mobility, rates declining as age increased. Hansen (1995) documented this young adult group as having a 29 percent mobility rate in 1993-1994. This compared to 17 percent for the US population (US Bureau of the Census 2000).

Focusing on an individual's life course, infancy is a time with high propensity for residential mobility (Kulu 2008). For example, around 1 million children migrate within Britain each year and most are local residential mobilities (Dobson and Stillwell 2000). Such moves predominantly express "need for additional space or the desire to live in a more pleasant environment to raise the children" (Kulu and Milewski 2007: 572). Whilst the former may be urgent-for example, if there is no bedroom for a new baby-the latter expresses the more voluntary or aspirational flavor of much residential mobility. An interesting further issue is the exact timing of the relocation and consequent possible causal linkages. Does relocation occur before the decision to have a child, after the decision-perhaps after the child has arrived-or do decision and movement occur more-or-less together? Evidence varies (Kulu and Steele 2013): some studies emphasized mobility triggered by childbirth (e.g. Clark and Davies Withers 2009); others suggested mobility triggering childbirth or, more likely, enabling a desire for a baby to be realized (e.g. Michielin and Mulder 2008; Mulder and Wagner 2001). Within all of this, housing qualities assume central roles, developed in the next section.

\section{ST PROOFS: NOT FOR DISTRIBUTION}




\section{FROM EVERYDAY TO RESIDENTIAL MOBILITIES}

In contrast to infancy, when a child gets older and established at school, residential mobility is reduced. The disruptive effect of changing school is frequently seen as too high a cost (Kulu and Milewski 2007). Where mobility does occur, it is likely to be associated with the same concerns of space and perceived neighborhood quality. Desire for access to a "good" school, for example, is especially important in the UK (e.g. Smith and Higley 2012; Smith and Jons 2015) and will be seen to exemplify residential mobility as a neighborhood matter in 6.3.4.

A third key life course stage, early adulthood, is associated both with migration, not least linked with accessing higher education and employment (Chapter 7), and residential mobility. Both kinds of mobility are expressed differently according to position within the family. For the young person attaining adulthood, space concerns and neighborhood quality issues that probably lay behind previous family-based residential mobility become supplanted by their own demands. These include desire for "independence" from the parental home and expressing emotional and sexual relationships through co-habitation, often also associated with marriage or other civil union (Kulu 2008). Indeed, both housing and environmental quality often decline following relocation (Rabe and Taylor 2010). From the parental perspective, in contrast, when a former child dependent leaves home, residential mobility may express the household's reduced space needs. Such moves to smaller properties are termed downsizing.

Fourth, in older age space re-asserts itself as an issue underpinning residential mobility. Besides being associated with the aforementioned children vacating the "family nest," mobility is common after bereavement of a family member (Bradsher et al. 1992). In a French study, Bonnet et al. (2010) attributed recent widows' residential mobility to their need to adjust housing consumption to available resources and to new or anticipated future needs, not least linked to health. Mobility can thus become a strategy to obtain some form of social support (Coulter et al. 2016). This can, of course, again be expressed through longer distance relocations, perhaps moving closer to family or returning to one's birthplace (8.4). Infirmities associated with old age frequently prompt more or less voluntary relocation, such as to live with offspring or move into care homes or other monitored or wardened accommodation (Rees and Hardill 2015). Using data from eleven European countries, Angelini and Laferrère (2012) distinguished elderly moves between private dwellings from those to a nursing home. Regression analysis revealed the former driven mainly by quality and cost issues, whereas institutionalization was determined by old age, poor health and absence of close family nearby.

Fifth, family dissolution is not just brought about by death. Relationship breakdown-perhaps somewhat paradoxically stimulated by the stresses of (frequent) migration (Boyle et al. 2008)-is, in fact, much more strongly associated with both residential mobility and migration than bereavement (Speare and Goldscheider 1987). One partner typically leaves the former family home, perhaps to establish a new family. More acutely, they may be fleeing domestic violence (e.g. Bowstead 2015; Menjívar 2013; Warrington 2001). Resulting moves again range from substantial geographical relocations to more localized residential mobility. To get further insight into this spatial breakdown, Table 6.5 presents regression modeling results of a complex study of survey data from the Netherlands (Feijten and van Ham 2007). The study had already shown that, compared to couples in a first relationship, separated people and people in a new relationship were more likely to have moved (also Rabe and Taylor 2010). Table 6.5 further reveals that "living arrangement has a considerable effect on the estimated moving distance" (Feijten and van Ham 2007: 639), with separation associated with relative short-distance moves. Most clearly, separated men with children moved shortest

Table 6.5 Distance moved by living arrangement and status for Dutch movers

\begin{tabular}{llc}
\hline Variable & $\begin{array}{l}\text { OLS regression } \\
\text { parameter estimate }\end{array}$ & Standard error $^{*}$ \\
\hline Single separated, with child(ren), male & -16.2 & 2.6 \\
Single separated, no children, female & -11.7 & 3.0 \\
New relationship, no children, male & -9.6 & 3.4 \\
Single separated, no children, male & -8.7 & 3.6 \\
Steady single, female & -6.7 & -4.5 \\
First relationship, no children, male & 1.9 & 5.59 \\
Single separated, with child(ren), female & -3. & 1.7 \\
First relationship, no children, female & -3.6 & \\
N=8399 & & \\
*: all significant at 1\% except \# significant at 5\% & & \\
\hline
\end{tabular}

Source: simplified from Feijten and van Ham (2007: Table 3). 
distances, linked by the authors to the men's strong ties to their previous place of residence, not least because children remain there in the custody of former partners. Nonetheless, single separated men also tended to move locally. For women, in contrast, shorter moves were most strongly associated with being separated but with no children. Residential mobility as opposed to longer migration was also positively linked with males in new and first relationships with no children, and with single females and those in a first relationship but with no children.

Expressing both how a life course perspective does not rigidly associate demographic changes with an irreversible and singular sense of time as flow (2.3.2.1) and how the era of mobilities has enhanced the kinds of movement encountered as well as their frequencies, recent years have seen more novel residential mobilities come to the fore. Coulter et al. (2016) recognized two families. Both again blur the distinction between migration and mobilities and indicate secular changes in the prevalent patterns of linked lives central to shaping individual life courses. First, residential itinerancy expresses "frequent shifts between multiple maintained residences" (p. 8). Examples include children moving, perhaps within the week, between homes of separated or divorced parents (Kelly 2007) and the commuter marriages and living-alone-together arrangements noted in Section 6.2.2. Second, residential transience (p. 9) expresses movement in much less structured ways between residences, highly prompted by fluctuations in monetary resources. Especially notable amongst young adults, the classic case is ex-students moving in and out of the parental home after graduation (Sage et al. 2013).

The extent to which residential mobility is the family matter suggested in this section is, of course, highly variable. Two particular qualifications are merited. First, ability to undertake any form of mobility is typically linked strongly to income and thus to characteristics such as social class. This is certainly true for residential mobility. However, as will be shown in all the migration chapters, it is not simply the case that high or increased income facilitates and promotes residential mobility. Instead, the reverse can be the case, as seen through housing tenure's association with mobility in the next section. Nonetheless, for more "voluntary" expressions of family-focused residential mobility, income clearly oils its wheels. Second, culture is also very important in linking (or not) life course transitions to residential mobility. Both between the Global North and the South but also across continents and even within countries, traditions or cultures (5.5) of residential mobility vary. As one example, European seniors' relocation to nursing homes was noted by Angelini and Laferrère (2012) as almost absent in Italy and Greece. These countries have "few such institutions and the elderly tend to stay at home or with their children" (p. 560). The life course, in short, does not provide any simple template for residential mobility behavior (Tyrrell and Kraftl 2015).

Finally, this section has shown that whilst residential mobility may be associated with the changing demands of the family within the life course-branding it a family matter-it is also linked with issues around space and place that merit attention in their own right. The former provides one core element of residential mobility seen as a housing matter, discussed next, whilst both experienced and imagined place characteristics position residential mobility as a neighborhood matter, covered in 6.3.4.

\subsubsection{Housing matters}

As noted, much residential mobility linked to the family is also associated with a range of housing issues, especially space demands (Clark and Huang 2003). Here, "space" is not restricted to the internal property dimensions (number of bedrooms and so on) but can encompass the outside too. For example, there might be desire to have a garden for growing vegetables, children's play or general relaxation. Indeed, obtaining private external space has long been regarded as one of the attractions of the suburbs (3.3.3).

Reiterating from a housing emphasis the implications of Section 6.3.2, as an individual or family progresses through their life course, housing needs and aspirations change considerably. Jones (1987) summarized this in a six stage life course typology:

- Pre-child-cheap, central city apartment;

- Child-bearing-rented single family dwelling close to apartment zone;

- Child-rearing-owned single family suburban home;

- Child-launching-owned single family suburban home;

- Post-child-smaller, high quality home;

- Later-life-institutional apartment or live with children. 


\section{FROM EVERYDAY TO RESIDENTIAL MOBILITIES}

Each stage can be revisited within a specific life course, as a result of a entering a new relationship for example. Similarly, Michelson (1977) found that as size of household increases, the likelihood of living in a house rather than an apartment and the location of the dwelling (downtown or suburban) also changes. For both these outlines, whilst residence-type might in part be understood as a proxy for size or space issues, this clearly does not present the full picture. Place or location (central city, suburb) also comes into it, as does tenure (below). These aspects may themselves be further entangled with the residential satisfaction that particular housing experiences provide. Residential mobility for housing matters, in short, is about much more than accessing suitably sized "boxes for living."

Housing tenure refers to the financial arrangement through which the right to live in a house or apartment is gained. It has three main forms: owned outright or in the process of being purchased though a mortgage loan (owner-occupied); rented from a private owner or landlord (private-rented); or rented from the state or a non-profit body (social housing). Each tenure's relative importance geographically varies considerably. This is illustrated in Figure 6.4 for Europe. At one extreme, almost all Romania's population live in houses already paid for, whilst, at the other extreme, such housing is very rare in Switzerland where more than half of housing is rented. Such tenure structures have considerable implications for migration behaviors, demonstrated both here and in Chapter 7. Reflecting supply constraints, not least relative affordability, specific tenures also tend to be associated with key divisions within some of the principal socio-economic life course arenas. Across much of the Global North, owner-occupancy is positively linked with being employed, white, middle class, middle aged and well educated; private-renting links to being unemployed, young and living in non-family households; whilst social housing is linked strongly with social disadvantage (Blunt and Dowling 2006: 90-91).

The pattern of housing tenure reflects a snapshot of what are known as housing careers. Defined by Kendig (1990: 133) as "a succession of dwellings occupied by individuals over their lives," he drew a parallel between such a career and other "careers," such as employment. As he saw it, the aim-"the apogee of the housing career" (Clark et al. 2003: 145)-was outright homeownership. Life course sensitivity, however, adds complexity to any such aspirational trajectory through linking the content of a housing career to life course stages and transitions, which are complex, diverse and often non-linear. As with employment careers, housing careers do not necessarily follow an upwards trajectory but may decline, stay relatively flat, or rise and fall considerably over time. Crucially though, progression through a housing career is generally undertaken via migration and residential mobility (e.g. Clark et al. 2003, 2006). With each move, the possibility exists for tenure change to occur, from renter-to-owner or owner-to-renter.

Individual and household characteristics such as age, income, race and family size are important in determining who changes tenure (Hassan et al. 1996). Groups likely to move from renter to owner status include new couples, two-parent families with children and newly employed higher status workers, whilst those more likely to shift from owner to renter include new single person households, single-parent families, and the elderly.

Within the playing out of the housing career, a strong empirical finding has been that periods of often considerable residential mobility tend to be associated with private-renting, whilst owner-occupancy is associated with staying put (e.g. Böheim and Taylor 2002; Clark and Dieleman 1996; Helderman et al. 2006). The latter again points to the importance of recognizing non-migration within mobility studies. Home owning has been shown to prevent residential relocation in various ways. First, purchasing a home, usually through a loaned mortgage, is a major investment for an individual or family (Figure 6.5). As Helderman et al. (2006: 112) put it, owner-occupiers often just cannot afford to relocate:

[a] large share of most homeowners' savings is tied up in their home, so moving is harder for homeowners than for renters. When they move, homeowners are confronted with higher transaction costs than renters. The transaction costs involved in selling a home contain both financial and non-financial costs. There are moving costs, taxes to pay, the services of an estate agent, as well as the effort involved in selling the home.

On top of this emphasis on financial costs, investment in property is also often investment in place. It is about making a home (Blunt and Dowling 2006). Development of local ties discourages subsequent relocation, from sending children to local schools to making friends with neighbors. In contrast, private-renting has been associated with a more superficial connection to place, both promoted by and promoting high population turnover. The short-term, unstable and changeable contracts that often comprise renters' tenancy agreements encourage such high turnover. In short, via reinforcing positive feedback loops, owner-occupancy promotes settlement promotes owner-occupancy, whilst private-renting promotes transience promotes private-renting.

\section{ST PROOFS: NOT FOR DISTRIBUTION}




\section{Diversity of Population by Housing Tenure accross Europe, 2013}

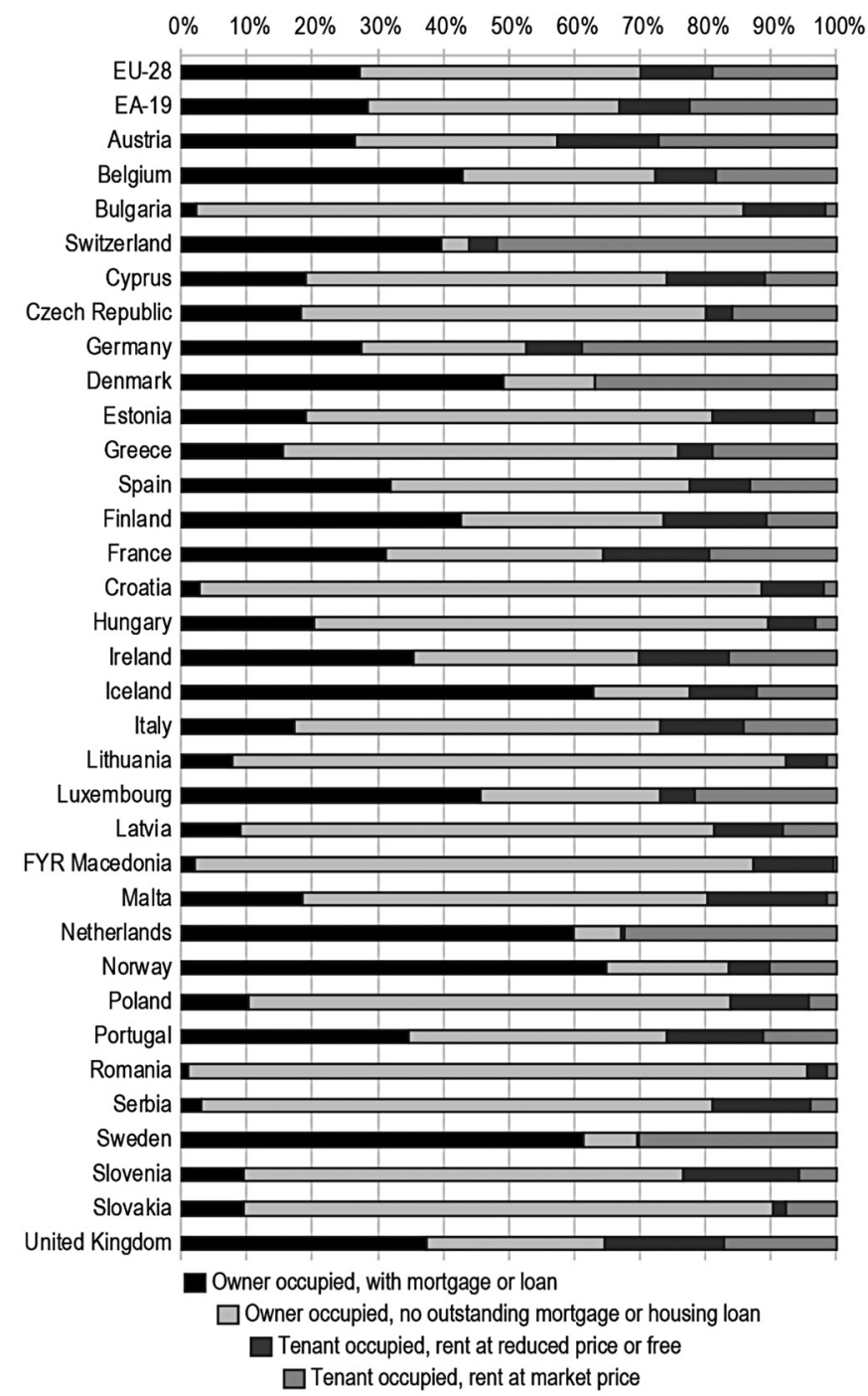

Figure 6.4 Diversity of population by housing tenure across Europe, 2013.

Source: European Union Open Data Portal: https://open-data.europa.eu/en/data/dataset/fAtiyJCKzLXUhNX3wZUw. Metadata for file ilc_Ivho02 found on Eurostat: http://ec.europa.eu/eurostat/cache/metadata/en/ilc_esms.htm.

Nevertheless, one must again be wary of over-generalizing or assuming these associations. For example, where owner-occupancy is (increasingly) the usual tenure, one might expect it to decline in terms of its significance within a housing career as a marker of being emplaced. Evidence from the Netherlands supports this (Helderman et al. 2006). Similarly, in countries where renting is more commonplace, it may be regarded as less "transitional" within a housing career and consequently be more associated with building place.

Culture is also a critical consideration within the complex relationships between tenure and mobility. In the US, for example, homeownership is generally regarded as something highly desirable to achieve, especially within specific locations. Both tenure (ownership) and the location of dwelling unit confer a particular level of social and economic status (Morrow-Jones 1988). Moreover, different tenure configurations are typically seen as culturally appropriate for different life stages. An accepted order of transitions through status levels exist, from the private-renting of young single adults to the owner-occupancy of "respectable" families with children. For older age groups, a change in tenure

\section{ST PROOFS: NOT FOR DISTRIBUTION}


142 FROM EVERYDAY TO RESIDENTIAL MOBILITIES

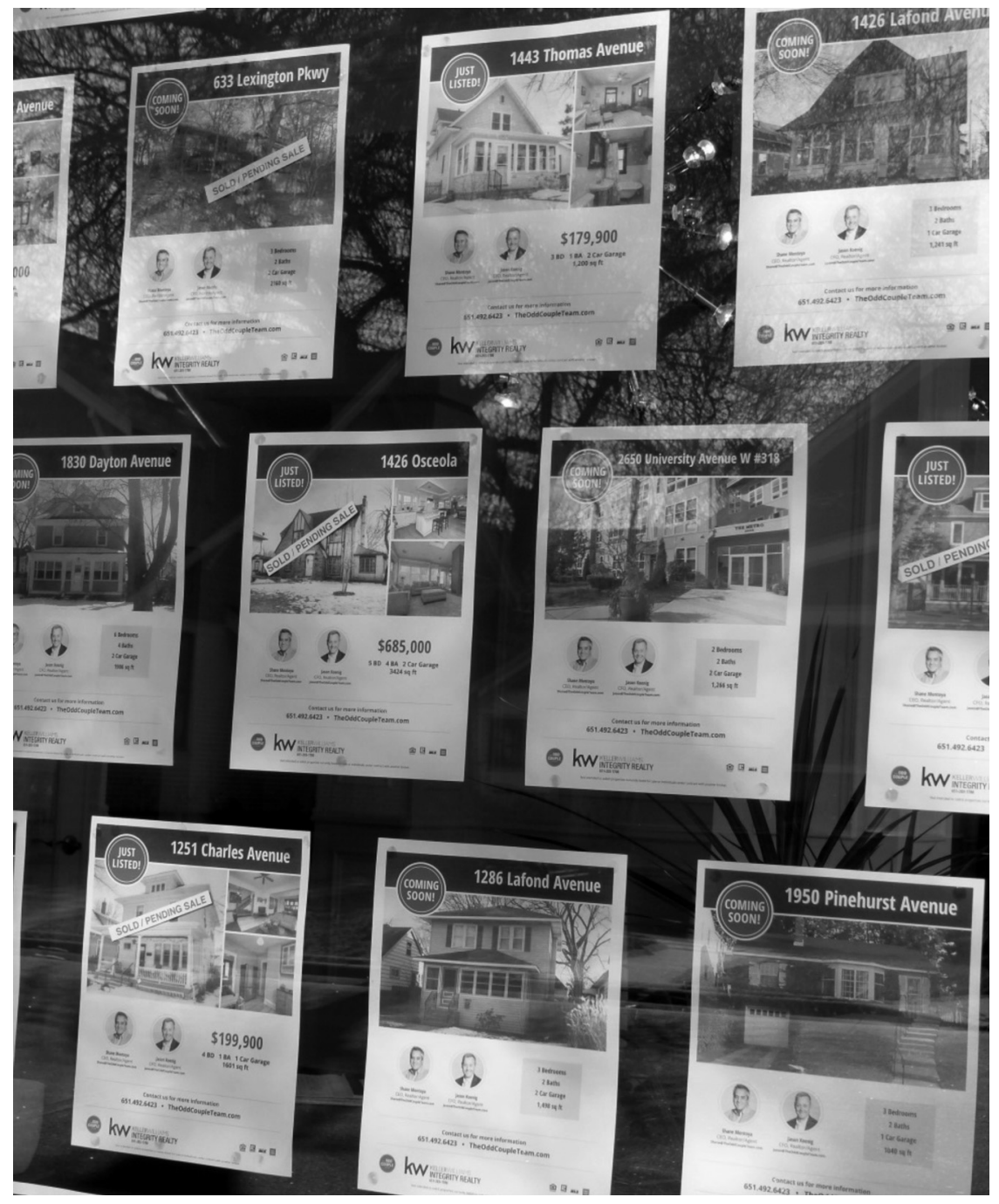

Figure 6.5 Buying a first home is a major financial commitment

Source: author.

from owner to renter status is often culturally normative. Clark and Davies (1990) illustrated this from disaggregated US tenure data for 5-year age groups from the age of 55 years. They found that through to age 69 , about 80 percent maintained ownership status. By age 79, however, percentage of owners declined to about 70 percent, with 68 percent over 80 years maintaining home ownership.

Emphasizing the potential two-way movement of the housing career, a tenure shift from owner-occupancy to private-renting, with attendant residential relocation, can occur for a range of financial considerations. First, tenure downsizing can signify attempts to save money or to bring out equity invested in "bricks and mortar" for use elsewhere. For example, Angelini and Laferrère (2012) found low income households especially likely to move into both smaller homes and shift to renting. Second, some of Gough's (2008) young owner-occupiers in Lusaka-who had often become owner-occupiers due to the death of their carers-were forced "rent out their homes, then rent cheaper accommodation for themselves" (Gough 2008: 251) in the highly negative relational context of Zambia's generally deteriorating economic situation. Third, where a property is still being paid for, such negative economic context can combine with a family having taken on too high mortgage payments to result in them losing their property through foreclosure (Box 6.4).

\section{ST PROOFS: NOT FOR DISTRIBUTION}




\section{BOX 6.4 MOBILITY CONSEQUENCES OF HOUSING BUSTS}

An owner occupied house is typically the greatest economic asset a family owns. There is thus a huge incentive within many countries across the world to purchase one's home. Unfortunately, this can go badly wrong. Both the purchase of the property and even the consequences of owning a property that has been paid for completely can become a very negative economic experience. This, in turn, can have considerable implications for family migration and residential mobility.

Three terms are of special significance here. First, a housing bust occurs when the market through which mortgage loans are obtained in order to buy houses collapses. This occurred in the US in 2007, following several years where low interest rates and the granting of mortgage loans to buyers without secure means to pay them back prompt a house buying boom. Consequently, house process increased dramatically until a peak was reached and price decline set in. Pandit (2011: 83) now takes up the story:

homeowners who had risky mortgages were unable to avoid a potential crisis by refinancing or selling their homes to repay their mortgages. Loan performance fell significantly, especially for subprime ... mortgages ... given out with large initial discounts, often to people without solid documentation of their income and assets.

And the housing market collapsed.

Accompanying a housing bust will be a rise in negative equity, a second key term. Roughly speaking, this is where present market value of a house has fallen below the value already paid or still being paid for it. Third, another certainty within a housing bust is a rise of housing foreclosures. Here, the individual or family paying the mortgage default on the loan to the extent that the lender begins legal proceedings to seize the property in lieu of debts. In the recent US housing bust it was estimated that for the second quarter of 2009 alone there were nearly 1 million filings for foreclosure. This translated to around 240,000 repossessions. (Many of the remaining houses were sold at auction, probably at considerable loss to those forced to sell.)

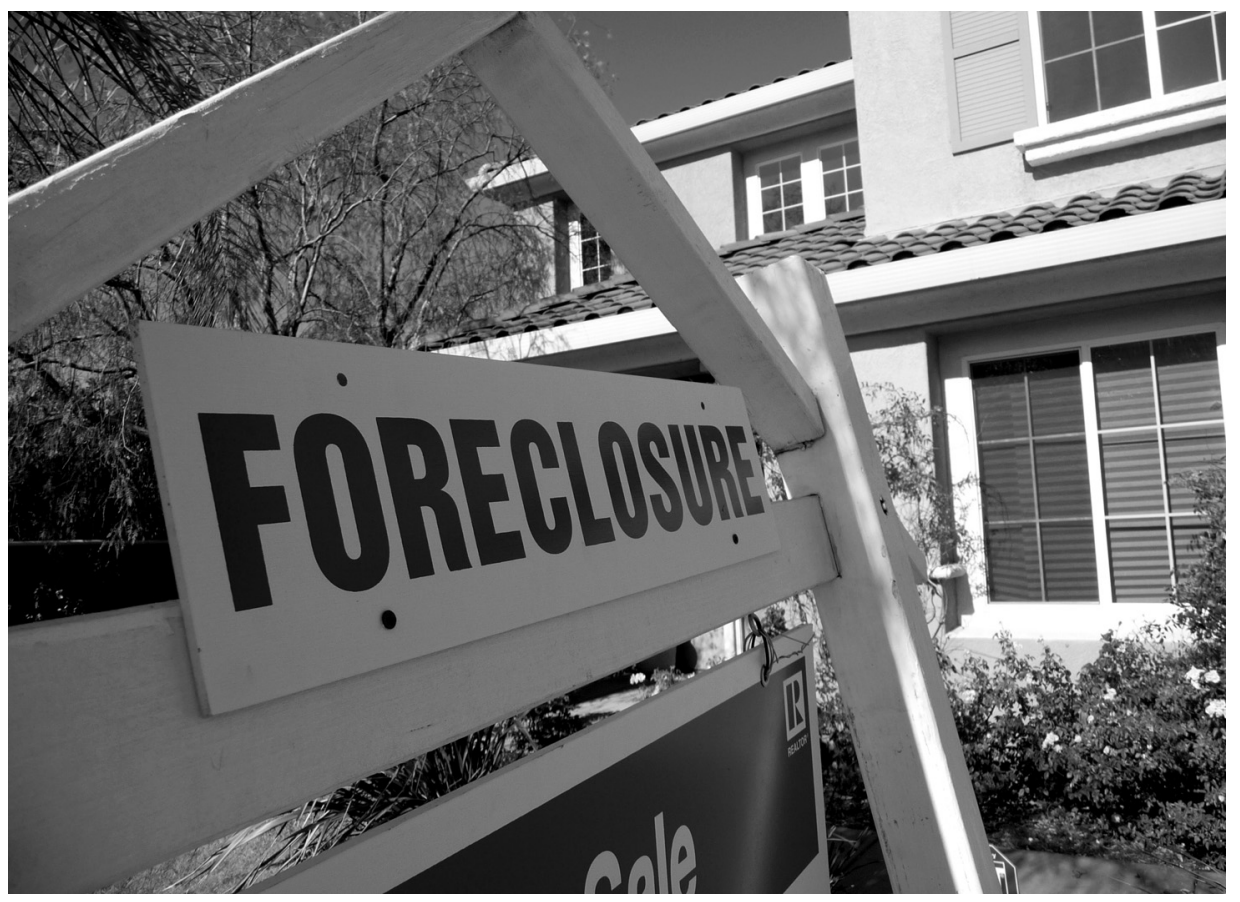

Source: https://upload.wikimedia.org/wikipedia/commons/a/a9/Sign_of_the_Times-Foreclosure.jpg 


\section{FROM EVERYDAY TO RESIDENTIAL MOBILITIES}

Clearly, a housing bust is a tragedy for anyone caught up in it. It is also a highly politically charged event. Critical questions are soon asked about both the ethics of the mortgage lending market and of its regulation by the state. Moreover, it should be noted how housing busts tend to be socially selective, with the recent US collapse typical in that it was most acutely felt in low-income areas, especially those populated by ethnic minorities.

A housing bust has a range of mobility implications. At first sight, it might be expected to promote residential mobility, as a consequence of enacted foreclosure is eviction from the property if relocation has not already occurred. However, although "[d]efault-induced moves are always the first mobility-related impact observed during a downturn" (Ferreira et al. 2010: 34), they are rarely the last. Instead, the fall in house prices (rise in negative equity) and increase in interest rates charged on mortgage loans tend to "lock-in" families to their homes, stifling mobility even when desired.

Based on econometric modeling work with American Family Survey data between 1985 and 2007, Fernando Ferreira and colleagues investigated the relationship between mobility and both negative equity and higher interest rates. They found clear relationships after controlling for other variables associated with mobility. Specifically, higher interest rates lowered mobility but negative equity had still more of a effect. In short, following a housing bust, both experienced economic factors-such as having to find more money to service debts or not wishing to downsize housing after moving due to negative equity from the house being sold-and risk aversion during a period of perceived housing financing uncertainty and crisis appear strong mobility deterrents.

A final mobility consequence of the kind of housing bust that occurred in the US from 2007 concerns neighborhood quality issues. Areas of housing bust may well become generally environmentally depressed. This can provoke out-migration of those relatively unaffected by the bust and also deter new in-migrants, thereby reinforcing an area's decline.

(Sources: Crump 2008; Ferreira et al. 2010 ; Pandit 2011)

In summary, housing has a multitude of complex relationships with residential mobility. Many, although not all, of these relationships are linked to diverse family practices. Housing's links to migration over longer distances is equally marked and will be covered in Chapter 7. For both, further associations with place characteristics are also important, indicating how residential mobility can also be seen as a neighborhood matter.

\subsubsection{Neighborhood matters}

Whilst usually linked with family and housing considerations (Figure 6.3) (Clark et al. 2006), residential mobility can occur when neighborhood dissatisfaction comes to the fore within an individual or family everyday life course. This third perspective, residential mobility as neighborhood matter, reinforces the need to take aspects of place into account for understanding relocations (Rabe and Taylor 2010). These aspects concern both place of origin and place of (potential) destination. Here, it is often how they are perceived by the potential mover that is key. Furthermore, although residential relocation for neighborhood matters can come about from little meaningful choice, usually it can be tied to aspirational and voluntaristic life choices. Constraint and choice are not as clear cut categories here as may first appear, however.

Some neighborhood matters initially very much down to choice, such as relocations relating to accessing key services. Consider schools. Worldwide, parents frequently invest much effort, emotion, time and finances in their children's education. In the richer countries of the Global North, one reflection of this engagement is striving to place offspring in "good" schools, where they are more likely to flourish to the best of their academic potential. Accessing such schools, however, may require more than good levels of motility but necessitate familial residential relocation, whether locally, considered here, or more intra-nationally.

Mobility relating to accessing good schools in the UK has become a common focus of investigation (Smith and Jons 2015). This is because recent political developments, notably producing "league tables" that rate and position schools, have raised the public profile of the educational geography of British schools. Table 6.6 gives an example of such a tabulation, with the schools showing a wide range of GCSE (examinations usually taken around age 16) results.

\section{ST PROOFS: NOT FOR DISTRIBUTION}


Table 6.6 School league tables: secondary schools on Devon-Somerset border, England, 2014

\begin{tabular}{|c|c|c|c|c|c|c|}
\hline School name & School type & \multicolumn{4}{|c|}{$\begin{array}{l}\% \text { achieving } 5+A^{*}-C \text { GCSES } \\
\text { (or equivalent) }\end{array}$} & $\begin{array}{l}\% \text { achieving } \\
\text { grades } A^{*}-C \text { in } \\
\text { English \& Maths }\end{array}$ \\
\hline England-all schools & & 53.5 & 59.0 & 59.4 & 59.2 & 59.9 \\
\hline Tiverton High School & Community & 46 & 55 & 54 & 54 & 56 \\
\hline Uffculme School & Academy: Converter & 77 & 85 & 80 & 80 & 80 \\
\hline Kingsmead Academy & Academy: Converter & 67 & 69 & 67 & 63 & 64 \\
\hline Court Fields & Community & 47 & 46 & 41 & 57 & 58 \\
\hline Wellington School & Independent & 49 & 84 & 86 & 94 & 96 \\
\hline
\end{tabular}

Source: www.education.gov.uk (accessed March 2014).

Utilizing such data on school performance and other sources of knowledge, middle class parents, in particular, use relocation to make sure their children reside within the catchment areas of supposedly good schools (Smith and Jons 2015).

Residential mobility to access services is not just a middle class preoccupation. Studies have shown the relative absence of everyday services within usually poor neighborhoods. This situation may prompt relocation to save on the costs and general inconvenience in accessing them. For example, food deserts are "areas of relative exclusion where people experience physical and economic barriers to accessing healthy food" (Reisig and Hobbiss 2000: 138). They are neighborhoods where gaining access to "healthy" foods at "affordable" prices is problematic. Although precise definition may be difficult, and with some cities seemingly not having such neighborhoods (e.g. Apparicio et al. 2007 on Montréal, Canada), presence of food deserts has been linked to poor health and social exclusion (e.g. Sharkey 2009; Wrigley 2002). One response to living in such a neighborhood is to seek to relocate, especially for the poor and physically immobile. Of course, whether this proves possible is a further necessary consideration strongly linked to available resources.

Desired and sometimes practiced relocation might also be expected with residential experience of other service "deserts," such as those where there is significant spatial exclusion from health care (e.g. Gatrell and Elliott 2015) or financial services (e.g. Leyshon and Thrift 1995). Further, even if residing in a "desert" is inadequate alone to prompt residential mobility, it can feed into the overall residential stress levels acquired from such a location.

The second area where residential relocation becomes a neighborhood matter is much less ambiguously not a matter of choice, although this eventually blends into a third area where choice reasserts itself. Section 6.3.5 argued that immobility may be partly a product of fear. If this situation becomes too extreme, one might anticipate relocation or escape to occur; albeit only if possible. One outcome may be to segregate-to concentrate and set apart residence within a defined area. Residential segregation has been studied widely with reference to race and ethnicity, where there are many controversies over its extent, causes and effects. A key debate, for example, concerns the degree to which urban neighborhoods in different countries are becoming increasingly ethnically homogenous (Box 6.5). Importantly, a degree of ethnic segregation need not be an inherent problem. Besides potentially providing relatively safety and solidarity from racist attack, for example, it can facilitate cultural development and survival (Finney and Simpson 2009).

Segregation is not solely a matter of race, ethnicity or the experience of relatively recent in-migration. Politics and religion may also be involved. This is apparent within the ongoing (c. 2016) horrors of the Syrian civil war. Communities, in part defined according to which branch of Islam they follow, have segregated within individual cities, such as within the capital Damascus (Keller 2013). Similarly, a legacy of the sectarian strife that followed the US-led invasion of Iraq in 2003 was an increasingly segregated Baghdad (Figure 6.6). Elsewhere, polito-religious residential mobility was also a key geographical feature of the conflict in Northern Ireland, not least since the 1960s. So-called "peace walls" were constructed to separate Protestant and Catholic communities into sharply delineated neighborhoods. 


\section{BOX 6.5 ETHNIC SEGREGATION IN THE UK}

The Director of the UK's Commission for Racial Equality caused a major stir within academic and media circles in 2005 by claiming Britain was "sleepwalking into segregation." Certain UK cities-many of which had experienced disturbances involving predominantly young Pakistani men in 2001 following Far Right provocations -were characterized as having areas increasingly numerically dominated by particular ethnic groups who had been key immigrant flows to the country since 1945. Within such areas, "White British" resident numbers were in marked decline. The UK, in short, was developing ghettoes.

Ethnic segregation can be measured in many ways, frequently utilizing statistical indices. Indeed, Peach (2009) wrote of an "Index War" raging in the US between 1930 and 1955 by advocates of rival measures. One widely used measure is the Index of Dissimilarity $\left(I_{d}\right)$, calculated as follows:

$$
I_{d}=1 / 2 \sum_{i=1}^{N}\left(\frac{b_{i}}{B}-\frac{W_{i}}{W}\right)
$$

In other words, for each of $\mathrm{N}$ spatial units, the fraction of the total White population $(\mathrm{W})$ for the spatial unit $\left(\mathrm{w}_{\mathrm{i}}\right)$ is subtracted from the fraction of the total Black population $(B)$ for that spatial unit $\left(b_{i}\right)$. The magnitudes of all of these are then summed, and the final total halved. $I_{d}$ value, on a scale between 0 and 1 , is usually then multiplied by 100 for ease of use.

Put simply, $l_{d}$ value refers to the percentage of a segregated population-usually an ethnic minority groupwho would need to relocate if the group were to mimic spatially a reference population-usually the white majority. Values of 30 or below are generally interpreted as low segregation, whilst values of 60 or more signify high segregation.

The following table shows the $I_{d}$ for seven UK cities for census years 1991 and 2001. It reveals, except where highlighted in bold, how $I_{d}$ actually declined over the decade, indicating segregation reducing not increasing. It was also relatively low.

\begin{tabular}{|c|c|c|c|c|c|c|}
\hline \multirow[t]{2}{*}{ City } & \multicolumn{2}{|c|}{ Caribbean } & \multicolumn{2}{|l|}{ Indian } & \multicolumn{2}{|c|}{ Pakistani } \\
\hline & 1991 & 2001 & 1991 & 2001 & 1991 & 2001 \\
\hline Birmingham & 40 & 35 & 48 & 42 & 62 & 55 \\
\hline Bradford & 39 & 32 & 49 & 42 & 54 & 51 \\
\hline Leeds & 63 & 35 & 42 & 42 & 61 & 55 \\
\hline Leicester & 29 & 39 & 42 & 44 & 47 & 46 \\
\hline London & 43 & 39 & 46 & 44 & 48 & 46 \\
\hline Manchester & 49 & 38 & 39 & 35 & 52 & 48 \\
\hline Oxford & 32 & 33 & 25 & 27 & 46 & 32 \\
\hline
\end{tabular}

Source: modified and simplified from Peach (2009).

What then of the idea that the UK is developing ghettoes? One should first note how the very term is problematic. It originates from the historical pattern of Jewish communities living in walled and legally restricted areas. Today, however, ghettoes are, in Peach's (2009: 11) words, more generally seen as "the product of exclusion, compulsion, enforced separation, individuation, dysfunctional family structures, and substance abuse." This description very poorly depicts the UK's ethnic communities.

Ghettos also imply extreme segregation. Peach examined this by focusing on the wards within two cities where ethnic minority groups comprised at least 70 percent of the population. In both Leicester and Bradford around a third of all ethnic minority residents lived in such areas in 2001. This is not especially high. (It compares to three quarters of the Black population of Chicago in the US in 2000 living within equivalent concentrations.) Looking next at specific key ethnic groups within each city, just 12 percent of Leicester's Indians lived in 70 percent+ Indian wards, and none of Bradford's Pakistanis in equivalent concentrations. Such evidence and other sources refutes the idea the UK is becoming "a country of ghettoes" (Finney and Simpson 2009: 115). 
Two further relevant socio-demographic features of ethnicity and mobility within the UK's cities also merit attention. First, ethnic minority people are migrating out of these cities, frequently expressing similar "aspirational movement" (Finney and Simpson 2009: 127) to the majority population. This promotes population dispersal rather than concentration. Second, of note is the relative youth expressed by ethnic minority population pyramids. This results, inevitably, in strong population growth via demographic momentum (3.2.2).

In summary, detailed empirical studies reject the suggestion that Britain is sleepwalking into segregation and producing ethnic ghettoes. Instead, ethnic dispersal is occurring, a trend projected through migration modeling to continue over the next half century. UK inner cities are diverse, urban and open, not segregated, ghettoized and closed:

Indians are leaving Leicester, Caribbeans are leaving Lambeth, Bangladeshis are leaving Tower Hamlets and Pakistanis are leaving Bradford, and within each of these areas the settlement areas are acting not as magnets but as generators of both population and migrants .... [T] The degree of separation between ethnic groups in Britain is not high, in the sense that those areas with relatively few White people are diverse and metropolitan in nature rather than exclusive" (Finney and Simpson 2009: 127, 136).

(Sources: Bailey 2012; Finney and Simpson 2009; Peach 2009; Rees et al. 2011; Robinson 1998)

These had become increasingly homogenous through selective residential mobility, often forced through minority terrorization (Boal 2002). Where such forced segregations upscale, the consequence is the ethnic cleansing discussed in Chapter 9.

Moving away from the violent extreme of ethnic cleansing, a central message from studies of residential segregation is how group concentration can come about both due to prejudice and from a greater or lesser degree of choice. It may express what Savage (2010) termed elective belonging. This is a third area where residential relocation as neighborhood matter can be expressed and links to Chapter 8's lifestyle migrations. Elective belonging represents a desire to "live somewhere appropriate for 'someone like me'" (Savage 2010: 132), or not to live somewhere where a person or household feels "different from their neighbours" (van Ham and Feijten 2008). Loosely put, this tendency might be seen as underpinning this whole sub-section (Box 6.6).

Investigations of elective belonging emphasize the class dimension of residential segregation. On the one hand, this life course arena intersectionally cross-cuts the racial, ethnic and religious expressions of segregation already covered, the patterns observed generally being also skewed towards poorer social classes. On the other hand, although it is not just the middle classes who desire elective belonging, their relocations are especially prominent. They are most clearly demonstrated by migration rather than residential mobility (Chapter 8 ) but the latter does also occur. For example, it was noted above how families may move to access better schools. One consequence of such population sorting (Bailey 2012), whether or not desired, is to make certain areas more middle class overall. This feeds through into and, in turn, reinforces emerging social geographies by prompting rises in house prices, for example. Mobility for elective belonging thus contributes to middle class social reproduction or how this class as a defined group reproduces itself across generations.

A stark geographical expression of class-delineated elective belonging that emerges from both residential mobility and longer distance migrations is the rise of gated communities. These are "walled and gated residential developments that restrict public access" (Atkinson and Flint 2004: 875), sometimes via security guards as well as physical barriers. Widely noted across the US (e.g. Blakely and Snyder 1997; Marcuse 1997; Sorkin 1992), they are found across both Global North (e.g. Atkinson and Flint 2004; Rofe 2006; Rosen and Grant 2011), even in its poorer nations (e.g. Stoyanov and Frantz 2006), and South (e.g. Atkinson and Blandy 2013; Coy 2002; Leisch 2002). Motivated in part by security considerations and class-linked fear (Davis 1998) within urban areas and countries sharply marked by strong socio-economic inequalities, they frequently "also meet ... an apparent desire to avoid day-to-day incivilities and random social contact" (Atkinson and Flint 2004: 880). Indeed, the desire for privacy motivation is taken further by Atkinson and Flint $(2004: 889,888)$ positioning residence in a gated community within "attempts at social extraction ... [where] the only remaining public realm for residents ... may be the space between the car and the shop or office door." Residential mobility to a gated community can combine with high levels of private personal mobility for an elective 
148 FROM EVERYDAY TO RESIDENTIAL MOBILITIES
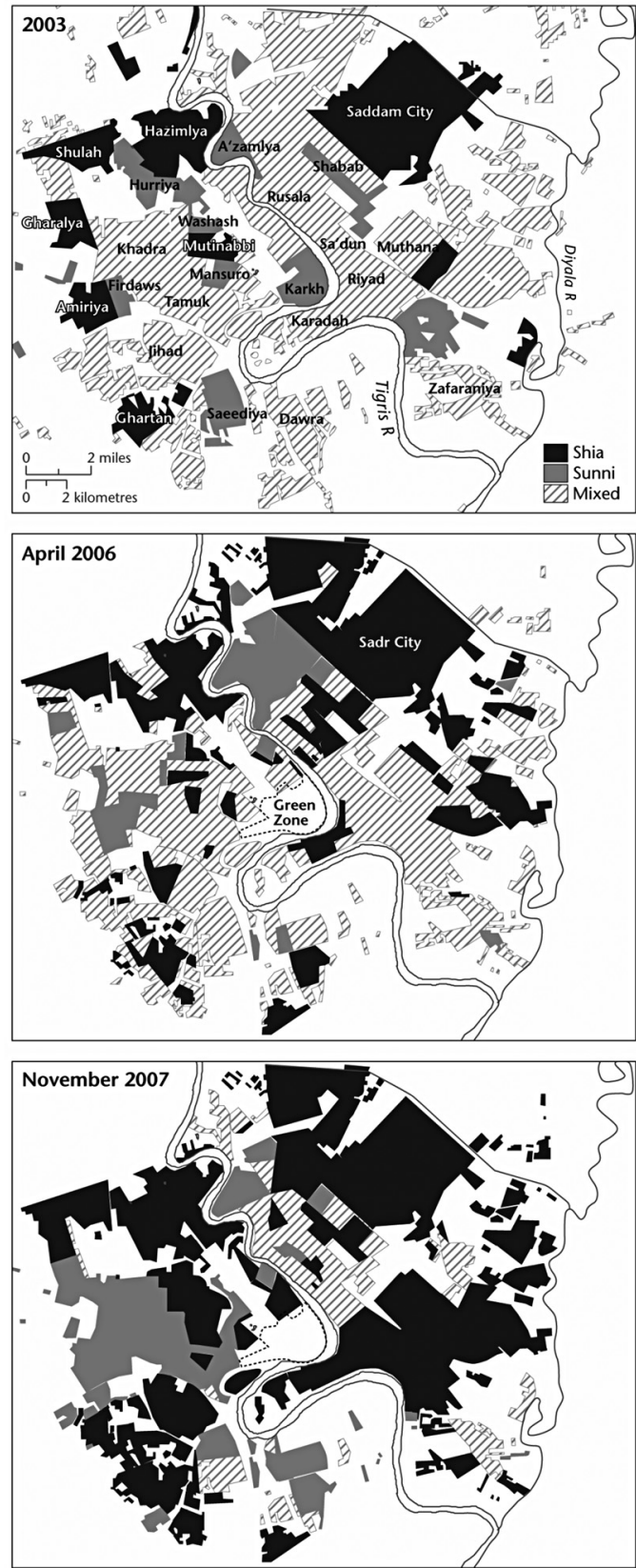

Figure 6.6 The growing sectarian divide in Baghdad, Iraq, 2003-2007

Gregory (2008).

belonging strongly exclusive and exclusionary.

Finally, social reproduction of a class-exemplified by gated communities-or of an ethnic, cultural or other group identity does not necessitate segregation or even geographical concentration. Thinking briefly about this returns to the importance of mobilities generally and of everyday accessibility implications of living in an era of mobilities. Useful is Zelinsky and Lee's (1998) concept of heterolocalism, developed to express how US ethnic minority communities were neither approaching some final state of cultural and ethnic absorption or assimilation nor becoming isolated

\section{ST PROOFS: NOT FOR DISTRIBUTION}




\section{BOX 6.6 CHINESE SUBURBIA AND GATED COMMUNITIES}

A 2008 USA Today headline proclaimed "Modern Suburbia not just in America Anymore," referring to the emergence and growth of suburban landscapes in China. The story went on: "[a]cross China, entire suburban cities are being built at a dizzying speed to keep up with population growth" (El Nasser 2008: np). Furthermore, as it has increased the form of suburban development has also changed, stressing the importance of cultural taste and status within housing choice. Wu (2010: 1) suggested that "Chinese suburban residential developments have recently seen the emergence of ostentatious, decorative and 'western'-style built forms" and that many are now gated communities. Packaging and branding of suburban residential space has been utilized to appeal to a growing consumer market, even embracing strategies such as naming new developments after foreign places (e.g. Yosemite, Napa Valley) as a means of conveying particular social status.

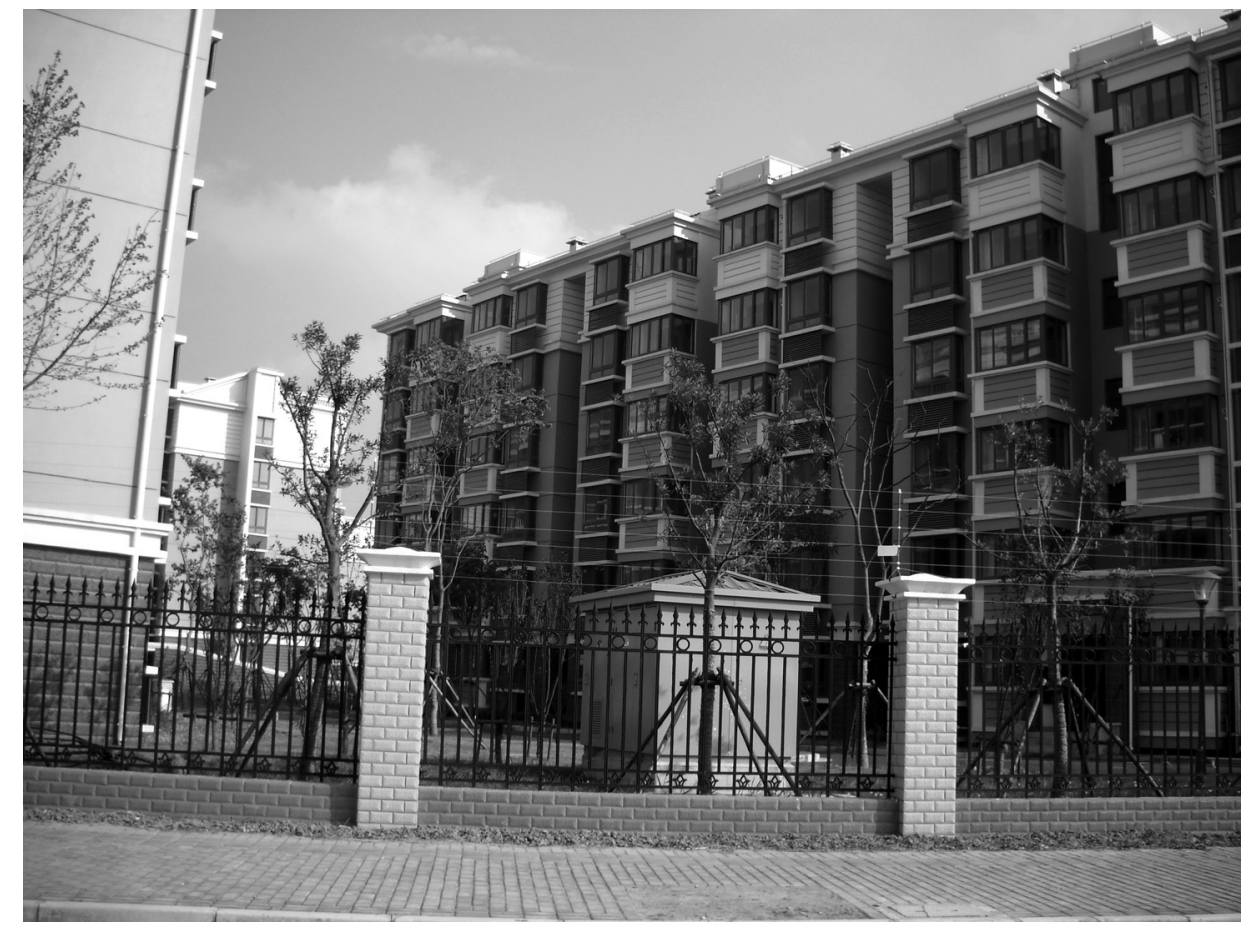

Gated community at Chongming, Shanghai, China

Source: I-Chun Catherine Chang (2012).

China's economic restructuring and opening to the outside world from 1978 began a period of significant urban change within the country. As it evolved towards a more market-oriented economy, changes in the spatial structure of urban areas accelerated. Zhou and Ma (2000: 217) identified five major processes of institutional and spatial restructuring that continue to influence urban China's spatial structure:

- Urban land use change;

- Infusion of foreign and domestic capital;

- Urban deindustrialization and tertiarization;

- Improvement in urban transportation;

- New housing development.

These processes have promoted growth of suburbanization in China's large cities, with associated residential mobility and housing career consequences.

\section{ST PROOFS: NOT FOR DISTRIBUTION}


In evaluating early 1990s suburbanization in four major cities, Zhou and Ma found urban core populations declining, while population was increasing in inner suburbs. Prior to reform, "congestion, high population density and declining housing quality were chronic problems in the urban core" (p.223) primarily from minimal state investment. Emergence of suburban housing corresponded with broader economic restructuring but also with incentives for urban dwellers to move to new suburban areas, including "more living space, agreement to allow [migrants] to return to the city later, relocation subsidies and reduced cost in purchasing suburban housing" (p.223).
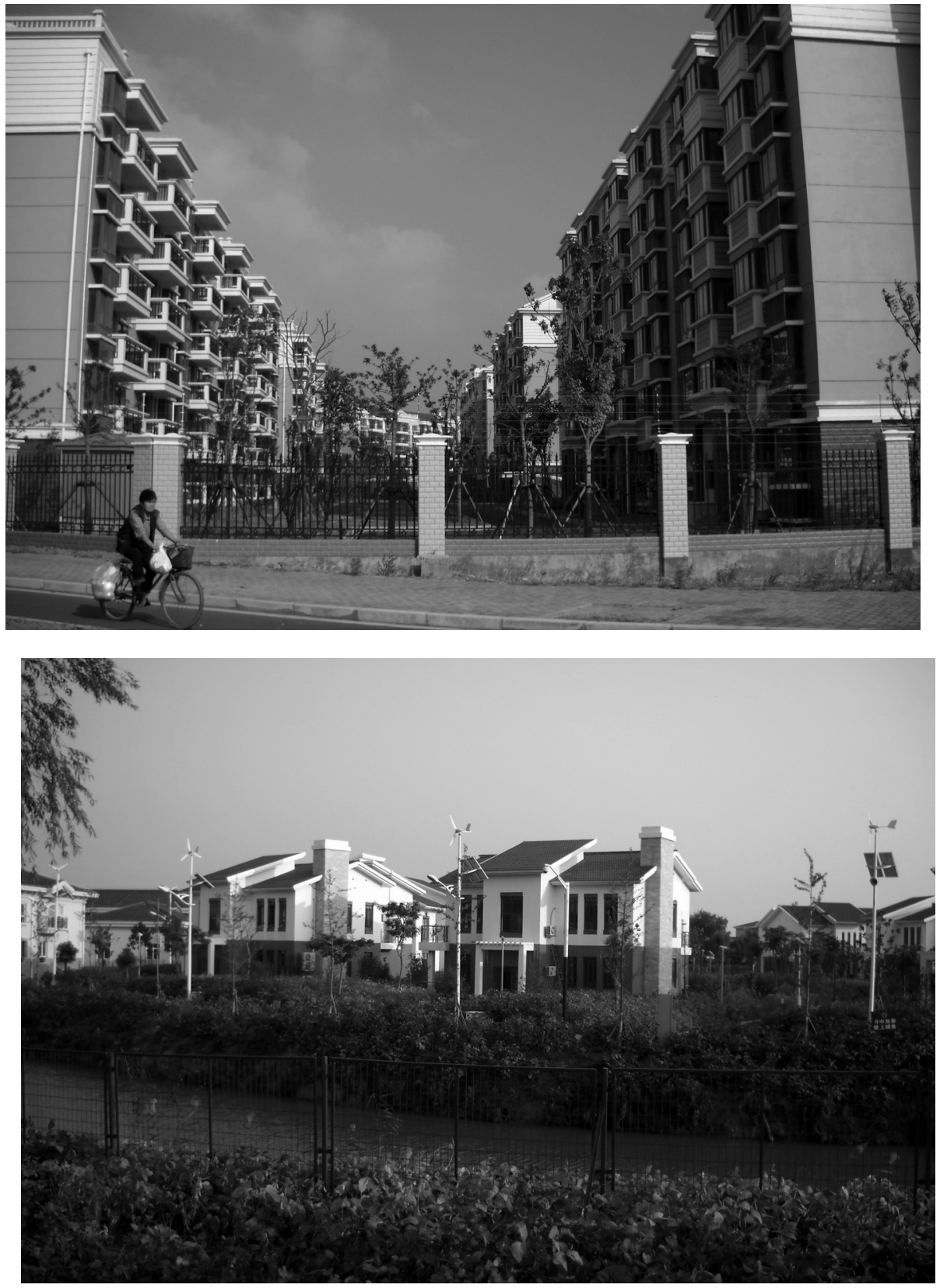

Suburban developments at Chongming, Shanghai, China

Source: I-Chun Catherine Chang (2012). 
China suburban landscape revolution can certainly be assessed from the perspective of urban restructuring. However, it is also a result of housing choice and mobility decisions made by individuals and families. The decision to relocate from one unit to another, or from one area of a city to another, is influenced by household characteristics, such as life course stage, level of education and income. Broader institutional and place specific factors are also important. Of course, it is insufficient simply to assume the same processes of residential choice apply in all national contexts. In China, gradual transition from state controlled to open housing market is involving a complex set of interests. Accommodation is allocated by a range of actors-state, work place, private marketand an individual's ability to negotiate housing location and tenure is strongly influenced by these institutional factors and by household characteristics. With several different categories of housing, including open-market, resettlement, work-unit subsidized, and municipal housing bureau, a range of options and constraints confront the typical family.

Global cities are increasingly experiencing suburban forms of development. However, it is important to continually re-evaluate residential mobility models such as Rossi's and all theories associated with housing choice and residential mobility in new, often very different contexts. This is very clear from the Chinese example.

(Sources: El Nasser 2008; Wu 2004, 2010; Zhou and Ma 2000)

cultural and ethnic islands. Instead, they had adopted a dispersed pattern of residential location at the metropolitan scale but retained ethnic and community identity. The result has been emergence of "communities without propinquity" (Webber 1964), expressed geographically as a sequence of "spatial disjunctures" (Zelinsky and Lee 1998: 287) between specific places of strong everyday significance, such as religious buildings, shops selling "ethnic" foods and denominational schools. Heterolocalism brings a transnational (7.5.6) sensibility to recognize a geographically dispersed "home" and complex entanglement between settlement and mobility at the intra-national scale (Halfacree 2012a; Smith 2001). For example, in the UK's north London suburbs, the Jain temple at Potters Bar and the Hindu Bhaktivedanta Manor in Hertfordshire are both in locations that allow the motorway system to bring together dispersed faith communities (Dwyer et al. 2013). In other words, transport mobility rather than residential mobility facilitates faith community reproduction.

\subsection{CONCLUSION: FROM MOBILITIES TO MIGRATIONS}

This chapter developed the importance of everyday mobilities to everyday life course (re)production and then recognized how these can merge into more "permanent" expressions of migration, with a specific geographical focus here on residential mobility. Building on the pioneering work of Rossi, the chapter demonstrated how taking both a family and a more individual life course perspective is critical for understanding and explaining both everyday and residential mobilities. At the same time, the chapter resisted suggestions of the presence of deterministic relationships between life course and mobility. There are definitely associations and relationships but these always remain more complex in their relational construction than would be done justice by deterministic law-like statements.

The extension of everyday mobilities further into migration will be carried on in the subsequent migration chapters, reinforcing the lack of definitive boundaries between them. Together, these build up a picture that shows how issues pertaining to often seemingly mundane mobilities of everyday living, from the perspective of the individual person's relational life course, cannot be separated from those underpinning less everyday, more noteworthy migrations. This reinforces once again the value of recognizing a mobility continuum. Yet, it also reiterates residential mobility's position as often being rather "ordinary" empirically-speaking, in the way commuting, for example, is generally regarded. In contrast, aspects of migration's less "everyday" and more eventful character will be pursued across the next three chapters. Finally, these accounts also strive to give greater attention than has been managed in the present chapter to the Global South, the relative imbalance here mimicking the primary association made in the literature between the era of mobilities and the Global North. 


\section{CHAPTER 7}

\section{Employment migrations}

\section{$7.1 \quad$ INTRODUCTION: BEYOND COMMUTING}

[T]he search for a better livelihood is a main cause of migratory movements.

The Model Migration Schedule (MMS), developed mostly by Andrei Rogers and colleagues (e.g. Rogers et al. 1978; Rogers and Castro 1986; Rogers et al. 2005) over four decades, expresses a set of mathematical functions to depict associations between migration intensity and age (Rees and Hardill 2015). A recent iteration is shown in Figure 7.1 (Wilson 2010). It is the composite of the six elements shown on the right of the figure. In summary, migration generally declines over childhood, not least reflecting the ties noted in Chapter 6, but peaks for students, labor force participants, the newly retired and the very elderly. The first two of the latter are the focus of this chapter.

"The major causes of migration are economic" was one of Ravenstein's core nineteenth-century "laws of migration" (5.3.1). Economic factors have been given predominant and privileged explanatory position ever since within migration scholarship (Halfacree 2004a). It is for economic or financial reasons that most relocation beyond residential mobility occurs within a country not beset by war or other life-threatening emergency (Fielding 2012). Moreover, relocation of the labor force is essential to the operations of the global capitalist labor market (Green and Shuttleworth 2015). Hence the labor curve's prominent position within the MMS.

Investigating the association between migration and the economic more closely requires asking what exactly comprises "economic reasons" for migration. Consider three readings. In some accounts, reasons are defined through

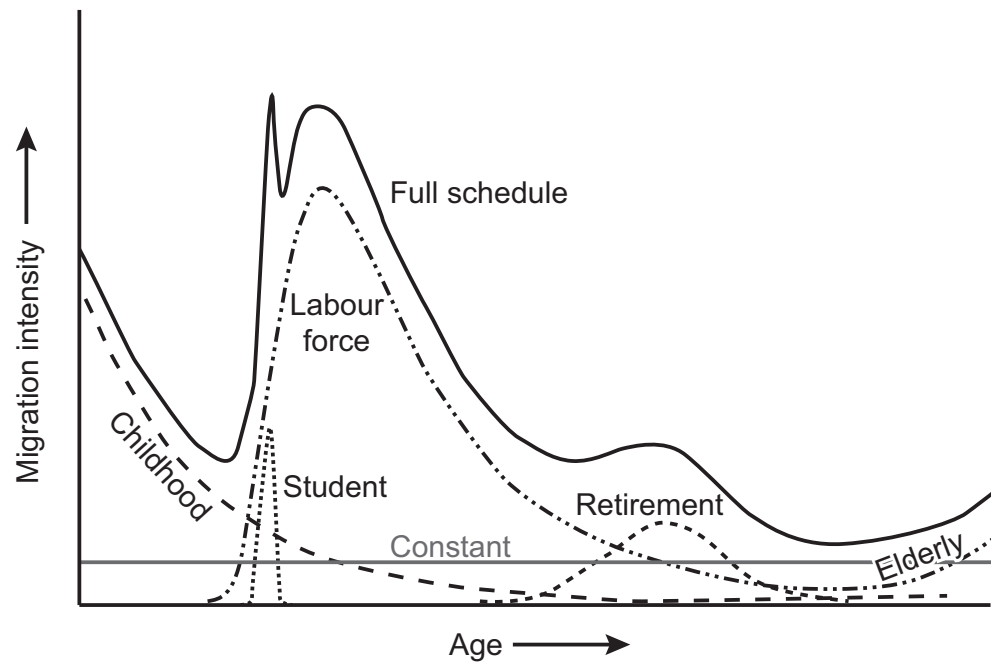

Figure 7.1 The model migration schedule.

Source: Wilson (2010: Figure 4); Rees and Hardill (2015: Table 9.2). 
work opportunities, migration seeking access to a better or more suitable job. Elsewhere, the economic is located more "structurally," expressing overall relative "health" of an economy usually defined at national or regional scale. Geographical differences in such health prompt flows of migrants towards more economically buoyant locations. The economic can also be read as more experiential. Relocations may be underpinned by a search for status, success or capital gain, or driven by economic hardship, seeking cheaper housing for example (6.3.3).

Whilst all migrations represented in the previous paragraph are underpinned by economic or financial reasons, the third category also indicates how the "economic" frequently interacts and entangles itself with "non-economic" or more-than-economic motivations (Halfacree 2004a). This important qualification allies employment migrations with wider search for a better quality of life. Likewise, from the other direction, Chapter 8's discussion of lifestyle migrations will show them entangled closely with class and income, and thus with employment issues; as will Chapter 9's overview of forced migrations. In short, the boundaries and categories needed to dissect migration into three manageable thematic chapters in this book are not definitive or necessarily consistent, and certainly not mutually exclusive (Halfacree 2001).

Notwithstanding these qualifications, the connection between accessing employment through migration remains as true today as when Ravenstein composed his laws. It remains paramount across both Global North and South. Even in an era of mobilities, mobility is frequently insufficient to displace migration for work reasons. Such employment migration, also known as labor migration, is addressed in this chapter. Building on Chapter 6, it can occur either when a job becomes too demanding to be accessed by commuting or through adopting an alternative household structure, or simply when starting a new job. The latter is most usual but varies, for example, between countries and through time, reflecting the multiply-scaled geographies and multiply-timed histories of the capitalist economy, respectively (Fielding 2012).

The rest of the chapter proceeds through four overlapping expressions of employment migration: student migrations (7.2); general employment migrations (7.3); specialist employment migrations (7.4); and employment migrations as family and community practices (7.5). Each has particular resonance within key arenas of life course constitution. With class a defining element of the economic, this arena unsurprisingly predominates. Nonetheless, other arenas, notably gender and ethnicity, feature notably. History and, of course, geography are essential contexts throughout. Of the latter, employment migrations have both similarities and differences between Global North and South and often transcend an unhelpful internal/international dichotomy. Age is also a crucial arena for employment migrations, reflecting employment's dominant position across a significant span of the life course. Starting and leaving work (retiring) are key life transitions strongly associated with migration. Lastly, employment migrations fluctuate by degree of practical everyday "choice" underlying the relocation, although Chapter 9 addresses the most involuntary "employment migration": slavery.

\subsection{STUDENT MIGRATIONS}

\subsubsection{Employment migration?}

A first expression of employment migration may not be regarded thus by some readers at all. It may not even be seen as migration. These are relocations that occur in order to study at university, other higher education establishment or center for advanced learning. They are included in the present chapter because study is a form of work and the consequences of obtaining resultant qualifications can impact considerably on subsequent employment migrations. Indeed, education level is frequently presented as the second strongest predictor of migration likelihood after age. For all age groups, as total years of education increases so does mobility (Long 1988).

Student relocations can additionally be usually regarded as migrations if interpretation avoids over-emphasis on "permanence" as a defining hallmark of migration, as argued in Chapter 5. Whilst students often return "home" during vacations and even after completing studies (Sage et al. 2013), not only do some remain but even those who do not are still resident for substantial periods of time in their place of study, with all the life course consequences that entails. Thus, a student curve features in the MMS (Figure 7.1). 
154 EMPLOYMENT MIGRATIONS

\subsubsection{Moving away to university}

Globally, education generally and higher education in particular have become increasingly important politically to promote and develop. National production of an "educated" population is increasingly "seen to underpin national economic competiveness in global knowledge economies" (Holloway and Jöns 2012: 483). Consequently, increasing numbers of young adults are taking up university education (Figure 7.2), the OECD average approaching two thirds (OECD 2012). Likewise, within the OECD it is estimated that just under a third of adults have some form of tertiary qualification, whether through university or more vocational education (OECD 2012).

Whilst higher education is about much more than job training, this depiction immediately makes its links to economic matters clear. Furthermore, studies of student migration frequently engage with the subject from the point of view of relative geographical gains or losses of human capital. One can deploy a home-university-labor market transition (HULT) model (Hoare and Corver 2010) to track both migrants and redistribution of human capital. This approach (e.g. Faggian and McCann 2009; Krabel and Flöther 2014; Venhorst et al. 2010) demonstrates, first, how university enrolment is associated with high levels of migration, both to attend university and then to obtain employment after graduation. For example, Smith (2009: 1796) estimated that "the annual internal migration of students in the UK, prior to onset and at the end of the academic year, is approximately equivalent to the de/repopulation of Birmingham," a city of just over a million people. Such substantial migrations have interesting consequences for the social geographies of university cities, such as growth of studentified areas (Box 7.1) and processes of gentrification (8.2.3).

Second, HULT models reveal geographical winners and losers in terms of human capital. Generalizing considerably, the former tend to be the main university cities and regions, whilst the latter tend to be remoter and rural areas and cities with relatively little university provision. A more international representation of this will be covered in Section 7.4.3.1's discussion of global brain drain.

Mobility and migration of both students and staff are central constituents of the "complex networks" (Holloway and Jöns 2012: 484) that comprise the institution of higher education (Smith 2009; Smith and Jöns 2015). Such

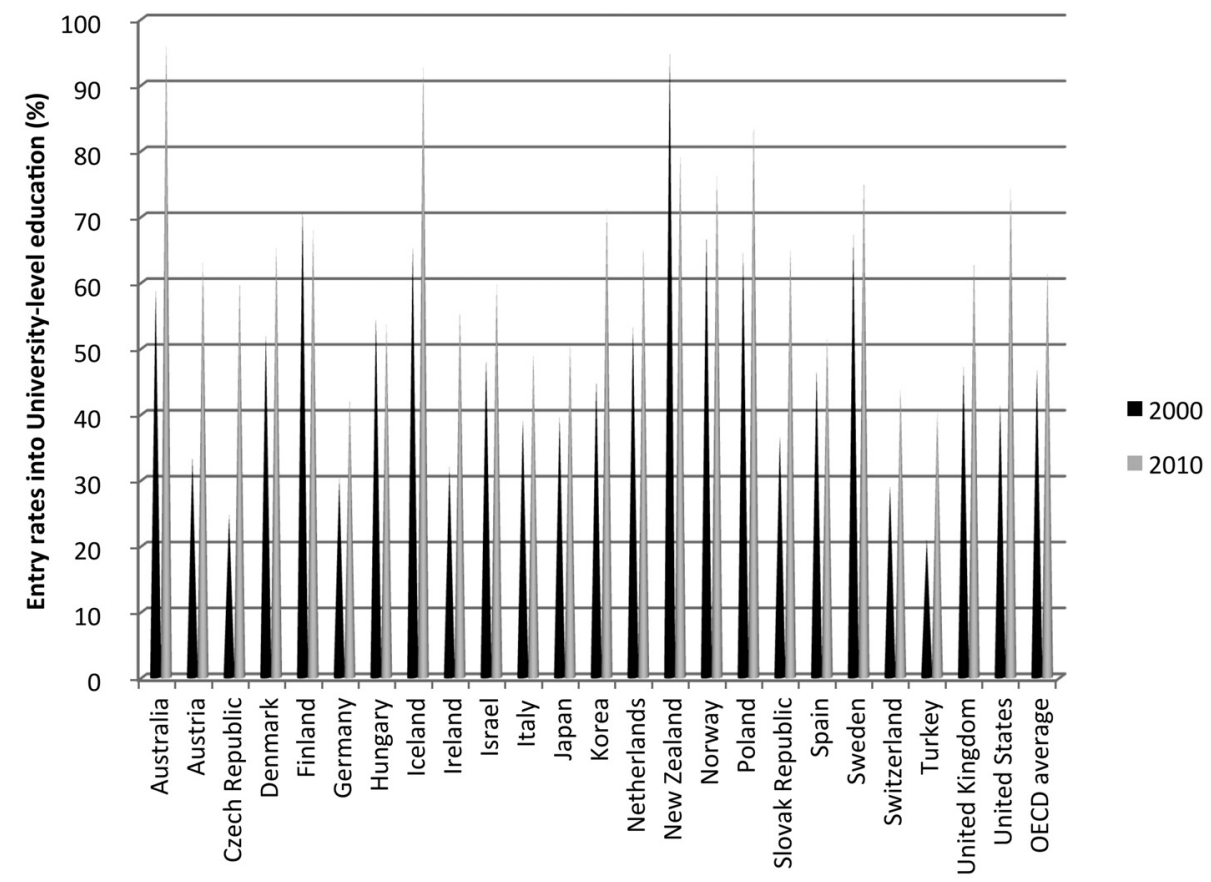

Figure 7.2 Entering university education, selected OECD countries, 2000 and 2010.

Source: Data from http://www.oecd-ilibrary.org/education/education-at-a-glance-2012_eag-2012-en, accessed 04/2014. 


\section{BOX 7.1 LANDSCAPES OF STUDENTIFICATION}

The concept of studentification was coined in 2002 by British Population Geographer Darren Smith. This timely academic neologism sought to call attention to some key geographical consequences of the ongoing mass expansion of higher level-mostly university-education then gathering pace. It is now an especially notable feature across the UK but occurs to varying degrees across the Global North generally. It comes about in part as a result of the strongly life course inscribed migration of mostly young adults to university towns and cities. Studentification illustrates clearly how a Population Geography phenomenon-migration-also has notable Urban, Social, Cultural, Economic and Political Geography consequences.

Studentification refers to "changes resulting from an influx of students within privately-rented accommodation in particular neighbourhoods" (Smith 2002: 6). A number of features merit reiteration. First, it mostly reflects "non-local" students who move to be accessible to their university. "Local" students also express studentification but not to the same extent. Second, the housing tenure involved is overwhelmingly private renting, seen in Section 6.3.3 to be most strongly associated with migration and residential mobility. Additionally, it is private rented accommodation provided largely by private individuals or companies. Accommodation provided by universities themselves-halls, often on campus-has also increased in quantity but residents are not usually classed as studentifiers, the consumption practitioners of studentification. Third, there is a concentration of studentification within small clearly bounded geographical areas. Re-stating, therefore, studentification expresses student migration to quite distinctive urban districts, in order for them to reside in private rented housing during university terms. Whilst long expressing the "classic" practice of UK student migration, studentification now merits its own label because of its heightened geographical impacts.

What, then, are these geographical impacts and consequences? The following diagram depicts some. They are both positive and negative, although it is the latter that are mostly highlighted in local press stories and expressed often quite vehemently to researchers:

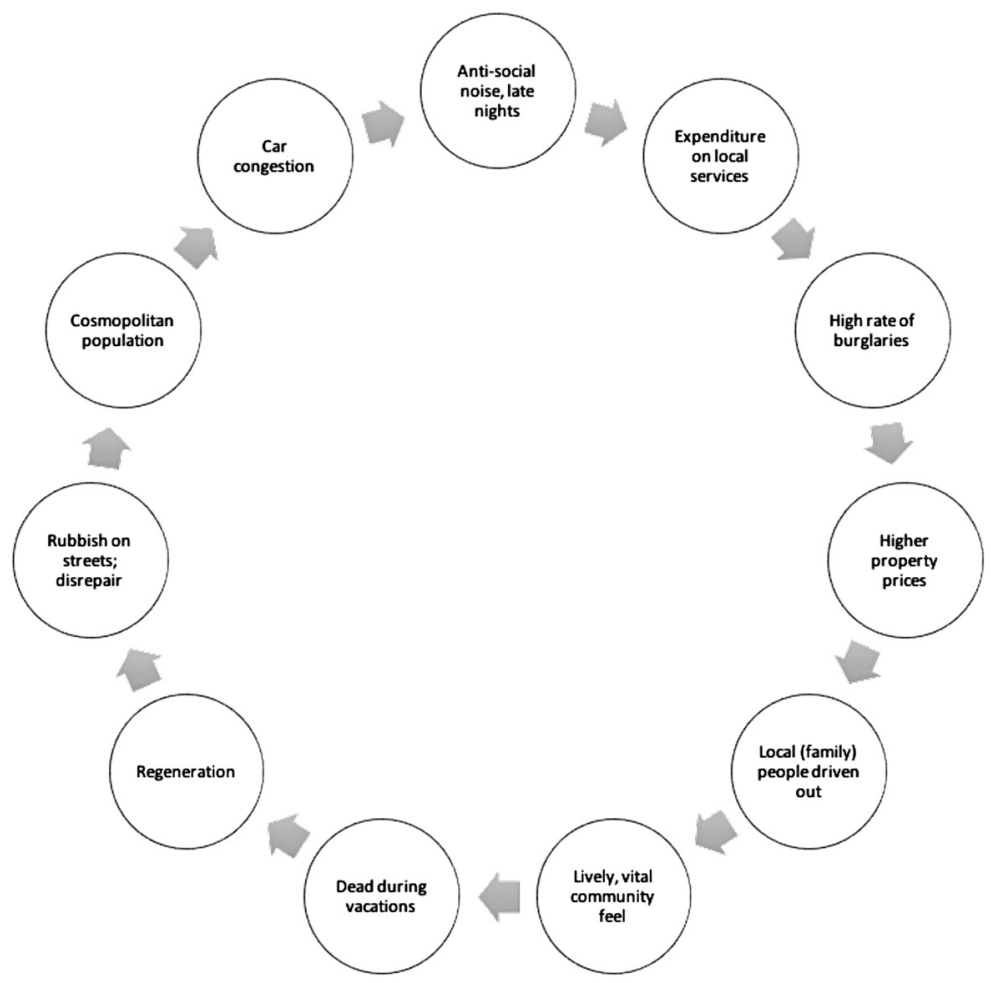

From these impacts, three themes stand-out. First, studentification produces increasingly homogenous urban neighborhoods according to various criteria: housing tenure (reversing the general trend towards increasing owner 


\section{EMPLOYMENT MIGRATIONS}

occupancy), occupation of residents (students), other characteristics of residents (young adults, middle class). Second, there are clear parallels between studentification and gentrification (8.2.3), including class displacement of local residents, perceived local community decline with high population turnover, and enhanced house prices from the economic potential of renting to students. Third, studentification has been noted by local media and politicians. Reports emphasize negative dimensions: late-night noise, general anti-social behavior, community decline, litter on the streets and lack of care and attention paid to house and garden maintenance.

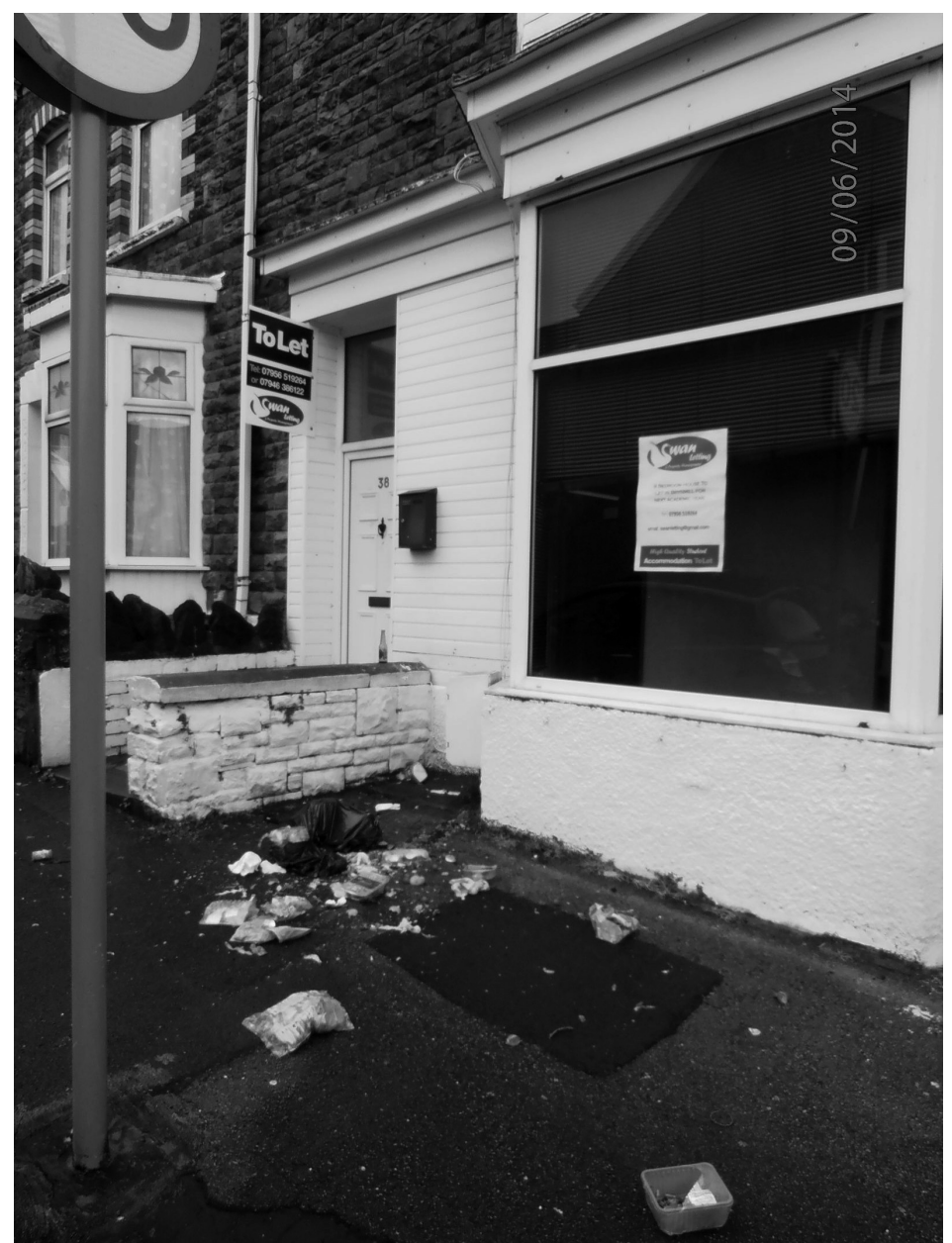

Source: author

Is studentification here to stay? On the one hand, there are attempts to rein it in by building more university halls, strengthening local planning controls on construction of purpose-built student accommodation or conversion of family homes to houses of multiple occupation (HMOs), and strictly enforcing existing planning conditions and other health and safety regulations. On the other hand, the proportion of young adults attending higher education is unlikely to decline in the emerging global knowledge economy. Whilst there is a trend towards attending more local universities (7.2.2), life course sensitivity recognizes strong desire for young adults to express the key life course transition of leaving the family home. It seems, in short, studentification is here to stay, albeit possibly in more regulated and moderated spatial forms. Universities, students, property owners and the local community thus need to reach some compact if studentified urban neighborhoods are to become sustainable.

(Sources: Allinson 2006; Hubbard 2008; Smith 2002, 2009; Smith and Holt 2007) 
mobilities are influenced strongly by policy. Considered below in terms of international student migrations, this is also relevant nationally. Student finance is key. For example, introducing fees or raising existing fees may suppress demand for university places and, hence, overall quantity of internal student migrations. Such developments may also have more complex geographical impacts. In a Canadian study, Greene and Kirby (2012) suggested Memorial University of Newfoundland's relatively low fees as a factor in attracting relatively larger numbers of students than its regional Atlantic coast neighbors with higher fees. Increasing costs may additionally develop an emerging trend away from migrating to university towards studying locally, where possible, or through distance-learning (Waters and Leung 2012). Here, mobilities-material and/or virtual-can displace migrations.

The trend towards local university study in the Global North is in tune with that of growing engagement with university education across the South. For Africa, Teferra and Altbach (2004) estimated that up to 5 million students were enrolled in tertiary educational institutions, demand growing rapidly. Cost factors impinge-both for students and universities-even more acutely than they do generally across the North. This again has both migration and broader mobility consequences.

\subsubsection{International student migration}

As recently as 2003, King and Ruiz-Gelices could claim that student migration was little researched. This is no longer the case, largely due to rapid development of scholarship examining international student flows (Smith and Jöns 2015). Although existing as long as there have been universities (Rivza and Teichler 2007), flows have been increasingly recognized. Indeed, Raghuram (2013) made the argument that as international migration is increasingly governed by whether or not one has the certain skills and knowledge particularly desired by the intended destination country (7.4), "student migration" consequently becomes a much less clear-cut sub-category of such migration.

There are at least five types of international student migration (Rivza and Teichler 2007: 63-64; Waters 2012). First, there is movement to very high status, internationally recognized universities. Detailed choice of destination often reflects (post-)colonial and political ties between origin and sender countries. This type is expressed in students from Asian countries seeking English-medium education in the US, UK, Canada, Australia and New Zealand. Second, there are students who decide to study in a neighboring country, often closely allied by culture and history. Migrations between countries within the EU or East/Southeast Asia express this well. Third, there is short-term study abroad, for a semester or year at most. Typically, the same subject is studied as at the migrant's "home" institution, grades obtained abroad counting towards the home degree. Fourth, there are international student flows stimulated by the destination university's desire to raise funds. Overseas students typically pay higher fees, although they sometimes save money by studying abroad. Fifth, some international student migration is underpinned by (perceived) failure within the domestic system. This may be more relative than absolute, such as UK students migrating abroad after failing to get into the elite Oxford and Cambridge Universities (Brooks and Waters 2009).

Growth of international student migration both reflects and reinforces an internationalization of the higher education sector, understood as "an increase of border-crossing activities amidst a more or less persistence of national systems of higher education" (Teichler 2004: 7). Border-crossings encompass both student migrations and relocations or short- and long-term visits of academic staff (e.g. Leung 2013). All these mobilities, in turn, can be set within (and help produce and reproduce) globalization and mobilities generally (Findlay et al. 2012). Countries respond differently to the challenge of internationalizing, from asserting the independence and distinction of their university sector to cooperating and collaborating with foreign providers (Chan 2013).

International student and staff mobility is strongly facilitated by various charitable, national and transnational schemes. Since 1987, the EU has sought to integrate universities more closely across member countries to enhance the skills of its labor force, promote cultural integration and build a European identity through its Erasmus program. During its first 21 years, 2 million students moved around the EU under this program (Rodríguez González et al. 2011).

Importantly, attention must be drawn to the selectivity of student migrations and their consequences. In spite of the global expansion of higher education, university attendance remains strongly underpinned by social class. In short, going to university remains most closely associated with people from higher status backgrounds. Whilst this link partly reflects monetary resources and costs of attending university, it is also a matter of culture. And student migration, international student migration still more particularly, is still more sharply class delineated for the same reasons. Even within funded schemes such as Erasmus, costs and cultures play crucial roles (Rodríguez González et al. 2011). 
Politics

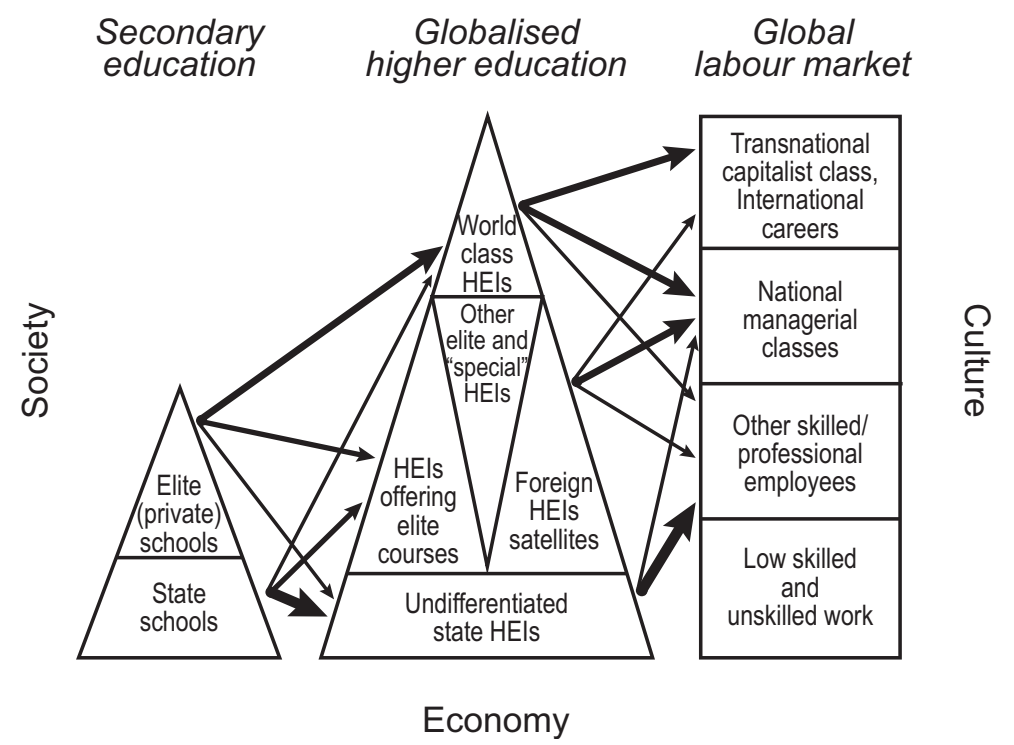

Figure 7.3 Student migrations in a differentiated and differentiating educational and labor market context.

Source: Findlay et al. (2011: Figure 1).

Figure 7.3 expresses the encultured class selectivity of student migration. It positions both internal and international student flows in relation to a differentiated secondary education sector, higher education sector and global labor market. The figure is arranged with privileges and status increasing upwards within the three shapes. The width of the areas gives a qualitative indication of numbers involved. It suggests privilege begetting privilege-elite schools to elite universities to elite jobs-but also how migration reinforces and consolidates these life course paths. For elites, migration and mobility positively shapes their life course. From attending private school, probably as a residential boarder, to studying at a world-ranking university, possibly abroad, to developing an international career with spells in many countries (7.4.3), mobility becomes normal and normative. As Findlay et al. (2012: 122) expressed it, "migrating to learn [becomes] part of the process of learning to migrate," grounding a culture of migration (5.5) presenting mobility as key to career and life course success.

In conclusion, although taking care not to over-generalize, migration for education, whether at university or school (6.3.4), promotes accumulation of educational and other social and cultural capital that facilitates subsequent career development (Waters 2006, 2012). Not only is it shaped strongly by the differential life course arena of class, it is also molded socially by the arenas of gender, age and others, whilst clearly being also a product of history and geography. Thus, also note Figure 7.3's contextual variables of Society, Politics, Culture and Economy.

\subsection{GENERAL EMPLOYMENT MIGRATIONS}

\subsubsection{Speculative versus contracted migration}

The established neoclassical approach to explaining and modeling migration presents potential workers as responding economically rationally to variations in wages across space, migrating from countries or regions with low wages to those offering higher remuneration (5.3.2). It at least implicitly assumes such employment migrants to be speculative (Silvers 1977). In actuality, even in an era of mobilities, contracted migration, where a job at the destination is prearranged (Silvers 1977), is much more common across the Global North (Flowerdew 1992). Even within the US, where speculative migration is assumed to be more established, Cooke's (2011) observed decline in migration is hardly indicative of a flexible, speculative workforce. 
Across the Global South speculative employment migration is more commonplace. It may even involve migrating into unemployment, where just a small chance of eventually obtaining a better job at the destination is worth the economic risk involved (Todaro 1969). This reflects not only the often acute economic hardship experienced by those involved but also economic structural reality, both within the home country and internationally, of a world multiply divided geographi=cally in terms of employment opportunities and prosperity. Increased workplace insecurity or precarity plays an additional role here (Tyner 2015b), undermining any place security provided by stable employment. Whilst leaving home to seek one's fortune may exist mostly in fairy tales across the Global North, it is often commonsense practice in much of the world.

Finally, conclusively distinguishing speculative from contracted migration is frequently very difficult in practice. Most of the relocations covered below will be to a greater or lesser extent "contracted" in terms of employment obtained. Furthermore, Section 7.3.2 next considers some considerable barriers to "free" speculative movement of labor, which qualify any suggestion of it becoming resurgent within an era of mobilities. It also indicates how a relational perspective requires consideration of non-migration as well as migration for gaining a fuller picture of the complex relationships between migration and employment (Green and Shuttleworth 2015).

\subsection{2 "Free" movement of labor?}

\subsubsection{Location-specific capital}

A life course perspective proves extremely useful for helping understand why speculative migration is not more commonplace. Its focus on lives lived within and through diverse contexts highlights the living biographical person rather than the disembodied abstract unit free to move at any time, implicit within neoclassical theory. Entanglements or moorings (Moon 1995) especially reduce potential for speculative migration of all kinds (Coulter 2013; Coulter et al. 2016; Schewel 2015). This is even true for the unemployed.

Politicians largely from the political Right have long advocated the unemployed undertaking speculative employment migration (Southall 1991; Tannock 2015). Being mobile becomes a feature of a person's potential "employability" (Green and Shuttleworth 2015). For example, in a speech to the Conservative Party's 1981 conference, UK Trade Minister Norman Tebbit declared:

The figure of three million unemployed ... is an appalling total. Whether it is measured in economic terms ... or in the equally vital terms of social pressures, the human misery, the frustration and indignity that come with the feelings of rejection and alienation as real job seekers are faced day after day with a fruitless search for work, we cannot ignore the price that unemployment today is exacting ... I know those problems. I grew up in the 30s with an unemployed father. He... got on his bike and looked for work and he kept looking until he had found it.

(quoted in Southall 1991: 272, emphasis added).

That the unemployed should become more proactive in seeking employment through deploying mobility and migration typically links to broader depictions of them as complicit in their situation (Smaje 2013). In turn, this forms part of a morally and politically loaded rhetoric of "deserving" versus "undeserving" poor (Valentine and Harris 2014; Jones 2012). Noting just two of its key features, first, it is frequently expressed spatially (e.g. Tannock 2015). For example, Lewis's (1969) highly influential account of "cultures of poverty" located them within urban "ghettoes," a geographical focus reiterated in the US more recently by Wilson $(1987,1996)$ (Small et al. 2010). Second, such emplaced cultures are noted for immobility in all socio-spatial dimensions, including employment migration. Revisiting both these points, the rest of this section asks not whether such cultures can be recognized but why lack of speculative migration away from geographical unemployment hotspots may, in fact, be a rational strategy for the poor and unemployed, rather than signifying fecklessness.

Staying put, even when unemployed, can express the geographical hold of the diverse ties with which people are entangled within everyday life (Fischer 2002; Moon 1995; Schewel 2015). Three kinds of entanglements stand out. First, if the unemployed person is in a family household then either someone else in the family may still have a job, which could be lost with migration, or someone else may be able to find work locally, the added worker effect. The latter is often gendered, with a husband's unemployment stimulating women's labor-market participation (e.g. Prieto-Rodríguez and Rodríguez-Gutiérrez 2000).

\section{ST PROOFS: NOT FOR DISTRIBUTION}




\section{EMPLOYMENT MIGRATIONS}

Second, personal and family ties are as important for non-migration as they are acknowledged for migration (Mulder and Cooke 2009). Family, friends and even "weak ties" (Granovetter 1973) of regular acquaintances serve to connect people to place in numerous ways. On the one hand, they give everyday emotional support and social interaction. On the other hand, "location-specific advantages" (Fischer and Malmberg 2001) may provide a range of resources for the unemployed person, from casual work opportunities to diverse monetized and non-monetized support, such as child-care, food provisioning and building maintenance work (e.g. Gibson-Graham 2006; Scott 2000; Smith and Jehlička 2007). Local ties seem especially important for those with low skills and qualifications. In an increasingly "flexible" employment context (Green and Shuttleworth 2015), being "just-in-place" can make a potential worker "justin-time," "just-in-case" they are needed (Tunstall et al. 2012).

Third, one must recognize a range of more institutional ties. Some blend into the personal, such as children attending specific schools and families not wishing to break this link (6.3.2). Others are more externally constructed, notably involving housing (Blanchflower and Oswald 2013; Boyle et al. 1998). For example, living in an owner-occupied house in negative equity (Box 6.4) can make moving house, even when unemployed, extremely financially disadvantageous. Likewise, migrating somewhere with significantly higher house prices (often where there are more jobs!). For non owner-occupiers, although generally more mobile than owners (6.3.3), staying in an area can facilitate gaining suitable accommodation. A stayer can acquire local knowledge of geography (where [not] to live) and renters (who [not] to rent from), and also become rated as "local" enough to be eligible for social housing (Hughes and McCormick 1981).

Overall, the concept of location-specific capital is vital for understanding the frequent immobility of labor and its otherwise paradoxical rationality. It refers to "aspects of homes, communities, and networks of friends ... not easily replaced [elsewhere] in the short run" (Paxson and Rouse 2008: 38). Developed over time, such capital accumulation helps explain why immobility is often positively linked to length of residence (Fischer and Malmberg 2001). Speculative employment migration might bring a household economic capital but this may be at the cost of losing location-specific capital. Whilst different forms of capital are not somehow equivalent (Bourdieu 1984) and unemployed (or other) families rarely make precise calculations of whether to move based on weighing up such values, it seems clear that locationspecific capital accumulated from ties and entanglements makes place much "stickier" than neoclassical migration theories suggest. At times, it would even be relatively irresponsible for even the unemployed to migrate speculatively.

\subsubsection{International migration policy}

An international policy perspective on employment migration turns the undeserving poor/culture of poverty "explanation" of the relative lack of speculative employment migration amongst the unemployed still further on its head. In spite of dire ongoing political rhetoric across the Global North of countries being "swamped" by (employment) migrants, actual numbers are far smaller than one might expect from pure neoclassical theory, given the clear South-North economic divides persisting globally (Zaiceva and Zimmermann 2008). In addition, the vast majority of international migrants60 percent according the United Nations (UNDP 2009: 21)-move between nations of similar developmental status. This all reflects the political fact that global free movement of labor is a myth (Samers 2010), at both national and international policy levels. As Raghuram (2013) pointed out, international migration is increasingly governed by whether or not one has certain skills and knowledge particularly desired by the intended destination country. Even if one does, it is often a further requirement to have a specific job to take-up, making it more contracted than speculative employment. Table 7.1 demonstrates such a policy context for New Zealand. It is typical across the Global North in terms of the range of rules and schemes that legitimize certain types of labor migration only. None of these policies favor speculative labor migrants, with the possible exception of the Working Holiday Scheme for young adults.

Useful in organizing understanding of the quantity and quality of international employment migration is the concept of international labor market segmentation (ILMS). Developed by Samers $(2008,2010)$ from Marxian perspectives of migration within a capitalist world (5.4.1), it has three dimensions of labor force division. First, labor is divided globally by individual countries' or groups of countries' (e.g. EU, NAFTA) immigration policies. These favor some countries' migrants but exclude those of others. Second, policies divide labor within countries by favoring migrants with specific skills or characteristics. Table 7.1 demonstrated this. Third, even when recruited, migrant workers are divided from non-migrants both socially and spatially, typically having more precarious contracts and being concentrated into certain locations.

\section{ST PROOFS: NOT FOR DISTRIBUTION}


Table 7.1 Migrating to work in New Zealand

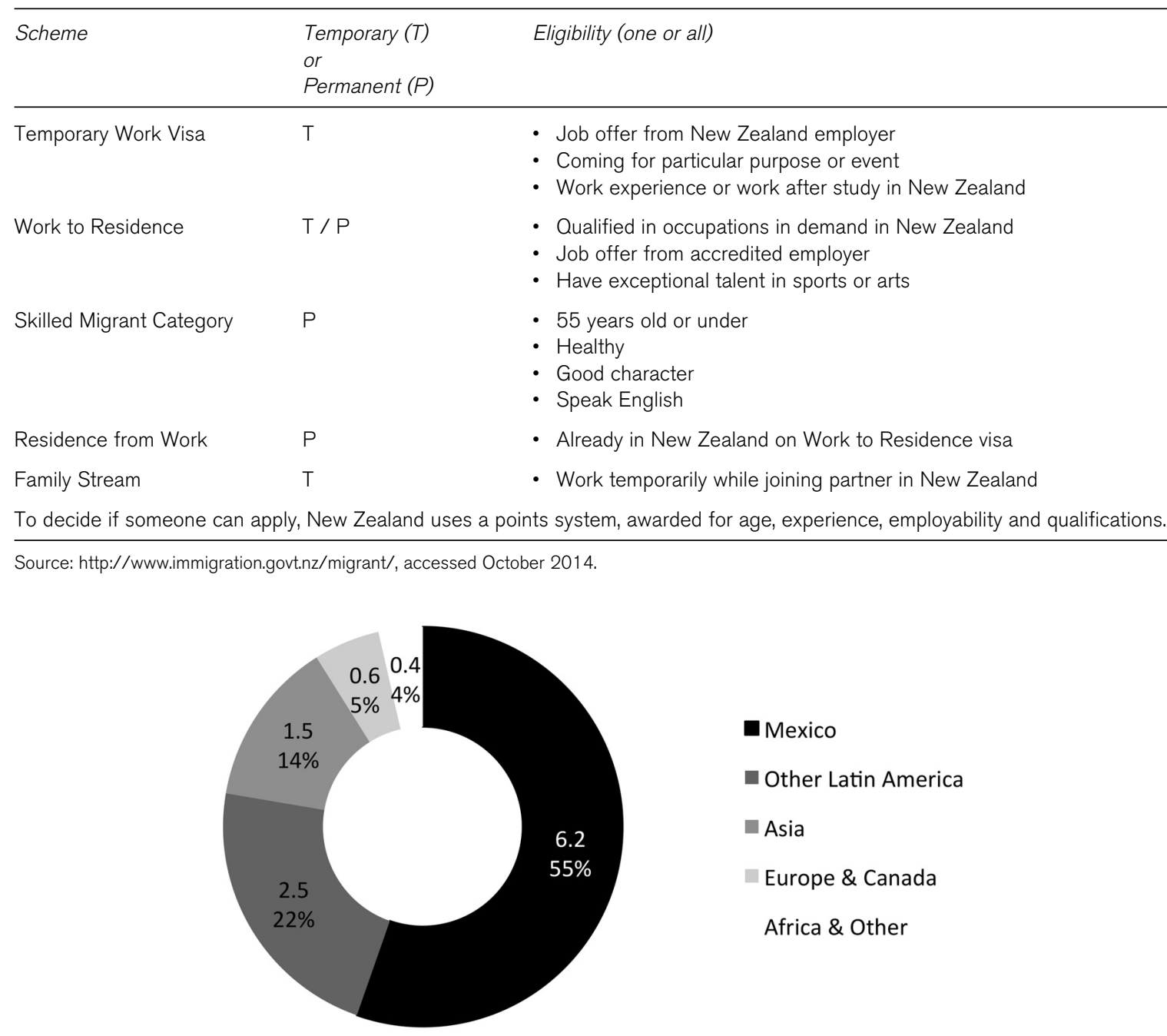

Figure 7.4 Birthplace of US undocumented population, 2005 (Millions).

Source: redrawn from Passel (2006: Figure 4).

ILMS emphasizes the importance of recognizing structural (global economic) governance of all international migration. It qualifies the extent to which employment migration is ever likely to be fully and truly "speculative" in a geographical sense. Yet, even the most ill-informed labor migrant is likely to have some idea where the jobs are and will head that way (Figure 7.1). Employment migration may thus better be regarded as more-or-less speculative and more-or-less contracted, as will become clear from the examples presented below.

\subsubsection{Examples of general employment migrations}

\subsubsection{Within the South}

Across the Global South, labor migration from rural to urban locations overwhelmingly predominates. This largely explains, at least at one level, the rapid increase of urbanization across the South (3.4.1). From the favelas and barrios of Latin America, to the townships and bidonvilles of Africa, to the wasti, kampong liar and katchi abadi of Asia (Asia 


\section{EMPLOYMENT MIGRATIONS}

Society n.d.), promise of better-or even some form of-employment fuels urbanization. This was the case for the equivalent rapid urbanization, slum development and shanty town settlement across the emerging Global North during the Industrial Revolution (Williamson 2002).

Examples of employment migration to major Southern cities (King et al. 2010; Williams et al. 2009: 165-170) could be taken from across the region but there is only space here to develop two illustrative cases. The first involves the substantial labor migrant flows that have demographically transformed China's population geography since the 1980s. The second focuses on the specific but yet more ambiguous fate of young female migrants in Ethiopia.

Feng Hu and colleagues (2011: 64) characterized China's huge migration of labor from rural to urban areas since the late 1970s as "history's largest flow of internal migration." Similarly, for Tilly (2011: 676) it was "the largest and most momentous migration in the world today." In part, this reflects the size and huge population of the country but it is also a response to relaxation of strong official barriers to such migration within the hukou household registration system (Bosker et al. 2012; Poston et al. 2012). Statistics suggest that around half of China's population is now urban, most growth in cities coming from rural to urban migrants, whose numbers had grown to 150 million by the mid2000s (Connelly et al. 2011). These huge migrations reflect, simply, economic disparities between urban and rural China (Poston et al. 2012). They are facilitated by support networks of family, friends and others from the same origin locations (Zhao 2003), a finding that will be shown as commonplace for employment migration.

Interestingly, people's movements have tended to be studied separately from China's urbanization because migrants are assumed to be temporary (Connelly et al. 2011). They are known as the "floating population" (Poston et al. 2012). Again, one witnesses another problematic legacy of defining migration as permanent relocation. Presence of "temporary" migrants in such large numbers, now seemingly institutionalized into cross-generational repeated sets of practices and behaviors, at least in the mid-term, cannot be separated from debates on, for example, the future of China's urbanization. Additionally, substantial numbers of formerly temporary migrants are, as Connelly et al. (2011) put it, "not 'floating' anymore" but have become "permanent" urban residents.

Conventionally, the floating population was thought of as dominated overwhelmingly by men, who left wives and children behind to better themselves economically in the city. However, women now make up around half of these migrants. Furthermore, and challenging another stereotype, they are not all young unmarried women, who will return to the countryside after a few years to complete their adult life course transition to wives and mothers (Connelly et al. 2011).

Analysis using the 2006 Chinese General Social Survey gave more early insight into China's rural-to-urban labor migrants (Hu et al. 2011). Table 7.2 shows, first, migrants still comprising under a fifth of the hukou-designated "rural laborer" population. The vast majority were classed as floating population rather than permanent (urban-resident) migrants. Second, migrants were more likely to be male and younger, and less likely to be married or illiterate than non-migrants. However, being a migrant did not necessarily translate into higher net income (also noted by Zhao 1999). ${ }^{1}$ Comparing permanent with circular (floating population) migrants, shows the former more likely to be married and with higher incomes but less likely to have moved between provinces. Confirmed through modeling work

Table 7.2 Characteristics of China's "rural laborers" according to migrant status, 2006

\begin{tabular}{|c|c|c|c|c|}
\hline & Non-migrants & All migrants & Permanent migrants & Circular migrants \\
\hline Male (\%) & 48 & 65 & 64 & 65 \\
\hline Married (\%) & 89 & 61 & 73 & 60 \\
\hline Illiterate/Semi-illiterate (\%) & 17 & 2 & 1 & 2 \\
\hline Per capita net income (Yuan) & 1,904 & 1,698 & 2,203 & 1,653 \\
\hline Number $^{1}$ & $\begin{array}{l}11,130 \\
(83 \%)\end{array}$ & $\begin{array}{l}2,274 \\
(17 \%)\end{array}$ & $\begin{array}{l}182 \\
\text { (8\% migrants) }\end{array}$ & $\begin{array}{l}2,092 \\
\text { (92\% migrants) }\end{array}$ \\
\hline
\end{tabular}

1 Total number of "rural laborers" $(13,404)$ are adults aged 15-64 not in education or with at least a Bachelor degree.

Source: adapted and simplified from Hu et al. (2011: Table 1). 
(Hu et al. 2011), the small set of permanent migrants suggest a degree of stability and prosperity, as previously more speculative temporary labor migration is overtaken by settlement (Connelly et al. 2011). This apparent "success" is in spite of earlier studies suggesting the floating population often experienced lower occupational attainment and wages than established urban counterparts, possibly partly due to discrimination (Meng and Zhang 2001).

A second example also involves rural-to-urban relocation, reasserting its geographical pre-eminence across the South. Especially in regions such as sub-Saharan Africa, mostly young people undertaking migration to urban centers is not only to search for work-and to escape economic hardships, war and/or the AIDS pandemic (van Blerk and Ansell 2006)-but also to signify the key life course transition to adulthood. By migrating, young adults assume responsibility for their everyday lives (Lauby and Stark 1988; van Blerk 2008). Responsibility may also not be just for themselves. Even relatively young teenage migrants may have dependent family members in their home rural areas, if not the full families of the Chinese floating populations.

Van Blerk's (2008) study of teenage Ethiopian female migrants illustrated the situation well. A number of reasons prompted their moves from rural origins. Negatively, these included escaping traditional cultural practices, failed marriages and the stigma of having sex outside of marriage. More positive was the attraction of engaging in paid work, so they could support themselves and their families, and the lure of an apparently more glamorous urban lifestyle, perhaps encouraged by experiences related by friends or relatives who had already migrated. However, reality could hit immediately. For example, after being met by one of the employment "brokers" based at the bus stations where most teenage girls arrive, 16 year old Berhane was soon introduced to prostitution ("doing business"):

I came by bus to Addis Ababa looking for a job. At the bus station I met a girl who said she could find me a job babysitting. She took me to her room and her friends gave me clothes and eyeliner. They told me to stand outside and a man came to talk to me. That is how I started to do business.

(quoted in Van Blerk 2008: 248)

Evaluating such speculative labor migration gives ambiguous results. Not all migrants enter the sex industry but most do typically experience low wage employment at best, often in domestic or factory contexts (e.g. Camacho 1999; Lauby and Stark 1988). Yet, migration facilitates transition to adulthood and economic independence in the medium term. Even sex work may be regarded as "success." Two of Van Blerk's (2008: 251) respondents, Helen and Alem, expressed ambiguity well:

I come from far and from a very poor family. My mother and I used to sell grains. I came to find work but ended up doing business. I know it can't change my life but it can help me a little and I can help my family.

(Helen, aged 16, Nazareth)

Before we lived a different life-we were so poor. Now I am doing business I can send money home and my family live a better life.

(Alem, aged 18, Addis Ababa)

\subsubsection{From South to North}

Across the Global South, migration to its major cities is not the only expression of more-or-less speculative employment migration. Just as eighteenth and nineteenth-century Europeans sought new work and lives through migration to the settler colonies of North America and Australasia (King et al. 2010), many in the South today seek to migrate North, mostly driven by economics (Samers 2010; Tilly 2011). Indeed, it is a key marker of the Age of Migration (Castles et al. 2014).

Whilst many South-to-North labor migrants will be relatively unskilled, thus largely ineligible for support under officially sanctioned labor recruitment schemes, others will be highly skilled and qualified but may struggle to be allowed to apply these skills in a new political-economic environment, just as they were frustrated from being able to benefit economically from them sufficiently in origin countries (Conradson and Latham 2005; Parutis 2011). Iredale (2001) found this downgrading of professional training and skills often industry or occupation specific, and also gendered. For example, registered nurses (largely female) trained in countries such as the Philippines and Bangladesh who 


\section{EMPLOYMENT MIGRATIONS}

emigrated to countries such as Japan or the US often found themselves working as nursing assistants, receiving "much lower rates of pay than they are entitled to" (Iredale 2001: 15). In contrast, largely male IT professionals were less economically downgraded.

Given a policy context restricting speculative labor migration from South to North especially (7.3.2.2), it is perhaps unsurprising that many of these migrants will be undocumented. Unlike legal migrants, who have "express authorization of [a] national government to reside in the country of destination" (although often not permission also to work; 9.4.3), undocumented migrants "cross international boundaries either without being detected by authorities ... or . . . overstay their visas" (Samers 2010: 11). They are also known as irregular, clandestine, unauthorized or illegal migrants, although the latter term is politically disputed by campaign groups which challenge the idea of migrants being "illegal" (e.g. No One is Illegal 2016).

Undocumented labor migrants can be very attractive to employers, who often seek "workers who are cheap, docile, reliable, productive, and ... [have] no 'baggage' whatsoever, including children" (Samers 2010: 147). They epitomize the precariousness of much contemporary employment even in the world's richest countries (Tyner 2015b). Their employment is typically undertaken under relatively "informal" conditions, with employees lacking the (limited) rights and security associated with having appropriate documentation, such as social insurance numbers or identification cards.

Sectors most associated with undocumented employment overlap with both documented and contracted employment within "ethnic/immigrant niches" (Samers 2010: 150). They include:

- agriculture: from meat packing and processing to intensive market gardening

- clothing/textiles;

- construction;

- landscape gardening;

- food processing;

- retail, notably small grocers and street selling;

- care, cleaning, catering-the "three Cs";

- sex work; and

- $\quad$ anything dirty, dangerous, (physically) difficult or demeaning.

Examples from this list have already been noted and others will emerge later. Two now illustrate how hard it is to separate conclusively speculative international labor migrants from all migrants, yet also how economically important such migrants nonetheless are. Both examples demonstrate:

[m]igrant workers ... caught in the cross-fire of contemporary capitalism. On the one hand, advanced capitalist economies can't live without devouring the rich resources of cheap labour located in, and coming from, the poorer parts of the world. On the other hand, (so called) advanced capitalist polities find it hard to deal with the consequences of living with strangers. Politically at least, foreigners are best and most easily exploited abroad.

(Wills et al. 2009: 257).

Presence of labor migrants originating from Mexico and other Latin American countries is a widely noted feature of the US's contemporary population geography (Martin and Midgley 2003; Massey 1995a; Shrestha 2006). The US acts as a magnet to all countries to its south. It is primarily the prospect of work within the world's largest economy that draws their migrants. Consequently, whilst Mexico is the main country of legal immigration to the US, with Mexicanborn migrants comprising nearly one fifth of that total in 2004 (Shrestha 2006), the Mexican-US border is also a key location for attempted illegal entry (Andreas 2009).

Flow of undocumented labor migrants from Mexico-not least younger, less qualified males from rural areas (Sanchez and Pancheco 2012)-expanded rapidly after 1976, due to rising US wages and a weak Mexican Peso (Martin and Midgley 2003). This was boosted from 1982 by the collapse of the Mexican economy. US employers "assemble[d] crews of Mexican workers to harvest crops, clean buildings and work in construction jobs for low wages" (Martin and Midgley 2003: 32; Passel 2006), becoming reliant on this "cheap, reliable, and disposable labor force" (Andreas 2009: 37). Even establishment of the North American Free Trade Agreement (NAFTA) in 1994, supposed 


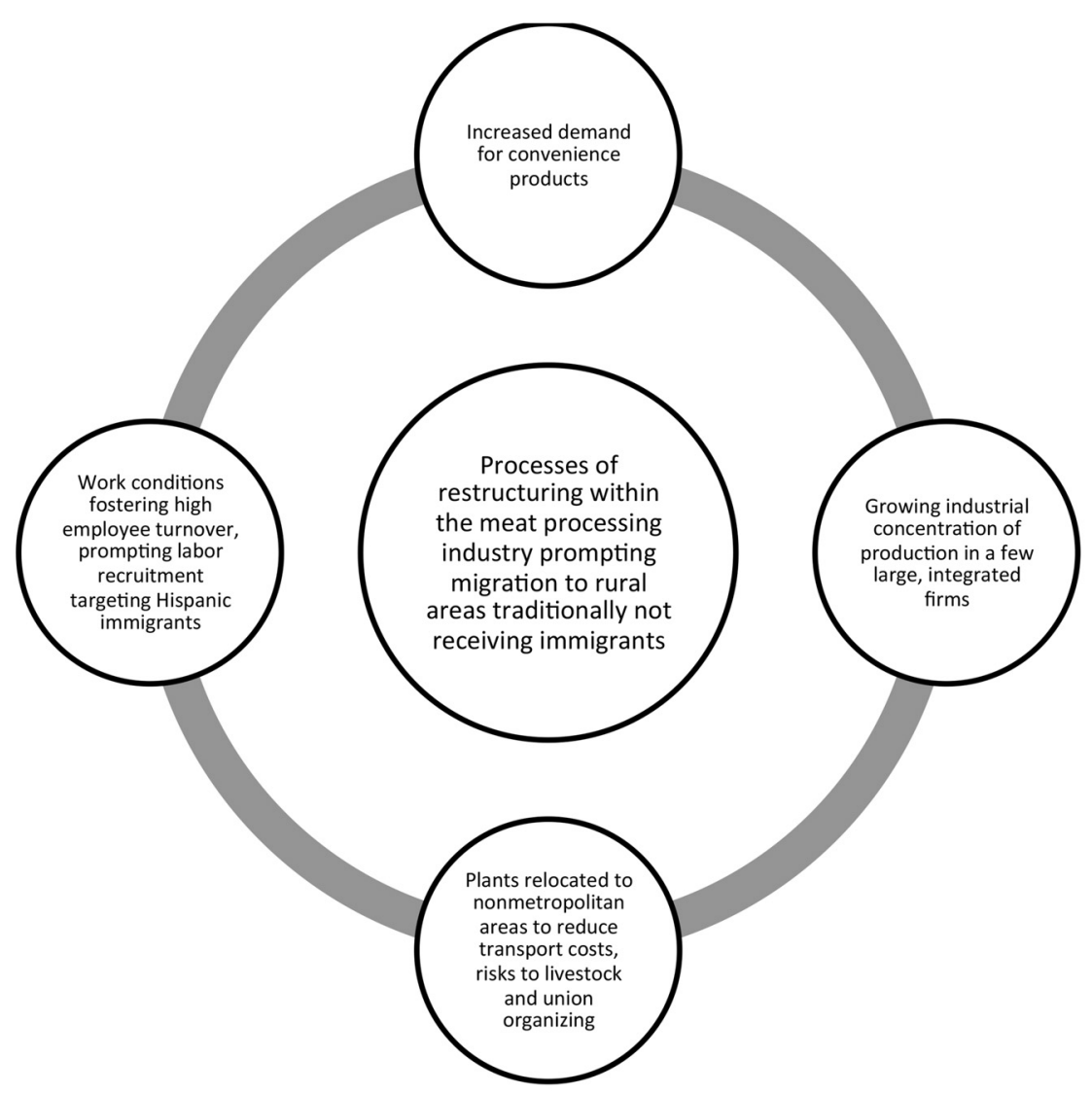

Figure 7.5 The restructuring US meat industry's labor migration consequences.

Source: summarized from Kandel and Parrado (2005: 452).

to boost employment opportunities in Mexico (although the country fell into recession in 1995), and stricter border controls did not significantly dampen immigration (Andreas 2009; Martin and Midgley 2003). In 2005, shown in Figure 7.5 there was an estimated 6.2 million undocumented Mexican immigrants in the US, over half the undocumented total, with a further 2.5 million from the rest of Latin America (Passel 2006). By 2012, it was estimated that this had risen to 6.7 million, 59 percent of the total (Baker and Rytina 2013).

The Population Geography impact of Mexican and other Hispanic immigrants in the US is no longer predominantly just associated with border states such as California, Arizona and Texas. As part of broader migration into the US South and beyond (Winders 2005)-into "the heartland," as journalist Rubén Martínez (2001: 186) put it-an interesting development is migration to nonmetropolitan towns (Vias 2012). These towns are characterized by historically entrenched population decline, brought about by out-migration of young adults and consequent population ageing (3.4.3). Yet, since the 1990s, rural population loss has been checked and reversed in some locations. This is not through lifestyle counterurbanisation (8.3.2) but from immigration of mostly Hispanic labor and their families. The Hispanic population of the nonmetropolitan US rose from 2.6 to 3.8 million between 2000 and 2010, from 5.4 percent to 7.5 percent of the total (Carr et al. 2012). Migrants were pulled in by employment opportunities from restructuring agribusiness operations, such as meat processing (Kandel and Parrado 2005; also Tannock 2015) in states such as lowa. This was at least initially supported by local politicians. Such is the demographic change that the question has been raised as to whether such immigrants-predominantly young, of child-bearing age and often with children, thus supporting local services such as schools-might be the "savior" of dying rural communities (Carr et al. 2012). Longterm revitalization, requires, however, re-imagining of US rural and Southern cultural and ethnic identity and belonging. 


\section{EMPLOYMENT MIGRATIONS}

This is proving extremely challenging within the intense debates about borders and immigration in the wake of 9/11 (Winders 2005, 2007).

A second example introducing something of the practices of more speculative and often undocumented Southto-North labor migration comes from a survey of 341 low paid workers within the economically polarized global city of London, UK. These workers were engaged with cleaning, hospitality and catering, home care and food processing (Y. Evans et al. 2005; May et al. 2007). The survey found 90 percent born outside of the UK, half arriving in the previous 5 years. A wide range of circumstances brought them, mostly directly, to London-asylum seeker, au pair, working holidaymaker, student, partner of British citizen-but around a quarter came directly in search of work. Whilst some fitted the classic profile of the lone labor migrant, most were not living alone, over half were aged 30 years or older, and up to a quarter lived with dependent children.

On arrival in London, networks of family and friends usually helped migrants find work. Such channeling also reinforced ethnic and gender segregation within the labor market, suggested by the ILMS (Hagan 1998; Samers 2010). Moreover, it is important to reiterate how such workers typically experience the most precarious employment situations, "involving job insecurity, limited statutory entitlements, both in the workplace and to social benefits, low wages and high risks of ill-health" (McDowell et al. 2009: 7). Table 7.3 gives a further sense of this precariousness through a profile of five undocumented Pakistani men smuggled into the UK (Ahmad 2008). Furthermore, as in the US, presence of especially undocumented labor immigrants from the Global South-"the non-European, the non-white and those who arrive without the papers to work" (Wills et al. 2009: 257)-has been subject to widespread political debate. A 2008 opinion poll placed immigration as the top concern for over 40 percent of the UK electorate and all recent Governments have sought to impose ever tighter immigration controls (Anderson 2010; Wills et al. 2009). Nevertheless, speculative labor migrants still arrive daily and the political debate continues.

\subsubsection{Within the North}

Migrants from Eastern Europe featured prominently in the previously discussed London study. It is to such employment migration within the Global North, itself strongly internally differentiated economically, that the chapter now turns. With increasing legal barriers to migrants entering from the South, already having legal residence within the North, whether through being born there or acquiring immigration documents allowing both residence and work, gives international labor migrants a head start. The outcomes of their migrations, however, are often little different from those of the undocumented, as the London study implied.

In 2004, the European Union (EU)-EU15-expanded to include the so-called A8 states-Czech Republic, Estonia, Hungary, Latvia, Lithuania, Poland, Slovakia, Slovenia-and in 2007 to embrace the A2-Bulgaria and Romania. All were formerly part of the Eastern European bloc dominated by the Soviet Union. In 2004 they retained varying degrees of economic hardship, both in absolute terms and relative to existing EU members (Boeri and Brücker 2005: 633). Consequently, although free movement of labor is supposedly one the EU's principles, it was feared that A8 and A2 accession would see a huge influx of labor migrants into richer EU countries. Individual member states were allowed to impose temporary restrictions-transitional arrangements-on A8 and A2 workers for up to 7 years (Boeri and Brücker 2005).

Table 7.3 Profiles of smuggled male Pakistani migrants in London, UK

\begin{tabular}{llllll}
\hline Name & Age & Education & Marital status & Occupation before migration & Current UK employment \\
\hline Cha-Cha & 50 & & Married & Owner of several clothing shops & Meat shop/garment factory worker \\
Aaloo & 28 & & Married & Factory worker & Chicken shop worker \\
Rashid & 28 & BA & Single & Property development & Supermarket/newsagent \\
Wasim & 30 & Metric & Single & Small shop owner & Chef in take-away restaurant \\
Hamid & 23 & Up to age 11 & Single & Agriculture (rice/wheat) & Construction \\
\multicolumn{2}{ll}{ All were smuggled to the UK in 2003-2004 and were now legally resident but working illegally. }
\end{tabular}

Source: simplified from Ahmad (2008: Table 1). 
Whilst Eastern European migrants were far from absent within the EU prior to joining, accession saw a boost in numbers notwithstanding transitional arrangements (Green and Shuttleworth 2015). A8 and A2 migrants into the EU15 increased from accession levels of about 1 million from the A8 in 2004 and nearly 2 million from the A2 in 2007 to 2.3 million and 2.6 million, respectively, in 2009 (Holland et al. 2011; Kahanec 2012). Figure 7.6 illustrates this boost with the steepening of the graphs. By 2009, however, A8 and A2 nationals comprised just 1.2 percent of the EU15 population, although representing nearly 5 percent of the populations of the A8 and A10 countries.

When considering migrant origins and destinations in greater detail (Kahanec 2012), Poland and Romania were by far the most important sending countries, accounting for three quarters of A8/A2 migrants in the EU15. This in part reflected the large populations of these countries. Germany and the UK were principal destinations, hosting nearly two thirds of A8 and A2 emigrants, although the latter also favored Italy and Spain. Over time, A8 citizens have increasingly favored the UK, Ireland and Spain, and A2 citizens Spain and Italy, both at the expense of Germany and Austria, especially. Kahanec (2012) suggested this may reflect, in part, the growth and power of immigrant networks in migration decision-making, a key theme reiterated throughout this chapter.

Attention now turns to consider two of the most prominent of these migrant streams in more detail. First, Polish migration to the UK was an option taken up early on, as the UK, unusually amongst EU states, allowed A8 nationals to enter the country with few restrictions (Drinkwater et al. 2006). Studies of Polish migration to the UK are now numerous. They provide answers to the "what" and "why" questions: numbers of migrants, spatial patterns, and motivations (e.g. Drinkwater et al. 2006; Parutis 2011; Trevena et al. 2013).

Research has also explored "how" Poles migrate (e.g. Ryan and Sales 2013) and "how" their lives play out as migrants (e.g. Szewczyk 2013). Parutis (2011), for example, showed Poles highly mobile within the labor market but roughly seeking to follow a trajectory that shifted from speculative "any job" to "better job" and eventually, hopefully, to "dream job." Elsewhere, Callender (2012) focused on the lives of young adult Poles in Northamptonshire. He revealed the transformative embodied experience labor migration presents, with relocation reinforcing identities simultaneously being constructed through contextualized life course transitions such as taking up paid work, getting married or having children. Callender (2012: 227) went on to propose a "turbulent transition," defined as occurring when there are "major changes within biographies as a result of migration ... made quickly with scarce access to support structures and in environments that contain high levels of perceived risk." Although now a well-trodden path-after 2004 migration to the UK became strongly normative for many young Poles-there remains much uncertainty for the migrant "performer" (Halfacree and Merriman 2015) to negotiate. Labor migration always represents a potentially risky "stepping-stone" (after Szewczyk 2013) within a life course journey.

Other research on Polish migration to the UK includes studies of migrant place-making. Attaining a comfortable sense of place, aided by heterolocal (6.3.4) features in the landscape, such as the Catholic church and Polish clubs,

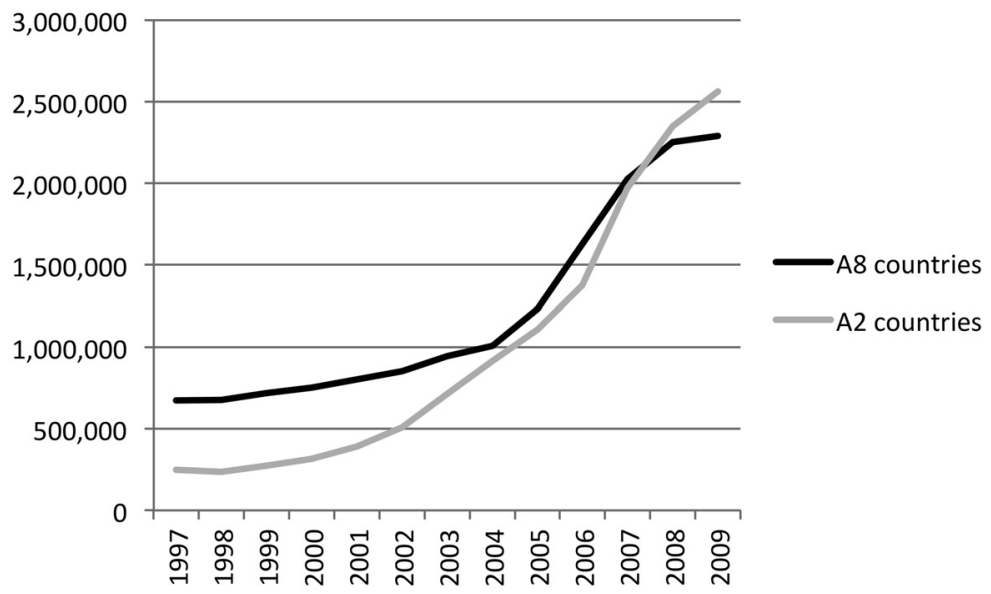

Figure 7.6 Eastern Europeans resident in EU15, 1997-2009.

Source: from data in Kahanec (2012: Table 1). 


\section{EMPLOYMENT MIGRATIONS}

is a highly skilled and negotiated accomplishment (Gill 2010; Szewczyk 2013). It also involves developing complex links with family and places in Poland (Ryan and Sales 2013; Ryan et al. 2009). Back in the UK, Gill and Bialski (2011: 246) argued that weak associational ties so vital for Poles to "get by" (jobs, housing, social life) typically emerge from "a tyranny of micro-geography and happenstance." Migrants improvised quickly within a "chain reaction" of friendshipmaking:

You go to work, you can search for some work, and it turns out that they gave you that work because you live above that hotel. And you're lucky enough to live with some Slovak guy or a Polish guy. The Polish guy already has some friends somewhere. You go somewhere out for a beer with him and you meet some other friends of his, and that network quite quickly gets created. And if you don't know any Polish people then obviously you create these connections with some English people.... So really, you can quite quickly create some acquaintances. I don't know anyone who would be alone or live alone.

(Grzesiek, quoted in Gill and Bialski 2011: 246)

As a second example, Spain has been a main destination for Romanian employment migrants before and after 2007 (Box 7.2). Its shift from a country of net emigration to one attracting international labor migrants occurred from the late 1980s (Arango 2000), when the economy boomed. This buoyant economic context combined with an ageing domestic population, a large informal sector and highly segmented economy, plus inexperience in managing immigration, attracted labor migrants from the youthful populations of North Africa, former colonies in South America and elsewhere (Şerban and Voicu 2010). Romanians took advantage of this situation.

\section{BOX 7.2 THE MIGRANT "FLOOD” THAT NEVER WAS}

On 1 January 2014 the UK lifted the transitional arrangements that had restricted migration from the EU's newest two members, Romania and Bulgaria, to the UK. Reflecting the "hot" political status of migration, right-wing tabloid newspapers were up in arms. Headlines included:

- Draw a Red Line on Immigration or Else! (The Sun, 18 December 2013)

- Cheeky Beggars

- Romanians' crime vow on first bus to the UK

- $\quad$ Migrant: 'England is our country as well now' (The Sun 31 December 2013)

- I'm from Romania, I came to Britain for the hospital": beggars sleeping rough around Marble Arch are arrested by UK Border police just a day after immigration laws are relaxed (Daily Mail 2 January 2014)

- Now voters turn against EU in record numbers as floodgates open for Romanian and Bulgarian migrants (Daily Mail 4 January 2014)

A reader of such stories could have been forgiven for concluding that the entire population of these two countries-around 28 million people-were about to pack their bags and emigrate to the UK! Reality, however, was very different. It reinforces the impression throughout this chapter that it usually takes more than just potential for higher wages, perhaps backed up by the benefits of a social welfare system (such as health care), to bring about international residential relocation. International employment migration comprises a more complex and nuanced quality of life experience than simplistic headlines suggest.

Focusing on Romanians, joining the EU in 2007 did see considerable employment migration to other EU countries where transitional measures were either not in place or less strict than in the UK. That year alone, over 500,000 left the country and only 100,000 returned home. However, emigration then declined, with pent-up demand satisfied. By 2012, only 170,000 emigrated; nearly as many returned. Economic problems in popular destinations, such as Italy and Spain, "tarnished" any "dreams of a better life" (Fontanella-Khan 2014) for many. And changing economic conditions in Romania, including export success with automobiles and agriculture, improved economic prospects at home considerably. On top of this, perception of the UK is a prejudiced country- 
derived not least from the same media reports and right-wing political rhetoric noted above-deterred Romanians from contemplating coming to the UK.

Consequently, the predicted "flood" never came. Instead, there was the absurd media spectacle of journalists, TV crews and even MPs waiting at Luton Airport for the arrival of the first flight from Romania-Wizz Air W63701 from Târgu Mureş-following the UK's lifting of restrictions. The overwhelming majority of the 146 passengers on the three-quarters full plane were returning from Christmas break to jobs they already had in the UK. Only a handful were taking advantage of the lifting of border controls. Of this handful, all were arriving to work rather than to take advantage of the UK's social benefits system-of which they often knew little-and had clear intentions ultimately to return home. Victor Spirescu was one such arrival from the village of Pelisor in Transylvania, where his wife awaited his return "very soon with a lot of money." Bemused by the media reception, he summarized his position thus: "My God. Man, I don't come here to rob your country. I come to work. ... To make money to go home."

As to the future, it is likely that substantial numbers of both Romanians and Bulgarians will continue to undertake international employment migration within the EU, just as will nationals of the other EU states. This assumes extremist political parties are unable successfully to impose exclusionary policies. By far the majority of this migration will be for employment, institutionalized within the sorts of migration networks noted throughout this chapter. Yet, such migration is rarely ultimately one-way and substantial, near-balancing flows of return migrants are also likely to continue. More generally, one should also note-not least in the context of the considerable economic inequalities across the EU-that labor migration within Europe is, in fact, relatively modest and sharply skewed towards the young and better educated. It is appropriate to leave the final word to Florin Alexandru, a 30 year old Romanian chef:

I love my country. I don't want to live abroad all my life, so I go for a few months to work in a restaurant or on a cruise ship and then I come home... Tell the Brits to relax: we are not coming to steal your jobs and welfare.

(Sources: Davies and Malik 2014; Fontanella-Khan 2014)

The 2002-2006 period consolidated Spain as the second most attractive destination, after Italy, for Romanian labor migration, accounting for around a quarter of emigrations (Şerban and Voicu 2010). By March 2008, nearly 665,000 Romanians were registered in Spain, 16 percent of all immigrants and the second biggest group after Moroccans (Elrick and Ciobanu 2009). This exceeded 800,000 by 2011 (Rodríguez-Planas and Farré 2014). Of central importance in establishing, consolidating and expanding Romanian employment migration to Spain-which continued even after the Spanish economic collapse of 2007-2008 and later temporary restriction on accepting Romanian labor migration (Buck 2014; Rodríguez-Planas and Farré 2014)-have been the already flagged migrant networks. These often link specific villages in Romania to destinations in Spain (Elrick and Ciobanu 2009; Şerban and Voicu 2010). Entering Spain legally with a tourist visa, Romanians then overstayed and were helped by already established relatives and friends both to live (e.g. providing floors to sleep on) and to enter the labor market. They, in turn, became established, often aided by Spain's legal regularization of undocumented immigrants, attaining a position themselves to assist newer migrants.

Networks established between Romania and Spain also "trigger" migrations (Șerban and Voicu 2010: 101-102). First, they provide the kind of detailed information about the destination widely recognized as vital for migration decisionmaking (5.3.3). Second, a culture of migration (5.5) becomes established. This encompasses such things as consumption and other lifestyle behaviors of migrants at the destination that may be quite different from behavior at home but is only accessible through migration (also Marinov 2015 for Bulgarian Roma). "Migration" becomes part of the "behavioural repertoire at the community level," prompting "simple imitation to rite of passage" (Şerban and Voicu 2010: 102). Third, monetary remittances impact on the social order of the home community. Households receiving monies from relatives abroad can rise in prosperity and status, prompting households not involved in migration to send their family members to earn money abroad.

\section{ST PROOFS: NOT FOR DISTRIBUTION}


170 EMPLOYMENT MIGRATIONS

Table 7.4 Immigrants in Spain 2000-2011 in low-skill occupation groups (\%)

\begin{tabular}{|c|c|c|c|c|}
\hline & \multicolumn{2}{|c|}{ A8/A2 Immigrants } & \multicolumn{2}{|c|}{ Other immigrants } \\
\hline & Men & Women & Men & Women \\
\hline Food services & 3 & 18 & 6 & 11 \\
\hline Sales & 1 & 3 & 3 & 6 \\
\hline Construction & 31 & $<1$ & 17 & $<1$ \\
\hline Manufacturing & 19 & 7 & 16 & 4 \\
\hline Domestic services & 1 & 35 & 2 & 25 \\
\hline Agriculture and fishing & 4 & 1 & 2 & $<1$ \\
\hline Other unskilled & 27 & 25 & 21 & 20 \\
\hline
\end{tabular}

Source: Simplified from Rodríguez-Planas and Farré (2014: Table 3).

In summary, for Romanians in Spain, the net result of such network agency is that, "with every new migrant, the network resources become available to a new set of relatives and friends ... [and] migrants came one after another, repeating and proliferating the positive experience of the predecessors" (Şerban and Voicu 2010: 101, 105). Every new migrant adds to the human capital of the network, even if their personal involvement eventually wanes (Şerban and Voicu 2010: 102).

Diverse Romanians have come to Spain in search of work. However, detailed analysis by Ambrosini and colleagues led them to describe more recent flows as "characterized by negative selection" (Ambrosini et al. 2011: 6). They have been skewed in favor of the low skilled and less educated, often with lengthy migration histories and frequent engagement with informal or illegal employment. Combining Romanians with immigrants to Spain from other A2 and A8 nationalities, Rodríguez-Planas and Farré (2014) discovered from Labor Force Survey data that only around 5 percent of men and 6 percent of women worked in medium- or high-earning occupations. Table 7.4 profiles the types of low skilled jobs most were involved in. A key role was played by construction for men-often a "gateway" or Parutis's (2011) "any job" for speculative labor migrants, not least when there is an ongoing building boom, as in Spain in the early 2000s. For women, the key sector was domestic service (nannies, nurses, housekeepers). For both sexes, the second main employment category was miscellaneous "other unskilled jobs," encompassing janitors, drivers, warehouse workers and so on. The table also shows the gendered skew in favor of certain jobs much stronger for A8/A2 immigrants than for other immigrants.

Finally, working on southern strawberry farms has been a form of employment associated with Romanian labor migrants to Spain. There is rural-focused labor migration besides relocation to urban centers (Green and Shuttleworth 2015). Many of these migrants were recruited through guest worker programs, which emphasized the employment's temporary nature. As with Poles in 2004, however, many strawberry workers left the fields for better jobs once Romania became an EU member. They have increasingly been replaced by Moroccans, demonstrating ethnic dynamism within an ongoing international labor market segmentation (ILMS) system (Mannon et al. 2012).

\subsection{SPECIALIST EMPLOYMENT MIGRATIONS}

\subsubsection{Introduction: from speculation to contract}

Although initially distinguishing speculative from contracted employment migration, Section 7.3 ultimately adopted a loose interpretation of the former. This was both because truly speculative labor migration is very rare, especially when the constitutive roles of networks and political constraints are taken into account, and due to great difficulty in sharply distinguishing speculative labor migrants, whether in terms of occupations or workplace experiences. Indeed, except in life-critical situations, covered in Chapter 9, wholeheartedly speculative migration is likely to be regarded as rather socially irresponsible, expressing lifestyle migration (Chapter 8) more than conventional forms of employment migration. Consequently, most labor migration involves some element of "contract," whether or not expressed through formal employment agreements entered into prior to the move or in terms of encultured expectations of success, life course transition and development. 
This section focuses on expressions of employment migration unambiguously contracted. It turns from less skilled employment outcomes-if not necessarily migrants themselves-to illustrate mostly, but not exclusively, more middle class experiences. All involve high levels of specialism, whether indicated by formal credentials or more practical skills. Attention also shifts from migrants born mostly in the Global South or the poorer parts of the North to those from richer countries.

\subsubsection{Employment careers and spiralism}

Developing a distinctive employment career became crucial to a typical middle class life course since at least the emergence of capitalism and the boost it received with the Industrial Revolution. Following Greenhaus et al. (2010: 9), such a career expresses "the patterns of work-related experiences that span the course of a person's life." For long-term "success" in such a career, commitment, planning and flexibility are required from the person concerned in shaping life course experiences (Hall 2002; Shaffer et al. 2012). ${ }^{2}$ Developing a successful employment career typically includes willingness to engage in residential migration: "[m]oving away for a new job or another opportunity ... can often mean moving up the social ladder" (Adey 2010: 37). Locating this link within a broader mobilities context, Adey (2010) also cited Morley's (2000: 202) observation of how mobility "is increasingly seen as a social good-[,whilst] immobility increasingly acquires ... connotation of defeat, of failure and of being left behind."

Spiralism (Watson 1964) is a concept linking residential migration to employment career development, specifically to upward social mobility or positive change in social status or position. Most associated with managerial and professional fractions within the middle class (compared to less mobile small business owners and other self-employed), spiralism is the practice of frequent residential relocation to develop one's career (Fielding 2012; Green and Shuttleworth 2015). Indeed, numerous relocations are often expected of such careers, especially in early years. Each migration should either directly or indirectly lead to economic betterment and potential promotion within the workplace. It is facilitated by, just as it serves to develop, a class-based culture of migration that normalizes relocation, in contrast to sedentarist cultures of non-migration more relevant in other social contexts (6.3.3). As already noted, a spiralist culture of migration is often initiated by moving away for university study (Fielding 2012: 112), possibly abroad.

Looking closer at spiralism, it can be linked to the first two of Brown's (1982) three career strategies: organizational, occupational and entrepreneurial. Whilst not mutually exclusive, organizational strategies involve obtaining upward social mobility through promotions within an employer's internal labor market (ILM), whilst occupational strategies involve moving upwardly within a profession, often changing employers. Entrepreneurial strategies involve becoming self-employed or an employer and, in contrast, are often grounded in non-migration.

The classic geographical location of "organization man" (sic.) (Whyte 1956) is Japan, with its lifetime employment system granting tenure of employment within an organization to elite employees (Boyle et al. 1998). Wiltshire (1990) highlighted the importance of personnel transfers within Japanese organizations. Considerable mobility, both occupational and spatial, was the price tenured employees paid for "protected" status, expressed in long chains of linked moves. Wiltshire gave an example from the Employment Security Bureau of the Ministry of Labor, whereby ten moves resulted from a section chief's retirement. Recent years, however, have seen the lifetime employment system decline, especially among highly educated workers (Hamaaki et al. 2012). This reflects, not least, reduced "credibility of implicit promises of future wage increases" (Hamaaki et al. 2012: 840) stemming from Japan's recent economic problems.

Japan's changes also reflect more general decline in organizational strategies within a more "flexible" economic environment, whereby neo-liberal ideas such as the "boundaryless career" (Arthur and Rousseau 1996) have been proposed (Roper et al. 2010). Consequently, whether or not actively chosen, occupational career strategies for the middle class involving frequent relocation remain commonplace. Within the university sector (Kim and Locke 2010), for example, Table 7.5 gives a clear sense of the importance of international migration for seven UK-based career academics. Note, though, how both career and migration were also entangled with other life course aspects.

With a focus on geography, a further valuable concept for understanding combined socio-spatial mobility is the escalator region. Proposed by Tony Fielding to account for patterns of migration to and from South-East England (Fielding 1992b), it has broader geographical applicability (Champion et al. 2014; Green and Shuttleworth 2015). Fielding (2012: 107) defined escalator regions as "regions/countries that usually contain large metropolitan cities (for example, global cities such as London, New York and Tokyo), and that act as 'engines' of promotion into

\section{ST PROOFS: NOT FOR DISTRIBUTION}


172 EMPLOYMENT MIGRATIONS

Table 7.5 Migration backgrounds of jnternational academics working in universities in North West England 2011

\begin{tabular}{lllll}
\hline Participant & Subject & Origin country & Other countries & Initial reason for coming to UK \\
\hline A & Physics & Nepal & Japan, Italy, Germany & 5-year research fellowship \\
B & Biosciences & Germany & US & Lectureship \\
C & Computing & Iraq & UAE & Doctorate, later IT consultant \\
D & Social care & Iraq & & Escape atrocities and persecution \\
E & Psychology & Germany & & Lectureship \\
F & Psychology & Greece & "Middle East" & Student \\
G & Business & Nepal & Banking work \\
\hline
\end{tabular}

Source: simplified from Pherali (2012: Table 1).

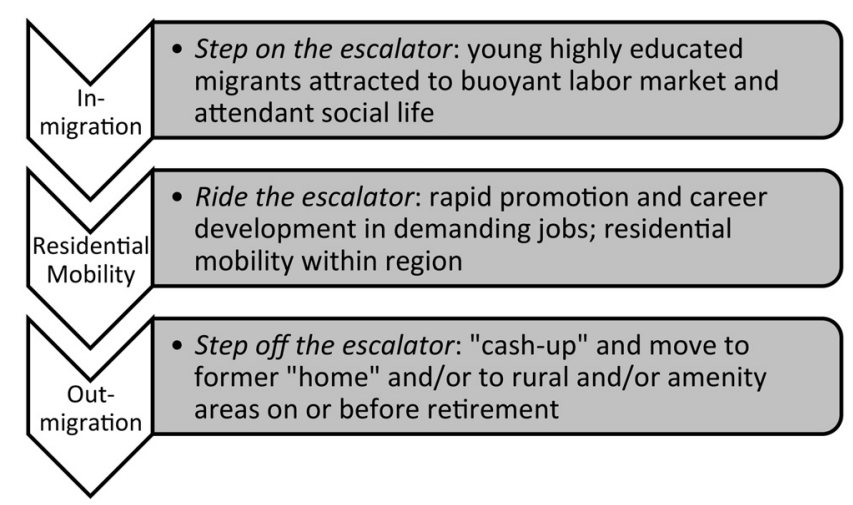

Figure 7.7 The escalator region concept.

Source: elaborated from Fielding (1992b, 2012).

middle- and upper-class jobs." Figure 7.8, outlines how escalator regions work, sucking in the young, highly qualified and ambitious, before losing them later when different priorities from high paying employment in the culturally vibrant big city become more significant in their lives. Where out-migrants go when they "step off the escalator" will be considered in Section 8.3.2.1.

\subsubsection{International elite employment migrations}

\subsubsection{A global brain drain}

Whilst both spiralism and the escalator region concept have been mostly investigated within the Global North, sociospatial mobility is very much apparent across the South. Mention of university students, for example, immediately invokes the socio-spatial mobility phenomenon known as brain drain. This refers predominantly to "movement of high-level experts from developing countries to industrialized nations" (Teferra and Altbach 2004: 41). It is usually perceived in terms of "lost" human capital of qualified graduates emigrating for anticipated higher salaries and better quality of life in the Global North. Such graduates may have been educated in their home countries before emigrating or they may have migrated abroad to study and simply not returned. Such flows-think of medical doctors, engineers or academicscan also, as Teferra and Altbach (2004) also noted, be regionally expressed (Skeldon 2008). All are part of the general geographical redistributions of human capital discussed in the previous sub-section.

Flow of highly skilled workers has increased since the 1970s from most regions but the implications for sending communities are unevenly distributed. Defoort (2008), for example, found that the number of highly skilled international migrants increased from 4.3 million in 1975 to nearly 17.3 million in 2000. Yet it was regions with lowest percentage of skilled workers, such as much of Africa, Central America and Eastern Europe, that had highest emigration rates. Elsewhere, notably Asia and South America, highly skilled emigration rates remained relatively stable. China and India, 
while exporting a large number of skilled workers, have large human capital reserves to draw upon, in contrast to smaller countries which may have more disproportionate skilled labor emigration.

Figure 7.8 gives a further sense of brain drain emigration's geography. Its shows developmental level of origin, year and gender of highly skilled (with post-secondary education) emigrants aged 25+ years then resident in rich OECD counties. Between 1990 and 2000, numbers rose from 12.5 million to 20.5 million. Rates, though, only increased from 5.1 to 5.5 percent, reflecting the rise in worldwide education. The graph also shows that whilst the proportion of highly skilled migrants who relocated from high-income countries (usually other OECD) actually declined slightly over the decade, the higher proportion from less developed country categories rose, especially from low-income counties. Rates in 2000 were approaching the 8 percent of the post-secondary educated from the lower-middleincome counties then living in the OECD.

Figure 7.9 also shows highly qualified women displaying higher rates of migration to the OECD than men, most strikingly from the poorer countries. Such a gender bias in favor of women has been increasing, reflecting women's rapid global increase in post-secondary schooling, an increased demand for their labor in health care, leisure and other services (Green and Shuttleworth 2015), enhanced family reunion programs, and socio-cultural attitudinal changes towards female emigration (Docquier et al. 2009).

As for destinations, consider the UK National Health Service (NHS) (Guardian 2014b). It is estimated that 11 percent of NHS staff are not British. Figure 7.9 shows 37 percent of UK-registered doctors did not train in the UK. The Philippines, in addition, is noted for its supply of largely female nurses.

Emigration of relatively elite graduates from Global South to North is typically discussed in terms of its impact on the economic development of the migrants' home countries (Docquier and Rapoport 2011; Skeldon 2008). Interpretations of this issue are split between optimists and pessimists (Samers 2010: 80-85; Docquier and Rapoport 2011). Optimists note how having relatively little market demand for their skills, the highly qualified may remain un(der)employed if they do not emigrate. Attention is then given to the potential transformative impact of the monetary remittances (7.5.5) emigrants typically send back to extended families (Skeldon 2008). Such monies can promote internal development within the South, it is argued. This will eventually see more highly skilled jobs created in these countries, ultimately cutting off the need for the most qualified to emigrate. Besides money and emigrants themselves (Iredale 2000), ideas about "modern life" can also "return" home to promote development, as noted earlier for

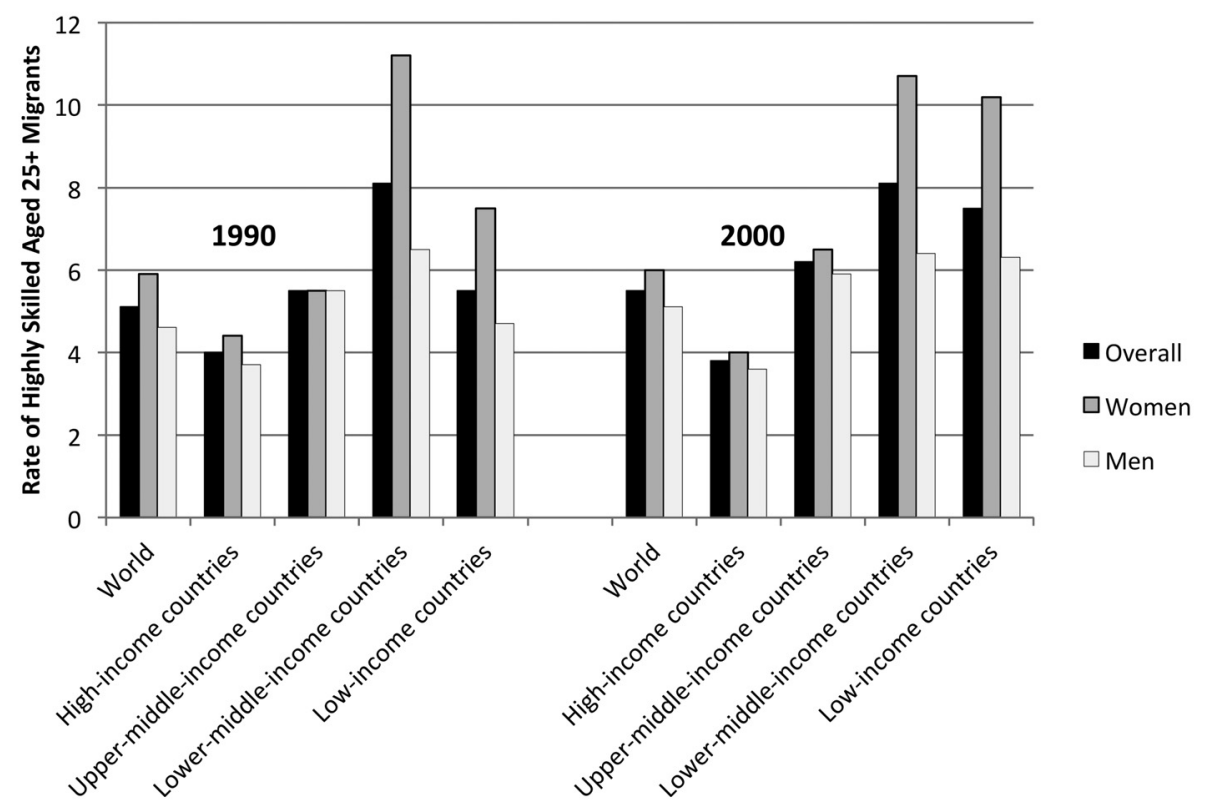

Figure 7.8 Highly skilled emigrants to OECD countries, \% of total, 1990 and 2000.

Source: derived from data in Docquier et al. (2009: Tables 1 and 2). 


\section{EMPLOYMENT MIGRATIONS}

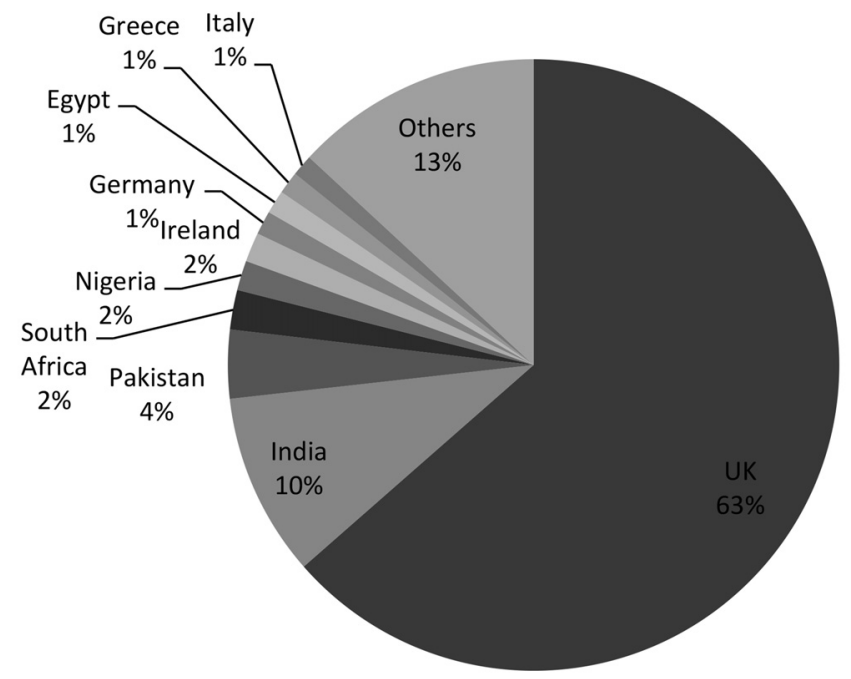

Figure 7.9 Countries where UK medical doctors trained.

Source: based on data from (Guardian 2014a).

Romanians in Spain. For optimists, brain drain migration is thus poorly named, migrants instead representing "international development agents" (Faist 2008).

Pessimists, on the other hand, focus on the loss of human capital from a country. They set much less store in the developmental potential of financial remittances, return migrants and socio-cultural ideas. Indeed, uneven distribution of the former may create social and spatial divisions within migrant origin communities (Skeldon 2008) and remittances may be spent on luxury imported goods that do little to develop Southern economies. In terms of the size of remittances, some studies have shown these decreasing with the level of a migrant's education (e.g. Niimi et al. 2010), although this is disputed (e.g. Bollard et al. 2011). If correct, less skilled speculative emigration may thus benefit the South more than elite emigration. This, in turn, is immediately complicated however by Mountford's (1997) finding that simply developing a highly skilled workforce promotes development, even if many recipients emigrate. As he put it:

a brain drain, either temporary or permanent, may increase the long run income level and income equality in a small open economy, and ... may even be preferable to a non-selective 'general' emigration.

(Mountford 1997: 302-303).

\subsubsection{From brain drain to brain circulation}

Notwithstanding debate as to overall costs and benefits of brain drain (Docquier and Rapoport 2011; also 7.5.4), there is considerable brain circulation within the global labor market. This involves "back and forth movement of skilled people between various countries of emigration and immigration" (Samers 2010: 82) or "brain drain and brain gain ... going in all directions: north-north, north-south, south-north, and south-south" (Mahroum 2000: 23). Figure 7.9 gave a suggestive snapshot of this dynamism. It is reinforced by Table 7.6, which gives the "Top 10" countries of at least 4 million inhabitants exporting academically elite adults around the turn of the millennium. Looking at absolute numbers, whilst many poorer countries' graduates have strong representation within the OECD-notably the Philippines, India and Mexico-circulation within the OECD is also very prominent-notably from the UK and Germany. Considering rates gives a different picture. Besides the astonishing 83 percent of Haitian graduates resident in the OECD in 2000, African countries' graduates have a very strong tendency to migrate for work within the OECD.

Bringing together these trends, numerous studies have explored circular migration of highly skilled and qualified elites around the world (Olwig and Sørensen 2002; Samers 2010; Shaffer et al. 2012). Whilst such elite circulation has always existed (Scott 2006), scholarship only took off from the 1980s. It was stimulated by economic globalization 
Table 7.6 Top 10 countries exporting highly skilled people to OECD, 2000

\begin{tabular}{lrlr}
\hline $\begin{array}{l}\text { Highest stocks } \\
\text { (Highly skilled emigrants, }\end{array}$ & Aged 25+) & $\begin{array}{l}\text { Highest rates } \\
\text { (Percent graduates emigrating) }\end{array}$ \\
\hline United Kingdom & $1,479,604$ & Haiti & 83.4 \\
Philippines & $1,11,704$ & Sierra Leone & 49.2 \\
India & $1,035,197$ & Ghana & 44.7 \\
Mexico & 949,476 & Kenya & 38.5 \\
Germany & 944,579 & Laos & 37.2 \\
China & 783,881 & Uganda & 36.0 \\
Korea & 613,909 & Eritrea & 35.2 \\
Canada & 523,916 & Somalia & 34.5 \\
Vietnam & 507,200 & El Salvador & 31.7 \\
Poland & 456,337 & Rwanda & 31.7 \\
\hline
\end{tabular}

Source: selected and simplified from Docquier and Rapoport (2011: Table 2).

and associated economic forms, such as the transnational corporation (TNC), promoting such migrations, greater recognition of the diversity of employment migrations, and how "temporary migration was the evolving norm" (Koser and Salt 1997: 285). Ensuing scholarship revealed elite brain circulation as highly organized, managed by individual states and multi-state bodies such as the EU, employers and intermediaries, including recruitment and relocation agencies (Koser and Salt 1997). Elite circulation also generates much less political controversy (Scott 2006), especially where migrants are nationals from the Global North, than employment migrants entering the North from the South. Favell (2003), with a European focus, talked of "Eurostars in Eurocities," further reflecting the strongly urban orientation of such migrations, notably to world cities (Sassen 1991).

Based on Scott's (2006) work with British middle class residents of Paris, France, Figure 7.10 identifies six types of highly skilled migrant resident in world cities. This classification takes into consideration motives for migration, migrant commitment to the host city or country, and family status. All three themes reiterate recurring themes in this chapter: how employment migration can blend into other expressions of migration, and vice versa; the potential significance of place ties; and the relevance of migration's family context. Furthermore, from a life course perspective, Scott also noted how individuals often changed type as a result of transitions, such as having children, children leaving home, or meeting a life partner in the new country.

Lastly, it must be reiterated just how selective international brain circulation (Box 7.3) clearly is (Beaverstock 2005; Skeldon 2008). Besides concentration within a limited range of occupations, membership is strongly gendered in favor of males and biased towards those more senior within occupations. There are also costs associated with this exclusivity. A disembedded life outside work is indicative of experiences of isolation, reinforced by long working hours and local cultural mismatch (Amit 2002; Beaverstock 2005). For married couples, the (usually female) partner of the labor migrant may be unable to work legally in the host country, promoting them either to remain in the home country or become a trailing spouse (7.5.2.1), their own employment career put on indefinite hold. Finally, an increasingly "flexible" work context (Amit 2002) raises the prospect of increased job insecurity within expatriate employment.

\begin{tabular}{|c|c|c|c|c|c|}
\hline $\begin{array}{c}\text { Motive for } \\
\text { migration }\end{array}$ & \multicolumn{3}{|c|}{ Career-path } & Relationship \\
\hline $\begin{array}{c}\text { Commitment to } \\
\text { host country / city }\end{array}$ & Permanent 'lifer' & \multicolumn{2}{|c|}{ Circulator or indefinite } & Permanent \\
\hline Family status & $\begin{array}{c}\text { Empty nest / } \\
\text { retired }\end{array}$ & $\begin{array}{c}\text { School-age } \\
\text { children }\end{array}$ & \multicolumn{2}{|c|}{ Single / partner } & Local family \\
milieu
\end{tabular}

Figure 7.10 Classifying highly-skilled migrants. 


\section{BOX 7.3 INTERNATIONAL RELOCATIONS OF FINANCIAL WORKERS}

Banking and financial services is an economic sector strongly associated with international migration to world cities. Studied extensively in a range of papers, Jonathan Beaverstock has shown conclusively the specific importance of transient migration to banking organizations and its more general role in both driving as well as responding to globalization. Thus, in a 1993 survey of banks in the City of London, international postings out of and into them were acknowledged as vital. Around half of those surveyed sent staff abroad to work in "their global-city office networks" (Beaverstock and Smith 1996: 1383) for at least a year, whilst over 80 percent received staff from the same networks. Common destinations for postings were other key financial centers, such Hong Kong, Singapore, Washington, Toronto, Paris, New York and Tokyo, but also more unusual "offshore" financial destinations, such as Jersey and the Isle of Man. Individuals' locations within these networks are revealed clearly by the spatial trajectories of the two financial workers in New York in 2000: 1. Male investment banker, 2. Female chartered accountant:

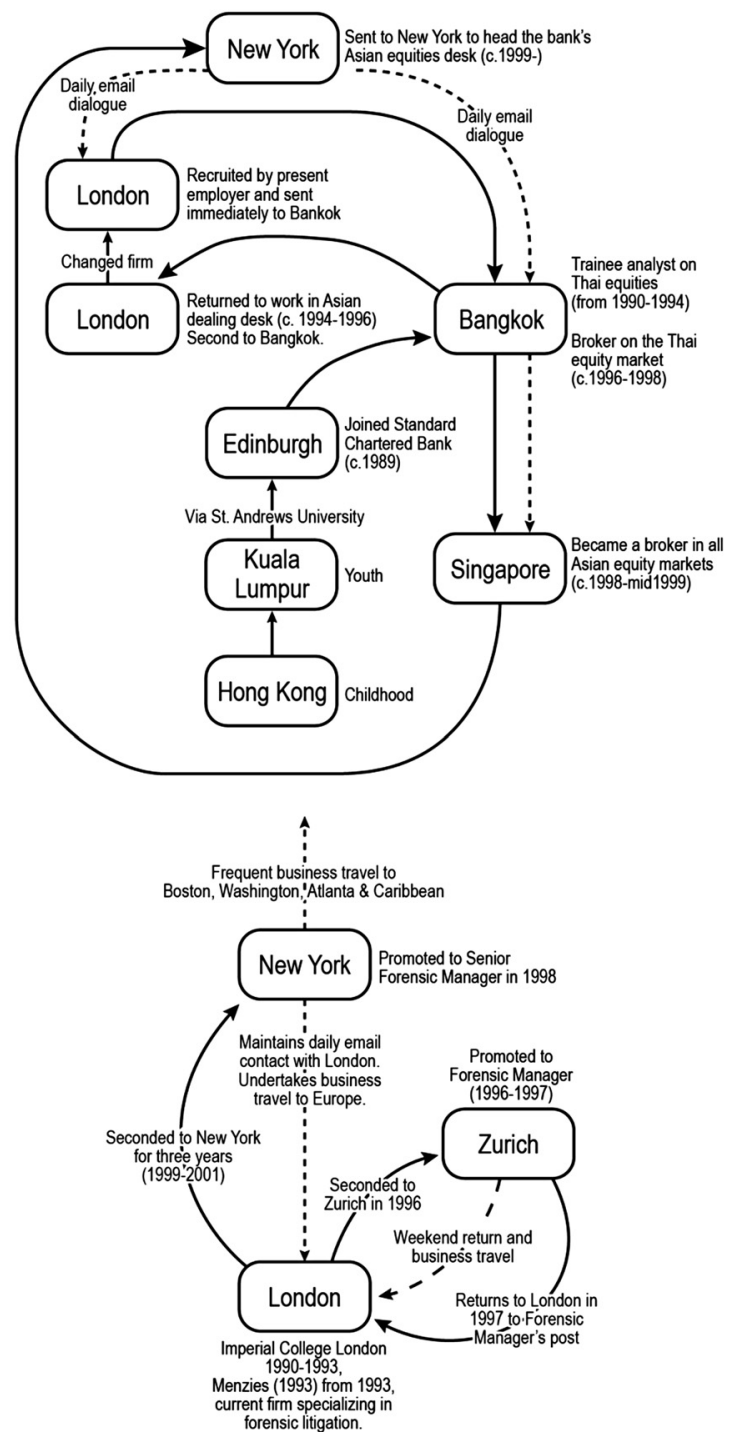

Source: Beaverstock (2005: Figure 2). 
Despite the expense for the banks and developments in information technologies and business travel, case studies of British expatriate financial elite workers in both Singapore and New York found them still playing vital roles for global banks. They helped, in particular, to promote "global-local knowledge network formation" (Beaverstock 2002: 537) and transfer. This occurred within a range of "meeting places"; not just the office but also bars, restaurants, sporting clubs, social clubs and business associations. However, in Singapore, whilst expatriates engaged with Singaporeans at work, they rarely socialized with them, thus making the British both "embedded" and "disembedded" in the city.

The success of transnational financial specialists is rooted within a highly selective and specialist culture of migration that centers on transnational occupational mobility. It is the culture already introduced in the chapter. It embodies the globalized network society (Castells 2000) of transnational everyday existence and flows within networks that appear almost "independent" of national and international migration governance. The latter helps explain their relative "invisibility" (Findlay 1996).

(Sources: Beaverstock 1994, 2002, 2005; Beaverstock and Boardwell 2000; Beaverstock and Smith 1996)

\subsubsection{Circular migrations for extractive industries}

Circular forms of contracted employment migration are not solely associated with people with noted educational credentials. In particular, there are a number of expressions globally linked with the labor needed for mineral extraction and other primary industries. These expressions again problematize the scope of "migration" and the boundary between it and "mobility" (Chapter 6).

Extractive industry concerns itself with mining, quarrying, dredging and obtaining oil and gas. It has always been difficult and dangerous work and today some has moved to such remote locations that the principal workforce has to be sourced mostly from outside the immediate area. Migrant labor is brought in, works a roster of a given number of days, then returns "home" for a break, before the cycle is repeated. It is a fly in fly out (FIFO) or, sometimes, drive in drive out (DIDO) labor force.

FIFO was pioneered in the Gulf of Mexico oil and gas fields. It quickly spread to other offshore extractive areas, such as the North Sea, and to on-shore sites such as the Canadian tar sands (Storey 2001). It has been recognized within popular culture, such as the Delines's (2014) Oil Rigs at Night, ${ }^{3}$ where the singer tells of the 23 days her (soon to be ex-) lover will still be away on the rigs, whose "golden lights" she sees from their home.

Academic scholarship has focused on FIFO associated with mining, especially in Australia. By 2000, the state of Western Australia had around 50 types of minerals being extracted (gold, iron, nickel, diamonds, bauxite), making it one of the world's principal mineral regions. A boom prompted by huge rising demands from Chinese and Indian economies (McKenzie 2010; Storey 2001) underpinned considerable labor migration to the state and expansion of male full-time jobs. Direct employment growth in mining was strongest in the state capital Perth, rising by 105 percent between 1996 and 2006. This compared to 29 percent growth for regional Western Australia. With Perth very far from the remote mineral extraction sites, many of the jobs comprised FIFO long-distance commuting.

The FIFO employment mobility structure is encouraged not just by remoteness but also by the relatively short life-span of some ore bodies, and by employers' provision of basic low-cost accommodation and ability to fill some gaps in local services (Cheshire 2010). Most FIFO workers are now housed in already established or purpose-built settlements (McKenzie 2010), whose conditions have improved considerably in recent years. They often paralleled increased state intervention, settlements losing their former "company towns" character.

Besides impacting ambiguously on already-existing rural communities (Storey 2010)-providing highly paid jobs but disrupting established community life-pressures associated with the FIFO lifestyle should again be noted. FIFO migrations are predominantly male. Migrants typically leave behind partners, children and wider family when they live away for often considerable periods. This can impact negatively on family relationships. At the workplace a migrant may "experience loneliness, homesickness and a sense that they are 'missing out'" (McKenzie 2010: 365). Yet, FIFO can avoid the trailing spouse (7.5.2.1) by allowing a partner to develop their own career in locations with more diverse 


\section{EMPLOYMENT MIGRATIONS}

Table 7.7 Migrants' perceived impacts of FIFO, Queensland, Australia, 2009

\begin{tabular}{ll}
\hline Domain & Perceived impacts \\
\hline Social life at home & $\begin{array}{l}\text { Enjoy time to socialize on days off } \\
\text { Avoid social contact due to fatigue } \\
\text { Hard to make and maintain friendships } \\
\text { Social life at mine site }\end{array}$ \\
Enjoyed \\
Drinking culture-fitting in \\
Less able to participate in team sports \\
Finances & Provision of facilities at site helps maintain fitness \\
Fatigue/mood & Financially rewarding \\
Job satisfaction & Compromise in lifestyle to maximize income \\
\hline
\end{tabular}

Source: simplified and reorganized from Torkington et al. (2011: Table 2).

labor markets, such as Perth or even Sydney or Melbourne in the Australian case. Table 7.7 lists positives and negatives identified by FIFO migrants in Queensland (Torkington et al. 2011), another Australian state where the practice is common.

Overall, one can question the desirability and long-term sustainability of employment mobilities so entwined with long-distance travel and ultimately transient operations (Cheshire 2010). Nonetheless, as with other forms of "flexible" employment, FIFO/DIDO mobilities are spreading. McKenzie (2010), for example, noted that in Western Australia it was increasingly common within construction, home maintenance, medicine and management.

Whilst FIFO/DIDO represents a form of circular migration that blurs the migration/commuting divide, such blurring is not, however, especially novel. Nor is it peculiar to the Global North. Circular relocation is expressed, for example, within historically embedded patterns of transhumance, the seasonal movement of pastoral farmers with their herds (Niamir-Fuller and Turner 1999). Other more recently developed expressions across the Global South, however, are again frequently associated with mineral extraction, as in Southern Africa.

Employment migration across Southern Africa has been widely studied (Crush 2000), not least due to the circular migration system initiated when gold and diamonds were discovered in the nineteenth century. The system became firmly established under the racist apartheid politics of South Africa in the twentieth century (Boyle et al. 1998; Crush et al. 1991). Migration for work in South Africa's numerous mines involved both internal and cross-border migrations (Callinicos 2005; Collinson et al. 2003; Posel 2004). It was extremely tightly controlled by the apartheid state as part of its broader racial engineering project. Internally, for example, apartheid forced black rural South Africans to live in so-called "homelands," with little access to land or an agrarian lifestyle. Consequently, many men, in particular, undertook circular migration to work in the country's mines. This labor was supplemented by recruitment from outside the countryfrom neighbors such as Swaziland, Lesotho and Botswana-although contracts were very limited and highly restrictive. Overwhelmingly again, men were involved and dependents largely prevented from joining them in prison-like hostels (Boyle et al. 1998). It is therefore perhaps unsurprising that circular labor migrants are also frequently associated with high HIV/AIDS prevalence, an association that extends beyond South Africa (Clark et al. 2007).

With the end of apartheid and introduction of mass democracy in the early 1990s, it might have been expected that South Africa's circular labor migrant system would be displaced by more permanent forms of labor migration (Posel 2004), as is emerging in China (7.3.3.1). This has not happened significantly, though labor streams are now more feminized (Collinson et al. 2006) and occupationally diverse (Crush 2011). Of employment migrants to South Africa in 2006, half were mine workers, reaching 87 percent of those from Botswana (Crush 2011). Circular migration has become strongly institutionalized, Posel (2004) suggesting households "back home" provide security for migrant workers within an insecure, unstable work environment (an interesting reversal of the usual relationship; 7.5.3). Again, the importance of family ties (7.3.2) and distributing risk (5.3.2) is clear. 


\subsection{EMPLOYMENT MIGRATIONS AS FAMILY AND COMMUNITY PRACTICES}

\subsubsection{Not just work ...}

Employment or labor migration, as its name suggests, emphasizes the economic underpinning of the migration it represents. Yet, this chapter has demonstrated how any detailed study of such migration discovers simple economics alone rarely explains all dimensions taken by resulting relocations. Thus, Parutis (2011) noted how the Poles studied as employment migrants to the UK (7.3.3.3) also came to try life abroad, see the world, and/or learn English. Adopting a biographical life course approach that stresses lives lived in context (Chapter 2) anticipates such more-thaneconomic, broader quality of life "additions" to employment migration. It particularly indicates the need to place such migration within community and, most especially, family contexts. Thus, not only should "a great deal of what has been traditionally thought of as individual migration ... be more appropriately conceived of as family migration" but "family migration should move front and centre" (Cooke 2008a: 262) even within employment migration.

More-than-economic dimensions of employment migration (Halfacree 2004a) can be glimpsed in numerous ways and noted under five headings below. First, is recent heightened feminization of migration flows, notably at the international scale. Second, the importance of ties for understanding both migration and non-migration (7.3.2) is reemphasized, focusing on how they structure inequality within actual migration outcomes. Third, return migration foregrounds both family and community considerations. Fourth, the critical role of remittances from international migrants, already noted, is developed. Fifth, financial ties between people and places established by labor migrants and those they leave behind may develop into transnationalism.

All together, employment migration frequently expresses a family strategy to attain a better overall quality of life. For example, Waters (2006) spoke of family strategic migration of Hong Kong Chinese to Canada, with adults relocating not just for their own employment but also for children to accumulate educational and cultural capital. Nonetheless, any such family strategy is as prominently cut-through by divisions of class, gender, ethnicity and so on as family lives and strategies overall. Supposedly seeking to serve the family, it becomes entangled with broader debates around what exactly is meant by "best interests" and who benefits most in specific circumstances (BeckGernsheim 2002). Acknowledgement of an active female voice within migration helps prompt such debate.

\subsubsection{Feminization of migration}

One of the major migration developments since the late 1960s is its "feminization." This refers, first, to simple genderaware recognition of migration of women in/on their own terms. Of course, female migration takes many forms. These include relocations for marriage (6.3.2), as part of a family unit or by trafficking (9.3.1). Yet, rapid and significant demand for female workers has led to a growing number of women migrating for their own employment (Castles et al. 2014). The significance of their gender immediately asserts itself. For example, Raijnan et al. (2003) highlighted challenges facing undocumented Latina migrants in Israel. Besides having to accept lower status jobs than they would in their home country and negotiating the complexities of being undocumented, the authors discussed the implications for mothers having to leave children behind while they seek employment abroad. Elsewhere, Morokvasic (2004) describes the experiences of women from Central and Eastern European countries who face utilizing temporary labor migration for jobs in health care, domestic work and informal trade as economic adaptation strategies during the transition years.

Consider Asia. An initial surge in female labor migration came with migration to the Middle East. Then, beginning in the 1990s, it reflected greater demand for female labor within Asia generally (Castles et al. 2014). Such migrants are concentrated in "female" jobs: domestic workers, hotel and restaurant staff, "entertainment" (often prostitution), assembly line workers for textiles and electronics, and health and care. Yeoh et al. (1999) highlighted domestic laborers moving from countries such as the Philippines, Sri Lanka, Indonesia and Bangladesh to the Middle East and elsewhere in Asia. Flows continued to increase and take on new, often illegal forms. O'Neill (2001) argued that dual processes of increasing globalization and greater restrictions on migration opened avenues for trafficking domestic laborers, as with Nepalese girls "sold" to Kuwait. Growing demand for paid domestic work in the Gulf States and Asia has drawn more and more Southern female migrant labor generally to fill these positions (O'Neill 2001). 


\section{EMPLOYMENT MIGRATIONS}

Both heightened internal and international "independent" migration of women has important implications for constitution of both families/households and communities of origin and destination. No longer at any scale simply "holding the fort" at home, women's heightened residential mobility is a major motor behind, for example, the rise of transnational links and cultures across the globe. Not all women's moves, of course, are so independent, even if the overall family move may be seen as economic migration. Much of it remains tied.

\subsubsection{Tied migration}

\subsubsection{The ties that bind}

Tied migration is an established concept within the migration literature (Cooke 2008b; Halfacree 1995a; Van der Klis and Mulder 2008). It usually references the gendered consequences frequently expressed within an employment migration taken for the benefit of one partner's career. As Cooke (2008b) noted, its origin lies in human capital theories (5.3.2). Its usual gendering was articulated by Mincer (1978: 758):

[with] smaller gains from migration, wives are more likely to be tied movers ... while husbands ... are more likely to be tied stayers than tied movers.... [T] The larger the wife's contribution to family earnings, and the stronger her job attachment, the greater the deterrent effect on family mobility.

In other words, tied migration in practice usually refers to experiences of female partners of men relocating for work reasons, often within a developing employment career. Women predominately comprise the trailing spouse, prompting to the idea of wives "married" to husbands' jobs and migrations (Finch 1983).

There are consequences from being a trailing spouse. Whilst the move may further the male's employment career and be seen as economically "rational" from the perspective of maximizing family income-fitting a "logical" family strategy-it may do little to further the trailing spouse's career. It frequently undermines it. Indeed, Halfacree (1995a) suggested such outcomes work to undermine women's careers more generally within an overall patriarchal environment.

In a tied migration context, "ties" refer less to moorings (Moon 1995) connecting people to place than to interpersonal bindings that bring a partner (and often the rest of family, notably children: Bushin 2009) along with the migrant on relocation. Historically, such tied migrants were largely invisible collateral within perspectives on the migration process that focused almost exclusively on one (male) household member. Children and other family dependents were equally invisible. Necessarily shifting from this overly narrow focus has required engaging again with the more-than-economic (Halfacree 2004b).

Noting work of numerous authors, Van der Klis and Mulder (2008: 2) summarized the overall situation and tied migration's challenge to the family:

Family migration decisions are especially difficult because of the simultaneous impact in residential, work, and family domains for all the family members.... Both individual preferences of partners and their common interests influence the decision whether or not a family migrates; as do external circumstances.... Furthermore, there is a major influence of gender dispositions on priorities and concessions made by individual partners.... If couples do migrate, one ... likely follows the other as a trailing spouse (also called tied mover); if they do not, the other partner may become a tied stayer.

Thus, although we see the emergence of other family forms $(6.2 .2,7.4 .4)$, such as the dual-location household, commuter marriages or FIFO working, tied migration remains a prominent consequence of employment. This is irrespective of whether or not it is strongly gender-biased, as presently constituted. It represents another potential experiential life course cost of labor migration (Halfacree 2004b). How much of a cost, though, depends in part on whether the household is dual-career or simply dual-employment. 


\subsubsection{Dual-career versus dual-employment households}

The consequences of experiencing tied migration are likely to be greatest-in terms of earnings but, especially, career development-if the tied migrant is trying to develop a distinctive employment career as compared to just seeking remunerative work. Falkenberg and Monachello (1990: 340) expressed the situation:

Dual-career families are those in which work has a high degree of salience for both marital partners; that is, both spouses are highly involved in their jobs and seek personal growth and development through their work. In dualincome families, work is of low importance to one or both spouses; thus, at least one spouse is working only to improve the family's economic status.

Gender roles adopted by family members work to shape adoption of either the dual-employment or dual-career form (Cooke 2008b). Simplifying considerably, within the "traditional" patriarchal household, husbands assumed "breadwinner" and wives "homemaker" roles. This promoted the dual-employment (better, single-career) form that prioritized the husband's career and migrations favoring this. The wife likely became a tied migrant, often also dropping out of the labor force on migration. However, adoption of more equal mixed gender roles has helped dual-career households emerge. Here, a wife's career assumes, in principal, equal importance to that of her spouse. Employment migration decisions become more complex, human capital theory (5.3.2) anticipating gender-neutral calculations of economic benefits to the family overall of moving as compared to staying. Even men with a distinctive employment career could become tied migrants.

Much research has investigated these issues and others concerning the gendering of migration (e.g. Boyle and Halfacree 1999; Chant 1992; Willis and Yeoh 2000), especially within the US and Europe (e.g. Cooke 2008a; Halfacree 1995a; Van der Klis and Mulder 2008). For example, Paul Boyle and colleagues matched large census-based sets of microdata from the UK and US to produce two samples of linked married or cohabiting couples. These were investigated through modeling techniques and confirmed the hypothesis that long-distance family migration frequently impacted negatively on female partner's labor market status (e.g. Boyle et al. 2001, 2002, 2003). To such work has been added a stronger life course perspective examining "temporal interdependencies between migration decisions, employment, and life course events" (Cooke 2008b: 259) or transitions. Having children is especially important (6.3.2), not only "amplify[ing] gender role behaviours" (Cooke 2008b: 259; Cooke 2001) but also often cross-cutting women leaving paid employment after migrating as a trailing spouse. The overall conclusion has been that tied migration remains strongly gendered-mostly experienced by women-with this gendering not necessarily economically rational from an overall family human capital perspective. It demonstrates the continued practical salience of patriarchy within everyday life but also how other life course priorities, such as having children, complicate the gender-employment-migration issue (Halfacree 2004b). In short, employment migration for partnered couples widely remains a patriarchal practice but also requires appreciation of the more-than-economic life course context in which labor migration is set.

\subsubsection{Bonded and un-free labor}

Tied migration takes a further, more explicit, form in the guise of debt-bonded or other largely un-free employment migrations. Whilst not quite slavery (9.3.2), bonded labor is defined by Anti-Slavery International as follows:

A person becomes a bonded labourer when their labour is demanded as a means of repayment for a loan. The person is then tricked or trapped into working for very little or no pay, often for seven days a week. The value of their work becomes invariably greater than the original sum of money borrowed. Often the debts are passed on to next generations.

(www.antislavery.org/english/slavery_today/bonded_labour/default.aspx).

Falling into such a position is often expressed through migration, both internal and international, whilst having migrant status in a destination can exacerbate any un-free labor situation.

Bonded forms of labor, both historically and today, are especially associated with the Indian sub-continent, notably India and Pakistan, although also found elsewhere in different forms (Box 7.4). It is a widely-researched topic (Carswell 


\section{EMPLOYMENT MIGRATIONS}

and De Neve 2013), spurred on by campaigns from groups such as the International Labour Organization (ILO) targeting all forced labor (Lerche 2007).

Within India, legislation that both defined and banned bonded labor was only passed in 1976 (Srivastava 2005). Such labor still affects between 250,000 to 20 million people, this huge discrepancy in estimates reflecting the challenge of both defining its exact parameters and then revealing its actual extent (Lerche 2007). The larger independent estimate equates forced labor with any debt bondage, however short-term, whilst the smaller figure refers to officially defined long-term debts. Since the 1950s, there has been a shift from traditional feudalist debt bondage to forced labor associated with capitalist economic relations (Carswell and De Neve 2013).

Indian bonded labor is especially associated with what Breman (1994) termed neo-bondage, typically involving seasonal migration. Laborers-men, women and children, mostly from the lowest, landless caste groups and tribes (Box 2.3)-endure long working hours and very low wages. Indeed, there are around 30 million seasonal migrant laborers (6-7 percent total) across India-not all bonded-mostly working in the unregulated informal economy within sectors as diverse as agriculture, brick kilns, cigarette making, construction, fish processing, mat weaving, mines, rice mills, salt pans, silver works, sugar cane harvesting and processing, stone quarries and weaving (Lerche 2007; Srivastava 2005). Neo-bondage:

involves the tying in of prospective labourers through loans/advances given during the lean season before the start of the (mainly) seasonal employment relation. During the employment period against which the labourer has pledged his/her labour power, the labourer may only be paid a minimum allowance, while the overall payment is settled at the end of the seasonal employment. This compels the labourer to stay in the employment relation after the advance has been paid off.

(Lerche 2007: 439).

Lerche (2007: 440) noted Indian male bonded laborers entering neo-bondage primarily for economic reasons. Furthermore, "they tend to do so with open eyes, well aware of the pay and conditions which await... [within] the best option available to them." Nonetheless, this is often with a strong sense of helplessness. Thus, in their study of bonded labor within the village-based power-loom industry of the Tiruppur region of Tamil Nadu, Carswell and De Neve (2013: 446) quote Raja:

there is no other way: if I have an urgent family problem and I need Rs 10,000, I ask my employer for the money and he will add it to my outstanding debt. I know that my kadan (loan) will go up and that I won't ever be able to repay, but there is no other way.

Networks again play vital roles, laborers following patterns well established from their village, for example. Finally, female and child bonded laborers are often even less "free" than men, experiencing a most extreme form of tied migration.

There are still other forms of employment migration where the degree of "freedom" allowed the migrant is questionable. This is exemplified by experiences of many domestic servants, who "in the Middle East are like luxury goods ... indispensable for maintenance and upkeep of the large homes and comfortable lifestyle which the population is used to now" (Shah 1995: 1017). "Need" for such servants reflects entangled relations of gender, class, religion and ethnicity, with local (higher class, Islamic, Arab) women "discouraged" from employment in general (Malecki and Ewers 2007).

Migration of female domestic workers occurs both to initial employment locations, notably the Gulf States, and to accompany employers often internationally and to global cities such as London and New York. In all cases, extreme examples of exploitation and abuse-"torture, rape, sexual assault, overwork, and non-payment of wages" (Silvey 2006b: 23)-have been noted. Servants are often virtual prisoners of employers (Murray 2012; Parreñas 2001). Ratih, an Indonesian maid in Saudi Arabia, expressed her severe constraint on movement, reinforced by labor and migration laws (Box 7.4):

My boss and madam never let me go outdoors. Whenever they went out, they would shut me inside the house. My friend, a woman from Gringging, received even worse treatment from her employers. Once she was done with her chores, they would lock her up in her bedroom, furnished with a TV and a bathroom.

(quoted in Nurchayati 2011: 484) 


\section{BOX 7.4 BUILDING QATAR'S WORLD CUP STADIA}

In September 2013, headlines in the UK's Guardian newspaper announced: Revealed: Exploitation of Qatar's World Cup "Slaves" and Qatar World Cup Construction "Will Leave 4,000 Migrant Workers Dead." Thus began a campaign, still ongoing in 2016, seeking to draw attention to the plight of employment migrants in the small Gulf state of Qatar. The journalistic "hook" was that these workers were employed in part in constructing infrastructure for the 2022 FIFA soccer World Cup, with the final in the new Lusail City.

The 2013 stories originated from an investigation that revealed 44 Nepalese migrant workers to have died over just 2 months that summer, half from heart failure or workplace accidents. The International Trade Union Confederation (ITUC) estimated from such evidence and additional cases of Nepalese, Indian and other deaths within Qatar's huge 1.2 million labor migrant workforce that 4,000 could die-around one per day-before "a ball is kicked" in 2022.

What is the cause of this terrible situation? Immediate contributing factors included: laboring in $50^{\circ} \mathrm{C}$ heat, exhaustion generally, non-payment of wages, retention of passports (preventing return home), denial of drinking water, endemic sickness, and overcrowded and insanitary living conditions. All reflected, in turn, the migrant labor tradition that operates in Qatar and some other Gulf states, the kafala system.

In order to be granted a visa to work in Qatar, a prospective migrant must obtain a kafeel or sponsor. The resulting relationship binds the migrant to the employer, in clear contradiction of any idea of the "free" laborer. As International Labour Organisation (ILO) migration specialist Azfar Khan (2014: 17) depicted it, a system originating in the 1930s "in the best tradition of Arab hospitality" had become thoroughly exploited by "unscrupulous kafeels."

Within the "structured violence" (Gardner 2010) of the kafala system, abuses have included: kafeels failing to pay medical insurance and fees for residence and employment permits, charging the workers instead; withholding wages; retaining passports; violence and general harassment and exploitation of workers. Although most are illegal under Qatari and other Gulf State laws, workers typically fail to complain. They fear the consequences, such as deportation or other sanctions. Not only have families often borrowed money from agencies and loan sharks to send a worker abroad, thus incurring debt, but remittances often become vital for a family's everyday survival back home. A clear sense of this comes from the tragic case of Noka Bir Moktan, a 23 year old who supposedly died of "sudden cardiac arrest" in October 2013. His impoverished family had paid substantial amounts to send him to Qatar, including travel and agency fees. Unfortunately, this was money borrowed from a loan shark. Consequently, the family is now afraid he "will demand that Moktan's two sisters, aged 14 and 16, who were collateral for the loan, be sent to work in brothels in Mumbai to pay off the debt" (Doward 2014: np).

Increasingly aware of such abuses, both labor sending countries and Qatar have made varied responses. The former have been relatively muted, however, reflecting the importance of remittances to their economies. Whilst Indian workers, notwithstanding high numbers of deaths, are seemingly treated better because a long history of sending guest workers to the Gulf has enabled support networks to be established, Nepalese prominence in the initial news stories reflects even greater dependence. Most workers are first-time emigrants, many with little previous family experience of such migration. This naivety helps explain why they are the lowest earners and most exploited. Yet, demonstrating the importance of this system to Nepal (around one third of Gulf labor migrants originate from this country), when the Nepali Ambassador described Qatar as an "open jail" for Nepalese migrants he was immediately recalled.

By 2014, the Qatari authorities, pressurized by media stories and the resulting bad publicity (also exposing exploitation of female domestic workers), campaigns by ITUC, ILO, Amnesty International and others, and by the World Cup governing body FIFA, were talking of significant reforms to the kafala system. To what extent this occurs remains to be seen but it seems clear that labor of male migrants will continue for the foreseeable future to underpin mega construction projects, fuelled by oil revenues, which are seeking to transform Qatar and other Gulf States into twenty-first century world city states.

(Sources: Booth 2013; Doward 2014; Gardner 2010; Gardner et al. 2013; Khan 2014; Malecki and Ewers 2007; Pattisson 2013; Rizzo 2014) 


\section{EMPLOYMENT MIGRATIONS}

\subsubsection{Return migrations}

Circular movements feature strongly within various forms of employment migration. Recognition of such spatial forms both challenges the idea of migration as "permanent" relocation and emphasizes more-than-economic aspects inherent within almost all labor migration. Both matters emerge from consideration of employment migrations' links to subsequent return migrations, where out-migrants relocate back to an area resided in before, especially during childhood. Whilst expatriate return to the home country might be regarded as return migration, usually the destination's spatial scale is seen as smaller; return to a "home" village, for example. An element of "return" concerns some sense of re-establishing the subject's past life course everyday geographies.

The scope and extent of return migrations has only been recognized widely since the 1960s within migration scholarship (Peterson 2013). However, such flows have always been present. For example, as a counter-current to the Great Migration from Europe to the US (King et al. 2010), it is estimated that a third of the 52 million Europeans emigrating between 1824 and 1924 eventually returned (Wyman 2005).

As re-emphasized below with respect to remittances and transnationalism, keeping links with "home" or "origins"the terms are difficult to define precisely-is widely recognized within all forms and scales of migration scholarship. Relatively rare is the migrant who completely severs all ties with their origin, at least where that origin has provided geographical context for a significant part of their life course. Moreover, today, technologies of travel and communication associated with the era of mobilities make the sustaining of connections much easier, more affordable and everyday.

Yet, in spite of such mobilities, "permanent" return migrations are still commonplace. Indeed, mobilities technologies facilitate return both directly and through their ability to enable migrants to maintain links with home (Peterson 2013). Table 7.8 presents a typology derived from international return migration that led Cassarino (2004) to emphasize the importance of returnee "preparedness" and "resource mobilization" for the outcome of a return migration. Also important is where return migration fits into the migrant's actual and anticipated life course biography. The outcome, as Table 7.8 suggests, is not always a success story, whether financially or socially.

Return migration may be associated with downward rather than upward social mobility. Such downward mobility is unlikely to be a problem where return is largely for lifestyle reasons, as with professional workers "stepping off the escalator" (Figure 7.8). It may well be welcomed. However, where return is for more personal reasons, such as marriage breakdown (Feijten and van Ham 2007), or from some sense of "failed" labor migration, downward experience can be most problematic. For example, for China's floating population (7.3.3.1), return can cause severe embarrassment and loss of face (Poston et al. 2012). Silvey (2001) likewise noted how young women who had sought employment in Indonesia's factories but then became unemployed viewed returning to rural origins as a failed transition to adulthood. Some even engaged in prostitution rather than return.

Whilst presence of return migrations warn not to over-state the degree of choice within original employment outmigrations, being able to enact a desire to return does not always lead to hoped for outcomes. "Return" is often very hard socially (Rigg 2007)-it is a challenging "role" to perform (Halfacree and Merriman 2015)-as the biographies of those who return are inevitably marked by experiences away, with both positive and negative effects. For example, Koning's (2005) encounters with young rural women returning to their village in Java, Indonesia, found them stuck between two worlds-rural-traditional and urban-modern-at home in neither. As Olwig and Sørensen (2002: 14) put it:

returnees draw on life experiences and resources gained abroad when returning to their place of origin. They therefore do not return to the same place they left behind when they migrated, but rather to the place of origin that they have constructed abroad as migrants.

Table 7.8 Types of return migration

\begin{tabular}{ll}
\hline Return of failure & Inadequate economic rewards in destination; inability to integrate or adapt \\
Return of conservatism & Always aimed to return; gained enough away for modest life "back home" \\
Return of retirement & Return home for closing phase of life course \\
Return of innovation & Dynamic return to change things with resources from time away, monetary and non-monetary \\
\hline
\end{tabular}

Source: adapted from Cerase (1974) and Cassarino (2004). 
Ireland provides good illustration of the complexities within the return migration experience. Long (in)famous for emigrations driven by extreme economic hardship, as around the Great Famine of the 1840s (Gráda and O'Rourke 1997), throughout the twentieth century it experienced huge out-flows of labor migration, especially to the UK, North America and Australia (Duffy 1995; King 1991). The 1980s were no exception, high unemployment prompting very high emigration rates peaking at over 70,000 in 1989 (Courtney 2000); the country's population was just 3.5 million. However, this situation reversed when the success of the so-called Celtic Tiger economy from the mid-1990s prompted high rates of immigration. Amongst the diverse people coming to live and work in Ireland (MacÉinrí and White 2008) were return migrants, especially in the late 1990s (Ní Laoire 2007).

As part of a larger return migration project (Ní Laoire 2008), Caitríona Ní Laoire interviewed 14 migrants aged in their 30s and 40s in 2004-2005 who had returned to rural Ireland. Prefacing her analysis, she noted:

dream of return is a classic theme of Irish emigrant discourse. It has a mythic quality in diasporic culture, from the classic exile ballads to films like John Ford's The Quiet Man (1952), which suggest that the ultimate aim of the emigrant is to return to Ireland and some kind of alternative to the modern urban immigrant lifestyle.

(Ní Laoire 2007: 337)

Respondents expressed this "dream" well, being drawn (back) to a perceived Irish rural idyll and to family and community. Yet, they also demonstrated disappointments. Experiences of return did not always live up to expectations. Perhaps this was to be expected since they typically moved from urban centers where they had sought higher education and employment (Corcoran 2002). Negative issues included loneliness, loss of anonymity and general "culture shock." Overall, as with Koning's (2005) Indonesians, Ní Laoire's Irish returnees could find themselves stuck between two worlds, fully at home in neither. As Irish writer Joseph O'Connor (1993: 14) put it: "You're home in Ireland but you're not home really. London is in your head, or New York, or Paris. But you're in Ireland."

Finally, Table 7.8's "return of innovation" category comprises a dynamic group who return to change something in their home place using resources gained from time away. These resources may be both monetary and non-monetary. Of particular note are return migrants who create jobs, especially in rural areas where employment decline has long prompted out-migration. Returnees represent a small group within what Bosworth (2010) termed "commercial counterurbanisation" (8.3.2.2), whereby a migration flow easily depicted solely in lifestyle terms is also underpinned by economic considerations (Halfacree 2008). Often only by leaving rural areas do young adults acquire the necessary skills, finance and other resources to start a successful business. If attracted back later in life-when, as emphasized, other life course priorities than those directly focused on employment often become more significantthey may become a powerful force for rural endogenous economic development (Stockdale 2006). Case study work in Scotland, England and Denmark, for example, revealed such migration and job creation occurring (Bosworth and Willett 2011; Findlay et al. 2000; Herslund 2012; Kalantaridis and Bika 2006). However, its scope remains limited, counterurban return migration more associated with self-employment than employing others (Stockdale 2006). It also requires migrants to understand, engage with and embed themselves within the local community (Bosworth and Willett 2011), not always easily achieved.

\subsubsection{Remittances}

There are other ways migrants keep connected or reconnect with their past homes, wider families, and origin communities than attempting to return "home." As widely noted in this chapter, a principal means of doing so is through remittances. These are "transfers, of money and goods, across geographic boundaries, often internationally by migrants in a destination country to their family, friends, or communities in a source country" (Pena 2013: 1).

Monetary value of remittances is far from trivial. World Bank data estimate their value to the Global South in 2011 at US $\$ 372$ billion, having climbed steadily from about US $\$ 25$ billion around 1990 (Ratha and Silwal 2012). They were projected to reach nearly US $\$ 500$ billion by 2014. Table 7.9 gives further breakdown for 2011 and change over the 2008-2011 period, revealing sharply growing significance to South and East Asia in particular (Box 7.4). Countries across the Global North also receive substantial remitted sums.

In terms of the practice of remitting money, Julca (2013a) reported on fieldwork in New York with Latino immigrants. First, there was a strong sense of obligation to send money, even when the migrant was struggling to

\section{ST PROOFS: NOT FOR DISTRIBUTION}




\section{EMPLOYMENT MIGRATIONS}

Table 7.9 Remittance flows to Global South, 2011 estimates

\begin{tabular}{llc}
\hline & $\begin{array}{l}\text { Remittances 2011 } \\
\text { US \$ Billions }\end{array}$ & $\begin{array}{l}\text { Change 2008-2011 } \\
\%\end{array}$ \\
\hline East Asia and Pacific & 107 & +26 \\
South Asia & 97 & +35 \\
Latin America and Caribbean & 63 & -3 \\
Middle East and North Africa & 42 & +17 \\
Europe and Central Asia & 41 & -9 \\
Sub-Saharan Africa & 22 & 0 \\
TOTAL & 372 & +15 \\
\hline
\end{tabular}

Source: selected and simplified from Ratha and Silwal (2012, Table 1)

make a living. This was an obligation to siblings (e.g. for education back home); parents, husbands or wives; extended family (e.g. where there is sickness) and close friends; and/or those owed money, such as funders of the costs of migration (airfare, visa, other transportation). Labor migrants even delayed paying household bills or worked extra shifts to keep up remittance payments, such was their significance.

There is little doubt that an emigrant sending remittances can be a great asset to a family or even a more broadlydefined community at the destination. It clearly expresses Stark's (1991) labor migration as risk reduction strategy (5.3.2), reducing family vulnerability by allowing members to buy food and drink, secure accommodation, health care and education, and even invest in businesses (Julca 2013a, 2013b). Jones (1998: 9) expressed it thus:

unlike other international flows into such regions, remittances reach the hands of thousands of migrant families, rather than families of a few entrepreneurs or social leaders. The decision-making power behind migration and behind the spending of remittance earnings is similarly dispersed.

Numerous case study examples are found in the literature (also 7.3.3). As one example, Singh and Velásquez (2013) noted the importance of remittances within Ecuador and Bangladesh in alleviating household poverty, with 40 percent spent on purchasing scarce land.

Whilst the potential of remittances for individual families may thus be predominantly positive, this chapter emphasizes the need to set employment migration within broader contexts in line with a life course perspective. Here unfortunately, the role and potential of remittances become more ambiguous. Even at local community level, unevenness of distribution can promote socio-spatial divisions, inequality and envy (Julca 2013a; Skeldon 2008). Generally, remittances benefit lower middle-income families more than the very poor, reflecting the former's greater ability to fund sending someone abroad (Singh and Velásquez 2013). In Ecuador, Calero et al. (2008) estimated that 57 percent of recipients came from the richest 40 percent of the population and just 22 percent from the poorest 40 percent.

Much debate has focused on the potential of remittances for promoting socio-economic development within poorer countries (Ratha 2013; Skeldon 2008; UNDP 2009). On the one hand, they represent relatively stable forms of foreign exchange earnings, supplies of currency that can pay off national debts, vital help for countries hit by natural or other disasters, job creators and prompts to family investment. On the other hand, remittances may soon depart the receiving country if spent on imports. They can also stimulate local inequality and lead to dependency on international employment migration continuing indefinitely, as from the estimated 11 million foreign workers presently employed in the Gulf States (Gardner et al. 2013). Additionally, whilst the current scope of remittances is global, distribution is very uneven by country and within countries (Skeldon 2008). Figure 7.11 shows both the top 10 countries in receipt of remittances and the top 10 for remittances as percentage GDP. Whilst India and China dominate receipts, Tajikistan and Lesotho are most dependent. Note Nepal's position, which helps explain its government's "modest" response to the scandal discussed in Box 7.4. 

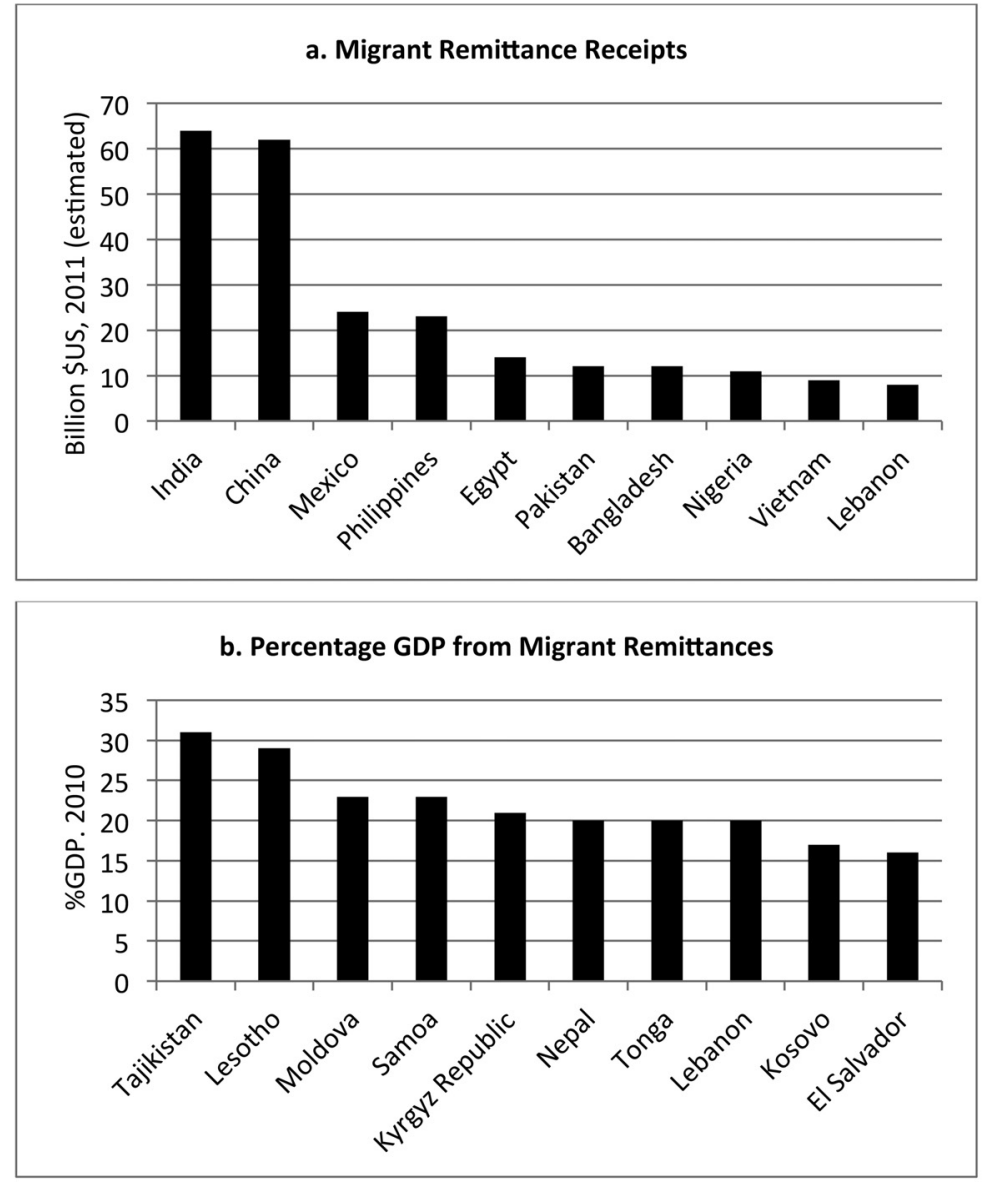

Figure 7.11 Top 10 Global South recipients of remittances, by amount (a) and \%GDP (b).

Source: re-drawn and modified from Ratha and Silwal (2012, Box 1).

\subsubsection{Transnational communities}

Sending remittances "back home" sharply expresses employment migrants' continued connections with origin people and places. Such sustained linkages frequently lead to return migration. When this does not/has not yet occur(red), emigrants' and even internal migrants' retention and development of often detailed and complex links with somewhere lived before is another huge topic within the migration literature. It reinforces critique of regarding migration as "permanent relocation," a supposed Year Zero brand new start in life. Instead, uneven, often irregular and yet sustained diverse networks, flows and interconnections can be mapped between "destination" and "origin." This is excellently expressed through the concept of transnationalism (Castles et al. 2014).

Transnationalism refers to "processes by which immigrants forge and sustain multi-stranded social relations that link together their societies of origin and settlement.... An essential element ... is the multiplicity of involvements that transmigrants sustain in both home and host societies" (Basch et al. 1994: 7, emphasis added). It involves establishing multiple place attachments, usually "bifocality" (Vertovec 2004) between "origin" and "destination," within an increasingly interconnected mobile globalized world (also Dunn 2010; McEwan 2004; Vertovec 1999), and demonstrating clear processes of attachment between them. An example linking back to earlier (7.3.3.3) was Ryan et al.'s (2009) illustration of Poles in the UK linking back to Poland through transnational caring roles distributed between both countries. Transnationalism challenges any dualism implied within depictions of international migrants "cross[ing] borders, bringing their culture with them, and becom[ing] relatively less or more assimilated to prevailing

\section{ST PROOFS: NOT FOR DISTRIBUTION}




\section{EMPLOYMENT MIGRATIONS}

cultural norms of the new territory-[as] either sojourners or settlers" (McEwan 2004: 500). It can be top down, as encouraged by globalized transnational corporations, or more bottom-up, resulting from "grass-roots initiative by immigrants and their home country counterparts" (Portes et al. 1999: 221). The latter is usually emphasized (Castles et al. 2014).

Transnationalism can be associated with the idea of diaspora. This concept has a long history and is equally difficult to define conclusively. Today, it is typically expressed in two contrasting ways (Dufoix 2013). First, it can draw on "the paradigmatic Jewish case" (Dufoix 2013: 4)-the global scattering of the Jews following Roman destruction of Jerusalem's Second Temple in 70CE. Here, it signifies exile, nostalgia and a dream of eventual return to the homeland. Diaspora members retain traditions, customs and languages; they are, in effect, only sojourners. A second contemporary definition, however, does not emphasize settlement but, as McEwen suggested above, challenges any sojourner versus settler dualism. This perspective draws most strongly on Black African experiences of emigration. It was expressed by Stuart Hall (1990: 235):

diaspora does not refer us to those scattered tribes whose identity can only be secured in relation to some sacred homeland to which they must at all costs return.... [l]t ... is defined, not by essence or purity, but by the recognition of a necessary heterogeneity and diversity; by a conception of 'identity' which lives with and through, not despite, difference; by hybridity. Diaspora identities are ... constantly producing and reproducing themselves anew, through transformation and difference.

This second understanding of diaspora (also Gilroy 1993, 1994; Hall 1999) is closest to ideas of bottom-up transnationalism and transnational communities and cultures (Al-Ali and Koser 2002; Vertovec and Cohen 1999).

Unlike globalization, which focuses on the broad structures facilitating communication and movement, transnationalism incorporates both these macro-scale structures and micro-level activities and linkages, placing more emphasis on agency (Castles et al. 2014). Yet, it is not without disputation. For example, Samers (2010: 96) asked whether transnationalism is somewhat of a misnomer, given that individuals tend to form connections to specific communities or villages rather than to nations per se. Additionally, he suggested transnationalism implies too much homogeneity within a group of migrants that span different age groups, genders, generations, religions and social classes. In a study of Bulgarian Roma migrant identity, Marinov (2015) demonstrated complex individual connections to local home places in Bulgaria, cities and countries experienced through migration (e.g. Netherlands) besides a broader sense of a Roma nation.

How transnational or diasporic identities are practically enacted or performed (Halfacree and Merriman 2015) is a central theme within the literature. Besides rejecting the idea that culture is metaphorically "baggage to be packed, uprooted and transplanted" (McEwan 2004: 502), studies emphasize the embodied effort that migrants make to become transnational or to develop "a way of living in more than one place at a time" (Cravey 2003: 616). This helps qualify unduly celebratory accounts of transnationalism by demonstrating it as often difficult to achieve and sustain. A focus on "lived experiences" (McEwan 2004: 509) or "transnationalism from below" (Smith and Guarnizo 1998) explores: the what, why and how of remitting money; travel between countries; use of technological communications, such as Skype (Box 7.5); everyday living spaces, including adopting new gender roles in "makeshift households" (Cravey 2003: 616); and sustaining immigrant group identity within the destination country. Performing transnationalism involves "everyday 'mundane efforts' [that] include stress and dislocations, the 'trickiness' of getting bodies across borders, the costs of communication, and the impacts of those difficulties on identity, well being, and belonging" (Dunn 2010: 4).

Claire Dwyer's work on UK suburban migrant faith communities presented an informative example of transnational sustenance of group identity (Dwyer 2012; Dwyer et al. 2013; Huq 2013). She showed an outer west London suburb (Southall) representing a potential ethnoburb with its expressive South Asian ethnoscape (Nasser 2003). Prominent were Hindi, Islamic and Sikh buildings (Figure 7.13), religious belief and performance frequently being fundamental for inscribing transnational identity (also Ahmed 2013). An interesting aspect is how the buildings are constructed and utilized (Dwyer 2012). The Shikharbandhi Jain Deraser temple in Potters Bar, north of London, was constructed by the closely-knit Jain community, mostly migrants from East Africa since the 1960s (Dwyer et al. 2013). Material embodiment of bifocal transnational performance, the temple has 52 eucalyptus trees representing the 52 villages in Gujarat (India) from where most of the community traced their ancestry and is made from Indian marble and sandstone carved in India.

\section{ST PROOFS: NOT FOR DISTRIBUTION}




\section{BOX 7.5 SKYPE, SOCIAL MEDIA AND TRANSNATIONALISM}

Sustaining transnational identity is, has been and always will be highly dependent on technologies of communication and the virtual mobilities these supply, as well as on the corporeal mobilities of circular migrations, return migrations and short- or long-stay visits, with their associated technologies (cars, planes and so on). In the early days the letter took center-stage in the former, in the future it might be a hologram-type virtual presence. Today, it is a wide range of technologies, many of which have come into their own in the last few decades but have already become largely taken-for-granted aspects of everyday life. These technologies enable users to overcome some of the disruption (international) migration has brought about to their everyday life course routines, whilst also helping build a more transcendent transnational identity.

Three kinds of technologies are of particular note, whilst never forgetting the sustained importance of the humble letter (Box 5.4)! First, there is the telephone. Historically, landlines enabled those subscribing to a connection in their home or able to access a reliable and affordable public phone to maintain contact with relatives living away. However, growth of mobile phone ownership and usage, as they have become increasingly affordable and utilizable (due to increased network coverage), now truly makes the phone a global transnational technology. Across the Global South, in particular, where until recently there was often minimal access to telecommunications, mobile phones are becoming ubiquitous. There are now more mobile phones here than in the North; even by 2010, China, India, Brazil and Indonesia alone had one third of the 4.6 billion mobile subscriptions worldwide. As Ling and Horst (2011: 364) noted, "while the internet has seemingly stolen all the headlines, the mobile phone has quietly provided people at the bottom of the income pyramid access to electronically mediated communication; often for the first time."

A gendered transnational expression of the use of mobile phones has enabled Filipinas living and working abroad as domestic servants to maintain contact with children remaining in the Philippines. Routine use of the phone has allowed them to maintain an active role in their children's lives. It also justifies and legitimates their adoption of international employment migration, as they can now retain many gendered family responsibilities and a sense of being a "good" mother.

A second key contemporary communications technology is the personal computer, whether accessed from home or from public locations such as internet cafés. Use extends from the humble email to the diverse forms of social media. Not least amongst the latter is Facebook, an online social networking service founded in the US in 2004. Just a decade later it has around one billion subscribers. In terms of access, residents of wealthier countries are most prevalent, reflected in the regional geography of subscribers shown below. Nonetheless, whilst Africa, for example, may only have had just over 5 percent of subscribers in 2012, numbers had increased by nearly 50 percent within one year.

\section{Facebook Subscribers 2012}

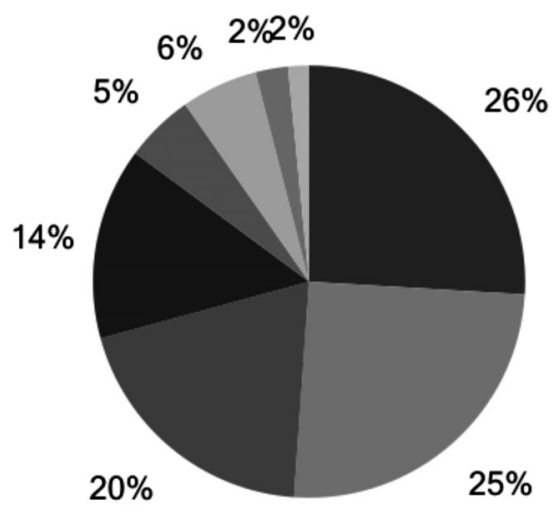

Europe
Asia
North America
- South America
Africa
Central America \& Caribbean
Middle East
Oceania




\section{EMPLOYMENT MIGRATIONS}

Case studies have begun to explore how social networking through Facebook and applications such as Twitter are being used to construct transnational identities. For example, a study of 26 international migrants to New York focused on their Facebook use. It was seen as very beneficial for maintaining relations with family and friends abroad but was also a signifier of prestige and a means to document everyday migrant lives. Nalan, a 32 year old Turk, for example, was most enthusiastic:

[It] is very useful, definitely. I keep in touch with my friends, a lot of friends, family. My ex co-workers.... I'm here, but we don't feel so far away from each other. We can see each other online.

(quoted in Lingel et al. 2014: 1505)

The telephone and personal computer are increasingly coming together, epitomized by smart phones. This development is represented in the third key contemporary communications technology, namely Skype. This voiceover-IP [Internet Protocol] service emerged from Scandinavia in 2003. Subsequently bought by the Microsoft corporation in 2011, Skype allows registered users to communicate with peers by voice (microphone) and moving image (webcam). Although phone calls may be placed on traditional telephone networks, the critical feature of Skype is that calls are free of charge. By the end of 2010, Skype had 663 million registered users.

Research has again shown Skype invaluable for maintaining transnational identities. For example, in a study of 53 Chinese professionals living in London, 49 (92 percent) used it to keep in touch with overseas parents, well ahead of the next most used internet tool, Instant Messaging (36 percent). Ed, a 26-year-old long-term resident accountant, summarized its appeal:

I use Skype to contact my family.... Before Skype, I bought those cheap phone cards for international calls. Since I had Skype, I have not made calls from my landline. ... For international calls, Skype alone is enough. It's free. Also, the quality is ... even better than those telephone cards.

(quoted in Kang 2012: 150).

Finally though, as argued in the main text, performing transnational identities through communication technologies is never a done deal, without problems or challenges. Thus, for the Filipina mothers, children were often ambivalent about the consequences and effectiveness of transnational communication, critical of migrants' ability to reconstitute their mother roles. For the New York Facebook users, fatigue with the medium could set in. Lastly, the Chinese Skype users identified a group of "silenced mothers" who lacked the digital skills and confidence to utilize the application. This could result in what Kang (2012: 152) termed "[t]he supposedly feminine task of affection and care ... reassigned to men." Technologies are not neutral tools but active elements within the always negotiated identities that comprise transnationalism.

(Sources: Fesenmyer 2014; Internet World Stats nd; Kang 2012: Ling and Horst 2011; Lingel et al. 2014; Madianou and Miller 2011; Metykova 2010)

Finally, transnationalism, as its name implies, has been mostly developed to understand emigrant communities. However, in line with problematizing international versus internal dualism within migration scholarship (5.7), one may also recognize domestic forms of transnationalism (Halfacree 2012a). This will be developed in discussion of challenges to sedentarist assumptions of a single "home" within lifestyle migrations (8.3.3.2) but even internal labor migrants may develop quasi-transnational or transregional forms of identity and expression within everyday lives. 


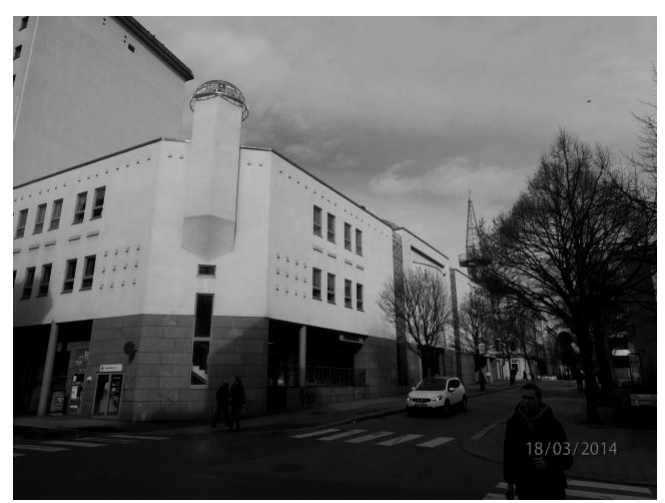

Figure 7.12 The Urtehagen Mosque: part of the transnational landscape of Osto, Norway.

\subsection{CONCLUSION}

This substantial chapter has born witness to just how considerably the economic still underpins human migration in the twenty-first century, just as it did when Ravenstein elaborated its role within his laws of migration (Fielding 2012). All geographical forms of migration, whether internal or international, are of central importance both to all national economies and to the Global Economy overall. Demands for and of labor within the opportunities and constraints presented by a capitalist world system are key to understanding this significance. Demand for labor of specific forms, whether expressed at the macro-scale of the economy or at the micro-scale of the individual employer stimulate migrant flows crisscrossing at all scales from regional to global (3.4). The demands of labor, for economic resources to enable survival or prosperity, or to experience a meaningful, rewarding and/or challenging job or career, frequently prompt employment migration within a life course. All consequent moves present potentially important life transitions (2.2.1), often even events (5.6.3).

Nonetheless, while accepting the fundamental significance of the economic within employment or labor migration, the chapter also made clear that actual expressions of such migration are immensely complex and diverse in constitution and content (Box 7.6). Abstract models based on "rational" (human) capital maximization or optimization calculations may confidently predict general flows-for example, from high to low regions of unemployment, or from low to high wage countries-but what specifically plays out "on the ground"-arguably, the focus of Population Geography-may ultimately quite poorly express such logics. Lived context is everything, as a life course approach emphasizes. Purely "economic" migration is largely a heuristic abstraction, at least one remove from how biographies develop according to both spatio-historical framings of place and time and bio-social positionings of class and status, health and differential ability, race and ethnicity, religion and belief, sex and gender, sexuality, and individuality (only some illustrated in this chapter). Additionally, noted throughout the chapter were the crucial roles played in shaping migration by institutional intermediaries: family, (im)migration policies, linkages and networks, and cultures of migration. To conclude, in line with Olwig and Sørensen's (2002: 1) opening quote for this chapter: "the search for a better livelihood is a main cause of migratory movements." But as these authors acknowledged, the key word is "livelihood": employment migration is always economic but it is also about more-than-economic parameters central to livelihood or quality of life. Other migrations focus almost exclusively on these latter parameters and comprise the lifestyle migrations considered next. 


\section{BOX 7.6 THE COMPLEXITIES OF MIGRATIONS AND MOBILITIES: THE CASE OF MONGOLIAN KAZAKHS}

Kazakhs form an ethnic minority population within Mongolia, comprising about four percent of 2.8 million residents. Originally arriving in the nineteenth century, this Kazakh minority traces its ancestry to populations living in Xinjiang province in western China. It now resides primarily in the remote, arid and mountainous western province of BayanUlgii Aimag, although Hovd Aimag and Ulaanbaatar, the capital, are also nodes of concentration. Although BayanUlgii Aimag is close to Kazakhstan, Mongolia and Kazakhstan do not share a common border. Consequently, movement between the two countries requires either passage by land through China or Russia or an international air flight.

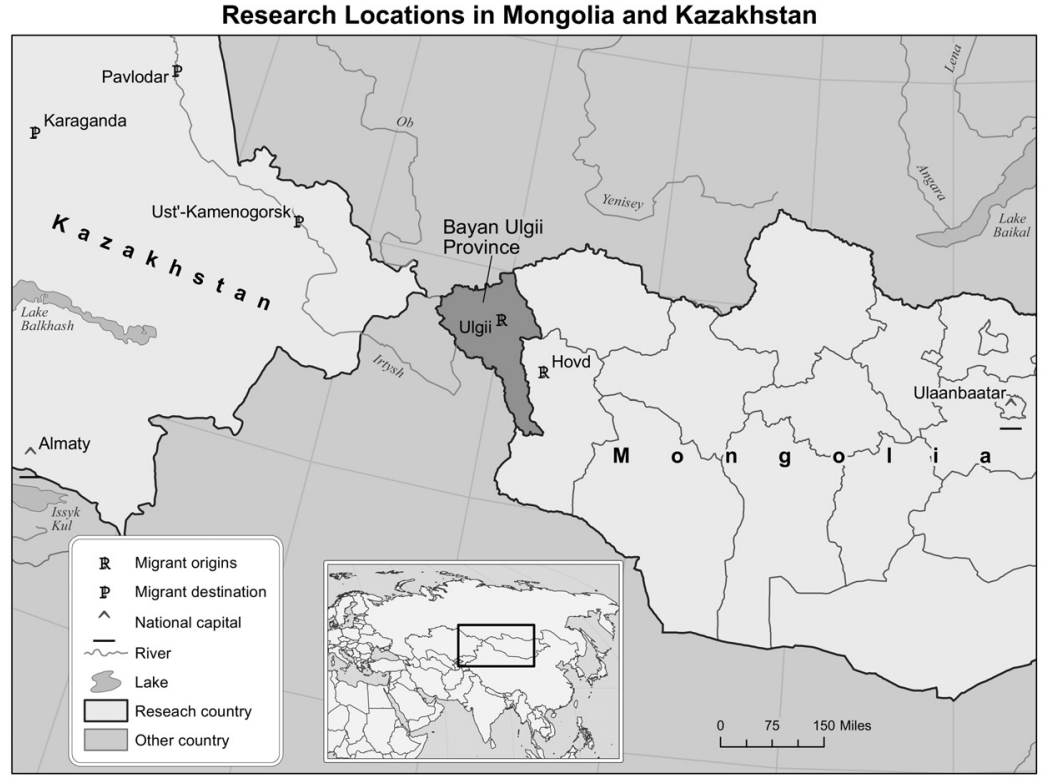

Following disintegration of the USSR and consequent opening of borders across this once closed region, this diasporic Kazakh community was welcomed back to Kazakhstan in the early 1990s as a strategy to increase the proportion of Kazakhs living in Kazakhstan. President Nezerbayev declared his country homeland for all Kazakhs and created policies aimed to repatriate diaspora members. At the same time in Mongolia, decline of trade with Russia was creating food shortages and a weak economy. The combination of cultural pull factors and economic push factors led to large-scale out-migration of Mongolian Kazakhs to Kazakhstan.

The post-1991 Mongolian Kazakh migrations to Kazakhstan provide an interesting case study of the importance of entangled micro- and macro-scale factors in influencing migration. It can be conceptualized from several different theoretical perspectives. Drawing on more integrationist theories, three distinct periods of migration can be identified. Within them, macro-scale structural factors, such as immigration policies and incentives in Kazakhstan and Mongolia, as well as economic constraints in both countries, influenced migration options and changes in behavior. These combined with micro-scale contexts, such as desires of Mongolian Kazakhs to return to their homeland and Kazakhstan's general cultural pull.

In the first period, 1991-1996, dire economic conditions in Mongolia combined with new immigration laws in Kazakhstan which included incentives for migration, such as housing allowances, 5-year work contracts and one-time payments for family members. This facilitated large scale out-migration from Mongolia. Nearly 60,000 Kazakhs (of approximately 120,000 in total) resettled in Kazakhstan. However, by 1997 the Kazakhstani government had reduced the quota and benefits were more limited for new migrants. Additionally, early migrants' 5-year work contracts were beginning to expire. Many Mongolian Kazakhs, nearly one-third of the original migrants, had returned to Mongolia by 2001. In addition to the changing economic climate, the cultural "pull" of 
Kazakhstan, while still important, had proven less rewarding than anticipated. Rather than being accepted as equals, Mongolian Kazakhs were broadly seen as backward, country-folk who could not speak Russian (the dominant language in Kazakhstan) very well and who were primarily herders rather than city residents. Other migrants, however, found success in Kazakhstan and never returned, and new migrants from Mongolia continued to move in despite earlier migrants' experiences. During the third period, 2001-2010, Mongolian Kazakhs remained rooted in both Mongolia and Kazakhstan. With few families lacking relatives residing in Kazakhstan, mobilities across the border evolved significantly. At the macro-scale, immigration policy in Kazakhstan became more restrictive, albeit still relatively liberal. Economic conditions had also improved in Mongolia generally and in Bayan-Ulgii specifically. Mongolian Kazakhs in this period moved across the border as individual migrants intending to take up permanent residence in Kazakhstan, as temporary labor migrants for seasonal work or as students seeking to attend Kazakh universities. Mongolian Kazakhs also migrated beyond Kazakhstan to Turkey, Russia, US, Germany and other European countries, albeit in very small numbers.
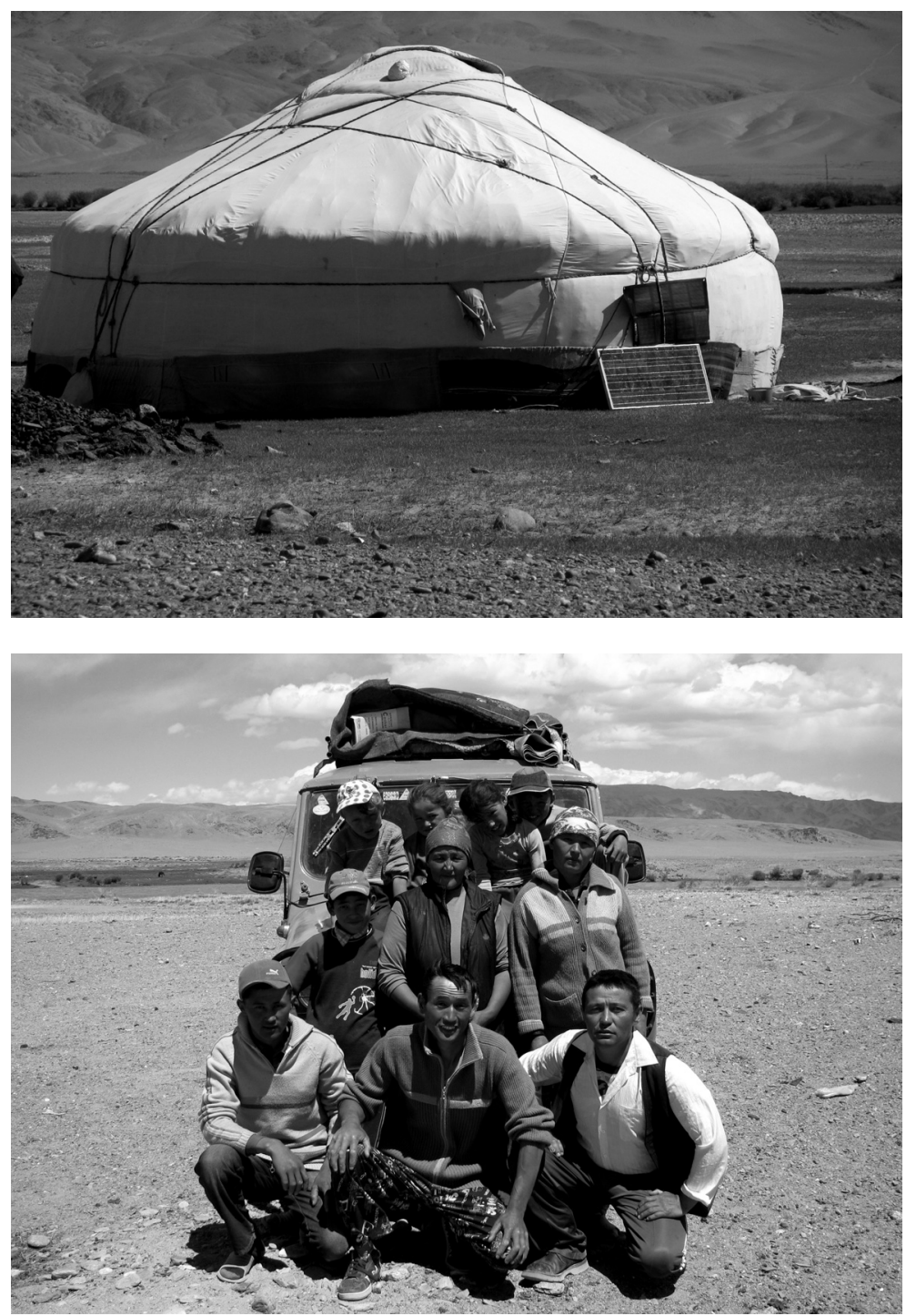

Seasonal migration amongst Kazakhs in Bayan Ulgii, Mongolia

Source: Holly Barcus and Cynthia Werner (2008).

\section{ST PROOFS: NOT FOR DISTRIBUTION}


194 EMPLOYMENT MIGRATIONS

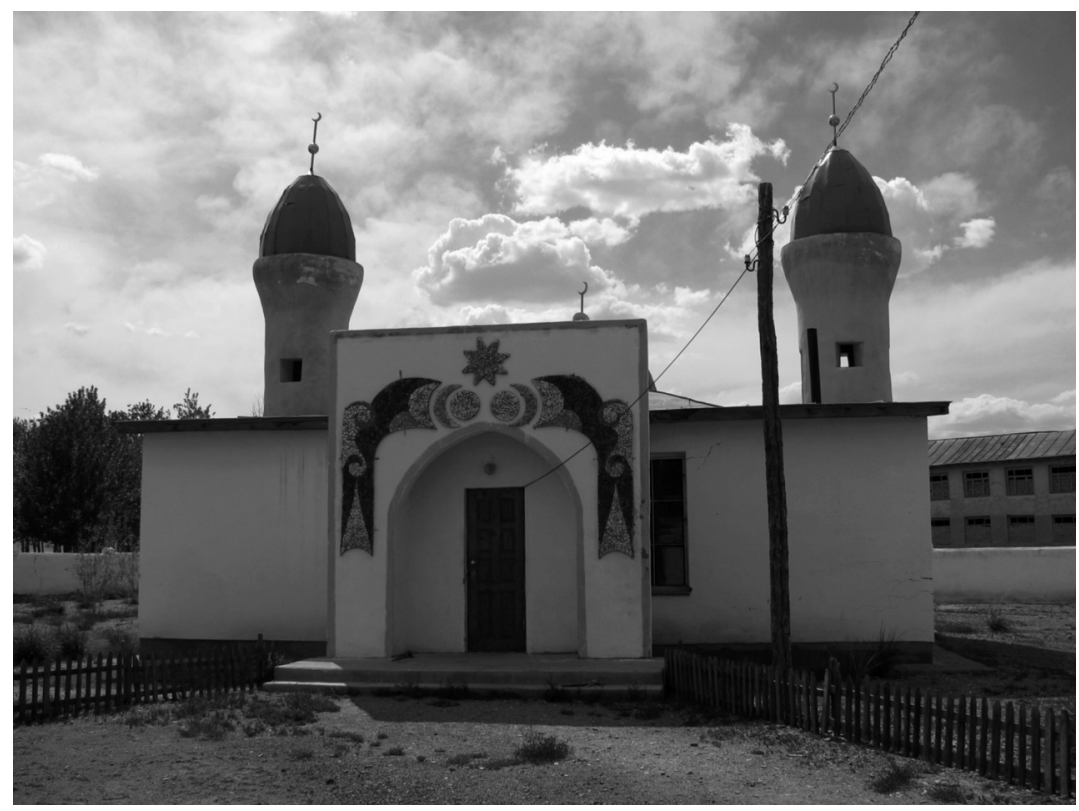

Mosque in Bayan Ulgii, Mongolia

Source: Holly Barcus and Cynthia Werner (2008).

A second way the flows of ethnic Kazakhs can be conceptualized is through the lens of transnationalism and the importance of social networks in facilitating information and migration flows between the two countries. Early 1990s waves of migrants now provide extensive social and familial networks across many of the urban and rural areas of Kazakhstan as well as in new destinations of Mongolia. These networks facilitate both long term migration as well as temporary labor migrations. With rapid development of transportation and communications technologies in these regions, especially the cell phone, information about work opportunities travels rapidly influencing flow of labor migration.

Gendered perspectives on migration offer a third lens through which to view the migrations, which have profoundly different effects on men and women. As a patrilineal society, marriage between a man and women results in the woman becoming part of her husband's family. Thus, when the husband or husband's broader family migrates to Kazakhstan, the wife loses social and familial networks in Mongolia. She most likely moves to a location in Kazakhstan in close proximity to the husband's family, where she is unlikely to have friends and family. Likewise, if a husband decides not to migrate but his wife's extended family migrates, she also loses her networks. Despite these constraints, many women view Kazakhstan as a modern and desirable place. It is viewed as a place in which women have both family and a career because of easier access to education and employment opportunities than in Mongolia and because of modern conveniences (running water, electricity, household appliances) streamlining household tasks.

(Sources: Barcus and Werner 2010; Brubaker 1993; Diener 2009;

Finke 1999; Werner and Barcus 2009)

\section{NOTES}

1 Note that the table excludes more qualified residents, many of whom were probably migrants.

2 Here it may be noted how the word career originates from the Latin word for wagon-carrus.

3 Written by Willie Vlautin, copyright El Cortez Records. Available on Colfax by the Delines (Decor Records DECOR033CD).

\section{ST PROOFS: NOT FOR DISTRIBUTION}




\section{CHAPTER 8}

\section{Lifestyle migrations}

\subsection{INTRODUCTION: MIGRATION FOR THE PROJECT OF THE SELF}

\subsubsection{More-than-economic migration}

Chapter 7 made a strong case for continuing to recognize employment factors as the principal driving force behind migration at scales beyond residential mobility (Chapter 6). Nonetheless, it also argued that even moves categorized as "employment migration" may be inadequately served by such designation. Whilst vital and necessary, economic factors relating to paid work rarely express fully specific relocations. For a richer, less monochrome and more threedimensional representation, other priorities and considerations must enter the equation (Halfacree 2004a; Stockdale 2014). Such factors often come to the fore with specific life course stages, such as when there is a need for a bigger house when children are born. In other words, placing migration within the biographical life course context frequently requires engaging with more-than-economic as well as economic priorities. Chapter 6 has dealt with some of these, expressed in relocations for housing, family and neighborhood matters. Additionally, and certainly within supposed consumer societies (Baudrillard 1998), the more-than-economic expressed by aspired lifestyle is entangled in migration decisions. This chapter illustrates where it becomes pre-eminent within a move.

The remainder of the chapter is organized as follows. This section continues by defining lifestyle, lifestyle mobility and lifestyle migration. The latter as distinct category is relatively new in the academic literature. It was only very implicit within Ravenstein's nineteenth-century laws (Table 5.5), when as a category of migration it was much less socially inclusive than today. Nonetheless, lifestyle issues have long been recognized as important forces driving migration to improve quality of life. The chapter then proceeds through three broad and internally differentiated families of lifestyle migration: the lure of the city (8.2), the lure of the country (8.3) and lifestyle retirement migration (8.4). Each shows particular resonances with key arenas of life course constitution, class and age standing out notably. The families again transcend any internal/international migration dichotomy. All represent more unambiguously expressions of individuality and "choice" than employment migrations, albeit with noted qualifications. The chapter conclusion (8.5) reiterates the importance of acknowledging lifestyle migration-both act of relocation and process-as inextricably relational.

\subsubsection{Lifestyles, lifestyle mobilities and lifestyle migrations}

Lifestyle is one of many words used within everyday life which when scrutinized by academics is seen as difficult to pin down precisely. However, a consensus has emerged that it is now not simply a "trivial" aspect of personal consumption but a key constitutional force within contemporary society. Engaging early within this debate, British sociologist Anthony Giddens (1991: 81) defined lifestyle as:

a more or less integrated set of practices which the individual embraces, not only because such practices fulfill utilitarian needs, but because they give material form to a particular narrative of self-identity.

A number of comments can be made about this definition. First, lifestyle is foremost about practices-things done or lived-which include but go beyond acts of consumption. Second, one must not exaggerate the extent to which a 


\section{LIFESTYLE MIGRATIONS}

lifestyle is a neat and total package (in contrast to "lifestyles" marketed in glossy magazines, for example). Instead, lifestyles have what Lorenzen $(2012: 96,98)$ termed "provisional coherence," always part of "an ongoing, dynamic, and piecemeal process." Third, whilst Giddens emphasized the scale of the individual, this individual is always relationally constructed. Lifestyle, too, is an ongoing relational production. Of critical importance will be individual position within the bio-social arenas prominent within the life course (2.3.1), including their coalescence within institutional forms, notably the family. Fourth, lifestyles are rooted in both the needs and desires through which self (individual) is constructed.

An overall sense of self-construction-active and (self-)reflexive making of personal identity-is argued to bring lifestyle to the forefront of everyday life today. In brief, although wary of exaggeration (Chapter 9), the era of mobilities (reflexive modernity; Beck 1992) incorporates the idea that a person constantly has to make choices about who they are, what they do, what they aspire to, and so on throughout a life course. There is continual "quest for an individual identity ... [a] need to be someone" (Greenfield 2008: 150). Within this project of the self, everyone chooses as "we have no choice but to choose" (Giddens 1991: 81). One outcome is expression of lifestyle mobilities (also Cohen et al. 2015) or the ability to choose and move between lifestyles.

Lifestyle mobilities have at least four dimensions. First, they express the sense that-except in extreme cases (such as cults)-lifestyles are never simply taken off-the-shelf and adopted for life. They must be continuously practiced or mobilized-reproduced and produced. Within their mobilization, a degree of "messiness" inevitably "mak[es] inconsistencies typical rather than problematic" (Lorenzen 2012: 98), for example. Second, inconsistencies and other changes, often relating to key life course transitions (marriage, childbirth), can prompt lifestyle change. This requires adopting new everyday practices and re-imagining "narrative[s] of self-identity" (Giddens 1991: 81) in a search for renewed relative existential coherence. Third, lifestyle mobilities usually rely heavily on various technological mobilities for enactment: transportation, communications and virtual mobilities (Urry 2007). Fourth, lifestyle mobilities may be globally spread but they remain strongly socio-economically marked. They are practiced most, since they are more of a practical everyday option, by wealthier people, where the resource-defined ability to construct the self is most apparent. All four dimensions of lifestyle mobilities are expressed through residential migration.

Migration can immediately be seen as active under the second dimension of lifestyle mobilities-lifestyle change. Contra much of everyday life, which is less "contemplative" (Thrift 2007: 114), undertaking relocation is usually carefully thought through, albeit often utilizing imperfect or incomplete information (Chapter 5). This can include re-thinking self-identity narratives. Furthermore, the simple fact of being in a new place almost inevitably requires adopting new everyday practices. Migration also engages strongly with the third dimension of lifestyle mobilities. Again almost by default, it becomes entangled with technologies of movement, which also in an everyday sense require reconfiguration at the destination (new commute, update Facebook page). Lifestyle mobilities' fourth dimension-socio-economic selectivity-will become readily apparent through this chapter. Finally, it is initially harder to see migration's link with the first dimension of lifestyle mobilities-continuous practice. Conventionally, scholarship has focused on the specific act of relocating from "A to B." However, reimagining it as a potentially unfolding biographical event (5.6.3) focuses attention on migration as life course practiced into the future in a new place. In summary, migration is strongly allied with lifestyle mobilities and signals the kind of "[c]hange ... in life [that] afford[s] opportunities to rethink problems, choose between the means to address them, and set new paths" (Lorenzen 2012: 112). This comes together in this section's final concept: lifestyle migration.

Lifestyle migration expresses a wish to "live somewhere appropriate for 'someone like me'" (Savage 2010: 132). On the one hand, this allies it with emerging expressions of socio-spatial segregation (Borsdorf and Hidalgo 2009), clearly shown by gentrification (8.2.3) but also apparent within strands such as amenity migration (8.3.3). On the other hand, lifestyle migration expresses a deliberative attempt to attain elective belonging, place of residence selected to fit "biographical life history ... [and be] congruent with one's life story" (Savage et al. 2005: 29, 54) or project of the self.

Lifestyle migration was explicitly defined and delineated by Michaela Benson and Karen O'Reilly less than a decade ago (Benson and O'Reilly 2009a, b) although the general subject area had long been researched (Benson 2015). Thus, Rossi (1955) noted long ago in the context of urban residential mobilities (6.3.1), residential movement taking place to access suitable social milieu for children finding marriage partners! For Benson and O'Reilly (2009b: 1, emphasis added), lifestyle migration expressed "a route to a better and more fulfilling way of life, especially in contrast to the one left behind." It predominantly comprised "relatively affluent individuals, moving either part-time or full-time,

\section{ST PROOFS: NOT FOR DISTRIBUTION}


permanently or temporarily, to places which ... signif[ied] ... something loosely defined as quality of life" (Benson and O'Reilly 2009a: 621). Six key elements develop this outline.

First, in the idea of "route" there is an emphasis on ongoing practice rather than immediate, discrete instrumental acts (Benson 2011; Halfacree 2014a; Salazar 2014). Migration, in other words, is again presented in an event-like manner, playing out within a continuing life course.

Second, undertaking lifestyle migration tends to stress change within projects of the self (e.g. Hoey 2005, 2006). Whilst some relocations may seek to retain or rediscover some aspect of lifestyle perceived as (being) lost-seeking to regain elective belonging-mostly indicates a desire for lifestyle change and a new elective belonging. It typically occurs following a "watershed event" (Salazar 2014) in one's life course; significant life transitions, such as bereavement, job loss or retirement.

Third, Benson and O'Reilly showed similar hesitancy to the present authors to seeing migration as somehow denoting "permanent" and "full-time" change. This enables lifestyle migration to encompass forms of leisure mobility, such as second home consumption (8.3.3.2), rather than be strictly separated from them (also Cohen et al. 2015).

Fourth, emphasis on "places which ... signify" highlights the key role of culture and cultural representations of place within lifestyle migrations. Differential geographical imaginaries of what everyday life is like in particular (types of) places both spurs relocation to desirable locations and encourages movements away from undesirable ones. Almost all case studies of lifestyle migration, therefore, stress the importance of representations (Halfacree 2014a).

Fifth, the definition of lifestyle migration also recognizes the slipperiness of defining what actually comprises (improved) quality of life. Whilst quality of life arguably underpinning all migrations, quality of life is an extremely relational product of culture, individual experiences, tastes and subjectivity, life course position, and so on. It also involves economic factors and in this respect lifestyle migrations should not be unduly separated from employment migrations, demonstrated in often similar responses to economic downturn, for example (Huete et al. 2013).

Sixth, it is necessary to reiterate how lifestyle migration tends to be associated with "relatively affluent individuals." It is biased towards higher social classes and richer countries. Whilst lifestyle migration is undertaken by the less affluent and, more strongly, lifestyle elements enter migration decision-making processes in general (as with economic considerations), there are good reasons for emphasizing socio-economic selectivity. First, it qualifies the potential liberating rhetoric of any era of mobilities (5.2.2) by drawing attention to class-bias in this representation of the social world's everyday dynamics (Croucher 2012). Second, affluence is closely associated with privilege and the ability to shape destination places and the lives of those within them (Benson 2014; O'Reilly 2014). Third, associated with such privilege is the element of "choice," central to lifestyle migration but again not socially uniform. Choice is always itself relationally constructed-never completely "free"-but practical enactment is more an option for some members of society than others. Benson and Osbaldiston (2014b: 3) summarized this contextualized choice in a quote also highlighting difference in emphasis between lifestyle migration and migrations covered in Chapters 7 and 9 :

Rather than a focus on production [Chapter 7] and the involuntary nature of many migrations [Chapter 9], lifestyle migration appears to be driven by consumption and is optional and voluntary, privileging cultural motifs of destinations and mobilities. In part, this drive to migrate to particular places is framed by social and mediatised constructions of particular destinations as offering an improved way of life.... Beyond this ... the better way of life sought often embeds existential and moral concerns, with the expectation that through migration these will be, in some way, resolved.

(also-Osbaldiston 2015)

Lifestyle migration research, building on pioneering studies such as The Amenity Migrants (Moss 2006), has now "developed into a healthy field of scholarly enquiry, generating its own corpus of literature" (Benson and Osbaldiston 2014b: 1; Benson 2015). This has acknowledged lifestyle migration's emerging significance for demographic and socio-economic restructuring both within and between countries (Eimermann 2015; Hoey 2014). The rest of this chapter can only begin to touch on this body of work, whilst also drawing on older literatures and well-defined research areas. Class and age, in particular, come through strongly in terms of lifestyle migration's relational constitution, with noted cameos for sexuality and gender. Expressions of individuality are also notable. Culture's role is central, shown via popular culture quotes and representations of space and place. A final theme is reiteration of the connections and uncertain boundary between migration and other forms of mobility (Cohen et al. 2015). For example, lifestyle migration 


\section{LIFESTYLE MIGRATIONS}

often stems from desire to access "good" places more "permanently" than via leisure mobilities (6.2.4). Finally, the chapter exhibits lifestyle migration's strong pro-rural flavor (Benson and Osbaldiston 2014b) but turns first to relocations to that preeminent destination for employment migration: the city.

\subsection{THE LURE OF THE CITY}

\subsubsection{Bright lights, big city ...}

I have no relish for the country; it is a kind of healthy grave.

The economic draw of the city for migrants at all geographical scales looms so large across the globe that it is easy to reduce causes of urbanization almost exclusively to economic factors. However, within the employment moves to the city covered in Chapter 7 were also noted the presence of more-than-economic factors. Indeed, one would soon discover more if the examples were considered in greater depth. These factors involve family needs but also, in many cases, seeing the city as presenting an alluring quality of life, often at odds with what is perceived to exist at the migrant's origin.

Central here-and throughout this chapter-are contrasting social representations of space (Halfacree 1993), such as city, suburb or countryside (e.g. Hill 2010; Stevenson 2003). Social representations of space are culturally inscribed expressions of geographical settlement categories that exist as "organizational mental constructs which guide us towards what is 'visible' and must be responded to, relate appearance and reality, and even define reality itself" (Halfacree 1993: 29). They comprise both concrete images and their associated concepts, values and emotions. A product of culture, the "social" in the name also emphasizes how social position, such as class or ethnicity, influences individual representational repertoires.

Table 8.1 presents two rival representational readings of both city and countryside. In one, the city is a metaphorical Jerusalem, a glowing beacon of civilization and associated enlightened progress. In the other, it is a Babylon of "dark satanic mills" imagery and squalid drudgery. In contrast, the countryside represents the city's mirror opposite (Williams 1973), broken peasants and reactionary attitudes or healthy community and rustic harmony, respectively.

Jerusalemic representations of the city provide strong migratory draws for lifestyle migrants, not least those for whom the countryside and small towns, in contrast, seem boringly moribund, impoverished or even oppressive. Draws are not merely symbolic, however, as the city appeals in terms of what is actually found within it (Hall 1998). More quantifiable lures include, of course, paid employment and (relatively) good work and career prospects (Chapter 7). However, they additionally encompass the diverse assembly of things that together comprise the "pulse of city life" (Borgegärd and Murdie 1993: 274): education (schools, universities, even a structured education system); housing (supply, if not always affordability); medical and general health services; other services (entertainments, shops, restaurants); social and cultural life (crowds, diverse peoples, cultural institutions); space(s) of safety, and for exploration and self-realization; more liberal and open attitudes; and so on. Once more, lifestyle issues overlap and merge with those concerning employment, family life and simply being able to live: quality of life.

From a life course perspective, lifestyle migrations to the city are strongly associated with young adults and the life transitions (2.2.1) of entering paid work and establishing life and identity largely independent of (parental) childhood

Table 8.1 Contrasting representations of city and country

\begin{tabular}{llll}
\hline City as Jerusalem & Country as Peasantry & City as Babylon & Country as Arcadia \\
\hline Community & Society & Mob & Community \\
Freedom & Repression & Disorder & Order \\
Progress & Regress & Work & Retreat \\
\hline
\end{tabular}

Source: from Short (1991: 81). 
home (Arnett 2000). They are much less strongly associated with other key transitions, such as marriage, having children or retirement. In terms of relational construction, age seems paramount, albeit often cross-cut with sexuality, gender, ethnicity and so on. Although class-related strands certainly occur within pro-urban lifestyle migration (8.2.3), it is less class-defined overall than its pro-rural equivalent.

\subsubsection{Urban lifestyles and self-realization}

Self-realization appeared within the diverse list above of draws of the city. This idea-developing and, hopefully, fulfilling one's potential; being, in short, (true to) oneself-is widely noted as an important underpinning of lifestyle migrations (Benson and Osbaldiston 2014b; Osbaldiston 2012, 2015). Relocation, in line with developing a reflexive project of the self, is "aspirational, not only in terms of what it holds in store for [the migrant], but also in terms of what [the migrant] can become" (O'Reilly and Benson 2009: 5). This takes migration beyond simply escaping a life and/or place seen as stifling or oppressive and towards seeing it as opening the possibility of more radical, even eventful life change. Of course, as O'Reilly and Benson (2009: 5) warned, it is frequently "not so easy in practice for ... migrants to shed the skins that are their past lives." One must thus be wary of falling for overly strong rhetoric of change that often accompanies lifestyle migration. Nonetheless, especially when escaping immediate personal crisis, a degree of selfrealization can come through with the move (Halfacree 2014a; Nowok et al. 2013).

Self-realization through pro-urban lifestyle migration will be illustrated with help from three British Indie Rock songs of the 1980s, although almost any genre of popular music could have been chosen from across the globe. The heterogeneous lures of the big city for young adults, in particular, have strong and ubiquitous presence within popular culture (Connell and Gibson 2003; Stevenson 2003: Chapter 7).

First, in The Smiths's London, ${ }^{1}$ a young working class man leaves Manchester in northern England for a new life in the capital. The move is clearly of great significance, although only two hours away by train, as he wonders in the song if he has made the "right decision," apprehensive of the trials and tribulations ahead. Moreover, whilst such a migrant will clearly require employment in London-he seems a speculative labor migrant-it is life transition and lifestyle that come through most strongly. This is reflected by the songwriters' emphasizing the "[j]ealousy in the eyes" of the family this emerging adult (Arnett 2000) leaves behind.

Second, more "disreputable" attractions also lure younger adults to the urban lifestyle, where the city should be seen more as Babylon than Jerusalem (Table 8.1). This is suggested by Shane MacGowan's gendered and sexualized depiction of the city in London You're a Lady. ${ }^{2}$ Whilst the city may have a "heart of gold," the scarred thighs and sad eyes suggest its "ring of lights" present a dangerous lure for the unwary. It is one, however, many follow. Far from the typical lifestyle migrant pre-eminent in this chapter, cities such as Berlin, London, New York and Paris have long attracted those seeking life outside the perceived oppressions and conformities of the mainstream (Griffiths and Maile 2014; Scott 2006). Celebrated (by others, reviled) Bohemian quarters of cities-Fitzrovia and Soho, London, 1900-1955; Greenwich Village, New York, between the wars; Montparnasse, Paris, 1910-1930 (Wilson 2000)-are populated largely by migrants for whom the lures of drink, drugs, freer sexuality, music and art are typically much stronger than promises of work and career (e.g. Moraes 1995).

London's Soho is also now noted for its vibrant gay cultural life, which came thoroughly to prominence from the mid 1980s (Collins 2004). It may well be to this district, therefore, that the third song's protagonist, Bronski Beat's Smalltown Boy, ${ }^{3}$ is heading. Escaping small town homophobia, where even parents are unsupportive, the young man is taking the train to a place where he hopes to find both the relative freedom and love that can never be found at home (Box 8.1). Other cities' districts have similar migratory appeal, such as Castro in San Francisco (Weston 1995) or Kreuzberg in Berlin (Heebels and van Aalst 2010).

Although London long had a noted gay cultural and entertainment presence, the emergence of Soho's so-called "Gay Village" arose from a specific set of circumstances (Collins 2004; Mort 1995; also 6.5). This indicates a general point that emergence of distinctive socio-cultural geographies through migration must never be attributed solely to migrants' residential demands. In Figure 8.1's model of "alternative" place development, it is not only a matter of demand but also of supply, whereby geographies are produced to act as particular lures to specific population sub-groups. The figure expresses co-production between sub-group members and profit-orientated capitalist interests, members of both groups sometimes overlapping. Typically, ascendancy of the latter eventually leaves little more than a superficial "alternative" veneer (Stage 5) and one must search elsewhere for a new alternative place (Fenton 2004).

\section{ST PROOFS: NOT FOR DISTRIBUTION}




\section{BOX 8.1 THE RURAL AS DESTINATION FOR LGBT MIGRATIONS}

Whilst sexuality is not reducible to lifestyle choice, its everyday cultural performance has lifestyle expressions. For example, think of the cultural rituals and performances that help construct, sustain and reproduce heterosexual identities. However, such lifestyle expressions are always relationally constructed. In particular, a high degree of heteronormativity (2.3.9), even aggressive heterosexuality, across much of the world both reflects and reinforces certain sexualized lifestyle forms and practices at the expense of those not wishing and/or unable to conform to such lifestyles. In short, the practice of everyday sexual lifestyles is undoubtedly relational to a "civil society [that] can be conceptualized as a heterosexual (as well as patriarchal and racist) construction that serves to make entry into the public realm very difficult for those whose sexual lives are judged 'immoral'" (Hubbard 2001: 55).

For the LGBT community, one consequence of such intolerance and repression is migration to locations seen as more "tolerant" or even supportive of lifestyles associated with their sexuality. Whilst one may label such relocations "lifestyle migrations" in that they are clearly seeking places promising a better quality of life, where (sexual) authenticity can be realized within projects of the self, one must also recognize such movement as a possible response to immobilities due to fear (6.3.5) and demonstrating overlaps with Chapter 9's focus on forced relocation.

Larger cities or quite well-defined districts within these cities are usually presented as the places most attractive for LGBT lifestyle migrations, as the main text suggests. However, numerous studies since the mid1990s have discussed rural locations of relative sexual inclusivity. They reveal how rural LGBT sexual identities and lifestyles are (re)produced, with "rural spaces ... sexed and rural sexualities ... spaced" (Smith and Holt 2005: 314). In other words, one must not "take for granted that lesbian and gay lives are lived in the urban environment" (Binnie and Valentine 1999: 178). Moreover, LGBT people, as with heterosexuals, often have nonsexualized lifestyle preferences for rural places. Again, a life course related change from young adult preference for the city to more middle-aged desire for the rural may occur. In summary, Binnie and Valentine (1999: 178) noted:

a cartography of lesbian and gay rural existence and experience ... [T] he rural in the sexual imaginary [is] simultaneously utopian and dystopian - a place of escape or becoming, as well as a place to escape from ... For those raised in rural communities, the city is most likely to be seen as a place to escape to in order to define oneself as lesbian or gay. For those who actively chose a rural life the attractions are manifold.

Consequently, greater attention should be paid to "the significance of migration in lesbian and gay lives and identities" (Binnie and Valentine 1999: 179).

Consider Hebden Bridge in Yorkshire, northern England. Since the 1970s, this town has experienced rural gentrification stemming mostly from lifestyle in-migrations and market response to these, with property prices rapidly rising, poorer households displaced and the social composition of the former industrial, cloth-manufacturing town changing markedly in favor of the white-collar middle and upper middle classes. However, change has also been underpinned by a developing representation of Hebden Bridge as an "alternative" rural place. Further development led to the town being nominated "Sapphic Capital of Britain" within national and local media. The Observer (2000: np) newspaper, for example, claimed "the proportion of lesbian to heterosexual residents [now outstrips] that of [noted gay communities within] Manchester, London or Brighton."

Darren Smith and Louise Holt investigated the migrations and lives of lesbian residents in Hebden Bridge in the early 2000s. They found "two key place-specific appeals of rurality [to be] fundamental" (Smith and Holt 2005: 316). First, in contrast to the heterosexually aggressive city, idyllic rural representations suggested a site of safety and security. Second, Hebden Bridge's noted alterity and lifestyle diversity "promote[d] the inclusion of lesbian households, who are not marginalised or othered within wider social relations and practices" (Smith and Holt 2005: 316).

Evidence more generally suggests that connections between lifestyle migration, LGBT sexualities and gentrification made in the urban gentrification literature are equally applicable to such seemingly "out of the way" rural places as Hebden Bridge. Thus, Smith and Holt (2005: 321) concluded that: 
lesbian households represent a conspicuous and powerful social grouping which influences the socio-cultural construction of (semi-)rural spaces and places, and their sexualised and socio-cultural practices must be incorporated into analyses of rural change.

And lifestyle migrations appear to lie at the root of and to power this process.

(Sources: Binnie and Valentine 1999; Hubbard 2001; Robehmed 2012; Smith and Holt 2005; Smith and Phillips 2001)

Spaces of difference morph into spaces of exclusion (Zukin 2008), especially when gentrification is involved, considered next.

\subsubsection{Contested geographies of gentrification}

Migration to the city, as to any place, can have major consequences for those already living there. This is especially the case with gentrification. As with "alternative" relocations to the city, potential market demand from such migrations is picked up by entrepreneurs, place promoters and other capitalist interests. With the migrants, they co-produce reshaped urban places and their population geographies.

Ruth Glass coined the term gentrification in 1964. It emerged both from her research and life experiences within a London of "accelerating rehabilitation of Victorian lodging houses, tenurial transformation from renting to owning, property price increases, and the displacement of working-class occupiers by middle-class incomers" (Slater 2011: 571). In other words, gentrification signifies urban geographical class transformation, expressed via differential flows in class terms of in- and out-migrants. It has subsequently been demonstrated as widespread geographically, initially across the Global North (e.g. Butler 1997; Rose 1984; Slater 2011; Smith 1979, 1996; Smith and Williams 1986) but now encompassing many cities in the South (e.g. He 2007; Whitehead and More 2007; Visser 2002). Consequently, it has attracted substantial academic scholarship and there is only space to pick up some aspects.

Whilst gentrification takes place in areas of the city typically rundown due to abandonment and disinvestmentfrom both state and private sectors and the resulting socio-economic marginalization and poverty-it is notably propelled

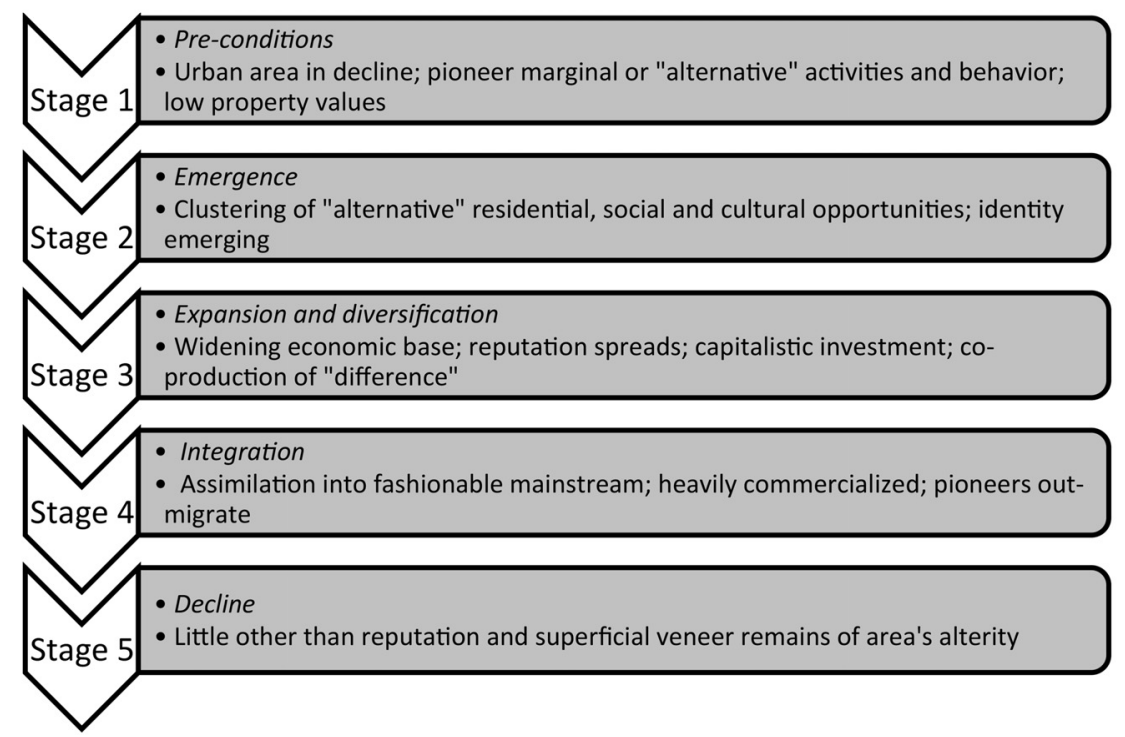

Figure 8.1 Model for the development of "alternative" urban districts.

Source: modified from Collins (2004: Figure 2). 


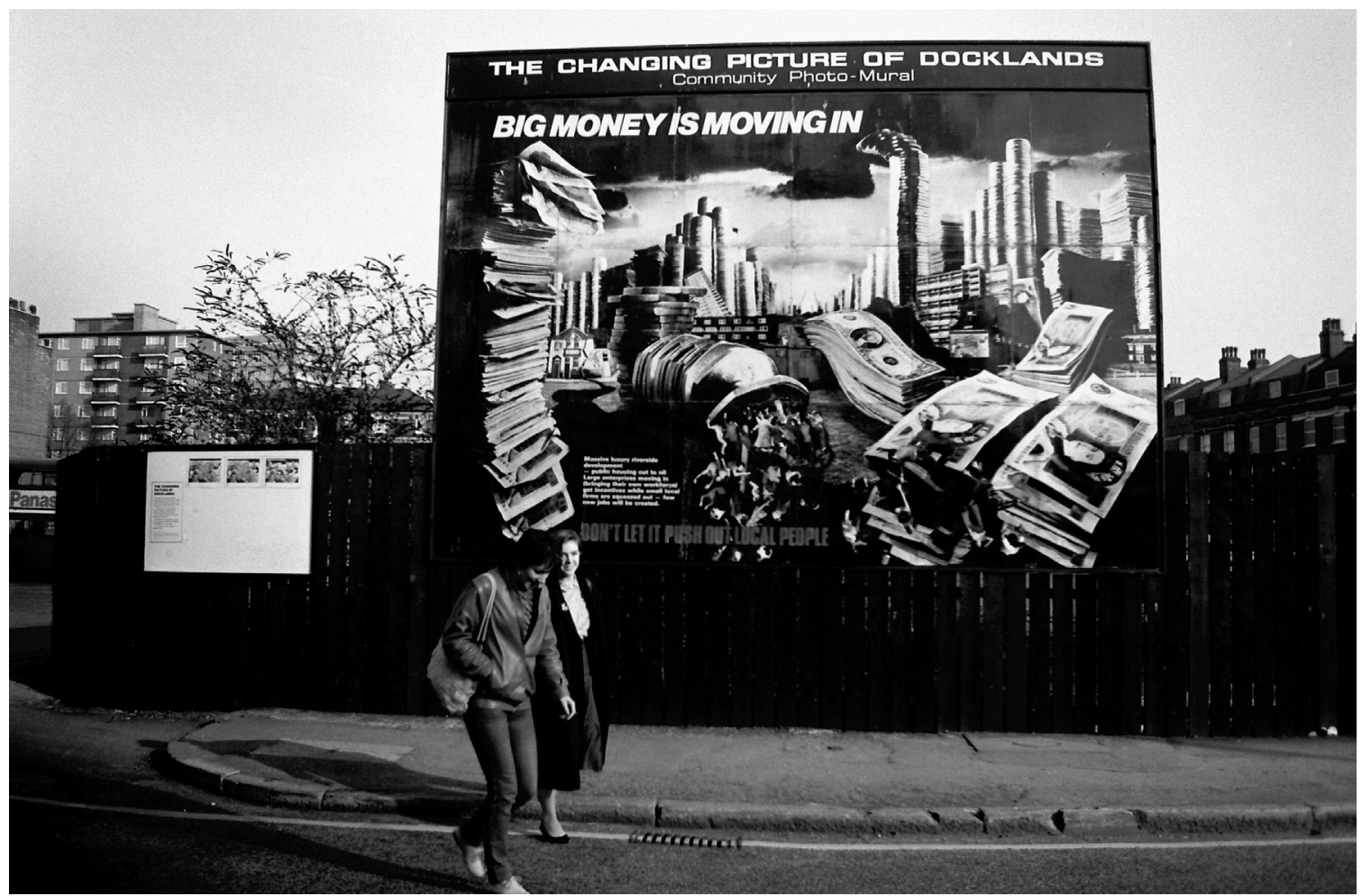

Figure 8.2 London's Docklands in the 1980s, just before gentrification.

Source: Bill Pearson.

forward by profit-seeking housing and place producers as much as by consumers and their lifestyle choices (Davidson 2007) (Figure 8.2). Thus, whilst British sculptor Grayson Perry (2014: 96) posited artists as "the shock troops of gentrification," enlivening forgotten neighborhoods and making them suddenly fashionable, their Bohemian agency was soon displaced by that of the property developer. Indeed, lively debate between rival supply-driven and demandled explanations of gentrification, rooted in contrasting philosophical positions, has been a constant if divisive theme within gentrification scholarship (e.g. Hamnett 2010; Slater 2011).

Focusing on gentrifiers, the in-migrants, their age and socio-economic position give clear clues why they migrate to inner-city neighborhoods to live and what they hope to obtain from relocation. First, they tend to be young adults, often recent graduates. In fact, Smith and Holt (2007) proposed studentifiers (Box 7.1) as "apprentice" gentrifiers. If not directly bringing about gentrification with their residence patterns (this happens in some areas that shift from studentified to gentrified), higher education students can acquire lifestyle predilection for inner-city living. They also have the cultural and educational capital that, after graduation, allows them to obtain the high salary jobs needed to become gentrifiers. This leads to the second key feature of gentrifiers, their tendency to be from higher social classes, in professional jobs. Inner-city gentrified neighborhoods have high cost housing, whether owned or rented, so moving into such accommodation requires high-paying employment.

Gentrification, therefore, predominantly expresses migration of young professionals to both refurbished and new build gentrification (Rérat et al. 2010) properties in formerly impoverished urban neighborhoods. Both convenience and culture are important (Hjorthol and Bjørnskau 2005). Inner-city location facilitates such everyday needs as relatively easy access to employment, typically within the same city and often demanding long working hours (making long commutes particularly onerous). However, lifestyle issues generally are central to the gentrification experience. This includes extending student lifestyle traits within the life course, such as visiting late night bars and clubs, and "acceptance of 'less-traditional' norms of sexuality, gender, and ethnicity relations" (Smith and Holt 2007: 156). It also increasingly includes less desired house-sharing with fellow young professionals, due to high housing costs (Smith

\section{ST PROOFS: NOT FOR DISTRIBUTION}


and Holt 2007). Nonetheless, gentrification goes further than a studentification "hangover." Tim Butler's work (e.g. Butler 2003, 2007; Butler and Robson 2003), for example, has demonstrated how middle class migrants to districts across central London strove to build the place-based sense of rootedness they perceived lacking within highly "globalised" work lives. Behaviors expressed projects of the self, involving "build[ing] a local community within the global city that maps onto their particular set of values, backgrounds, aspirations and resources" (Butler and Robson 2003: 1795). In short, young professionals can aspire to become grounded, emplaced and "authentic" (Benson and Osbaldiston 2014b) in gentrified places. However, this effort at self-realizing emplacement often occurs "in a bubble," oblivious to everyday lives of other groups within the neighborhood, a finding commonplace for lifestyle migrations generally. It is reflected, for example, in sending children away to school, whereby they have almost no contact with working class children in their area (Butler 2003; also Lees 2003 and Butler and Lees 2006 on super-gentrifiers).

Gender has also been identified as a central element in the gentrifier lifestyle (Bondi 1999). This is associated with increased feminization of the professional workforce (Hjorthol and Bjørnskau 2005). For example, where adopting a dual-career structure (7.5.3.2) necessitates renegotiating domestic responsibilities, gentrification offers a middle class female everyday life strategy to reduce the time-space constraints of dual spheres of waged labor and domestic responsibility (Bondi 1991; Rose 1989; Warde 1991).

Gentrified urban locations have also been seen as more conducive than villages, small towns or suburbs to less conventional lifestyles (England 1991), from single parents (Rose and Le Bourdais 1986) to the gay community (Knopp 1990; Weston 1995). Thus, Doan and Higgins (2010: 3) spoke of gentrification reflecting "the collective aspiration of the LGBT community to find neighborhoods in which they can actualize their quest for an otherwise marginalized identity," although they qualified the extent to which this occurs and its degree of permanence. Indeed, their Atlanta (US) study found a new wave of gentrification had led to formerly-strong LGBT neighborhoods becoming less tolerant of LGBT people and their businesses, an example of what is described below as urban revanchism.

Gentrification rarely just adds socially selective urban residential numbers to a city district. Whilst new build and refurbishment of derelict properties may provide new spaces for gentrifiers and thus not directly impact on fellow residents, other gentrifiers inevitably take accommodation previously owned or, more likely, rented by working class households. Moreover, gentrification sees the class texture of a place change considerably, with negative implications for existing community structures and practices. Reflecting this, out-migration is also a principal demographic feature of gentrification. Gentrifiers themselves demonstrate high population turnover-often being spiralists (7.4.2)-but net out-migration from gentrifying neighborhoods typically express working class people increasingly unable to afford to live within fragmenting communities. Neil Smith (1996) regarded such displacement as part of a "reclaiming" of urban space by the middle class that formed part of a larger revengeful political project-urban revanchism. This sought to turn-back more liberal efforts after 1945 towards economic redistribution, civil rights and affirmative action in favor of the discriminated and dispossessed. Instead, Smith recognized increasing policies and practices of social and spatial banishment to the margins for "public enemies" of the dominant classes. Such an appreciation links lifestyle migrations of gentrification to the kinds of forced migrations covered in Chapter 9, not least via evictions and dispersion of public housing tenants and their replacement by the middle class and very rich (Lees 2014; Slater 2013).

Drawing attention to such negative class-specific consequences and effects lay at the core of Glass' original coining of gentrification. It is the reason researchers such as Slater $(2006,2011)$ continue to advocate using the term, as against alternatives such as residentialization (Bromley et al. 2005), even if the latter acknowledges the sense of lives lived by gentrifiers themselves. Finally, beyond academia the term gentrification is deployed widely by those campaigning against and resisting displacement of working class people from inner-city neighborhoods (Figure 8.3; London Tenants' Federation et al. 2014). These neighborhoods may look today much the same as a century ago but "the ways they are used and the people who use them have entirely changed" (Zukin 2008: 725). Thus do population geographies evolve through migrations.

\subsubsection{The lure of the city in the Global South}

This chapter's coverage and examples are predominantly from the Global North, since it is there that overall social prosperity has developed most fully and generally, to facilitate the option of lifestyle migration within overall projects of the self. All the case studies in Benson and O'Reilly (2009c) and Benson and Osbaldiston (2014a), for example, are either set within the North or involve international migrants from the North. People across the Global South are

\section{ST PROOFS: NOT FOR DISTRIBUTION}




\title{
We Call These Projects Home
}

\author{
Solving the Housing Crisis from the Ground Up
}

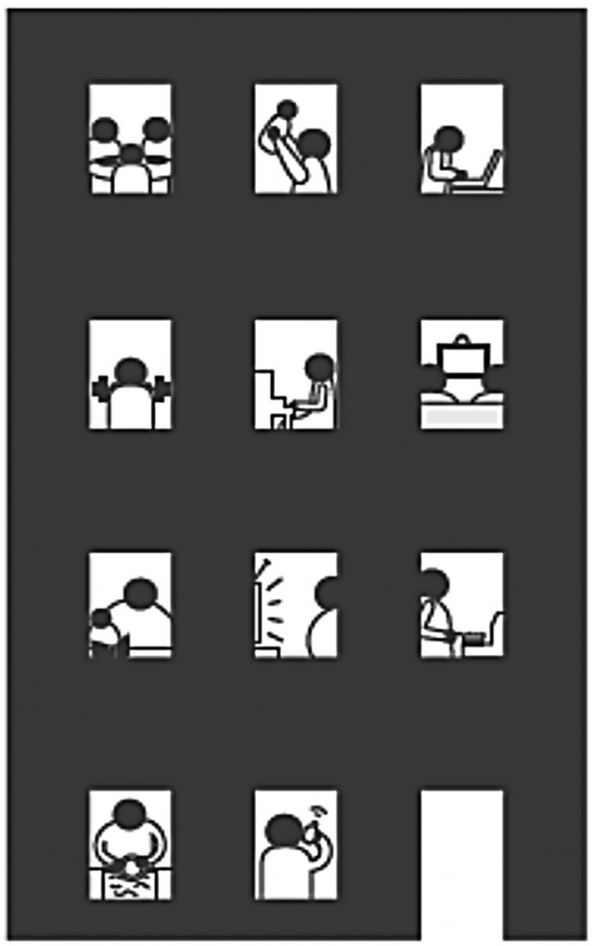

\section{A RIGHT TO THE CITY ALLLANCE REPORT ON PUBLIC HOUSING MAY 2010}

Figure 8.3 Resisting gentrification displacement.

Source: designed by Roston Woo. Online. Available HTTP: <http://righttothecity.org/wp-content/uploads/2014/02/We_Call_These_Projects_Home2.pdf>, accessed May 2016.

equally engaged with projects of the self but overall lack of resources, especially monetary, see explicit lifestyle migration featuring less prominently. Nonetheless, it would be wholly wrong to suggest that lifestyle factors do not feature frequently and often prominently within the migration decision processes of even some of the poorest in the Global South. As noted in Chapter 7, migration to the city may be driven largely by need to obtain paid work but the city also has other attractions: supply of general services, life opportunities and collective consumption (provision of public services-electricity, water, sewerage). Better quality of life is seen as more attainable in the city.

As in the Global North, a lifestyle element within pro-urban migration is especially strongly expressed by young adults. Once more, the attraction of the city's perceived association with relatively less oppressive and more tolerant attitudes towards diverse lifestyles and greater political freedoms is also important (whatever the reality; Williams et al. 2009), not least for women. Even the overall emotional or affective feel of a dynamic, vibrant city for a newcomer can prove attractive, not least for emerging adults (Arnett 2000) beginning to construct independent life course

\section{ST PROOFS: NOT FOR DISTRIBUTION}


identities. In sum, "[c]ities have appeal, . . . urban vibrancy and vitality . . . offer . . . residents' lives infused with innovation, difference, sociability, anonymity, rapid change and variety" (Williams et al. 2009: 168, emphasis added; also Boyle et al. 1998: 130-134). An additional migratory attraction of cities of the Global South is post-colonial assertion of progressive imagined futures. Often the socio-spatial crucibles of independence, Southern cities are eften mobilized more broadly as sites of civil society protest and assertiveness (Box 8.2).

\section{BOX 8.2 THE CITY AND PROTEST MOBILIZATION}

The quality of life lure of the city for migrants across the globe combines a strong economic element-the city as the principal site of "modern," relatively lucrative and abundant employment (Chapter 7)-with a broader set of everyday lifestyle appeals, as noted in the main text. Yet, it is important not to leave pro-urban migrants as they arrive in the city but to follow them a little further through their life course. In particular, it is useful to investigate the extent to which various positive imaginative offers of the city are subsequently realized. Does the city live up to its promises and perform its "enabling function[s]" (Jaffe et al. 2012: 644)? If unrealized, how do migrants respond?

Whilst most migrants may have little realistic choice but to manage as best they can when faced with unrealized positive urban experiences, not least due to lack of resources, others may take part in various forms of protest. This usually brings them into alliance with non-migrant urban residents, as "migrant" status becomes decreasingly relevant within their life course practices. Although rural protests remain prominent across the Global South, the (relatively) public spaces of the city both historically and in the present-day are widely recognized as primary geographical sites of mass political contestation. Within these manifestations, urban in-migrants' mobilities of (unfulfilled) residential relocation again become entangled with those found elsewhere on the mobility spectrum (Figure 5.1). This is expressed in protests for mobility and mobilities for protest.

First, urban residents express protests for mobility. These take many forms but together reiterate the ubiquity of mobilities in underpinning the everyday needs and demands of the life course. Examples include protests:

- For food and basic sustenance-"food riots" against the failure of food mobilities to reach the most poor;

- Against high housing costs-highlighting the stifling of both residential mobilities (Chapter 6) or even mobilities into any form of housing (homelessness);

- For employment mobilities-when the political-economic geography of the city stands firmly in the way of the upward social mobilities normatively associated with urban living;

- Against urban elites blocking residential and everyday mobilities-highlighting such exclusionary developments as gated communities (6.3.4) and inadequate affordable public transportation;

- For more individual and social control over life course mobilities-manifestations challenging the perceived hegemony of neo-liberal globalization's control, via institutions mostly based within cities, over everyday life;

- $\quad$ Against exclusion from political power and decision making within the city-political mobilities perceived as being over-ridden in contexts such as loss of public or green spaces.

Second, large scale urban protests in any of the categories above also require mobilities for protest. These not only encompass the physical movement of bodies to protest sites, itself often a major undertaking where transport infrastructure is poor, but also more virtual forms of mobility. This addition highlights the ever-present link between physical and imaginative mobilities. For a powerful urban protest to be enacted today-from the anti-neo-liberal globalization manifestations of Seattle in 1999 or Genoa in 2001 to the mass youthful political uprisings of the Arab Spring of 2011 (4.3.2) -not only will cultural resources, internet and telecommunications technologies be widely deployed to promote and circulate the event but so too will less tangible aspects such as solidarity, hope and passion need effective mobilization.

Overall, therefore, new urban arrivals' lives are likely soon to feed into the highly mobile mix of politics, passion and potential that is encapsulated by the normative political expression Right to the City. Whether that right goes on to be realized through their life courses, however, remains a very uncertain and highly relationally dependent outcome. Mass protests can, perhaps, sometimes help.

(Sources: Bush 2010; Jaffe et al. 2012; Köhler and Wissen 2003; Mayer 2009) 


\section{LIFESTYLE MIGRATIONS}

Finally, whilst the overall lifestyle lure of the city may be strong generally and assumes many forms across the Global South, specific and selective manifestations are also present. This includes gentrification (e.g. He 2007, 2010 on China; Whitehead and More 2007 on India). In South Africa it has been widely observed (Garside 1993; Visser 2002). This has been linked, in part, to changes in how city centers have been managed by the state. Redevelopment initiatives, such as central-city improvement districts (CCIDs), have kick-started rapid inner-city change in places such as Cape Town. As Visser and Kotze (2008: 2566) observed, "[o]nce the state decided to facilitate [city center] regeneration ... the market, in its many guises, immediately "came to the rescue'." The result has been less displacement of poorer people as focus is on new build and office conversion. However, city centers are still becoming class-defined exclusive spaces, whereby official talk of "mass settlement of the urban poor in the [city center] is utopian and empty rhetoric" (Visser and Kotze 2008: 2586). Overall, as Smith (2002) recognized over a decade ago, gentrification-with both in- and out-migration consequences-presents global urban revanchism.

\subsection{THE LURE OF THE COUNTRY}

\subsubsection{Being able to see the stars ...}

New York was a city where you could be frozen to death in the midst of a busy street and nobody would notice. Bob Dylan, Chronicles, Volume 1 (2004: 210)

[L]ife in the country is the only state which has always completely satisfied me... Now I ... know the joys of six or seven months a year among fields and woods of my own, and ... the deep joy of communion with the earth.

Edith Wharton, A Backward Glance (1934: 124)

In contrast to Jerusalemic representations of the city, typically juxtaposed by representations of the countryside as the domain of a reactionary quasi-peasantry, many people across the Global North in particular regard the city in Babylonian terms (Table 8.1). In contrast, the rural or countryside is read as a scenic enfolding and relaxed Arcadian community. It is a place where musician Laura Veirs ${ }^{4}$ can, both literally and metaphorically in terms of self-realization, "see the stars" (also Halfacree 2009, 2016). The key demographic consequence of this association is burgeoning migration seeking to access such experiences.

Assuming central prominence within residentially alluring representations of the countryside is what Cloke (2003b: 2) described as the powerful "centripetal force" of rural idylls. Highly positive rural representations are found across the world. Bell (2006: 150) recognized three ideal types: pastoral (farmscapes), natural (wildscapes) and sporting (adventurescapes). All have strong historical roots (Bunce 1994; Mingay 1989; J. Short 1991; B. Short 2006; Taylor 2011). They generally present the countryside as "relaxed and relaxing, scenic yet human scale, organic and natural, authentic and rooted, and somehow external to or otherwise distanced from (the negative features of) modern [urban] society" (Halfacree 2012b: 391). In sum, from analysis of a Canadian newspaper story:

Picturesque, farming, community, recreational, bucolic: these are the words of the conventional rural idyll, of the aesthetics of pastoral landscapes, of humans working in harmony with nature and the land and with each other, of a whole scene of contentment and plenty.

(Bunce 2003: 14)

Idyllic rural representations are resources strongly implicated in lifestyle migrations, demonstrated below. However, they also feature in detailing other types of migration when one rejects over-simplified "sound bite" (Stockdale 2014) explanations. For example, a new job might bring about relocation if not commutable (Chapter 7) but precise choice of where to move is often influenced (affordability is clearly significant; e.g. Osborne 2014) by lifestyle considerations. Thus, in a case study of urban-to-rural relocation within late 1980s England (Halfacree 1994), "employment" was the primary reason given by over half longer-distance movers but almost all cited aspects of the "environment" at a secondary level when explaining the move's full geography. Furthermore, potential for idyllic countryside representations 
to play a role within even seemingly non-lifestyle moves is facilitated in the era of mobilities, where personal mobility is typically very high (6.2). Their still greater potential for facilitating explicitly pro-rural lifestyle migrations is even clearer.

The demographic significance of countryside representations does not end with acts of relocation (Benson 2011; Halfacree and Rivera 2012; Halfacree 2014a). Where rural places do not meet migrants' expectations, for example, efforts may be made within "post-migration life" (Benson and Osbaldiston 2014a: 3) to re-shape them, environmentally, socially and affectively into conforming to the desired image (Halfacree 2012b; Murdoch and Marsden 1994).

Besides physical landscape features (Halfacree 1995b), rural idylls typically embody assumptions about domesticity (Little and Austin 1996). Although there are exceptions (on rural challenges to heteronormativity, see Bell and Valentine 1995; Valentine 1997; Box 8.1), these imaginaries generally reinforce conventional heterosexual nuclear family households (Little 2003):

the rural idyll [presents] very conventional images and expectations of women's place in rural society; at the heart of the family, the centre of the community. There can be no doubt that the woman of the rural idyll is the wife and mother, not the high-flying professional, the single childless business entrepreneur.

(Little and Austin 1996: 106)

Idyllic life also tends to be pro-natalist, promoting child-bearing within conventional nuclear families. Emphasis on the nuclear family as the basis of rural community also circumscribes care of the aged and sick to immediate (female) relatives (Hughes 1997).

Whilst idyllic representations of the countryside may predominantly be expressed through "the bourgeois imaginary" (Bell 2006: 158), they may nonetheless "speak of a profound and human need for connection with land, nature and community" (Bunce 2003: 15; Halfacree 2012b). This possible need can be linked to the search for authenticity-of self, place, everyday life. Authenticity is widely regarded as underpinning much lifestyle migration but especially that involving rural destinations (Benson and Osbaldiston 2014b; Osbaldiston 2012, 2015). Seeking out a rural place is a dominant strategy for gaining greater authenticity for the project of the self. Although often having to be worked at following migration (Benson 2011; Jobes 2000):

[t]he individual lifestyle migrant often utilises place as a toolkit to capture authenticity in their accounts of lifestyle migration; post-migration experiences are mobilized in the affirmation of such authentic places.

(Benson and Osbaldiston 2014b: 9)

Some sense of this utilization is covered within the vast literature on counterurbanization, whose story is now introduced.

\subsubsection{The counterurbanization story}

\subsubsection{Introducing the story}

At least until the 1970s, the dominant demographic image of rural populations throughout the Global North was one of decline (e.g. Johnston 1966). People migrated away from rural locations towards growing towns and cities, a loss captured evocatively by the phrase "drift from the land." It is an image that persists and, indeed, still accurately depicts the demographic experience of many rural areas (3.3.4). However, based initially on evidence from US census data (e.g. Beale 1975; Berry 1976), Demographers and Population Geographers from the mid 1970s began to noticein some parts, of some rural areas, in some countries-rural populations increasing through net in-migration. Together with optimistic talk of "population turnaround" (Brown and Wardwell 1980) and "rural renaissance" (Morrison and Wheeler 1976), the term counterurbanization was coined (Berry 1976). It may be defined simply by Figure 8.4's negative linear correlation between population growth and settlement size (Fielding 1982), reversing urbanization's positive correlation.

Counterurbanization ${ }^{5}$ soon attracted considerable research attention. It has developed into what one of its leading researchers, Tony Champion (1998), termed "the counterurbanisation story." This metaphor is highly apposite. The Concise Oxford Dictionary (1964) defined "story" as: "[p]iece of narrative, tale of any length told or printed in prose 
208 LIFESTYLE MIGRATIONS

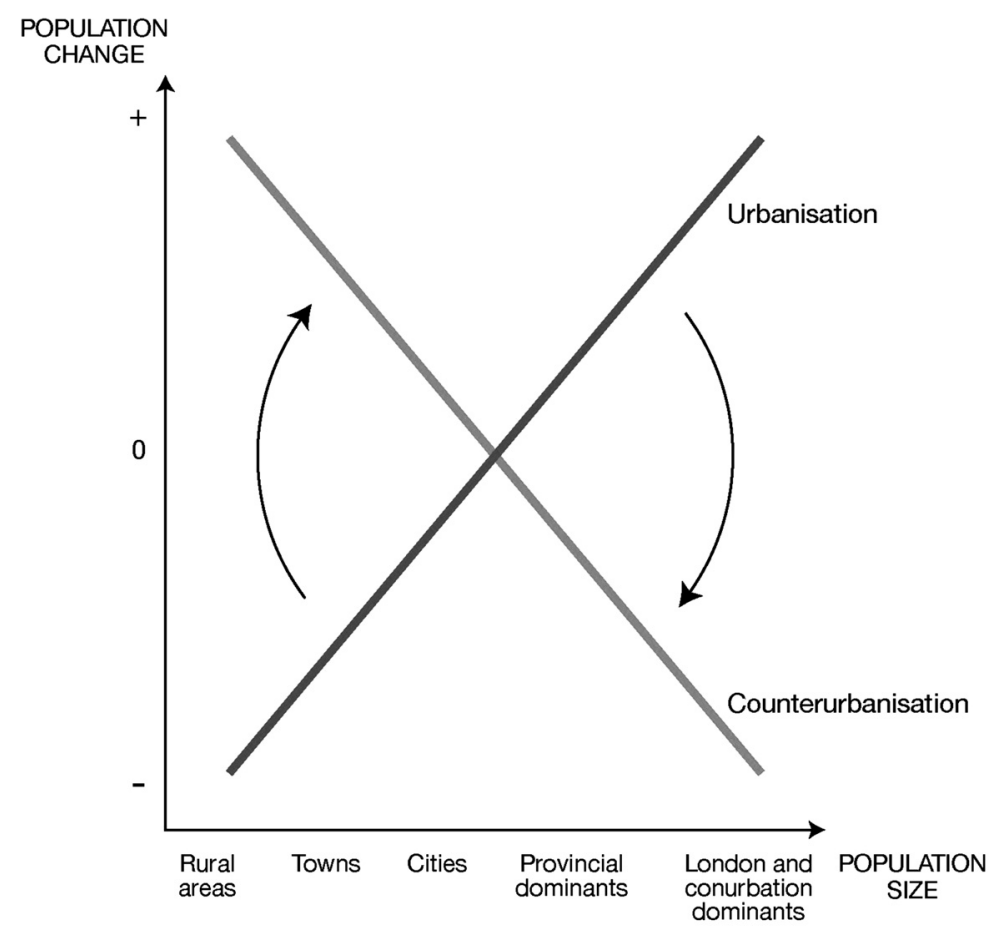

Figure 8.4 Graphical representation of counterurbanization

Source: based on Fielding (1982).

or verse of actual or fictitious events, legend, myth, anecdote, novel, romance." All of these angles have featured within counterurbanization's academic and popular telling (Halfacree 2008). It is now, in short, a saga!

Researchers soon recognized what was happening with counterurbanization was not simply metropolitan expansion into the countryside, although exurban growth of "urban-connected country living" (Taylor 2011: 323), producing "amenity-driven sprawl" (p.335), was and remains commonplace. Instead, people "voting with their feet" and migrating to live within more rural residential environments could relocate far from the commuting ranges of major cities. Furthermore, this need not simply be relocation from city to countryside (Champion 2001; Halliday and Coombes 1995; Mitchell 2004) but express a broader "pro-rural" (Halfacree and Rivera 2012) inclination. Champion and Atkins (1996) suggested a "counterurbanization cascade," migrants taking multiple moves (largely) down the urban hierarchy, ultimately reaching unambiguously rural destinations.

Much academic effort has been expended trying to explain counterurbanization (Halfacree 2014b; Mitchell 2004). Early attempts, reflecting Jeffersonian agrarianism and the US "frontier thesis" (Bunce 1994), presented it as a "natural" human evolutionary phenomenon. It was then recognized as enabled by technological developments in line with the emerging era of mobilities (private transportation, labor-saving devices in the home, commuting) that facilitated residence distant from workplace. Cultural factors were also strongly emphasized, as Berry's (1976: 24) initially hypothesized "reassertion of fundamental predispositions of the American culture ... antithetical to urban concentration" became more critically nuanced (Hoey 2014; Jacob 1997; Jobes 2000). Elsewhere, counterurbanization acquired a "wholly darker, more hard-edged, materialistic and realistic explanation" (Fielding, 1998: 42) linked to the dynamics of the capitalist class structure (Fielding 1982). Notably, rural areas increasingly presented both economic opportunities and a geographical milieu for service class identity to develop (Thrift 1987). Within all this debate, representations of rural (and urban) places were accorded a key role in mobilizing the migrations (e.g. Bijker 2013; Dirksmeier 2008; Halfacree 1994; van Dam et al. 2002).

An unfinished chapter in the counterurbanization story brings it squarely into the lifestyle migration orbit through emphasizing process and outcomes, but also cautions not to give too much weight to representations (Halfacree 2013a, 2014a; Halfacree and Rivera 2012). In short, there is more to moving to and living within the countryside than 
just linking it to social representations of space. Beyond the "armchair countryside" (Bunce 1994), more-thanrepresentational encounters are active within counterurban relocations (Box 8.3). This recognizes how an experiential and embodied sense of what it feels to be in the countryside must not be lost. Features such as feeling closer to nature or isolated and alone can moor a migrant in their "authentic" rural place or prompt them to move away, respectively. As with many migrations, counterurbanization does not essentially "end" with residential relocation but is, like lifestyle, an ongoing practice.

\section{BOX 8.3 RURALITY: FROM REPRESENTATIONAL TO MORE-THAN- REPRESENTATIONAL}

[L]andscape ... is not the passive object of our gaze, but rather a volatile participant - a fellow subject which arches and bristles at us, bristles into us.

(Macfarlane 2012: 254)

In the Handbook of Rural Studies, Paul Cloke (2006) drew attention to the social construction perspective for understanding rurality. This approach, informed strongly by social science's late-twentieth-century "cultural turn," has sought to articulate "the fascinating world of social, cultural and moral values which have become associated with rurality, rural spaces and rural life" (Cloke 2006: 21). Rurality, in other words, was regarded primarily as a representation of socio-cultural issues.

However, in a subsequent review of the sub-discipline of Rural Geography, Michael Woods (2009: 850) observed a "creeping back into discussions of questions about the definition and conceptualization of rurality." A key reason for this, he argued, was renewed desire to re-materialize rurality, to recognize it as more than a socio-cultural product. Indeed, a critical reflection on the cultural turn is that it dematerialized social life, neglecting "more thingy, bump-into-able, stubbornly there-in-the-world kinds of matter" (Philo 2000: 13). There are thus now calls to rise from the "armchair countryside" (Bunce 1994: 37) and "get hands dirty" by (re-)engaging directly with the earthy material rural of soil, rocks, water, animals, plants, insects, weather, temperature, and so on.

Desire to refocus on what it is like existentially and sensuously to be "in" the rural does not come (solely) from some quasi-libidinal impulse but also more soberly reflects interest in non-representational theory (Thrift 2007). This wide-ranging body of scholarship builds on principles such as emphasizing action over contemplation, and recognizing ourselves as being entangled in the world rather than somehow standing aloof. A core concept is affect, which broadly refers to the feelings, emotions and actions brought about through these bodily engagements. Taking affect seriously in the context of rurality requires paying direct attention to the rural's own forces-including agencies of non-humans: from other animals and plants to inanimate objects and physical forces. All can be summarized as the more-than-human (Whatmore 2002).

Appreciating the non-representational resurrects the lively affective aspect of a "natural" rurality conceptually killed through overconcentration on "dead" (Carolan 2009: 1) representational rurality. However, it is unproductive to set up representation and non-representational in binary opposition. Both require adequate attention. Rurality should be seen as neither predominantly representational nor non-representational but as embracing both through the notion of the more-than-representational (Lorimer 2005).

Exploring pro-rural lifestyle migration through a more-than-representational rural lens reiterates the conclusion that the physical act of relocation usually regarded as signifying a migration provides too limited a focus. On the one hand, representations such as rural idylls may prompt migrations, and any mismatch between them and what is found "on the ground" is increasingly recognized as forming a crucial post-migration element for negotiation within many lifestyle relocations. On the other hand, taking the non-representational on board also requires consideration of post-migration lives at destinations. It demonstrates, in particular, how the rural world at the destination can work to engage affectively the migrant in their everyday life.

More-than-human expressions of rurality, in particular, can come into their own following relocation. They can "scape" a migrant as they "press hard upon and into our bodies and minds, complexly affect[ing] our moods, our sensibilities" (Macfarlane 2012: 341). Several interlinked consequential themes are suggested: 


\section{LIFESTYLE MIGRATIONS}

- $\quad$ Slowing down within a less explicitly hyper-mobile landscape;

- Feeling life by attuning to the rhythms of the more-than-human world and the seasons;

- Acknowledging connectedness through rhizomatic links with plants, animals, inanimate objects, other people;

- Developing a place-based $d$ welling by becoming and sensing embeddedness or rootedness within everyday life;

- Learning by doing through practices promoting a re-focused, authentic sense of one's life and project of the self.

Overall, a more-than-representational rural perspective indicates how the rural environment or landscape is not just an "object" to be acquired through lifestyle migration. It is itself a far from passive but an "animated" (Rose 2006: 538) and lively "zone of entanglement" (Ingold 2008: 1807) that works to shape the life course in often novel directions. Such reorientation may be linked, in language used in this chapter, to efforts to attain more authentic projects of the self.

(Sources: Halfacree 2013, 2014a; Halfacree and Rivera 2012)

Scholarship telling the counterurbanization story soon revealed complex patterns. Whilst it covered much of the Global North (e.g. Champion 1989; Boyle and Halfacree 1998a), it "created a population mosaic, not a monochrome painting" (Halfacree 2014b: 518). Generalizing considerably, there developed a consensus that counterurbanization demonstrated at least four kinds of selectivity.

First, it is life course selective. Counterurbanization is predominantly expressed by middle aged and retired adults, and by households with two or more adults. This positions it, in general, further along the schematic life course than Section 8.2's pro-urban migrations. Section 7.4.2 introduced the concept of the escalator region, whereby large cities such as London, Paris or New York suck in young, highly qualified and ambitious people early in their career development but then often lose them later in their life course, when different everyday priorities become more significant. Counterurbanization often occurs. While declared in diverse ways, such as being "for the sake of the children," providing a better quality upbringing environment, it principally represents parental lifestyle priorities (Bushin 2005,2009 ). Additionally, retirement from the spatio-temporal demands of paid employment often allows latent residential preferences to be realized (8.4.1), again resulting in counterurbanization at or just before retirement (Stockdale 2006, 2014; Stockdale and MacLeod 2013). For all counterurbanizers, however, relocation to a rural residence generally signifies a major life course transition (e.g. Hoey 2005, 2006).

Second, counterurbanization is socially selective. As with gentrification-and for similar reasons of expense and cultural resonance-it is biased in favor of members of the higher social classes. A service class association has been especially noted (e.g. Boyle and Halfacree 1995; Thrift 1987). It is also associated with being a housing owner-occupier and with being economically self-employed. Most counterurbanizers are also not return migrants.

Third, counterurbanization is historically selective. This is not just in terms of its mid-twentieth-century blossoming as a quasi-mass phenomenon but also because it fluctuates with the state of the economy (Fielding 1998). With noted exceptions (below), it is strongest in buoyant times when resources required for the move are generally more abundant and people less locationally risk averse.

Fourth, counterurbanization is highly geographically selective, both intra- and inter-nationally (Boyle and Halfacree 1998a; Champion 1989). As with much international employment migration (Chapter 7), it expresses strong path dependency, with present migration patterns routed in migration patterns made earlier by others (Brown et al. 2011). In terms of intra-national selectivity, counterurbanization is strongest in more accessible countryside, with isolated, remote rural areas still typically experiencing net out-migration (Halfacree 2008, 2014b). In terms of international selectivity, it remains unevenly developed even across the Global North. Grimsrud (2011), for example, demonstrated how the counterurbanization story did not travel well to Norway, where what pro-rural migration there was typically reflected family and economic reasons more than any lure of country living. Regression analysis showed key counterurbanization reasons, such as "physical environment" and "social milieu," only notable in underpinning moves to exurban (Taylor 2011) rather than rural destinations (Grimsrud 2011).

\section{ST PROOFS: NOT FOR DISTRIBUTION}




\subsubsection{Chapters within the story...}

Looking briefly at case studies of different chapters within the counterurbanization story reveals further the phenomenon's scope and diversity (Bijker 2013). First, consider pro-rural internal migration within specified countries, or domestic counterurbanization. Leaving the UK-US focus of much scholarship, European expressions have been demonstrated within Spain (e.g. Pallarès-Blanch et al. 2014, Paniagua 2008, Solana-Solana 2010), for example. Research conducted by María Jesús Rivera Escribano between 2000 and 2004, based predominantly on in-depth interviews, explored migrants' rationales for relocating from urban to rural areas in the northern Navarre region (Rivera 2004, 2007). It showed considerable heterogeneity in both reasons given for moving and those involved. Migrants demonstrated various positions, from relocating for pragmatic and adaptive reasons (space, cost, access) to making a deliberate choice to reside in a rural environment. Despite such heterogeneity, pursuit of a better lifestyle, expressed within a shared sense of rural as the sphere of community, stillness and regeneration, was commonplace.

Second, as with most migration expressions (Chapter 5), counterurbanization does not confine itself within national borders. There is international counterurbanization, regarded from Sweden as expressing globalization (Hedberg and Haandrikman 2014) for example. An excellent expression is migration of Britons to rural France (e.g. Buller and Hoggart 1994; Neal 2013). Michaela Benson (2011) examined such migrants through ethnographic fieldwork in the Lot department. Rural France seemed transformed by them into Britain's "lost" rurality, an "Arcadian dream" (Benson 2011: 166) that still provided opportunities for the committed to adopt an authentic rural lifestyle. Benson went on to examine how the British lived in rural France and how they faced up to "disjuncture between ... expectations and the local culture" (Benson 2011: 61). Rural France often fell short of romantic representations, being neither "idealized village life characterized by authentic conviviality" (Benson 2011: 87) nor any off-the-shelf "remedy for an unsatisfying present and an unpromising, uncertain future" (Benson 2011: 39). Consequently, migrants had to adapt, performing often tough work of "negotiating locality" (Benson 2011: 45-65): its people, more-than-human inhabitants, climate, landscape, and so on (Halfacree and Merriman 2015).

Third, although presented under such a heading here, all counterurbanization is not reducible to lifestyle migration (Halfacree 2008; Hedberg and Haandrikman 2014). Lifestyle is typically entangled with other quality of life priorities and a few or several may be expressed through the move. For example, for counterurbanizers not of retirement age employment considerations are almost always of strong significance within the move. Even retirement age counterurbanizers may not, in practice, disengage from paid work completely. Paralleling calls within gentrification research to focus on "corporate creation" (production) as much as on "class formation" (consumption) (Davidson 2007), Gary Bosworth (2010) coined the term commercial counterurbanization to shift some attention from counterurbanizers to "growth of rural economies stimulated by inward migration" (Bosworth 2010: 977). In line with the counterurban consensus, Table 8.2 shows the types of employment created in Northeast England through commercial counterurbanization skewed in favor of those where self-employment is relatively high: hospitality and business and domestic service (also Bell and Jayne 2010; Herslund 2012). Bosworth saw general potential for such employment stimulating further growth in the rural economy and "neoendogenous development" (Bosworth 2010: 977). Others qualified this potential, not least due to the high rates of self-employment rather than wider job-creation (e.g. Stockdale

Table 8.2 Ownership of rural micro-businesses by migrant status, NE England 2007 survey

\begin{tabular}{lll}
\hline Sector & Ownership (\%) \\
\cline { 2 - 3 } & In-migrant & Local \\
\hline Hospitality & 28 & 14 \\
Retail & 20 & 29 \\
Business and Domestic Services & 19 & 12 \\
Manufacturing & 12 & 9 \\
Land-based Business & 4 & 6 \\
Construction & 3 & 14 \\
Other & 14 & 16 \\
$N=$ & 698 & 561 \\
\hline
\end{tabular}

Source: simplified from Bosworth (2010: Table 1). 


\section{LIFESTYLE MIGRATIONS}

2006) and commuting to work and services outside the residential area (Findlay et al. 2001). Bosworth and Willett (2011) further observed how commercial counterurbanization's employment effectiveness could be influenced by representations of rural and rural people. Where the countryside represents a slower pace of life from urban living and/or rural residents seen as out of tune with a fast-paced, globalized economy, migrants may be less inclined to job creation than when less narrowly representationally constrained engagements with rural people and places occur.

Fourth, counterurbanization research has moved from initial focus on the US and then the UK to acknowledge the rest of the Global North (Boyle and Halfacree 1998a; Champion 1989). All the time, new spaces of counterurbanization are emerging. For example, Greek crisis counterurbanization (Gkartzios 2013) is one response to ongoing economic crisis, whereby especially younger adults not only engage in brain drain migration (7.4.3.1) but also adopt pro-rural moves, particularly out of Athens. These are in response to unemployment and fear/experience of crime and to seek out extended family, new starts and opportunities. As Gkartzios (2013) observed, there are many counterurbanization stories in Greece today. Eastern Europe also demonstrates new spaces of counterurbanization, Šimon (2014: 136) exploring its expression in the Czech Republic. Although with strong employment dimensions, Czech counterurbanization appeared "primarily non-economic migration driven mostly by lifestyle oriented motivations." This differed from other East European countries where economic factors were more prevalent (e.g. Brown and Schafft 2002 on Hungary). Šimon proceeded to construct a four-fold typology that distinguished chapters based on differing household motivations, preferences and employment expressions within any overall counterurbanization tale (Figure 8.5). Lastly, although the Global South has been largely overlooked within the counterurbanization story, this too is changing. Besides studies in South Africa (e.g. Ingle 2013; Smailes 1997), Potts (2005) showed that over the previous two decades Zambia had been experiencing a "quiet" counterurban population shift. This, she argued, was driven by economic decline and consequent poverty within the cities more than by positive lure of the countryside, making it closer to the Greek experience than the Northern consensus. Similar conclusions came from counterurban expressions in Ghana, Tanzania and Zimbabwe, Rigg (2007: 140) concluding that "[c]ounter-urbanisation in Africa is not, usually, driven by choice, but by necessity."

\subsubsection{Rural amenity migrations}

\subsubsection{Birds flying south ${ }^{6}$ and seeking a sea change}

Bell (2006: 150) labeled one of his three ideal types of rural idyll (8.3.1) the sporting idyll of adventurescapes, suggesting desire to be near and able to access regularly amenity or leisure resources may be a key driver of pro-rural lifestyle
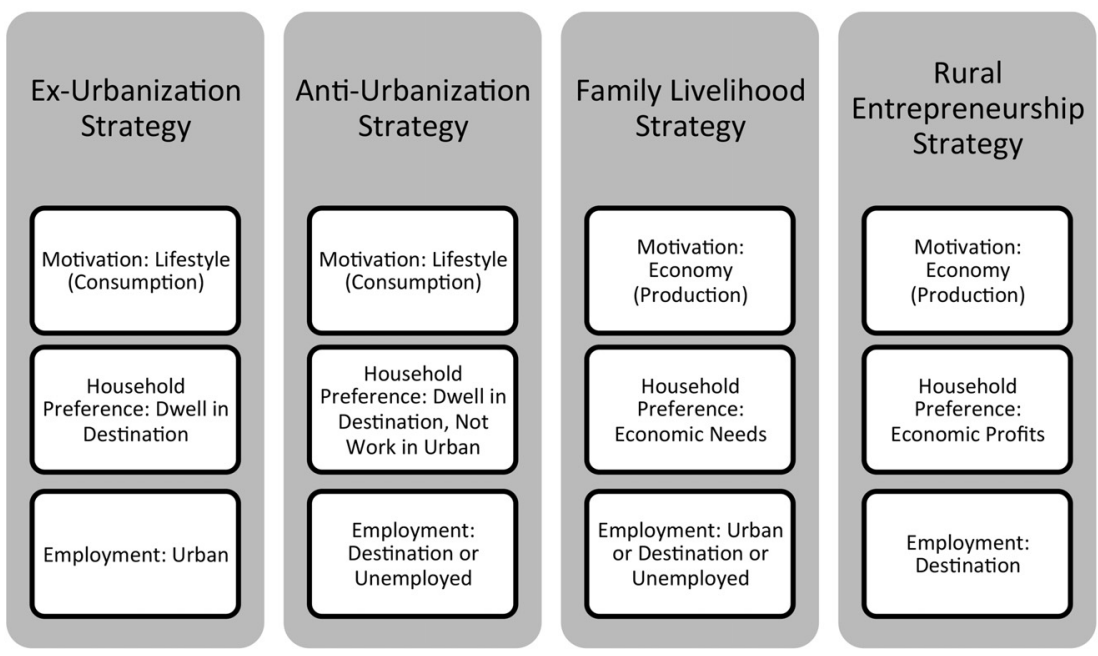

Figure 8.5 Counterurban household strategies in the Czech Republic.

Source: adapted from Šimon 2014 : Table 5. 
migrations. This is indeed the case. Consequently, a body of scholarship focusing on amenity migration, or "the movement of people based on the draw of natural and/or cultural amenities" (Gosnell and Abrams 2011: 303; Moss 2006; Osbaldiston 2010a), has a strong rural flavor. This originates not least through amenity associations with nature, landscape, mountains and coast. There are also overlaps with retirement migrations (8.4). As with lifestyle migration in general, amenity migration has been increasing in recent decades. This has marked implications for the environmental and social composition of rural places (Borsdorf and Hidalgo 2009), with an "increasingly large footprint on rural landscapes worldwide" (Gosnell and Abrams 2011:314).

Besides North America (e.g. Jobes 2000; Loeffler and Steinicke 2007; Moss 2006), Australia has witnessed strongly the amenity strand of rural lifestyle migration (Osbaldiston 2010a). Cutting across accounts of counterurbanization has been the idea of Sea Change migration. Initially named after a TV series (SeaChange) which featured urban residents migrating to a small coastal town (Burnley and Murphy 2004; Murphy 2002; Osbaldiston $2010 b)$, the term resonates with Elizabethan English playwright William Shakespeare's origination of "sea change" as a major life change associated with the sea. ${ }^{7}$ It is primarily used to characterize migration to coastal areas with high amenity offer (Gurran and Blakely 2007), although sometimes used as an alternative to counterurbanization to describe leaving Australia's cities for non-metropolitan areas (Murphy 2002).

Focusing on the coast, Sea Change migration has demographically transformed localities beyond reasonable commuting range of large cities. However, once again, besides an affinity for coastal lifestyles, different types of amenity place shape and are shaped by different Sea Change strands, reflecting diversity even within relocation to the coast. Murphy (2002: 5-6) expressed this diversity along the New South Wales coast away from the large city of Sydneynorth of Newcastle, south of Nowra (Figure 8.6)-when he noted:

distinction ... between large towns such as Port Macquarie and Coffs Harbour and smaller ones such as Evans Head and Harrington. The larger places offer better commercial and social services but have the urban 'feel' that many leave the city to escape from. Urban centres can also be distinguished according to the lifestyles that they offer. Byron Bay ... has been transformed from its seaside industrial character, based on an abattoir that was operating up until the early 70 s along with a Greek café that closed at $6 \mathrm{pm}$ and a couple of rough pubs, into a kind of ersatz yuppie, 'waxhead' (surfie) and hippie paradise. It's atypical and contrasts sharply with places like Evans Head, south of Ballina, which has a character that resonates... [with] family holidays in the 50s and 60s. .. Bateman's Bay, with its middle-class Canberra influence, has different characteristics to the more democratic and industrial Nowra and its hinterland around Jervis Bay and St George's Basin.

As with counterurbanization, amenity migration frequently transcends any Northern intra-country confine (cf. Borsdorf and Hidalgo 2009) and becomes international amenity migration. Adding to Benson's (2011, 2013a) research on the British in rural France, international amenity migration scholarship includes accounts of: North Americans relocating to Ecuador (Hayes 2014), Mexico (Croucher 2009) and Panama (Benson 2013b); Belgians moving to Tanzania and Chile (Salazar 2010, 2014); "global nomads" (D'Andrea 2007) whose former lifestyle mobilities took them through "new age" locations such as Goa in India (and Ibiza in Spain) before becoming lifestyle migrants to similar locations (Korpela 2009, 2014); Jews making an international "homecoming" to Jerusalem, Israel; and Japanese migrating to Malaysia (Ono 2015). Within expressions from North to South, strong feelings that more "authentic" and supposedly "freer" (cf. Korpela 2014) lives can be lived in these poorer countries are notable. International amenity migration between the countries of the North and from North to South is also expressed strongly within international retirement migrations (8.4.2.4).

A key issue for international amenity migration is the extent to which expatriates integrate or even just associate with the established population of the destination country. They may lose the "touristic" practices of amenity mobility after relocating (Weidinger and Kordel 2015) but, as with international employment migrations (7.4.3.2), often remain relatively isolated. International amenity migrants often live in an expatriate bubble (Fechter 2007), using generally high levels of privileged mobility (Croucher 2012) to connect back more to origin countries than to others within their destinations. For example, Gustafson's (2001b) Swedes in Spain associated the latter country with amenities, climate and other (often similarly expatriate) residents but kept Sweden associated with continuity, family, familiarity and security.

\section{ST PROOFS: NOT FOR DISTRIBUTION}


214 LIFESTYLE MIGRATIONS

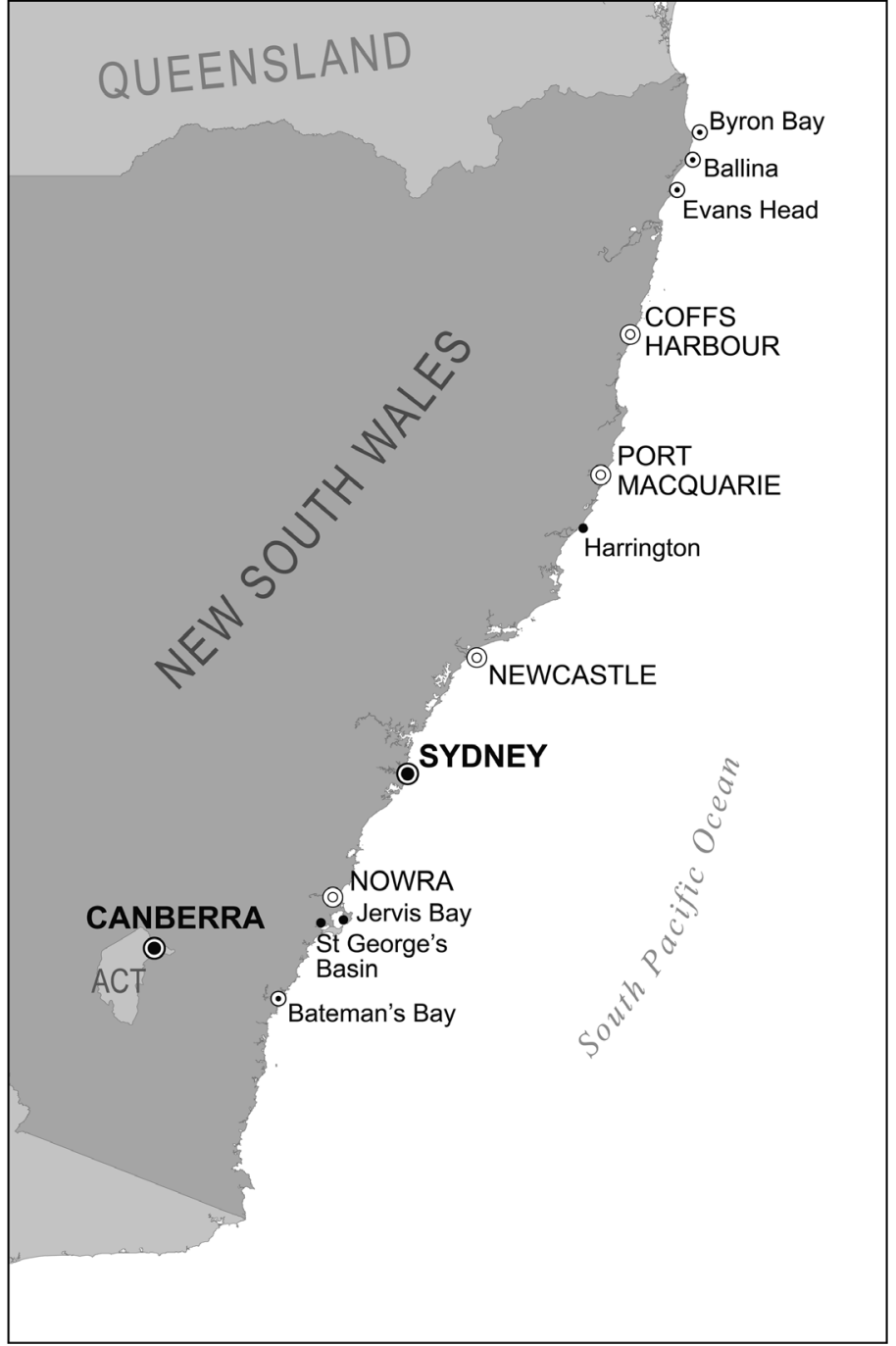

Figure 8.6 Sea Change coastal towns in New South Wales, Australia

Transnational (7.5.6) lifestyles again blur distinction between migration and mobility and query the former's reliance on assumptions of "permanent" relocation. As further illustration, the "surfie" Sea Change lifestyle may be embraced as fully by urban residents accessing the beach regularly as by actual relocators to the coast, whilst such a lifestyle may have diminishing appeal when Sea Changers age, even prompting out-migration. In the first case, amenity mobility seems more significant from a life course perspective than amenity migration. In the second, the amenity migration is unlikely to be "permanent." Engaging such concerns further is now demonstrated through second home consumption.

\subsubsection{Rural second homes}

In common sense it is obvious: ... a person is either here or there, and not in two places at the same time.

(Callon and Law 2004: 3)

A holiday or second home may be defined as an "occasional residence of a household that usually lives elsewhere and which is primarily used for recreation purposes" (Shucksmith 1983: 174). They are found across the world, in 
cities as well as scenic rural, wilderness and coastal environments (Hall and Müller 2004; Paris 2011). They range from simple shacks to mansions of the super-rich, and are transnationally as well as domestically owned (Paris 2011). Numbers grew through the past century, recent upsurge in academic interest reflecting this growth, their increased spatial reach (Williams et al. 2004) and recognition of their potentially heightened significance within the era of mobilities (Halfacree 2011a, 2012a; Hall and Müller 2004; Mclntyre et al. 2006). In life course terms, second homes are associated with leisure and family life with children, whilst their often considerable expense-purchase and maintenance, and in temporal as well as monetary terms-makes them strongly class-specific.

There is only space to consider rural second homes and attention will focus on Nordic countries, a key site for both presence and academic study. In contrast to countries such as the UK and US, where second home owners typically represent an adventitious and exclusive rural population with little strong established connections with their second home locations, second homes in the Nordic countries (and elsewhere; e.g. Barke 2004 and Querol 2010 on Spain) are more socially diverse and may reflect sustained bonds between urban populations and specific rural places (Gallent et al. 2005; Hall and Müller 2004) (Box 8.4). Particularly informative is the considerable intensity, emotional outlay and diversity of engagements between owners and both properties and surrounding environments. Whilst detailing leisure use predominates (e.g. Hall and Müller 2004; Kaltenborn 1998; Vepsäläinen and Pitkänen 2010), their relatively uncontroversial character has prompted more rounded investigation of everyday practices (Halfacree 2012a).

Research has revealed second homes frequently comprising a meaningful and central element within consumers' everyday lives. They become an extension of "home." This may ultimately lead to "permanent" relocation to the second home, possibly on retirement, but even where not the case the property is rarely simply "secondary ... in an elite landscape for the privileged" (McIntyre 2006: 13). Beyond escapism (Halfacree 2011a, 2012a), the second home can comprise a key node within a family's "geographically extended network of social relations and ... multiplicity of dispersed people and regions" (Williams and Kaltenborn 1999: 227). Within such a heterolocal (6.3.4) way of life, where place expresses "routes" more than "roots" (Gustafson 2001a, 2014), the "second" home can supply complementary "aspects or dimensions of lifestyle that are not offered in [the] primary home or 'ordinary' life" (Bjerke et al. 2006: 88). Second home consumption can thus express "an act of connecting rather than an act of distancing" (Rolshoven 2007: 17), the property "a solid partner in a fluid world" (Halfacree 2011a). Notions of "usual residence," so central to migration scholarship (5.1.1), become decentred. Second homes may even provide a "line of flight" (Halfacree 2011a), critically presenting life at the second home (rural, connected to nature, time rich) as a better, more authentic, "alternative" reality and source of self-realization to that experienced at the first home (urban, capitalist market, time pressured). This "challeng[es] the dominant refrain rather than harmonis[es] with it" (Halfacree 2011a: 151) even if such consumption is nonetheless highly (financially and psychologically) dependent on the dominant refrain of the first home lifestyle.

In summary, research supports a case for not regarding second home consumption as peripheral to migration or Population Geography because it inscribes temporary relocation. This perspective can be extended, Halfacree (2012a, 2014b) argued, to other forms of mobilized rural place and amenity consumption. Lifestyle mobilities (Cohen et al. 2015; Mclntyre 2009) blur boundaries of travel, leisure and migration. The rural result is a diversity of counter-urban populations that encompass movements across the whole mobility spectrum (5.2.2). Modeled in Figure 8.7, it has as a first axis the extent to which "pull of rurality" (especially representations) underpinned relocation, as compared to more "instrumental" considerations, such as employment or family support in old age. A second axis expresses "intensity" of time spent within the identified rural environment. This seeks to capture the extent a person/household becomes entangled with this environment (Gallent 2007) or how important the rural environment is for their project of the self. Time spent or duration (Bell and Ward 2000) is an imperfect proxy to represent such connectedness.

Figure 8.7's 14 slices represent different "counter-urban encounters" (Halfacree 2012a: 220). These range from rural engagement as an incidental and minor (in-transit visitor) to daily lives inscribed by their rural environment (noncommuters (in situ)). The conventional "mainstream" counterurbanizer now becomes dispersed across diverse expressions of counter-urban consumption. No longer is permanent relocation versus temporary sojourn the key categorical axis distinguishing rural consumers (Halfacree 2014b). Everyday emplaced mobilities (sic.) (Osbaldiston 2014) instead assume overall priority. 


\section{BOX 8.4 BALANCE QUICKLY RESTORED: CABIN LIFE IN NORWAY}

In 2009, researcher Kjell Overvåg estimated that around 40 percent Norwegian households have access to at least one second home. There are now upwards of 450,000 of these properties, numbers growing by around 5,000 per year, in a country of only 5 million people. Most are located in rural or coastal locations, with fastest growth in the mountains. The quality of the properties is also changing, with general upgrading of housing and facilities, as well as an increase in size. Overall, the largest and most sophisticated second homes are found in the east of the country. This geography reflects their association with urban residents and urban places, notably Oslo, even if ownership by Norway's rural population is almost equally common. Another key development is emergence of second home resorts, spatially separate from existing mountain settlements. As elsewhere, Norway's second homes are principally used for leisure and recreation and they are also known as recreational homes.
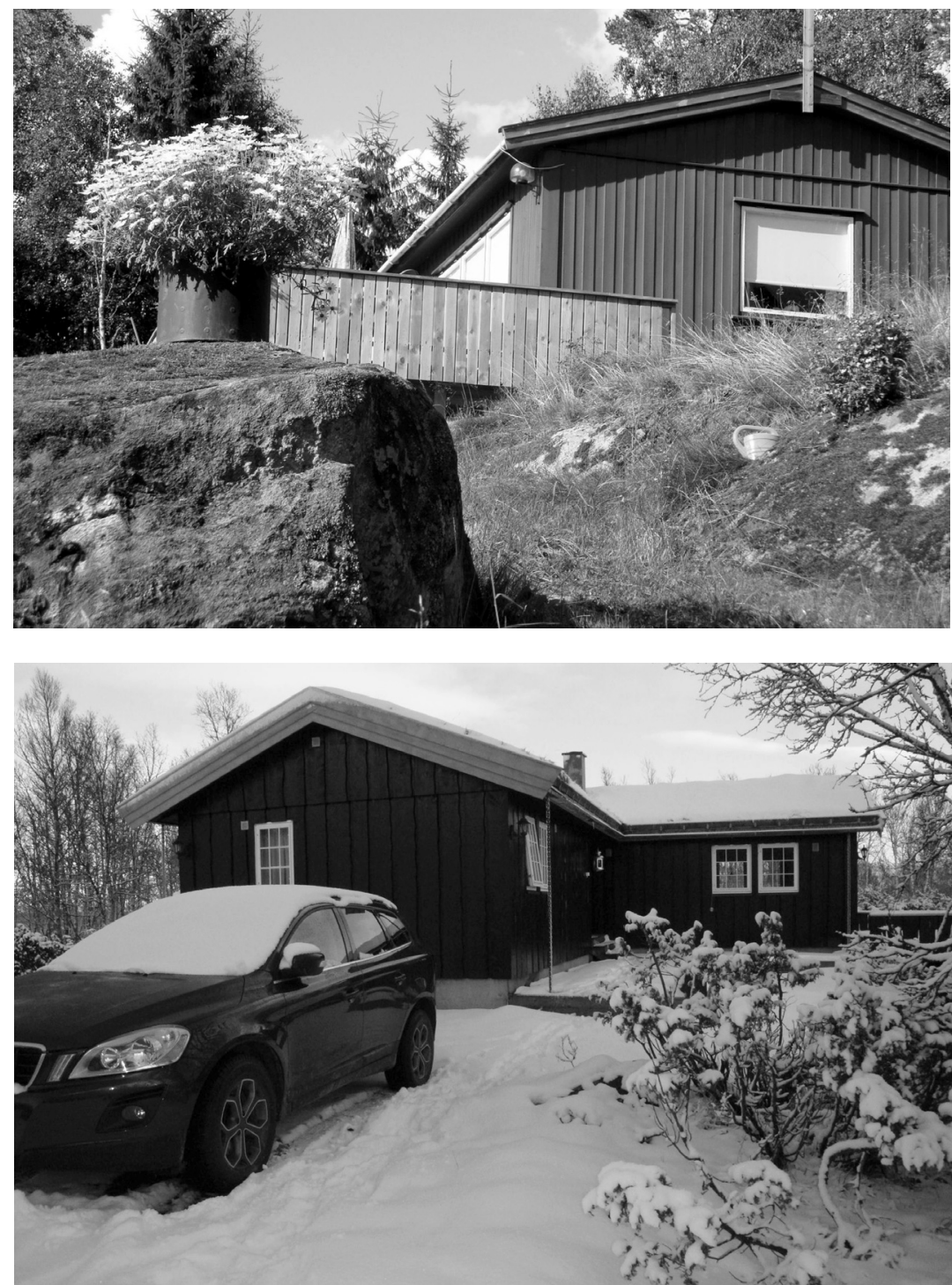

Two relatively modest Norwegian second homes.

Source: Nina Gunnerud Berg. 
Demonstrating both similarities and contrasts with gentrification, Norwegian second home growth in mountainous areas, in particular, is leading to significant social geographical change. As Overvåg (2009: Paper $2,13)$ summarized:

the rough picture is that large parts of the mountain municipalities are experiencing simultaneous decline and ageing of the permanent population in combination with decrease in the primary industries [such as farming] on the one hand, while on the other hand there is a considerable increase in temporary in-migration of rather wealthy middle-aged second home owners.

With mountain areas much less impacted by "permanent" population shifts than in many other European countries, there is a very different counter-urban rural population composition. However, often intensive use of second homes at weekends and during holidays means that Norway still experiences significant redistribution of urban populations to the rural, albeit seasonal and temporary.

The hytte (cabin) has deep cultural roots within Nordic folklore, national identity and Romanticism (as in ether Nordic countries). Emerging as a decidedly bourgeois lifestyle element in the nineteenth century, their social base broadened after 1945, such that by the 1960s they were a leisure option even for many working class families. Facilitating further recent growth, besides higher disposable incomes, have been key signifiers of the era of mobilities: raised personal mobility, developments in communications and technology more generally, and increased leisure time.

Particularly noteworthy from numerous Norwegian studies of second home consumption is the considerable intensity and diversity of engagements between users and both properties and surrounding environments. Many second home consumers interact considerably with the local permanent population and often their properties are located in areas with strong family connections. Such findings support the argument in the main text that second homes are not some simple off-the-shelf "leisure commodities" but have much greater life course significance. Nonetheless, the increasing role of the capitalist market in both constructing and promoting second homes and related developments in rural Norway can pressurize such relationships.

Desire to develop economic opportunities in these quite impoverished Norwegian rural areas has been found often to unite local and second home populations, rather than divide them as elsewhere, but only when development takes place outside their immediate vicinity. In other words, they "are united in their wish for a rural idyll in their own backyards" (Farstad and Rye 2013: 50). It is the backyards of existing second home owners that are especially threatened with new developments. Moreover, such developments include the most economically lucrative and profitable luxurious and sophisticated purpose-built properties. Their consumers will likely have a very different relationship to their environments than this middle-aged female interviewee from Sjodalen in the late 1990s:

Cabin life is tradition. It has always been my favorite existence. Away from crowds, time pressures and the regulations of civilization and technical things. Experience, enjoy and protect "untrammeled" nature. Live a simple life materially and in agreement with a few, chosen people. Physical - and not the least psychological - balance is quickly restored during a stay at the cabin"

(quoted in Williams and Kaltenborn 1999: 225)

(Sources: Farstad and Rye 2013; Halfacree 2012b; Overvåg 2009; Williams and Kaltenborn 1999) 

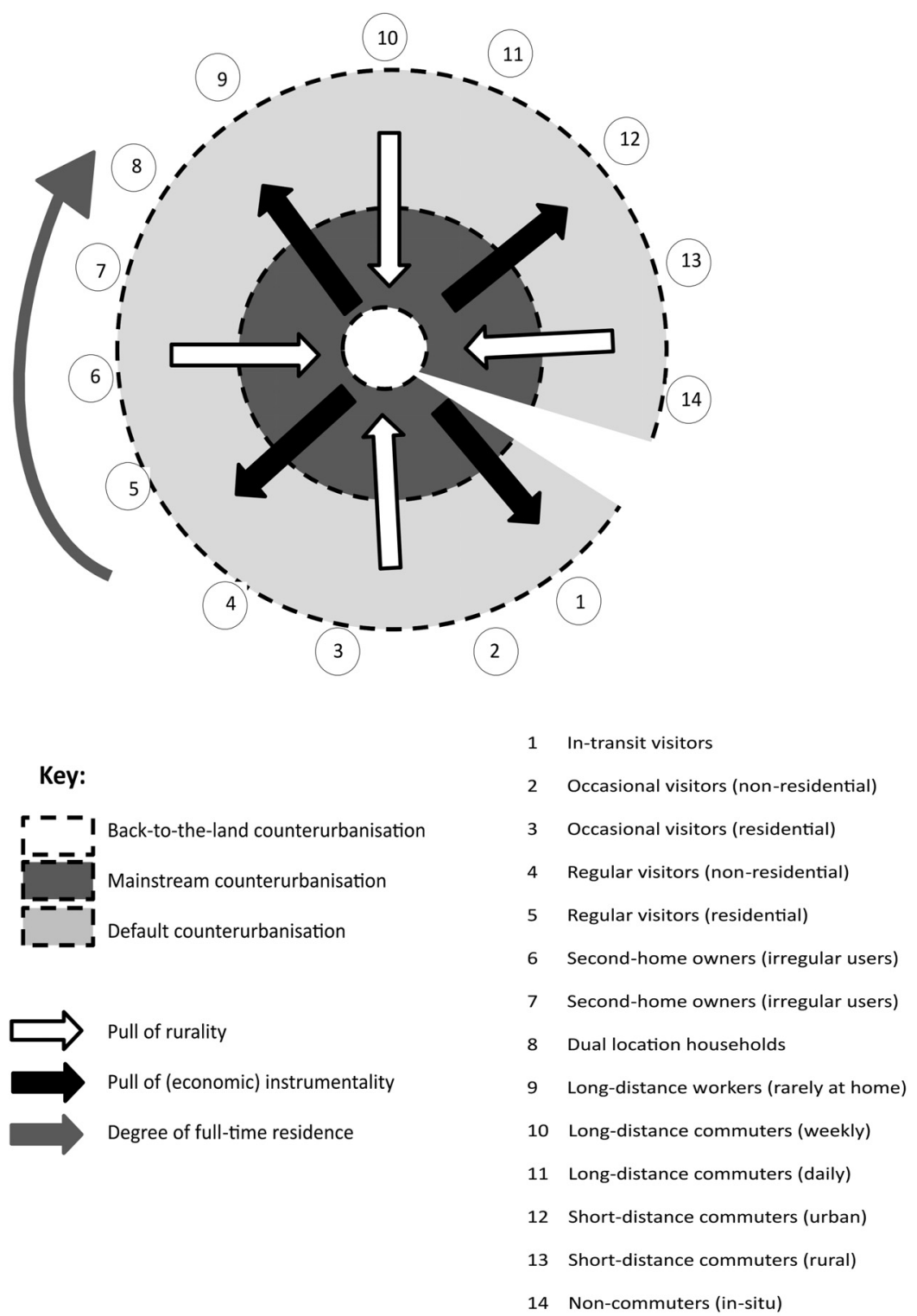

Figure 8.7 Model of rural counter-urban populations

Source: Halfacree (2012b: Figure 1).

\subsubsection{Going "back-to-the-land"}

\subsubsection{Utopian relocations to the countryside}

The most "alternative" or radical expressions of counterurbanization are back-to-the-land moves (Halfacree 2006, 2007a, forthcoming; Jacob 1997; Jobes 2000). For these movers, the destination's rural or "natural" qualities are extremely far from incidental. Back-to-the-land involves people moving from usually more urban origins to a rural location in order not just to access rurality but to establish deeper living and working connections with the land. Such movers were largely overlooked in Section 8.3.2.2 but are included in Figure 8.7, again dispersed according to rural place entanglement, which is likely to be strong. 
Before considering some present-day back-to-the-land migrations, an historical contextual perspective demonstrates their "alternative" or countercultural character as deeply rooted (Coates 2001). It is a part of long-standing tradition whereby migration, both internal and international, seeks a suitable location to establish an ultimate expression of aspirational lifestyle migration: utopian community. Migration seeks "fundamentally different and [hitherto] unobtainable everyday reality" (Halfacree 2013b: 1)-eu-topia or a "good place." Rural locations feature strongly in such searches, both on account of their practical provision of potential space and again through how Arcadian countryside representations (Table 8.1) present "a way of life, or a model of an alternative society inspiring a social project that challenges contemporary social and economic ill" (Mormont 1987: 18). The supposed "authenticity" of the rural is critical here.

Illustrating historical international back-to-the-land migration, following the pioneering seventeenth-century Pilgrim Fathers, numerous often persecuted religious minorities sought out the supposed terra nullius of North America to establish utopian experiments. For example, the Shakers were a celibate communistic group founded in the UK by the messianic Ann Lee. With eight companions, Lee emigrated to the US in 1774. Shaker communities sprung up throughout New England, believers peaking at 6,000 in 1840 before dwindling (Montgomery 1962). They produced what Philo (1997) termed "other rurals." Similarly but staying within the UK, Hoare (2006) told of Mary Girling (1827-1886) and her followers. Initial relocation from rural Suffolk to London saw consolidation of her Children of God sect but it took subsequent back-to-the-land migration to the New Forest (southern England) for their communistic, prayer-based vision to be most fully, if briefly, realized.

Embracing secular as well as quasi-religious projects, social historian Alun Howkins (2003: 204) observed how "[d]ropping out of modern society and going back to the land ... has characterised all radical movements in the last 400 years." Again within the UK, this was expressed in the practices and ideology of the seventeenth-century Diggers (Marshall 2001) and by later Romantics, Chartists and Guild Socialists (Coates 2001; Hardy 2000). Unsurprisingly, therefore, the 1960s counterculture, an "international" (Suri 2009) cultural expression of dissent, expressed practically a similar urge to get back-to-the-land within its broader utopian quest for simpler, more natural and authentic life (Edgington 2008; Suri 2009; Young 1973). Global North examples ranged from musicians and artists to numerous communal and household experiments (Halfacree 2006, 2009, 2016, forthcoming). Moreover, it was not all ephemeral but often left an enduring legacy within rural places (Box 8.5). This consequently prompted path dependency, such places continuing to attract more countercultural lifestyle migrants today (e.g. Burmeister 2013; Longhurst 2013).

\subsubsection{Contemporary back-to-the-land expressions}

Back-to-the-land lifestyle migration has seen resurgence across much of the Global North in recent years (Halfacree 2006; Wilbur 2013), not least among younger adults. This ranges from intentional community, "deliberate attempt[s] to realise a common, alternative way of life outside mainstream society" (Meijering et al. 2007: 42) (Table 8.3), to individual household or family scales. A recent major survey of the literature from the late 1960s onwards (Halfacree forthcoming) concluded that it is both a reactive and proactive phenomena but has shifted over time in favor of the latter (Figure 8.8).

As reaction, back-to-the-land remains one response from the counterculture expressing spatialized rejection of key aspects of everyday life within (urban) mainstream society. It involves attempts to escape or "drop out" from that mainstream. It is rarely solely escapist, however. More proactively, back-to-the-land is motivated by perceived feelings that elements of a fulfilling life course are lacking within mainstream life but can either be found and/or be realizable

Table 8.3 Key attributes of intentional communities

1. No bonds by familial relationships only

2. A minimum of three to five adult members

3. Members join voluntarily

4. Geographical and psychological separation from mainstream society

5. A common ideology adhered to by all members

6. Sharing (part of) one's property

7. Group interest prevails over individual interests

Source: simplified from Meijering et al. (2007: Table 1). 


\section{BOX 8.5 COUNTERCULTURAL WALES}

The 2009 edition of the influential travel book series The Rough Guides focusing on Wales included a dedicated "Alternative, New Age and Green Wales" section. It was introduced thus: "Possibly more than any other part of Britain, Wales-the mid and west in particular-has become something of a haven for those searching for alternative lifestyles" (Le Nevez et al. 2009: 58).

This claim is readily backed-up by evidence. For example, one of the authors recently went to the small settlement of Newport, on the west Wales coast, to take part in a monthly 7-mile ramble billed as an eco-homes walking tour.

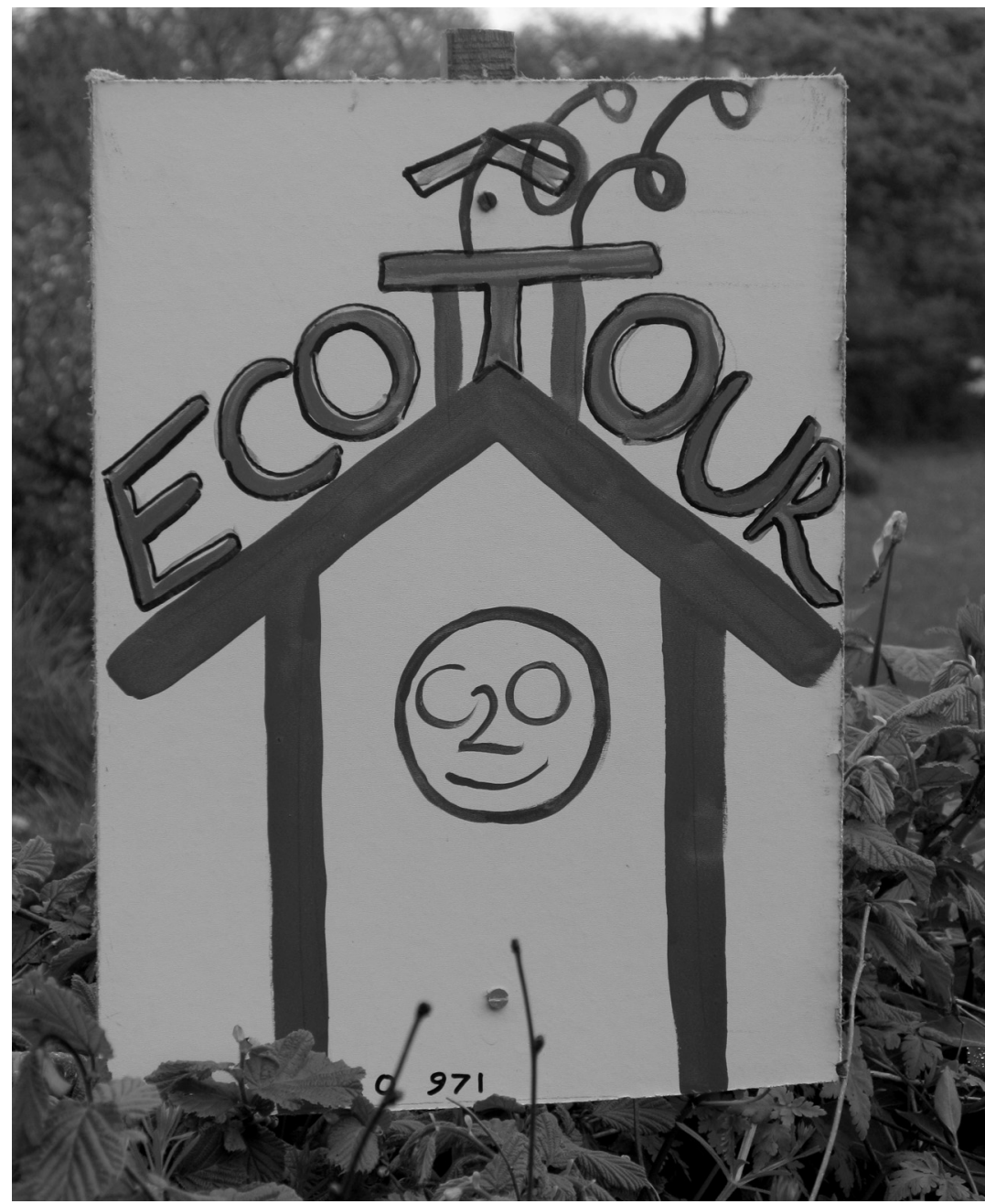

The tour was fully subscribed by a wide range of people, including Wales-based academics, local residents thinking of building their own eco-homes, a builder and even a couple from southeast England. Starting at the house of an ecological architect, it took in eight "eco-homes," where a desire to reduce carbon footprints and other general negative impacts on the planet's ecosystem formed a central consideration in design and everyday functioning. The group spoke with occupants and saw a wide range of green technologies and materials in action within their scenic environmental context. Many residents, moreover, were lifestyle migrants. Sites visited included the former home of self-sufficiency pioneer the late John Seymour and the Brithdir Mawr back-to-theland intentional community. Some further sense of what was visited is seen in the following:

\section{ST PROOFS: NOT FOR DISTRIBUTION}



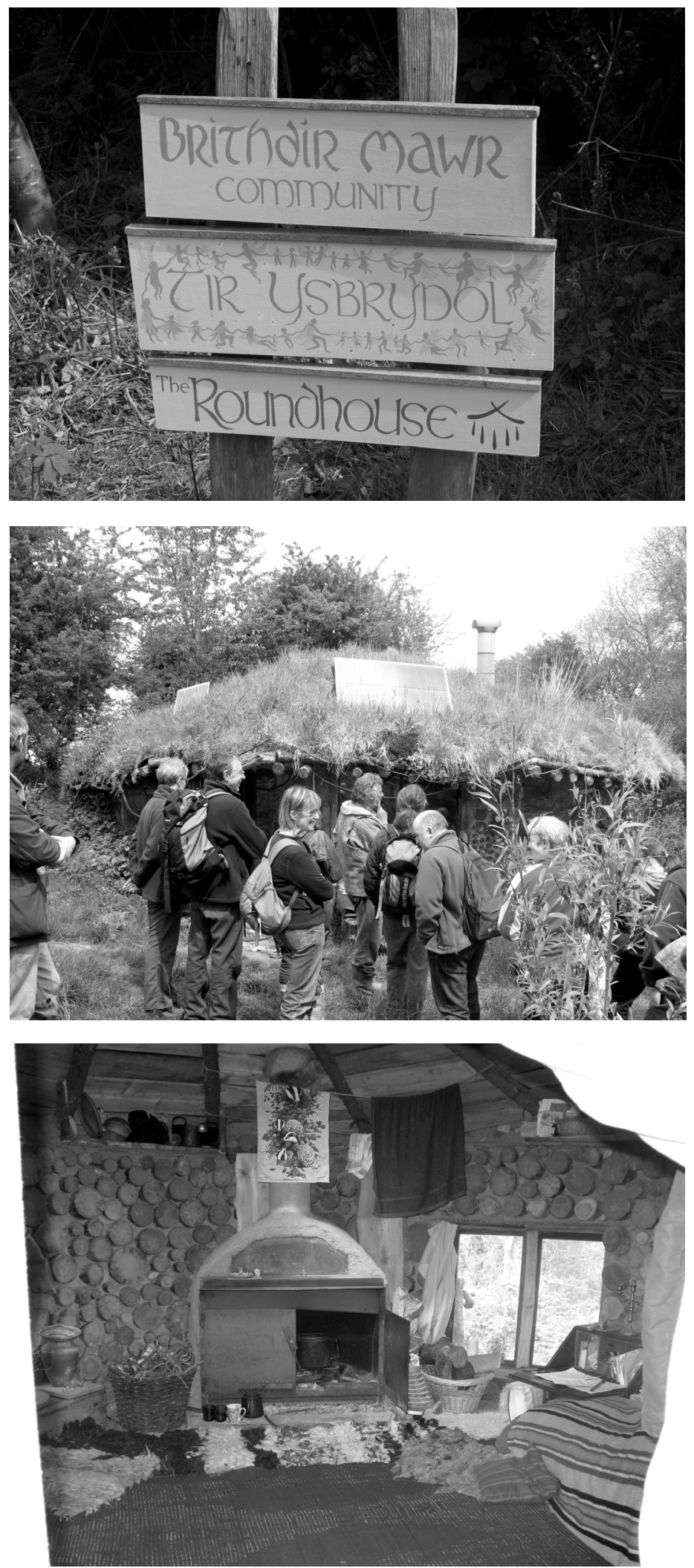

1ST PROOFS: NOT FOR DISTRIBUTION 


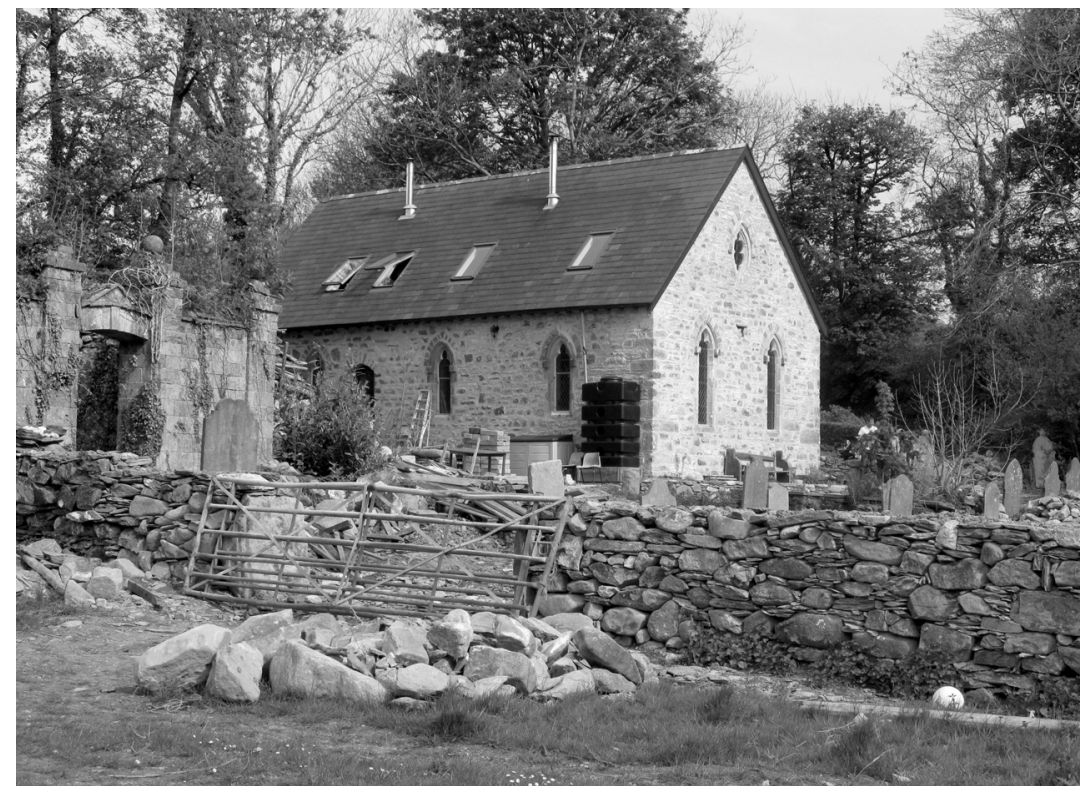

Source (all images): Keith Halfacree.

Why has rural Wales this "alternative" character and appeal to past and present "alternative" lifestyle migrants? Three broad overlapping and reinforcing explanations come through. First, one can note Wales's longstanding historical cultural differences from its dominant English neighbor. On offer: rural Wales's strong sense of community (gwerin), its overall cultural richness and resilience (language, art, song), and its powerful Romantic "natural" appeal (mountains, coasts, hills). Consequently, "[e]xcept in the most Anglicized urban enclaves ... in fundamentals everything is different in Wales even now. The history is different, the talk is different even in English, and so is the manner of life" (Morris 1986: 245).

Second, what is found "on the ground" is also both attractive and facilitating for back-to-the-land and other countercultural lifestyle in-migrations. This includes: a low population and population density (space); land and property both relatively available and affordable; a social environment usually relatively welcoming or at least nonplussed towards non-assertive newcomers; and good higher education facilities (accessible Universities). Attention can also be paid to rural Wales's existential physicality and environment, its abundant more-than-human nature and an enveloping affective dimension (Box 8.3). Finally, many back-to-the-land migrants tend to be relatively impoverished-unlike "mainstream" counterurbanizers-and this marginal socio-economic position can resonate with many within the local, agriculturally-based population.

Third, rural Wales was a site of many back-to-the-land and related experiments in the late 1960s/early 1970s. This legacy lives on in the twenty-first century. Pembrokeshire was the base of aforementioned John Seymour from 1964, whose presence partly inspired a range of 1970s "alternative realities" (Rigby 1974), including:

- Selene Community, who set up the Commune Movement in 1968, originally located in Pembrokeshire and then on a dilapidated hill farm further north in Gwynedd;

- $\quad$ Penwern (near Newport) commune base of the musically successful Incredible String Band in 1968;

- Tipi Valley intentional community, formed in 1976 near Llanfynydd, Carmarthenshire and now "one of the longest-serving alternative traveller lifestyle sites in Britain" (McKay 1996: 53);

- Organic farming pioneers, such as Patrick Holden (later Director of the Soil Association) at Tregaron from 1973;

- Nant-Y-Cwm Steiner school in Rhydwilym, Pembrokeshire; 
- World-renowned Centre for Alternative Technology (CAT), founded in Machynlleth on the southern edge of Snowdonia in 1973;

- Even Richard Kemp, who with partner Christine Bott moved to Tregaron in 1975 "to practice ... hippie ideas and manufacture high quality LSD in seclusion" (Roberts 2008: 181)!

In other words, what Noel Longhurst (2013) termed an "alternative milieu" has become woven into the geography of rural Wales and continues to inspire not only the writers of the Rough Guide but also prompts sustained path dependency within "alternative" lifestyle migrations today.

(Sources: Halfacree 2010; Le Nevez et al. 2009)

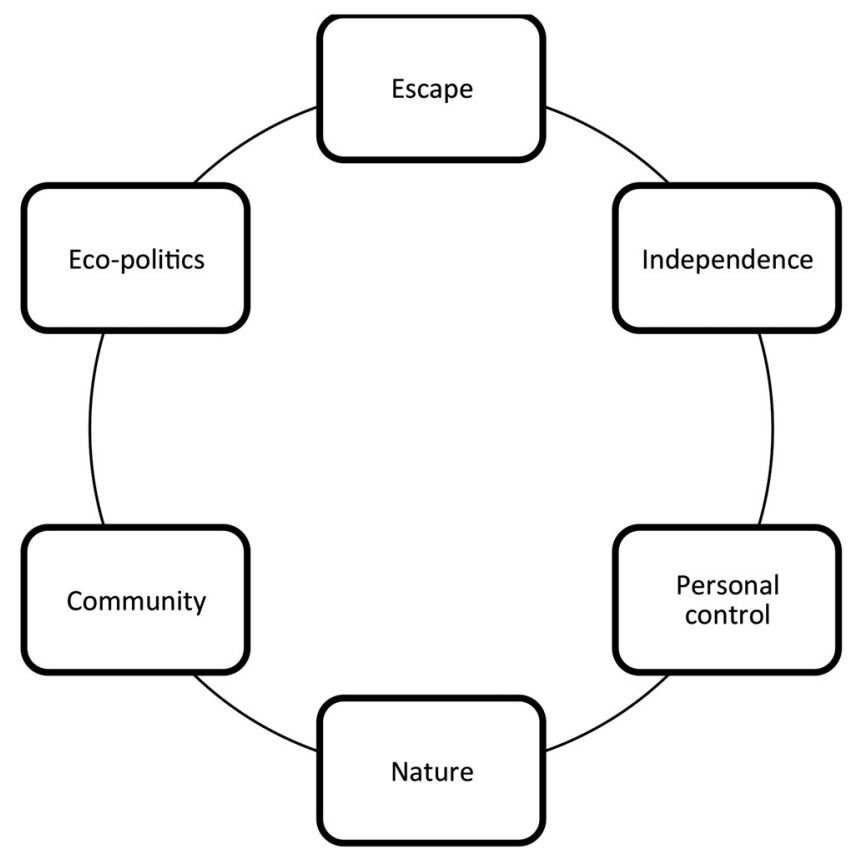

Figure 8.8 Goals within back-to-the-land.

Source: Halfacree (forthcoming)

within a rural environment. Authentic projects of the self are spatialized. There is a quest to attain more independence from mainstream society; to gain greater personal control within everyday life, and to become more connected and in tune with the rest of the sentient world (nature). These three elements are reflected in the importance given to gaining some degree of self-sufficiency, usually in food production. There is also search for community, expressed in a desire to connect with all (not solely human; Box 8.3) inhabitants of local place.

For all of these goals, a rural or wilderness location is rarely incidental. Rural representations encompass these desired elements (Halfacree 1995b; Holloway and Halfacree 2006) configured into Table 8.4's "radical ruralities." They provide cultural resources facilitating attainment of countercultural goals. The countryside is appreciated in both aesthetic and experiential terms. Whilst "radical" lure of the rural does not always translate into engagement with land work (Chapter 7 2009), a clear trajectory within back-to-the-land from the late 1960s is increasing foregrounding of eco-political priorities, notably the quest for more sustainable living (e.g. Wilbur 2013, 2014). The search is on for lifestyles that not only reject the city and embrace the country but which both engage on a daily basis and actively promote to social models that strive towards embedded environmental, social and economic sustainability. This is demonstrated by promotion of eco-villages (Andreas and Wagner 2012; Dawson 2006; Meijering et al. 2007; Sargisson 2001, 2007), rooted in radical rurality and principles of low impact development (Fairlie 1996; Pickerill and 


\section{LIFESTYLE MIGRATIONS}

Table 8.4 Elements of "radical rural" representations

\section{$\checkmark$ Diverse home accessible to all $\checkmark$}

- Localisation: "set of interrelated and self-reinforcing policies that actively discriminate in favour of the more local whenever it is ... reasonable and conveniently possible" (Lucas 2002: np)

- Community discourse incorporating more-than-human

- Eco-centric/deep ecology beliefs
- Rural rooted in land-based activities: "rural economy ... has its foundation in the land and what it produces-animal, vegetable and mineral. A rural culture is distinctive because it grows out of the land ... rooted in the earth" (Fairlie 2001: 9-10)

- Diverse meanings of land beyond means of production: empowerment, sustainability

- Celebration of physical labor. attuning to our humanity and place within natural world

$\checkmark$ Distinctive and challenging lifestyle choices $\checkmark$

Source: based on Halfacree (2007b).

Maxey 2009a, 2009b). An example is the settlement of Sieben Linden in Germany, comprising around 135 people, which "aims to use only as much energy, space, and raw material as necessary, so that sufficient resources remain for every other person on the planet to also have the possibility of living in such a way" (Würfel 2012: 12).

Countercultural back-to-the-land migration occurs across the Global North. Although its heartlands remain-as in the 1960/1970s-North America, Northwest Europe and Oceania (Meijering 2006; Sargisson 2001; Wagner 2012), developments further afield merit attention (Halfacree forthcoming). In part, this geographical expansion reflects rural inaccessibility within countries such as the UK due to high costs (land, property), lack of opportunities (space taken up), exclusionary planning regulations and countercultural intolerance (Dearling 1998). Thus, one sees international countercultural back-to-the-land migration from Germany, the Netherlands and the UK to countries such as Spain and Portugal. In these destinations, rural depopulation remains the norm and access to land and property consequently somewhat easier. For example, Francisco (2010) studied "ecomigration" to northeast Algarve, Portugal. With a poor and ageing depopulating demography, this area contrasted strongly with the more socially "conventional" tourist paradise and key international retirement location of the Algarve coast (Williams and Patterson 1998). However, it attracted Northwest European back-to-the-land migrants. Typically initially residing on boats moored in the Guadiana River, there was a trend towards more permanent land settlement. As with counterurbanization generally, these migrants may have a role in revitalizing declining rural areas, "neutralizing the grave demographic imbalances" (Camarero et al. 2009: 125).

Eastern Europe also gets a look-in. Macintyre (2010) told the fascinating story of the village of Romashki in the Ukraine, whose diverse eco-community expressively combines political, practical and more idealistic strands of twentyfirst century back-to-the-land. Change in the village started with an urban doctor and lawyer couple relocating "to live a more 'beautiful life' closer to nature" (Macintyre 2010: 17). They subsequently cultivated land to grow food and become as self-sufficient as possible. Other back-to-the-land minded people came to visit these pioneers. Again showing path dependency, some stayed to create a varied community that "share[d] a love for the natural beauty of Romashki and the space to be active and creative with their hands and minds" (Macintyre 2010: 89).

Although related to relational contexts such as class (back-to-the-land migrants tend to be well educated and middle class), gender (back-to-the-land often re-inscribes patriarchal practices-e.g. Conlon 2014; Maxey 2002; Wilbur 2014), and age (being mostly young adults, back-to-the-land expresses the importance of individuality for shaping differential life course experience-2.3.1). Lives "speak on [their] own terms" (Shanahan and Macmillan 2008: 236) within these projects of the self $\mathrm{f}_{\mathbf{I}}$ reflected unambiguously within a final back-to-the-land strand.

Off-grid living, although something of "an abused expression" (Vannini and Taggart 2014a: 188), refers to a lifestyle whereby households are:

disconnect[ed] from the electricity grid and natural gas network serving an area ... [and] also on their own for other typical domestic needs like water and sewage. It is also common ... to grow some food, to be far removed from the highway grid, and to be selectively connected to the media of communication."

(Vannini and Taggart 2014a: 188) 
In other words, lifestyles studied through in-depth ethnography on the Atlantic Coast and across Canada by Phillip Vannini and Jonathan Taggart (e.g. Vannini and Taggart 2013, 2014a, 2014b, 2014c) sought to reject almost the whole infrastructural framework of the era of mobilities. Off-grid mobility is consequently paradoxical, undertaken in order to reject present-day mobilities, act on the "pull of remove" (Vannini and Taggart 2013: 308) and attain the "stillness" (Bissell and Fuller 2011) of a "New Quietism" (Vannini and Taggart 2014a). Nonetheless, for daily living, stillness still requires everyday mobilities to fulfill everyday needs. Moreover, even the most radical off-gridder soon comes to realize that "off-grid life can never be lived in complete disconnection and autonomy" (Vannini and Taggart 2014a: 202). This may be particularly noted when migrants age.

\subsection{LIFESTYLE RETIREMENT MIGRATIONS}

\subsubsection{The locational "freedom" of retirement}

Whilst the lure of the city was seen to connect primarily with younger adults and the lure of the country with middleaged families and the newly retired (plus a more youthful back-to-the-land strand), older people's lifestyle migrations also merit specific attention (Longino and Bradley 2006; Rees and Hardill 2015; Rogers 1992a; Warnes and Williams 2006; Walters 2002). Whilst rural lifestyle migrations, in particular, may be claimed to be undertaken "for the sake of the children," actual agency of future adults within such moves is often very limited (Bushin 2005, 2009). The same cannot be said for many moves by the retired.

Retirement from waged labor and/or commencement of receipt of pension monies (public or private) represents a key life transition for most individuals and often their households. In the "ideal case," locational constraints imposed by need to access and hold down paid work are lifted. This allows pension payments and, probably more significantly, other financial resources (savings, selling one's home) to be mobilized to facilitate lifestyle migration. Consequently, the Model Migration Schedule (Figure 7.1) has a distinctive retirement curve (Rees and Hardill 2015). Rogers (1989) also proposed an elderly mobility transition (EMT) to model the geographical distribution of the elderly population over time (Boyle et al. 1998: Box 5.4).

The idea of lifestyle retirement migration must be utilized carefully, however. First, retirement rarely necessitates migration. Treating it as a "reason for moving," commonplace in academic literature, is problematic (Halfacree 1994). Second, the status and significance of retirement as a life transition varies globally, attaining most distinction in the Global North where "employment" is most formalized. Nonetheless, whilst "in the 1950s retirement was a one-way, one-time, irreversible exit made primarily by men, almost always at age 65 ... the gateway to old age" (Moen 2003: 273), today it is becoming once again less sharply life course delineated (Ekerdt 2010). Third, actual retirement date from paid work should not be over-stressed. Warnes and Williams (2006: 1258) prefer the term "later-life migration," in part "because many 'retirement migrations' occur well before the conventional delimiter (65 years) of sociallyconstructed old age." In addition, many "retired" people continue undertaking paid employment, even if not within their established career, and thus may be affected in very similar ways to economic migrants when economic downturn occurs, for example (Huete et al. 2013). Fourth, in contrast to the ideal, retirement for many people leads to considerable economic hardship. The retirement experience is strongly shaped by class and family structure, in particular (Ekerdt 2010; Radl 2012). Fifth and building on the last point, retirement migration may not be lifestyle migration at all (Litwak and Longino 1987; Rees and Hardill 2015) but instead be forced by economic hardship, health or frailty problems, or from having to vacate housing "tied" to a job (e.g. farm workers, caretakers). In sum, lifestyle retirement migration is a tricky category to delineate precisely. A range of its expressions will now be considered.

\subsubsection{Expressions of lifestyle retirement migrations}

\subsubsection{The diversity of retirement migrations}

Much has already been covered in this chapter regarding lifestyle migrations of older people. In particular, whilst not a major strand within pro-urban migrations, except to urban sheltered housing or nursing homes, the retired are a key group within counterurbanization, both domestic and international. In addition, Chapter 6's movement of retired people 


\section{LIFESTYLE MIGRATIONS}

to live near family (6.3.2) also has a strong lifestyle element. This can be extended to migrations to retirement homes (Angelini and Laferrère 2012; Croucher et al. 2006) and purpose-built retirement villages (e.g. Bernard et al. 2009; Evans 2009; Streib 2002) or co-housing communities (e.g. Brenton 2008; Glass 2012), where leisure and amenity priorities typically feature prominently (Bernard et al. 2009; Katz and McHugh 2010). Return migration (7.5.4) also has a noted lifestyle retirement migration element. All these forms cannot be revisited here. Instead, further types of both lifestyle mobility and migration by the retired are introduced.

\subsubsection{Lifestyle retirement mobilities}

The locational "freedom" of retirement for some people is evocatively expressed in the lifestyle retirement mobilities of the recreational vehicle (RV) phenomenon (Jobes 1984; Simpson 2008). Annually, hundreds of thousands of Americans and Canadians take flight south as Snowbirds, driving in RVs (Figure 8.9) to winter across a network of camping sites, resorts and parks in US States such as Florida, California, Arizona and Texas. Phoenix, Arizona, a city popular for these seasonal lifestyle mobilities, was the site of a 1988 questionnaire-based study of managers, activity directors and residents (McHugh and Mings 1991). It found "RVers" overwhelmingly retired and white. Most were married couples, with a few Loners on Wheels (as played by Jack Nicholson in the 2002 film About Schmidt). Middle to upper-middle income households with high levels of education predominated. For 90 percent, their nomadism was seasonal, permanent housing maintained elsewhere.

The RV phenomenon is closely allied with second home mobilities (8.3.3.2) and is equally challenging to quantify (Happel and Hogan 2002). It is not confined to North America. In Australia, Grey Nomads have been shown as still more mobile (Mings 1997). They may be sub-divided into those resembling Snowbirds, generally moving around relatively established sites, and those more resistant to such organization (Onyx and Leonard 2005). The latter, strongly valuing independence, emphasized values of freedom, adventure and new experiences. Within Europe, thousands of French retirees, often from "modest social classes" (Viallon 2012: 2085), annually explore Morocco's Atlantic coast in RVs. In a lifestyle described as "contemplative," unlike Grey Nomads they take "pleasures ... primarily from within rather than from experiencing new places" (Viallon 2012: 2085). Even within the global RV population, therefore, mobile projects of the self are being differentially constructed.

\subsubsection{Domestic lifestyle retirement migrations}

Intra-national or domestic lifestyle migration around retirement has long been acknowledged within academic literature. Warnes and Williams (2006) even referenced a paper noting it as a mass phenomenon in Europe and the

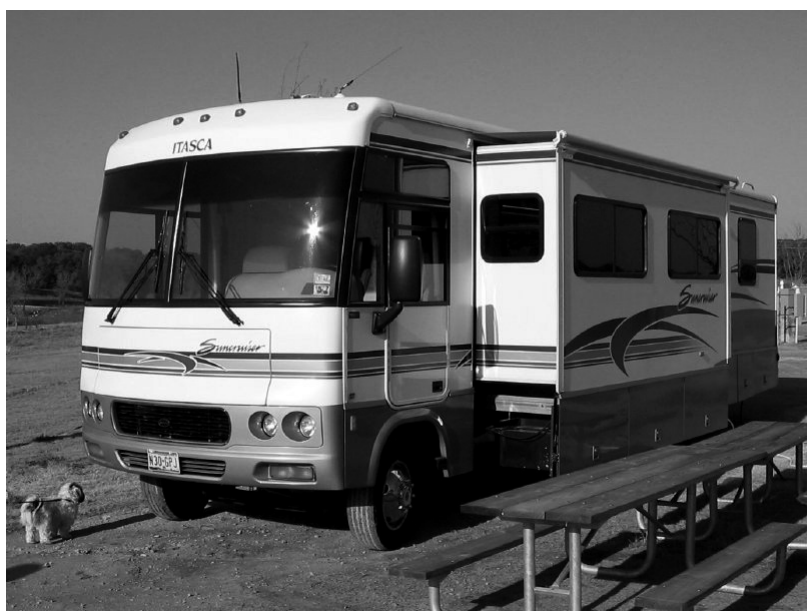

Figure 8.9 A recreational vehicle (RV): Class A motorhome with slide-out extended floors.

Source: http://commons.wikimedia.org/wiki/File:Rv_classa.jpg, Public Domain. 
US by the 1920s (Gilbert 1939). Emphasis has been on relocation to coastal areas (and spas), a geographical focus that has remained (Raymer et al. 2007; Walters 2002). Thus, Cribier (1987) noted that around a third of the Paris population and a quarter of residents of other French conurbations migrated on retirement, with about 15 percent settling in coastal resorts. Increasingly, coastal locations have been joined by rural destinations, reflecting the prominent role of the retired within counterurbanization. Today, across much of the Global North, there are thus two dominant strands of domestic lifestyle retirement migration, each with its own principal geographical form: "restricted coastal zones, in which they cluster, and rural areas of exceptional landscape value, in which they generally disperse" (Warnes and Williams 2006: 1263).

Focusing on the coast, a predominant destination of UK lifestyle retirement migration has long been England's southern and southwestern coastal towns and surrounding areas. Migration from London and the inner Southeast region has been especially notable (Champion 2005; Law and Warnes 1976). Utilizing 2001 Census data, Dennett and Stillwell (2008) showed patterns of migration by retirement age people resembling those of the middle aged, emphasizing a blurring between these groups pursued below. Net out-migration from London and other built up areas and net in-migration to rural areas, especially coastal resorts, stood out. The only relatively high retirement-age inmigration areas were districts along the English south coast and the mainly rural counties further northeast ef Norfolk and Lincolnshire.

Complicating the twin draws of amenity-rich coasts and rural areas for domestic lifestyle migrations are other entangled strands. Within the US, the key RV destinations of Arizona, Arkansas, Nevada and New Mexico (Rogers 1992b) indicate the importance of sunny and dry climates-Sunbelt-for retirement migrants (Longino et al. 2002). In contrast, within Norway, a country with less of a tradition of retirement migration, flows are dominated by losses from Greater Oslo towards the southeast and coastal areas of Oslo Fjord and Skagerrak (Myklebost 1989). Domestic expressions of return migration (7.5.4) represent a significant element of these flows.

\subsubsection{International retirement migrations}

Research on domestic expressions of retirement migration appears to have declined in recent years. It has been eclipsed by studies of international retirement migration (IRM) (King et al. 1998). Crossing the internal/international migration divide, IRM is nonetheless underpinned by similar concerns to much domestic lifestyle retirement migrations for access to desirable weather and climatic conditions. Not least engaging northern Europeans, migrations seek the mild and warm climates of southern Europe (Casado-Díaz et al. 2004; Huber and O'Reilly 2004; Rodríguez et al. 2004), with notable exceptions including Nordic and other Europeans moving to rural Sweden (Hedberg and Haandrikman 2014). Coastal and rural destinations again predominate, Warnes and Williams (2006: 1263) claiming Spain's Costa del Sol and Italy's Tuscany "epitomise" climatic and rural retirement destinations, respectively.

IRM has been facilitated by numerous developments characteristic of the era of mobilities. These include, first, the relative ease and affordability of international travel (Warnes and Williams 2006). For example, Table 8.5 shows countries and cities sending flights to Alicante airport, used to access Spain's Costa Blanca, on just one day (3 July 2014). Note the prominence of northern Europe, especially the UK. Second, IRM is facilitated by monetary mobilities or the relative portability of migrants' financial resources, including a common currency (Euro) across much of Europe. Third, diverse telecommunications, not least mobile phones and the internet (Box 7.5), enable migrants to retain strong transnational links (7.5.6) with family and friends (e.g. Banks 2009; Hedberg and Do Carmo 2012). Fourth, relative absence of immigration controls within the EU reduces bureaucratic hurdles for migrants, even if domestic regulations and paperwork can be onerous (e.g. Benson 2011).

Diverse mobilities may facilitate IRM but it remains far from unproblematic (Lizárraga Morales 2010; Warnes and Williams 2006). First, often quite concentrated redistribution of relatively elderly people to new locations can raise challenges for supplying suitable health and social care, not least when migrants age (e.g. Hardill et al. 2005). For example, whether or not they are covered by the destinations' welfare system may depend on immigration status. This is a growing issue given the demographic ageing of the populations of the Global North (10.2). Second, physical transformations of retirement destinations, such as the Costa del Sol, prompt numerous environmental concerns (e.g. Jennings 2004; Zasada et al. 2010). Substantial building of homes and associated infrastructure (e.g. golf courses) not only deplete scarce water resources but can impact negatively on the area's ecology. It also impacts on lives and everyday practices of "local" inhabitants, from raising property prices to marginalizing local traditions. Such issues can

\section{ST PROOFS: NOT FOR DISTRIBUTION}




\section{LIFESTYLE MIGRATIONS}

Table 8.5 Origins of flights to Alicante, 3 July 2014

\begin{tabular}{llc}
\hline Country & Cities & Total cities served \\
\hline United Kingdom & Belfast; Birmingham; Blackpool; Bristol; Cardiff; Edinburgh; Glasgow; Leeds; & 15 \\
& Leicester; Liverpool; London; Manchester; Newcastle; Southampton; Southend & \\
Spain & Barcelona; Bilbao; Ibiza; Madrid; Palma; Santiago de Compostela & 6 \\
Germany & Bremen; Düsseldorf; Hamburg; Karlsruhe; Stuttgart & 5 \\
Norway & Oslo; Rygge; Stavanger; Trondheim & 4 \\
Denmark & Ȧrus; Billund; Copenhagen & 3 \\
Netherlands & Amsterdam; Eindhoven; Rotterdam & 3 \\
Sweden & Göteborg; Stockholm; Umeå & 3 \\
Switzerland & Basel; Geneva; Zurich & 3 \\
Finland & Helsinki; Tampere & 2 \\
Algeria & Oran & 1 \\
Belgium & Brussels & 1 \\
France & Paris & 1 \\
Iceland & Reykjavik & 1 \\
Ireland & Dublin & 1 \\
Italy & Milan & 1 \\
Lithuania & Kaunas & 1 \\
Russia & Moscow & 1 \\
Total & & 1 \\
\hline
\end{tabular}

Source : www.alicante-spain.com/alicante-airport-arrivals.html, accessed 3 July, 2014.

feed into a third area of contention, that of political conflicts between (some) retirees and (some) local interests where social integration is poor (Borsdorf and Hidalgo 2009; Janoschka 2011).

Within Spain, a key destination for IRM, whilst overall only a third of resident foreigners are European, over threequarters of elderly foreigners (65+) are from elsewhere in the EU, rising to over 90 percent in provinces such as Alicante and Málaga (Rodríguez et al. 2004). The Costa del Sol, in the latter, is especially favored by the British, Germans predominate on the Balearic and Canary Islands, and the French choose Catalonia and Valencia (Rodríguez et al. 2004). Again expressing path dependency (Brown et al. 2011), nationals from each country tend to cluster following migration. This is often reinforced in subsequent constrained social networks (e.g. Casado-Díaz 2009).

One of Spain's "Little Norways" (Haug et al. 2007) is Torrevieja on the Costa Blanca, well connected to Norway itself (Table 8.5) and hosting a Norwegian school and other "familiar" services. Research revealed reasons for moving to this town, shown in Table 8.6 (Casado-Díaz 2006). Besides the overwhelming significance of the warm climate, perceived health benefits (not least on account of the climate) and explicit lifestyle issues stood out. German respondents especially emphasized health and the British cost and access factors, whilst Nordic retirees largely ignored any perceived slower pace of life or high accessibility as reasons for moving. Note how previous holidays frequently signaled some prior connection to the area. Spain is also a key location for second homes (Paris 2011), leading to seasonal migration by retired Swedes (Gustafson 2009), for example, which again blurs any migration/ mobilities divide.

The importance Britons gave to lower costs in explaining IRMs to Torrevieja reiterates how these relocations, as with lifestyle migrations in general, are rarely unconstrained. Broader quality of life factors always have a role, too. This qualification is clear within IRM of North American citizens to Central and South America (Jackiewicz and Craine 2010; Lizárraga Morales 2010; Migration Policy Institute 2006), previously depicted as amenity migrants (8.3.3.1). A survey of 211 retirees in the state of Guadalajara, Mexico, found low cost-of-living cited by almost all as a reason for retiring to Mexico besides climate and environment (Sunil et al. 2007). Focusing on these favorable economic conditions, the percentage "agreeing" or "agreeing strongly" with a range of statements was as follows (Sunil et al. 2007: Table 2):

- $\quad$ "My income allows more leisure spending than in the US"

- "I feel financially secure in Mexico"

- " "I live here because housing is affordable"

81 percent 
Table 8.6 Reasons for retiring to Torrevieja, Spain.

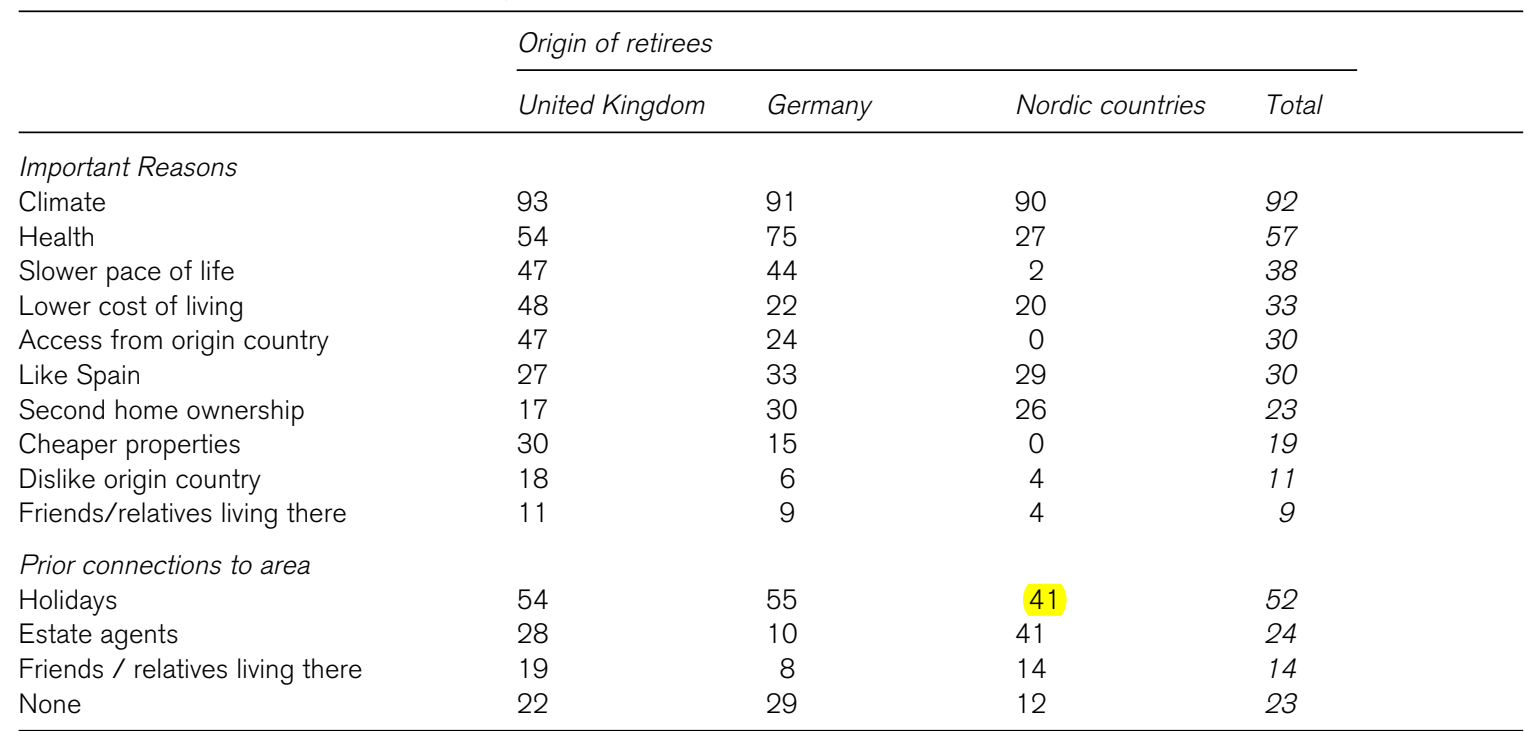

N.B. multiple-response allowed; only reasons given by $10+$ percent respondents in one category shown.

Source: simplified from Casado-Díaz (2006: Table 3).

- " "l live here because health-care is affordable"

- "My decision to move to Mexico was mainly economic"
59 percent

54 percent

Note, in particular, over half presenting economic reasons as the principal cause of relocation.

Finally, as with counterurban lifestyle migrations by younger adults, despite relatively low costs, desirable climates and a more relaxed way-of-life, adapting to the everyday realities of living at an IRM destination can be challenging. Besides the simple fact, despite good air links, of living far away from one's "home" country, acquiring language competence can be a major challenge. Unlike international counterurbanizers (e.g. Benson 2011) however, differences felt between immigrants and the local population may be more accepted, albeit sometimes in quite patronizing and/or class- and ethnicity-specific ways (Benson 2013, 2014). As with Viallon's (2012) French RVers, the pleasures of living in the new place may be appreciated primarily from within. Returning to North Americans in Mexico, ethnographic research concluded how "expatriate retirees acknowledge cultural differences and strive to preserve their home cultural values," which ultimately resulted in "the central form of intercultural relationship [being] the employer-employee contract, Mexicans are subservient, while expatriates are in control" (Banks 2004: 377). This aligns lifestyle retirement migration with a rather uneasy, unethical and unsatisfactory project of the self.

\subsubsection{Midlife lifestyle "retirement" migrations}

As noted above, exact date of finishing paid employment should not be over-prioritized in studying lifestyle retirement migrations. Just as it is often useful to regard migration as a process rather than as a punctual action (5.6.3.2), so too is it useful to recognize retirement "as a process that occurs over time" (Moen 2003: 269, emphasis removed). As such, it incorporates changes not only in work but also in other aspects of life course, such as health, household structure and leisure interests.

This more open idea of retirement has increasingly led researchers to note that pre-retirement migration, recognized widely within counterurbanization research, may be indicative of socio-demographic emergence of a "new life stage" (Moen 2003: 274). This may be denoted the midcourse or midlife. Migration is a key associated component of this stage but remains under-researched (Wulff et al. 2010). Study of pre-retirement migrants to the German spa town of Bad Fuessing demonstrated them transitioning from certain "touristic" practices as relocation became "permanent" (Weidinger and Kordel 2015). Elsewhere, midlife migration to the Glens of Antrim in Northern Ireland 
was strongly shaped by past experiences (not least childhood memories), aspirations and expectations, with the desire to seek roots again coming through (Stockdale et al. 2013). This reinforces, once again, the importance of placing migration within its biographical life course context and associating (lifestyle) migration with ongoing attempts to fulfill overall projects of the self.

\subsection{CONCLUSION: RELATIONAL LIFESTYLE MIGRATIONS}

This chapter introduced the huge range of lifestyle migrations increasingly receiving prominent billing within the migration literature. It revealed both city and, especially, countryside to hold notable attractions-expressed representationally and "on the ground"-that can prompt residential relocation more-or-less independent of employment considerations. Such lifestyle migrations are strongly associated with class in most circumstances and most prominently expressed by citizens of the Global North. In life course terms, transition to "independent" adulthood, raising a young family, seeking fundamental everyday lifestyle change, and retirement from an employment career appear especially significant prompts to different geographical expressions of such migrations.

At least across the Global North, both lifestyle migration and lifestyle mobilities more generally have come of age, in practice and scholarship, within a facilitating era of mobilities (Cohen et al. 2015). Nonetheless, the chapter ends with three vital qualifications. First, somewhat paradoxically, while mobilities facilitate both access to aspired lifestyles (e.g. amenities) and lifestyle migrations, much of the rationale underpinning such migrations seemingly qualifies and even challenges any emphasis on the "freedoms" presented to cohorts living through these times (Gustafson 2008; Sager 2006). Indeed, the lifestyle migration literature attributes much of the underlying cause of this migration to geographical search for and subsequent attempts to cultivate authenticity-through-place or roots (Box 8.6). Seemingly, whilst one may reject any assumption of a sedentarist metaphysics to everyday life (Cresswell 2006) (5.2.1), active striving for emplacement by lifestyle migrants supports Osbaldiston's (2014: 182) conclusion "that the 'sedentary' social sciences still have much to say about phenomena like lifestyle migration."

\section{BOX 8.6 AUTHENTICITY-THROUGH-PLACE IN AMENITY MIGRATIONS}

The lifestyle migration literature emphasizes strongly the importance of "authenticity" within these expressions of aspirational behavior. Authenticity is a very difficult concept to pin down precisely. It is, as Nick Osbaldiston (2010a: 2) described it, "a powerful cultural paradigm that is born through myth and narrative" but refers to what is somehow "real," "genuine," "true" and "verifiable." It is an overall character that some critical commentators, from different political positions, feel is increasingly lacking within the contemporary (post-)modern world, where an inauthenticity of superficial depthlessness and fake performance is more the everyday norm.

For Sociologist Anthony Giddens, a search for authenticity featured strongly within the self-reflexive projects of the self which began this chapter. He noted how "[t]he project of the self remains one of control, guided only by a moral of 'authenticity' [but also with] a remoralising of daily life" (Giddens 1991: 225-226). For this to succeed, one needs to be an "authentic person." Such a person "knows herself and is able to reveal that knowledge to the other, discursively and in the behavioural sphere" (pp. 186-187) and has successfully established "a framework of basic trust by means of which the [life course] can be understood as a unity against the backdrop of shifting social events" (p. 215).

As Giddens suggested, authenticity is firstly about the person. Lifestyle migration thus reflects the (newly) "authentic" self or someone who aspires to become more "authentic" seeking a suitable location to develop, sustain and/or further cultivate that desired existential condition. However, as lifestyle migration explicitly reveals, self-authenticity is closely connected to and facilitated through place-authenticity.

Osbaldiston linked such place authenticity with late nineteenth-/ early twentieth-century Sociologist Émile Durkheim's notion of the "sacred." Both, he contended, acquire exalted evaluation not on account of some "intrinsic value" but due to "underpinning narratives or myths" (Osbaldiston 2010a: 3). Developing this perspective through Smith's (1999) four-fold model of places as mundane, profane, sacred or liminal, Osbaldiston argued that a sacred 
evaluation of destination places is prominent within amenity migration. Whilst cities and the (sub)urban represent mundane or often profane places (albeit attractive to some lifestyle migrants: 8.2.2), the narratives amenity migrants accord to "natural" rural or coastal destinations align them with the sacred. They are certainly not represented as liminal places of transgression and uncertainty! Amenity places thus present geographical sites where performance of self-authenticity can be grounded and facilitated through sanctified credentials, an argument that can be extended to lifestyle migration destinations generally, especially with a rural focus.

To support his argument, Osbaldiston (2010a: 4) suggested three "themes that sacralize/authenticate amenity-rich places against profane [and mundane] worlds." He illustrated each with empirical material, confined here to one indicative quote from documents produced by the Clarence Valley Council in New South Wales, Australia, a noted Sea Change (8.3.3.1) destination:

\section{Authentic nature: pristine, untouched natural worlds}

The countryside is breathtakingly beautiful. Whether you're exploring the rugged peaks and gentle valleys of the high country or cruising the wide open river plains, every turn in the road reveals something new and spectacular.

\section{Authentic past: cultural heritage as the sacred}

Much of the Clarence hinterland retains its rustic, early pioneering feel. The graveyards and ruins of gold rush settlements lie crumbling in the grass and beyond the abandoned town of Dalmorton, you can pass through a tunnel hewn from the solid rock by convict labour.

\section{Authentic communities: local culture as sacred}

Then there are the communities. Each town in the Valley, from the tiniest rural hamlet to the vibrant commercial and cultural hub of Grafton, has it's (sic.) own distinctive character and strong sense of "place" and community. The people of the Valley take pride in who they are and where they are.

Generally, amenity landscapes, as "nature," are "constructed in discourse to be pristine, untouched, and spectacular ... juxtaposed implicitly against the city as a dirty and aesthetically unpleasing arena"; they are "connect[ed] ... to the past"; and have "local communities not only warm, friendly, and sociable but also creative and individual" (Osbaldiston 2010a: 10). Finally, sacredness of amenity places is not just relationally constructed within discourse but grounded and everyday existence is always precarious. In particular, "profaning development and consumer capitalism" (Osbaldiston 2010a: 11), with "counter-narratives" of gentrification, commodification and unsustainability, are ever-present threats, not least, paradoxically, as a consequence of the popularity of amenity places to lifestyle migration (Box 8.4).

(Sources: Benson and Osbaldiston 2014b; Giddens 1991; Osbaldiston 2010a, 2010b)

Second, one might ask how effective lifestyle migrations ultimately prove to be in fulfilling people's desires within their projects of the self for "a better and more fulfilling way of life" (Benson and O'Reilly 2009b: 1). On the one hand, the overwhelming impression from the literature is of the migrations being "successful" overall. This is to some extent supported by longitudinal modeling study of migration generally, which has tended to see it associated with increased subjective well-being (e.g. Nowok et al. 2013; also Melzer 2011; Sloan 2013). On the other hand, the latter studies did not focus on lifestyle migrations specifically and research tends only to engage with migrants who remain resident in the destination, overlooking any who have left because the move has not worked out (Halfacree and Rivera 2012). Further, lifestyle migration scholarship now gives more attention to post-migration lives (Benson and Osbaldiston 2014b; Halfacree and Rivera 2014) and the "adjustments" migrants come to make, both in terms of attitudes and representations and of behavior and practices (e.g. Benson 2011; Halfacree 2013a, 2014a). This reiterates once again how migration is not a "done deal" with residential relocation.

Finally, it is important to reiterate that although lifestyle migration is underpinned by individual or household "choice," albeit a choice rooted in power and privilege accorded by class, refracted through age, gender, ethnicity and so on, such choice is rarely unconstrained. Certainly for the middle classes, enacting lifestyle migration still relies strongly on the kinds of "necessary conditions" (Fielding 2012: 123) noted in Figure 8.10. For all, lifestyle migration's relational construction must never be overlooked or forgotten. Thus, whilst Ní Laoire (2008) concluded a study of

\section{ST PROOFS: NOT FOR DISTRIBUTION}




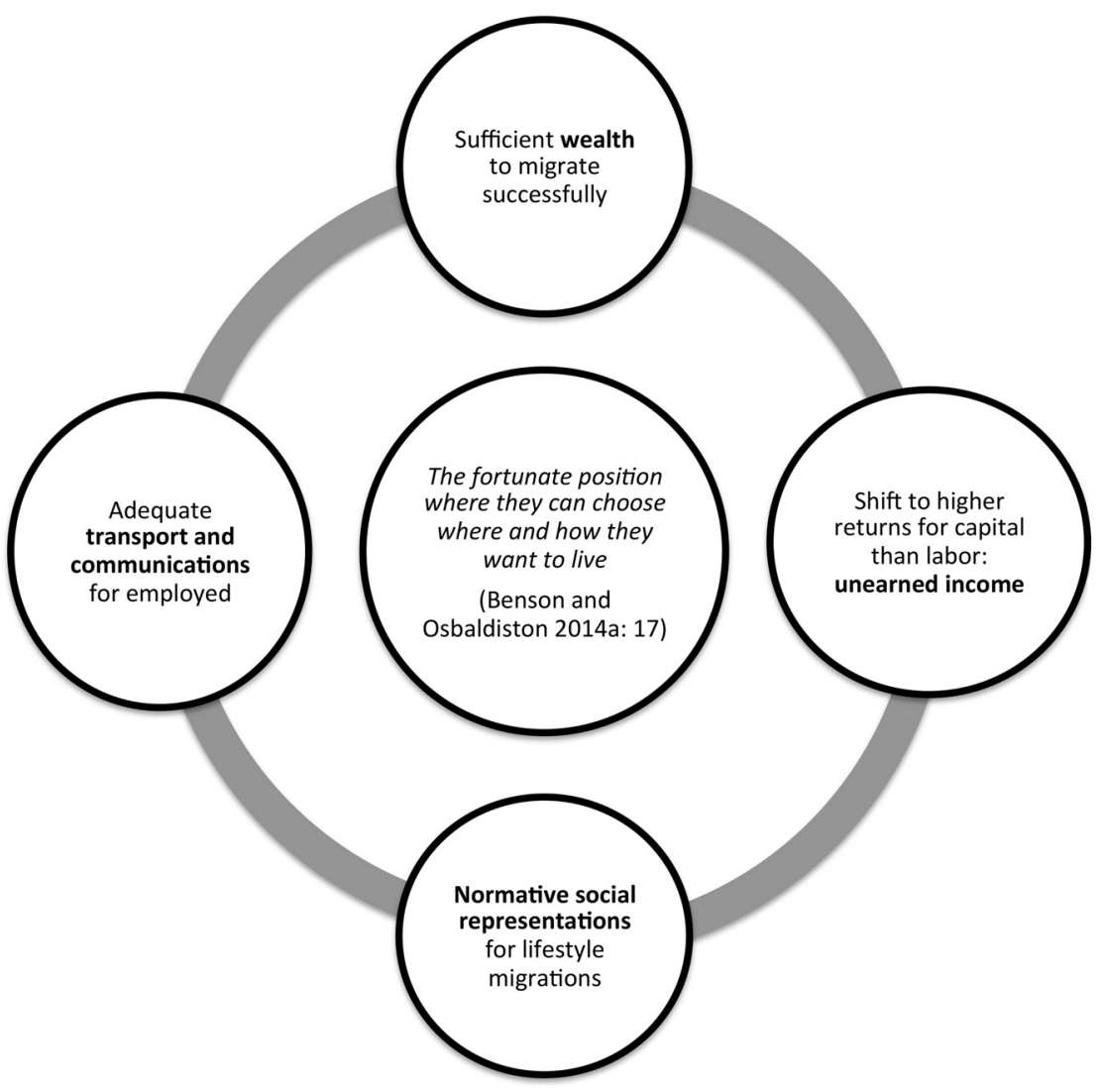

Figure 8.10 Necessary conditions for undertaking lifestyle migration.

Sources: derived from Fielding (2012: 123) and Benson and Osbaldiston (2014a: 17-18).

return migration to Ireland by highlighting a later life course desire for "settling back," this was complexly constructed. Lifestyle priorities were intertwined with issues of family and relationship formation and dissolution, child-rearing, caring for elderly parents, reconnection with siblings and/or friends, career changes, "new starts," and so on (also Corcoran 2002). As Huete et al. (2013: 346) argued when rejecting undue separation of lifestyle migrations from employment migrations, there is no "one-size-fits-all model" for lifestyle migration.

\section{NOTES}

London-The Smiths (1986), written by Steven Morrissey and Johnny Marr, taken from The World Won't Listen (WEA).

London You're a Lady-The Pogues (1989), written by Shane MacGowan, taken from Peace and Love (WEA).

Smalltown Boy-Bronski Beat (1986), written by Bronski Beat, taken from The Age of Consent (MCA).

To The Country-Laura Veirs (2007), written by Laura Veirs, taken from Saltbreakers (Nonesuch).

5 Also spelt counterurbanisation, counter-urbanization or counter-urbanisation.

6 Inspired by Birds Fly South-The Mastersons (2012) written by Chris Masterson and Eleanor Whitmore, taken from Birds Fly South (New West).

7 A body transformed after drowning in The Tempest! 


\section{CHAPTER 9}

\section{Forced migrations}

\subsection{INTRODUCTION: THE IMPORTANCE OF LABELS}

\subsubsection{Defining forced migrations}

$[\mathrm{H}]$ ow restricted or undesirable must one's options be to justify the conclusion that it is reasonable for someone to reject those options and choose migration?

(Bartram et al. 2014: 70)

Even as Michael Samers (2010: 11) noted " "[a]prominent academic distinction in the migration literature ... between forced and voluntary migrants," he immediately qualified this by emphasizing in practice "a continuum between forced and voluntary, and ... determining precisely who is 'forced' and who is 'voluntary' is difficult." This caution is endorsed within this chapter. Whilst illustrating experiences of migrants located very strongly towards the "forced" end of the continuum, definition of "forced migration" first needs further consideration. This leads to emphasizing the considerable importance of labels and categorization throughout the chapter (9.1.2). Following this positioning, Section 9.2 discusses the modern history of "defining refugees" and the principal categories of forced migrants and forced mobilities. Section 9.3 addresses the latter, mostly in a critical discussion of present-day slavery. Section 9.4, the heart of the chapter, then illustrates the complex processes and experiences of forced migrations of refugees, internally displaced persons and asylum-seekers. Causes, from political persecution to environmental degradation, are covered, as is the political challenge of finding durable solutions to allow refugees to return to a life course less immediately shaped by flight and danger. Section 9.5's short conclusion once again raises questions of human agency and definitions.

Human agency, stressed within the humanistic tradition of Population Geography (1.2.4) and forming a central element of the life course perspective (2.3.10), is central to the question of defining forced (or non-forced/voluntary) migration. For Sociologist Anthony Giddens (1984: 9), "[a]gency concerns events of which an individual is the perpetrator, in the sense that the individual could, at any phase in a given sequence of conduct, have acted differently." This authorship of action, importantly, need not be carefully thought through or even mentally conceptualized at all but is still, ultimately, the outcome of an individual. Giddens's definition thus positions a migration as only truly forced when the person(s) involved had no influence on its initiation, actual occurrence and, probably, even subsequent playing out. From this strict definition, to be completely forced is likely to be very rare indeed. Consequently, Castles et al. (2014: 51) regarded the term "forced migration" largely as "an oxymoron, because people need to have a certain level of agency in order to move" and it is those with the least agency, the most deprived, who are "the ones "forced to stay'."

Reiteration of agency and individuality within all migration practice, however, does nothing to undermine the need to recognize the presence of more-or-less forced migrations worldwide. Indeed, approaching the problem from the other direction, critically examining any proposition that migrations are largely "voluntary" soon reiterates the necessity of recognizing force or coercion. Whilst Chapters 6-8 outlined a diversity of mobilities and migrations largely classifiable under the "voluntary" label, only the most naive observer would describe the majority as completely "free." Thus: commuting mobilities are required to access suitable employment; residential mobility may be the only realistic way for a family to keep a roof over its head; relocating from one end of a country to another, even half way round 
the world, may be the only means a person can imagine making a living; and even lifestyle migration to the countryside may be undertaken in part because it is seen as the responsible thing to do at the migrant's life course stage. In other words, a degree of coercion is expressed via everyday economic, cultural, political, family and other relational life course pressures "encouraging" residential relocation. Within what Archer (2003) terms internal conversations, "the "internal dialogue' or 'self talk' of individuals as they debate with themselves how they might want to modify themselves and "the world as a consequence'" (Evans 2014: 308), migration often becomes the only practical move (sic.) to make.

From an everyday practical perspective, therefore, both force and choice-structure and agency-are expressed within all migrations. However, the balance varies considerably between types of migration and within social and embodied variations of each type. In this chapter, forced migration refers to geographical displacement whereby individuals, families or larger groups are compelled unwillingly to leave their home and/or region or country as a result of actual or feared severe negative threats. These threats are diverse but typically result in what Fullilove (2004) characterized as "root shock" or "the traumatic stress reaction to the loss of all or part of one's emotional ecosystem" (Fullilove 2014: 142). They range from immediate risks to life, such as war; through perilous racial, ethnic, or political persecution, discrimination or intolerance; to more environmental challenges to life and livelihood, such as droughts, volcanic eruptions, extreme soil degradation and climate change. Once displaced, forced migrants might eventually return "home," or they might never return but find themselves creating new lives in new locations often distant and culturally distinct from their homes.

Forced migration is always constructed relationally. Thus, for Castles et al. (2014: 50), it depended where migration was positioned relative to "capabilities and aspirations ... within a given set of structural constraints." In terms of the differential arenas of life course experience (2.3), especially notable are class, geography, gender, ethnicity and history. Age, whilst important in the context of forced migrations of children (Box 9.1) and trafficking (9.3.1), is less prominent than in the previous migration chapters. Many causes of forced migration, in short, are relatively insensitive to age. In contrast, politics at both national and international scales looms very large indeed, instigating, molding and shaping all aspects of forced migrations.

\section{BOX 9.1 UNCERTAIN PLACES OF CHILDREN WITHIN FORCED MIGRATION}

Children comprise one of the most vulnerable segments of the global population, not least because they are highly dependent on adults for both basic survival and social, emotional and physical development. Forced displacement exacerbates vulnerability, often exposing them to violence, exploitation, abuse and neglect.

Children comprise nearly half the population of concern for the UNHCR. Prior to and during their initial flight, they may become separated from families, also leaving many as caretakers and protectors of remaining members of fragmented families. Even in refugee camps, although these may provide food, shelter and basic education, young people face an uncertain future. Many will little access to higher education, land, or other means to create and sustain their lives economically. Such loss of ability to create a viable livelihood means that even basic life stages, such as family formation may be delayed or curtailed. In Malawi's Dzaleka refugee camp, Rashid reflected:

In my country you become a man when you marry and have children of your own. Your family gives you a plot of land and you go about your business. Here, no, I am afraid to marry. Where will we go-what will we do? I cannot marry.

(quoted in Healy 2012: 6)

This 18-year-old Congolese man was far from alone in his worries. There was wide agreement that marriage was not an option, whilst early pregnancy and parenthood and dropping-out of school very much was.

Not all child or youth migrants are found in camps or in other protected circumstances. Some end up or even set out on their own as asylum-seekers, becoming especially vulnerable to human smugglers and traffickers, violence and abuse while being illegally transported across borders. Ukraine, bordering the EU, is a country in which large numbers of unaccompanied asylum-seeking children have been identified. Most are Afghan and 
Somali males aged 15-17 years. Many are suspected of entering the EU with the assistance of organized smuggling rings. How such young people are treated by the asylum system-and how they should be treatedis an area of much debate within both policy and scholarship. They often comprise some of the "bureaucratically problematic people" (Griffiths 2012: 715) state asylum systems find hard to deal with.

At least two core related issues stand out with respect to youthful asylum-seekers arriving unaccompanied by a "responsible" adult. First, authorities frequently doubt the arrivals are indeed children, not least when they lack corroborating evidence (e.g. passport) but even when they do have documentation. Consider Astrit, a 17year-old arrival in the UK from Kosovo:

When I told her my age she said did I have any documents. I . . gave her my birth certificate. She called [someone who] said 'I don't believe you because you don't have a photo or a stamp'. But in Kosovo our birth certificates don't have a photo or a stamp....

(quoted in Crawley 2007: 23)

Within a "culture of disbelief" (Crawley 2007: 26), youthful migrants are strongly suspected of pretending to be children in order to obtain more favorable treatment within the asylum process. Such suspicion is reinforced by

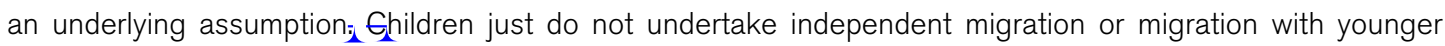
siblings.

Age-related disputes are correspondingly commonplace within the asylum process. They have prompted ever more ingenious and intrusive methods for determining age, such as $\mathrm{x}$-raying bone or teeth or assessing levels of physical development. A study for the UK Home Office found half of those seeking asylum who claimed to be under 18 years of age had their ages disputed. Within grueling and often highly intimidating interviews, any expression of political views or more general agency was used to argue that they cannot be children. The ultimate result of such engagements is to find children placed into some form of detention, often in a state of limbo rather than facing imminent deportation.

A second core theme in terms of independent asylum-seeker children is the need to recognize their agency fully, not just in terms of their ability to relocate. The everyday dependency of children on adults must not be automatically assumed. This extends even to trafficked children, where any sense of them expressing agency is often denied and they are instead portrayed solely as victims. Raising "[d]ifficult questions about consent and agency" (O'Connell Davidson 2011: 466) and about the key life course transition to adulthood, children must be more fully recognized as potential agents of their own life course. Such experiences may be difficult and perilous, nevertheless. This may involve them with the same interactions with traffickers as adults, for example, if such people are the only "practical means by which many children [can] access a range of benefits that would otherwise be unavailable to them" (O'Connell Davidson 2011: 468).

In summary, whilst "chronological age" categorizing a person as child or adult may have "huge implications and is highly significant" within the bureaucracy and logic of the asylum and immigration process, it may be "much less significant for children and young people themselves" (Crawley 2007: 18). Instead, the need to flee danger or to earn income is foremost, and the struggle to avoid the same kinds of exploitation and abuses that impact on "not-so-moving children" (O'Connell Davidson 2011: 460).

(Sources: Bhabha and Schmidt 2008; Crawley 2006, 2007, 2010; Healy 2012; Maguire 2012; O'Connell Davidson 2011; Tyler et al. 2014; Williamson 2011)

\subsubsection{Not (just) on the label}

If asked for a category of forced migrant, "refugees" immediately spring to mind, recent news headlines reporting, for example, Most refugees since second world (Guardian 2014c). This default term triggers images of camps of blue or white tents emblazoned with the United Nations (UN) symbol located in remote and often harsh landscapes. But defining refugees cannot be reduced to the visual imagery of Figure 9.1: at the end of 2014, of the 85 percent of refugees for whom accommodations were known, only just over $1 / 3$ were living in refugee camps, with nearly $2 / 3$ 


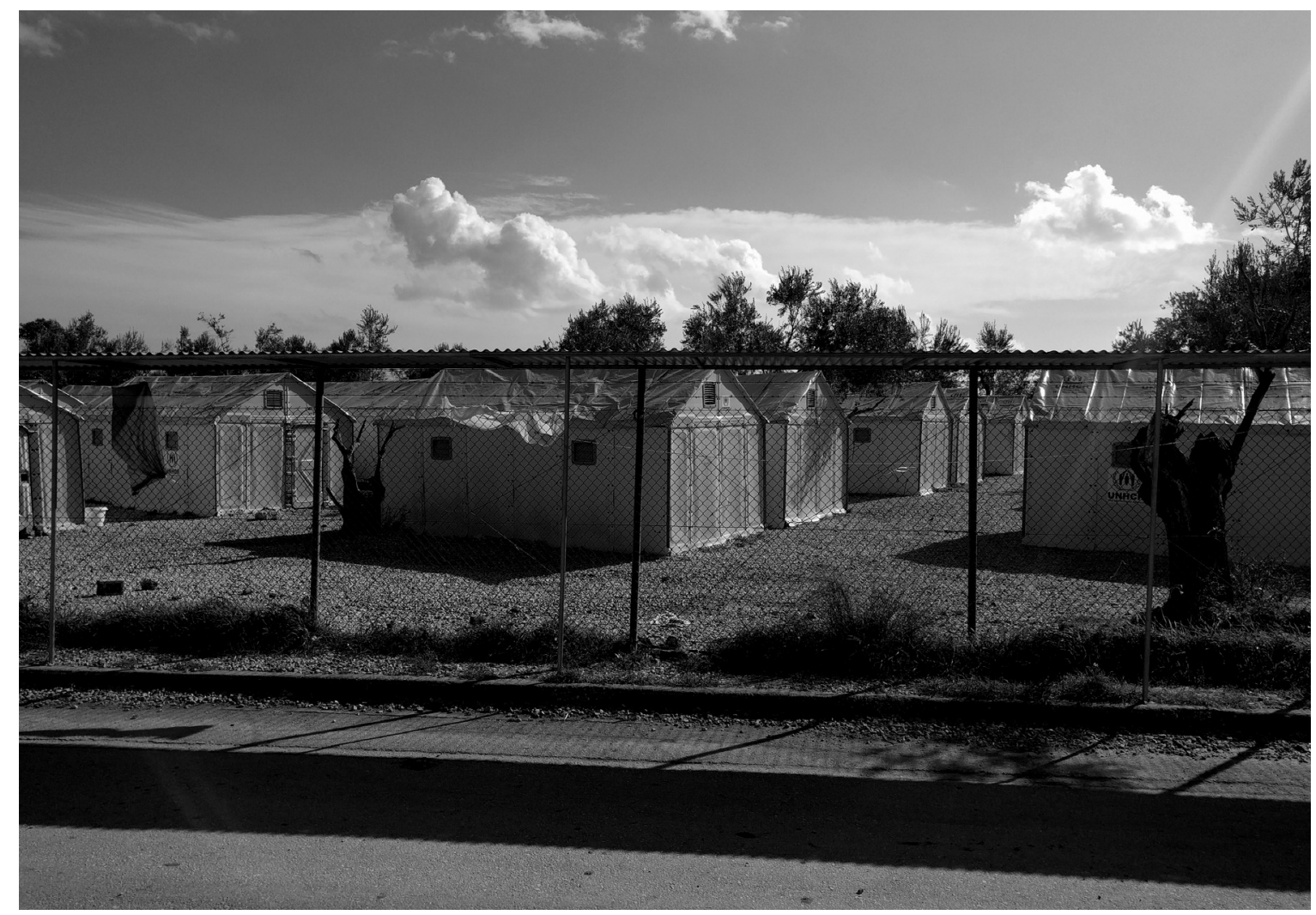

Figure 9.1 Refugee camp: Lesvos Island, Greece.

Source: Creative Commons, Public Domain.

elsewhere, mostly in urban areas, a trend that was on the increase (UNHCR 2015a: 41-43). Instead, defining refugees and other categories of forced migrant is a complex, contested and highly political matter. Its outcome strongly influences subsequent life course experiences of officially-classified forced migrants and those failing to acquire this status. Thus, in a paper noting a "challenge of categories" in respect of defining Palestinian refugees, llana Feldman (2012: 388, emphasis added) wrote how official:

determinations about what sort of displaced person someone is shape how and whether [they] can receive assistance and protection [and overall] experiences of displacement. People live their lives in part through the categories into which they fall and as those categories shift, so too do the possibilities of those lives.

Two decades earlier, Roger Zetter (1991) argued that social and lived experiences of refugees was linked to the public policy practices and procedures of institutions that defined the labels applied to displaced persons. These labels, he argued, were not merely bureaucratic instruments. Instead, by considering "how labels are created and applied, we can better understand how patterns of social life and cultural norms, straining under the intense pressure of forced displacement, are mediated, impacted and ultimately controlled and reformulated by institutional agency" (Zetter 2007: 173). This later paper also noted how the refugee label had evolved, transformed via processes of globalization and increasingly complex causes and patterns of forced migration. The result was that it "ha[d] become politicized, on the one hand, by the process of bureaucratic fractioning which reproduces itself in populist and largely pejorative labels whilst, on the other, by legitimizing and presenting a wider political discourse of resistance to refugees and migrants as merely an apolitical set of bureaucratic categories" (Zetter 2007: 174). This changing meaning-fractioning-of the "refugee" label by governments acts, for example, as a dynamic tool to discriminate between "genuine refugees"

\section{ST PROOFS: NOT FOR DISTRIBUTION}


and "economic migrants." It may also transform migrants' behavior, such as prompting them to (be able to) endure trafficking or "illegal" employment.

Whilst there is clear administrative necessity for categories such as "refugee," and the label can powerfully draw attention to their plight (cf. Masquelier 2006), Feldman's and Zetter's arguments reinforce the need to be thoughtful and reflective when interpreting the forced migration experience. Statistics on official "refugee numbers," for example, tell only a partial if quantifiable part of the story. Whilst official labels must certainly be noted, not least on account of their often considerable life course consequences for those marked (Griffiths 2012), the politics of their definition, in particular, means that their ultimate authority in defining migrant types must always be recognized as provisional and limited.

\subsection{RECOGNIZING FORCED MIGRATIONS AND MOBILITIES}

\subsubsection{Developing categories of forced migration}

Taking an historical perspective, through the twentieth century the displacement of individuals, households and larger populations or settlements increasingly involved a wider and more varied range of countries, ethnic groups and types of displacement. This diversity of circumstances and the growing number of people affected demanded international understanding and cooperation to address the varied needs of those displaced. Consequently, formal creation of the United Nations High Commission for Refugees (UNHCR), the international body responsible for providing humanitarian assistance to individuals in need, came in 1950 in the aftermath of dramatic population upheavals of World War II, when nearly 40 million persons became displaced (Castles et al. 2014). This agency is now not only tasked with providing humanitarian assistance but is also prominent within the "key matter of debate" (Bartram et al. 2014: 72) regarding defining or categorizing who counts as a displaced person.

Outlined in Table 9.1, UNHCR identifies three primary types of forced migrants: refugees, internally displaced persons (IDPs) and asylum-seekers. In addition to these categories, it also recognizes stateless persons and other persons of concern. As emphasized above, the interplay between the label attached to a displaced individual and subsequent implications for their life course prospects following displacement, including immediate aid and resettlement

Table 9.1 UNHCR definitions of displaced persons

\begin{tabular}{|c|c|}
\hline Category & Definition \\
\hline Refugee & $\begin{array}{l}\text { Persons recognized under } 1951 \text { Convention Relating to the Status of Refugees, } 1967 \text { Protocol Relating } \\
\text { to the Status of Refugees, } 1969 \text { OAU Convention Governing the Specific Aspects of Refugee Problems } \\
\text { in Africa, the UNHCR Statute; and persons granted complementary forms of protection or temporary } \\
\text { protection. }\end{array}$ \\
\hline Asylum-seeker & $\begin{array}{l}\text { Persons who have applied for asylum or refugee status but have not received a final decision on their } \\
\text { application. Only asylum-seekers with cases pending at the end of the year (stock) are included in } \\
\text { UNHCR's total population of concern. }\end{array}$ \\
\hline $\begin{array}{l}\text { Internally displaced } \\
\text { person (IDP) }\end{array}$ & $\begin{array}{l}\text { Persons forced or obliged to flee or leave their homes or places of habitual residence-in particular as a } \\
\text { result of armed conflict, generalized violence, violations of human rights, or natural- or human-made } \\
\text { disasters-but who have not crossed an international border. }\end{array}$ \\
\hline Stateless person & $\begin{array}{l}\text { Persons not considered as a national by any State under the operation of its law. Most stateless persons } \\
\text { have not fled their country of habitual residence. UNHCR seeks to avoid and reduce statelessness. }\end{array}$ \\
\hline Returnee & $\begin{array}{l}\text { Persons who have been displaced but have returned to their country or place of origin. UNHCR seeks to } \\
\text { reintegrate former refugees and IDPs as soon as possible. For statistical purposes, only refugees and } \\
\text { IDPs who have returned during the past } 12 \text { months are included in these UNHCR populations of } \\
\text { concern but in practice operations may assist returnees for longer or shorter periods. }\end{array}$ \\
\hline
\end{tabular}

Source: UNHCR (2006: 16-17) 


\section{FORCED MIGRATIONS}

options, is very close. However, development of these categories and the reaction of the Global North, in particular, to the displaced people they cover has changed considerably since 1945.

Subsequent to mass movements of people across national borders following World War II, Boyle et al. (1998) recognized three relatively distinct periods in post-1945 refugee policy evolution: 1945 to the 1970s; 1980s to the early 1990s; late 1990s to the present. These broad periods contextualize historically how individual countries have contended with refugee flows. They map a trajectory of ever-tightening policy in regards of official recognition of a "forced migrant."

During the first period, 1945-1970s, refugee movements were initially recognized in Europe as legacy of World War II. The world, broadly speaking, was relatively open and sympathetic. Northern nations were willing to receive refugees and provided reasonable services to them. The UNHCR was established (1950) and guiding principles developed for countries receiving refugees. In 1951, the UN approved the Convention Relating to the Status of Refugees, continuing a process of developing a body of international law, conventions and guidelines to protect refugees, The 1951 Convention or Geneva Convention, as it is also known, not only defined a refugee but contained 46 articles which established minimal refugee rights, including types of assistance to be provided by host nations and obligations of refugees to host governments (Gallagher 1989).

Later in this first period, in 1967, the Protocol Relating to the Status of Refugees (the New York Protocol) increased the scope of the 1951 Convention to include displaced persons worldwide. It also expanded the definition of a refugee to include large groups of people fleeing from their country of residence, a category specifically not included in the 1951 Convention. Next, the Organization of African Unity's Convention Governing the Specific Aspects of Refugee Problems in Africa (1969) was adopted to address mass movements of people needing humanitarian assistance on the African continent, the Cartagena Declaration (1984) later giving similar recognition to Latin America (Castles et al. 2014). As of April 2011, 147 states had signed either the 1951 Convention or 1967 Protocol, most (142) signing both (UNHCR 2011). Today, as a result of these post-1945 developments, the UN considers a Convention Refugee as:

a person residing outside his or her country of nationality, who is unable or unwilling to return because of a 'wellfounded fear of persecution on account of race, religion, nationality, membership of a particular social group, or political opinion.

(UNHCR, quoted in Castles et al. 2014: 222).

However, such a definition excludes a large number-the majority-of forced migrations.

A second period of post-war refugee policy emerged through the 1980s. With perceived mounting immigration pressures and increasing numbers of displaced persons, the Global North began to experience so-called "compassion fatigue" and moved to tighten restrictions on resettlement (Boyle et al. 1998). The preceding period had witnessed development of new refugee flows, reflecting "escalation of struggles against white colonial or settler regimes in Africa from the 1960s, resistance against US-supported military regimes in Latin America in the 1970s and 1980s, and long-drawn-out political and ethnic struggles in the Middle East and Asia" (Castles et al. 2014: 225; Zolberg et al. 1989). For example, following the Vietnam War (which ended in 1975), nearly 3 million "boat people" fled Vietnam, Cambodia and Laos, more than 1 million resettling in the US, Australia, Canada and western Europe (Castles et al. 2014). The new flows were less individuals fleeing explicit political persecution and more large groups escaping generalized violence and seeking humanitarian assistance.

By the 1980s, large flows of asylum-seekers were moving directly to North America and Europe, followed in the early 1990s by populations displaced by collapse of the USSR. Overall, the sheer number of displaced persons-in addition to increasing numbers of other migrants seeking to move internationally (Chapter 7)-undoubtedly placed great strain on the capacity of many countries to process adequately all applications for asylum and manage growing pressure for immigration. At the time, Gallagher (1989: 594) argued that the then current system of addressing refugee movements "represents an extraordinary evolution during the latter half of this century of capacities to cope with such movements of people." Yet, he further suggested problems inherent in the system, notably its emphasis on state sovereignty and a willingness of countries to grant asylum. Notably, whilst signatories to the 1951 Convention are not obligated to grant asylum, they are prohibited under the key principle of non-refoulement from returning refugeesbut not failed asylum-seekers-forcibly to their country of origin (Gallagher 1989). 
A third policy period emerged from the late 1990s, framed by continued growth in numbers of displaced persons but with Northern countries increasingly averse to all immigrants. Increasing efforts to curb immigration resulted in more restrictive policies and acute politicization of migration issues (Castles et al. 2014). On the one hand, this period saw the aforementioned fractioning of refugee categories (9.1.2) and, on the other hand, rising state and public hostility towards immigrants of all types. International policy emerging in this period was pioneered by the Dublin Convention (1990). Seeking both to restrict and to "ease and share the burden" across Europe, it comprised two main elements. First, confinement, which:

sought to distinguish between 'economic migrants' and 'true political refugees' by imposing visa restrictions on .... potential ... source[s] of asylum-seekers ... enhanced by the use of 'carrier sanctions' ... fines ... to be levied on ferry companies, airlines, and other 'carriers' for bringing in an undocumented migrant.

(Samers 2010: 211)

Confinement, in short, made it considerably harder to enter a European country in order to claim asylum. Second, the principal of refoulement (compare 9.2.1) sought to confine asylum-seekers to the first officially designated "safe" country entered when leaving the home country. This aimed to counter supposed "asylum shopping," whereby asylumseekers sought countries most likely to grant asylum, but effectively reduced choice in terms of where asylum-seekers lived.

Consolidating this third period of refugee policy, present-day attitudes to forced migration across the Global North must be seen in the context of migration management/managed migration. This comprises a set of policies designed to regulate carefully the type and number of all migrants, whether asylum-seekers, refugees, family members or employment migrants. Such "management" requires the sustained attention of a range of agencies and border control entities, as well as governmental infrastructure, to prioritize the entrance of some migrants over others. Samers (2010: 186) argued that "migration management reflects government's concern with other issues including security, the dictates of foreign policy, trade liberalization, promoting the international standing of a country, creating ethnic or racial homogeneity, nation building and advancing an 'imagined future'." A similar, related term utilized by the European Union (EU) is border management, which Wolff (2008: 254) described as "a solution to the geographical proximity with neighbors that are a source of instability and thus a way to ensure the EU's own internal security." While managed migration refers to the composition of migration flows, border management addresses issues of security. These include, but are clearly not limited to, the composition and magnitude of migration flows, with an eye on potential terrorist threats in particular. An interesting case study is that of Frontex (Box 9.2).

Into the twenty-first century, the policy and societal challenges posed by forced migrants have certainly not gone away (Guardian 2014c). Northern policy, in particular, largely remains focused on excluding all but the most "deserving" (sic.). On the one hand, for UNHCR, numerous conflicts, droughts, famines and pervasive inequalities "mean that vulnerable, desperate and persecuted people continue to seek sanctuary in another country, and continue to face barriers, hostility, and often rejection" (Goodall 2011: 3). Yet, on the other hand, the current international political "mood" on this issue reflects both the changing dynamics and ethno-geographical composition of forced migrant flows. In terms of the former, perceived further increased pressure on recipient states comes not just from increased numbers of Convention Refugees, for example, but somewhat ironically from greater recognition of the potentially expanded scope of "forced migrants."

\subsubsection{Expanding definitions of forced migration}

\subsubsection{Recognizing forced migrations beyond refugees and asylum-seekers}

People involuntarily pushed from their homes today include not only those displaced by war and political instability, as traditionally defined, but increasingly by more novel causes, including environmental disasters, development projects and fragile economies. All are largely human of forced migration are rising numbers of Internally Displaced Persons (IDPs). This acknowledges how forced migration is not just a matter of international migration but often unites international and intra-national flows under common causes. 


\section{BOX 9.2 FRONTEX AND EUROPEAN UNION BORDER SECURITY}

Frontex is an agency representative of integrating and intensifying border security trends in Europe. This is a process not without problems both for Member States and migrants/asylum-seekers. The European Council established Frontex in 2004 as the European Agency for the Management of Operational Cooperation at the External Borders of the European Union. Its primary mission is to "promote, coordinate, and develop European border management" through six key tasks for EU Member States:

1. coordinating operational cooperation in the management of external borders;

2. assisting training of national border guards, including establishing common training standards;

3. carrying out risk analyses;

4. following up on research relevant for the control and surveillance of external borders;

5. assisting in circumstances requiring technical and operational assistance at external borders;

6. providing necessary support in organizing joint return operations.

As an EU agency, Frontex is a depoliticized, technocratic and managerial body supposedly supporting and linking national and supranational authorities. Pollak (2009: 906) labeled it a "network agency" due to its objective of developing "coherence, durability, and stability among the EU network of institutional actors at multiple levels of governance, hence reducing the transaction costs of coordination." Since inception, Frontex has gained financial independence and its budget has risen almost exponentially, highlighting EU will to invest in enhanced external border management. Despite these investments, ultimate responsibility for control and surveillance of external borders still lies exclusively with Member States. Frontex does not perform independent operations, nor gather intelligence.

Frontex's development occurred in the context of European border integration, EU cooperation on migration and asylum, and a post-9/11 climate which heightened tension between border control issues and terrorism/security. With European integration and the principle of free movement, internal borders have been greatly diminished. EU nationals can travel relatively easily between countries, as can goods and information. While these borders have diminished, however, others have been both created and intensified. This has led to a popularized concept of Fortress Europe.

Frontex reflects the effort to multiply sites and modes of border management. The agency has created mechanisms for intensifying management activities, notably establishment in 2007 of rapid border intervention teams (RABITS). These comprise officers of national law enforcement bodies that deal with border management in each Member State. They are available to these countries as a tool to respond to "extreme" border conditions. There has only been one deployment to date. In 2010, 175 guest officers from 24 Member States were deployed to the Greek-Turkish land border when Greece was facing increased undocumented migration flows.

Frontex's intensified border management initiatives have intensified consequences for migrants and asylumseekers. The most readily identified issues stem from concerns over its legality, particularly involving the practice of non-refoulement, the prohibition of returning refugees to places where they are at risk without considering their proposal for asylum. The right to non-refoulement raises particularly problematic questions when applied to interception of migrants at sea. For instance, should rescued persons be considered under Europe's refugee law if intercepted in the territorial waters of a third state? This ambiguous legality has led scholars to posit that such crucial decisions are often made ad hoc in Frontex's operations. Furthermore, actors making these decisions are not democratically elected governments or EU institutions but military and security officials on the Mediterranean. Thus, despite Frontex's supranational framework, individuals in localized spaces essentially shape refugee law operations. Indeed, despite emphasis on human rights in Frontex's rhetoric and regulation, its internal structure indicates a lack of strong mechanisms for accountability. The Frontex management board primarily controls its activities and consists of one representative from every Member State and two representatives from the European Commission. The board appoints the Executive Director, who is then accountable to them. Migrant and human rights groups have criticized this structure because without representation from other groups it is solely the interests of Member States that are represented in decision making processes. 


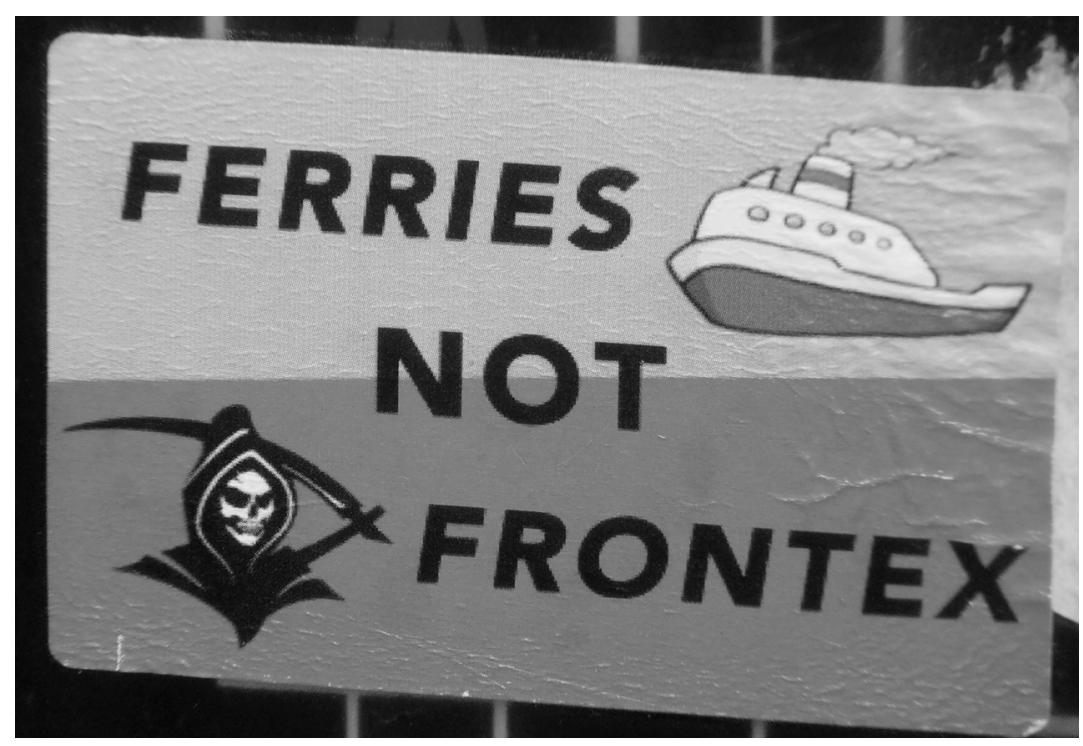

This slogan "Ferries not Frontex" emerged after 800 adults and children drowned whilst trying to cross the Mediterranean on 18 April 2015.

In sum, Frontex illustrates well some of the considerable and contentious challenges facing efforts to create harmonized and harmonious international border controls that can manage both forced and more voluntary international migrants. Yet it is a challenge rising ever higher up the political agenda of both national and extranational governments across the Global North within this age of migration.

(Sources: Council of the European Union 2004; Frontex 2014; Klepp 2010; Leonard 2008; No Frontex Days Catania 2016; Pollak 2009; Tholen 2010)

Within political opinion and migration policy throughout the Global North, one key but vigorously disputed category within any expanded understanding of forced migration is that of forced economic migration. As Chapter 7 demonstrated, economic reasons are the prime cause of migration at almost all geographical scales but should some expressions be seen as more forced than voluntary (Bartram et al. 2014)? Are not, for example, "economic" factors such pervasive poverty, malnutrition, or long term environmental deterioration that disrupts or precludes the continuation of adequate livelihoods, "sufficient" push factors to define resulting migrations as forced? Such people have usually been regarded more as "economic migrants and welfare seekers in disguise" than refugees (Castles et al. 2014: 113). This clearly has negative consequences in terms of how their treatment by countries they (attempt to) migrate to. Samers (2010: 13) again summarized the situation well:

those who are "forced" by poverty are imagined by governments as "voluntary" and so-named "economic migrants". [Moreover,] [t]he term "economic migrant" and "economic migration" may have negative connotations and assume a pejorative tone; that is, economic migrants are often imagined in relation to "more deserving" refugees.

Without refugee or asylum-seeker status, forced economic migrants face the same obstacles to international migration as employment migrants generally (Chapter 7). Countries across the Global North, in short, are increasingly reluctant to accept them, in spite of their reliance upon international migrant labor power.

Underpinning one family of potential forced migrants are negative experiences of the physical environment. This suggests a category of forced environmental migrants. Thus, in 2008, the journal Forced Migration Review took up

\section{ST PROOFS: NOT FOR DISTRIBUTION}




\section{FORCED MIGRATIONS}

the question of environmental migration, first defined by a United Nations Environment Programme (UNEP) researcher nearly a quarter of a century earlier as:

people who have been forced to leave their traditional habitat, temporarily or permanently, because of a marked environmental disruption (natural and/or triggered by people) that jeopardized their existence and/or seriously affected the quality of their life.

(El-Hinnawi 1985: 4)

In the journal, Dun and Gemenne (2008) contemplated issues surrounding the various terms now associated with such migrations, shown in Table 9.2. These fluctuate between stressing climate or environment more generally, and between describing those concerned as migrants or refugees. While some migrations associated with the environment result from acute disasters, such as hurricanes, tsunamis or volcanic eruptions, other chronic environmental disasters occur much more slowly, such as desertification. These provide a life course temporality that brings into question whether the final decision to move is forced or more voluntary. Paralleling debates around forced economic migration, at what point in a degraded natural environment, from which an individual derives their livelihood, is a decision to migrate "forced"? Numerous estimates of the number of people currently or potentially displaced by climate change, for example, range from 25 million currently to nearly 200 million by 2050 (Urdal 2008: 5; also Black et al. 2011). Are such people environmental refugees? Thorny questions such as this prompted Bates (2002) to call for better definitions than overly loose generic definitions of climate or environmental refugees that fail to distinguish variations in choice or necessity.

Definition becomes even more contentious from a policy perspective. Some scholars pushed for expansion of the 1951 Convention definition to include environmental migrants, often also seeking instruments to protect them in ways similar to refugees. Others regard environmental relocators as economic migrants and not entitled to refugee protection (Stavropoulou 2008). The outcome of such debates is vital for the lives of those involved. If a country views environmental migrants as refugees, it is likely to be more open to resettlement options and provision of aid. In contrast, if viewed as voluntary migrants moving primarily for economic reasons, probability of support is considerably lower.

A further related type of forcibly displaced migrant is someone involuntarily resettled because they previously resided in an area "favored" for a major development project such as a dam. Those displaced may again resemble refugees or IDPs. Displacement is most often of groups already poor or marginalized, thus lacking resources to relocate successfully. Their poverty, for Scudder (2005), also helps explain their relative neglect within forced migration scholarship.

\subsubsection{Recognizing mixed migration}

An important consequence of recognizing diversity amongst the causes of forced migration is to note that almost all migrants, legal and undocumented, economic and forced, increasingly use the same routes and even methods of migration (Box 9.3). For example:

[p]eople who are on the move from one country to another, even when they meet the criteria for refugee status, increasingly engage in unauthorized or undocumented movement, making use of similar routes, employing the services of the same smugglers and obtaining fraudulent travel documents from the same suppliers.

(Riera 2010: 26)

Table 9.2 Terms associated with environmental migrants and migration

Climate-induced migrants

Climate change migrants

Climate refugees

Environmentally-induced forced migrants

Environmental refugees

Ecological refugees

Source: expanded from Dun and Gemenne (2008). 


\section{BOX 9.3 REFUGEE MODES OF TRANSIT: USE OF BOATS}

The concept of transit migration highlights the routes taken and the multiple motivations and origins of those following them within international migration generally. In particular, it draws attention to how the direction and experience of migration can be shaped strongly by the practical modes of transit taken by refugees and other forced migrants to reach a hoped-for country of asylum.

First, specific countries may be only practically accessible via certain modes of transit, whilst certain modes of transit may only access particular countries. Second, many modes of transit are frequently associated with considerable dangers for migrants, both from those controlling them, notably people smugglers, and from their specific form. A good illustration of both points is the use of coyote guides to take undocumented migrants across the Mexico-US border, often through very harsh desert environments where more than 6,000 migrants have died since 2000 (Schultz 2014). The composition of such migrant groups leads to a third point, namely that they typically express mixed migration. Forced migrants escaping wars, famines, ethnic strife and so on are often, quite literally, placed in the same boat as economic migrants seeking livelihoods abroad.

Boats are very commonly used globally as a mode of transit for mixed migrants. For example, many Rohingya, discussed in Section 9.4.1.2, risk their lives at sea to try to reach Thailand or Malaysia rather than take the land route to Bangladesh. Charged around $\$ 2000$ by people smugglers, they risk being imprisoned for ransom or being sold into debt bondage (7.5.3.3) if they survive the hazardous trip but are unable to pay up on arrival. Elsewhere, although an island state remote from the main sources of refugees, asylum-seekers have frequently sought to reach Australia by boat. The negative response of Australia to such efforts is described in Section 9.4.2.3.

An island features in a third example of forced migrants using boats to seek a country of asylum. Lampedusa is the largest of the Pelagie Islands but still a tiny place of just 20 square kilometers and 5,000 people. Strategically, however, it is located half-way between Tunisia and Sicily (Italy). Still more critically, it is part of Italy and thus the EU. Given this geopolitical position, Lampedusa is a magnet for undocumented migrants arriving by boat from North Africa. Tragically, the crossing is extremely hazardous, often not helped by the lack of seaworthiness and overcrowding of the boats deployed. Consequently, it has often featured in European news stories that have focused both on numbers of migrants arriving and on the deaths of many at sea. It is also cited in academic papers that focus on detention and subsequent deportations of failed asylum-seekers and unwelcome economic migrants from the island.

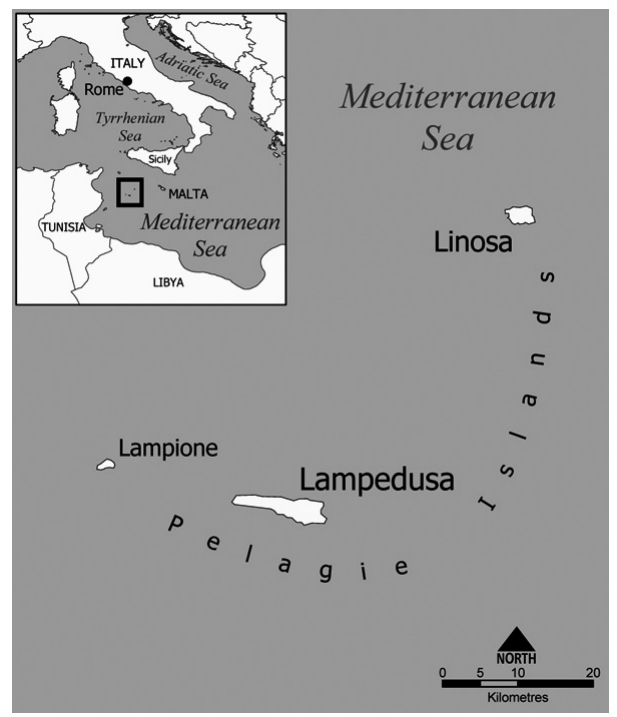

Source: Pelagie Islands map. Licensed under Creative Commons Attribution-Share Alike 3.0 via Wikimedia Commons. Online. Available HTTP: <http://commons.wikimedia.org/wiki/File:Pelagie_Islands_map.png\#mediaviewer/File:Pelagie_Islands_map.png> 


\section{FORCED MIGRATIONS}

In terms of migrant arrivals, between 1999 and 2011 there were over 150,000 landings on Lampedusa, comprising the majority of irregular migrants apprehended at Italy's sea borders overall. Composition of arrivals has been complex. It has fluctuated considerably over time in terms of the balance between asylum-seekers and economic migrants (notwithstanding difficulties defining categories) and between ethnic origins (Egypt, Libya, Tunisia, Horn of Africa, Nigeria), strongly dependent on relational circumstances. At times, the tiny island seemed overwhelmed by the scale of arrivals, whilst also showing considerable humanitarian concern for those involved. Such concern has been prompted, not least, by the high cost in human lives paid by those not making it to Lampedusa alive.

In October 2013 a news story broke that more than 100 people were dead and around 200 missing after an overcrowded boat carrying migrants caught fire and sank just off the Lampedusa coast. This latest tragedy in what Italian President Giorgio Napolitano called a "succession of true slaughters of innocents" saw a final death toll of 366 out of a total of the 518 people, each of whom had paid $\$ 1,600$ to make the crossing from Libya. The tragedy unfolded when, with land in sight, the boat's bilge pump failed and in trying to restart it the Captain started a fire. As waking passengers "scrambled for safety, people stampeded to one side of the overloaded vessel, which listed and began to capsize in a terrible, slow roll. Those below deck stood no chance. Those on top were thrown into the sea, or tried to cling on as the boat went over" (Nelson 2014: np). Subsequently, almost all those who survived left Italy, many seeking asylum in Germany, Norway and Sweden. For example, 18-year-old Fanus from Eritrea avoided being registered as an asylum-seeker in Italy, where she would then have had to stay under Dublin Convention refoulement rules. Instead, she purchased a fake passport and an airline ticket and sought asylum in Sweden.

Finally, mode of transit in general has fed into how forced and other migrants are depicted, not least by the media. For example, there are the now ubiquitous images of border fence scaling or overhead shots of desperate people huddled together in overcrowded boats. Sometimes these depictions evoke sympathy and support but often have the opposite effect: "[b]oats of migrants evok[ing] xenophobia, racialized, well-rehearsed fears and moral panics about the 'other'" (Hyndman and Mountz 2008: 257).

(Sources: Andrijasevic 2006; Ciabarri 2014; Collyer and de Haas 2012; Cuttitta 2014; Ellingwood 2009; Guardian 2013b; Hyndman and Mountz 2008; McMahon 2007; Motlagh 2014; Nelson 2014)

Diverse international migrants, in short, frequently converge when migration as process is focused on. This is recognized by what UNHCR labels mixed migration, where forced and voluntary categories blur and overlap in numerous ways (van Hear 2009) as indicated in Table 9.3. Consequently, as with human trafficking (9.3.1), mode of transit must not be equated with a specific category of migrant. Likewise, clandestine entry may be the only practical way both asylum-seekers and employment migrants can enter a particular country. Yet, under international law, each of the officially-defined migrant categories identified within a mixed migrant group is to be afforded a different type of protection and access to aid. Thus, following arrival at their destination, subsequent fates of a mixed migration group may diverge considerably.

Table 9.3 Four kinds of mixed migrations

\begin{tabular}{ll}
\hline Motivationally mixed & $\begin{array}{l}\text { Reasons for leaving home country may be very diverse, both between migrants and even for an } \\
\text { individual migrant } \\
\text { Mobility mixed }\end{array}$ \\
$\begin{array}{l}\text { Same routes, modes of transport, agents (e.g. people smugglers) used by refugees and employment } \\
\text { migrants }\end{array}$ \\
Geographically mixed & $\begin{array}{l}\text { On acquiring at least asylum-seeker status in one country, migrants may move on to another country, } \\
\text { possibly for more "voluntary" reasons not least related to life course issues }\end{array}$ \\
Historically mixed & $\begin{array}{l}\text { An institutionalized international migration flow shifts in character from being dominated by refugees } \\
\text { to being more characterized by employment migration (or vice-versa) }\end{array}$
\end{tabular}

Source: developed from van Hear (2009: 10). 


\subsubsection{Recognizing forced mobilities}

Besides issues stemming from recognizing the ever-broadening diversity of forced migrations and their entanglement with other migrations, the privileged status of migration within all human forms of mobility again merits critical scrutiny. The present book has sought to position migration within a spectrum of mobilities that includes other forms of movement (5.2.1). Such a perspective is valid here. Even if the idea of forced migration may not always hold as a robust categorization for a migrant, whether due to political (re)definition as "voluntary" or through recognition of the presence of notable human agency within the move, recognition of more everyday aspects of forced mobility can reinforce the sense of overall lack of human agency within mobilities taken as a whole. In other words, by again not limiting the idea of migration to geographical relocation, the idea of forced or determined mobility becomes more conceptually sustainable. However, forced mobilities are also beyond the scope of UNHCR. Within most states across the Global North they remain below the radar until, for example, revealed by media exposés of contemporary slavery. They are, nonetheless, the associates of the forced immobilities introduced in Section 6.2.5. Together, both further qualify any conceptual framing of the present period as a liberal and democratic era of mobilities (5.2.1).

\subsection{FORCED MOBILITIES}

\subsubsection{Human trafficking}

Both highly newsworthy and emotionally distressing, forced relocation of mostly young girls and women for sexual and other forms of extreme exploitation is likely to strike the reader as clear-cut expression of forced migration rather than forced mobility. As such, it may seem incongruous here. However, with the former reading challenged by critical commentators it is argued below that the latter is often a more appropriate depiction of the unsavory mobilities involved. This, in turn, allows links to be made between some migrants involved and some of Chapter 7's supposedly voluntary employment migrants.

Human trafficking involves clandestine movement of migrants, "treated as objects" (Bartram et al. 2014: 80), usually between countries by a "trafficking agent." Subsequent forced employment is then either to repay costs of being smuggled or from (quasi-)enslavement (after Samers 2010: 328). It is defined formally by the UN (2000: Article 3a) as:

recruitment, transportation, transfer, harbouring or receipt of persons, by means of the threat or use of force or other forms of coercion, of abduction, of fraud, of deception, of the abuse of power or of a position of vulnerability or of the giving or receiving of payments or benefits to achieve the consent of a person having control over another person, for the purpose of exploitation. Exploitation shall include, at a minimum, the exploitation of the prostitution of others or other forms of sexual exploitation, forced labour or services, slavery or practices similar to slavery, servitude or the removal of organs.

Exemplifying mixed migration (Box 9.3) by overlapping with the more general clandestine immigration routes of undocumented migrants (Samers 2010; 7.3.3.2), human trafficking is a global phenomenon. According to the United Nations Office on Drugs and Crime (UNDOC), between 2007 and 2010 victims were of 136 different nationalities and it was reported in 118 countries. Women and girls comprise 75 percent of the total number of trafficked individuals, with children comprising 27 percent of victims (UNODC 2012: 7).

Looking at East Asia specifically, Figure 9.2 is based on information compiled from numerous news stories. It indicates some principal trafficking flows across the region. Japan, South Korea and China stand out as key recipient countries, with Thailand, the Philippines and the Russian Federation major sources. Importantly, whilst this map expresses mostly international movements, there is also trafficking within countries, notably China, again blurring any international/internal divide.

Human trafficking is overwhelmingly underpinned by extreme economic exploitation (Scarpa 2008). Drawing on African evidence, Fitzgibbon (2003) identified three types. First, there is trafficking within conflict zones, notably of children, for recruitment to work in mines or to become child soldiers, for example. Uganda's Lord's Resistance Army 
246 FORCED MIGRATIONS

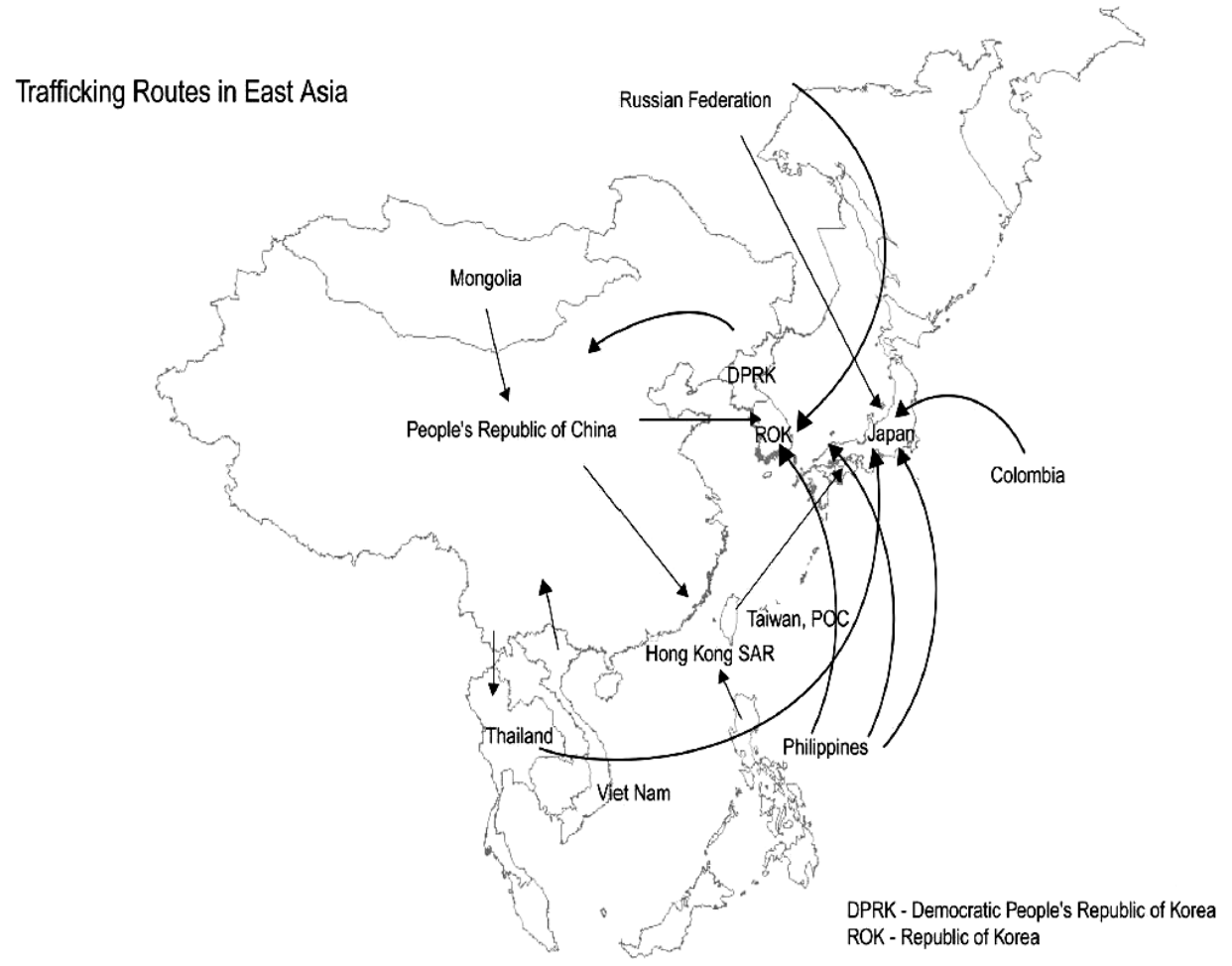

Figure 9.2 Human trafficking in East Asia.

Source: Lee 2005: Figure 1.

has been accused of abducting over 10,000 children for the latter purpose. Second, there is trafficking in forced labor more broadly. Again, this often involves children (also Box 9.1). In Sub-Saharan Africa, trafficking is typically for farm and domestic work, occurring both within and across countries and often involving relocation from rural to urban places (Adepoju 2005). Third, the most pervasive and global form of trafficking is for sexual exploitation. This overwhelmingly involves women and girls. In Africa, trafficking is especially directed towards Europe and the Gulf States, although South Africa also receives trafficked migrants from East Asia and Eastern Europe (Adepoju 2005). Besides these three main types, there is trafficking for forced marriages-often as child brides-and adoption (boys), with both apparent within China and the latter within Nigeria, for example (Guardian 2013a; Lee 2005).

Overall, it is estimated that sex trafficking accounts for nearly 58 percent of all cases globally, while forced labor accounts for 36 percent (UNODC 2012: 7). Geographically, trafficking for sexual exploitation is more common in Europe, Central Asia and the Americas, while Africa, the Middle East, South and East Asia and the Pacific are more commonly associated with forced labor trafficking (UNODC 2012: 7).

In terms of its practice, "[h]uman trafficking can be understood as a process by which people are recruited in their community and exploited by traffickers using deception and/or some form of coercion to lure and control them" (UNODC 2012: 16). Based on evidence of sex trafficking from states of the former USSR, especially Ukraine, Hughes (2000) identified three main recruitment methods. First, advertizing in newspapers offered supposedly lucrative salaries overseas in jobs such as nannies, waitresses, dancers and hostesses. Second, bogus marriage agencies sought mailorder brides for prospective husbands in Western Europe. Third, women were recruited via friends and acquaintances, most of whom had been trafficked themselves previously. This was the main method.

While the magnitude of trafficking is undeniable, research in this field remains challenging (Bartram et al. 2014). Two dimensions are particularly hard to overcome. First, there is identifying those of interest, who are largely hidden: prostitutes, traffickers, victims/survivors and undocumented immigrants. Second, being associated with politically and 
socially stigmatized or illegal behaviors, these groups are both difficult to locate and may be disinclined to cooperate reliably with researchers (Tyldum and Brunovski 2005). Nonetheless, it is not difficult to find individual accounts of sex trafficking in particular.

First, consider these four BBC news headlines:

- 22 May 2012: "Trafficked: Sex slaves seduced and sold" (BBC 2012a);

- 12 October 2012: "Human trafficking to UK 'rising'" (BBC 2012b);

- 18 January 2013: "Twelve arrested in US raid on Latin sex-trafficking ring" (BBC 2013a); and

- 8 January 2013 "The girls stolen from the streets of India" (BBC 2013b).

The stories span the globe-US, UK, Mexico, India-a snapshot of the range of occurrences globally. Second, numerous books tell of the phenomenon, ranging from memoir to academic accounts (Scarpa 2008). Table 9.4 lists a small selection. The following graphically expresses some of the horrors involved:

In November 2005, Gavril Dulghieru, a Moldovan living in London, was convicted for conspiracy to facilitate illegal immigration, misuse of stolen credit cards, forgery, money laundering and conspiracy to traffic for prostitution and sexual exploitation. The women he trafficked worked 20-hour shifts in brothels in Park Lane, Mayfair and Soho, were fed only one meal a day, and charged for the use of cutlery:

"They were forced to have sex with up to 40 men a day for as little as $£ 10$ a time to pay off $£ 20,000$ debts each-the price for which they were 'bought'. They were charged rent, and subjected to fines if they refused anal or unprotected sex or a client was not attracted to them. ... One 23-year-old described how she had to pay £300 per day to live locked in a shared basement and her captors threatened to kill her family. Like many trafficking victims, the computer graduate was lured to Britain by promises of a respectable, well-paid job in a hotel or restaurant but ended up in a brothel: 'I believed they would kill my family,' she told the court. 'I thought I hadn't a way out of this situation. I didn't think I had a life in front of me. I wanted to escape but everything was locked. We were locked up all the time. I was told I need to go with clients and I needed to do sex with them. I felt very bad. The first time I wasn't able to talk afterwards' (Cowan 2005)."

(O'Connell Davidson 2006: 11).

Whilst the latter account and all cited in Table 9.4 emphasize the suffering and abuse typically accompanying human trafficking, there have nonetheless been critical calls to re-think how human trafficking is represented. This has been driven forward most forcefully by feminist thinkers. Critique begins to question the overwhelming emphasis on the migrations' "forced" character. A powerful example comes from Julia O'Connell Davidson (2006, 2010), just quoted depicting the horrendous consequences of being sex trafficked. Whilst not downplaying the appalling experiences of the exploited women, O'Connell Davidson's particular concern is that by equating sex trafficking with "modern slavery," small numbers of "deserving victims" are separated from a mass of women with similar experiences who are consequently labelled "undeserving". " The latter would include those working in the sex industry who have not been trafficked, potentially including non-immigrants. All may well experience horrendously exploitative working conditions (e.g. Anderson and Rogaly 2005). For O'Connell Davidson (2010: 245), therefore, "the idea that 'trafficking' can be clearly marked off from other violations of human and labour rights ... [expresses] a discourse of depoliticization." It is not only the trafficked who are victims, whose everyday work mobilities are forced.

O'Connell Davidson $(2006,2010,2011)$ also took issue with any taken-for-granted depiction of trafficked women as forced migrants, rooted in an over-simple dualism of voluntary/consensual versus involuntary/non-consensual. In short, in all but the most limiting cases, trafficked women are not entirely without agency. From contexts such as recruitment by friends to offers of becoming mail-order brides, hostesses or dancers, many trafficked women clearly have some knowledge they will be engaged with the sex industry at their destination. The critical issue is that most "do not expect the sexual exploitation and violence that awaits them" (Hughes 2000: 636), the confiscation of travel documents or extreme debt bondage (7.5.3.3). All come together in many cases-but certainly not all-to position the trafficked as experiencing everyday forced mobilities (and forced immobilities, such as when imprisoned by employers) rather than forced migrations. 
Table 9.4 Book-length accounts of human trafficking and modern slavery

\begin{tabular}{|c|c|c|}
\hline Author & Date & Title \\
\hline Rutvica Andrijasevic & 2010 & Migration, Agency \& Citizenship in Sex Trafficking \\
\hline Alexis Aronowitz & 2009 & Human Trafficking, Human Misery: the Global Trade in Human Beings \\
\hline Kevin Bales & 1999 & Disposable People: New Slavery in the Global Economy \\
\hline Kevin Bales & 2005 & Understanding Global Slavery: A Reader \\
\hline Kevin Bales \& Ron Soodalter & 2010 & Slave Next Door: Human Trafficking \& Slavery in America Today \\
\hline Karen Beeks \& Delila Amir & 2006 & Trafficking \& the Global Sex Industry \\
\hline Parosha Chandran (ed.) & 2011 & $\begin{array}{l}\text { Human Trafficking Handbook: Recognising Trafficking \& Modern-Day Slavery } \\
\text { in the UK }\end{array}$ \\
\hline Anthony De Stefano & 2007 & The War on Human Trafficking: US Policy Assessed \\
\hline Kathryn Farr & 2004 & Sex Trafficking: The Global Market in Women \& Children \\
\hline Sharon Hendry & 2010 & Radhika's Story: Surviving Human Trafficking \\
\hline Siddharth Kara & 2009 & Sex Trafficking: Inside the Business of Modern Slavery \\
\hline Andrea Parrot \& Nina Cummings & 2008 & Sexual Enslavement of Girls \& Women Worldwide \\
\hline $\begin{array}{l}\text { Kamala Kempadoo, Jyoti Sanghera } \\
\text { \& Bandana Pattanaik (eds.) }\end{array}$ & 2011 & $\begin{array}{l}\text { Trafficking \& Prostitution Reconsidered: New Perspectives on Migration, } \\
\text { Sex Work, \& Human Rights }\end{array}$ \\
\hline Nicholas Kristoff \& Sheryl WuDunn & 2009 & Half the Sky: Turning Oppression into Opportunity for Women Worldwide \\
\hline Rhacel Parrenas & 2011 & Illicit Flirtations: Labor, Migration, \& Sex Trafficking in Tokyo \\
\hline Andrea Parrot \& Nina Cummings & 2008 & Sexual Enslavement of Girls \& Women Worldwide \\
\hline Vidyamali Samarasinghe & 2009 & $\begin{array}{l}\text { Female Sex Trafficking in Asia: The Resilience of Patriarchy in a Changing } \\
\text { World }\end{array}$ \\
\hline Silvia Scarpa & 2008 & Trafficking in Human Beings: Modern Slavery \\
\hline Louise Shelley & 2010 & Human Trafficking: a Global Perspective \\
\hline Benjamin Skinner & 2008 & $\begin{array}{l}\text { A Crime So Monstrous: a Shocking Exposé of Modern-Day Sex Slavery, Human } \\
\text { Trafficking \& Urban Child Markets }\end{array}$ \\
\hline
\end{tabular}

\subsubsection{Twenty-first century slavery}

More unambiguously than human trafficking, but often overlapping with it in the accounts exemplified by Table 9.4, the institution of slavery-human beings bought and sold as commodities for exploitation, who exist solely as others' property-suggests a clear combination of forced migration and forced mobility. For example, forced migration is the only way to describe the "export" of slaves from Africa to the Caribbean and the US-the Middle Passage across the Atlantic-from the sixteenth to the nineteenth centuries. And forced mobilities is the only way to characterize their exploitation on the cotton, sugar or tobacco plantations and elsewhere, as depicted, for example, in Solomon Northup's (1853) recently celebrated account of his 12 years as a slave in the US.

Surely, however, slavery no longer exists in the twenty-first century? The answer, unfortunately, is "yes" (Weissbrodt and Anti-Slavery International 2002). Whilst nowhere is it formally still legal, "slavery endures across the globe ... in ... different guises ... [a] transition ... to an Orwellian doublespeak wherein it is forbidden everywhere, yet practiced nearly everywhere" (Androff 2011: 210). Difficult to pin down exactly, for reasons again to do with defining free and un-free, estimates of present-day slave numbers in the world vary enormously. Androff (2011:216) suggested a range from up to 27 million people claimed by the "advocacy camp," such as Bales (2007), to "just" 800,000 from the "government camp," such as USDOS (2008). Emphasizing this definitional dilemma, whilst the UN estimated up to 20 million people held in bonded labor globally (Androff 2011), how many are "slaves" is uncertain (7.5.3.3). Representing this complexity, Table 9.5 outlines seven expressions of modern slavery (also Weissbrodt and Anti-Slavery International 2002). Additionally, depicting slavery as a form of "management practice" (Crane 2013), Figure 9.3 expresses the wide range of contexts enabling it to flourish today.

As with human trafficking, it is not hard to find accounts of modern slavery, despite the challenge of accessing both slave locations and slaves. Many of the books listed in Table 9.4, for example, cover the often horrific experiences involved. Media stories are numerous. As this chapter was being written, the UK's Guardian newspaper had a front-page exposé on slavery's involvement in providing cheap foodstuffs for Northern supermarkets. The story began thus: 
Table 9.5 Forms of modern slavery

\begin{tabular}{lll}
\hline Type of slavery & Description & Key locations \\
\hline Chattel slavery & Complete ownership of one human being by another & Mauretania, North Africa and Middle East \\
Debt bondage & Enslavement due to debt & South Asia \\
Contract slavery & Coercion into unpaid work after being lured to remote & Extractive industries, fishing, farming, \\
& locations by contracts guaranteeing work & factories, plantations, \\
State slavery & State captures citizens and forces them to work & North Korea, Myanmar \\
Domestic servitude & Women and children forced into domestic work & Households worldwide \\
Religious slavery & Slavery maintained through religious practice & Africa, India, Nepal \\
\hline
\end{tabular}

Source: derived from Androff (2011: 213-215).

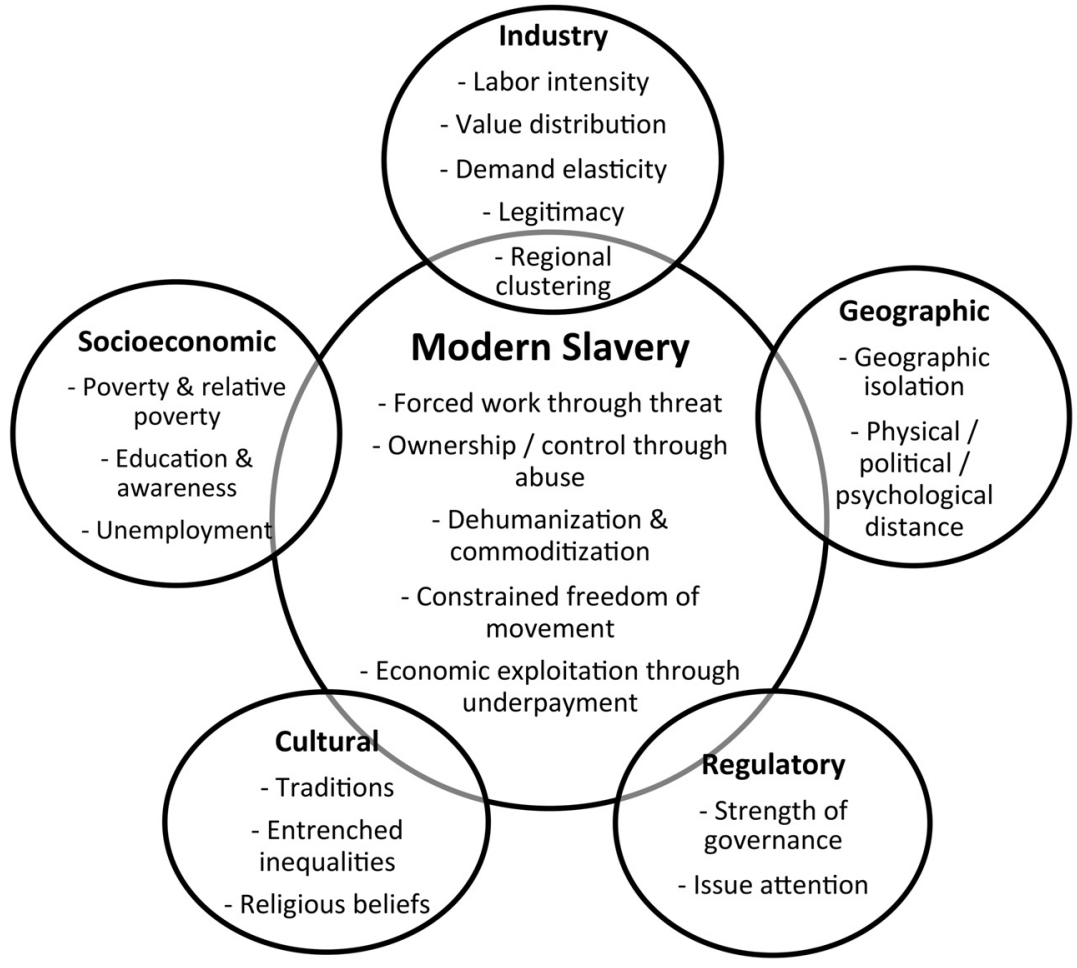

Figure 9.3 Contexts of modern slavery.

Source: substantially simplified from Crane (2013: Figure 1).

Slaves forced to work for no pay for years at a time under threat of extreme violence are being used in Asia in the production of seafood sold by major US, British and other European retailers.

(Guardian 2014d: 1)

It went on to name "the world's largest prawn farmer," Thailand's Charoen Pokphand (CP) Foods, as buying fishmeal for farmed prawns from "suppliers [with] fishing boats manned with slaves." Escaped slaves told journalists of:

20-hour shifts, regular beatings, torture and execution-style killings, [being] at sea for years, [using] methamphetamines to keep them going [and seeing] fellow slaves murdered.

(Guardian 2014d: 1) 


\section{VIEWING POPULATIONS SPATIALLY}

Notwithstanding the horrors of such tales, some critics are again wary of singling out slavery as inherently unique and distinctive as an economic practice within the context of everyday working lives across the globe today. For instance, again turning to O'Connell Davidson (2006: 6), she has argued for "no clear line between [slavery] and 'free' labour." She emphasized both historical and present-day constraints on the latter (O'Connell Davidson 2010), including on its migration and mobility, consequently preferring to stress a "continuum of exploitation" (O'Connell Davidson 2006: 6). Whilst acknowledging how some domestic slaves are brought to UK by "employers" (Craig 2007), O'Connell Davidson (2010) noted how their employment and everyday conditions were not necessarily so different from, for example, some Western expatriates' domestic servants in Bangkok, Thailand (7.5.3.3, Box 7.4). Whilst modern slavery does clearly involve considerable coercion in its migrations, to see it as unambiguously delineating "forced migration" as against default "non-forced migration" by non-slaves is too certain a divide to draw. It is clear, however, that modern slaves' everyday mobilities are, indeed, highly forced.

\subsubsection{Everyday wage "slavery"}

Continuing the O'Connell Davidson critical line, she also argued that within working lives:

economic and/or other impersonal forces can lead people to consent to "severe economic exploitation" and to an employment relationship that entails close restrictions on their freedom of choice and movement.

(O'Connell Davidson 2010: 250-251)

In other words, beyond extreme cases of slavery and human trafficking, there are economic aspects of the everyday life course across the world promoting forced mobilities, if not necessarily forced migrations.

Whilst employment migration (Chapter 7) might generally be regarded as non-forced, the mobilities that attend it may be seen differently. This idea underpins the culturally populist pejorative idea of wage slavery, whereby having to sell one's labor in the capitalist marketplace to survive is seen as analogous with being actually owned by someone else (chattel slavery). Articulated especially by anti-capitalist political currents, US Beat writer Charles Bukowski put it directly if somewhat crudely when he asked how someone could:

enjoy being awakened at 6:30 a.m. ... leap out of bed, dress, force-feed, shit, piss, brush teeth and hair, and fight traffic to get to a place where essentially you made lots of money for somebody else and were asked to be grateful for the opportunity to do so?

(Bukowski 2009 [1978]: 97)

Even if one does not equate working in the capitalist economy with slavery, elements of everyday employment experiences speak of forced mobilities. They are mobilities individuals see little choice but to accept within the internal conversations (Archer 2003) and practices that construct their everyday lives and overall life course. On the one hand, there are the profound, numerous and comprehensive but largely taken-for-granted controls over workers exercised by employers and state (often to the worker's benefit) that certainly extend to mobilities to, from and within the workplace-from the discipline of clocking-in, through being in certain places at certain times, adhering to health and safety constraints, to business travel. On the other hand, the broader economic relational context of working lives also has numerous forced mobility outcomes. This is especially the case with commuting (6.2.2). For example, in the UK the extreme expense of living in London-notably very high house prices-is often poorly compensated by earnings. This encourages migration out of the city, in turn necessitating forced mobility of lengthy commutes to and from employment within the city (Guardian 2014a). From forced mobilities, however, the chapter now turns to situations where geographical relocation becomes imperative, even if the destination and subsequent playing out of the resulting forced migration is more open. 


\subsection{REFUGEES, INTERNALLY DISPLACED PERSONS (IDPS) AND ASYLUM-SEEKERS}

\subsubsection{Scale and causes of present-day forced migration}

\subsubsection{Refugees, IDPs and asylum-seekers globally}

Following the UNHCR (9.2.1), forced migrants fall into three main categories: refugees, internally displaced persons (IDPs) and asylum-seekers. Looking at statistics on these displaced persons, it is easy to get lost amongst the different figures by year, category, and so on. However, at least three important stories are apparent (UNHCR 2015a). First, for most of the twenty-first century the total number of displaced people has fluctuated around the 40 million mark. Second, since 2012 this figure has increased notably, reaching nearly 60 million by the end of 2014. Third, the number of asylum-seekers is dwarfed by the number of officially recognized refugees, which in turn is overshadowed by the number of IDPs. By the end of 2014 the respective figures were 1.8 million (3 percent), 19.5 million (33 percent) and 38.2 million (64 percent). Each of these three groups will be considered in turn.

First, the refugee population has grown steadily over the past half century (UNHCR 2010, 2015a). In 1960 it was 1.7 million globally, growing to 8.5 million in 1980, 12 million in 2000, dipping to just over 10 million in 2009 but rising to nearly 19.5 million by the end of 2014. This overall growth, plus fluctuations, partly expresses changing UN definitions but more strongly reflects relative occurrence of wars and lesser conflicts. Demonstrating this, the top five source countries for refugees in 2014 were engaged in such conflicts: Syria (3.9 million), Afghanistan (2.6 million), Somalia (1.1 million), Sudan (0.7 million) and South Sudan (0.6 million) (UNHCR 2015a: 13-14). The first three countries alone accounted for over half global refugee numbers. Excluding 5 million Palestinians registered with the UN Relief and Works Agency, Asia and Pacific (including Syria and Afghanistan) with 8.7 million refugees and Africa with 4.6 million predominated.

Looking at where refugees are to be found presents a different geographical impression. Figure 9.4 shows the picture for 2010, highlighting countries adjacent to conflict zones, such as Ethiopia and Jordan, and countries receiving those relocating further, such as the US, Canada, Sweden and Germany. Overall, however, four out of five refugees are found in the Global South, in spite of the North "gripped by panic about asylum since the 1980s" (Castles et al. 2014: 224). This is reinforced by the six countries hosting nearly half the global refugee total at the end of 2014: Turkey (1.6 million), Pakistan (1.5 million), Lebanon (1.2 million), Iran (1.0 million), Ethiopia (0.7 million) and Jordan (0.7 million) (UNHCR 2015a: 12). Each provides varying degrees of safety.

Second, whilst refugees and asylum-seekers grab headlines across the Global North, they are far outnumbered globally by internally displaced people (IDPS), those involuntarily displaced to a secondary location within their home country (Hampton 1998). IDPs in 2010 are mapped in Figure 9.5 and are seen to be still more concentrated into a small number of Southern countries than refugees. At the end of 2014 , of 38.2 million IDPs worldwide, largest numbers were located in Syria (7.6 million), Colombia (6 million), Iraq (3.6 million), Democratic Republic of the Congo (2.8 million) and Ukraine (0.8 million) (UNHCR 2015a: 23-5). This reinforces the point that forced migration is certainly not just international South-to-North migration but is primarily experienced within the South.

Like refugees, IDPs have been forced to leave their homes and livelihoods but, unlike refugees, have not crossed an international border and are therefore not protected in the same way under international law (UNHCR 2006: 153). For example, during a civil war IDPs may become displaced because their race, ethnicity, religion or political ideology differs from one of the warring groups. They "may be trapped between opposing sides ... or come under direct attack by insurgents.... Competition over scarce resources or land often aggravates such conflicts, with the displaced bearing the brunt of the violence. When states disintegrate into anarchy ... some of the worst atrocities have been inflicted on the internally displaced" (UNHCR 2006: 156). IDPs leave behind access to food, housing and land, and are often involuntarily separated from families and communities.

Third, asylum-seekers are individuals claiming sanctuary in another country. They represent an intermediate or liminal group, displaced from their home countries but not (yet) officially recognized as refugees. Geographically, it is instructive to compare the origin countries of asylum-seekers with the countries where asylum is claimed. Figure 9.6 shows using 2010 data that the former are predominantly in the Global South and associated with armed conflict. 


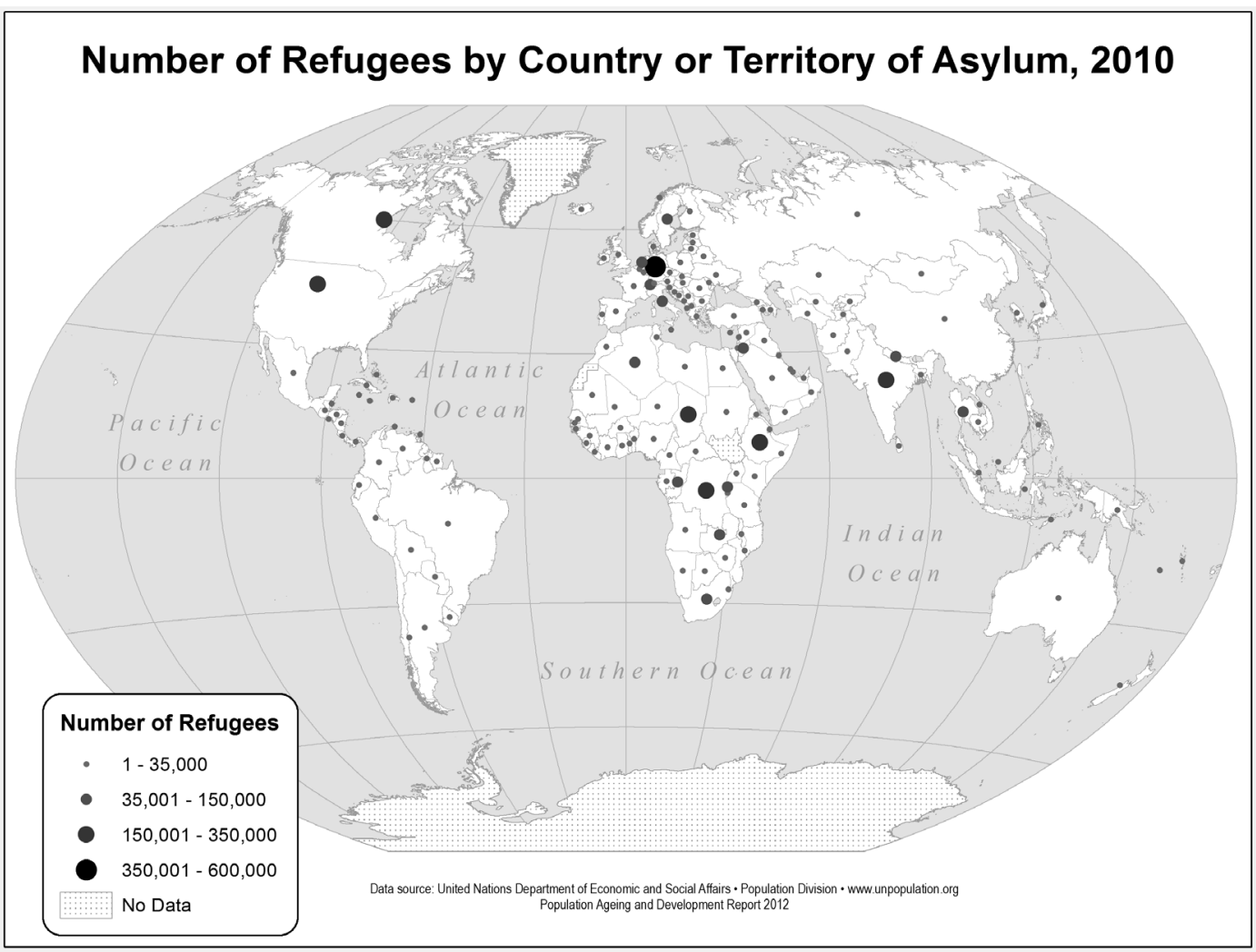

Figure 9.4 Number of refugees by country or territory of asylum, 2010.

Source: United Nations Department of Economic and Social Affairs, Population Division, Population Aging and Development Report, 2012.

Of 1.47 million asylum applicants in 2014 , only three countries sourced over 100,000 claimants: Ukraine (0.3 million), Syria (0.2 million) and Iraq (0.1 million) (UNHCR 2015a: 30-31).

Turning to countries where asylum is sought, Figure 9.7 shows these, in contrast, more dominated by the Global North, especially Europe. This impression is reinforced by the top five for 2014: Russian Federation, Germany, US, Turkey and Sweden. In other words, asylum-seekers frequently favor the same countries as international employment migrants (7.3.3.2), an insight reinforcing the perception and potential for mixed migration overlap between the two types of migrant flow. It is only with asylum-seekers, however, whose numbers are dwarfed globally by refugees and IDPs, that the Global North has a predominant presence. This once again contextualizes the North's present-day overall and overwhelming "exclusionary regime[s], designed to keep out asylum seekers from the South" (Castles et al. 2014: 227).

\subsubsection{Causes of forced migration today}

While the multitude of factors displacing refugees, IDPs and asylum-seekers are often unique to geographic locations, populations and political situations, scholars have developed models or typologies to synthesize them. Kunz (1973, 1981) proposed forced migrants grouped into anticipatory flows, in which the displaced leave as conditions deteriorate but before being forced out, and acute flows, in which groups flee immediate dangers.

More recently, Wood (1994), within a broader model of forced migration, recognized three overlapping spheres prompting forcible displacement. Once again, he emphasized the challenge of labeling people within any of these spheres conclusively "forced" or "voluntary." His three categories were: war, political instability and persecution ("conditions usually blamed for causing refugees"); ethnic, religious or tribal conflicts ("conditions that give rise to intense territorial and nationalistic emotions, intolerance of foreigners and ethnic cleansing"); and ecological crises/life 


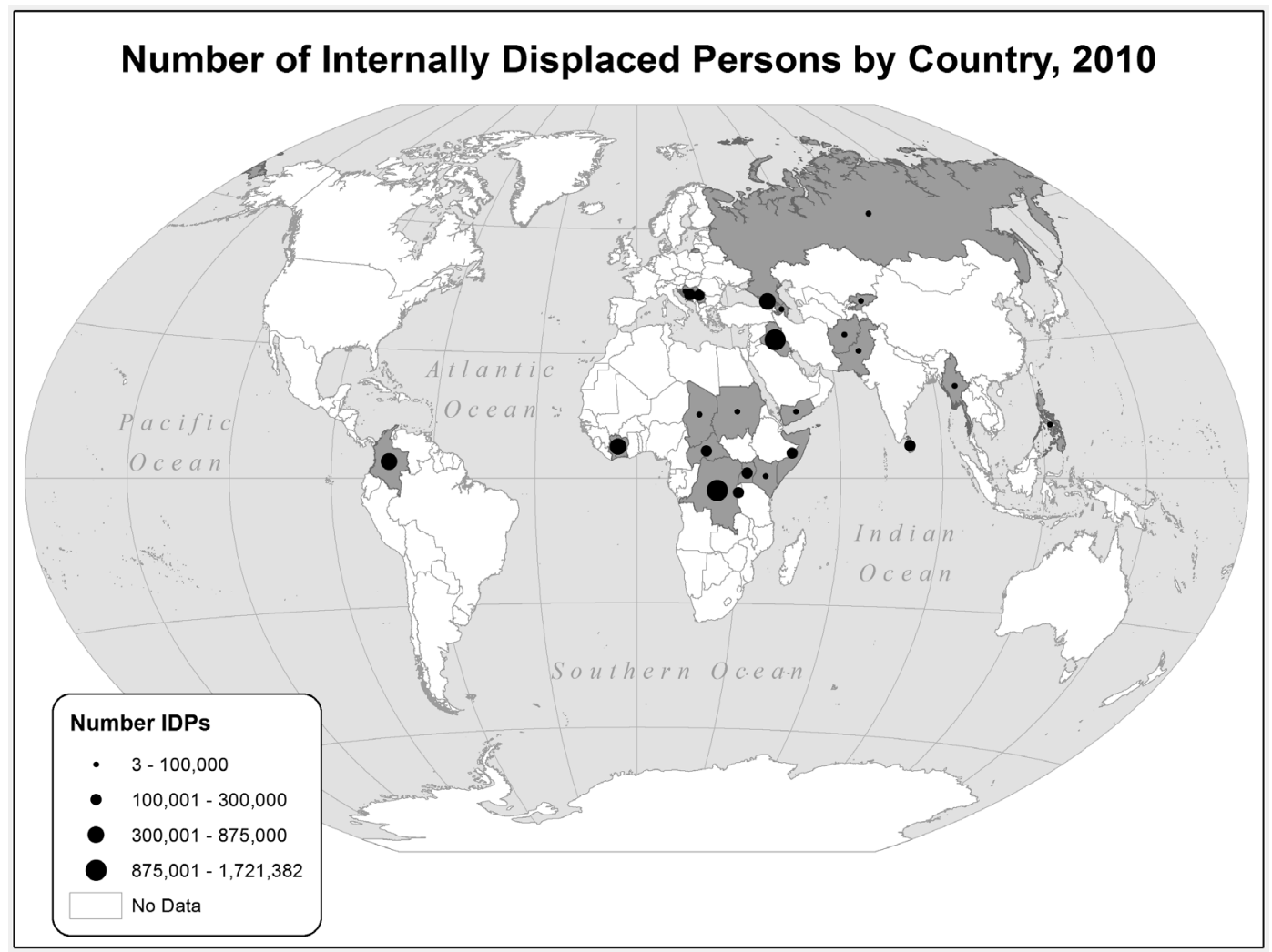

Figure 9.5 Number of IDPs by country, 2010.

Source: UNHCR Global Trends, 2011.

threatening economic decline ("conditions usually blamed for causing international 'economic' migrants"). It is a robust classification. Almost 20 years on, one can easily identify national and regional crises creating forced migrants in each category (e.g. Castles et al. 2014; Moorehead 2005; van Hear and McDowell 2006; Whittaker 2006).

First, populations displaced because of violent conflict remain an immediate and preeminent expression of forced migrations. Wars, both civil and cross-national, regularly prompt sudden and huge migration flows. The ongoing cases of Afghanistan, Democratic Republic of Congo, Iraq and Syria all too clearly bear this out (e.g. Becker 2013; Refugees International 2003; Sassoon 2008). There is space here to consider one example.

The Syrian conflict, begun in 2011, soon developed into what at the time of writing (July 2015) had become a largely stalemated civil war. Estimates placed well over 4 million persons as having fled the country from around 9 million displaced overall (UNHCR 2014, 2015a). The latter figure is equivalent to half the country's population. In terms of geography, almost all refugees moved to immediately neighboring countries (UNHCR 2014, 2015b)-as they have from continuing conflicts in Afghanistan and Iraq (Romano 2005; Sassoon 2008). By late 2015, Lebanon accommodated 1.1 million Syrian refugees, equivalent to a quarter of its own population, prompting worries about the impact of such huge absolute and relative numbers on social and political stability. Large numbers were also located in Jordan (0.6 million), Iraq (0.2 million) and Egypt (0.1 million). However, Turkey saw refugee numbers soar to 2.2 million through 2015, as the situation in Syria deteriorated. Moreover, whilst prior to 2015 few Syrian refugees made it further afield, Northern countries generally very reluctant to take them, this all changed when waves of desperate Syrian and other migrants-mixed migration again-began to move into Europe, in particular heading for Germany and Sweden to seek asylum. As this huge migration of desperate people continued, countries in Europe hastily reintroduced border controls, toughened security and built fences to try and keep them out and to respond to estimates that up to 3 million more could arrive in Europe seeking sanctuary or jobs by 2017 (Cook and D'Emilio 2015). 


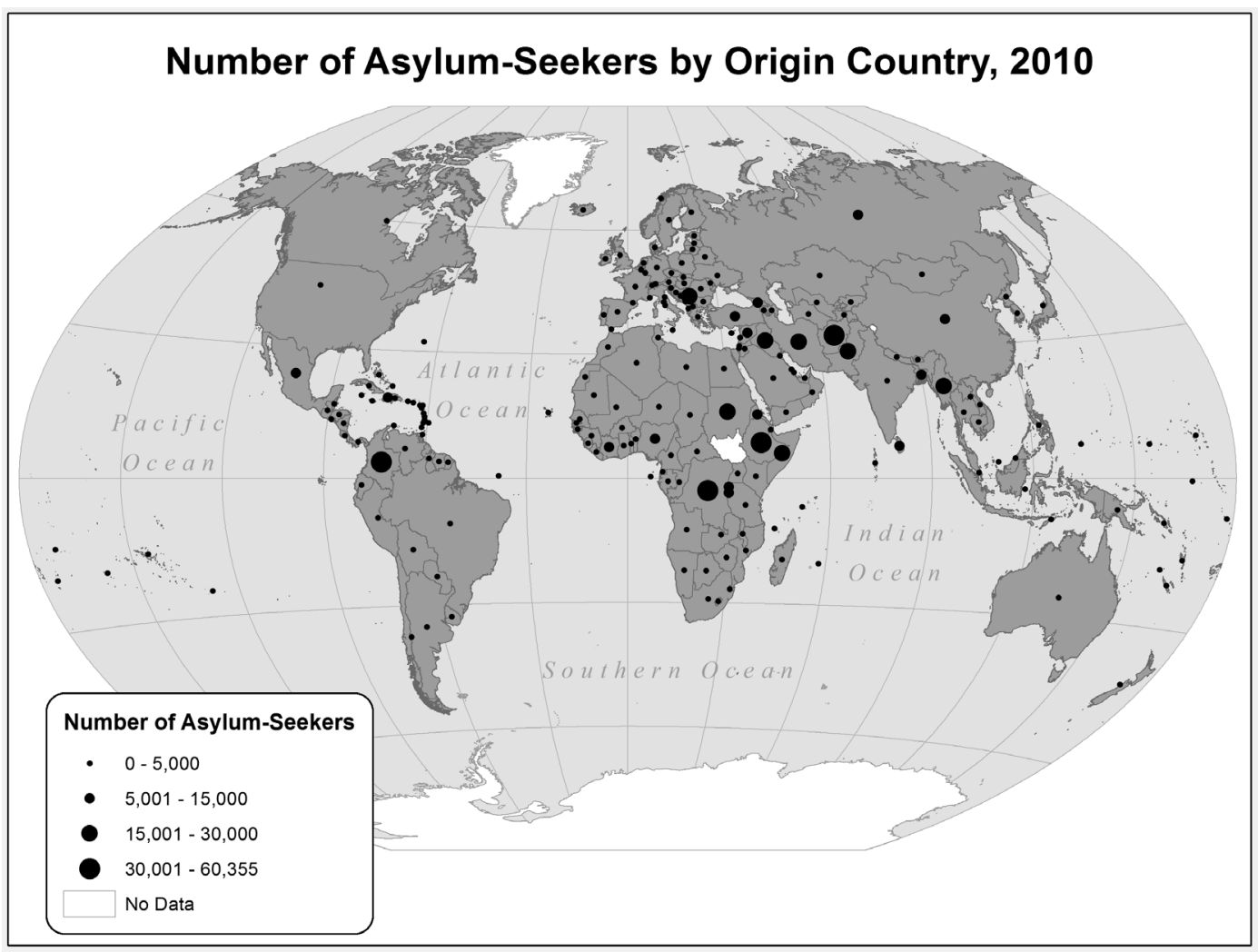

Figure 9.6 Number of asylum-seekers by origin country, 2010.

Source: UNHCR Global Trends, 2011.

Besides these striking numbers and the distinct geographies they are producing, numerous distressing stories of the Syrian refugee experience have emerged through the media and beyond. News stories reported Syrians perishing or barely surviving trying to cross the Mediterranean to reach Europe in overcrowded and unseaworthy boats (e.g. Al Jazeera 2015; Telegraph 2015; also Box 9.3). Elsewhere, within the medical journal The Lancet alone, stories have included: reports of poor support for refugees, especially women, who have experienced sexual violence in the war (Ouyang 2013); appeals by the Jordanian Minister of Health for help to tackle the challenge of providing basic refugee services, especially for the majority living outside refugee camps (Murshidi et al. 2013); more general calls to support the needs of women and girls (Sami et al. 2014); and highlighting refugee health needs broadly (Dewachi et al. 2014). Further research elsewhere considered, for example, potential impacts large refugee numbers have on destinations such as Lebanon and Jordan (e.g. Becker 2013), whilst press reported young refugee girls being married off to provide them a degree of safety from rape and kidnap (Guardian 2014e).

Forced migration from war overlaps with displacements caused by ethnic, religious and tribal conflict, including those recent or ongoing in Central African Republic, Myanmar, Nigeria, Rwanda and South Sudan. Often more individually expressed "intolerance" can also be expanded from Wood's model to include forced migration due to other expressions of prejudice, such as political repression and homophobia (6.2.5).

As an example, consider the plight of the Rohingya from Myanmar (formerly Burma). This ethnic group is associated with Arakan, the historic name of the region bordering Bangladesh to the north and known officially since 1989 as Rakhine State. The Rohingya's Sunni Muslim religion and mixed ethnic ancestry have long been regarded negatively by Myanmar's Buddhist majority. The consequence has been repeated persecution, discrimination and exploitation since Arakan was invaded by Burma in 1784 (Cheung 2011; Kiragu et al. 2011). As 


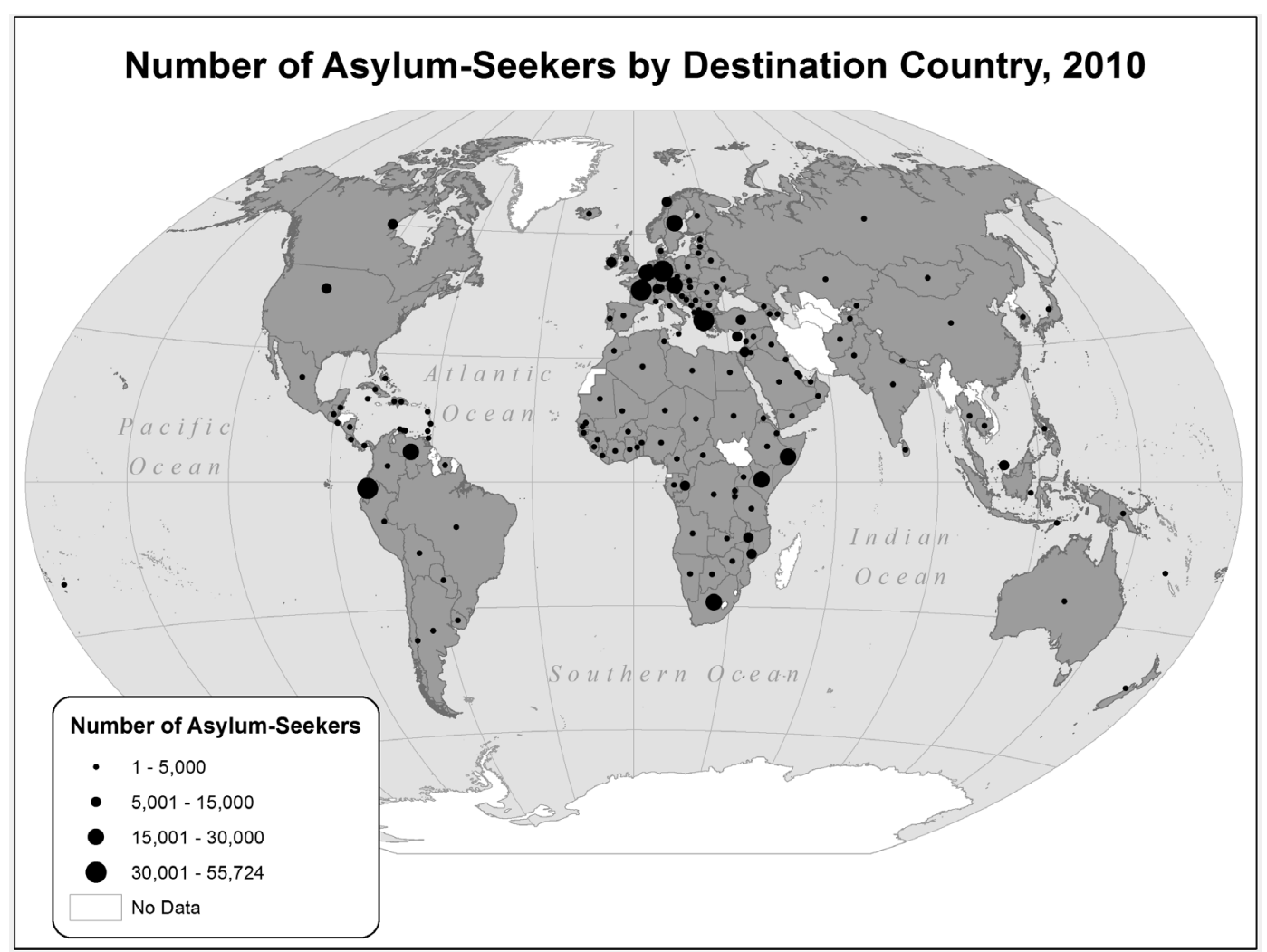

Figure 9.7 Number of asylum-seekers by destination country, 2010.

Source: UNHCR Global Trends, 2011.

a result, Rohingya have been excluded from Myanmar citizenship since independence in 1947, depicted as illegal Bengali immigrants, and now experience severe restrictions on marriage, employment, health care and education (Motlagh 2014).

Simmering tensions between the Rohingya and other Rakhine residents flared up in June 2012, following the rape of a Rakhine woman. Over a hundred lives were lost, thousands of homes destroyed and tens of thousands displaced (Kipgen 2013). Both sides suffered but the Rohingya most especially, resulting in both internal displacement and refugee exodus. On the one hand, an estimated 750,000-1.3 million Rohingya resident in Myanmar (Kiragu et al. 2011; Motlagh 2014) were by 2014 "confined to a wasteland of camps, ringed by armed guards" (Motlagh 2014). A humanitarian crisis, described as a "crime against humanity" by advocacy groups, had a devastating impact. For example, there was a blockade on critical aid to over 100,000 Rohnigya around the city of Sittwe (Motlagh 2014). On the other hand, over a million Rohingya fled Myanmar and became refugees. Around 400,000 went to the Gulf States, 200,000 to Pakistan, 20,000 to Thailand and 15,000 to Malaysia but the 400,000 in Bangladesh stood out (Kiragu et al. 2011). The vast majority of these were not resident in refugee camps, especially those administered by UNHCR, but lived undocumented in the wider community. Although common adherence to Islam promoted toleration by the Bangladeshis, the country was poorly set-up to help, not least in refugee areas, had high population growth and was highly prone to natural disasters and impacts of climate change (Kiragu et al. 2011). However, and despite Bangladesh not being a signatory of the 1951 Convention or 1967 Protocol, Cheung (2011: 66) observed that Rohingya refugees were secure from refoulement and demonstrated "capacity to carve out their own protection space and achieve a level of de facto integration, even in the face of punitive immigration enforcement measures and sometimes beyond what international actors or the formal refugee frameworks can offer." 


\section{FORCED MIGRATIONS}

Almost equally prominent today to the previous two categories of forced migrant are flows originating from more social-environmental causes and expressing various strands of forced economic migration. Typical are migratory responses to devastating famines and droughts, ongoing in Sub-Saharan Africa (IDMC 2014), but also recent massive population relocations due to environmental catastrophes, such as Thailand's 2004 tsunami, the 2005 Hurricane Katrina in the US, and Japan's 2011 earthquake. Additionally, development projects displace populations, including building large dams for hydroelectric power and flood control, such as China's Three Gorges project (Box 9.4), India's Sardar Sarovar scheme or Botswana's Southern Okavango Integrated Water Development initiative (Scudder 2005). Population pressures in a concentrated or primary settlement might also prompt governments to create new settlements to relieve pressure. Using the cases of Bengali settlement in the Chittagong Hill Tracts and Javanese settlement of western New Guinea, Penz (1992) described displacement of indigenous people. Large groups of forced migrants resulted from "state-sponsored colonization of territories of indigenous people by settlers from the overpopulated heartlands of [Bangladesh and Indonesia]" (Penz 1992: 106).

Forced migrations stemming from these diverse socio-environmental causes are especially hard to estimate. And this does not really begin to cover forced economic migrations. Those affected are not considered for official refugee status under the 1951 Convention and the UN is unable to keep count of total numbers (UNHCR 2011). Nonetheless, they exhibit quite a marked global geography, whereby what Perez (2010: 32) rather charitably labeled "[a]ccidents of geography" lead, for example, to:

countries least able to prevent climate change ... becom[ing] the most vulnerable to its earliest effects... [Their] populations-often the poorest of the world's poor-are more likely to occupy dangerous locations, such as coast lines, flood plains, steep slopes, and settlements of flimsy shanty homes.

Even within rich countries, the poorest are affected disproportionately by socio-environmental forced migration. Consider the impact of Katrina, a Category 1 hurricane which made US landfall in August 2005. It brought about storm surges which destroyed many coastal settlements in Louisiana and Mississippi and inundated 80 percent of New Orleans. The city was especially vulnerable due to both geographical location and human causes. The latter included intensive use of the Mississippi river, removing soil that kept the area above sea level, and clearing and draining barriers to flooding such as coastal marshes. The consequence was 1 million IDPs who relocated inland to escape the devastation. New Orleans's city population plummeted from nearly 0.5 million to around 93,000 just after the storm (Brown 2006; Reuveny 2008).

The fate of Katrina's IDPs has been widely studied, not least the slow return that saw New Orleans's population still only at 0.2 million in mid 2006. In an examination of the determinants of return migration, Groen and Polivka (2010) noted how, in terms of race, whites returned more than blacks, which they attributed largely to the neighborhood patterns of storm damage to the city. An increased presence of Hispanics was also observed, labor migrants responding to the demands of clean-up and reconstruction (Campanella 2006). For non-returnees, attention has been given to resettlement areas, such as parts of Texas, and to how well IDPs assimilated (e.g. Reuveny 2008). Other studies concerned themselves with the resilience of New Orleans (e.g. Campanella 2006), and problems of IDPs' posttraumatic stress disorder (PTSD) and of returnees' obtaining new property insurance (Brown 2006).

Katrina's IDPs are generally regarded in the literature as exemplifying climate or climate-change induced forced migration (Black et al. 2011). Celebrated environmentalist Lester Brown (2006) went so far as to term them elimate refugees, However, in a similar critique to that f $_{\mathbf{L}} \mathrm{O}^{\prime}$ Connell Davidson regarding sex trafficked women and presentday slaves (9.3), Masquelier (2006) took issue with the latter depiction. She noted, first, how many US commentators were uneasy with using a term which challenged both the US's self-image and the "correct" position of refugees within the Global South. For Masquelier, however, the term was problematic because it became a racialized discourse separating out poor African-American victims as a unique group, a discourse that often also condemned them for their experience. As she put it, "refugee became the 'R-word': an ethnic slur that . . f functioned to exclude them from the rest of the United States" (Masquelier 2006: 742). This example of the fractioning of "refugees" distracted attention from more directly confronting widespread experiences of poverty and disempowerment in the US today that are certainly not confined to those forced to evacuate New Orleans. Indeed, "the poor of New Orleans had been 'left behind' long before the storm hit the city" (Masquelier 2006: 736). 


\section{BOX 9.4 POPULATION DISPLACEMENT BY THE THREE GORGES DAM IN CHINA}

Examples of large scale river dams, built for flood control, irrigation, urban water supply, recreation and generating power, can be found across the globe. From the Itaparica Dam in Brazil, to the Aswan Dam in Egypt, to the Kainji Dam in Nigeria, such structures are, however, the source of much controversy. They bring not only the positive benefits listed above but often also displace and require resettlement of large numbers of people.

In 1994, amongst heated debates surrounding the decision, China began constructing the world's largest dam-the Three Gorges Dam on the Yangtze River. Standing 185m high and stretching 2300m wide, it was finally completed in 2006. Built to help control devastating floods and to generate electricity for China's rapidly growing economy, the project attracted considerable criticism for extensive ecological and environmental damage, potential species extinction, and for the thousands of acres of prime agricultural land covered by the rising waters. News media, academics and international policy and governance groups alike have thus written of the impacts of the Three Gorges Dam project. One further key issue is its displacement of many people.

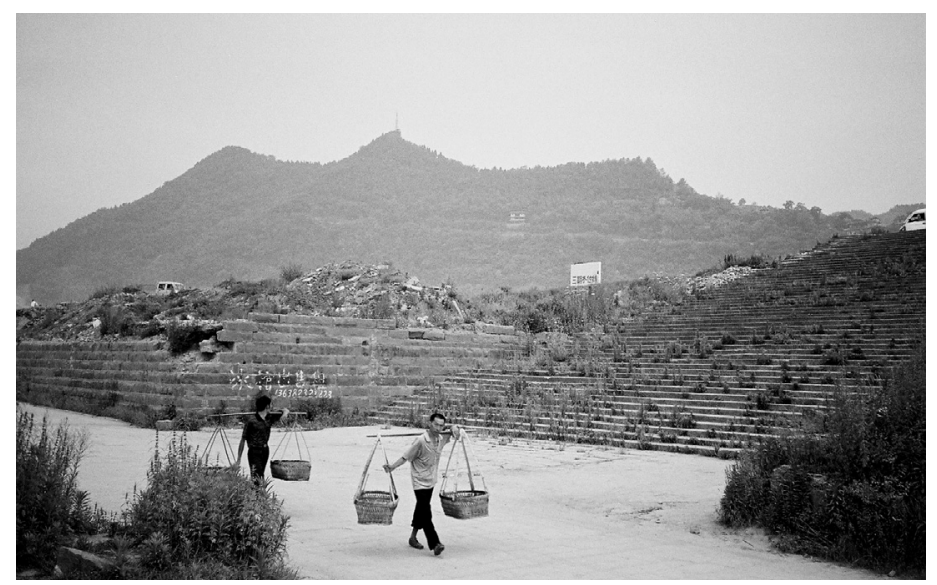

Former inhabitants walk through a town demolished in anticipation of rising water levels.

Source: Barcus (2006).

Displacement and resettlement of local populations during the building of large dams is common but the sheer numbers displaced by the Three Gorges project was unprecedented. Estimates range from 1.2 to 1.4 million people, from 13 cities, 140 towns and 1,350 villages. UNHCR Human Rights Watch wrote "the million-plus population of the Three Gorges Dam area is to be subjected to forced and involuntary relocation to areas that are, for the most part, infertile and already overpopulated hill-slopes high above the proposed reservoir waterlevel" (UNHCR 1995: 8). The largest portion of the population to be resettled comprised farmers where relocation to higher lands or alternative villages meant existing agricultural land lost and inadequate compensation.

Using a "developmental relocation" policy, the Chinese state provided local government bodies with compensation funds for investment in resettlement zones rather than compensating individual families. This was a strategy UNHCR Human Rights Watch claimed "falls short of acceptable standards, both monetarily and in terms of transparency and accountability" (UNHCR 1995: 9), leaving families unable to rebuild lives to preresettlement well-being levels.

An in-depth study of two townships in Yunyang County found that the twin impacts of the Three Gorges and an ongoing conservation project increased population pressure. Floodwaters covered the most productive farmlands and conservation efforts towards reforestation reduced farmlands on steep slopes. Out-migration of labor offset some pressure but the study concluded that the projects "reduced carrying capacity and brought economic hardship in impoverished areas" (Jim et al. 2010: 243).

(Sources: Handwerk 2006; International Rivers 2009; Jim et al. 2010; Scudder 2005; Sutton 2004; Topping 1995; UNHCR 1995; Wu et al. 2004; Yardley 2007) 


\subsubsection{From arrival to durable solutions: processes of forced migration}

\subsubsection{Forced migration as process}

In conceptualizing forced migration, one can learn from Section 9.3's recognition that for human trafficking and modern slavery the elements of coerced movement are not just-and, arguably, often not principally-concerned with the decision to relocate but come through at least as strongly when relocation has taken place. This emphasis is in line with more processual, lived or biographical understanding of migration (5.6.2). Forced migration of refugees, asylumseekers and IDPs can thus be viewed not just as involving relocation but encompassing displacement and initial flight, arrival at safe(r) location, and resettlement (Boyle et al. 1998: 191-194; Moorehead 2005): antecedence-uprootingtransition-resettlement (Fullilove 2014: 143).

As explained above, forced migration encompasses a wide range of relocations: international refugees and asylumseekers, internal IDPs and others of concern to UNHCR, including stateless persons (Table 9.1). Regardless of the label, all share in common experiences of displacement and need to resettle somewhere safer. While those remaining behind must often cope with discrimination, economic uncertainly, environmental degradation and/or political instability, those leaving typically become dependent on international aid agencies and host countries (Wood 1994: 615). Yet, whilst human agency is compromised in all situations, it is still apparent. This is also the case within resettlement, which also takes different forms. Therefore, the next two sections consider two broad categories that describe the process of forced migration subsequent to initial causes of flight: arrival at a safe location/initial resettlement; and later resettlement.

\subsubsection{Arrival at safe(r) location: camps, urban areas and the case of IDPs}

First priority for a forced migrant once they have fled is to find a relatively safe destination. This in itself can be very difficult. On the one hand, information concerning possible destinations (5.3.3) may be very partial, inaccurate, outof-date and even non-existent. This inadequacy plus the sheer need simply to get away can lead to migration to places as dangerous as those left behind. For example, Moorehead (2005: 169-170) told how Guinea had seemed a place of safety from various West African conflicts, notably in Sierra Leone and Liberia, but was attacked from 2000 by disparate forces from such countries, who accused Guinea of harboring enemies. On top of this, Guinea's President showed no support, describing refugees in his country as "little better than cockroaches," prompting Guineans to attack and harass them.

On the other hand, the mode of transit taken by the forced migrant may itself constrain choice of destination (Box 9.3). This can present acute dangers and reduced migrant agency. For example, increased reliance on people smugglers due to heightened border controls and ever-stricter immigration policies (Castles et al. 2014) not only makes those fleeing intolerable living conditions prone to becoming trafficked (9.3.1) but practically means choice of destination is largely left to routes established by smugglers.

Nonetheless, assuming a degree of both knowledge and agency in the relocation process, once in flight migrants must decide where to go. Do they stay within their home country or cross international borders? The former will become IDPs, the latter asylum-seekers and eventually refugees if the destination country grants this status. Besides whether to cross national borders, forced migrants also must decide from further settlement "options." If available, they might settle collectively in designated encampments serviced by international aid agencies, such as UNHCR; they may be more dispersed, either relocating to smaller towns and villages, typically along a national border; or they may make their way to urban settlements. Options available are specific to particular conflicts but offer different advantages and disadvantages.

As noted earlier, while the iconic image of refugees is a giant tent city, the reality is that only about one-in-three of the displaced population globally lives in this settlement type. Refugee camps take various forms, ranging from small informal settlements to much larger encampments housing thousands. The type of camp depends in part on the policies of the host country, which can vary over time. For example, in the 1990s the Thai government evolved its policy from one favoring a large number of dispersed small encampments along its border, which resembled and operated as small villages, to a policy of fewer and larger encampments that concentrated refugees (Bowles 1998). This shift in policy reflected the changing nature of the conflict in Myanmar, from where refugees were fleeing, and the Thai government's desire both to contain the refugees and provide a level of protection. 
Encampments, in general, offer aid agencies a cost effective way to administer food aid, provide protection and deliver health and education programs. Among the challenges, however, are overcrowding, social and organizational issues and potential environmental problems resulting from high population densities (Black 1998). These challenges have drawn criticism of encampments as policy. Black (1998), however, contended that settlement type should not be over-emphasized. It is policies that promote participation by both refugees and local hosts in project design and implementation that are key to success, regardless of settlement type.

In contrast to refugee camps, most of the recently displaced migrate to and settle within urban areas. Their settlement tends to assume a more distributed pattern. Such migrants face a different set of challenges than those in encampments. For example, service provision, whether education, health care or food aid, is more difficult to distribute to a population that might be largely hidden and hard to identify in an urban area. Zetter and Deikun (2010: 5) added overcrowding, poor living conditions and lack of clean water, inadequate sanitation and high levels of food insecurity to the refugees' list of challenges. For host communities, there may be additional burdens. Tibaijuka (2010: 5) argued that urban refugees may "exacerbat[e] the vulnerability of the already resident urban poor" through increasing competition for resources, potentially heightening social tensions in the new location. There was indication of this with the Rohingya in Bangladesh (9.4.1.2). Echoing these concerns and similar to Black's earlier point, Goodall (2011) wrote that relationships between hosts and guests are influenced by discourses that surround their arrival. Negative or hostile discourses can undermine integration and resettlement efforts (also Finney and Robinson 2008). In counterpoint, Grabska (2006) argued for refugees being seen as positive social agents rather than economic burdens for host communities. Using Sudanese refugees in Cairo as an example, she suggested that despite being "socially, economically, culturally, and politically marginalized, refugees participate and contribute to the transformation of urban spaces" (Grabska 2006: 287). Likewise, refugees located in Global Northern cities, for example, can stimulate development of diverse culinary and cultural provisions (Zukin 1996).

Finally, women arriving at supposedly safe locations must often not only face up to the trauma of exile but additional challenges stemming from displacement, including increased vulnerability to sexual abuse and violence (experienced by Syrian refugees; 9.4.1.2) and often increased workloads as they assume a new status within a family as both breadwinner and caretaker of often small children and the elderly (Gururaja 2000: 13). Yet, while taking on these new roles, women often simultaneously face severe restrictions on access to resources, limited education or training, and minimal participation in decision-making. This question of highly constrained agency has sparked debate on the importance of gender within displaced groups. For example, El-Bushra (2000: 4) argued that instead of focusing exclusively on the role of women and implications of displacement, scholars should also ask "where men fit," suggesting a more comprehensive perspective that incorporates the "web of relationships," inclusive of power and powerlessness, and young and old dimensions, between the sexes.

\subsubsection{Moving on: subsequent resettlement and durable solutions}

No one, in the end, wants to be a refugee. Exile is an unhappy state. Refugees seldom want to leave home, and ... they dream of the day they can return. The best "durable solution" ... is to go home ... if that is not possible, the next best option is resettlement.

(Moorehead 2005: 290-291)

First stops for refugees, whether encampments or locations within local communities or urban areas, are not necessarily long-term settlement options. While providing a degree of immediate safety, they may be highly unsuitable for long-term residence. Initial flight from a dangerous situation can result in family members left behind or lost en route, and these must now be located. Furthermore, within the initial sanctuary there may be long-term questions about sustained access to food, drink and shelter, and then to education, employment, and so on. First stops, therefore, are quite often seen as "temporary."

Long-term settlement solutions are as vital for IDPs as for refugees and asylum-seekers. However, their fate has again been somewhat neglected within scholarship and political practice. In contrast, refugees fall under UNHCR and its policies can have major influence on resettlement practices and patterns. The UN and other advocates have outlined several strategies or policies by which countries can address the long-term settlement of refugees that would enable them to live more "normal" everyday lives. These are referred to as durable solutions. Initially comprising three 


\section{FORCED MIGRATIONS}

strategies-local integration, third country resettlement and repatriation-a fourth option, in line with present prominent political attitudes towards forced migrants, has become increasingly popular across the Global North-containment and exclusion (Boyle et al. 1998, Robinson and Coleman 2000). Each durable solution merits considering, asylumseekers also featuring in the latter half of the discussion. However, first, a non-durable solution but all too commonplace occurrence must be noted.

In spite of initial intention, refugees often remain in camps for lengthy periods. These are termed protracted refugee situations by UNHCR. At the end of 2011 they involved over 7 million refugees in 26 countries (Castles et al. 2014: 229-230). For example, of nearly 5 million Palestinian refugees (Castles et al. 2014: 183), 3 million lived in 2006 in around 59 refugee camps maintained by the UNHCR (Whittaker 2006: 73). Whole lives are lived in these camps, which may come to resemble mini-cities but are still, at the end of the day, temporary residential sites. For many refugees, in other words-and asylum-seekers (discussed below)-place of initial safety comes to assume more than a temporary, transient liminal site between two places of more sedentarist settlement. Indeed, it is from fear of such permanent temporariness (sic.) that the governments of Jordan, Lebanon and Syria encouraged Iraqi refugees to return home in the early 2000s (Sassoon 2008).

Returning to durable solutions, the idea behind the first, local integration or local settlement, is that the forced migrant is "here to stay." S/he thus assumes the status of a "permanent" immigrant, even if not necessarily assuming complete naturalization or assimilation (Crisp 2004). Under this scenario, once the migrant (and his/her family) has crossed an international border to a neighboring country, s/he will be re-settled or self-settle in this same country.

Integration into a new community is, however, often very complex and achieving "full integration" difficult to determine or measure. Moreover, given the highly uneven geography of refugees (Figure 9.4), high concentrations pose a major challenge for local integration. Thus, whilst Africa historically showed high rates of integration (Boyle et al. 1998: 195), ever-increasing numbers tax severely Kliot's (1987: 115) earlier depiction of "African refugees ... seen as fellow men and the local population, most likely kinfolk ... usually friendly and generous." Nevertheless, a degree of local integration almost always occurs within every refugee situation, whether or not officially sanctioned.

In contrast to local integration, the years immediately after World War II saw third country resettlement increasingly embraced by European and other Northern countries such as the US, Canada and Australia. The idea was to resettle refugees permanently in countries other than those to which they first fled. The increasing challenge was to find resettlement not just for relatively small numbers of European and Russian refugees but for those displaced from and within the Global South, particularly Africa and Asia during the 1960s and 1970s. Thus, beginning in the late 1970s, third country resettlement policies developed and are reflected in Figure 9.4's geography. As of 2014, 27 countries are counted among the resettlement base of UNHCR, numbers of resettled running at nearly 1 million per decade since 1995 (UNHCR 2015a: 21).

An indicative example of third country resettlement comes from the consequences and fall-out of the Vietnam War (1955-1975), where there was mass exodus of refugees to neighboring countries. With hosts such as Laos experiencing significant problems with social, ethnic and economic integration, third country resettlement became widely utilized (Boyle et al. 1998: 195-198). This was not an easy decision and the results not unequivocally successful:

Many [Northern] countries that resettled Indochinese refugees note[d] the refugees' severe ethnic and cultural problems of adjustment as well as their own limited absorptive capacity, economic problems, xenophobia, and compassion fatigue as reasons why resettlement is a durable solution of last resort.

(Stein 1986: 268)

Similar conclusions can be reached from more recent examples of third country resettlement, not least when it is combined with dispersal (discussed below) to often less hospitable parts of a country. Moorehead (2005) gave the example of Dinka refugees from Sudan experiencing resettlement in Oulu, a city northern Finland. Oulu presented a series of "scarcely imaginable contrasts" (p. 272) for the Dinka, including an almost total lack of potential to develop a significant employment career within their geographically relocated and disrupted life courses. In sum:

there is no work; and it is very unlikely that there ever will be. A few may find employment eventually as cleaners or looking after old people, if their Finnish is judged good enough ... [F]or the engineers and ... teachers, the nurses and ... would-be university graduates, there is nothing. 
Moorehead (2005: 274-275) thus went on to suggest how third country resettlement may consequently be far from a durable solution, with those involved often eventually moving on elsewhere:

so the Dinkas in Oulu wait. [T] hey prefer to start dreaming again, to plan for the day when they will become Finnish citizens, with Finnish passports, and will be able to move on, to the life they hope for, in another country.

Once again, the importance of a person's official category-here, being a Finnish / EU citizen-appears central in shaping (future) everyday life course experiences.

Consequent of problems with third country resettlement, it was increasingly seen as the "least desirable and most costly" (Stein 1986: 278) durable solution. This perception is enhanced when the Global North's rising resistance to all immigration is noted. The years 1979-1982 marked the peak of third country resettlement (Stein 1983) and it has since declined in popularity. Between 2005 and 2014 only 0.9 million refugees were resettled but 5.2 million returned to their country of origin (UNHCR 2015a: 20).

The latter figure indicates the importance of the third durable solution, repatriation. This involves refugees returning to their home country. It can be voluntary or involuntary, organized or spontaneous. While repatriation may seem an ideal solution to the unwanted life course stage of being a forced migrant, safely returning is often very difficult to orchestrate successfully. It is most controversial when involving forced deportations, mostly of so-called "bogus" or "failed" asylum-seekers, a practice which may seem to challenge the underlying general principle of non-refoulement (9.2.1) within refugee policy generally.

Drawing on evidence from repatriation of Southern Sudanese in the early 1970s, Rogge and Akol (1989) outlined the plethora of problems refugees may face upon returning "home." Life courses are once again disrupted by relocation. First, for refugees that have spent a considerable time away, there may be challenges re-acculturating to the home society after spending long periods in a different community with different cultural norms. This can be particularly problematic for children, who often converse in the host country language. Indeed, a second generation may never have lived in the "home" country. Second, economic challenges include costs of repatriation. For those who have lived in a host country for many years, repatriation can involve leaving behind new livelihoods and often requires starting over in the home country. Refugees accustomed to infrastructure and services of the host country may also experience reduced standards of living and economic opportunities. Third, political challenges for repatriated refugees include being regarded as subversive to the home country government, which may also not welcome them because of the expenses associated with their resettlement and rehabilitation needs. Finally, there may also be a gap in perception of safety: how safe the repatriated feel versus how safe the host country believes their home country to be. Other challenges facing repatriated refugees involve property or land ownership disputes, homelessness and broken-down home communities (Marsden 2005; McDowell 2006; Romano 2005). Afghan refugees in Pakistan, for example, gave as principal reasons for not returning lack of suitable shelter (57 percent), inadequate livelihood (18 percent) and security fears (18 percent) (Kronenfield 2008). This led Kronenfield (2008: 59) to advocate local integration, especially in protracted situations where "it is often impossible to return to the status quo ante."

In the context of Iraq following the fall of the oppressive Saddam Hussein regime in 2003, Romano (2005: 436) outlined five conditions that ought to be met before repatriation occurs. These are shown in Table 9.6. It was considered that only the fifth condition had been met in Iraq by 2005-a situation that hardly improved in the subsequent decade as near-constant strife tore apart a society "transformed by violence and the pervasiveness of deep mistrust amongst the population" (Sassoon 2008: 4). Nonetheless, in spite of refugees and IDPs having been advised not to return, much repatriation had occurred (Romano 2005), demonstrating the pull of return. For example, around 60,000

Table 9.6 Five requirements for ideal refugee and IDP return.

1 Authorities agree to returns at all levels

2 Availability of necessary resources to support a comprehensive return program

3 Relevant expertise to assist return program

4 Security of returnees assured

5 Displaced populations' desire return to places of origin

Source: derived from Romano (2005: 436). 
returnees were living in temporary shelters around Kirkuk. Some, however, may well have been IDPs evicted from "squatted" government buildings or land, suggesting that of this return migration might not have been so freely chosen.

Repatriation assumes a clear sense of a "home" to return to, defined at whatever geographical scale. This is not necessarily a simple matter and again raises the problem of sedentarist assumptions of everyone having a single "home" (5.2.1). Consider this seeming paradox from Afghanistan's continuing refugee situation (Kronenfield 2008). Between 2001 and 2007, it was estimated that over 3.5 million Afghan refugees returned from neighboring Pakistan, with 2.5 million remaining in the latter. Yet, it was estimated that there had only been 2 million Afghan refugees in Pakistan in 2001. How was this possible? While refugee populations typically have high birth rates, this could not be the full story! Instead, Kronenfield (2008) emphasized the fluidity of populations and definitions such as "Afghan refugee." Individuals frequently moved to and from Afghanistan and Pakistan, sometimes being "refugees" and other times resembling "economic migrants," effectively living in both Afghanistan and Pakistan. This adds another dimension to the mixed migration concept (9.2.2.2).

Although this section has focused on refugees, repatriation is of considerable relevance to the fate of asylumseekers who ultimately fail to attain refugee status through not being regarded by the country to which they have applied as filling required criteria. For most forced economic and environmental migrants (9.2.2.1), in particular, forced repatriation is likely to be their ultimate fate if they do not agree to return home "voluntarily" (Blinder 2014). Here though, through the integrating lens provided by migration management and border management (9.2.1), one is forced to again address mixed migrants and the fate of undocumented migrants in general, rather than solely forced migrants.

Forced repatriation is not a new state practice (Bartram et al. 2014) and has as its mirror image the universally condemned expression of forced migration known as ethnic cleansing (Box 9.5). It is widely critiqued within both scholarship and advocacy (e.g. Collyer 2012; Gibney 2008; Schuster 2005). Nonetheless, there has been rapid rise in what Schuster and Majidi (2013: 1) depicted as "a practice acknowledged to be brutal, expensive, and ineffective." It should be seen as part of the broader growth of the triumvirate of "detentions, deportations, and dispersals" (Samers 2010: 216) within migration management, each now considered in turn.

\section{BOX 9.5 ETHNIC CLEANSING: THE DARK SIDE OF DEMOCRACY?}

The twentieth century saw a particularly notorious form of forced migration, ethnic cleansing, leap to global prominence within news stories of wars and conflicts. Through these accounts, the life course arena of ethnicity stood out particularly strongly, as ethnic cleansing may be defined as the attempt "to remove a people and often all traces of them from a concrete territory" (Naimark 2001: 3). Although not identical to the still more extreme "exterminist activity" (Dahlman and Ó Tuathail 2005: 573) of genocide, which aims to destroy part or the whole of a population, the practices have often been closely linked.

Sociologist Michael Mann (2005) began his influential book on the politics of ethnic cleansing with a typical account of the practice, taken from the Los Angeles Times. Originating from Kosovo in former Yugoslavia in 1999, this told of ethnic Serbian soldiers entering the house of an elderly ethnically Albanian couple. They beat and killed the husband, searched in vain for the couple's children, who would have probably met a similar fate, and then evicted the wife and burnt the house to the ground. The elderly widow survived and was eventually found by her daughter living as a refugee in a camp in Albania. As Mann observed, "[c]hange the names of the people and places and the incident could have occurred almost anywhere in the world over the past few centuries-in Australia, Indonesia, India, Russia, Germany, Ireland, the United States, Brazil" (pp. 1-2). In other words, ethnic cleansing may not be new historically but became more commonplace and assumed higher global profile through the twentieth century.

As a practice entailing often barbarous violence, one might think ethnic cleansing has little connection to modern liberal democracies. Mann, however, argued quite the opposite. For him, it expressed democracy's "dark side," consequent of the fact that "democracy has always carried with it the possibility that the majority might tyrannize minorities ... [a] possibility [with] more ominous consequences in certain types of multiethnic environments" (p. 2). This first "thesis" was supplemented with a further seven, including (pp. 5-9): 
- $\quad$ Ethnic hostility rises where ethnicity trumps class as the main form of social stratification ... ;

- The danger zone of murderous cleansing is reached when ... movements claiming to represent two fairly old ethnic groups both lay claim to their own state over all or part of the same territory and ... this claim seems to them to have substantial legitimacy and some plausible chance of being implemented...;

- $\quad[M]$ urderous cleansing occurs where the state exercising sovereignty over the contested territory has been factionalized and radicalized amid an unstable geopolitical environment. . .;

- Murderous cleansing is rarely the initial intent of perpetrators. . ;

- There are three main levels of perpetrator: ... radical elites running party-states; . . bands of militants forming violent paramilitaries; and ... core constituencies providing mass though not majority popular support ... ;

- [O]rdinary people are brought by normal social structures into committing murderous ethnic cleansing".

Besides presenting and illustrating these theses, Mann presented a table that mapped degrees of violence and cleansing within ethnic relations: This located ethnic cleansing within a spectrum of violence and discrimination that all cause forced migration, many noted throughout this chapter. This illustrates how this dark side of democracy is not as singular as it may sometimes seem but blends into other forms of forced migration.

\begin{tabular}{llll}
\hline Type of violence & Type of ethnic cleansing & & Total \\
\hline None & None & Partial & Vulticulturalism or \\
equivalent & $\begin{array}{l}\text { Loss of aspect(s) of identity } \\
\text { (e.g. language) } \\
\text { Institutional coercion }\end{array}$ & $\begin{array}{l}\text { Official language restrictions, } \\
\text { segregation }\end{array}$ & Cultural suppression \\
Policed repression & Selective repression & $\begin{array}{l}\text { Partial repression of language } \\
\text { \& culture, policed settlement/ } \\
\text { displacement }\end{array}$ & $\begin{array}{l}\text { Total repression of language \& } \\
\text { culture, population exchanges, } \\
\text { deportations \& pressured emigration }\end{array}$ \\
Violent repression & Generalized repression & $\begin{array}{l}\text { Pogroms, communal riots \& } \\
\text { rapes, violent settlement/ } \\
\text { displacement }\end{array}$ & $\begin{array}{l}\text { Wild deportations \& emigration, } \\
\text { sterilization, forced marriage \& rapes }\end{array}$ \\
Unpremeditated & $\begin{array}{l}\text { Mistaken war, civil war } \\
\text { mass deaths }\end{array}$ & $\begin{array}{l}\text { Callous war, civil war \& } \\
\text { revolutionary projects }\end{array}$ & Ethnocide \\
fratricide & $\begin{array}{l}\text { Exemplary \& civil war } \\
\text { repression, systematic } \\
\text { deaths }\end{array}$ & $\begin{array}{l}\text { Forced conversion \& mass } \\
\text { killings of leading (political) } \\
\text { classes }\end{array}$ & Genocide \\
\hline
\end{tabular}

Dark shading: murderous ethnic cleansing core; middle shading: borderline zone.

Source: simplified from Mann (2005: Table 1.1).

(Dahlman and Ó Tuathail 2005; Mann 2005: Table 1.1)

Detention involves use of removal or accommodation centers. These "prison-like structures usually surrounded by razor (barbed) wire and tight security" (Samers 2010: 217) are found globally. Their operations are often out-sourced to private companies (Bartram et al. 2014). Moreover, although their main task is warehousing immigrants who have fallen foul of the legal terms of their status (such as having been convicted of a serious crime) and asylum-seekers deemed to have "failed" to make their case to be labeled refugees or who are awaiting legal appeal decisions contesting immigration status (Hyndman and Mountz 2008), they may also accommodate asylum-seekers on arrival. This is especially likely for "bureaucratically problematic people" (Griffiths 2012: 715) effectively criminalized for not having any officially recognized identity (e.g. passport or identity card). Time spent in such centers is limited in some countries but not in others. Worldwide, various forms of abuse have been reported in removal centers, from Malaysia (e.g. Hedman 2008) to the US and Europe (e.g. Welch and Schuster 2005). They are centers of despair, as in the tragic case of 


\section{FORCED MIGRATIONS}

Manuel Bravo awaiting deportation to Angola from the UK, who hanged himself from both fear of return and with the hope that his death would allow his newly-orphaned 13-year-old son to remain in the UK (Athwal and Bourne 2007: 112-114).

Australia illustrates an extreme case of the increasing detention of asylum-seekers within what Hyndman and Mountz (2008: 250) recognized as a global tendency towards neo-refoulement or "a geographically based strategy of preventing the possibility of asylum." Following increased arrivals of asylum-seekers smuggled by sea from East Asia (but often originating much farther away) in the 1990s, a series of remote, inaccessible detention centers were built in mainland outback locations such as Woomera (Mares 2001). In spite of their location and harsh regimes, even these were soon deemed inadequate by the Australian state, which instead turned to a "Pacific solution" (Mountz 2011). Detention centers were established on remote islands, such as Christmas Island and the tiny impoverished Pacific island state of Nauru. Subsequently, asylum-seekers, usually intercepted at sea under "aggressive interdiction policies" (Hyndman and Mountz 2008: 261), were sent to these centers. Even if they made it to Australian islands they could be transferred to these centers since the Australian government had made "several hundred small islands no longer part of Australia for the purposes of migration law" (Hyndman and Mountz 2008: 259-260). Within these remote centers, asylum-seekers are especially isolated, even from advocates and human rights monitors (Mountz 2011), and conditions harsh; in Nauru, water supplied just once a day, for just one hour (Hyndman and Mountz 2008).

Besides detention, deportation of criminalized migrants, failed asylum-seekers and other undocumented immigrants has been growing (Ellermann 2009; Gibney 2008). For example, the US increased annual deportations between 1995 and 2005 from about 40,000 to over 200,000. Explaining such rises, Samers $(2010: 217,220)$ argued that this strategy of removing migrants may be to placate public opinion, deter other similar potential immigrants and encourage voluntary departures, although its actual impacts are more doubtful. It signifies a government "doing something" (Gibney and Hansen 2003), exerting sovereignty (Bartram et al. 2014) in an era of mobilities and globalization, when such state agency is increasingly challenged.

However, looked at differently, whilst deportation rates have increased, it can be questioned why it is not more commonplace. Actual numbers of "unwanted" migrants forcibly repatriated remains quite low for most countries (Bartram et al. 2014). Attention must thus be paid to: presence of "liberal norms" (p. 46) which, not least through legal challenges, prevent deportations due to fears for the deportee's subsequent safety; origin countries' reluctance to take emigrants back, not least as this may lose them valuable remittances; and high deportation costs, from legal fees to the expense of chartering special planes to transport migrants.

Paying attention to experiences following deportation-a neglect within scholarship (Schuster and Majidi 2013) that reiterates how migration is often over-simply reduced to relocation alone (5.6.3)-reveals not only very uncertain and often highly dangerous futures (e.g. Peutz 2006, on Somalis deported from the US and Canada) but also how deportation as a policy fails to live up to advocates' expectations as a durable solution. Repeat migration, in particular, is very common (Hagan et al. 2008). Schuster and Majidi (2013) argued that deportation often promotes re-migration, not just from continued risks in the "home" location, probably enhanced by deportees' raised public profiles, but due to deportees often not having paid off the costs of initial emigration, having developed transnational ties, and the "shame" of having failed as migrants and of possibly being seen as "contaminated" by another culture. Of course, some deportees succeed on return, often helped by skills picked up abroad (Schuster and Majidi 2013).

Where neither detention nor deportation is considered "appropriate" for asylum-seekers and refugees, dispersal may occur. The idea is to "lessen the burden" locally and avoid concentrations of asylum-seekers or refugees developing. Whilst advocates argue that one further benefit is reduction of racial tensions, this blames the victims for causing such tensions (Samers 2010). Dispersal typically relocates forced migrants both locally and nationally to areas of cheaper housing. Unfortunately, these are also often areas of economic hardship, high unemployment and little previous experience of immigration (Spicer 2008). Dispersal may also take "asylum seekers away from kith or kin and access to support services" (Athwal and Bourne 2007: 108)-and heterolocal networks (6.3.4)-and relocate them in perceived places of exclusion. Such places are often characterized by racism and general hostility, even at school (Spicer 2008). However, negative experiences of dispersion can be moderated through local practices, such as portraying asylum-seekers and what they have to offer positively in local media (Finney and Robinson 2008).

State-sponsored dispersal is often not the fate of "failed" asylum-seekers who are neither detained nor deported. Instead, they may be forced to scrape out a meager existence day-by-day with little or no state support (Crawley et

\section{ST PROOFS: NOT FOR DISTRIBUTION}


al. 2011). Schuster (2011) engaged with young Afghan men in Paris swelling the ranks of the Sans Papiers (McNevin 2006) by being transformed from refugees to undocumented migrants. This was especially due to the laborious bureaucracy of the French asylum system. She found:

France's inhospitality ... evident to new arrivals, who are shocked and disheartened to find fellow Afghans sleeping in parks, under canal bridges, and on the streets, suffering from lice and scabies, or accommodated with alcoholics and drug addicts in the bedlam of emergency hostels.

(Schuster 2011: 1400)

Finally, developing from repatriation is a fourth policy family, increasingly embraced by recipient countries in the Global North: containment and exclusion. This is part of managed migration (9.2.1) and works hand-in-hand with detention and deportation. With rising domestic political concerns and intolerance over (imagined) volume of refugees-and immigrants broadly-Northern governments "have moved to both exclude asylum seekers and contain potential refugees in their place of origin" (Boyle et al. 1998: 199). Forms of exclusion such as fining airlines for bringing asylumseekers, erecting physical barriers, insisting on visas for travel, and legislating on location where asylum claims must be made are represented in the Dublin Convention. Australia's aggressive interdiction is part of this exclusionary armory. Robinson and Coleman (2000: 1219) saw this policy further operationalized through military intervention and the production of supposed "safe havens" and "safe corridors" where at risk populations can shelter. Increasingly, the aim of Northern governments is seemingly not to deal with asylum-seekers and refugees, however harshly, on their "home turf" but to make sure they never reach that soil.

Whatever, the impact of punitive policies of deterrence, world news daily tells clearly how refugees are still ubiquitous. They will remain so until the only truly durable solution-eradication or at least substantial reduction of causes of forced migrations-is more fully enacted and focused on than has been managed to date.

\subsubsection{Forced migrant adjustment, adaptation and integration}

Section 9.4.2.3's first three durable solutions share in common the need for the displaced to (re-)establish livelihoods, networks, and communities in their ultimate destination locations. Whether at "home" or in a new country/community, the processes through which individuals re-build lives and reconfigure their life course involve often considerable adaptation in everyday practices. Scholars of refugee studies have turned their attention to understanding these processes, which in varying degrees apply to all "permanent" international migrants. In other words, this final section ultimately enfolds forced migrants within the broader long-distance migrant category.

Before such migrant reconciliation, however, one must note continued exceptionalism for asylum-seekers compared to officially recognized refugees. Impoverishment and poor housing are not just experienced by "failed" asylum-seekers but are commonplace for asylum-seekers in general. Indeed, Allsopp et al. (2014: 35) depicted the UK asylum system as "a poverty producing machine," with multiple pathways into poverty. Consolidating the impression given by the young Afghans in Paris (Schuster 2011), they found "[t]he main cause of enduring poverty ... delays in the asylum system leading, for many, to long periods of dependency on benefits with no right to work" (p. 21). Such a situation was even acknowledged by the official Joint Committee on Human Rights (2007: 110) as deliberately prompting departures and deterring others from coming.

A key issue, as noted, is prohibition on asylum-seekers undertaking paid employment, in spite of frequently having skills in high demand in asylum countries (e.g. doctors, engineers) (Phillimore and Goodson 2006). This can prompt engagement with informal work, including sex work for (mostly) women, potentially endangering both themselves and their asylum claims. Yet, gaining "legitimate" employment is often difficult even for recognized refugees, with such barriers as lack of interpreters, delays in issuing official documents, and difficulties in dealing with disability and health issues (Allsop et al. 2014: 23) all discouraging economic integration.

Focusing on officially recognized refugees, while only a small portion is formally resettled, those who are often go to countries geographically and culturally distinct from their homes. The extent of their subsequent adjustment, adaptation and integration within the currents of everyday life in these destinations requires multi-dimensional and multi-disciplinary analysis. ${ }^{2}$ From such a perspective, Ryan et al. (2008), with an emphasis on psychological well-being, 


\section{FORCED MIGRATIONS}

proposed a resource-based model of migrant adaptation. This emphasized a diversity of resources that facilitate adaptation to another culture: personal, including health, social skills, and individual traits (e.g. self-esteem, optimism, self-efficacy, hope); material, including property, money, and personal possessions; social, including social networks and personal relationships; and cultural, including skills, knowledge and beliefs from one's own culture. All these resources are unequally possessed but are strategically (re)deployed by migrants as they come to terms with the country of resettlement. Ability to call upon them, in part, helps explain differential capacity of individual migrants to adapt. Resources may also change through time, consequently affecting individual adaptation at different life course stages.

In a study of Vietnamese refugees settled in northern Alberta, Canada, Montgomery (1996) acknowledged three dimensions of adaptation: economic, socio-cultural, subjective. Regression analysis of the predictors of each revealed particular affiliations: economic adaptation with length of time in Canada, being male, being married or cohabiting, and length of time in formal education or vocational training in Canada; socio-cultural adaptation with length of time in Canada, length of time in formal education or vocational training in Canada, and younger age; subjective adaptation with living in smaller settlements, being married or cohabiting, and involvement in ethnic networks. Again, he concluded that "refugee adjustment and adaptation are best conceived as having multiple components" (Montgomery 1996: 695). Strong economic adaptation, for example, did not necessarily also mean strong socio-cultural and/or subjective adaptation.

Ultimately, even when acculturation (Portes et al. 2009)-change in cultural patterns groups experience when coming into regular contact with other groups-does not lead to adopting the cultural patterns of the host population or broader assimilation between migrants and hosts does not occur (Bartram et al. 2014), settled refugees eventually become an integral component within the recipient country's ethnic diversity (Castles et al. 2014: Chapter 12). Whilst sustained presence of racism globally means that refugees will probably never be entirely free of prejudicial experiences and pressures that, in extreme forms, prompt forced migration (6.2.5), a degree of integration within the host society will probably eventually be achieved.

"Integration" of resettled refugees and other migrants is a key policy topic, although exact definition of the term is elusive (Robinson 1998). It is used in a range of ways in varying contexts. Using the "Indicators of Integration" study commissioned by UK Home Office in 2002, Ager and Strang (2008) proposed ten "core domains" to integration, shown in Figure 9.8. Starting at a foundation of rights and citizenship, they recognized four markers and means of integration-employment, housing, education, health-mediated by the facilitators of language, culture, and safety and stability, and social connections that bridge, bond and link. Putting all these domains together in a robust and resilient manner is the normative challenge posed by the jigsaw of successful integration.

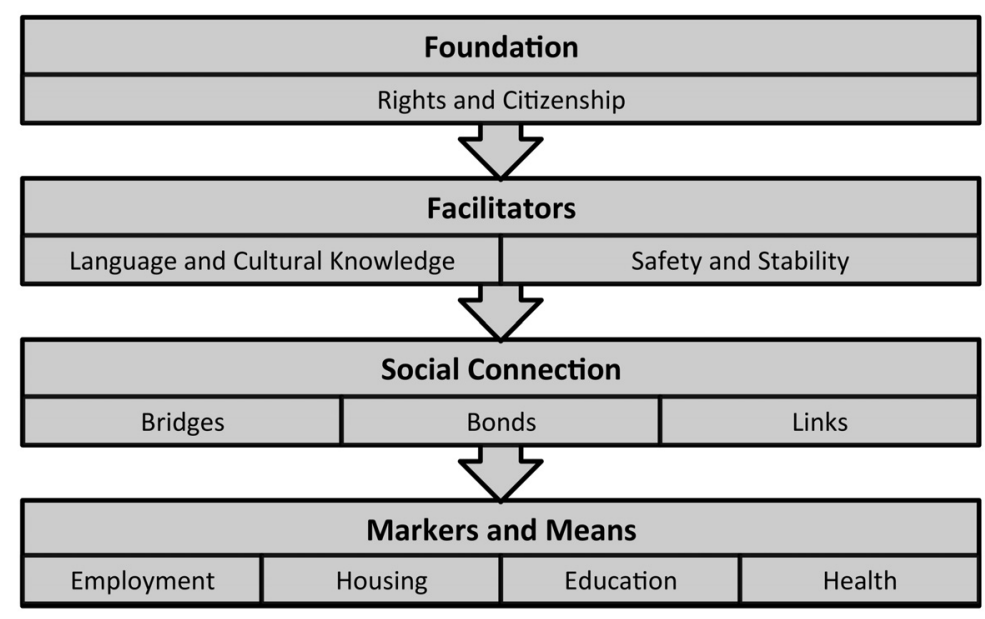

Figure 9.8 A conceptual framework defining the core domains of integration.

Source: adapted from Ager and Strang (2008: Figure 1). 
From an individual perspective, adaptation and integration-let alone assimilation-can not only be very difficult but require a very long period of time to achieve. Challenges are often sharpest in a context where large numbersreal or perceived-of refugees impact prominently on local resources or populations, not least in relatively poor host countries or communities. Notwithstanding xenophobia and racism, potential hosts may reject permanent settlement out of concern for anticipated impacts on their own populations. Challenges include ability to provide housing, land and/or employment for new populations (Stein 1986). Like migration, adaptation and integration are best seen as processes for which any idea of a final, conclusive outcome can often in practice be both limiting and inadequate. Instead, "how complex societies can reconcile differences and solve conflicts without violence ... [will and must remain] an ongoing concern for any society" (Bartram et al. 2014: 87, after Favell 1998).

Finally, this section's focus on adjustment, adaptation and integration reiterates the often common experiences of and issues facing both refugees settling in third countries and long-distance migrants generally. For forced migrants, experiences of being driven into exile can never be completely forgotten in a country of asylum but everyday life has then to be negotiated in much the same way as for any immigrant. Ethnic or other ties and networks can be very useful here, not least by connecting with those who have gone before (Chapter 7), often then touching on issues of (non-)assimilation and residential segregation (6.3.4) as life courses become newly-configured in new everyday life contexts.

\subsection{CONCLUSION: LOOKING BEYOND VICTIMHOOD}

This chapter provided introductory insight into the geographies and practices of forced mobilities and migrations present across the world today. It identified at least four key themes, all of which illustrate and expand appreciation of the forced migrant experience. First, forced mobilities and migrations must be seen to be brought about by numerous causes. The classic case may still be the war refugee fleeing conflict across national borders but this is far from the full picture. Causes are much more diverse than just war and conflict, as ubiquitous as these unfortunately remain, and can extend to environmental and even economic factors. Geographically, whilst refugee and asylum-seeker migrations are numerous, overall numerical predominance of IDPs, concentrated within the Global South, should be more fully recognized.

Second, as flagged from the start of the chapter, forced migrant definitions are of central importance to the forced migration experience. Not only must the diversity of causes be recognized, as noted, but as Anderson (2010: 307) observed "immigration controls produce status" and this is of considerable experiential significance not only for subsequent employment options (Chapter 7) but to migrant everyday lives generally. Being recognized as a "legitimate" asylum-seeker, transitioning from asylum-seeker to refugee, or being classified instead as an "economic migrant" all impact strongly on the individual's ability to develop their life course in desired directions in terms of employment, for example.

Third, increased political fractioning (Zetter 2007) of forced migrants globally but by governments of the Global North particularly has made the forced migration experience increasingly challenging for many involved. Notwithstanding the hardship of initial displacement, increasing intolerance to all immigration (except perhaps to that of the very rich) is being felt strongly by forced migrants. This results in fewer and fewer experiences meriting the invaluable official legitimizing label of "refugee." For the rest, migration management increasingly works to send them "home," subject them to detention or other strict state scrutiny or regulation, or prevent them ever arriving in Northern countries.

Finally however, in spite of this contemporary context of heightened exclusion, it is wrong to portray forced migrants solely as victims. On the one hand, fractioning them into small groups of the totally-disempowered (sic.) runs the risk-argued above in the context of sex-trafficking, modern slavery, child migration and New Orleans evacueesof presenting them as unique special cases. This neglects their relational construction through broader processes of exploitation and exclusion-notably racism-that not only impact on them but also on other groups of both migrants and even some non-migrants. On the other hand, even the most forced migrants still express some human agency in and through their moves. From lived experiences within Belgian detention centers (Lietaert et al. 2014) to Congolese women in Rwandan UNHCR camps (Pavlish 2005), human agency resists deleterious labels, restates identities and dignity, promotes communities and children, and so on. All migrants have some say in the construction of their life courses and lives across space.

\section{ST PROOFS: NOT FOR DISTRIBUTION}


268 FORCED MIGRATIONS

\section{NOTES}

1 There are parallels here with political fractioning of refugees, discussed by Zetter (2007). Indeed, the idea of fractioning pervades this chapter.

2 Migrants' experiences within a "new" society are neither just in their hands nor solely their own responsibility. The extent adjustment, adaptation and integration occurs is very much located within a two-way context between migrants and already resident populations. Space is lacking to examine this broader issue (e.g. Baubock et al. 1996) and its link to assimilation, multiculturalism and so on. 


\section{CHAPTER 10}

\section{Ageing and mortality}

\subsection{INTRODUCTION: THE AGEING BODY AND THE END OF A LIFE COURSE}

From a life course perspective, lives across space end with death (Figure 2.3). ${ }^{1}$ This final life transition (2.2.1), mortality, is the third element of Population Geography's classic triumvirate (with fertility and migration). Consequently, this transition and the ageing human body that inevitably eventually leads to such an outcome if not brought about by other causes are the subjects of this penultimate chapter.

Like fertility, mortality has been studied less by contemporary Population Geographers than migration (Boyle 2004). Nonetheless, there is considerable geographical scholarship, since death is very much a spatial matter. Often considered with morbidity-the "prevalence of sickness in a population" (Poston and Bouvier 2010: 391)-the spatiality of mortality is extensively covered by both Medical and Health Geographies and Health Studies (e.g. Brown et al. 2010; Gatrell and Elliott 2015; Gesler and Kearns 2002). Thus, in the absence of any fully-fledged life course geography (Chapter 2) engaging with all aspects shaping lives across space, these other sub-disciplines are drawn on considerably in this chapter.

Morbidity has a somewhat uneasy position within Population Geography, not least because most sickness does not, of course, end with (immediate) death. Nonetheless, it both reflects and is reflected in part in the life course truism that all bodies age or gradually wear out as one reaches "the limits of life" (Heath 2013: 20). The final consequent "climax of the ageing process" (Hunt 2005: 204) is death: the ultimate immobility. It is thus unsurprising that "the probability of dying is extraordinarily affected by age" (Gould 2015: 95), which is why (older) ageing and death are combined in this chapter.

There are qualifications to be made in associating mortality or morbidity with ageing, however. First, ageing does not start in middle or later life but at a body's physical and mental peak around 20 years. One can distinguish "primary ageing" throughout a life course from environmentally-related "secondary ageing." Comfortable living conditions (good housing, relaxation, healthy diet, exercise) can slow the latter, adverse conditions (poor housing, stress, bad diet, sedentary living) accelerate it (Hunt 2005). Second, becoming elderly is not just of significance in signaling a life course's latter stages, being much more than "sitting in God's waiting room"! Third, becoming elderly should not automatically be associated with increased morbidity or reduced mobility (Del Casino 2009: 248), even if these associations are present overall. Fourth, reduction of demographic ability, notably mobility, is not solely a function of older age. Mobility constraints are also apparent for children, women in some cultures, people with certain illnesses and the "disabled" (Box 10.1). They may be largely biological, as with severe long-term illness, but may equally be entirely social, as in patriarchal constraints on women's mobility. They are often complexly bio-social, as with those associated with disability or childhood. Fifth, although to die peacefully in bed is probably most adults' dream, the reality is that "ageing" will probably not be noted on our death certificates.

Before engaging with mortality, Section 10.2 introduces the (normative) end-of-life-course elderly life experience. Section 10.3 then presents how mortality is measured and outlines global patterns and trends. To begin to explain these mortality geographies, Section 10.4 outlines how they have been modeled, before Section 10.5 presents three grouped causes of mortality, relating each to key arenas of life course experience-particularly noting relative prevalence across the bodily ageing spectrum. Section 10.6's short conclusion first notes how a consequence of changing mortality geographies is a world population demographically ageing before re-emphasizing the importance of resources and access for understanding and explaining both senior lives and mortality. 


\section{AGEING AND MORTALITY}

\section{BOX 10.1 DISABILITY: SOCIAL AND EMBODIED (IM)MOBILITIES}

Reiterating that seniority is not a "disability," there are parallels between how old age impacts on mobility and accessibility and the consequences of being disabled. Both old age and disability are socially constructed, making their mobility and accessibility geographies also social products. Yet, both are also embodied, such that these same geographies cannot be reduced to the social sphere alone.

The "social model of disability" developed in the 1980s and 1990s became "the foundation stone on which social-scientific disability studies matured during the 1990s" (Hansen and Philo 2007: 494). In this model, the body, medicine and technology take a back seat to explicit focus on the consequences of living within a predominantly ableist society, whereby "ideas, practices, institutions and social relations ... presume ablebodiedness, and by so doing ... construct persons with disabilities as marginalised, oppressed, and largely invisible 'others'" (Chouinard 1997: 380). The model focuses on how society creates conditions in which disability results, not least in terms of accessibility and mobility. Geographically, for example, the modern city is (re)produced in ways that dis-able people with diverse impairments. As a Los Angeles urban planner put it, "the built environment is basically designed for the average human being, plus or minus half a standard deviation" (quoted in Hahn 1986: 273). The result, Hahn (1986: 279-80) went on to argue, is how the city's dispersed geography:

absence of a strong sense of community, and ... unattainable images of personal appearance and attractiveness ... [mean that] for a sizeable proportion of the disabled population, Los Angeles remains unexplored territory, an unending stretch of impenetrable geography that may never be experienced because of artificial obstacles imprinted upon the land.

This turn towards social and contextual models to frame academic scholarship was not just experienced by geographies of disability. The rise of post-positivist approaches generally (Chapter 1) and within Medical Geography specifically saw a shift towards understanding health as constituted through social and cultural environments. It led critics to suggest a Health Geography that would "go beyond the biomedical perspective of an absence of disease, ... framing ... health issues within critical-social-theoretical frameworks, [often using] qualitative methodology and mixed methods, and ... [attending] to the meaning of place" (Rosenberg 2011: 109).

Although similar developmental routes taken by Disability and Medical Geographies set the scene for subdisciplinary alliance, the unifying triumph of the social model has itself been increasingly challenged. Nearly two decades ago, Hughes and Paterson (1997: 326) lamented how the social model "concedes the body to medicine" at a time when it was increasingly being realized within broader scholarship how social and cultural the body really was. The body is not just a "site invaded by a disease with a specific etiology" (Dorn and Laws 1994: 107) or of a specific disabled condition or impairment. Instead, it is an active socio-cultural accomplishment. The social model of disability could thus even be accused of adding another dimension to the exclusion and "rejection" of the disabled body by the same ableist society this model so strongly critiques!

Central to an embodied sense of disability is acknowledgement of "the real, lived experience of changed/changing/painful/clumsy/immobile bodies" (Parr and Butler 1999: 21). Impairment frequently leads to pain and inconvenience, irrespective of presence or absence of disabling social forces. One cannot deny everyday difficulties involved in washing and dressing, reading small print, ascending or descending stairs, accessing toilets, preparing food, visiting shops and services, and so on. In short, "people are disabled by society, and by their bodies" (Shakespeare 2006: 56, emphasis added): no degree of social-cultural-political-geographical change can eliminate lived reality completely.

A consensus has thus been building on the need for the body to be brought back centrally into work on geographies of disability. Nancy Hansen and Chris Philo (2007: 493) heralded its "ongoing 'retrieval' ... in both disability studies and ... disability geography." Whilst a person impaired in some way(s) is likely to be disabled by ableist society, s/he also experiences impairment. Through the body, social disability and impairment meet, mix and manifest: articulately anticipated by Liz Crow (1996: 56, 59), herself disabled, discussing the enlightenment of the social model and its omissions in the context of her own experiences: 


\section{Enlightenment}

"My life has two phases: before the social model of disability, and after it. Discovering this way of thinking about my experiences was the proverbial raft in stormy seas. ... This was the explanation I had sought for years. Suddenly what I had always known, deep down, was confirmed. It wasn't my body that was responsible for all my difficulties, it was external factors, the barriers constructed by the society in which I live. I was being dis-abled-my capabilities and opportunities were being restricted-by prejudice, discrimination, inaccessible environments and inadequate support. Even more important, if all the problems had been created by society, then surely society could un-create them."

\section{Omission}

"So how is it that, suddenly, to me, for all its strengths and relevance, the social model doesn't seem so water-tight anymore? It is with trepidation that I criticise it. However, when personal experience no longer matches current explanations, then it is time to question afresh ... [T] prevents us from dealing effectively with the difficult aspects of impairment. Many of us remain frustrated and disheartened by pain, fatigue, depression and chronic illness, including the way they prevent us from realising our potential. ... [O]ur silence about impairment has made many of these things taboo and created a whole new series of constraints on our selfexpression."

(Sources: Barnes and Mercer 2003; Crow 1996; Hahn 1986; Hall 2000; Hansen and Philo 2007; Imrie and Edwards 2007; Kearns and Moon 2002; Parr 2002; Parr and Butler 1999; Rosenberg 2011)

\subsection{ELDERLY LIVES}

\subsubsection{What does it mean to be "elderly"?}

\subsubsection{Representations of older people}

Put simply, across the globe today there exist a vast array of late( $r)$ age life experiences, immediately qualifying any ultimate truism that becoming a senior signifies entry to the final stage(s) of one's life course. It refutes any idea of "retirement" as a clearly delineated life-cycle (2.2.1) category. Instead, multiple pathways must be recognized through the later years of life. Moreover, with life expectancy-roughly, the age to which a "typical" individual would expect to live (10.3.1)-continuing to increase, individuals may expect to be categorized as "aged" for nearly 30-40 years of their life course, at least across much of the Global North! This opens up the potential of senior diversity still further.

This sense of often very diverse experiences of old age immediately suggests key interpretive questions: who is "old"; what does it mean to be "old"; and how well does "old(er)" age mark life course experience? The UN categorizes all individuals 60 years and upwards as "old" people (UNFPA 2012b), although many countries in the Global North use 65 years to delimit the group since this is often the age individuals access pensions, social security, or other senior social benefits (Kinsella and Phillips 2005; Weeks 2005). But most observers now argue that "old age" is more than number of years accumulated by an individual (Del Casino 2009: Chapter 9; Hockey and James 2003). Rather, the term conveys a range of characteristics or social constructions associated with particular life transitions, (dis)abilities, and roles within specific societies. Scholars have consequently become increasingly dissatisfied with terms such as "elderly," "senior" or "senior citizen" because they too readily connote simply loss of physical and mental capacity, fragility, and decreased economic capacity (Kinsella and Phillips 2005). Stereotypes ignore active and increasingly healthy older adult populations and their diverse community contributions across both Global North and South.

How older age is experienced has much to do with how members of a particular society view ageing. Primary ageing may inevitably lead to older bodies but material evidence of ageing, perceptions and interpretations of ageing, and how ageing adults should act, for example, are all socially constructed (Calasanti 2005; McHugh 2003). Transition to becoming "old(er)," in other words-as with all life course transitions-is much more than a chronological issue. While "medical" perspectives within contemporary ageing discourses may focus on negative aspects of growing old (Hockey and James 2003)-such as decreasing physical and mental abilities and increasing morbidity-"sociological" 


\section{AGEING AND MORTALITY}

perspectives, called to mind through images perpetuated by television and magazines of older adults engaged in outdoor activities-such as cycling, gardening, hiking and surfing-challenge notions of declining health and compromised bodies. Hardill and Baines (2009) argued that older people can actively both create and resist particular age identities through use of space and place. In rural England, for example, they showed older residents active care givers and community volunteers, challenging any notion of them as only spatially entrapped recipients of care.

Generally across the Global North, activity or mobility is utilized to present positive images of body and identity for seniors. This is strongly boosted and manipulated by the market. As Calasanti (2005: 11-12, emphasis added) noted, "through their appearance and activities, and often with the help of the anti-ageing industry, ageing people increasingly challenge the stereotype in which old age equals disease and decline." A retirement industry utilizes images of older adults engaging actively with beautiful outdoor landscapes to invoke a sense or impression of health and vitality (McHugh 2003). A norm of the active older person has thus emerged, important not just for commercial purposes but because behaviors and attitudes considered acceptable for any particular age group rely on such social norms and expectations (Liefbroer and Billari 2010). Even the language used to describe older adults indicates how they are viewed and likely treated within a society or community. Terms such as "retiree" suggest financial flexibility, "empty-nester" connotes time flexibility, while "elderly" or "senior citizen" is more suggestive of frailty and declining health.

In spite of positive models of active retirees (Figure 10.1, Box 10.2), the overall language, norms and expectations associated with older people remain largely negative (Hockey and James 2003; Hunt 2005), particularly across the Global North (Box 2.2). Cultural representations and attitudes towards older people are strongly influenced by ageism. This negative conceptualization of ageing prompts discriminatory and patronizing behavior towards those who are older. It "includes categorization, stereotyping, and prejudice, but the most critical aspect is exclusionary behavior" (Calasanti 2005: 8). It is equivalent to sexism and racism in its systematic nature. Ageism is rooted in the social organization of societies, in which different age groups are more or less powerful, the powerful also shaping perceived negatives and positives accrued to members of different age groups (Calasanti 2005). Ageism constructs old age as a state to be avoided, or at least postponed, a rationale for face-lifts, anti-ageing make-up and hair transplants, for example. There is still far to go before Northern populations will (once again) be "comfortable with the reality of death" (Hunt 2005: 213).

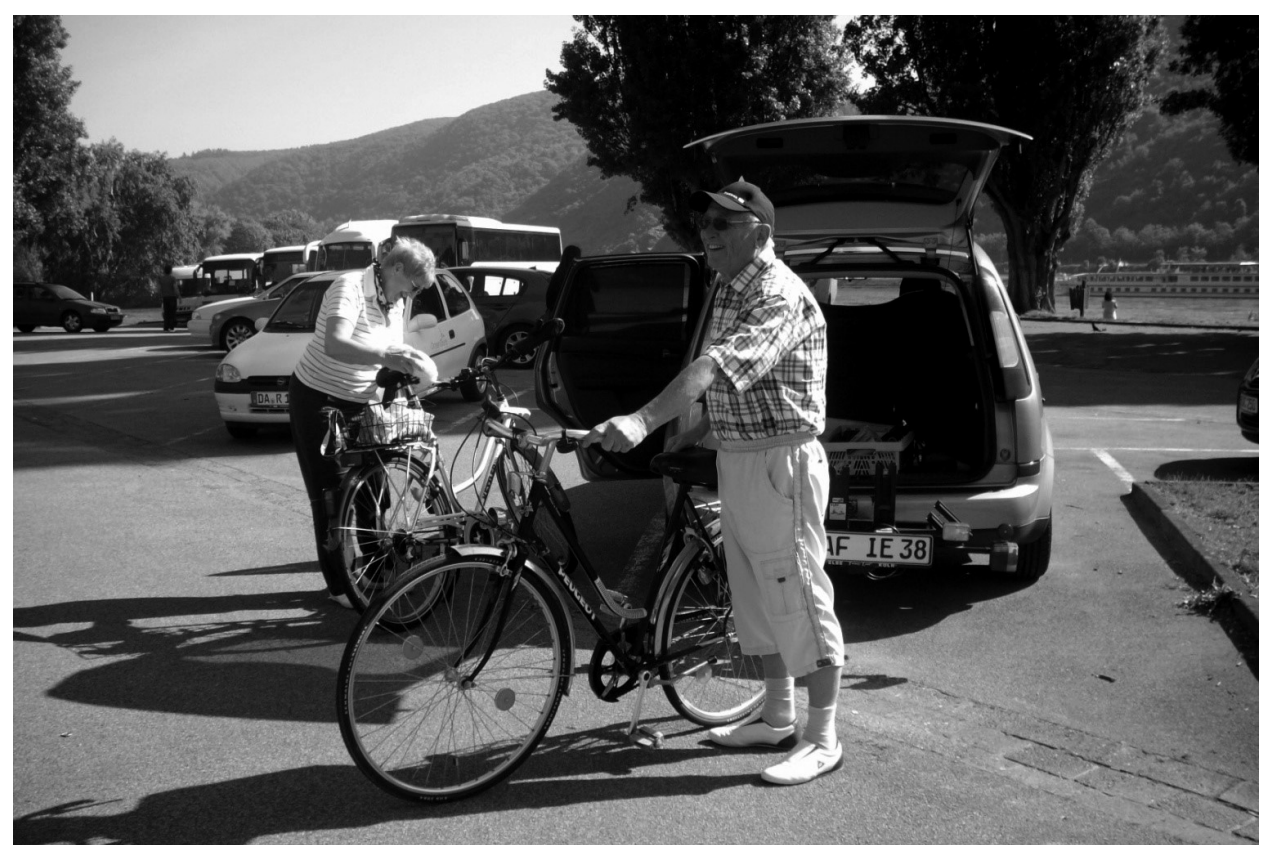

Figure 10.1 Active retirees.

Source: Wikicommons, Public domain. 


\section{BOX 10.2 IMAGES OF RETIREMENT AND THE PERPETUATION OF AGEISM}

Geographer Kevin McHugh challenged dualistic, positive versus negative, representations of ageing in analysis of images and scripts of Sunbelt retirement in the US (8.4.2). One the one hand, he described six images of ageing, retirement and place:

\section{Geographic cornucopia}

In the ideal sequence, the image and reputation stimulate the desire to visit Arizona, that leads to repeated visits and, if hopes are realized, the trips, in turn, lead to a more permanent relocation at the time of retirement. ... Seniors project idealized images and pleasures they experience in daydreams, and this halo-a romanticized, idyllic vision-tends to remain as residue, shaping long-lasting views about place as a discovered home.

Ageless selves

The retirement industry in Arizona and other Sunbelt locales offers a most alluring mask, the ageless self located in idyllic settings outside time and change.

\section{Near perfection}

The phrase "as close as it gets to perfect" is chosen carefully, because it would be unrealistic (and boastful) to claim outright perfection.... In addition to perfection, one often finds the utopian invocation to escape in advertisements for Sunbelt retirement communities: "Escape to the unspoiled natural beauty of Arizona's high desert and Sierra Vista, Arizona, the 'nicest little town under the sun'."

(pp. 172-173)

The right stuff

A critical aspect of retiree attraction programmes is luring the "right" sort of seniors, meaning the affluent and comfortable middle class with the appropriate cultural cachet.

Down home living

A marketing campaign from Mississippi "developed a personalized programme that plays upon its 'down home' (or friendly and welcoming) nature. Rather than encouraging migration to all parts of the Magnolia State, the programme concentrates on 20 towns officially certified as great places for retirees.... In terms of marketing, each town creates an identity and related slogan that is employed in promotional and advertising literature ... McComb enlists the help of the Mississippi Highway Patrol in recruiting retirees: its troopers are instructed to stop retirement-age couples on the interstate highway and invite them as special guests to 'McComb Hospitality Day', where the honoured couples receive lunch at the Dinner Bell restaurant."

(pp. 174-175)

Nomads of desire

Retirement is the serious business of choosing a style of leisurely living (from many choices), pursuing your choice with gusto and, in the process, finally discovering who you really are.

Yet, one the other hand, rather than perpetuating positive aspects of ageing, McHugh concluded that these images actually reinforced negative stereotypes, contributing to ageism. He wrote: "so-called positive views signify cloaked denials and repressions of the facts of human ageing and old age, are rife with happy delusions that adulate youth and productive adulthood, and fail to accord meaning to the third age and beyond" (p. 180). Despite the positive light in which retirement is commercially portrayed, underlying ageist sentiment suggests ageing in any other form than so portrayed is undesirable.

(Source: McHugh 2003) 


\section{AGEING AND MORTALITY}

Pervasive across the Global North, ageism is less apparent in the South. Here, attaining "elder" status is more associated with acquiring knowledge and wisdom, albeit often not unambiguously (e.g. Geest 2002). With becoming elderly also less associated with the distinctive and major life transition of retirement from (paid) work than in the North, there is less a sense of an older population "dependent" (10.2.2.5) on those of working age (Gould 2015). In sum, anywhere in the world, "[a]geing happens in a cultural context" (Fry 2000: 751) and attention must be paid to this context and the more general relational construction of older age to appreciate how it is understood.

\subsubsection{The relational construction of old(er) age}

"You're only as old as you feel" is an adage often lightly quipped but which gets to the heart of what individuals consider as "old." Crucially, this "feeling" is not just a biological matter but is also shaped by the societies in which people live. Contemporary Northern societies emphasize the individual, emotional and embodied personal (Markus and Kitayama 1991), as the chapter has suggested. This is an appreciation mere counting of years cannot apprehend or convey. Foregrounding "cultural variability and dissociation from bodily ageing" (Hockey and James 2003: 54), how "old" a person feels is conditioned relationally by many of the key arenas shaping differential life course experiences (2.3).

First, there is the historical time period through which an individual lives (Shanahan and Elder 2002); one's cohort. For example, being aged 65+ in 2016 is a very different experience than being 65+ in 1916, wherever one is in the world. In general, history witnesses changes in composition of populations and in how behaviors, roles, and expectations for individuals are considered within and for age groups. Ageing adults often experience striking contrasts between their own expectations for retirement and ageing and those of their parents or grandparents. From a Northern perspective, King et al. (2000: 5) recognized five sets of twentieth-century changes affecting deeply the "social construction" of old age: "spread of retirement, increases in survival, rising affluence, greater education and improved housing."

Second, Section 10.2.1.1 indicated how geography is extremely relevant at different scales. For example, visualize being a senior in rural France compared to urban Benin. Expectations and experiences of becoming old(er) vary considerably by country, in part reflecting cultural and socio-economic variations. Additionally, local scale factors, such as urban versus rural variations in accessibility to services (Woods 2005), also shape older lives, not least when individual immobility is high. Space is also critical in reinforcing identity formation. In a US study of retirees in Arizona, McHugh (2007: 293) wrote "it is through congregating, interacting and conversing on a daily basis that seniors in retirement enclaves affirm and reaffirm who they are, both to themselves and outsiders." Spaces in which older populations reside help cultivate, shape and reaffirm a sense of identity within generations, as well as create identities consumed or perceived by others (McHugh 2007). Central to such construction and presentation are, once again, mobilities and migrations.

Third, class and status strongly differentiate experiences of being elderly. Retirement, for example, becomes a time for lifestyle migration for many across the Global North (Chapter 8) but for others it is a life transition characterized by struggle for everyday survival (Grundy 2006). Income and wealth, in particular, translate into very different lifestyles in older age, just as they do throughout the life course, fundamentally shaping and determining the possibilities laid out in Box 10.2, for example.

Fourth, health and disability clearly shape the ageing experience (Box 10.1). Distinction is often made in the Global North between two life course phases for the older person. These are the Third Age, or young-old, and the Fourth Age, or old-old (Laslett 1994). The former is "a time when we are still healthy enough to engage in all of the normal activities of daily life, but are able to be free of regular economic activity," while "the distinguishing characteristic of [the Fourth Age] is an increasing susceptibility to senescence, increases in the incidence of chronic disease and associated disabilities, and, of course, death" (Weeks 2005: 386, 395). Or, reflecting relative material resources as well as health, the Third Age is "potentially a period of self-realization and ... empowerment," whilst the Fourth Age is "when 'real' old age sets in" (Hunt 2005: 193, 202), often associated with institutionalization. Weeks (2005) suggested 65 and 85 years of age as indicative markers of the beginning of each stage, respectively.

Fifth, ethnicity and race mold experiences of old age. Strongly linked to culture, spatial location (for example, whether or not immigrants) and individuality, Blakemore and Boneham (1994) suggested three categories of ethnic elderly: Self-Reliant Pioneers, who tended to acquire strength from their (ethnic) community; Gradually Adjusting Migrants, slowly adapting to host society; and Passive Victims, who felt discriminated against, not least on account of

\section{ST PROOFS: NOT FOR DISTRIBUTION}


(black) skin color. Within ageist (Northern) societies, elderly ethnic minorities may feel confused: empowered by origin cultures that value positively the elderly, whilst marginalized by the host society's dismissal of the elderly.

Sixth, religion and belief particularly comes into the picture in respect of awareness of the ultimate bodily demise of the elderly. Religious beliefs that hold to life after death or reincarnation can induce greater acceptance of the idea of old age than secular beliefs for which death is regarded as definitively a life's end. This helps explain the psychology behind "death-denying societies of the West" (Hunt 2005: 205), for example.

Seventh, experiences and to a lesser extent representations of ageing are strongly linked to sex and gender within most societies. Globally, women tend to have longer life expectancies, outliving men particularly in the oldest age groups: a "feminization of ageing" (UNFPA 2012b: 27). For example, in 2012, at age 60, there were 84 men for every 100 women but for age 80 years there were only 61 men per 100 women (UNFPA 2012b). Feminization marks ageing societies (10.6.1), not least because older women have been shown to have stronger social networks and to receive more support from adult children but also to provide care to children and the sick. Older men, in contrast, having principally had economic roles, can suffer when losing these on retirement, including becoming isolated (UNFPA 2012b: 28). Such notable gender differences in roles, social connectivity and economic resources present different gender-based challenges for supporting ageing populations. Such challenges are further influenced by different representations of older men and women, often influenced by sexist or patriarchal assumptions, such as men being more in need of looking after.

With sexuality having little immediate direct connection with ageing, the eighth and final life course arena to note is individuality. As with all aspects of lives across space, it must not be forgotten that one is ultimately dealing with individual people, albeit usually entangled intensely and multiply with others. Individual older people can just as much buck trends, expectations and assumptions as those of younger ages!

Summarizing at a societal level, attaining old(er) age is often associated with particular social behaviors, such as performing a grandparent role (UNFPA 2012b: 20), but such behaviors and how they are perceived vary considerably between and within cultures. For example, UNFPA (2012b: 21) noted how older adults in South Africa associated old age with accumulated life experiences but increasing dependency on others, physical and mental capacities used as markers of age rather than chronological definitions. Elsewhere, in Botswana, "elderhood is a status attained by becoming a grandparent, which often happens to mothers in their 30s" (Johnson and Climo 2000: 684). Even within societies, perceptions vary. In a US study, Montepare and Lachmen (1989) found respondents at different points in the life course holding different subjective age identifications, with younger adults often identifying as older and older adults sometimes holding younger age identities. Personal fears of ageing and overall life satisfaction influenced subjective and actual age perceptions.

For the Population Geographer, the relational construction of old(er) life is of particular empirical note in respect to movement. Older individuals' lives tend to generate particular (im)mobilities and migrations, just as particular (im)mobilities and migrations shape these same lives. Although largely covered in earlier chapters, these links are now re-flagged before addressing mortality, the ultimate immobility.

\subsection{2 (Im)mobile lives of the elderly}

\subsubsection{Geography, gerontology and the ageing body}

Geographers have long contributed to debates on ageing from both positivist and critical traditions (Hardill 2009). Furthermore, whilst a wide range of disciplines engaged with ageing have actively employed a spatial perspectiveDemography, Sociology, Environmental Psychology-others have more recently (re-)embraced such perspectivesEpidemiology, Social Medicine, Public Health (Andrews et al. 2007: 152). Geographical Gerontology, while not always cohesively defined, now encompasses a range of studies that foreground space and geography (Del Casino 2009: Chapter 4). Perspectives range from broad geographic delimitations, such as "urban" or "rural," to more micro-spaces, such as clinical settings (Andrews et al. 2007). Rooted in landmark studies by Golant (1972), Rowles (1978) and Warnes (1982), Geographical Gerontology has evolved from primary concern with abstract spatial expressions of older people, such as location, movement and related services-a perspective that, as within Population Geography (Chapter 1), was criticized for over-empirical emphasis. It now engages with geography's role within experiential, theoretical, 


\section{AGEING AND MORTALITY}

and differentiated dimensions of ageing (Andrews et al. 2007; Rees and Hardill 2015). Scholarship now "emphasize[s] the importance of space in constructions of ageing" (Hardill 2009: 1, emphasis added), not reducing it to backdrop.

Focus on senior lives across space must attend to the ageing body. Just as disability studies have been critiqued for ignoring the body in their desire to emphasize the social construction of disability (Box 10.1), studies of ageing must not neglect the corporeal. For example, besides ill health, bodily ageing is the main way energy impairment or anergia-a condition of lethargy or lack of physical activity "analogous ... to fatigue but ... a more persistent state" (Maurer et al. 2009: 145)-occurs. Whilst lack of energy and fatigue in old age should not be assumed and where present often indicates serious health problems (Avlund 2010; Cheng et al. 2008), it is a truism that as people get older, bodies age (10.1). Parts may be replaced-artificial limbs, transplants, medical devices such as heart pacemakers-and technological aids such as mobility scooters facilitate mobility, but the overall effects of ageing see body parts gradually wear out. Consequently, ageing is likely to be associated with slowing down, loss of bodily resilience and increasing anergia. Whilst "old age" is no disability (Wiles and Allen 2010) and representations of ageing bodies are infused with ageism (Hockey and James 2003), processes of bodily ageing cannot be denied (Andrews 1999). For Population Geography, this appreciation links to older people's (im)mobilities and migrations and the overall importance of accessibility for everyday living. However, such mobilities do not reflect only such bodily concerns (Rees and Hardill 2015; Warnes 1992).

\subsubsection{Third Age migrations}

Third Age seniors are often difficult to distinguish from younger adults (Stockdale and MacLeod 2013). Both can be predominantly represented as mobile and healthy. Even an assumption of Third Age seniors experiencing time supposedly unshackled by work obligations and filled with hobbies and leisure-mostly only relevant to social welfare societies of the Global North, of course-is increasingly problematic. Even in the wealthiest countries and/or those with highest levels of social welfare, older people are frequently engaging with paid employment. Indeed, such engagement is increasingly regarded as necessary as rising elderly dependency (10.2.2.5) impacts on the ability of social welfare to support the senior population (Green 2009). Besides paid work, many older adults also provide productive but volunteer services (Droogleever Fortuijn et al. 2006). Hardill and Baines (2009) categorized such work in "Brightville," England, as falling within two categories: "giving alms," helping "others" perceived to be in greater need or needing greater assistance, and "giving to each other," supporting neighbors within the same community who share similar experiences.

Yet, in spite of the geographical constraints that both paid and unpaid work may impose, a distinctive feature of the Third Age in the Global North is prevalence of lifestyle migrations, both internal and international (Warnes 1992). Chapter 8 introduced such migrations, reflected in a distinctive retirement curve (Rees and Hardill 2015) within the Model Migration Schedule (Figure 7.1). They revealed more rural, coastal and climatically warmer (Sunbelt) destinations having special appeal for the retired, expressed in "permanent" relocations. Return migration (7.5.4) was also seen with a retirement dimension. Chapter 8 further noted older people's involvement in more "temporary" mobilities, such as accessing second homes and the novel mobilities of Snowbirds or Grey Nomads. Nonetheless, all of sometimes several later life migrations are unlikely to relate solely to lifestyle issues. Consequently, Plane and Jurjevich (2009) argued that the term "retirement migration" applied to all migration of those past their main working years was often inappropriate. It failed to convey the diversity and sequencing of moves ageing adults make in later life, not least in the Fourth Age.

\subsubsection{Fourth Age mobilities and immobilities}

In contrast to the Third Age, Fourth Age elderly are most likely to be unambiguously economically retired. They can also be predominantly represented as relatively immobile and less healthy overall. With bodily ageing, the microgeography of the home is likely to assume heightened significance within everyday life (Milligan 2009). This is reinforced, for example, by otherwise independent adults forfeiting driver's licenses. The home is likely to require reconfiguration to fit more limited mobility and work potential, as well as care demands (Dyck et al. 2005). Table 10.1 lists various housing options for adults needing assistance, due to such things as declining eyesight and impaired physical mobility. In the US in 2009, around 4.1 percent of the population aged 65+ years lived in institutional settings and 2.4 percent in housing with at least one supportive service for seniors (Administration on Aging 2010). 
Table 10.1 Residential options for adults needing regular assistance

\begin{tabular}{ll}
\hline Residential form & Description \\
\hline $\begin{array}{l}\text { Continuing-care Retirement } \\
\text { Communities }\end{array}$ & $\begin{array}{l}\text { Living within distinct community, moving into increasingly supportive accommodation as } \\
\text { needs increase } \\
\text { Accessory Dwelling Units }\end{array}$ \\
$\begin{array}{ll}\text { Independent living units attached to another property, usually of family member ("granny flats") } \\
\text { Congregate Housing }\end{array}$ & $\begin{array}{l}\text { Public housing, typically with shared spaces, such as living rooms, dining areas and laundry } \\
\text { rooms }\end{array}$ \\
Board-and-care Homes & More institutionalized Assisted-living Facilities \\
Foster Care & Hosts care for small numbers not requiring continual attention \\
Nursing Homes & Institutions providing 24-hour services to very frail \\
\hline
\end{tabular}

Source: adapted from Brossoie (2008).

With everyday mobilities much more problematic than in the Third Age, there is the migratory tendency, again most notably in the Global North, for Fourth Age "downsizing" into smaller housing. Migration becomes more associated with residential mobility (Chapter 6) than lifestyle (Warnes 1992), although it encompasses repatriation of former international retirement migrants (Darlington et al. 2015; Hardill et al. 2005), for example. From Table 10.1, there is relocation into the Accessory Dwelling Units (Brossoie 2008) known as "granny flats," attached to but relatively independent of a son's or daughter's residence, or to explicitly-planned retirement communities. The latter range from independent single households to later-life care facilities, such as assisted living where residents live independently with help, to nursing facilities oriented to needing constant help. Within all such housing, much faith is often placed in a range of technologies embedded in mobilities, such as alarms summoning help easily triggered by residents or sensors measuring movement, kitchen safety or falls (Courtney et al. 2008).

Chapter 6 indicated the range of Fourth Age assistance migrations. Most notably, residential migration into nursing homes and other institutionalized care is common across much of the Global North. These often result from poor health, infirmity and lack of close family nearby combining with cultures less accepting of extended family arrangements than across much of the South (UNFPA 2012b). Cost considerations can play a key part, as can fears about living in particular neighborhoods. Overall though, whether chosen or more forced, life within retirement communities can be very rewarding and grounded. As McHugh (2007: 295) observed:

Retirement communities display exceptional propinquity, animated by the mutually reinforcing processes of generational consciousness and spatial proximity. Each day, senior citizens act, interact and converse in myriad settings in retirement enclaves. Of similar age, ethnicity and social class, they hold much in common. It is not only similar backgrounds, life histories and migration selectivity at work here, for engaging with each other on a daily basis forges a collective identity and spirit that is palpable. Residents act and speak of who they are, both to themselves and to "outsiders" via volunteer work, voting in local, state and national elections, and articulating and defending their beliefs and values.

To summarize, in a seminal article Litwak and Longino (1987) proposed a developmental perspective model of later life migration. They described three major types of moves made by older adults in the US: "(1) [R]etirement/amenity movers who are wealthier, married, and in better health, (2) [M]oderate/chronic disability movers who are poorer, widowed, and older and need informal caregiving, and (3) [M]ajor disability movers who are moving to an institution" (Conway and Houtenville 2003: 312). Testing Litwak and Longino's (1987: 268) findings that older movers to Florida were amenity seeking, "young" elderly, healthy, and often married, while moves from Florida were largely older, less healthy and/or disabled, and frequently widowed assistance migrants, Bradley (2011) found confirmation from 2005-2007 American Community Survey data. The Fourth Age largely involves the latter, Litwak and Longino's categories two and three. 


\section{AGEING AND MORTALITY}

\subsubsection{Accessibility, care and mobilities}

Accessibility to and within the residential environment, from community scale down to housing unit level, is a key consideration for ageing adults. Community scale challenges include accessing public spaces and resources, such as parks, community centers, shops, health care facilities and, often linked to all these, transportation. At the housing unit level, the (semi-)private space of the home must be accessible for everyday needs and uses. Inadequacy in any of these accessibilities may prompt residential relocation. Being more towards the forced than voluntary end of the spectrum, such migration is something socially responsible planning and provision would be expected to mitigate within overall landscapes of care (Milligan and Wiles 2010).

Particular spaces become associated with accessibility challenges: inner-city residence on higher floors of apartment blocks proves problematic if elevators are regularly out of order; living in a food desert (6.3.4) or a neighborhood largely devoid of specific services may prompt out-migration, especially if transport poor (6.2.1). Stafford (2009: xv) asked in Elderburbia if suburbs are "a good place to grow old?" While young families flocked to US suburbs and suburban homes in the 1950s (Box 3.3), families have now aged in place and Stafford argued that such locations are now unsuitable, reinforcing isolation if unable to drive. Development styles such as cul de sacs limit public transportation access and the ability to escape the isolation of their design. He observed: "where every yard is fenced, houses without porches are set back from the sidewalk, and garage doors open and close at the flick of a button, elders (and others) are totally relieved of the need for or opportunity of interacting face-to-face with neighbors" (Stafford 2009: xvi). Yet, older suburban residents are still expected to keep properties tidy and smart. Consequences of being alone can be even more acute for seniors living in remote rural areas, often in isolated houses. This is a worrying implication from counterurban retirement migration (8.4.2). Indeed, the UK has a campaigning website encouraging older people in the countryside to think through more proactively the practicalities of a rural retirement (Over the Hill 2014).

In spite of accessibility challenges, often exacerbated by death of a partner, independent senior living remains common across the Global North, even in the Fourth Age. Institutionalization or migration to retirement communities is not the majority experience (Hunt 2005). Instead, seniors remain in their own homes, with familiar place surroundings-environment, acquaintances, friends and possessions, often acquired over a lifetime. Rowles (1983) saw ageing identities strongly linked with place, "homes ... conceived of as ... containers of memories and a locus of the elder's identity" (Cutchin 2001: 31). Nonetheless, whilst independent living is often the preferred option, for others the financial burden of moving to a care facility is prohibitive and residential immobility is less freely chosen.

For all seniors, particular attention must be given to geographic accessibility to health facilities, which is often poor (Santana 2000). This is another area of scholarship in which Geographers make significant contributions (Andrews et al. 2007). Access to health facilities becomes a key driver of choice of living environments, as well as an important component of local and national policy. Where facilities are situated close to ageing populations, ability to access (transportation) and the types of services offered are decisions of considerable importance.

One may also contrast within eldercare formal caregiving, provided by clinics or other care facilities, and informal care, given by family and friends (Joseph and Hallman 1998). Geographically, countries at different stages of the Demographic Transition (4.2.2), with different socio-economic and geographical (e.g. urban versus rural) profiles and family cultures, face different challenges (or similar in different degrees) in formal versus informal health care provision. Climo (2000: 695) considered "[t]he first and most important difference in eldercare between rich and poor countries [being] that people from rich countries look to government and public agencies for assistance ... whereas in poor countries, the family in its many varieties assumes almost total responsibility." Relationships are complex within this generalization, however. In Canada, for example, 80 percent of care giving to ageing adults is informal (Joseph and Hallman 1998). Such assistance is also strongly gendered, women dominating provision.

Socio-spatial dynamics are also changing care provision (Milligan and Wiles 2010). With ageing parents often living long distances from employed children, care-giving challenges grow. Relatives living closest typically shoulder most of the load for daily activities, such as shopping and bathing (Joseph and Hallman 1998). Filial obligations to care for ageing parents, including co-residence or remittances, can significantly stress families separated through migration. Further, rapid urbanization and increasing rates of migration can undermine cultures in which elder status is associated with knowledge and wisdom. Consequently, "the elderly may become devalued as they lose control over information, religious ritual, and esoteric knowledge, all valued resources" (Climo 2000: 699). In Puerto Rico, for example, changes in family structure brought about by declining family size and increased migration has altered ageing

\section{ST PROOFS: NOT FOR DISTRIBUTION}


care. Increasingly spatially spread-out families and changing migration patterns of women prompt greater demand for non-family care that was once informally provided. This necessitates new policies to close the gap between family care and unmet need (Zsembik and Fennell 2005).

\subsubsection{A dependent population?}

Previous sections have demonstrated that whilst it is important not to over-generalize or be too definitive, senior life course stages tend to demonstrate various degrees of distinctiveness from those of younger adults. Consequently, statistical measures have been devised to represent anticipated impact of seniors on younger age groups within a population (Kinsella and Phillips 2005). Most neutrally, the ageing index expresses the number of people aged 65 years or older per 100 children under 15 years.

More controversial is the gerontic dependency ratio, which is the number of people aged 65 or older per 100 people aged 20 to 64 in a given population. This measure is used to suggest the economic consequences of having an aged population, being the ratio of the assumed "dependent" elderly population to the "independent" working age population (Weeks 2005).2 Table 10.2 gives the highest and lowest gerontic dependency ratios recently estimated by the World Bank. High ratios are dominated by Northern countries with enduring low fertilities (e.g. Italy), high life expectancies (e.g. Japan) and high out-migration of working age people (e.g. Bulgaria). In contrast, low ratios are found for countries with low life expectancies (e.g. Afghanistan) and high employment in-migration (e.g. Gulf States; Box 7.4).

There are a number of problems in interpreting the ratio of older to younger people in terms of dependency (Gould 2015; Kinsella and Phillips 2005; Spijker and Maclnnes 2013). Critically, it fails to account for older adults who continue to be economically active and are thus far from dependent. In this respect, age 65 years may be seen as much too low a threshold, and the index might be better focusing on Fourth Age seniors. The idea of elderly dependency can also be seen as very much a Global North perspective infused with ageism (Box 2.2), which assumes a degree of formal exclusion from waged labor-for seniors on "retirement" (and for children)-that is simply not a practice common across the Global South. Instead, where "multigenerational households are the norm" (Gould 2015: 188), seniors change rather than abandon their family contributions, taking important roles at harvest and other busy times, for example, and helping the extended family unit with childcare and so on. Integrated within the family unit, seniors are supported through it rather than given the more independent and separated position dependency suggests. Consequently, presenting "the elderly as 'burden' and 'dependent' is ... the exception rather than the norm" (Gould 2015: 187). Evidence from gerontic dependency ratios alone is insufficient to compare empirically the consequences for countries of having ageing populations.

In conclusion, whilst measurement and talk of dependency may not be a useful way to approach elderly lives, Section 10.6 .1 below will nonetheless emphasize how a geographically uneven ageing population still presents complex and major global challenges for the twenty-first century (Dorling 2011: Chapter 7). Both migration and mobility are implicated in numerous ways within this, as are changes in the geographies and other patterns of mortalities to which the chapter now turns.

Table 10.2 Selected gerontic dependency ratios by country, 2013

\begin{tabular}{llll}
\hline High gerontic dependency ratio 2013 & Low gerontic dependency ratio 2013 \\
\hline Latvia & 27.9 & United Arab Emirates & 0.5 \\
France & 27.9 & Catar & 1.2 \\
Portugal & 28.2 & Bahrain & 2.9 \\
Bulgaria & 28.8 & Kuwait & 3.2 \\
Finland & 29.5 & Oman & 3.8 \\
Greece & 29.9 & Eritrea & 4.1 \\
Sweden & 30.3 & Saudi Arabia & 4.2 \\
Germany & 32.1 & Rwanda & 4.3 \\
Italy & 32.6 & Burundi & 4.5 \\
Japan & 40.5 & Afghanistan & 4.6 \\
\hline
\end{tabular}

Source: World Bank (2014). 
280 AGEING AND MORTALITY

\subsection{MORTALITY: MEASUREMENT AND GLOBAL TRENDS AND PATTERNS}

\subsubsection{Measuring mortality}

[I]n our mortality, we collectively lay down a record of our lives.

Every day on Earth, approximately 153,000 people die (WHO 2014b): almost 6400 per hour! As with births (Chapter 4), deaths are major occurrences for all involved, significant at a range of socio-spatial scales: family, community, nation, global humanity. They are typically memorialized through ritual behaviors such as ceremonial gatherings marking burials and cremations. Death marks the end of an individual's life course, the culmination of a life across space, the ultimate bodily immobility.

Within Demography, mortality expresses "the frequency with which death occurs in a population" (Poston and Bouvier 2010: 391). As with fertility, statistics-based measures have been devised to understand better its patterns and trends (Holdsworth et al. 2013: 10, 76-78; Poston and Bouvier 2010: 112-120; Weeks 2005). Four are particularly useful but increase in both sophistication and data demands as one proceeds through them.

First, Crude Death Rate (CDR) expresses total number of deaths occurring in a specified population over a specific period of time. For a given year it is calculated thus: y

CDR uses the estimated mid-year population of the area of interest as denominator and, paralleling CBR for fertility (4.1.2), is usually presented per 1000 people. As a rate, CDR is comparable across countries and localities. However, it does not provide any indication of who is dying or why, nor does it allow for age composition of a population (expressed in population pyramids; 3.2.2).

To account for population age structure, mortality rates can be "adjusted" or "standardized." This gives a second measure of mortality: Age Specific Death Rate (ASDR). Again as with ASFRs (4.1.2), ASDRs are usually calculated for 5-year groupings. Of particular note is Infant Mortality Rate (IMR), the ASDR for those aged under 1 year. IMR is "one of the strongest indicators of a country's wellbeing, as it reflects social, economic and environmental conditions in which children (and others in society) live, including their health care" (Alderman and Behrman 2004: vii). It is returned to below.

The third measure, Age Adjusted Death Rate (AADR) is derived by adding together all the ASDRs weighted by the proportion of the total population within that age group. AADR gives a clearer picture of overall mortality across geographic areas because it allows two populations to be more meaningfully compared by accounting for the population structure of each country (Weeks 2005).

Table 10.3 brings CDR, ASDR and AADR together for the example of Norway. It reveals Norwegian men having lower CDRs than Norwegian women, which might lead to a suggestion that men are living healthier lives. ASDR analysis, however, suggests the opposite. It shows that except for the very young, where boys and girls have similar death rates, men have consistently higher death rates. This gender difference is commonplace globally. Here, it leaves much greater proportion of Norwegian women in the aged categories, where death rates are extremely high for both sexes. In other words, higher female CDR is explained by the skew in the Norwegian female population relative to the male population in favor of older age categories. This conclusion is reinforced by much higher AADR for Norwegian men.

A fourth statistical measure related to mortality is Life expectancy $\left(\mathrm{e}_{x}\right)$. This is calculated using life tables (Holdsworth et al. 2013: 78-84; Poston and Bouvier 2010: 120-125) that "express the chance of dying at particular ages and the numbers that would survive, if a particular set of ASDRs were held constant for a population" (Holdsworth et al. 2013: 79). From these tables, life expectances for particular ages can be calculated, the most utilized being life expectancy at birth $\left(\mathrm{e}_{0}\right)$ : years, on average, an individual would expect to live if their age cohort experiences existing AADRs. Importantly, $e_{0}$ does not indicate any maximum age for a country's population but average mortality risk. A country with low $e_{0}$ could still have many very elderly people, as the low value may be due to a very high infant mortality (IMR). Table 10.3 shows $e_{0}$ higher for Norwegian women than men, reinforcing the ASDR and AADR findings of consistently higher male death rates. 
Table 10.3 Mortality rates, Norway 2013

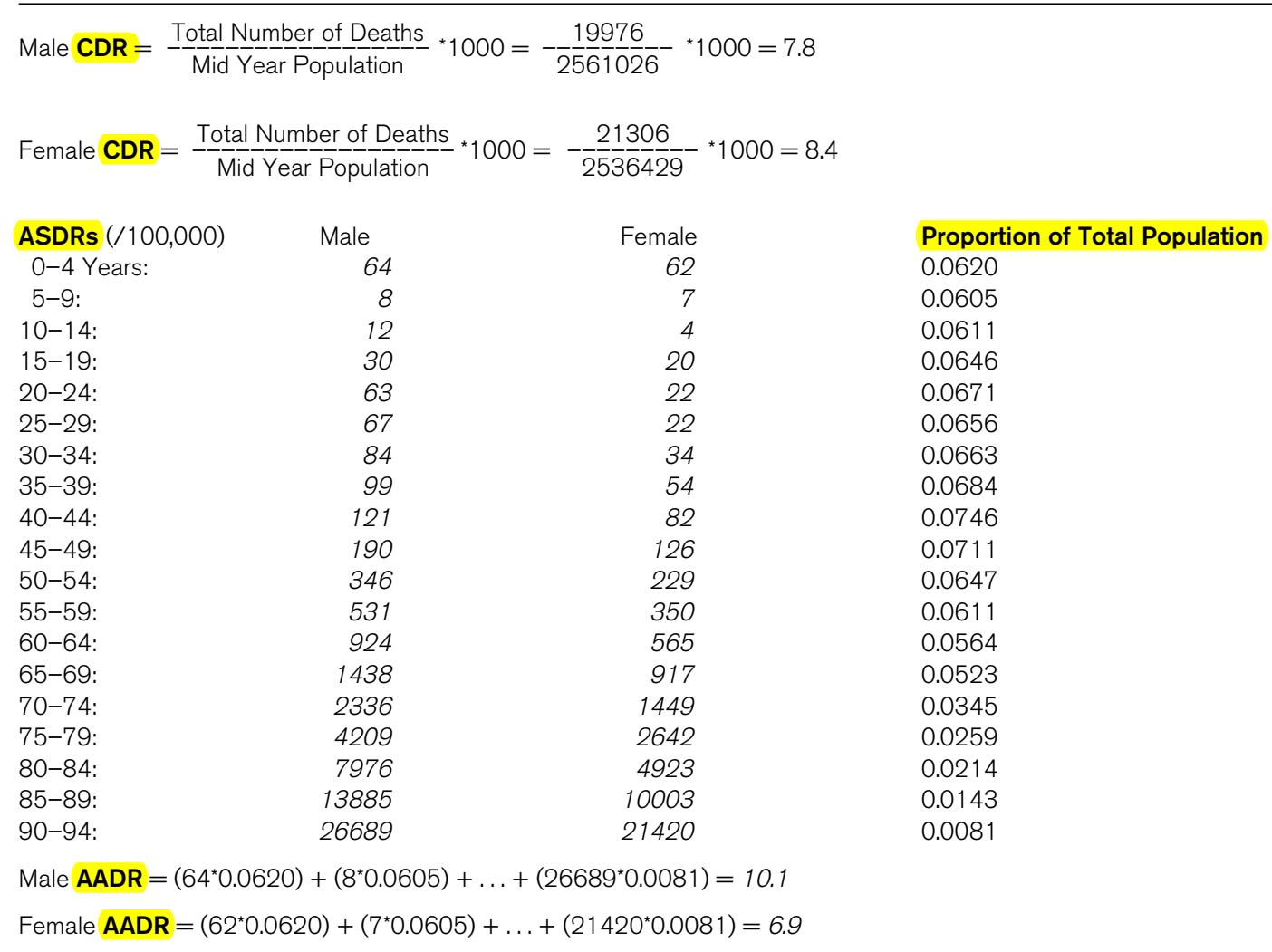

Life Expectancy at Birth (e0): Men $=79.7 ;$ Women $=83.6$

Source: http://www.ssb.no, accessed September 2014.

\subsubsection{Global mortality patterns}

\subsubsection{Global death rates}

Having addressed measurement, a next step is to explore how CDR and AADR vary globally. Figure 10.2 shows the national scale CDR pattern for 2010. It demonstrates geographical unevenness. Countries in Sub-Saharan Africa, Eastern Europe and Russia have relatively high CDRs, while those in North Africa and much of South America have low values. Western Europe, the US and Canada all have moderate CDRs. It suggests that whilst CDR is partly indicative of poverty, showing broadly positive correlation, this is far from the full picture. A country's stage within the Demographic Transition Model (DTM) (4.2.2) is a critical further consideration, revealed more sharply through AADRs.

Figure 10.3 displays global mortalities according to a country's AADR in 2012. This time a much sharper Global North versus South distinction is clear. The former is characterized by low AADRs. Northern countries with relatively high CDRs in Figure 10.2 but now showing low AADRs, such as Bulgaria, indicate presence of relatively older populations. This is typical of late-stage DTM countries and of substantial international employment out-migration. The Global South has high AADRs, Africa standing out especially strongly. Relatively young population age structures, reflecting DTM stage-which gave some countries low CDRs-are now controlled and the contribution of relatively high poverty in sustaining high death rates more clearly implied. 


\section{Crude Death Rate, 2010}

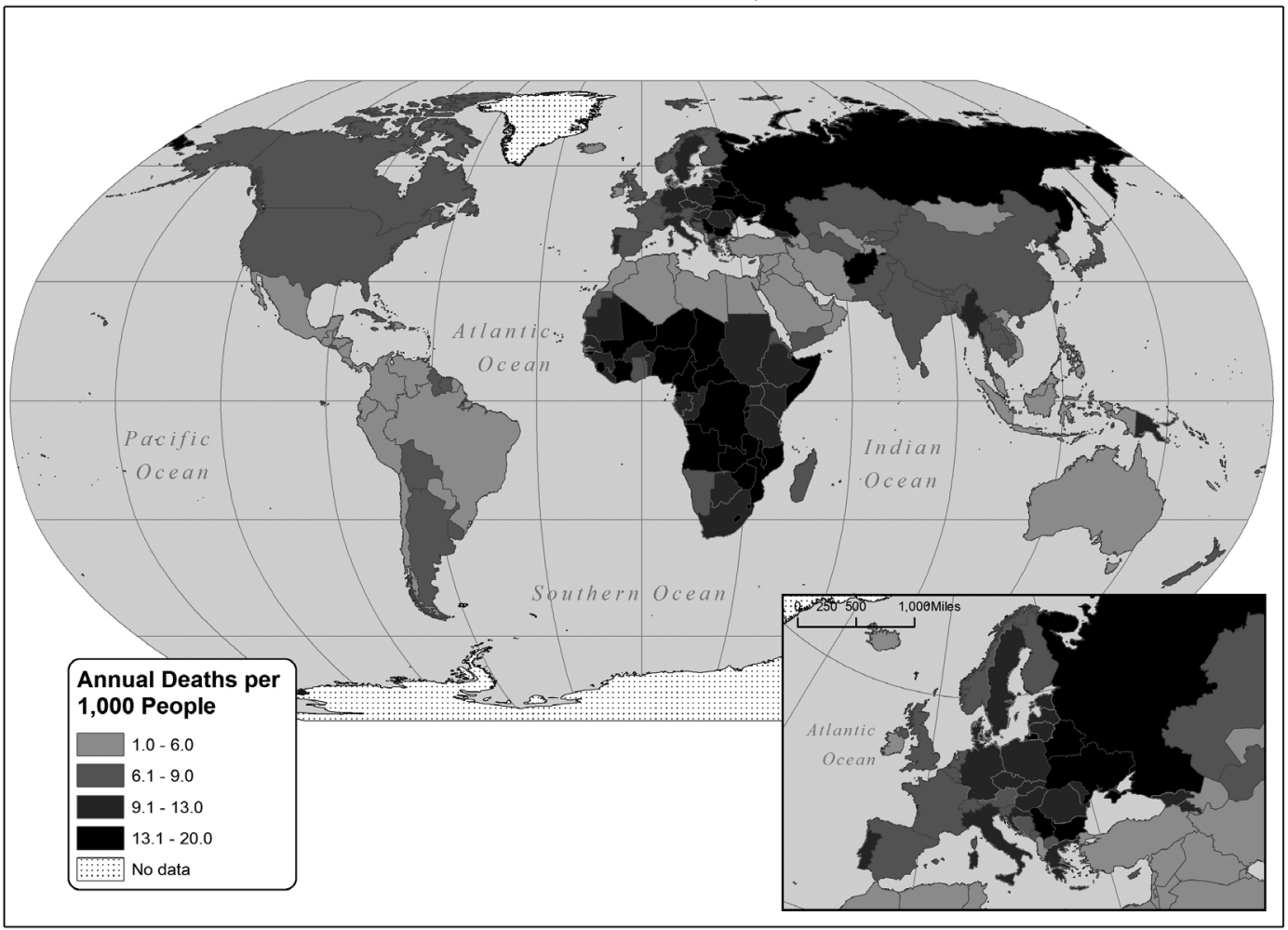

Figure 10.2 Global distribution of crude death rates, 2010.

\subsubsection{Age of death}

The importance of age in explaining death rates can be pursued further through examining global trends and patterns demonstrated by life expectancy, age of death and Infant Mortality Rate (IMR). Globally, life expectancy ( $e_{0}$ ) rose substantially from 48 years in 1950-1955 to 68 in 2005-2010 (UN 2012: xi; Wang et al. 2012). This increase, however, masks a complex global pattern, shown in Figure 10.4. More economically developed regions of the Global North experienced increases from an average of about 67 years in 1950-1955 to nearly 80 years in 2005-2010, compared to African countries' increase from about 37 to 55 years (UN 2012: xi). Such extended life expectanciesand corresponding decreases in premature mortality-are again indicative of progression through the DTM.

A wide range of factors may affect life expectancy (Gatrell and Elliott 2015). Many are considered in the remainder of this chapter. In summary, they can be considered at individual and more collective scales. Individually, experience of poverty is especially important. This is because of the multiplicity of connections between resources-particularly expressed via the arena of class and status-and almost all life course outcomes. Besides generally being required to access such factors conducive to good health as housing and residential environment, monetary resources are often directly required for treating adverse health conditions, including purchasing vital medicines and living aids.

Epidemics and natural disasters can have major localized impacts in reducing $e_{0}$. An ongoing illustration, returned to in Section 10.5.2.4, is the HIV/AIDS pandemic. This has been so severe that it prompted a life expectancy downturn in the 1990s against the twentieth-century upward trend (Wang et al. 2012). Collective declines in $e_{0}$ can also be brought about by economic problems reducing state expenditure on healthcare, rising levels of drug resistance, globalization's heightened interconnections of populations facilitating transmission of diseases and increasing urbanization of the global population (Gould 2015). Notwithstanding these causes of declines in $e_{0}$, Riley $(2001,2005)$ cited six very unevenly developed main factors driving a general trend towards rising life expectancy since 1800: public 


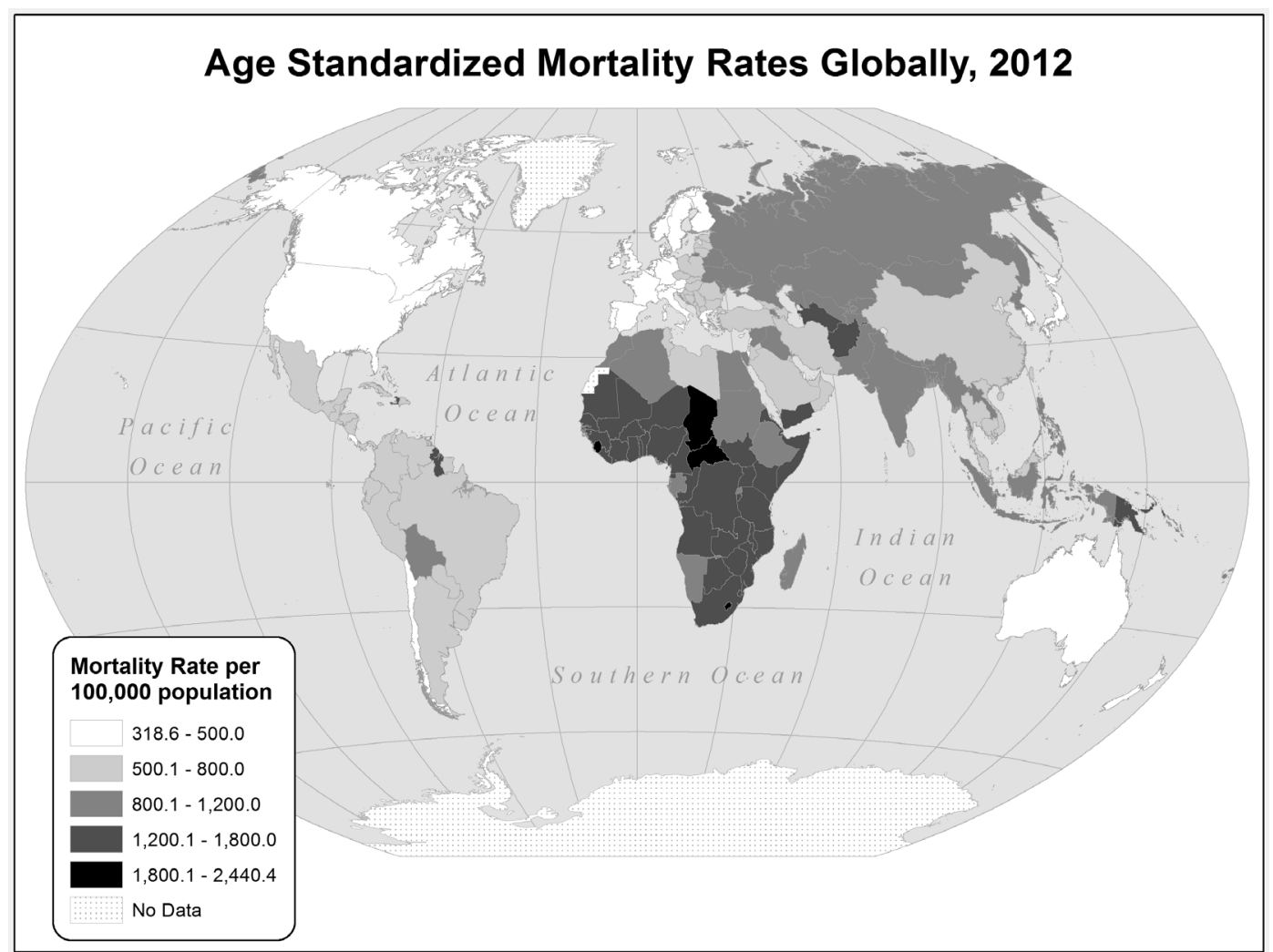

Figure 10.3 Age adjusted death rates globally, 2012

health measures; developments in medicine, including drugs and therapeutic technologies; increased wealth and income; better nutrition; more informed behavior; and increases in education generally.

In the context of this growing global life expectancy, it is unsurprising that most deaths occur at relatively older ages. This is demonstrated in Figure 10.5, which compares deaths by age group for the US in 2013 and South Africa in 2011. The US shows considerable mortality concentration in ages greater than 55 years, almost a third falling in the 85 years and over category. In contrast, South Africa's deaths were concentrated in the 25-74 years band, not least reflecting the devastating impact of HIV/AIDS on working age adults.

The significance of very early age deaths in Figure 10.5, notably for South Africa but still apparent in the US, draws attention to Infant Mortality Rate's (IMR) importance in underpinning life expectancy, both globally and within specific countries. Thus, despite advances in medicine and social support for the elderly, it is decrease in IMR that is most responsible for increasing global life expectancy, shaping its geography, and generating an ageing world.

Figure 10.6 presents a global picture of IMR in 2010, illustrating wide variation between countries (PRB 2015). This again reinforces the chapter's emphasis on strong links between mortality and poverty. Countries with highest rates of infant and child mortality are found in Sub-Saharan Africa, Democratic Republic of the Congo and Central African Republic having IMRs exceeding 100. In contrast, IMRs fell below 2 in Hong Kong, Iceland and Singapore, and were below 5 for most of Europe.

Despite these clear international contrasts, high IMRs are also found within countries with low IMRs overall, not least where there are large disparities in income and health care accessibility. Within the US for example, black infants are nearly 2.5 times more likely to die before their first birthday than white infants (Saenz 2007). Disparities in wealth, access to health care, nutrition and access to prenatal care explain much of this contrast. High IMR countries similarly often demonstrate significant variation by socio-economic status. Subramanian et al. (2006) found strong association in India, for example. 
284 AGEING AND MORTALITY

Global Breakdown of Life Expectancy at Birth, 1950-2010

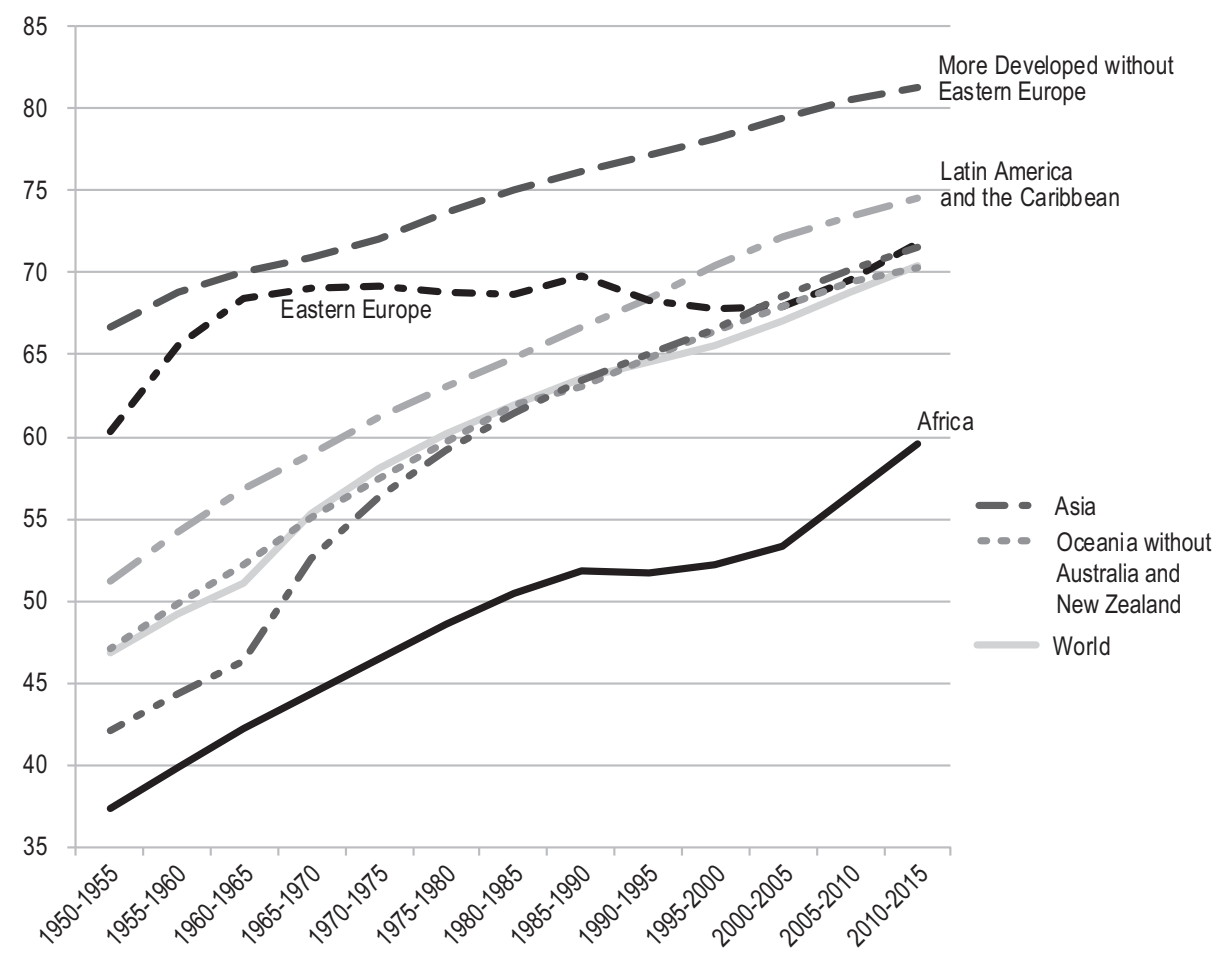

Figure 10.4 Life expectancy at birth by world region, 1950-2010.

Source: UN (2012: xi).

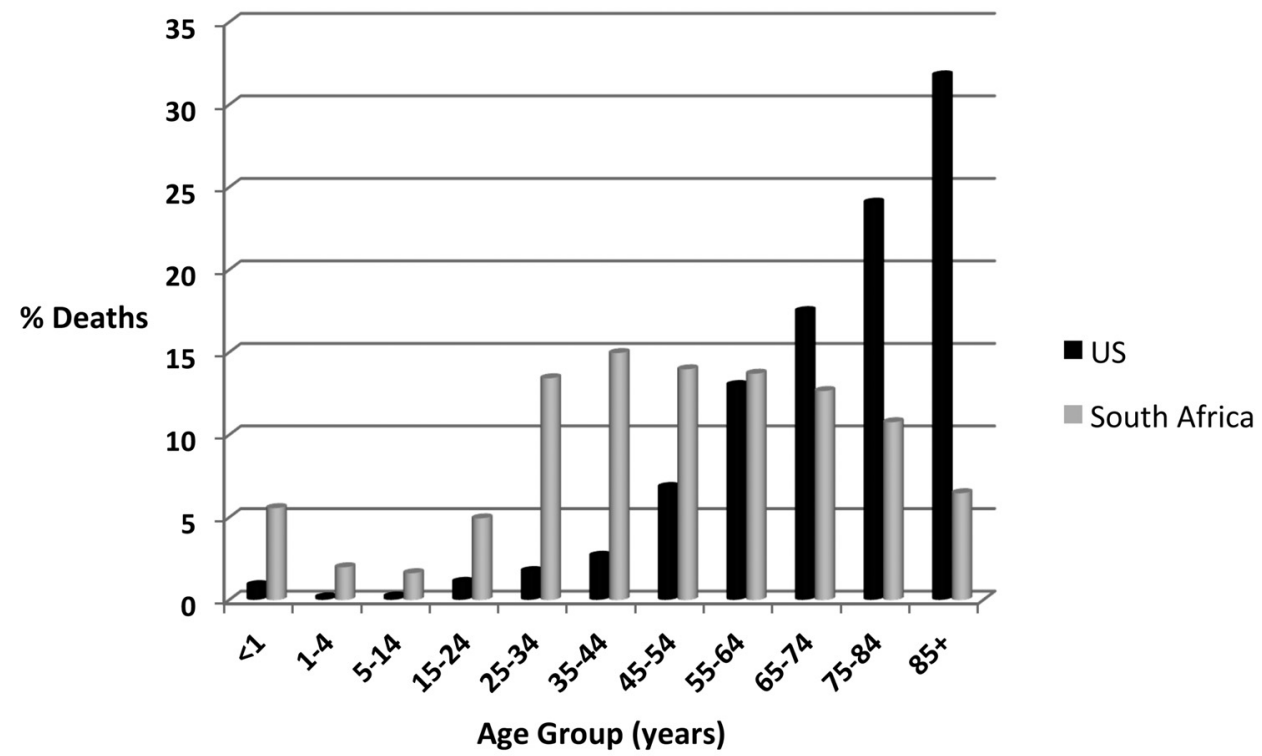

Figure 10.5 Deaths in the US (2013) and South Africa (2011), percentage total by age group. Sources: CDC (2015); Statistics South Africa (2014). 


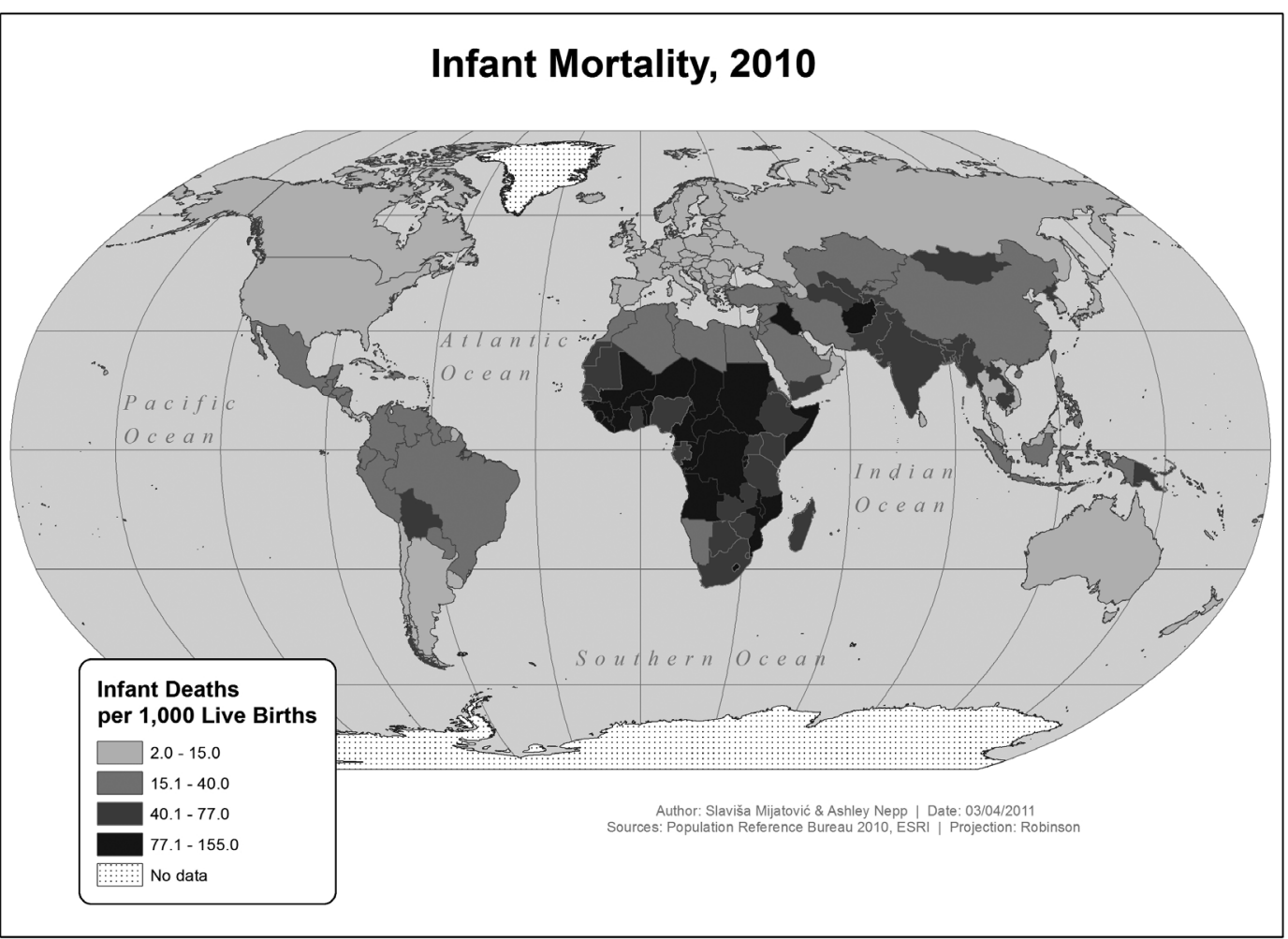

Figure 10.6 Infant mortality rates globally, 2014.

Looking at the causes of higher IMRs today, many infant (and child) deaths are from easily preventable or treatable diseases, including Malaria, dehydration caused by Diarrhea, and Acute Respiratory Infections (WHO 2016). WHO estimated nearly 2.5 million children could be saved annually if vaccinated against six standard preventable diseases: Whooping Cough, Tetanus, Measles, Tuberculosis, Polio, Diphtheria (also Zuehlke 2009). Clearly, achieving this goal is once again a matter of resources.

Circumstances within the home, particularly as they relate to the mother (in the context of mothers as principal child carers), are important influences on IMR (UNFPA 2007b). For example, mother's education facilitates access to and knowledge of information about nutrition, hygiene and health care. Domestic violence against women also has negative impact on children's well-being. Finally, experiences of "adequate antenatal care, delivery assistance, and attention to neonatal health, including breastfeeding, nutrition, rehydration, and immunization" (UNFPA 2007b: 251) are critical in shaping child health.

Away from IMRs, women in childbearing years face additional mortality risks related to childbirth. Factors such as lack of access to prenatal care, delivery outside of a hospital or clinic, and unsafe abortions all significantly increase a pregnant woman's risk of death. Whilst, globally, maternal deaths had declined 47 percent since 1990, they were still estimated at 287,000 in 2010 (WHO 2012). India (19 percent) and Nigeria (14 percent) alone accounted for one third. Thus, a general trend of maternal death rate decline can be attributed in part to improving health care systems, increased female education and access to health facilities (WHO 2012) but maternal mortality and morbidity remain critical human rights concerns (Pillay 2013). Approaches to women's health and health care must incorporate not just pathology-based solutions but also consideration of social determinants of health and mortality. They must, in other words, recognize the full context in which (un)healthy lives across space are produced, an appreciation also sought through attempts to model mortality geographies. 
286 AGEING AND MORTALITY

\subsection{MODELING MORTALITY GEOGRAPHIES}

\subsubsection{Epidemiological transition}

One way to represent mortality is to think about how death rates change historically. Presenting such change as a consequence of development and access to technology consolidates the mortality implications of the Demographic Transition Model (DTM) (3.3), already suggested in Section 10.3.2. Early DTM stages are marked by high death rates, short life expectancies and slow or no population growth (high mortality). Through time, with introduction of public health measures such as clean water and improved sanitation, and medical knowledge and technology, death rates decline. This leads to population growth, as fertility rates remain stable, producing an excess of births over deaths. As development increases, fertility rates decline and the population is characterized by longer life expectancies and slow population growth. However, as previously noted, the DTM is based largely on experiences of Northern countries and may be less applicable to developments across the South today.

Partly responding to critiques of the DTM's general applicability for health, as long ago as 1971 Abdel Omran proposed a specific Epidemiological Transition Model (ETM). Also known as the Health Transition Model (Holdsworth et al. 2013: 25-28), it focuses more on specific causes of death. As Gatrell and Elliott (2015: 129) summarized, the ETM "proposes that at an early stage of development causes of death such as parasitic, infectious, and nutritional diseases account for the burden of mortality, but then ... give way to non-communicable diseases" or "diseases of affluence" (Hunt 2005). This shift is caused by but also brings about increased socio-economic development, a "healthier population ... a cause of development as well as its consequence" (Gould 2015: 85). As Omran (1971: 510) originally expressed it, the ETM "focuses on the complex change in patterns of health and disease and on the interactions between these patterns and their demographic, economic and sociologic determinants and consequences." He recognized three categories of disease determinants: eco-biologic, reflecting environmental factors; socioeconomic, political and cultural, including standards of living and health habits; and medical and public health, a community's ability to prevent and combat disease (Omran 1971:520). Half a century later, each area remains essential for explaining mortality geographies.

An extended version of the ETM is shown in Figure 10.7. Stage 1, Age of Pestilence and Famine, is characterized by low life expectancies and high death rates. Mortality is largely caused by infectious disease, "determined by ... microbial exposures, dietary deficiencies ... illnesses due to inadequate food storage, [and] increased transmission rates and endemic disease as a result of increased population density" (McKeown 2009: 20S). By Stage 2, Age of Receding Pandemics, death rates begin to decline, epidemics become rarer, and life expectancies increase. While

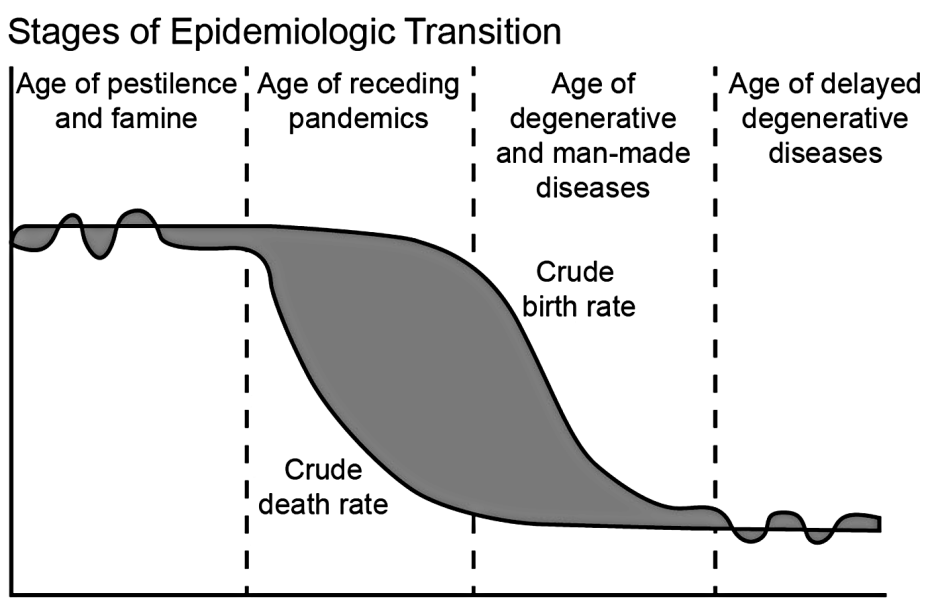

Stages of transition

Figure 10.7 Stages of the epidemiological transition model.

Source: Figure adapted from https://humangeography.wikispaces.com/Medical+Geography+and+Epidemiology. 
little population growth occurs in Stage 1, in Stage 2 it is notable. Changes reflect rising living standards: "improved nutrition, better personal hygiene, and less overcrowding; medical and public health advances and interventions, and ... adoption of antiseptics and pasteurization; lower fertility" (McKeown 2009: 20S), with greater infant and child survival. In Stage 3, Age of Degenerative and Man-Made Diseases, mortality declines to a low level and life expectancies continue to increase. Fertility begins to replace mortality as key driver of population change. Mortality results more from non-communicative degenerative (cancer, stroke, heart attack) sources than infectious diseases. Stage 4, Age of Delayed Degenerative Diseases, proposed by Olshansky and Ault (1986), is characterized by rapidly declining mortality rates for the elderly, deaths from degenerative diseases shifting to older ages. Within Stages 3 and 4 deaths relating to individual behavior and lifestyle become more prominent: "accidents, alcoholism, suicide, ... homicide, ... smoking and diet" (Poston and Bouvier 2010: 136).

Omran (1971: 532-533) also proposed two variations on the core ETM: "accelerated" and "delayed" models. The former envisaged a reduced period of time between high and relatively low levels of mortality, and comparatively rapid progression through Stage 2. Japan and Russia experienced such transition (Phillips 1994). For the delayed model, countries also experience rapid decline in death rates, largely catalyzed by imported public health initiatives, but other changes, such as overall development, improved socioeconomic conditions and fertility decline, lag this drop. Omran (1971) suggested Sri Lanka as an example, with several other countries in South Asia and Africa also representative (Phillips 1994).

Even with these nuances, the ETM is not without critics. First, Caldwell (2001: 79) argued that it focused on social and eco-biological change at the expense of adequately addressing the importance of scientific inquiry, medical technology and public health measures-water purification, sewage treatment, immunization programs-as contributors to mortality decline. Second, within-country variation in progression through the transition is notable. As McKeown (2009) pointed out, income disparities within a country can lead to the poor and poorly-educated experiencing a delayed peak in mortality from infectious disease, for example, as compared to more resourced counterparts. A third critique is closely related to the timing of Omran's original proposition. Originating in an era when infectious diseases were optimistically viewed as largely no longer posing major health threats, subsequent epidemic emergence of diseases such as HIV/AIDS and SARS underscores the need to conceptualize infectious and chronic diseases as enduring within societies, rather than consigned to discrete transition periods (McKeown 2009). Fourth, therefore, transition need not always be uni-directional (2.2.1). Mortality soon increases if standards of living decline (Holdsworth et al. 2013), Russia exemplifying such regression. After the break-up of the Soviet Union, living conditions deteriorated as the economy faltered and social safety nets disappeared. Life expectancy for men, in particular, decreased: 65 years in 1987, 61 by 1998, just 59 by 2000 (Hagga 2000; Heleniak 2002). This was largely attributed to unusually high rates of alcoholism (Shkolnikov et al. 2001), cardiovascular disease and injury (Hagga 2000). By 2013 male life expectancy had increased to 63 years (WHO 2015b), still below that of 1987.

\subsubsection{Spatial perspectives}

Not least due to their sustained prominence, mortality geographies can be imagined and modeled through explicitly spatial perspectives (Gatrell and Elliott 2015). First, maps, the basic tool of geographers, are used widely for understanding both location and movements of diseases. Maps of plague appeared as early as the seventeenth century, Filippo Arrieta creating one in 1694 in Bari in the Kingdom of Naples (Koch 2005: 21). Other famous maps included John Snow's 1855 plotting of cholera cases in London (Box 10.3). Various agencies now regularly produce atlases of disease (e.g. CDC 2016), and international bodies, such as the World Health Organization and United Nations, routinely utilize maps to track spatial inequalities in health, as well as disease diffusion. Novel forms of mapping have also been utilized, including cartograms, whereby a variable such as the number dying from a disease is used to scale a country's size instead of land area (e.g. Dorling 2005 [[AQ]], 2012; Mitchell et al. 2000; Shaw et al. 2008).

Second, while paper maps provided early examples of the importance of viewing disease and death spatially, Geographic Information Systems (GIS) now include sophisticated tools for visualizing and analyzing both disease and mortality patterns and diffusion of cases (e.g. Cossman et al. 2003). As just one example, GIS was deployed to detail clusters of Tuberculosis in Western Cape communities in South Africa (Beyers et al. 1996). Interest in the mapping of diseases and mortality is further stimulated by the flows of people and objects that are a hallmark of the era of mobilities (5.2.1). Koch (2005: 15) noted: "[i]t is no accident that medical mapping blossomed in the nineteenth

\section{ST PROOFS: NOT FOR DISTRIBUTION}




\section{BOX 10.3 EARLY EPIDEMIOLOGICAL MAPPINGS}

A now taken-for-granted way to begin to understand a particular disease and/or its associated mortalities is by mapping outbreaks or cases, whether or not epidemic. The practice began just over two centuries ago with Seaman's 1798 "spot map" of a Yellow Fever outbreak in New York City. The most famous pioneer of epidemiological mapping, though, is John Snow's representation of a Cholera outbreak in London, published in 1854. This map, reproduced from a recent format below, showed clear clustering of cases around a communal pump on Broad Street in Soho used for public water supply. The pump, as a consequence, became prime suspect for the source of the outbreak.

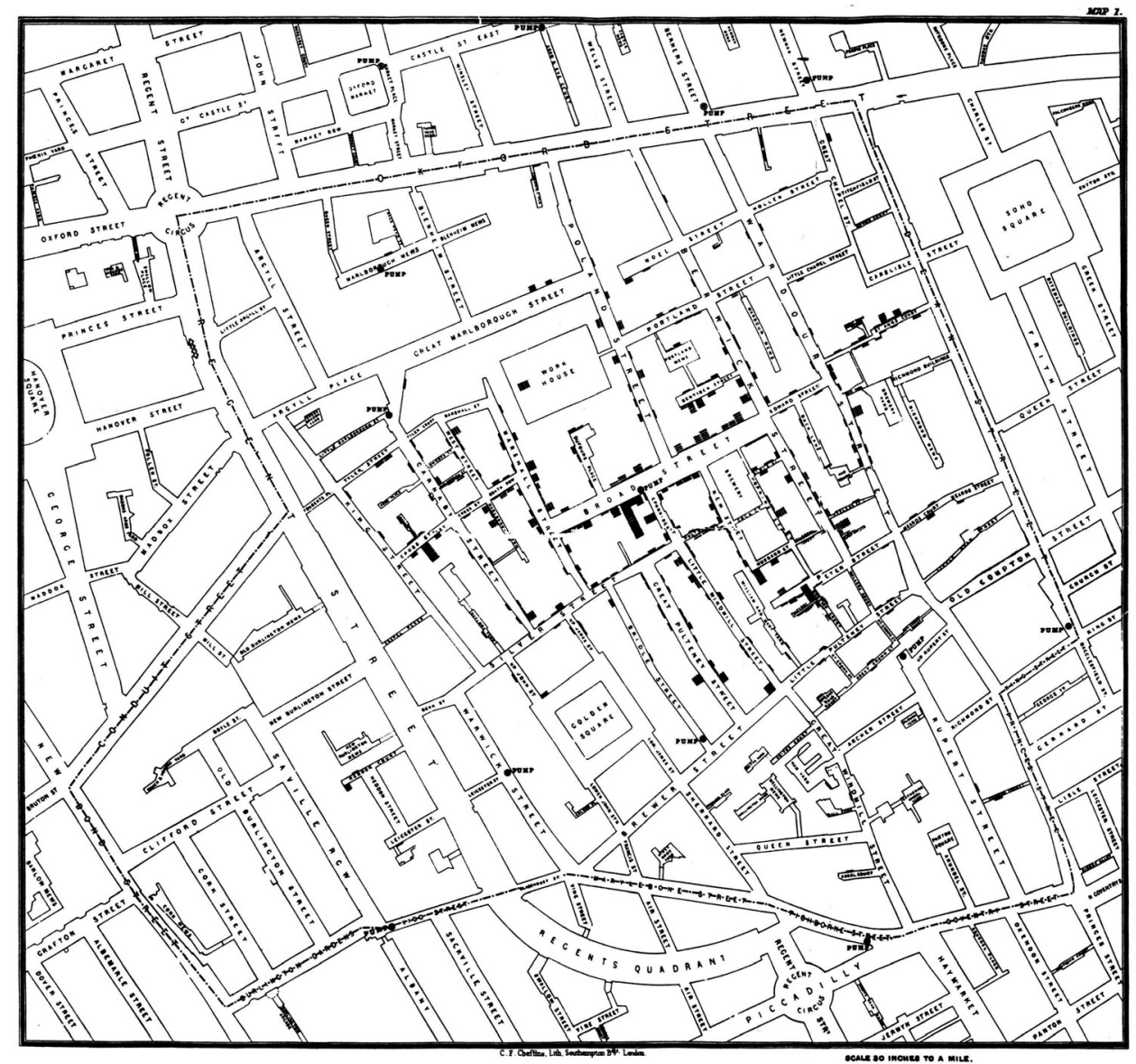

Location of 1854 cholera cases in Soho, London, and the location of water pumps.

Source: original map by John Snow showing cholera case clusters in London epidemic of 1854, drawn and lithographed by Charles Cheffins. From https://en.wikipedia.org/wiki/John_Snow, accessed 2 May 2016.

Snow's map gave support to the emerging idea that Cholera, a major cause of death, was spread via contaminated water. Consequently, the map was used to underpin calls for improved sanitation of Victorian drinking water. Although, contrary to myth, Snow's actions to get the pump handle removed (to stop mactually have done little to halt an outbreak already in decline, the incident presents a lasting "public health 'parable'" (Curtis et al. 2010: 327) on the vital importance of better everyday hygiene for health and to reduce mortality. Snow's map, in whatever form, has allowed him to remain "medical geography's hero" (McLeod 2000: 926)!

(Sources: Curtis et al. 2010; McLeod 2000; Rican and Salem 2010) 
century during a period of vastly increasing international trade and emigration, or that its renaissance began in the 1990s during a new era of globalization."

Third, to visualize the resulting connections between and within complex and extensive data sets, geovisualization tools provide increasingly powerful resources for researchers (Koua and Kraak 2004; MacEachran et al. 2004). These combine multiple ways of viewing the same data, linking tabular views with graphics such as charts and maps, to enable scholars to explore relationships within data. Hans Rosling's Gapminder (2014) provides a popular example of how dynamically linked data can both be presented in engaging ways and reveal new insights into mortality and health. Figure 10.8 shows a Gapminder representation of global Tuberculosis death rates, with the countries standing out with high rates towards the top of the diagram all located in Sub-Saharan Africa.

\subsubsection{Multivariate statistics}

As with fertility and, especially, migration, complex multivariate statistical techniques are also utilized to analyze the relative importance of variables anticipated to be associated with mortality (Gatrell and Elliott 2015). These enable researchers to explain statistically observed death rates and predict rates for other situations. Cluster analysis, for example, has been used to show up groups of places where from a mortality perspective it is unwise to live in England and Wales (Shelton et al. 2006)!

A further UK example analyzed successive waves of data from the British Household Panel Survey between 1993 and 2008 to discover if relocation to residential or sheltered housing for people aged 65 years or over was associated with enhanced death rates (Robards et al. 2014). Binary logistic regression examined the strength of associations between mortality and different housing transitions. For control, it also modeled mortality's associations with a variety of socio-demographic variables linked to death rates. Table 10.4 presents a summary of the results. Statistically significant odds ratios-chance of an event occurring in one group as compared to occurring in anothershowed mortality probability: increasing with age; lower for women than men; higher for never married than for those married or cohabiting; increasing as health declined; and relatively unrelated to past employment or access to a car.

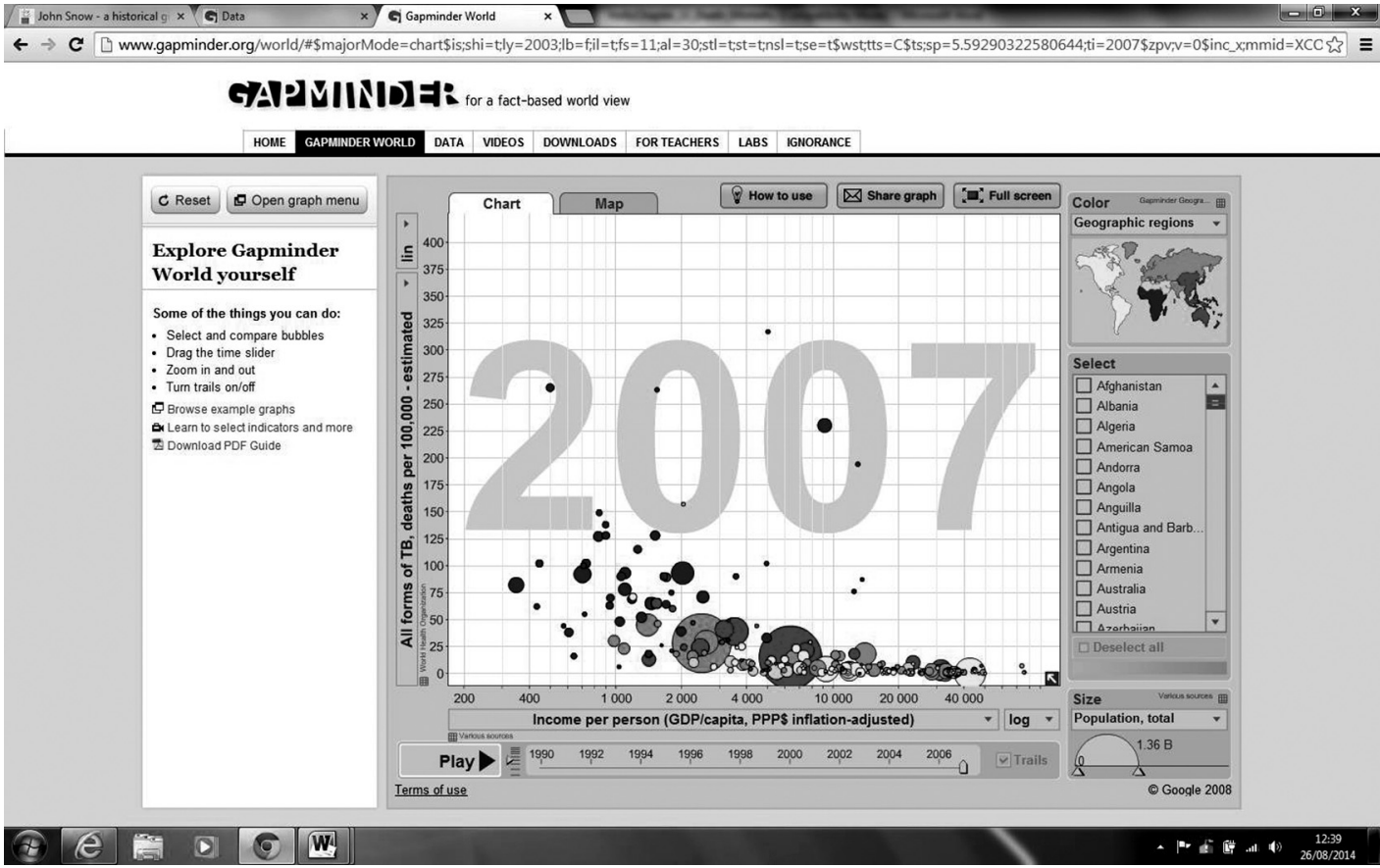

Figure 10.8 Screenshot of Gapminder graph for rates of tuberculosis deaths globally.

Source: www.gapminder.org/world/, accessed August 2014. 
290 AGEING AND MORTALITY

Table 10.4 Odds ratios for mortality by key mortality covariates

\begin{tabular}{|c|c|c|}
\hline Variable & Odds ratio & Significance \\
\hline \multicolumn{3}{|l|}{ Age } \\
\hline $65-74$ years & 1.00 & \\
\hline $75-79$ years & 1.87 & 0.000 \\
\hline $80-84$ years & 2.81 & 0.000 \\
\hline $85-89$ years & 4.97 & 0.000 \\
\hline $90+$ years & 8.34 & 0.000 \\
\hline \multicolumn{3}{|l|}{ Sex } \\
\hline Male & 1.00 & \\
\hline Female & 0.59 & 0.000 \\
\hline \multicolumn{3}{|l|}{ Marital status } \\
\hline Married or living as couple & 1.00 & \\
\hline Widowed, separated or divorced & 1.11 & 0.227 \\
\hline Never married & 1.38 & 0.014 \\
\hline \multicolumn{3}{|l|}{ Health status } \\
\hline Excellent & 1.00 & \\
\hline Very good/good & 1.13 & 0.364 \\
\hline Fair & 1.78 & 0.000 \\
\hline Poor/very poor & 3.62 & 0.000 \\
\hline \multicolumn{3}{|l|}{ Socio-economic employment } \\
\hline Non-manual & 1.00 & \\
\hline Manual and unskilled & 1.16 & 0.053 \\
\hline Never had a job & 0.75 & 0.095 \\
\hline \multicolumn{3}{|l|}{ Access to a car } \\
\hline Yes & 1.00 & \\
\hline No & 1.19 & 0.057 \\
\hline \multicolumn{3}{|l|}{ Housing transition } \\
\hline No transition-private housing all waves & 1.00 & \\
\hline To residential housing $2+$ years ago & 2.31 & 0.030 \\
\hline To residential housing $1-2$ years ago & 3.72 & 0.000 \\
\hline To sheltered housing $2+$ years ago & 1.78 & 0.121 \\
\hline To sheltered housing $1-2$ years ago & 0.98 & 0.950 \\
\hline
\end{tabular}

Source: simplified from Robards et al. (2014: Table 2).

These associations may be unsurprising but it is useful to have them confirmed statistically. Additionally, Robards et al. (2014) found that after controlling for these variables the probability of dying increased with transition from private housing. However, this association was only statistically significant for moving into residential housing as compared to more independent-living sheltered housing. The overall conclusion, therefore, was that transition from independent home living could prove a major life shock for older people, reflected in increased mortality likelihood.

\subsubsection{Proximate versus background mortality causes}

When examining causes of mortality, scholars emphasize how immediate causes, such as fatal illness, tell only some of the story from a life course perspective. Instead, it is consistently noted how these causes tend to be experienced unevenly within any specific population. In other words, death from pneumonia or suicide, for example, are not random occurrences but are instead rooted in the arenas of differential life course experience. Mortality, like fertility and migration, is highly relational.

The idea that specific causes of death are not for the most part randomly experienced can be represented in the model in Figure 10.9. The immediate cause of mortality-officially recorded on a death certificate, for exampleis known as the proximate cause. This is then seen as being shaped by a range of potential background causes. These 


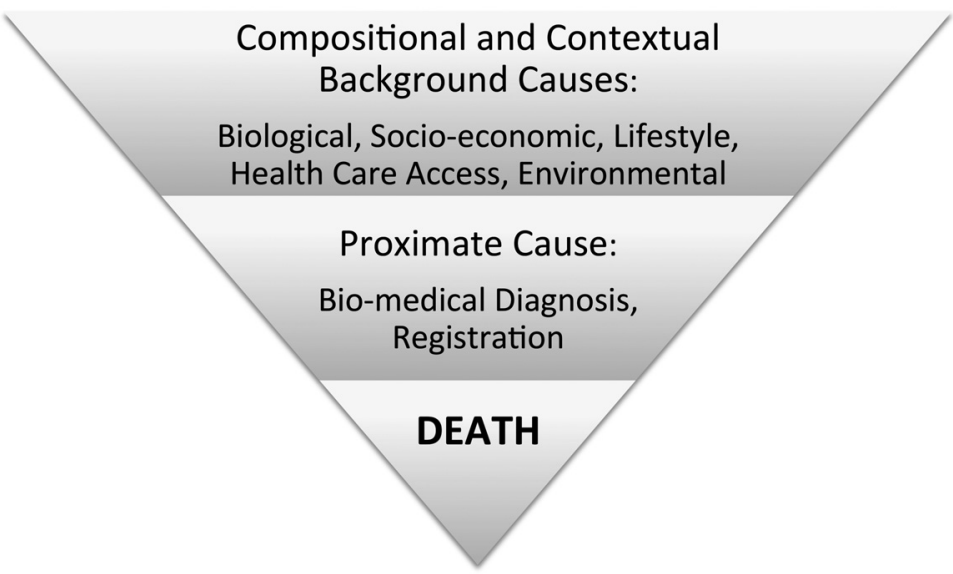

Figure 10.9 Differentiating causes of mortality.

Source: adapted from Gould (2015: Figure 4.4) and Sparks (2012).

express both compositional and contextual factors. The former "focus on individual attributes of people in certain places leading to differences in ... outcomes over space, [whereas the latter] highlight the importance of differences between places as they influence differences in ... outcomes" (Sparks 2012: 255). All background causes of death implicate both compositional and contextual factors, although the former seem most prominent overall (Pickett and Pearl 2001). The distinction is not without problems, however, and is revisited in Section 10.5.5.

In summary, how one dies-although itself not always correctly recorded (Myers and Farquhar 1998)-tends to a greater or lesser extent to reflect who one is and where and when one lives. The considerable importance of socioeconomic factors has already been indicated. Its prominence and also that of the other categories of background factor will now be elaborated on more fully through illustration of the interplay of background and proximate causes in differentiating global mortality today.

\subsection{DIFFERENTIATING MORTALITY: CAUSES OF DEATH}

\subsubsection{Multiple causes, unevenly experienced}

\subsubsection{Causes of global mortality}

Death, while universally experienced, varies dramatically from place to place and from person to person in cause. To get a handle on the numerous proximate causes of death (Poston and Bouvier 2010: 125-134), the World Health Organization (WHO 2014a) produced a three-fold classification: communicable diseases, perinatal, maternal and nutritional conditions; non-communicable diseases; and injuries and external causes. These categories are elaborated in Table 10.5.

Using WHO's grouping of countries (WHO 2014a), Figure 10.10 illustrates the global and regional breakdown of deaths according to the three groups for 2000 and 2012. Just three general observations are made here, as each group, its geography and life course associations are considered in greater detail next. First, in 2012, over two thirds of global deaths were from non-communicable diseases, with communicable diseases a distant second and injuries a further distant third. Death, in short, is mostly not caught from others! Second, between 2000 and 2012, deaths from injuries remained relatively stable, at around one-in-ten, but there was a notable shift away from communicable towards non-communicable diseases. Third, global figures and trends hide considerable regional variations. Each grouped cause of mortality is more prevalent in some regions than others. Notably, communicable diseases feature more prominently in Global South mortality than in the North, and vice versa for non-communicable diseases. 
292 AGEING AND MORTALITY

Table 10.5 World Health Organization classification of deaths

1. Communicable, maternal, perinatal and nutritional conditions

Infectious and parasitic diseases

Respiratory infections

Maternal conditions

Neonatal conditions

2. Noncommunicable diseases

Malignant neoplasms (cancers)

Diabetes

Mental and neurological disorders

Cardiovascular diseases

Respiratory diseases

Digestive disorders

Genitourinary diseases

Congenital anomalies

3. Injuries

Unintentional

Intentional

Source: simplified from WHO (2014a: Table 3.3).
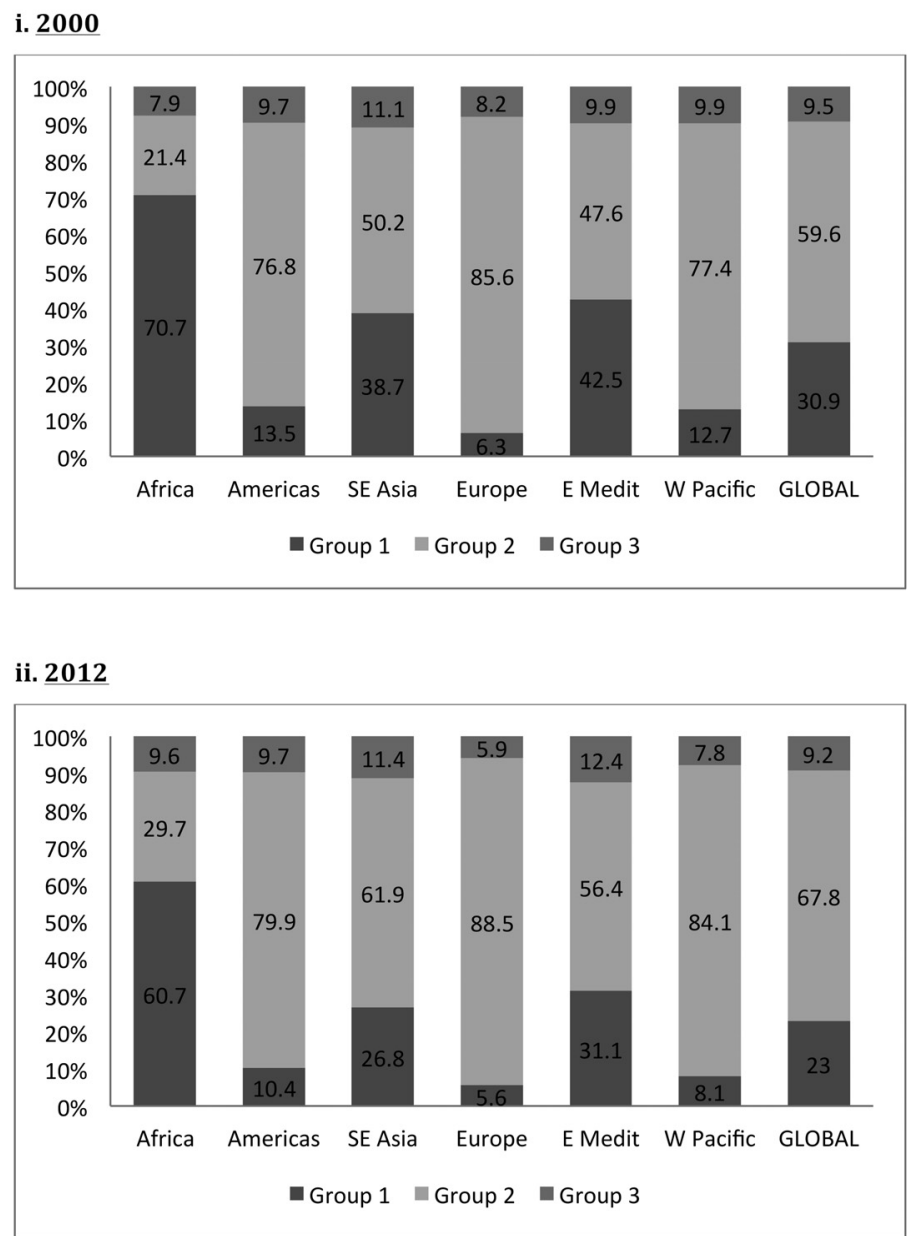

Figure 10.10 Global and regional distribution of deaths by group, 2000 and 2012.

Source: derived from WHO (2014b). 


\subsubsection{Mortality and the life course}

Death, by definition, concludes an individual life course. In aggregate, it is mostly associated with older ages but this relationship, illustrated by life expectancies, is stronger in the Global North than in the South. This immediately redraws attention to the almost overwhelming role in differentiating morbidity and mortality granted compositional and contextual effects of the life course arena of social class, status or other proxy of general wealth and prosperity (Dorling 2005 [(AQ]]; Dorling et al. 2000; Gatrell and Elliott 2015; Holdsworth et al. 2013: Chapter 9). Compositionally, class impacts on mortality directly-via, for example, differentiating ability both to avoid infections and life-threatening contexts and to access appropriate health care-and indirectly-via, for example, correlation with educational level. Illustrating the latter, analysis of US National Longitudinal Mortality Study and National Death Index data revealed each added year of education decreasing death risk (PRB 2011). This reflects higher levels of education's association with higher quality jobs, health insurance, housing, and standards of living; in short, higher incomes (PRB 2011). Contextually, prosperous countries have better capacity to host and provide access to comprehensive medical services. Indirectly, they further influence death rates through promoting everyday practices of healthier behavior.

The relationship between class/prosperity and mortality is not, however, straightforward. Nor is it only unidirectional. It is complicated, in particular, by the former's relational entanglement, illustrated throughout this book, with other arenas of differential life course experience (2.3). This has already been seen in this chapter in terms of geography and history. This sense of entanglement can be summarized by also emphasizing associations between mortality and individual behavior or lifestyle. As discussed for migrations (Chapter 8), whilst lifestyle can reflect individuality (agency) it has also been positioned in close association with social class (structure). This was demonstrated in the seminal work of French Sociologist Pierre Bourdieu (1977, 1984), for example (Cockerham 2005). Whilst this link mostly operates in the same overall direction as prosperity and mortality generally-positive association between healthy lifestyles and higher social status via education (Mirowsky and Ross 2003)-it can work in the other direction. The idea of "diseases of affluence" (Hunt 2005: 205) is in part indicative of a rise in risky behaviors, such as smoking, excess alcohol consumption, poor diets and sedentary lifestyles, with growing socio-economic development. For example, the US, despite considerable prosperity and high spending on health care, has comparatively low life expectancy (still only 76 years for men in 2013; WHO 2015b). While adding 10 years between 1950 and 2010 , other countries such as Japan and Canada made greater gains. Implicated in explaining this longevity "gap" are long-term negative health effects on adults who currently or previously smoked. The US exhibits relatively heavy tobacco usage (Kent 2010) and smoking is strongly associated with reduced life expectancy (e.g. Shaw et al. 2000). More generally, UN goals to reduce diet-related diseases such as diabetes and heart disease are undermined by a growing global trend towards being overweight, projected to see almost one billion adults obese by 2025 (Boseley and Davidson 2015).

Apart from class/prosperity and lifestyle, other dimensions of differential life course experience may be relatively independently associated with specific forms of mortality. For example, disease mortalities are more likely to impact on the less strong (younger, older) and less healthy; some diseases have genetic predispositions at least partly racial; religious belief can promote healthy or unhealthy behaviors (e.g. drinking alcohol); crimes of violence show a strong sex/gender divide between perpetrator and victim; and sexual behaviors can have health risks. Sections 10.5.2-10.5.4 expose some of these associations, either overall or in reference to specific proximate causes of mortality.

\subsubsection{Group 1: communicable diseases}

\subsubsection{Overview}

Deaths from infectious, transmissible or communicable diseases are notable throughout human history, strongly expressed in the high death rates of the ETM's first stage. The Plague or Black Death (1348-1350) is estimated to have killed 30-60 percent of Europe's medieval population (Austin Alchon 2003), exemplifying their potential impact. In more recent times there is the AIDS pandemic, new or emerging diseases, including SARS, E. Coli, Ebola and H1N1 (WHO 2013a), and even re-emerging diseases, such as tuberculosis, malaria and meningitis. Many of these diseases are concentrated in places experiencing high rates of urbanization, particularly where crowded or unsanitary living conditions are common, as well as in remoter places where humans are exposed to microbes (e.g. hantavirus, ebola) 


\section{AGEING AND MORTALITY}

previously either non-existent in human populations or very isolated (Olshansky et al. 1997). Human-induced climate change may be invigorating "old" diseases (Holdsworth et al. 2013; 11.3.4).

As defined by the WHO (2013a), communicable diseases "are caused by pathogenic microorganisms, such as bacteria, viruses, parasites or fungi; the diseases can be spread, directly or indirectly, from one person to another." They move from place to place via people connecting with other people or with other vectors-agents carrying and transmitting pathogens into another living organism-such as animals, birds and insects. Table 10.6 lists a wide range of infectious diseases, some more recognizable than others.

Table 10.6 is, however, far from exhaustive. New diseases are continually evolving, Jones et al. (2008) identifying over 335 infectious diseases emerging since 1940! Indeed, for Mayer (2000: 938), "the major lesson to be learned from the [AIDS] pandemic is that the assumption that infectious diseases are a phenomenon of the past, largely restricted as major health threats to developing countries, and that 'international health' consists of the study of problem ef developing countries, are all erroneous." Of factors prompting appearance of new pathogens, cross-species transfer is central (Mayer 2000). Most emerging infectious diseases (EIDs) (60 percent) are zoonoses, diseases transmittable from other animals to humans, such as influenza from swine and avian populations or yellow fever from monkeys. Once transferred, some new pathogens, such as HIV and ebola, can be passed from person to person and, by extension, move from place to place. Besides new pathogens, older, seemingly relatively dormant communicable diseases have also re-emerged in mortality tables (Sabel et al. 2010). Relevant here is antimicrobial resistance (Gatrell and Elliott 2015), the growing inability of antibiotics to kill harmful bacteria due to the latter changing structure, which is undermining potential treatment. This is helping return diseases such as tuberculosis to killer status (Guardian 2014f).

In the spread of communicable diseases, there is an important distinction between endemic and epidemic situations (Poston and Bouvier 2010: 127-33). Endemic diseases exist at fairly consistent levels within a population but epidemics represent "major increase or upswing of an infectious disease in an area that results in a large number of deaths, followed by a decline" (Poston and Bouvier 2010: 127). A classic historical example was the Spanish influenza that hit globally in 1918, following World War I, and is widely credited with causing upwards of 50,000,000 deaths. Indeed, Spanish 'flu was a pandemic, an epidemic that spreads to several countries. Such outbreaks demonstrate that whilst communicable diseases may be only second in Figure 10.10, historically and spatially they can be devastating. Being located geo-historically "within" an epidemic increases its threat of mortality substantially, reinforcing the importance of the cohort within life course analysis (2.2.2).

From a life course perspective, relative bodily vulnerability, not least on account of age (young, old) or other morbidity (healthiness), enhances the mortality risk of communicable diseases. Again, prosperity of an individual and his/her society is a paramount factor, playing a key role in determining access to pharmaceutical drugs to living in more therapeutic residential environments. This is clear when geographies of communicative diseases are considered in greater detail.

Table 10.6 Globally prominent infectious diseases

\begin{tabular}{ll}
\hline Acute encephalitis & Malaria \\
Acute infectious hepatitis & Measles \\
Acute meningitis & Meningococcal septicaemia \\
Acute poliomyelitis & Mumps \\
Anthrax & Plague \\
Botulism & Rabies \\
Brudellosis & Rubella \\
Cholera & SARS \\
Diphtheria & Scarlet fever \\
Enteric fever (typhoid or paratyphoid) & Smallpox \\
Food poisoning & Tetanus \\
Haemolytic uraemic syndrome & Tuberculosis \\
Infectious bloody diarrhea & Typhus \\
Invasive group A streptococcal disease & Viral haemorrhagic fever \\
Legionnaires' Disease & Whooping cough \\
Leprosy & Yellow fever \\
\hline
\end{tabular}

Source: taken from HPA (2013). 


\subsubsection{Mortality geographies of communicable diseases}

Increasing mobility, political upheaval, and environmental change and disaster all facilitate pathogen spread (Gatrell and Elliott 2015: Chapter 13). Thus, one would expect to see relatively high mortalities from communicable diseases in places, at times and for people experiencing such risk factors. Central is the role of spatial diffusion in developing epidemics and facilitating (re-)emergence of communicable diseases (Mayer 2000). The term refers to a geographic process by which something spreads from one place to another. Not least in an era of mobilities, in the absence of adequate biosecurity measures preventing spread, including vaccination (Box 10.4), infectious diseases can rapidly diffuse via long-distance transportation modes, such as airlines. However, diffusion can occur at a range of scales, although typically following migration or transportation routes. Factors covered in Chapters 5-9 that influence population movements-employment migration, war, urbanization-all diffuse diseases between places.

Figure 10.10 showed a strong overall global mortality geography for communicable diseases. At 61 percent, it was the principal grouped cause of death in Africa, of prominence in SE Asia and the Eastern Mediterranean, but of

\section{BOX 10.4 VACCINATION AND MORTALITY REDUCTION}

A key factor explaining rising life expectancy and decline in IMR from communicable diseases is growth of vaccination. This refers to administration into the body of material that is antigenic (a vaccine), in that it stimulates an individual's immune system to develop antibodies that subsequently resist and provide immunity to attacks by related pathogens.

Vaccination was pioneered in 1796 by British rural doctor Edward Jenner. He had noted country folklore that claimed milkmaids who caught the mild disease cowpox never later caught smallpox, a deadly killer. To test this connection, Jenner inserted pus from a cowpox pustule into a boy's arm. Whilst the boy experienced cowpox, he proved to be immune to smallpox. Although widely ridiculed at the time, results were published 2 years later and Jenner coined the word vaccine from the Latin vacca (cow).

When finally accepted as sound medical science, vaccination spread globally. It is now used to prevent numerous communicable diseases. So-called children's diseases have been especially targeted: polio, measles, diptheria, chicken pox. However, for success at group as opposed to individual level, vaccination must attain herd immunity, the rate required to protect the herd (group). Herd immunity enables the chain of infection to be broken and, ultimately, should result in disease eradication. Such an "outstanding success" (Gould 2015: 100) has been achieved globally for smallpox, whose last recorded case was in Somalia in 1977. Polio appears close to eradication, by 2012 still endemic only in Afghanistan, Pakistan and Nigeria. Indeed, whilst there remains a Global North-South vaccination divide, not least due to costs of provision, the twenty-first century presents a generally positive picture of growing uptake and acceptance of the practice. Vaccination programs are often financed and even delivered by international agencies and NGOs, such as Unicef and Save the Children.

However, this brief account of vaccination cannot end on a singularly positive note. Both deteriorating socioeconomic conditions and encultured beliefs, old and new, can seriously derail and impede vaccination practice. First, in countries experiencing serious socio-economic or political difficulties, from economic collapse to war and conflict, the health system is often an early casualty. Consequently, vaccination programs, often costly and complex in operation, may collapse. Second, culturally, there is a minority but nonetheless growing trend in both North and South to reject vaccination. For some this expresses resistance to being told what to do by the state or a fear that the state or international agencies are infiltrating their lives. Others believe vaccination harmful, such as making persons susceptible to other diseases. The life course arena of religion can come into the picture, with Old Order Amish in the US, for example, rejecting vaccination. Finally, even when vaccination programs are ongoing and popularly accepted, this must not be at the expense of other requirements for good health, such as clean water and air and effective waste disposal. Vaccination is just one component within normative networks of everyday healthy lives that can promote longevity in the life course and thus further reinforce the association between mortality and older age.

(Sources: Gatrell and Elliott 2015; Gould 2015) 


\section{AGEING AND MORTALITY}

relatively low significance in the Americas, Western Pacific and, especially, Europe. In sum, relative mortality from infectious disease is strongly concentrated into the Global South. Whilst this predominantly reflects the overall North-South socio-economic developmental divide between (Gould 2015), it is also indicative of the relational role of particularly configured environments in promoting or reducing mortality risks. For example, whilst the Ebola virus's deadly prevalence across western Africa in part reflected this region's poverty, its origin within cross-species transfer and subsequent survival within a particular bio-social environment also reflected a specific spatial ecology (Pourrut et al. 2005).

Figure 10.10 also showed that whilst percentage of deaths globally due to communicable diseases fell sharply from 31 percent to 23 percent of the total between 2000 and 2012, decline was uneven geographically. Biggest drops were in the African, SE Asian and Eastern Mediterranean regions, with almost no decline in Europe. There is, therefore, cautious suggestion of a degree of global convergence in causes of death, whilst reiterating that elimination of communicable disease mortality remains at best utopian ambition.

The epidemiology-patterns, causes and effects-and mortality geographies of each communicable disease vary considerably and clearly cannot begin to be covered here. They are a mainstay of Health and Medical Geographies. Just two will be considered in more detail, one of long-standing notoriety-tuberculosis-the other of recent infamyHIV/AIDS. For both, links between the diseases and their resulting mortalities and relevant arenas of life course shaping experience will be noted.

\subsubsection{Mortality geographies of tuberculosis}

Tuberculosis (TB) is caused by the bacillus Mycobacterium tuberculosis, which typically infects the lungs (pulmonary TB). It is spread in the air when those infected expel the bacteria, typically by coughing. Although only a relatively small proportion of people infected with M. tuberculosis develop TB, it remains a major global health problem (Gatrell and Elliott 2015) and the second leading cause of death from infectious disease worldwide. WHO (2013b) estimated 8.6 million new TB cases in 2012 and 1.3 million deaths. Most deaths are preventable, however, if those affected can access health care for diagnosis and subsequent treatment. Yet, while developments from the 1940s made TB a curable disease (Dorman and Chaisson 2007), it has resurged across the Global North since the late 1980s (Faustini et al. 2006; Rose et al. 2002) and continues to constitute a global morbidity and mortality burden (Corbett et al. 2003).

Figure 10.11 shows the global distribution of TB deaths estimated for 2012, excluding those dying who were HIV-positive. A marked geography is clear (Corbett et al. 2003), with the disease concentrated primarily within SubSaharan Africa and South/Southeast Asia. Note also moderately high values in Russia and much of Eastern Europe. This reflects the strong clear link between TB deaths and poverty (Faustini et al. 2006; Gatrell and Elliott 2015). This link is overlain by three key additional life course arenas: sex-men being most susceptible; age-a high number of child deaths, even if most victims are adults; and sexuality-due to a link to HIV/AIDS. Overlaying these compositional factors, however, are contextual considerations shaping death rates, such as presence or absence of health systems equipped for and focused on treatment.

Whilst expression of TB mortality varies between Global South and North, its link with poverty is overwhelming, especially for children. This is reflected in high rates of childhood TB in Afghanistan, Pakistan and Brazil (Nelson and Wells 2004), and explicit links to poverty in South Africa (van Rie et al. 1999) and Malawi (Harries et al. 2002), where treatment is typically also poor. Northern resurgence in TB deaths is associated with impoverished post-Communist Eastern Europe communities, indigenous populations of the Canadian north, and the inner city homeless in the US, for example (Gatrell and Elliott 2015). It is also linked closely to HIV/AIDS, immigration and drug resistance (Faustini et al. 2006). This HIV/AIDS link is global, the immunity-destroying effects of HIV both reactivating TB and opening individuals to new infections. Consequently, TB was the immediate cause of around 11 percent of AIDS deaths in 2000 (Corbett et al. 2003) and over a quarter of present-day TB deaths in the US are tied to HIV infection (Gatrell and Elliott 2015: 172). In the North, the HIV link is strongest for the homosexual white community and amongst heterosexuals within the Black African community (Rose et al. 2002). The latter association expresses the role of migration (Gatrell and Elliott 2015) and TB deaths within immigrant communities from the South. Finally, rising drugresistant TB (XDR-TB) is also found in high HIV areas, helping reinforce resurgence of this killer disease (Dorman and Chaisson 2007).

\section{ST PROOFS: NOT FOR DISTRIBUTION}




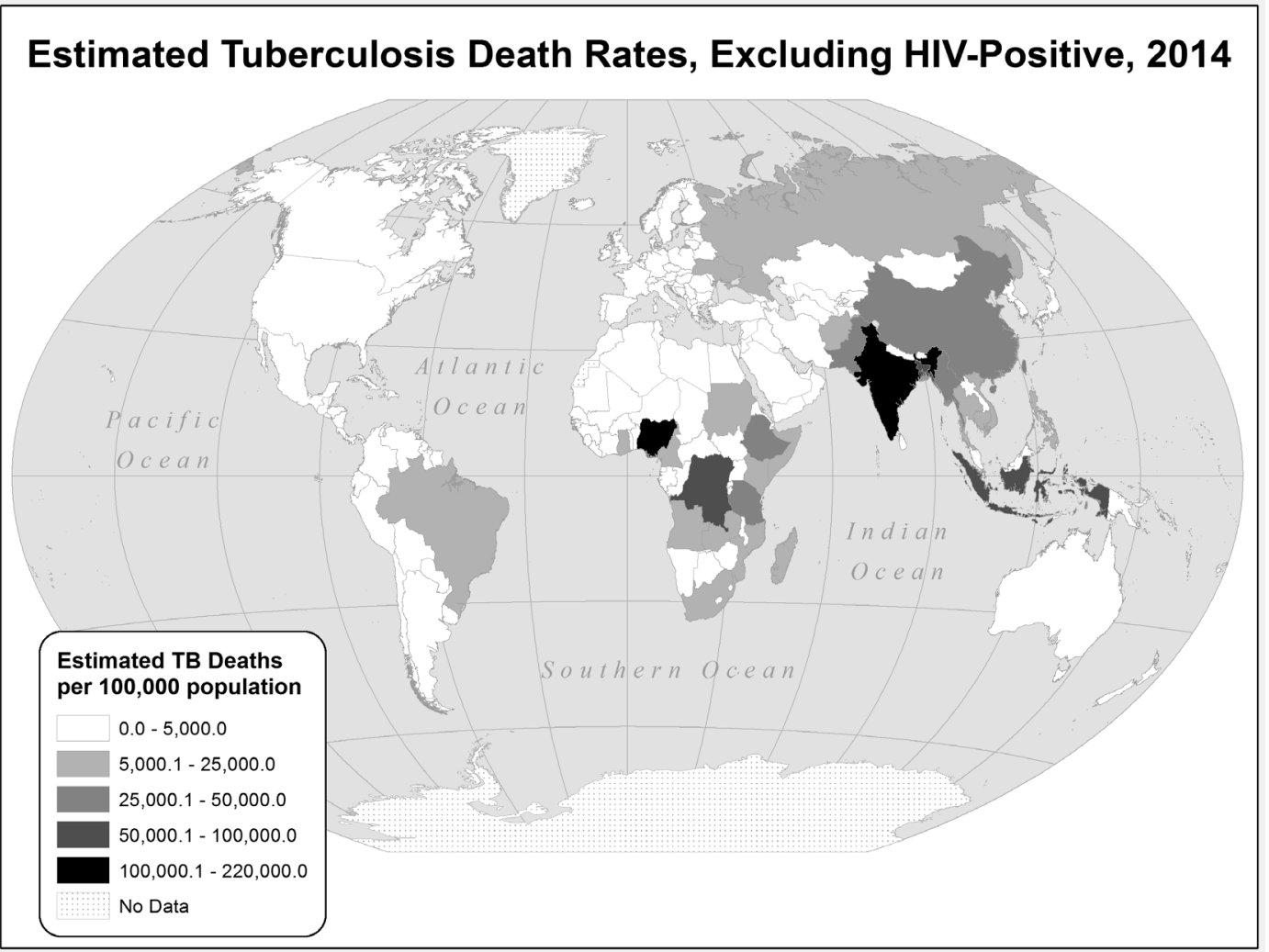

Figure 10.11 Global tuberculosis deaths, 2014

Source: WHO (2015: Figure 2.17), http://www.who.int/tb/publications/global_report/en/

\subsubsection{Mortality geographies of HIV/AIDS}

AIDS, Acquired Immunodeficiency Syndrome, already seen implicated in TB deaths, has redefined the landscape of disease and health care globally (Poston and Bouvier 2010: 129-133). Gould (2015: 22) characterized it "a truly exceptional epidemic disease" (also Gould and Woods 2003: Kalipeni et al. 2004). It became the principal cause of infectious disease mortality worldwide (WHO 2014b), although deaths are now in decline (1.2 million in 2014) after peaking at 2.3 million in 2005 (Gould 2015: 22).

AIDS is the most severe manifestation of the Human Immunodeficiency Virus (HIV) and is not a single disease. Rather, it is a complex of diseases and symptoms still not completely understood. A key feature is that it attacks the immune system, weakening the victim and opening them up to other infections (e.g. TB). This ultimately leads to death. There is no known cure or vaccine and, because it has a long latency period of between 5 and 10 years or even longer after initial infection, an individual can spread the disease without realizing they are infected (WHO 2004). HIV/AIDS has no vector spreading it but is overwhelmingly a behavioral disease (Gould 2015). Transmission is especially associated with sexual activity and intravenous drug use, although Table 10.7 shows its wider range of pathways.

Although commonly thought of as a new disease, it is hypothesized that AIDS actually existed for decades, possibly centuries, within West African primate populations. It likely underwent cross-species transfer to humans when infected animals were used for food (such as bushmeat) or other traditional cultural practices (Walters 2003). The population so infected, however, remained isolated until civil war erupted in the Democratic Republic of the Congo in the 1980s. Consequences of this war and its aftermath-refugees, starvation, disruption of normal settlement, movement of large numbers of young men in combatant groups and corresponding increase in sexually transmitted diseases-all facilitated the virus's emergence and diffusion. 
298 AGEING AND MORTALITY

Table 10.7 Major modes of HIV transmission

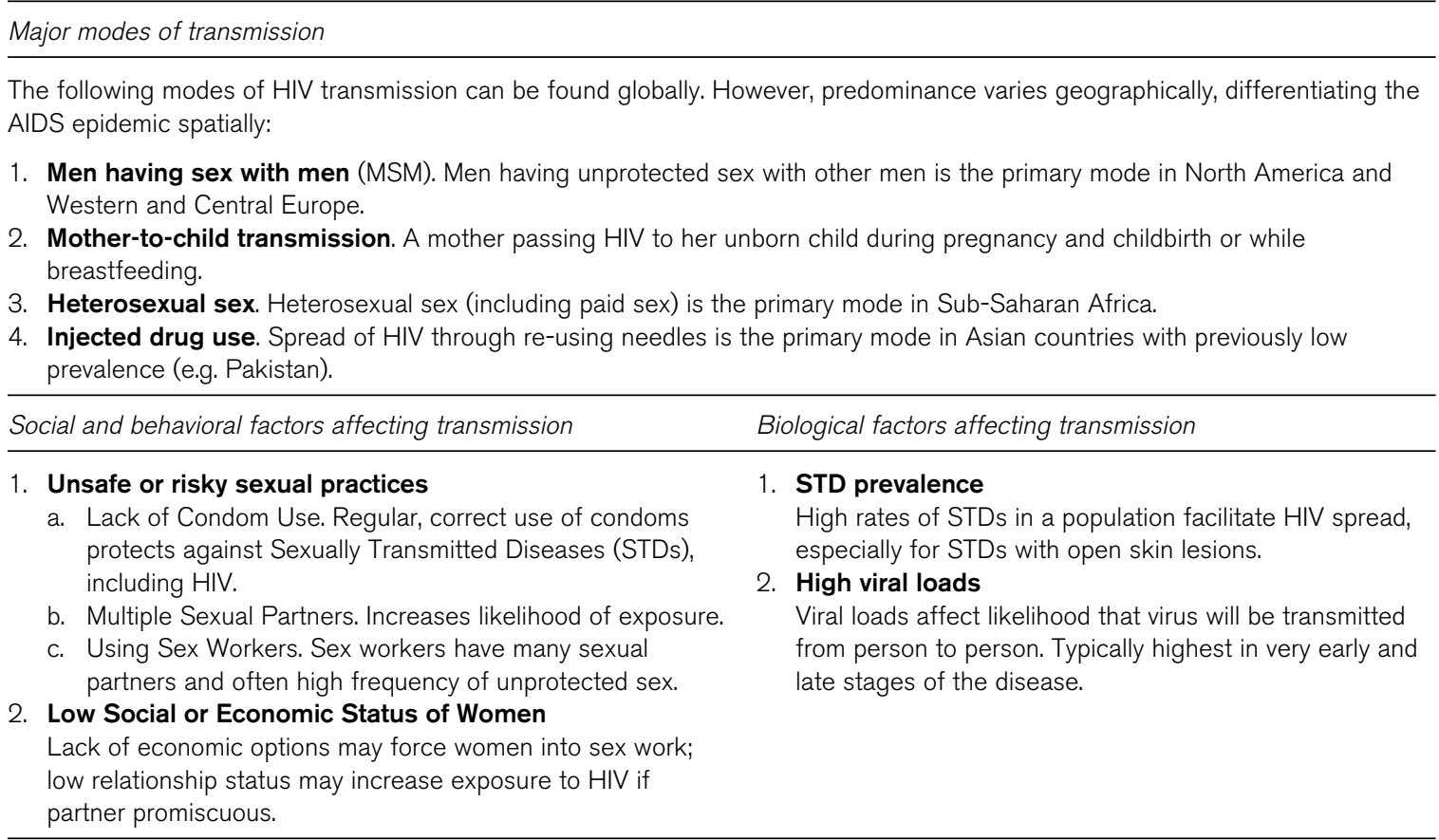

Sources: Mayo Clinic Staff (2013); UNAIDS (2010).

UNAIDS (2015) reported that 36.9 million people globally were living with HIV/AIDS and 1.2 million experienced AIDS related deaths in 2014. However, such headline figures hide considerable spatial variation. This reflects the fact that the rate and processes by which HIV/AIDS is transmitted (Table 10.7) is highly uneven geographically, differing considerably by world region and sub-populations within countries. In 2014, it was estimated that around 5 million people were living with HIV in Asia and the Pacific and 2.4 million in Western and Central Europe and North America. Corresponding AIDS deaths in these regions were 240,000 and 26,000, respectively (UNAIDS 2015). Death to infection ratios were 0.05 for Asia and Pacific and 0.01 for Europe and North America, representing often very different life course experiences for those with the virus, a finding returned to below.

It is Sub-Saharan Africa, especially the most southern countries, however, that has been most affected demographically and socially (Gould 2015) by HIV/AIDS, illustrated in Figure 10.12. In 2014, it had 25.8 million people living with HIV (UNAIDS 2015), 70 percent of the global total, a figure that had remained remarkably stable since 2000 (Lamptey et al. 2002: 10). Some 790,000 people died of AIDS related causes in 2014,66 percent of the global total (UNAIDS 2015). Over half of Sub-Saharan victims have been women, the disease spreading primarily through heterosexual transmission, women's generally low social status making them especially vulnerable (Lamptey et al. 2002; UNAIDS 2010).

Impacts of HIV/AIDS across Sub-Saharan Africa have been very severe. Unfortunately, early denial of the disease among some leaders, as in South Africa, delayed introduction of education and prevention programs (Dickenson 2004). Negative social and economic consequences for individuals, households and countries' development have been pervasive because the disease primarily affects those of child-bearing age and the most economically active (Gould 2015). One result is declining life expectancy, already suggested for South Africa in Figure 10.5. In Botswana, highlighted in Figure 10.12, life expectancy without AIDS would have been 74 years in 2010 but with the epidemic dropped to just 27; in Zimbabwe, it was 71 years without AIDS, 35 years with (Lamptey et al. 2002: 17).

As men and women in prime reproductive life course stage die, children are left orphaned. Many resulting extended families are now headed by grandparents (Gould 2015). Far from seniors living a life of "retirement" (10.2.1.1), they often lack resources to raise the children, leaving them facing potential malnutrition, dropping out of school and having 


\section{HIVIAIDS Prevalence in Africa}

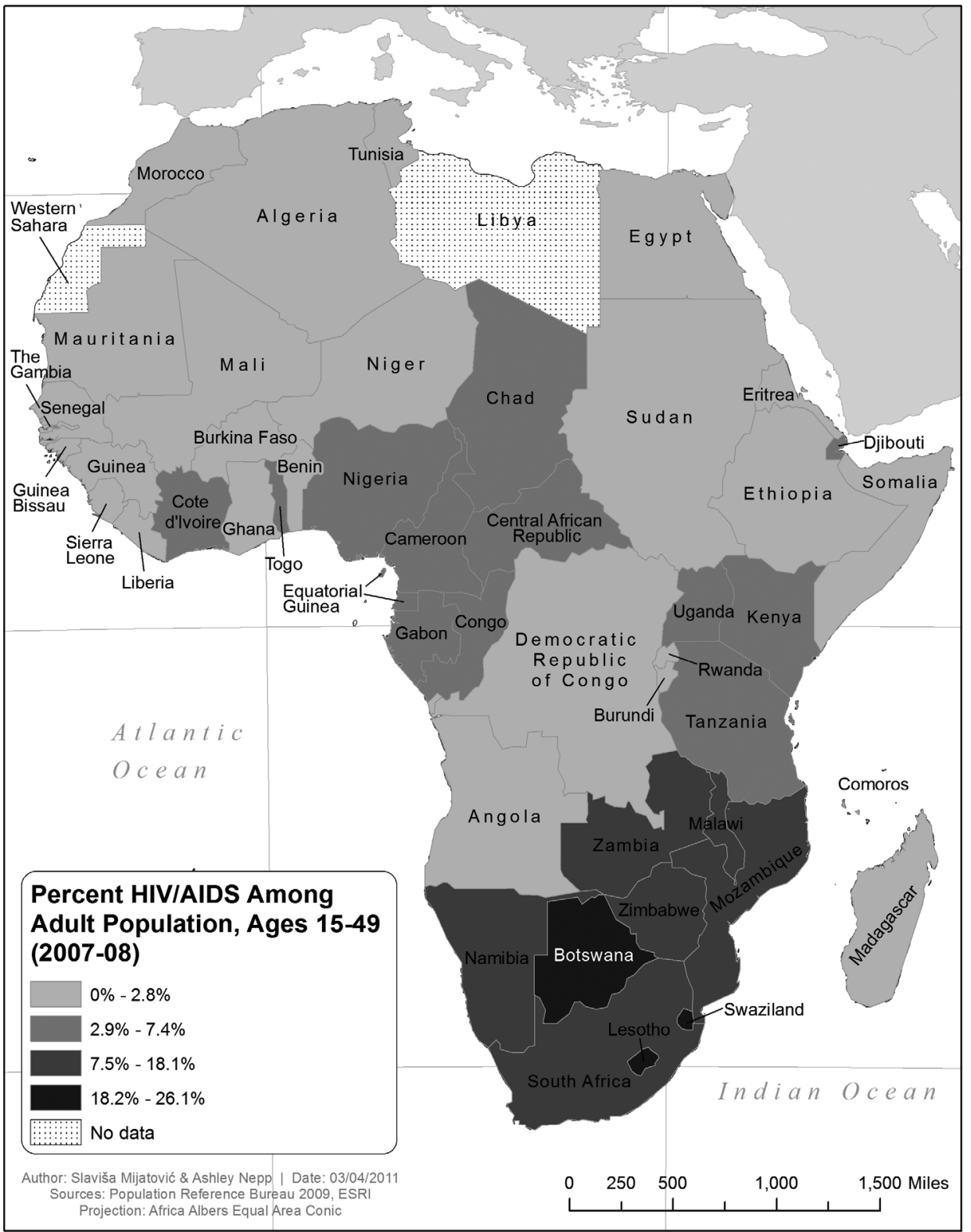

Figure 10.12 HIV/AIDS prevalence in Africa, 2007-2008.

reduced economic capacity throughout their lives. It also forces children to migrate for work, both to support themselves and siblings and parents sick with AIDS (Ansell and van Blerk 2004; Young and Ansell 2003). Michael Fleshman (2001) described such children as Africa's "silent crisis." A further migratory consequence of HIV/AIDS is a final journey in a history of circular migration (7.4.4) of migrant workers infected with the virus returning to South African homelands, for example, to die (Clark et al. 2007).

One hypothesis seeking to explain the prevalence of HIV/AIDS in Sub-Saharan Africa, sexual networking theory, posits the nature of sexual relations and socio-sexual norms in this part of Africa facilitating spread of the virus (Caldwell et al. 1989). Premarital and extramarital sex is common, women and men tending to have more than one partner. 


\section{AGEING AND MORTALITY}

Factors encouraging these sexual patterns include long periods of postpartum abstinence (4.4.2) for women, during which time males may seek additional female partners; practices of polygamy and wife inheritance, and the generally low status of women; and high rates of male employment migration, separating partners for long periods and increasing likelihood of additional sex partners. All contribute to rapid spread of HIV/AIDS and other sexually transmitted diseases (STDs). In a study of truck drivers and itinerant market women in Nigeria, Orubuloye et al. (1993) found drivers with sexual partners in multiple places, transmitting HIV over long distances, while market women also often had multiple partners, including the drivers.

The behavioral/lifestyle emphasis within HIV/AIDS transmission is reinforced further by other general features of the epidemic (Gould 2015). First, as noted, unlike most communicable diseases it is largely a cause of death for working age adults rather than younger children or seniors. In other words, it most impacts a group generally seen with most lifestyle agency within the life course. Second, HIV/AIDS prevalence is generally higher in urban areas, where more lifestyle choices are usually present (8.2). Third, the virus has impacted relatively more on the relatively educated and mobile than on the poorest. Fourth, displacement from clear association with poverty is also apparent at national scales, Botswana and South Africa having higher HIV/AIDS prevalence than poorer Sahel countries, for example.

From all the evidence one might thus conclude that deaths from AIDS, in contrast to those from TB, are not primarily shaped by poverty and/or class. Clearly, besides lifestyle, gender and gender relations molded by patriarchy are especially relevant, as is active sexuality. However, to deny the importance of class, status and resources would be naïve, since many of the problematic behavioral practices noted above are rooted strongly in relative poverty. In other words, a broad relational lens is required to appreciate the full set of background causes (Figure 10.9) underpinning AIDS deaths. Such a perspective is also instructive for interpreting the devastating position of AIDS deaths in Sub-Saharan Africa relative to those across the Global North. It reiterates the need to add contextual sensitivity to any compositional explanation for geographies of HIV/AIDS mortalities.

HIV/AIDS in the Global North is much more firmly associated with homosexual or bisexual men having sex with men (MSM) than in the South (Table 10.7). This is not, however, to represent it as a disease only applicable to the gay male community, as frequently presented in the epidemic's early years. Today, AIDS deaths across the North are still numerically significant-26,000 for Western and Central Europe and North America in 2014-but they do not reach the scale of those elsewhere. One main reason for this is greater and increasing access to antiretroviral drugs (ARVs). These drugs are increasingly being utilized to inhibit reproduction of the HIV/AIDS virus. HIV/AIDS may not yet have a cure, nor be reversible in its effects, but it can be controlled/stabilized through ARV use (Gould 2015). Better access to them in the North helps explain the earlier noted lower death to infection ratios in Europe and North America compared to Asia and the Pacific. Such uneven access, however, reflects not only the relative wealth of Northern countries (they are costly) and their substantial public health investments but also the ability of HIV/AIDS activism to promote adoption, disciplined use and underlying medical research.

Finally, returning to the Global South, whilst HIV/AIDS continues to devastate many regions, the prospect in 2016 is no longer as bleak as just a decade earlier. Consequently, threat of a still more overwhelming global HIV/AIDS pandemic is somewhat receding (Gould 2015), as already noted in terms of declining mortality if not in numbers infected. There is rapid growth in ARV use after early pioneering experiments (e.g. Galvao 2005). Consequently, 10.7 million Sub-Saharan Africans, 41 percent of those living with HIV now access ARVs, up from fewer than 100,000 in 2002. Five out of seven people on ARVs now live in this region (UNAIDS 2015). Additionally, ABC policies-abstinence, being faithful to one sexual partner, condom use-are having positive impact, as is political leadership by rulers such as Uganda's President Museveni (Gould 2005, 2015). Perhaps, then, the life course cohorts devastated by the virus will not ultimately become too prominent markers in the South's demographical history.

\subsubsection{Group 2: non-communicable diseases}

\subsubsection{Overview}

By 2012, over two-thirds of global deaths (Figure 10.10) were not caused by diffusion of epidemics but resulted from non-communicable diseases, which are not passed from person to person. Among this diverse group, further distinction can be made between chronic diseases, of long duration and usually progressing fairly slowly, and acute

\section{ST PROOFS: NOT FOR DISTRIBUTION}


diseases, "that start abruptly, last perhaps for only a few days and then settle, though perhaps developing into chronic conditions or leading to death" (Gatrell and Elliott 2015: 8). While most are potentially treatable, non-communicable diseases are largely incurable. A cure for cancer, for example, is one of the "holy grails" of medical science. There are four main groups of non-communicable diseases: cardiovascular, such as heart attacks and strokes; cancers; chronic respiratory, such as chronic obstructive pulmonary disease and asthma; and Ddabetes (WHO 2013c).

Non-communicable diseases are mostly associated with later ETM stages (Figure 10.7)-although this will be qualified-and with residents of richer countries in the Global North. This reflects how susceptibility to dying from them is related strongly to lifestyle and behavioral characteristics. Four primary lifestyle risk factors especially underpin noncommunicable diseases: tobacco use, physical inactivity, unhealthy diet, and harmful alcohol use (WHO 2013c). All are often strikingly manifest within richer countries. Non-communicable disease mortality is also principally associated with older ages-later life course-not least due their association with accumulating a lifetime's lifestyle "consequences," although even chronic disease is also still manifest among the young.

\subsubsection{Mortality geographies of non-communicable diseases}

Figure 10.10 shows a strong global geography overall for deaths from non-communicable diseases. They are the principal cause of death in all regions except Africa, being overwhelmingly dominant across the Global North: Americas (80 percent), Western Pacific (84 percent), Europe (89 percent). However, there is again the sense of global convergence, with 8 percent increase in the percentage of deaths globally from non-communicable diseases between 2000 and 2012 hiding larger increases in Africa, East Mediterranean and SE Asia.

It is important to note the increasing significance of non-communicable disease mortalities across the Global South. Countries experiencing rapid economic development and urbanization tend initially to be affected increasingly by both communicable and non-communicable diseases (Gould 2015). Changing lifestyles that accompany rapid transitions promote the latter, increasingly exposing people to the four principal risk factors. Wealth disparities result in growing prevalence of chronic disease among elite classes, whose lifestyles and diets often resemble those in more affluent countries (Boseley and Davidson 2015). However, within the same country, impoverished populations struggle with the effects of infectious diseases. This "double burden" places additional strain on fragile and developing health infrastructures (WHO 2013c).

The principal factor underpinning mortality geographies from non-communicable diseases-even more so than disease prevalence-is, once again, class or wealth (e.g. Borrell et al. 2010). This link is, nonetheless, cross-cut with other key arenas of life course differentiation. Although there are variations by sex for communicable diseases, gendered lifestyle factors underpinning prevalence to non-communicable diseases are especially important. Variations are attributable to differences in status, physiology and environment, and to lifestyle contributions that see more men adopting risky behaviors, such as smoking and excess alcohol consumption (Yin 2007). The global result is that women tend to live longer than men (Weeks 2005). However, this varies considerably, both by country and within countries, according to a wide range of social and cultural variables. For "Russia ... the difference between male and female life expectancy is 13 years (59 vs. 72), [for] the United States, [just] 5 years (75 vs. 80), [and for] Afghanistan ... no male disadvantage (42 vs. 42)" (Yin 2007).

Other arenas of life course experience also impact on the likelihood of dying from specific non-communicable diseases. For example, religious beliefs, such as not drinking alcohol (e.g. Islam) or not eating meat (e.g. many Buddhists) inevitably reduce the likelihood of mortalities associated with such practices (e.g. Hummer et al. 1999). More loosely, being generally healthy can promote resistance to dying from non-communicable diseases through, for example, feeding into more active lifestyles.

Just as it was significant for diffusing communicable diseases, population migration is a further complicating factor to be taken into account in recognizing mortality geographies (and morbidity) due to non-communicable diseases (Boyle and Norman 2010; Darlington et al. 2015; Gatrell and Elliott 2015). Migration reflects health inequalities, with both actually undertaking migration and distance moved being positively associated with degree of healthiness, and also re-writes geographies of health. For example, healthier people moving away from deprived areas can reinforce the unhealthiness of such places, raising their death rates. Migration thus tends to amplify mortality geographies (Dorling 2005) [[AQ]]. It also confounds analysis of environmental relationships between place and mortalities (10.5.5), making it hard to pin down where chronic diseases, such as cancer (e.g. Maheswaran et al. 2002),

\section{ST PROOFS: NOT FOR DISTRIBUTION}




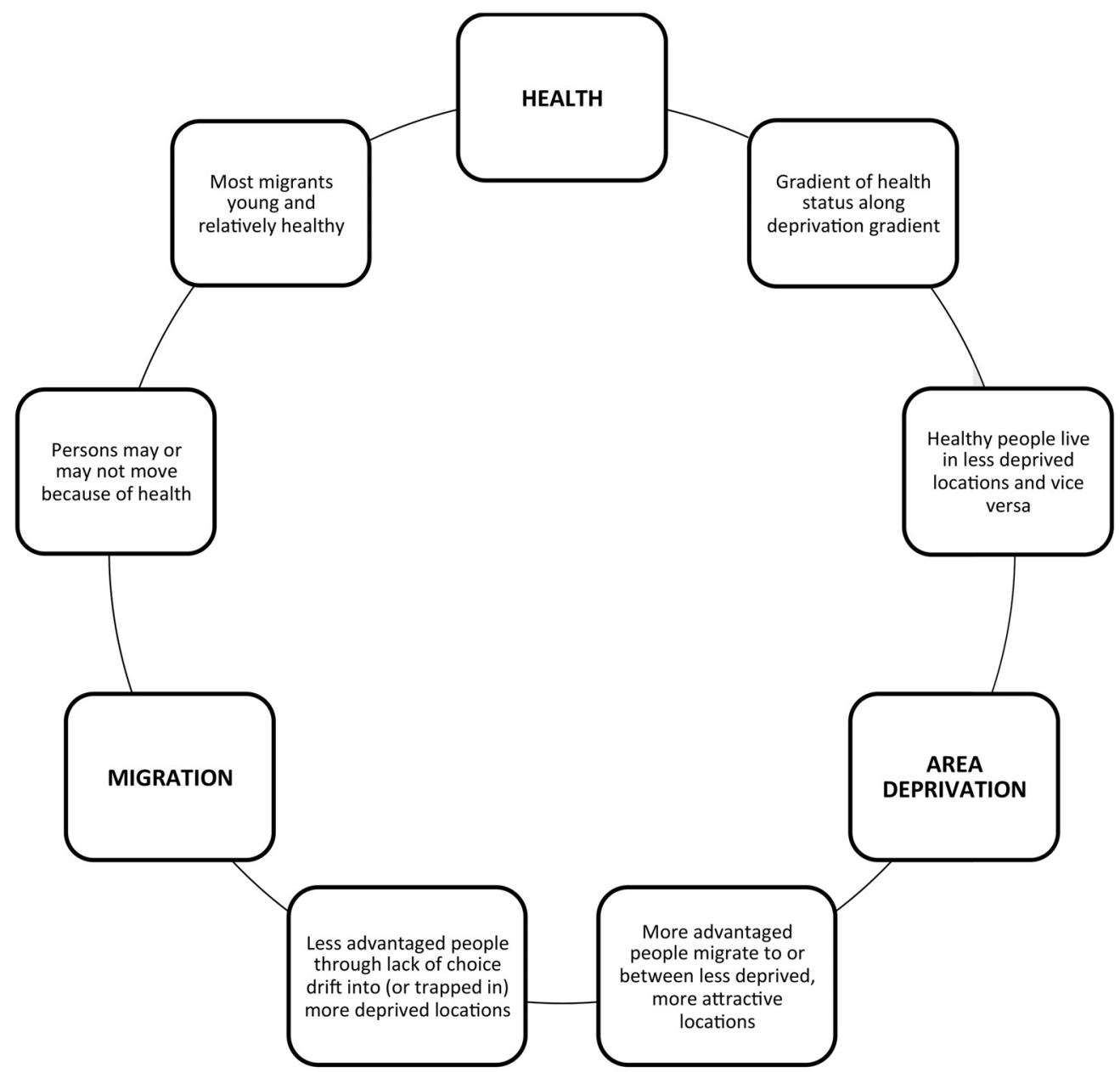

Figure 10.13 Interrelationships between health, migration and deprivation

Source: simplified from Boyle and Norman (2010: 356).

are contracted (present place of residence or somewhere lived before?). Now a significant body of scholarship, Figure 10.13 gives some sense of the many interrelationships between health, migration and deprivation.

As with communicable diseases, attention now turns to the more detailed life course mortality geographies of two specific non-communicable diseases. Both diseases are usually chronic but whilst the first, cancer, has numerous often illusive causes, reflecting complex interplay between genetics, environment and lifestyle, present-day prominence of the second, cirrhosis of the liver, almost entirely reflects harmful lifestyle practices consolidated through a life course.

\subsubsection{Mortality geographies of cancer}

Malignant neoplasms or cancers accounted for 8.2 million deaths in 2012, nearly 15 percent of the global total and 22 percent of non-communicable disease mortality. There are numerous forms of cancer but all involve emergence of a malignant growth or tumor as a result of uncontrolled cell division in part of the body. Some, for largely biological reasons, are primarily experienced by women, notably breast and cervical cancer, whilst others are mostly threats to men, notably prostrate and testicular cancer. Other apparent sex or gender variations are more explained by lifestyle factors, such as the greater incidence of lung cancer for men linked to higher rates of smoking (e.g. RuízGodoy et al. 2007). 


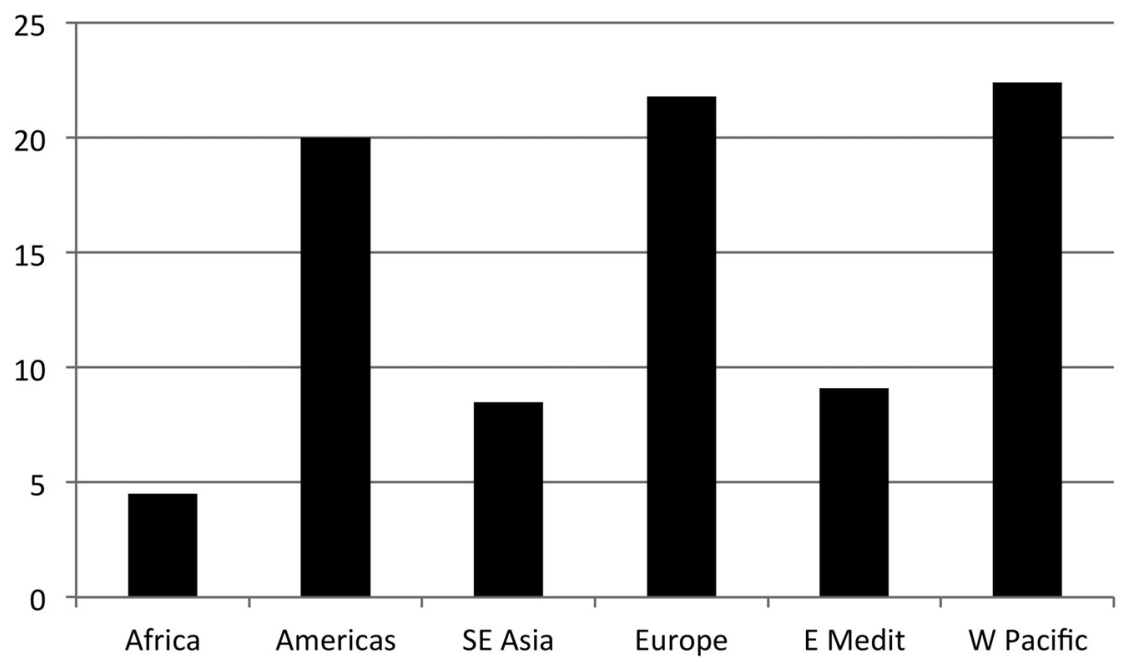

Figure 10.14 Global regional breakdown of deaths by cancer, 2012 (\% total deaths)

Source: derived from (WHO 2014b).

Figure 10.14 indicates the mortality geography of cancer across the world in 2012 according to WHO region. There is a clear binary divide between relatively low rates for Africa, SE Asia and the Eastern Mediterranean and high rates for the Americas, Europe and the Western Pacific. However, research on both individual cancers and/or countries consistently shows geographical variation not only found at this macro, approximately Global South versus North scale. Recognition and explanation of smaller-scale variations, such as the cluster of especially male cancer deaths within the Guangxi region of China (Deng et al. 2014), again suggests compositional and contextual factors combining to determine deaths.

Consider breast cancer, the main global cause of female cancer deaths. Its mortality trend is downwards, largely due to better and earlier detection, notably through screening, and treatment. However, detailed geography of this decline is complex and inconsistent, with increases in some areas. Diversity is largely explained by compositional life course factors. Risk factors associated with developing breast cancer include being of older age-the key variableliving in a richer country, having a young age menarche and an older age menopause (4.1.2), having a first child in one's 40s, being in a higher status group, and being obese and/or over-consuming alcohol (McPherson et al. 1994). In sum, and in contrast to most communicable diseases, breast cancer is especially associated with lifestyles most commonly found amongst women in the Global North (Tian et al. 2010). However, areas in the South where mortality from breast cancer has not fallen and even risen tend to be less prosperous, such as the poorer states of Brazil (FreitasJunior et al. 2012). In short, both lifestyle and relative poverty must be taken into account for explanation of breast cancer prevalence and death rates.

Distinguishing between morbidity and mortality, poverty adds a clear contextual dimension as to whether a breast cancer incidence proves fatal. This is because poverty is associated strongly with the unlikelihood of receiving both screening and treatment (Gatrell and Elliott 2015). Recognizing this dimension is crucial for interpreting ethnic or racial variations in Breast Cancer mortality within a specific country. In the US, mortality is higher for black than white women (Tian et al. 2010). Whilst this has led to theories suggesting susceptibility at least partially explained by differential biology, Whitman's team (Whitman et al. 2011) and others (e.g. Cooper et al. 2003; Sankar et al. 2004) rejected such explanations. They saw instead the results of access to screening, the quality of screening, and access to effective treatment differentiated according to race. In short, "disparities cannot be viewed outside the context of racism in the US" (Whitman et al. 2011: 594).

More generally, differences in cancer death rates generally across racial and ethnic lines are largely attributable to underlying social structures that advantage or disadvantage different groups within society (Dai 2010). Differences manifest in myriad ways: directly, in terms of who has access to resources, education or health care; and, indirectly, 


\section{AGEING AND MORTALITY}

through lifestyles associated with race or ethnicity. In summary, Rogers et al. (1996) evaluated racial and ethnic disparities in mortality relative to demographic, socioeconomic and health characteristics in the US. They found "Asian American mortality. . . low in part because of healthy behaviors and socioeconomic advantages; . . . Caucasian American mortality ... higher partly because of high prevalence and quantity of cigarette smoking; and ... Mexican, Native, and African American mortality ... higher partly from socioeconomic disadvantages" (p. 149).

The need for complexity in explaining cancer geographies and mortalities seems clear-cut (e.g. Henry et al. 2009; Schootman et al. 2011 on colorectal cancer; Ruíz-Godoy et al. 2007 on lung cancer). Consider cervical cancer, the leading cause of female cancer deaths in the Global South. Regression analysis (Drain et al. 2002) showed it positively associated with fertility, early age of first child and high teenage birth rate-compositional factors frequently associated with poverty-but also with low numbers of doctors per capita, less immunization, high rates of HIV and shorter life expectancies-contextual factors equally associated with poverty. Relationships between lifestyle and religion were also important. Christian and Hindu countries displayed higher rates than predominantly Buddhist, Muslim and Orthodox countries (Drain et al. 2002). Finally, a Mexican study of the geography of stomach cancer suggested that analysis by sex "can direct us toward etiologic clues" (Tovar-Guzmán et al. 2001: 317): men generally had higher death rates than women, reflecting unhealthier lifestyles, but in some poorer states this was reversed, reflecting relative female poverty.

\subsubsection{Mortality geographies of cirrhosis of the liver}

Lifestyle factors feature still more prominently with mortality brought about by cirrhosis of the liver. Alcohol consumption was estimated in 2000 to lie behind around 1.8 million (3.2 percent) global deaths but impacting on men ten times more than on women (Norström and Ramstedt 2005). Alcohol abuse underpins proximate causes of death ranging from homicide and suicide to heart disease but prominent is cirrhosis of the liver. It is the single most important cause of alcohol-related chronic disease and resulting mortality (Rehm et al. 2010). Studies reveal that increases in alcohol consumption, measured at either community or individual scale, increase cirrhosis deaths (e.g. Corrao et al. 1997; Ramstedt 2003; Norström and Ramstedt 2005).

Alcohol consumption-and subsequent Girrhosis mortalities-has a complex relation to prosperity. Instead, lifestyle has been more usually emphasized, historically gendered in favor of higher male alcohol abuse. However, Northern cultures are now experiencing some convergence, women consuming more alcohol (e.g. Young et al. 2005) and possibly experiencing larger negative health impacts reflecting, in part, biological factors (Rehm et al. 2010). Nonetheless, relative wealth does still enter the picture, both facilitating greater alcohol consumption, whilst also potentially facilitating better treatment: boosting and reducing deaths, respectively.

\subsubsection{Group 3: injuries}

\subsubsection{Overview and mortality geographies}

With disease accounting for over 90 percent of deaths in 2012, the remainder was down to "injuries." These ranged from "unintentional," notably road accidents, to "intentional," covering self-harm (suicide), interpersonal violence (e.g. murder), collective violence (e.g. war) and legal intervention (e.g. judicial execution) (WHO 2014a). To understand these causes of death and their geographies one has to move away still further from biologically-rooted epidemiology towards political considerations. As in Sections 10.5.2 and 10.5.3, considerable attention must also be paid to arenas shaping the life course, notably class, prosperity or socio-economic position.

Compared to disease mortality, deaths by injuries have been numerically more stable overall in recent times, both historically and geographically. Comparing 2012 with 2000 (Figure 10.10), their proportion is relatively static. Geographically, they varied from a low of 5.9 percent in Europe to a high of 12.4 percent in the Eastern Mediterranean. The latter reflects the impacts of wars and subsequent upheavals in Afghanistan, Iraq and Syria, in particular. Certainly, warfare, especially if assuming epidemic or even pandemic proportions, can cause major spikes in deaths, notably for individual countries. This was seen in Russia's 1950 population pyramid's (Figure 3.3) relative absence of adult males reflecting World War II losses. Accounting for the geographies of war and their associated death tolls is clearly beyond the scope of the present book. However, acknowledging them is not only important for understanding mortality 
geographies but vital for understanding many forced migrations (Chapter 9). Wars not only kill and maim and radically disrupt life course geographies, they also produce huge numbers of refugees and IDPs.

\subsubsection{Geographies of murder and suicide}

In contrast to collective deaths from warfare, many forms of injurious mortality are more individually caused and experienced. Two related examples are deaths from murder, homicide, and self-murder, suicide. The first is widely (mis)represented across popular culture but not researched as much as might be imagined (Massey 1995b; Shaw et al. 2005), whilst the latter has been of great interest to academic scholarship since Durkheim's sociological classic, Suicide, was published in 1897.

Homicide, covered under "interpersonal violence," accounted for just over half a million deaths globally in 2012, less than 1 percent of the total (WHO 2014b). This ranged from just 0.3 percent of total mortality in the European and Western Pacific regions, to 1.4 percent in Africa, and to a more substantial 2.9 percent in the Americas. Individual countries stand out (Richards 1999). In 2012, Honduras with its extreme rate of over 90 deaths per 100,000 population led the field, almost 1 in every 280 males aged between 30 and 44 years murdered annually. This was especially associated with gang violence (UNOCD 2014). However, whilst a complex global geography suggests a complex explanatory picture, geographies of murder at individual country or smaller geographical scale have emphasized a link with poverty and/or sex (Brookman 2005). In sum, as reiterated in a study of homicides in Finland between 1960 and 2000, poorer males tend to be both principal victims and perpetrators (Kivivuori and Lehti 2006).

Focusing on poverty, links between crime (murder specifically) and income inequality have long been acknowledged. In the 1940s, Cohen (1941) wrote how explanations of the geographies of crime were moving away from implicating physical environmental factors to recognizing increasingly the role "of personality, culture, and social organization" (p. 29). Connections strongly parallel those between ill health (and deaths) and such inequality (Wilkinson 1996). It is there at both compositional and contextual levels: poor people and people in poor places are most susceptible to death by homicide (Shaw et al. 2005).

Poverty also underpins other apparent associations. For example, in an overall context of late-twentieth century decline for most groups within the US (Blumstein et al. 2000), homicide rates rose for African-Americans (Massey 1995b). For Massey, this should not be explained by quasi-biological theories (e.g. Herrnstein and Murray 1994) but principally reflected US racism and discrimination. These promoted race-based segregation (6.3.4) and poverty. High rates of murder stemmed largely from how these "two features of metropolitan social structure ... interact to produce an ecological niche within which poverty is concentrated and crime is prevalent" (Massey 1995b: 1230; also Peterson and Krivo 1993). Demonstrating "place is a factor ... when trying to understand homicide" (Shaw et al. 2005: 47), African-Americans are forced within this niche to "adapt to a violent social world" (Massey 1995b: 1230) that normalizes violence, even murder.

Turning to sex, whilst men in general are much more likely to be murdered than women, it is young men, not least in their 20s, who are most at risk. Lifestyle factors are clearly pertinent here, including cultures and expectations of male behavior in conflict situations. A rise in such male risk can counter any general decline in homicide rates, as in England and Wales between 1981 and 2000 (Shaw et al. 2005). Awareness of murder as mostly (young) adult male experience should not downplay, however, other notable categories. These include: adult murders of infantsinfanticide (Brookman 2005: 192-194), especially of girls (e.g. Jones 2013); notorious "race hate" killings, from the lynchings in the US during the 1920s-1930s, to the stabbing of Stephen Lawrence while waiting for a London bus in 1993 (Dixon and Ray 2007); and killings of women by men, extreme patriarchal expression of how "men enact most 'domestic' and 'intimate partner violence'" (Hearn 2012: 590). All risk groups further demonstrate how sociogeographic expression of murder reflects what Shaw et al. (2005: 52) term "societal or public health."

A similar conclusion may be reached about suicide, whose complex geographies have been mapped for Europe at least since the late-nineteenth century (Middleton et al. 2008). Durkheim (1897) suggested numbers of suicides often to be in inverse relation to homicides, due largely to cultural differences. Such a relation is suggested by the global geography of suicides today but is more complex when studied in detail. In 2012, "self-harm" was estimated to have caused just over 800,000 deaths worldwide, 1.4 percent of the total, exceeding those from homicide. High concentrations were found in SE Asia (2.3 percent) and low numbers in Africa ( 0.7 percent) and the Eastern Mediterranean (0.8 percent). 


\section{AGEING AND MORTALITY}

Asia consistently stands out as the principal continent for suicide, accounting for around 60 percent of the global total. However, there is considerable internal geographical variation (Chen et al. 2012). Rates are especially high in Japan, South Korea and Sri Lanka but closer to the global average in China, Thailand and Singapore. Chen et al. (2012) went on to compare Asian suicides with those in Western countries, noting that apart from higher rates, death from self-harm in Asia is less skewed towards men (although still predominantly male) and involves relatively more older people. In terms of causes, acute life stresses appear relatively more important in Asia than mental illness. A further study in Taiwan also found less association with inner city areas than in the West but links to areas of low population density, high numbers of lone parent families and low incomes (Chang et al. 2011).

Looking in detail at causes of suicides, the link to low incomes is, unlike for homicide, more uncertain despite the Taiwan finding (Gatrell and Elliott 2015; Rehkopf and Buka 2006). On the one hand, economic shocks, such as Greece's recent economic collapse, have been linked to a sharp rise in male suicides (Guardian 2014g). On the other hand, various direct and indirect expressions of social isolation or fragmentation (Whitley et al. 1999)-which may again be linked to overall social and public health-tend to feature prominently. These may, of course, often be associated with poverty. Isolation is suggested in Taiwan's high rates of rural suicides, also noted in China (Chen et al. 2012), and in the association with lone parent families and marital break-up more generally (e.g. Hempstead 2006; Stack 2000). Established theories, after Durkheim, linking suicide to modernization's supposed erosion of ties between individuals and society (Stack 2000) further express this link. Individual isolation may also help account for the much higher rate of suicides for men than women in most Global North countries (Möller-Leimkühler 2003).

Finally, migration again comes into the explanatory picture, with higher suicide rates amongst immigrant populations (e.g. Lipsicas et al. 2012). An atlas of suicide mortality in England and Wales displayed high rates in inner city locations but also in coastal and remote communities (Middleton et al. 2008). Whilst the inner city clustering was associated with "psychiatric morbidity" (Middleton et al. 2008: 502), this was not necessarily down to mentally harmful effects of an inner city environment but could instead reflect "inward drift of individuals at increased risk" (Middleton et al. 2008: 502). This was also seen following closure of many mental health facilities in the 1980s (Dear and Wolch 1987). In contrast, remote and rural suicide clusters (also Middleton et al. 2003) may in part reflect migratory drift of healthier people away from such areas. Yet again, life course experiences of entangled compositional and contextual factors shape mortality geographies.

\subsubsection{Environments of mortality}

Of Omran's (1971) three principal categories of disease determinant (10.4.1), primary attention has been paid to socioeconomic, political and cultural factors thus far. Medical and public health factors, second, have also been mentioned, not least through association with class or prosperity. However, the third category, eco-biologic, has been relatively neglected, except in terms of disease structure. Yet, within both Population and Medical/Health Geographies (Macintyre et al. 2002) the "environment" is widely cited. Gatrell and Elliott (2015: 309) observed that as long ago as 400BCE Greek physician Hippocrates acknowledged the influence of "Airs, Waters, and Places" on human health and well-being. Building on this, entanglement of the physical environment with mortality clearly merits attention. When given, it leads ultimately to problematizing the distinction between compositional and contextual factors, again reflecting the multiple relationality of any life course.

Some causes of death can be seen as environmentally caused and others as highly environmentally constrained (Gatrell and Elliott 2015). An example of the former is cancer brought about by exposure to natural radiation, such as lung cancer from Radon in the home (e.g. Darby et al. 2005) or background terrestrial gamma radiation levels causing childhood cancers (e.g. Knox et al. 1988). Other causes of death may be found more universally but are nonetheless strongly constrained by the eco-biological environment. For example, Malaria is much more commonplace in countries with warm, wet climates (Tanser et al. 2003). Beyond such direct connections, however, links between the eco-biologic environment and mortality are often uncertain and complex (Box 10.5).

Consider urban versus rural contrasts in mortality. Sparks (2012: 257) observed a "rural mortality paradox" in the US, whereby after controlling for age, sex and race death rates were lower in rural than urban areas. This was paradoxical as rural areas appeared disadvantaged a priori in terms of lower incomes and education, more poverty, poorer housing and lower health insurance (Brown and Swanson 2003). There is also often stigma and exclusion experienced by rural residents (Watkins and Jacoby 2007). Yet, notwithstanding these negative factors, there

\section{ST PROOFS: NOT FOR DISTRIBUTION}




\section{BOX 10.5 MORTIFEROUS ENVIRONMENTS}

Scholarship having widely demonstrated by that both mortality and morbidity show positive geographical associations with areal deprivation levels, a question remains concerning how much of this pattern can be explained by non-positional characteristics of these environments. In other words, do high death rates in deprived areas reflect, in part, variables other than residents' poverty-such as pollution levels? This issue was investigated in UK and New Zealand contexts by a multi-disciplinary team utilizing multivariate analyses of diverse data sets.

For the UK, ward-scale measures of environmental factors known potentially to impact on health were used to construct a Multiple Environmental Deprivation Index (MEDIx). Eight variables were involved:

\begin{tabular}{lll}
\hline Environmental domain & Variable & Relevance for health \\
\hline Air pollution & $\begin{array}{l}\text { Particulate matter } \\
\text { Nitrous oxide }\left(\mathrm{NO}_{2}\right) \\
\text { Sulfur dioxide }\left(\mathrm{SO}_{2}\right) \\
\text { Carbon monoxide }(\mathrm{CO})\end{array}$ & $\begin{array}{l}\text { Respiratory disease } \\
\text { Cardiovascular disease }\end{array}$ \\
Average temperature & Locations of waste management \\
Industrial facilities & and metal production / processing & Cardiovascular disease \\
Ultraviolet radiation & $\begin{array}{l}\text { Cancer risk } \\
\text { Green space }\end{array}$ & $\begin{array}{l}\text { Protective effect against breast, prostrate and } \\
\text { colorectal cancers } \\
\text { Beneficial for self-perceived health, blood pressure, } \\
\text { excess weight and obesity }\end{array}$ \\
\hline
\end{tabular}

The main findings were, first, that five variables showed clear association with areal deprivation: three increased with rising deprivation- $\mathrm{SO}_{2}$ concentration, $\mathrm{CO}$ concentration, proximity to industry-and two decreased-area of green space, ultraviolet radiation exposure. Second, combining the eight variables into an overall MEDIx, the value increased roughly linearly as deprivation increased i.e. deprived areas had highest multiple environmental deprivation, and vice-versa. Third, MEDIx was similarly positively correlated with death rates for an area. This relationship stood when age, sex and socio-demographic profiles of areas were controlled. However, environmental deprivation had most independent impact in the least deprived wards, a geographical combination that, as suggested already, rarely occur together. This helped make the independent impact of environmental factors relatively modest overall. Thus, in conclusion, "the physical environment does not have a substantial influence on health inequalities as the effect of multiple environmental deprivation is greatest in the least income-deprived wards" (Pearce et al. 2010: 536).

The research team applied a similar approach to investigate the relationship between the environment and mortality in New Zealand. After investigating some additional potential environmental factors, these were not seen to be very significant in impacting on mortality. Therefore, the same variables were used as in the UK. Overall, less association between MEDIx values and areal deprivation was found. However, the positive link between MEDIx and death rates was reiterated, albeit once again "modest" in impact.

The conclusion from these studies is that the environmental quality of an area, whether or not associated with socio-economic status (usually it is), is linked to mortality rates, thus helping to explain the latter's geography $y_{\overline{\mathbf{i}}}$ However, the impact of environmental factors overall should not be exaggerated, reinforcing the priority accorded to compositional factors, notably class, in the main text. Mortiferous environments conducive to death exist but they are not usually principal causes of mortality geographies.

(Sources: Pearce et al. 2010, 2011; Shortt et al. 2012) 


\section{AGEING AND MORTALITY}

seemed to be something about the rural environment that promoted better health and fewer deaths. Reinforcing this proposition, a rural premium reducing mortality rates has also been found elsewhere (e.g. Jones and Lake 2013).

This rural premium idea also comes through within scholarship on so-called therapeutic landscapes, places where healthiness is experienced and/or promoted (Kearns and Gesler 1998; Lea 2008; Williams 2010). The concept has provided "a geographic metaphor for aiding in the understanding of how the healing process works itself out in places" (Gesler 1992: 743). It has frequently been linked to the health giving experience of embodied encounters with "physical" spaces, such as countryside and "nature," or it may be more explicitly socially mediated, as with claimed therapeutic potential of pilgrimage sites, such as Lourdes in France for Roman Catholics (Gesler 1996). For the homeless, a therapeutic landscape could involve: temporary experience of home comforts, such as baths or showers; care institutions, such as detoxification facilities or hospitals; even simply relatively tranquil and accessible public spaces, such as park benches (Petroskas 2011).

Can it be concluded, therefore, that rural living in particular is somehow life-promoting? This is perhaps a step too far. First, the geographical association is not found everywhere (Sparks 2012), suggesting that analysis to date has not controlled sufficiently for geographically-expressed non-"environmental" variables linked to mortality. Second and building on the latter, studies have found it difficult to control for the role of migration in contributing to spatial differences observed. For example, there is the generally healthy profile of rural in-migrants (counterurbanizers) and drift of the less healthy towards urban centers (Boyle et al. 2009; Darlington et al. 2015; Gatrell and Elliott 2015). Finally, even if rural areas do impart a positive premium on some lives (e.g. Halfacree 2014a), ultimately determining the exact "independent" role of the (physical) environment on health and mortality may be something of a misguided objective. The therapeutic landscape concept is less about places having intrinsically health-giving properties and more about the therapeutic outcomes emerging from encounters with specific places (Conradson 2005). For both health and mortality, but for the latter especially, abundant evidence-some presented above-suggests intervening "social" factors encountered through a life course more immediately influence health than the environment, whose impact may thus tend towards the trivial (Box 10.5). Reiterating Dorling (2005: 103) [[AQ*]], it must be recognized how mortality "lay[s] down a record of our lives," in all its complexity.

Such a conclusion might seem to leave contextual factors very much secondary to compositional factors for explaining mortality geographies. However, it is better to see supposed opposition between the terms as ultimately unsustainable. Geographies of the life course (Chapter 2) tell of lives, which acquire compositional characteristics through the life course (class, gender, sexuality, and so on) relationally constructed across space-time, making them also contextual. That the two aspects cannot really be separated lies at the heart of this book's relational framework. Who one is strongly determines likely mortality experiences (Dorling 2011:22-37) but most of who one is comes via "differential accumulation of exposures and experiences" (Lynch et al. 2000: 1202) of living a life across space.

\subsection{CONCLUSION: MORTALITY, RESOURCES AND ACCESS IN AN AGEING WORLD}

As global fertility rates continue to decline (from 5 children per woman in 1950-1955 to 2.5 in 2010-15) and, mostly on account of declining IMRs, life expectancy keeps increasing (from 48 years in 1950-1955 to 68 in 2005-2010), both number and proportion of older adults living in the world grows (Green 2009; UNFPA 2012b; Kinsella and Phillips 2005). We live, in short, in an ageing world.

One consequence of living in this ageing world is that in most societies today people have increasingly come "to view premature death as extraordinary" (Hunt 2005: 205). Instead, the expectation is to engage with at least some of the rich lifestyle opportunities of the Third Age before eventually succumbing to bodily ageing and consequent slowdown in the Fourth Age. Eventually, death will come from "old age" or, more likely, a non-communicable disease. This chapter sought both to present such a (Northern-biased) picture of the end of the life course as indeed being typical for some people but has also emphasized its selectivity. Overall, experiences of both older age and mortality have been shown, in short, as highly differentiated.

Distinctive and diverse spaces of both ageing and death are clearly present at a range of scales globally. When digging below initial patterns to explain them, however, the underlying causal theme consistently standing out has been a person's relative resources, represented directly or through such mediating terms as class, status, wealth, income 
or the developmental level of the country lived in (geography and history). Globally, continentally, by country, and within country, the relational constitution of ageing and mortality within a life course is defined prominently through resources (Gould 2015). In terms of life course arenas (2.3), class, geography and history have been most consistently stated, albeit always cross-cut by the potential of individuality, gender, sex and others to confound clear associations.

In close entangled tandem with emphasis on resources, accessibility has been a second recurrent theme found within the shaping of senior lives and constitution of death. Access to monetary resources is paramount but so too is: accessible homes; access to services; accessing lifestyle choices; access to medical services and appropriate medicines and care; access to clean water and adequate food; access to a supporting community; access to safe behaviors; access to therapeutic environments .... The list could go on. Such an emphasis thereby reinforces the overall conclusion that both compositional and contextual factors, inextricably entangled, shape the individual experiences and the aggregate geographies that denote the latter stages and then the end of lives lived across space.

\section{NOTES}

1 From other perspectives-religious, cultural or political impact, memories of family and friends-a life frequently does not end with death. Significant variation also exists between cultures and religions in how death is perceived and anticipated, and in rituals surrounding it. Whilst this chapter presents death as the end of a life course, this may be challenged by beliefs in reincarnation and forms of afterlife.

2 There is also a neontic dependency ratio from the population aged 0-19 years relative to those aged 20-64 (Holdsworth et al. 2013: 52), which seeks to express numerically the dependency of children and youth on working age adults. This can be critiqued in a very similar way to the gerontic dependency ratio, since many young people globally engage with waged work, for example (Gould 2015). 


\section{CHAPTER 11}

\section{Twenty-first-century lives across space}

\subsection{TWENTY-FIRST-CENTURY PERSPECTIVES}

Everyone is wrong about the future. Man (sic.) can only be certain about the present moment. But is that quite true either?

(Milan Kundera 2002: 148)

This book has introduced some key findings from the great diversity of contemporary scholarship that can be placed under a Population Geography sub-disciplinary heading. Chapters sought to present the results of studying the spatial expressions of population from the past 20 years in particular using an extended life course perspective as frame and guide (Chapter 2). This final chapter will now look briefly and cautiously to the future.

As a sub-discipline, Population Geography has evolved in two principal directions (also Bailey 2005). First, it has grown from one focused predominantly on descriptive, empirical and positivist approaches, utilizing primarily deductive methods, to one that has extended ever-outwards to incorporate diverse social science perspectives-feminism, Marxism, humanism, postmodernism, post-structuralism-often utilizing more inductive modes of inquiry (Chapter 1). Second, focusing primarily on those areas of scholarship directly tied to the focus of "traditional" Population Geography-the triumvirate of births, migrations and deaths-the sub-discipline has also increasingly pushed its explanatory frame outwards, in line with the inclusive holistic ethos of a life course approach. Consequently, the present book considered both longstanding ideas and emerging issues within each topic area, considering what has been investigated and suggesting new directions and often novel issues for consideration. The latter reflects a major theme of the book, namely how the ultimate shapes taken by lives across space are relationally constructed. They are shaped through key bio-social arenas of everyday life (2.3), including the geo-historical framing through which life course scholars denote cohorts (2.2.1). It is the latter, specifically the first half of the twenty-first century, which is given particular attention in this final chapter.

Of course, the future directions taken by the multitude of population geographies currently expressed across the globe are impossible to predict with any conclusiveness. Some sense of the academic challenge this presents was given a decade ago in a special issue of the journal Population, Space and Place. Most importantly here, Elspeth Graham (2004: 293) noted how several "big issues of the day" were intimately linked to Population Geography and so merited urgent attention: "relationships between population and resources, the movements of refugees and asylumseekers, the consequences of international migration and transnationalism, the implications of low fertility and ageing populations, and the gross inequalities in the risks of illness and death" (also Conway 2004). Many will be considered in the chapter, which similarly emphasizes how futures of both population geographies and Population Geography will be played out within broad societal parameters.

Section 11.2, therefore, lays out four key twenty-first Century Challenges faced by humanity as a whole and also largely caused by humanity. These are indicative of an era increasingly labeled even within mainstream media as the Anthropocene (e.g. Guardian 2014h) from the sheer extent of human environmental impacts. Within this context, not only must Population Geographers live and work but, more significantly, global to local population geographies are being made and shaped. Section 11.3 then presents some UN projections for the global population in 2050, making links in its anticipated expression to the four twenty-first Century Challenges. The book concludes with Section 11.4 reflecting briefly on the past, present and future of Population Geography, noting its strong contemporary position 
but also how continuing expansion in terms of scope, methodologies and engagement with social theory may be essential to understand detailed emerging population geographies. Indeed, there may be a case-flagged earlier in Section 2.4-for Population Geography to evolve into a more completely relational Life Course Geography.

\subsection{TWENTY-FIRST-CENTURY CHALLENGES}

\subsubsection{Anthropocenic contexts}

[A] new epoch of humanity ... defined by humanity's imprint on the planet"

In 2009, UN High Commissioner for Refugees António Guterrez named five mega-trends that would complicate humanitarian effort within his area of responsibility in the near future (UN 2009). These were: urbanization, migration, population growth, climate change, and food, water and energy insecurity. Whilst the first three refer to topics familiar and traditionally of central significance to Population Geography, the latter two reflect emerging contexts whose population geography impacts are thus relatively novel. Critically, however, all five trends have Population Geography resonances that extend far beyond their major consequences for refugees. They are also contexts that can be supplemented by others and together help justify the claim that the present period merits the label Anthropocene.

The term Anthropocene was coined at the turn of the Millennium by Nobel Prize winning Chemist Paul Crutzen and freshwater Ecologist Eugene Stoermer (Crutzen and Stoermer 2000). It has come to stand for "human impacts on the Earth's surface of such magnitude, scope and scale as to present an existential threat-or, perhaps, to offer transformative opportunities?-to people and other planetary life forms" (Castree 2014: 437). The suggestion is even that the geological epoch the Holocene is now ending (e.g. Crutzen 2002), although how this can be conclusively decided remains highly elusive (Castree 2014). Most significantly, as Castree's definition suggests, the Anthropocene is not just some neutral condition for scientists to debate at conferences and in journals but signifies a stepping-up of human impacts on the world that may be endangering the very survival of humanity and numerous other living things (Sachs 2012). Typically depicted is "an unknown and potentially inhospitable Anthropocenic future" (Castree 2014: 441 ) as transgression of a series of more or less determined global environmental boundaries that allowed "a safe operating space for humanity" (Rockström et al. 2009) increasingly occurs.

Four Anthropocenic contexts will shortly be introduced, three of which can clearly be linked to such boundary transgressions. Moreover, like the Anthropocene itself, each should not be regarded simply as life course backdrops but are strongly entangled relationally to key ongoing and emerging population geographies-births, migrations, deaths. The four global twenty-first century challenges noted are: inequality, global warming, resource depletion, and biotechnology. For each, a comprehensive account cannot be given but specific manifestations exemplify the general picture. Combined, each to a greater or lesser extent will shape presently emerging and evolving twenty-first century population cohorts.

First, however, it is instructive to return to António Guterrez's mega-trends. These not only chime with the twentyfirst century challenges but also, unsurprisingly given their source, are reiterated by the UN in its snappily-titled

Table 11.1 The UN's five priority areas for future population and development

\begin{tabular}{ll}
\hline Priority area & Includes \\
\hline Dignity and human rights & Tackling poverty, empowering women, overcoming discrimination \\
Health & Infant survival, sexual and reproductive health and rights, lifestyles \\
Place and mobility & Changing households, internal migration, urbanization, international migration, reducing place \\
insecurity
\end{tabular}

Source: derived from UN (2015a). 


\section{TWENTY-FIRST-CENTURY LIVES ACROSS SPACE}

Framework of Actions for the Follow-up to the Programme of Action of the International Conference on Population and Development Beyond 2014 (UN 2014a). The original Programme of Action was adopted in 1994 and the 2014 review largely reiterates its issues and ambitions. Priorities for the world's governments and peoples for action were grouped into five areas. These are listed in Table 11.1 and referenced in the rest of the chapter where appropriate. All reinforce the sense of challenge the Anthropocene presents if humanity is to be living in a world in 2050 at least as conducive to the development of diverse but rewarding life course geographies as it is in the present day. Absolutely central is the issue of (in)equality, considered next.

\subsubsection{Inequality}

Today, in the second decade of the twenty-first century, inequalities of wealth that had supposedly disappeared are close to regaining or even surpassing their historical highs. The new global economy has brought with it both immense hopes (such as the eradication of poverty) and equally immense inequalities.

(Thomas Piketty 2014: 471)

Given its central role accorded to humanity, when examining the impacts of the Anthropocene it is imperative not to confine attention to more physical environmental expressions. Instead, the often unhelpful human-environmental boundary (Castree 2014) itself merits frequent transgression. Thus, a first twenty-first century challenge shaping population geographies concerns the very "human" expressions of socially-produced inequality and the transgressing of boundaries of fairness and justice.

Throughout this book, inequality, a general, ubiquitous and multifaceted presence has shown itself absolutely central to underpinning and explaining population geographies. It expresses unevenness in the level of absolute and/or relative resources, measured between places, times, or people. Its significance has frequently been expressed throughout the book not least by the assertiveness shown by the key arena of life course differentiation labeled socioeconomic class (2.3.4), a foundational feature of the capitalist mode of production. Inequality is, however, also expressed through all the other arenas of life course construction: history; geography; health and differential ability; race and ethnicity; religion and belief; sex and gender; sexuality; and even individuality. Put slightly differently, present-day population geographies commonly express the variously socialized resource inequalities which remain apparent in the twenty-first century. Whether such inequalities and their relative size in particular are regarded as an inevitable consequence of human development or as ultimately unnecessary, and thus as a fundamental challenge to be overcome politically, they consistently appear as the critical context through which life courses are played out.

As the book has also abundantly demonstrated, inequalities in resources and access to them express themselves in a bewilderingly wide variety of forms. As already noted, they are not solely a matter of class, certainly not proximately. Some sense of this is shown by the myriad expressions of inequality illustrated on the inequality.org website, established by the Institute of Policy Studies (Inequality 2016). Almost like an actor wearing a series of masks, inequality's different facets-income, education, status, positionality, geopolitical location in the world, political authority-come to assume prominent roles in different Population Geography contexts for lives lived across space. However, all are underpinned, more-or-less directly, by the central lubricant of the capitalist world that is money and its central expressions as wealth (e.g. Dorling 2014; Harvey 2006; Stiglitz 2012). And, as Piketty's (2014) opening quote for this section suggests, wealth inequalities have shifted from (unevenly) declining post-1945 to (unevenly) increasing today.

From this brief reflection on a topic that is comprehensively and forensically discussed as a pressing global concern across academic, policy, NGO, media and civil society, it should thus be unsurprising that the first twentyfirst century challenge is that of tackling these inequalities in resources, not least but not confined to monetary resources, at various geographical and social scales across the world. To illustrate the scope of this challenge within limited space constraints, the global North-South articulation of promoting more even and equitable human development and the within-country task presented by inequality within one of the authors' home countries will be considered.

On 8 September 2000, 189 world political leaders assembled in New York and signed the United Nations Millennium Declaration. This sought to engage actively the world in that milestone year with the global challenge still presented by a general inequality that broadly divides the world in two in terms of overall human development: "richer" Global North versus "poorer" Global South. Within the "bold vision for the future" (Ban, quoted in UN 2014b: 3)

\section{ST PROOFS: NOT FOR DISTRIBUTION}


expressed in the New York declaration were Millennium Development Goals (MDG) (Gould 2015: Box 0.1). Ban KiMoon, UN Secretary-General, went on to write that these represented "a pledge to uphold the principles of human dignity, equality and equity, and free the world from extreme poverty." Table 11.2 lists the MDGs and the UN's own assessment of the extent to which they had been met by 2015 (UN 2015b). The (official) picture one sees is very positive but suggestive of considerable challenges remaining. This mixed assessment is reiterated more generally in commentary on the MDGs (e.g. Gould 2015; Sachs 2012). Indeed, it should also be noted that there have been numerous critical commentaries on the goals, in terms of how they have been framed, what they mean, the extent to which they are in fact being achieved, and even their potential negative consequences for poorer nations (e.g. Attaran 2005; Pogge 2004; Saith 2006). There is not space to deal with these here, suffice to note how, overall, they reinforce the extent of the challenge still facing the world to achieve any level of developmental equality that would make the Global North versus Global South distinction substantially redundant. Until this occurs-if it ever does-global population geographies are likely to continue to express the kinds of North-South contrasts this book has demonstrated widely.

The UN, in fact, has been very up front in regards of the challenge inequality still represents. They expressed it thus: "Millions of people are being left behind, especially the poorest and those disadvantaged because of their sex, age, disability, ethnicity or geographic location" (UN 2015b: 8). Inequality, once again, starts with poverty but then intersects (2.4) with other arenas of life course differentiation. Relationality is further expressed in the Framework of Actions document (UN 2014a), where the Dignity and Human Rights priority (Table 11.1) foregrounds poverty eradication as the highest imperative but also notes its entanglement with disempowering women and various forms of discrimination. In summary, "[w]hen growing inequality precludes human well-being for vast numbers of people, every part of society is impacted" (UN 2014a: 207).

In terms of geographies of inequality, the global or international scale is certainly not the only one of significance for everyday lives and thus for population geographies. Of particular note are sustained and often increasing resource inequalities existing within countries. These may again be expressed as occurring between places-notably between regions or between urban and rural locations-but are also articulated as inequalities between people within the same place. Access to resources is not just a factor of where one is but also of who one is-positionality (2.3.4). Abundant evidence of both such spatial and social expressions of within-country inequalities can be found within any country, albeit with considerable national variation. Attention is briefly paid here to the UK. Figure 11.1 shows it located towards

Table 11.2 Progressing the Millennium Development Goals

\begin{tabular}{|c|c|}
\hline Goal & Evidence of progress $(m=$ million, $b=$ billion $)$ \\
\hline 1. Eradicate extreme poverty and hunger & $\begin{array}{l}\text { Extreme poverty in developing countries: } 1990=40 \%, 2015=14 \% \\
\text { Global number of extreme poor: } 1990=1,926 \mathrm{~m}, 2015=836 \mathrm{~m}\end{array}$ \\
\hline 2. Achieve universal primary education & $\begin{array}{l}\text { Out of school children: } 2000=100 \mathrm{~m}, 2015=57 \mathrm{~m} \\
\text { Sub-Saharan enrolment: } 1990=52 \%, 2015=80 \%\end{array}$ \\
\hline 3. Promote gender equality and empower women & $\begin{array}{l}\text { South Asia enrolment: } 1990=74 \text { girls } / 100 \text { boys, } 2015=103 \text { girls } / 100 \text { boys } \\
90 \% \text { countries with more women in Parliament than in } 1995\end{array}$ \\
\hline 4. Reduce child mortality & $\begin{array}{l}\text { Under } 5 \text { s deaths: } 1990=12.7 \mathrm{~m}, 2015=6 \mathrm{~m} \\
\text { Global measles vaccination: } 2000=73 \%, 2013=84 \%\end{array}$ \\
\hline 5. Improve maternal health & $\begin{array}{l}\text { Maternal mortality ratio (deaths/100,000 live births): } 1990=380,2013=210 \\
\text { Skilled health personnel attending births: } 1900=59 \%, 2014=71 \%\end{array}$ \\
\hline 6. Combat HIV/AIDS, malaria and other diseases & $\begin{array}{l}\text { ART treatment: } 2003=0.8 \mathrm{~m}, 2014=13.6 \mathrm{~m} \\
\text { 900m insecticide-treated mosquito nets delivered to Sub-Saharan Africa } \\
2004-2014\end{array}$ \\
\hline 7. Ensure environmental sustainability & $\begin{array}{l}\text { 1.9b people gained access to piped drinking water } 1990-2015 \\
98 \% \text { ozone-depleting substances eliminated } 1990-2015\end{array}$ \\
\hline 8. Develop a global partnership for development & $\begin{array}{l}\text { Official development assistance: } 2000=\$ 81 \mathrm{~b}, 2014=135 \mathrm{~b} \\
\text { Global internet penetration: } 2000=6 \%, 2015=43 \%\end{array}$ \\
\hline
\end{tabular}

Source: UN (2015b: 4-7). 


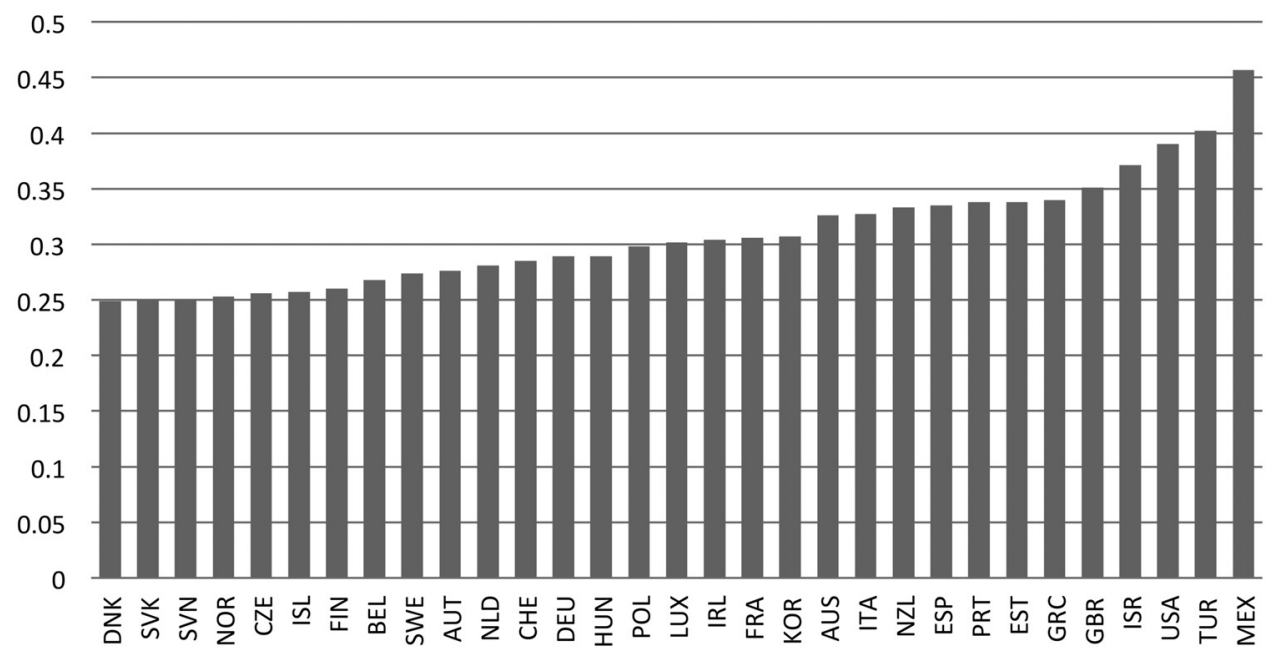

Figure 11.1 Gini coefficient of OECD income inequality 2012. (Gini coefficient compares cumulative proportions of population against cumulative proportions of income received. It ranges from 0 (perfect equality) to 1 (perfect inequality).)

(Source: data from OECD 2016)

the higher inequality end for OECD countries, for example, where Scandinavia demonstrates greatest equality and the US, Turkey and Mexico greatest inequality.

In 2013 , aided by the charity the Joseph Rowntree Foundation, a team of researchers from the Institute of Fiscal Studies published a substantial report on Living Standards, Poverty and Inequality in the UK (Cribb et al. 2013). The report analyzed net UK household income, reducing all income obtained from employment, investments, benefits and pensions by tax outgoings, controlled for household size and composition. It concluded that both mean and median net incomes had generally grown steadily over the previous 50 years but were intercut with falls in periods of economic hardship, such as when the report came out. However, against this broadly positive backdrop, expressions of inequality and poverty remained strong. In other words, there is clear parallel here with the predominant reading of the success of the MDGs. Specifically for the UK, since the 1980s income inequality had risen rather than fallen overall. "Relative poverty," measured as net income less than 60 percent of the overall median, showed a more complex trend, rising during the 1980s but then generally falling. In 2013, however, around 13 million people or 21 percent of the population were estimated to be in relative poverty when (generally rising) housing costs were taken into consideration. "Absolute poverty" had also been increasing.

Poverty in the UK has also shifted in terms of its population base. Since 1961, previous strong association with older people has declined, not least due to higher pensions and state benefits. In contrast, there has been a noted rise in poverty levels "among working-age adults without children" (Cribb et al. 2013: 7). This reflects, first, a rise in families without stable, consistent work bringing in a regular living wage that can support a family. So-called zerohours contracts, where the "employee" (sic.) is not guaranteed a set amount of work a week but has to be ready to take what is offered at often short notice, epitomizes this condition of extreme economic precariousness (Adams et al. 2015). Second, state benefits for those out of work have been consistently cut back (Dorling 2014). This is reflected in the rise of emergency food banks, increasing in number from 29 in 2009-2010 to 251 in 2013-2014, whose geographical concentration is found in places in the UK where welfare cuts have been highest (Loopstra et al. 2015). Together, precariousness and poverty are having very notable life course impacts, including undermining previous markers of the key transition (2.2.1) to adulthood such as moving into one's own home and being able to marry (Moore 2016) which are strongly linked to residential mobility in particular (Chapter 6).

Expressions of present-day inequality in the UK and its consequences have been highlighted in a number of publications (e.g. Davies 1998; Jones 2014; Meek 2014), including by Population Geographer Danny Dorling (2014). Dividing the population between the richest 1 percent and the remaining 99 percent-in line with the distinction made 
by the radical political Occupy movements that blossomed in 2011-2012 (Pickerill and Krinsky 2012)-Dorling noted how since return to economic recession around 2008, the richest 1 percent has grown still richer in absolute and relative terms. Austerity, the tough economic conditions resulting from reduced state expenditure, has been very unevenly experienced in the UK. Directly connecting to population geographies, Dorling emphasized again how inequality is not just a matter of money or economics by demonstrating how being born outside of the wealthiest 1 percent impacts negatively on lives in numerous ways, shaping childhood, work, lifestyle, health experiences and nearly all aspects of lives across space.

However, inequality in life opportunities is not solely a socio-political matter, as complex as this is in itself, but also involves environmental and biological contributions. The next two challenges illustrate the former and the third the latter. All three, though, must also be seen as highly social in how they are (not) experienced by different people in their lives across space. This once again reinforces the foundational status of social inequality and its undoubted status as a foundational twenty-first century challenge for humanity.

\subsubsection{Global warming}

This is an emergency. Immediate and transformative action is needed at every level: individual, local, and national; personal, political, and financial. Countries must set aside differences and work together ... for the common good .... in a way ... equitable and sensitive to particular challenges of the poorest countries and most vulnerable communities.

(David McCoy et al. 2014: 7)

Chapter 3 presented an overview of the geographical distribution of the global population-the Ecumene (3.2.1)-its considerable unevenness seen as a product of numerous factors. Amongst such factors are humanity's historical and present-day settlement responses to the opportunities and barriers presented to it by the physical environment. For example, coastal locations have long proved popular for settlement, not least because of the opportunities they provide for ship communication and food (fish). The physical environment, therefore, influences the Ecumene but this environment is far from static and most crucially is itself changed by the actions of humanity in particular. Thus, Conway (2004: 298) challenged Population Geographers to look at human-environment relationships from the perspective of humans driving environmental change and also in reverse, how environmental change has "significant impacts upon resource distribution and accessibility."

One of the key environmental factors influencing both human settlement overall and more individual playing out of lives across space is climate. This is demonstrated by its prominent role in the co-production of geographies ranging from crop production to lifestyle migrations to probabilities of catching communicable diseases. Climate change, therefore, has major implications for everyday human survival, quality of life and health, respectively, and thus on both direct and indirect expressions of Population Geography. Climate change has always occurred but today it is humanity's role in promoting it which has caught the world's attention.

Ongoing anthropogenic or human-induced changes to the climate are captured in the term global warming. Accorded central stage here are the multiple consequences of the warming of the Earth's atmosphere due to a buildup of carbon dioxide and other pollutants trapping the Sun's heat (NRDC 2014). The basic science is that these gases, produced from industrial, domestic and agricultural activities, not least burning fossil fuels, enhance the atmosphere's natural greenhouse effect, whereby energy emitted from the surface of the Earth becomes trapped in the atmosphere and consequently heats it up (Gatrell and Elliott 2015).

Any quick Google search of the terms "global warming" and "climate change" yields thousands upon thousands of results. Like inequality, therefore, this is far from an ignored topic but also like inequality it is politically controversial. Public debate continues to be heated and the exact science required to assess the precise anthropocentric contribution to global warming in particular is recognized as extremely complex. Nonetheless, the overwhelming global consensus is that global warming is a very real phenomenon and will impact substantially on climate, albeit very unevenly geographically (e.g. Monbiot 2006; Stern 2006). As with the Anthropocene concept (and inequality), therefore, global warming is not just a neutral term to note but a condition with potentially highly negative consequences for humanity. Countering and mitigating it thus definitely comprises a twenty-first century challenge.

\section{ST PROOFS: NOT FOR DISTRIBUTION}




\section{TWENTY-FIRST-CENTURY LIVES ACROSS SPACE}

Numerous formal attempts have been made to reduce global warming or at least lay down the grounds to do so. Again, parallels with the challenge posed by inequality can be noted. Especially notable is the Kyoto Protocol, an international treaty extending the more general 1992 United Nations Framework Convention on Climate Change (UNFCCC) to commit signatory countries to reduce greenhouse gas emissions. As of 2016, 192 countries have signed the Protocol (UNFCCC 2016). More recently, the twenty-first Conference of Parties to the UNFCCC (COP21), held in Paris in 2015, saw 195 governments set a target to limit global warming to "well below" $2^{\circ} \mathrm{C}$ as compared to preindustrial levels (UNFCCC 2016). COP21 requires at least 55 countries responsible for at least 55 percent of greenhouse gas emissions to ratify it before it becomes active. As of May 2016, just 18 countries responsible for 0.18 percent of greenhouse gases had officially ratified COP21... (UNFCCC 2016).

The science invoked within all these discussions is principally routed through the Intergovernmental Panel on Climate Change (IPCC). This is an international body established by the United Nations Environment Programme (UNEP) and World Meteorological Organization (WMO) in 1988 to provide a scientific perspective on the current state of knowledge of climate change and its potential environmental and socio-economic impacts (IPCC 2016).

One consequence of all this political and scientific concern has been numerous further endorsement of the reality and threat of global warming by various distinguished bodies. An example is that given by the eminent health professionals cited in this sub-section's opening quote from a British Medical Journal editorial. And within this environment of marked concern, Curtis and Oven (2012: 654), amongst others, have argued that Geographers are particularly well positioned to evaluate the "complex processes operating at various geographic scales," inclusive of the micro scales of daily action spaces as well as broader more aggregated areas, that global warming presents. Table 11.3 presents some ongoing areas of such enquiry just related to health.

For Population Geographers specifically it must be noted that within the broad climate change debate demographic implications do indeed loom large (Bailey 2011; Huguet 2012; IPCC 2007). Empirical linkages between climate change induced environmental changes and both fertility (e.g. Lockwood 1997; Rovin et al. 2013) and mortality and health (e.g. Huang et al. 2011; Nicholls 2009; Yang et al. 2015) have been made by Economists, Environmental Scientists, Demographers, Health Geographers and numerous others. Gatrell and Elliot (2015: 358-359) noted direct impacts-such as "increased number, frequency, and intensity of extreme weather events"-indirect impacts-such as ecosystem modification stimulating infectious diseases-and "impacts through other factors"-such as negative health effects brought on by loss of employment, property and income.

Actual and potential climate change induced migrations have also attracted increasing research attention (Bailey 2011; Castles et al. 2014) as an "environmental" variant of forced migration (9.2.2.1). Here it should however be noted that whilst areas particularly impacted by global warming in terms of loss of cultivable land and consequent increased food insecurity are likely to see population out-migration, such as many areas in Africa (FAO 2013), to be overly deterministic is problematic. Migrations typically have numerous complex relational causes, as Chapters 5-9 demonstrated. Thus, "[a] deterministic approach that assumes that all or a proportion of people living in an 'at risk' zone ... will migrate neglects the pivotal role that humans take in dealing with environmental change and ... ignores other constraining factors which influence migration outcomes" (Foresight 2011: 11). Further, as Castles et al. (2014: 211) observed after similarly quoting the UK Government's Foresight Report, there would still be considerable migration even without climate change "because it is driven by powerful economic, political and social processes." Second, not least through its links to heightened poverty, climate change may be associated with non-migration, being forced to

Table 11.3 Areas of enquiry for population geographers relative to health and climate change

- Issues of adaptation and resilience

- Sustainability

- Environmental justice and socially unequal impacts of climate change

- Psychological and physical impacts of environment on health

- Significance of affect and emotion for the perception and communication of hazard and risk associated with health impacts of climate change

- Understanding exposure to health risks of climate change requires knowledge of complex and individually variable daily action spaces and residential mobility over the life course

- Complex processes operating at various geographical scales

Source: Curtis and Oven (2012). 
stay put (6.2.5), as much as with migration. Third, political efforts to prevent migration stimulated by climate changenoted in Chapter 9-may be very detrimental for people affected and encourage undocumented migration. Indeed, Castles et al. (2014: 211) concluded that "[m]igration can represent a transformational adaptation to environmental change, and may be an effective way to build resilience." It will be nearly impossible to stop.

Intertwined with climate change's potential impact on populations is the issue of adaptive-response. This covers socio-political processes of mitigation and securitization, and more psychological perspectives on vulnerability and environmental hazards, much of it little covered to date within conventional Population Geography. Illustrative studies include political and cultural ecology frameworks of vulnerability (Robbins 2004), vulnerability perspectives (Cutter 1996), the role of social and cultural systems in mediating vulnerability (Adger 2006) and the earlier-mentioned emerging frame of securitization and fractioning (9.2.1) as it applies to climate change migrants (Oels 2013).

In summary, the Physical Geography environmental world of climate and climate change is clearly integrally entangled with human lives across space and their population geographies. This reinforces the threat and potential impacts that climate change prompted by anthropogenic global warming poses to all everyday lives. The UN's Framework of Actions report also clearly recognized this (UN 2014a). Population Geography as a sub-discipline must respond accordingly.

\subsubsection{Resource depletion}

[1]t is possible to envisage a long term process of peak oil leading to a move of economic activities ... closer to their markets, with the result that the global economy becomes less integrated but more regional ... [but also of] radical new conceptions of livelihood and economy.

(Peter North 2010: 586)

Also noted by the Framework of Actions report (UN 2014a) was the second environmental-social twenty-first century challenge with clear relational importance for emerging population geographies. This concerns unsustainable depletion of the resources that fuel all aspects of the globalised and mobilized (5.2.2) human world. This challenge is closely entangled with debates about the maximum or optimum size population the Earth can sustain, to be discussed in Section 11.3.2. These resources range from the inert physical components of the Earth used by humanity, notably oil and other minerals, to the approximately 8.7 million different forms of life (Goldenberg 2011) that share the planet with us humans. Both face severe risk of depletion, exemplified here through the cases of peak oil and the 2014 Living Planet report, respectively.

Explicitly put, oil in numerous forms underpins much of contemporary society and its geographies across the world. Figure 11.2's account of global petroleum use shows Asia now having overtaken North America in consumption (Plumer 2013) and also the relatively little that is consumed by Africa. Besides its key role in transport, industry and heating, oil is deeply implicated in the food system. As the summary of a critical 2001 report, Eating Oil, put it: "[within] the contemporary food system ... supply of even the most basic foods has become dependent on petroleum" (Sustain/Elm Farm Research Centre 2001: 1)-transport, processing, packaging, and so on.

A consequence of this dependency is that any sustained decline in the availability and affordability of oil and its derivatives will have major impacts on the everyday lives of everyone on the planet. Such impacts are likely to be similar to those anticipated from global warming. From a Population Geography perspective, deaths and considerable morbidity will undoubtedly follow subsequent political-economic everyday life disruptions. All more voluntary or everyday forms of migration and mobility could be reduced-the era of mobilities would be severely challenged-yet the disruptions also stimulate a rise in forced migrations, and in forced immobilities. Of particular note are the economic dimensions of future oil scarcity, including prompting substantial return migrations of labor from presently oil-rich Gulf states (Box 7.4), for example. At the same time, however, there will likely be a rise in FIFO-type labor migrations (7.4.4) to ever more remote sources of oil. Even fertility would be impacted through declining economic and political security reducing the confidence to have children, as well as via negative health impacts on fertility.

The concept of peak oil articulates this considerable potential threat to what are now in at least the Global North largely taken-for-granted oil-based lifestyles. It refers to "the point at which the global output of conventional oil reaches its maximum level and subsequently flow rates decrease ... [i.e.] when roughly half the world supply of oil has been produced and subsequent output falls" (Chapman 2014: 93). After peak oil, extraction costs are likely to increase

\section{ST PROOFS: NOT FOR DISTRIBUTION}


318 TWENTY-FIRST-CENTURY LIVES ACROSS SPACE

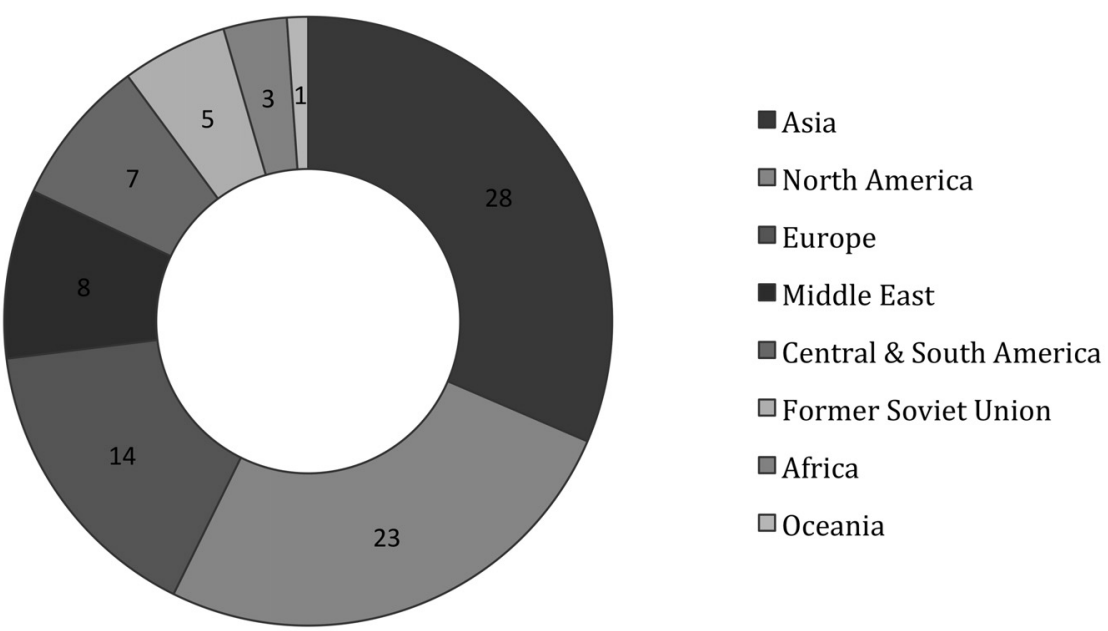

Figure 11.2 World petroleum consumption by region 1980-2012.

Source: based on data from US Energy Information Administration; Plumer (2013).

progressively as oil gradually runs out and what remains becomes harder and harder to access. As already noted, significant negative implications for the stability of the global economy and its potential growth are clear (Murphy and Hall 2011). Furthermore, the "peak" idea can, of course, be extended to the other fossil fuels-coal and natural gas (Maggio and Cacciola 2012)-and to other non-renewable resources.

As a broad idea but not yet so named, peak oil was first suggested in the 1970s but became popularized in the early 2000s as rival estimates of its likely date were proposed, ranging from 2005 to the middle of the twenty-first century (Chapman 2014). Variation reflected, in part, uncertainty over both the size and quality of available reserves, and the ability of new technologies to extract hard to reach sources. Non-fossil fuel alternatives to oil can also be factored in here. Overall, not least in a context of still rising demands for oil, Chapman (2014: 100) concluded that peak oil "should be acknowledged as part of a complex energy situation with the realization that cheap fuel is no longer available[,] ... prices will increase and high-energy based growth will be limited."

As a result of the negative implications of peak oil, both advocacy and practical attempts at less energy intensive lifestyles have been placed firmly on the political life course agenda (e.g. Bailey et al. 2010; Dorling 2013; North 2010). Localization, for example, whether in an "immanent" form of a "less globalised capitalism" (North 2010: 593) or in a more radical "intentional" form of a "normative [post-capitalist] political project" (North 2010: 589) both suggest reduced everyday mobilities and most forms of migration; a post-mobilities era. Certainly, recognizing the imprint of oil and its derivatives in so many everyday consumer products and either reducing their consumption or replacing the oil element with more renewable alternatives seems imperative. More positively, one potentially beneficial consequence of peak oil is how it may ultimately lead to reduced production of greenhouse gases. Indeed, if oil does not peak the danger is "that we will continue to find new reserves and burn so much more that climate change will be further accelerated" (Dorling 2013: 181).

Turning from depletion of the globe's physical resources-not it should be reiterated just oil-to its living populations, a number of campaigning groups headed by the World Wildlife Fund (WWF) published in 2014 its annual Living Planet report (WWF 2014). This report attracted considerable press coverage because it concluded the 1970-2010 period saw decline by over half within a sample of over 10,000 species of mammals, birds, amphibians, reptiles and fish representing all life on Earth. As Nijhuis (2014: np) sharply put it: "[t]wo human generations; half the animals gone." Principal causes according to WWF were over-hunting, over-fishing, loss and degradation of habitat, and climate change. Decline was strongest in tropical regions and freshwater environments, although generally apparent even within supposedly protected areas such as National Parks (WWF 2014).

The consequences for Population Geography of potential mass extinctions of many living species may not at first appear as immediate as with physical resource depletion. However, they may be even more severe, prompting

\section{ST PROOFS: NOT FOR DISTRIBUTION}


enhanced mortalities as the biological underpinning of food provisioning, in particular, is undermined. Here, attention must be paid to the ecological links, connections and networks that tie all global life together. Consequently, loss of what initially might be seen as an "insignificant" species can have ramifications reaching right up to the "top" predator, Homo sapiens. Bees provide an excellent if very worrying illustration of this entanglement. Numerous species, including honey bees and bumble bees, perform vital roles in pollinating plants, part of the diversity of insect pollinators that engage with around three quarters of agricultural crops (Greenpeace 2013). Their noted decline in Europe and North America, although poorly understood, is likely to be at least partly anthropogenic (e.g. habitat decline, possible role of insecticides). It has major potential implications for maintaining adequate food supplies in a century of continuing population increase (11.3.1) and, hopefully, greater equality in access to such supplies (Cameron et al. 2011; Greenpeace 2013; Klein et al. 2007).

\subsubsection{Biotechnology}

The whole discipline of medicine has colluded in the wider societal project of seeking technical solutions to the existential problems posed by the finitude of life and the inevitability of ageing, loss, and death.

(Iona Heath 2013: 20)

The three twenty-first century challenges presented so far have all set a very troubling context for the development of population geographies over the unfolding century. The final challenge, that posed by developments in biotechnology, paints a more ambiguous picture. It potentially provides both risks and opportunities that can impact on the human population, as well as ultimately raising deeper existential questions of the human life course. Again though, issues of inequality of consequences and outcomes loom large, this time presented through a bio-social lens.

Biotechnology is a major growth area within the twenty-first century economy, although actually as old as human history. As its name suggests, it seeks to combine biology and technology within new commodities. The Biotechnology Industry Organization depicted its subject as:

technology based on biology ... harness[ing] cellular and biomolecular processes to develop technologies and products [to] improve our lives and the health of our planet ... Modern biotechnology [helps] combat ... diseases, reduce our environmental footprint, feed the hungry, use less and cleaner energy, and have safer, cleaner and more efficient industr[y].

(Bio 2014)

In other words, numerous issues central to Population Geography when seen as lives across space-food, health, employment, energy-are and have always been central objectives within biotechnology's development and use. For the twenty-first century, this can be illustrated with respect to the quest for bodily improvement and the genetic engineering of food. Both show that whilst biotechnology may be strongly historically rooted, many of its forms today are qualitatively different and all are social and so relationally consumed. And most if not all may not be regarded in quite the unashamedly benign way that the Biotechnology Industry Organization presented them.

Notably since the middle of the last century and within affluent societies of the Global North in particular, the body has emerged as a crucial bearer of value within consumer culture and as a central expression of personal identity (Craddock and Brown 2010; Polivka 2011). Through various means, from the self-discipline of diet, fitness and exercise regimes, to the bodily adornment of piercings and tattooing, people strive to express themselves through their bodies. These techniques have increasingly been joined and some potentially displaced by biotechnological body modification via plastic surgery, laser, Botox, liposuction treatments, and so on (Brooks 2004). Working a body "into shape" is commonly part of an individual's life project but one where biotechnology plays an increasingly significant role.

Biotechnology's involvement with the diverse means of striving for the better body is not just motivated by the search for a form that somehow "naturally" feels better, such as one that is fit and energetic as compared to unfit and lethargic. Although this explicitly embodied aspect is vitally important, the aesthetic and symbolic aspect of the normative body also points to a potentially coercive role that may be played by socially and culturally inscribed bodily norms (e.g. Kinnunen 2010; Tait 2007). From the nineteenth century German philosopher Friedrich Nietzsche's call for humanity to strive to become Übermensch or "Supermen" (Box 2.5), to today's advertising ubiquitously bombarding

\section{ST PROOFS: NOT FOR DISTRIBUTION}




\section{TWENTY-FIRST-CENTURY LIVES ACROSS SPACE}

us with images of the ideal man and woman a person could become consuming their product, bodily improvement is inextricably social. It is also represented in neo-liberal terms as largely "matters of individualised consumerist responsibility" (Gilleard and Higgs 2013: 165; after Polivka 2011).

Reflecting the strong gendering of the bodily improvement project, women in particular with bodies that do not conform to slim, youthful, heterosexually desirable norms may be seen as "failing," not only socially but also individually. Although all people do not, of course, simply accept the goal of striving for an elusive socio-culturally inscribed "perfect" body (e.g. Orbach 1988), inability to meet what must be recognized as largely unattainable norms and ideals can lead to various forms of ill health. In particular, there are eating disorders such as anorexia and bulimia, plus often associated conditions of depression and other mental problems. Socio-culturally inscribed bodily projects can thus become extremely disabling, prompting morbidity or even mortality (e.g. suicide), with biotechnology consequently compromised.

With bodily improvement grounded in a healthy body, biotechnology's broader role in battling more general morbidity and mortality is also highly pertinent. With non-communicable diseases increasingly displacing communicable diseases as causing global mortalities (10.5.1.1), most notably across the Global North but increasingly in the South, considerable medical research is being invested in seeking the "holy grail" that is a cure for cancer (10.5.3.3), for example. And potential treatments may be increasingly individualistic, with drugs matched to the specificities of a particular tumor (Boseley 2016), for example. For older people too, especially in the Third Age (10.2.1.2), biotechnology can be harnessed for positive projects of the self, as in the "aspirational medicine" (Gilleard and Higgs 2013) discussed in Section 11.3.3.

There are further qualifications to the biotechnology-led bodily improvement trend, however. First, inequality must again be an ever-present consideration. Simply put, any form of bodily improvement is often costly (e.g. gym membership) but not least where biotechnology is involved. This was demonstrated sharply in Section 10.5.2.4 noting socio-spatial inequality in access to the antiretroviral drugs (ARVs) utilized to inhibit reproduction of the HIV/AIDS virus. It extends to less "critical" expressions such as plastic surgery.

Second, rival trends such as the rise of "diseases of affluence" (Hunt 2005) from a "globalization of unhealthy lifestyles" (UN 2014a: 120) are working to counter any bodily improvement trend. For example, UN goals on reducing diet-related disease are reportedly failing because of rising global obesity (UN 2014a). In 2010, 11.5 percent of adults worldwide (over 500,000) were obese but this has been predicted to reach 1 billion by 2025, with corresponding rises in diabetes, heart disease, cancers, and so on (Boseley and Davidson 2015), plus reduced everyday mobility. Consequences of rising air pollution from increasingly urbanized populations in notably South-East Asia, the Middle East and the West Pacific identified by the World Health Organization (Vidal 2016) also indicate lifestyle-related nonimprovement trends.

Third and most philosophically, medical doctor lona Heath (2013: 20), quoted at the start of this sub-section, warned in the British Medical Journal of the "medical technology industry" encouraging over-diagnosis, suggesting trivial expressions of ill-health and the corresponding need for bodily improvement where none is necessary. This feeds a tendency to "separat[e] ... notions of disease from the human experience of suffering," ultimately promoting existential life course failure to accept "the limits of life" and the inevitability of death. Such a cultural disposition is clearly unhealthy.

Intersecting with biotechnologists' search to produce an improved human body is the search for improved food supply through genetic engineering (Nicholl 2002). This technique refers in general to direct modification of an organism's genetic composition through, for example, adding genes from another species. The result is a genetically modified organism (GMO). Whilst humans have for centuries altered plant and animal species' genetic make-up through selective forms of breeding, it is only since the 1970s that direct genetic manipulation has been possible, rapidly expanding from bacteria to more complex organisms. Here again, biotechnology has "stepped up a gear" qualitatively.

Genetic engineering has been heralded as offering much potential for many areas of human life, not least in medicine and food supply (Azadi and Ho 2010). The former includes the mass production of drugs, hormones and insulin, plus replacing defective genes in living humans with working equivalents, known as gene therapy. Within food supply, emphasis has been on "improving" livestock and crops. This occurs through developing GMO versions of the specific animal or plant that enhances some desired property: higher yields, disease resistance, tolerance of herbicides, improved health qualities, changed color or shape, and so on (Woods 2011: 84). These "improved" versions could have significant positive impacts on food supply across the globe today, unequal access to which is a key indication of global poverty reflected in the MDGs (Gould 2015) and Framework of Actions report (UN 2014a).

\section{ST PROOFS: NOT FOR DISTRIBUTION}




\begin{tabular}{|c|c|c|}
\hline High yield & Virus / toxin creation & $\begin{array}{l}\text { Animal rights } \\
\text { concerns }\end{array}$ \\
\hline Reduced food quality & Limited seed access & $\begin{array}{l}\text { Threat to other } \\
\text { farmers }\end{array}$ \\
\hline Antibiotic resistance & Cheaper products & GMO becoming weed \\
\hline Potential toxicity & $\begin{array}{l}\text { Reduce genetic } \\
\text { diversity }\end{array}$ & Standardization \\
\hline Create allergenicity & Ethical concerns & Effecting other species \\
\hline $\begin{array}{l}\text { Unintended gene } \\
\text { transfer }\end{array}$ & Drought resistance & Others... \\
\hline
\end{tabular}

Figure 11.3 Some claimed benefits and risks from genetically modified crops.

Key: High yield = benefit; Reduced food quality = risk

Source: adapted and simplified from Azadi and Ho (2010: Table 1).

Unfortunately, genetic engineering is also mired in controversy over the safety and other ethical consequences of this direct manipulation of genetic structure. Besides equality-related questions of cost and control, deep moral questions have been raised about its use in medicine (Jonsen 2003). Controversy and fear have also led to widespread popular resistance across the world to the introduction of genetically modified crops, including crop-trashing actions (Doherty and Hayes 2012). Consequently, by 2007 almost all commercial cultivation was confined to just eleven countries, the US accounting for 58 percent, Argentina 19 percent and Brazil 15 percent of the total (Woods 2011). Figure 11.3 lists some of these concerns and the claimed benefits of genetically modified crops. Thus, whilst this aspect of biotechnology may have much to offer humans, both its potential and appropriate development is once again much more than simply a scientific matter. It involves a debate being reinvigorated across Europe as GMO advocates reiterate their case for lifting an EU ban on cultivation (e.g. Ghosh 2016).

\subsection{TWENTY-FIRST-CENTURY POPULATIONS}

\subsubsection{Global population size and geography}

Under the shadow of the four twenty-first century challenges facing humanity within the consolidating Anthropocene, this sub-section presents UN-derived estimates of the evolving global twenty-first century population and its geography (also Gould 2015: Chapter 10). This brief set of suggested demographic futures is based on population projections. This is a key statistical practice within Demography (Box 11.1) whose results are of interest to academics, politicians, policy makers and journalists alike. The section does not engage with the Population Geography triumvirate of births, migrations and deaths in detail, although some aspects of their futures are noted throughout this chapter. Instead, 


\section{BOX 11.1 POPULATION PROJECTIONS}

The button was pressed and the number that came out had eleven digits rather than ten.

(Dorling 2013: 36)

Dorling may have been warning of the perils of taking future population estimations too literally-indeed, this was one of the main themes of his book-but such estimates are widely promulgated and debated, and are arguably very important for humanity to be able to get a handle on where it is going numerically. Projections are also used widely in planning and policy making "to gauge future demand for food, water, energy, and services, and to forecast future demographic characteristics" (PRB 2001: 1).

In order to estimate future population at a given date for a given date-even just to present future possible population scenarios, perhaps for debate-complex statistical data modelling techniques are deployed. However, the figures produced are rooted predominantly in assumptions about each of the three components of the demographic equation: births, migrations, deaths. These assumptions are specified according to what is known at the time from past experiences and suggested from then active trends. Projections do not conventionally build in truly novel future demographic developments, since these are by definition unknown, and also tend to "incorporate very conservative assumptions about future development" (Gould 2015: 251), for example. This caution and limitation means they should be treated as best guesses of what seems to be happening. In this respect, the further ahead populations are projected, the greater is the chance of significant error.

$\S$ There are many sources of population projections but the most important is the United Nations (UN). More specifically, the UN's Population Division produces its World Population Prospects, which are updated every 2 years (UN 2016). These comprise not single sets of projections but a variety, each based on slightly different assumptions. In line with its status in the DTM, fertility is probably the most important variable, as even a small change can have major implications for future population size. It is also, unfortunately, very unpredictable, making it very hard to make the necessary assumptions. And its unpredictability but also its importance in predicting future populations is especially the case in high fertility Global South countries. It is generally assumed to be in decline when above replacement level for a country and when socio-economic development is taking place but the gradient of this decline is very uncertain. Consequently, different UN projections are typically differentiated mostly through different assumptions about future fertility decline.

The UN and other respected bodies producing projections have a good track record in predicting populations in the near future. However, to reiterate, the sheer impossibility of knowing conclusively how fertility and the other two core demographic components will change and fluctuate over time means that these projections should be always treated as very provisional. They certainly should not be regarded too respectfully as definite predictions. This qualification is especially to be noted in the context of a potential ten billion global population c.2050 discussed in this chapter.

(Sources: Dorling 2013; Gould 2015; PRB 2001)

after considering future global population numbers, sub-sections tackle two contentious issues, one longstanding, one more recently expressed: ideas of an "optimum" size of the global population and the dependency consequences of an ageing world.

According to the UN (2015a), the global population in mid-2015 was 7.3 billion, suggesting that the anticipated reaching of the 7 billion figure in 2011 (1.2.1) was probably premature. Table 11.4 goes on to show the UN's estimates of both the global population and those of the major world regions in 2050. They are based on numerous demographic assumptions which include fertility decline for countries with large families presently and a slight fertility increase where women now have fewer than two children on average. The global population for 2050 is given as almost 10 billion. It represents an increase in human numbers of almost a third, a very substantial growth for just 35 years, not least when put in historical perspective (Figure 1.1). Section 11.3.2 will reflect further on this figure.

Table 11.4 shows a strong geography of this substantial population growth, two striking contrasts standing out. First, whilst also noting the substantial predicted growth for Oceania, Africa will see spectacular change. Its population 
TWENTY-FIRST-CENTURY LIVES ACROSS SPACE

Table 11.4 UN estimates of world and regional populations, 2015 and 2050

\begin{tabular}{lccc}
\hline & \multicolumn{2}{c}{ Population (millions) } & \% Change \\
\cline { 2 - 3 } & 2015 & $2050^{1}$ & +32 \\
\hline WORLD & 7,349 & 9,725 & +20 \\
Asia & 4,393 & 5,267 & +109 \\
Africa & 1,186 & 2,478 & -4 \\
Europe & 738 & 707 & +24 \\
Latin America \& Caribbean & 634 & 784 & +21 \\
Northern America & 358 & 433 & +46 \\
Oceania & 39 & 57 & \\
\hline
\end{tabular}

Note: Based on medium-variant projection.

Source: simplified and modified from UN (2015a: Table 1).

Table 11.5 Top 10 countries by population size, 2015 and 2050

\begin{tabular}{|c|c|c|c|c|}
\hline \multicolumn{2}{|l|}{2015 ranking } & \multicolumn{2}{|l|}{2050 ranking } & \multirow[b]{2}{*}{ Change, 2015-2050 } \\
\hline Country & Population (millions) & Country & Population (millions) & \\
\hline China & 1,376 & India & 1,705 & +1 \\
\hline India & 1,311 & China & 1,348 & -1 \\
\hline USA & 322 & Nigeria & 399 & +4 \\
\hline Indonesia & 258 & USA & 389 & -1 \\
\hline Brazil & 208 & Indonesia & 321 & -1 \\
\hline Pakistan & 189 & Pakistan & 310 & - \\
\hline Nigeria & 182 & Brazil & 238 & -2 \\
\hline Bangladesh & 161 & Bangladesh & 202 & - \\
\hline Russian Federation & 143 & Dem. Rep. of Congo & 195 & +10 \\
\hline Mexico & 127 & Ethiopia & 188 & +3 \\
\hline
\end{tabular}

Source: derived from UN (2015a: Table S3).

is predicted to more than double. This reflects in particular the consequences of the demographic momentum (3.2.2) built into its presently young-aged population combined with continued high fertility rates in global perspective. In SubSaharan Africa in particular, cultural factors remain especially strong in promoting high fertility (4.3.2; Gould 2015). Further decline in deaths from the HIV/AIDS pandemic (10.5.2.4) is also of relevance for Africa's high predicted growth. Second, in contrast, Europe bucks the rising population trend with its population predicted to decline by 2050. Numerous European countries now have fertility rates below replacement level and its population notably ages (11.3.3). This all gives useful demographic context with which to critique present concerns widespread across Europe of the potential for its countries to become "overcrowded" from net international migration (Chapter 9).

Where a little more specifically will the world population be living in 2050? Table 11.5 gives some indication by comparing the top 10 countries by population size in 2015 with the expected top 10 for 2050 . Some notable changes include India replacing China as the world's most populous country, with the latter's population actually having declined (4.6.3). There is also the rise within and into the top 10 of three large African countries: Nigeria (third), Democratic Republic of Congo (ninth) and Ethiopia (tenth). This reflects the trend of Africa's demographic growth discussed above. Finally, the top 10 countries contained around 58 percent of the world's population in 2015, a percentage predicted to decline only slightly to 55 percent by 2050 (UN 2015a). Most of the world's people will continue to live within relatively few countries. But will the global condition in 2050 present an overpopulated world?

\subsubsection{An overpopulated world?}

We work out ways of living sustainably over time. We almost always have. If we don't, we don't survive.

(Dorling 2013: 136) 


\section{TWENTY-FIRST-CENTURY LIVES ACROSS SPACE}

One immediate response to the UN projection of the world's population by 2050 approaching 10 billion is to worry that this is simply too many people for Earth to accommodate and will thus have catastrophic consequences for humanity. Indeed, talk of even 7 billion inhabitants in 2011 provoked renewed speculation around this recurrent theme within human history. This was epitomized in several influential publications (Morgan 2003), such as 1968's The Population Bomb (Ehrlich 1968) and Tragedy of the Commons (Hardin 1968) and the slightly later The Limits to Growth (Meadows et al. 1972) assessment. In other words, the question of whether Earth's carrying capacity-the number of people that can be supported in terms of food and other basic needs-will be exceeded by 2050 is raised. Will the positive checks of a "Malthusian and neo-Malthusian nightmare" (Gould 2015: 251) of famine, war and other fatal disasters (1.2.1) likely come to pass?

Debates within both academia and well beyond on the topic of overpopulation are longstanding and often bitter and entrenched. This is not least because a vision of overpopulation is not only conjured up in terms of overwhelmed carrying capacity. It is also articulated by opponents of international immigration speaking rhetorically of destination countries and cities bursting with people (e.g. Pollard 2013) and by potentially racist calls for rigorous family planning policies to be adopted in the Global South to control its population growth (Hartmann 1995). Within the main overpopulation debate, for population pessimists on the one hand, "[n]othing is too bizarre when it comes to fears over future human numbers" (Dorling 2013: 9), while population optimists typically express near religious faith in technological advances-not least biotechnology-accommodating ever rising human numbers. Details of these debates cannot be reiterated here (for a Population Geography summary, see Holdsworth et al. 2013: 198-201). Instead, the present authors draw insights from a book specifically focused on the UN projections, Danny Dorling's Population 10 Billion (Dorling 2013). This charted, first, how humanity has coped throughout its turbulent history with the rise in population described in Section 3.2.1 and, second, then engaged with the issues entangled with its proposed continued upwards trajectory to 10 billion. Dorling concluded that the world should be more than able to cope with this many inhabitants, although also casting some doubt on whether this figure will be reached by 2050.

Dorling's book was not a simple argument to place relatively unreflexive faith in the ability of humanity to overcome potential crisis however. The author recognized how quite easily "[i]t could all go wrong" (Dorling 2013: 9) but, to reiterate, sought to present humanity not as "victim" of statistical numbers but as having the potential as a species to overcome whatever challenges the rising population is faced with. Moreover, post-2050 futures become still brighter as the global population is then projected to level and ultimately decline. The critical framing Dorling articulated is thus that, as stated earlier in Section 1.2.1, population matters such as the global total figure are never solely matters of numbers-in-nature but express lives relationally lived across space within a particular society. This emphasis on lives lived is developed a little further in the rest of this section.

In their summary of the debate around supposed overpopulation, Holdsworth et al. (2013: 200) quoted influential US Economist Jeffrey Sach's 2007 Reith Lecture. In this talk he asserted:

Our planet is crowded to an unprecedented degree.... It's bursting at the seams in human terms, in economic terms, and in ecological terms. This is our greatest challenge: learning to live in a crowded and interconnected world ... creating unprecedented pressures on human society and on the physical environment.

(Sachs 2007)

At first sight, Sachs's depiction of a crowded Earth seems almost completely contrary to the qualified optimism of Dorling's book. This is to misread it. As Sachs went on to imply, the large number may be(come) real but it is more effect than cause or at least better seen as a proxy for the real test that humanity must pass. This involves "learning to live" within our means within the Anthropocene-termed below sustainability. Or, as Dorling (2013: 2, emphasis added) put it:

the actual number of people on the planet is, to an important extent, incidental to the impact humans have on both the environment and each other.... [I]t's not how many of us there are but how we live that matters most.

Here, the diversity of lives lived must be fore-grounded. The book has sought to present people as fundamentally neither solely abstract, objective, equal and individual components of a "population" (such as neo-liberal subjects) nor as restricted to specific, cultural, unequal and grouped expressions of "populousness" (such as Global North versus 
Global South blocs) (Curtis 2002). Instead, people are both individual and grouped, coming together within categories of the Same but also and simultaneously (re)distributing into an indeterminate variety of Others (1.4.1). Life course biographies express this fluidity. This relational emphasis is demonstrated in the fact that how people live their lives across space-notably how they are born, how they mobilize and migrate, and how they become ill, aged and eventually die-remains immensely varied across the world today. This implies a very open and consequently irresolvable conclusion if seeking to depict the consequences of 10 billion global inhabitants in 2050. Nonetheless, for the cohorts' population geographies that ensue, the four twenty-first century challenges are likely to loom very large. And, as reiterated by David Malakoff (2011) in an article on overpopulation in Science, within this context geography is keenly important, place-specific local conditions essential considerations for relationally judging anything labeled "overpopulation."

First, there is inequality. The degree of equality in possession and access to resources, broadly defined, will be a critical factor in defining whether Earth 2050 appears overpopulated. It will likely do so if there is prominent presence of socio-spatial unequal expressions of premature mortality, in particular, but also of forced migrations and more generally morbid and/or repressed or substantially unrealized lives. Those living (sic.) such lives may be seen as inherently "too many" from an equality or "dignity and human rights" (UN 2014a) perspective. They will comprise "vulnerable" or "surplus" populations, whose biopolitics is severely compromised. Biopolitics expresses "the political negotiation of life; how life, its existence and vitality, is linked to the regulation and contestation of who has priority to live and flourish, and who might be left to wither and die" (Tyner 2013: 702). Tyner listed a range of categories of individuals who might be considered "surplus." He further argued that these populations are created not least by mechanisms of migration regulation creating categories of "'unauthorized', 'undocumented', irregular', 'illegal'" (Tyner 2013: 705), although many "population processes ad practices ... feature prominently in [producing] variegated geographies of dispossession" (Tyner 2013: 705-706).

What is fore-grounded within this brief emphasis of the contextual importance of inequality for future population geographies is not population numbers-as overpopulation is usually presented-but "how we live" in terms of a political enumeration of social fairness and equal life opportunities. It may also be suggested that it is not really the excluded who are the "problem" but that other "surplus" population, the 1 percent with extreme wealth (e.g. Dorling 2014; Williams 2015). Inequality's "overpopulations" are thus both the marginal and the over-privileged. Consequently and unsurprisingly, a need to overcome gross inequalities is given central and active priority in Dorling's analysis (Dorling 2013, 2014; also Wilkinson and Pickett 2009) and more indirectly in linking global demographic futures to "development," for example (e.g. Gould 2015).

Second, there is both global warming and resource depletion. The implications of the population chaos that may be caused by the twin hammer blows to humanity of global warming induced climate change-better climate disruption, unpredictability and instability-and ever-reduced affordable access to the resources that literally power the era of mobilities (5.2), notably oil and its derivatives, puts "simple" overall counts of people in the shade. As Dorling (2013: 369) sharply concluded in an Afterword: "better to worry about the climate than about 10 billion people." "Overpopulation" in this context indicates humanity's over-use of non-renewable resources, notably oil, and requires fundamental re-think of the Global North lifestyle model, including perhaps any normative assumption of a global era of mobilities.

Third, there is biotechnology. Attention may be drawn to what biotechnological advances can achieve in the context of the Demographic Transition Model (DTM) (4.2.2), not least given the latter's key role in underpinning population projections (Box 11.1). Specifically, in specifying projected future world populations, Dorling (2013) emphasized the critical role of fertility, where seemingly small tweaks one way or the other in the projection's model can have major implications in terms of population numbers and their geographies. Biotechnological support for ability to control fertility more reliably than at present can help to accommodate lower fertility demands. This has potential for seeing lower global populations than projected as more countries attain later DTM stages earlier. Biotechnology's more general ability to enhance certainty and reliability in the whole procreation process for individuals' and families' may have similar impact. Once again though, issues of affordability and (in)equality of access loom large. This is certainly also true at the other end of the life course, with biotechnology's ability to-both extend life and at least ameliorate the experience of morbidity. These are issues which will likely assume more significance when it is noted how the world in 2050 will not only be more populous but also more aged, considered in the next section. 


\section{TWENTY-FIRST-CENTURY LIVES ACROSS SPACE}

This sub-section can be pulled together through nominating the widely discussed and promoted integrating idea of sustainability (e.g. Adams 2005; Washington 2015; Whitehead 2007). Unfortunately, from the outset this term is very hard to pin down (e.g. Kuhlman and Farrington 2010; Williams and Millington 2004), Washington (2015) reporting a study supposedly finding over 300 definitions (Harris and Throsby 1998). Indeed, he only defined sustainability at the end of his book with an emphasis, as in the present text, on human lives as lived. For Washington (2015: 195), sustainability involves "creating a culture that lives in harmony with Nature (and each other) into the future ... [It involves] healing the planet, along with our society and our maladjusted economy."

In other words, sustainability necessitates a critical reflective audit of the emerging Anthropocene, which is seen to be having a multi-dimensional self-destructive impact. Unlike, for example, the MDGs which were "targets mainly for poor countries" (Sachs 2012: 2208), sustainability sets a major challenge for all of humanity to develop and practice normative models of lives across space. These models must consistently fall within a triple bottom-line of being economically, environmentally and socially sustainable. Initially calling on corporations to focus "not just on the economic value that they add, but also on the environmental and social value that they add-or destroy" (Elkington 2004: 3), Elkington's term has expanded in scope to become a necessary consideration within all that humanity undertakes. And in the context of the present book and this sub-section in particular, sustainability must always have a population numbers component but by its very holistic and multidimensional nature it involves so much more.

The UN's (2014a) Framework of Actions also ends with recognizing the challenge to humanity that the sustainability goal presents. The report reiterates earlier calls for "a new development environment that is shaped by, and must respond to, a need to reconcile rising levels of consumption, threats to the environment, and growing wealth and income inequality" (UN 2014a: 203). If not quite establishing specific Sustainable Development Goals (Sachs 2012), the UN then advocated the seven "paths to sustainability" listed in Table 11.6. Almost all can clearly be seen to have Population Geography resonances. The future shaping of the global population, whatever its (maximum) size, through the relational practices of births, migrations and deaths, needs also to be framed by these priorities. They are all, of course, intensely political.

\subsubsection{An ageing world-from dependency to global demographic opportunity?}

Ageing is not what it once was.

(Gilleard and Higgs 2013: vii)

The senior population, defined as those aged 60 years and over by the UN, comprised 11.5 percent of the global population in 2012. It is projected to nearly double to 21.5 percent by 2050, exceeding for the first time the number of children aged below 15 years (UN 2015a). People aged 80 years or more, roughly the Fourth Age (10.2.1.2), will grow from 1.7 percent to 4.5 percent of the population-125 to 434 million people-as global median age rises from 29.6 to 36.1 years (UN 2015a). Consequently, well over a decade ago scholars such as Johnson and Climo (2000: 531) acknowledged "an extraordinary demographic shift" occurring in the global population. Furthermore, absolute and relative growth of seniors is not projected to be limited to socio-economically developed Northern countries, where the baby boom generation (Box 3.3) will have all attained retirement ages, but will spread throughout the world. Whilst countries such as Australia, the US and those in Europe are now beginning to develop policies to address the implications of these ageing populations, much of the Global South will need to do likewise within a few decades.

\section{Table 11.6 The UN's paths to sustainability}

1. Strengthen equality, dignity and rights

2. Invest if lifelong health and education, especially for young people

3. Achieve universal access to sexual and reproductive health and rights

4. Ensure security of place and mobility

5. Build sustainable, inclusive cities

6. Change patterns of consumption

7. Strengthen global leadership and accountability

Source: UN (2014a: 209-214). 
As Chapter 10 has already suggested, a high proportion of seniors in a population, represented by a relatively large top bulge on population pyramids (3.2.2), has important practical and political implications for a country. It has given rise to a second key demographic concern to that of overpopulation: rising elderly dependency. Indeed, some commentators have suggested that the real "population time bomb" is not numbers of people but numbers of elderly people (Harris 2016). This articulates fear of the consequences of a rising elderly population seen as being increasingly "dependent" upon a shrinking "independent" working age population. It is expressed statistically in indicators such as gerontic dependency ratios (10.2.2.5). By 2050 it is projected that besides 24 European countries, seven Asian and four Latin American countries will have fewer than two people aged 20-64 years for every person aged 65 or above (UN 2015a).

However, as also noted in Chapter 10, simply focusing on numbers is once again not very helpful. In particular, it assumes a very twentieth-century Global North model of population demographics. In this model, people aged over 60 years are overwhelmingly economically inactive and, even at younger senior ages, rather frail. Both assumptions are increasingly untrue in the North, just as they are largely misrepresentations of senior lives across the Global South. A Third Age versus Fourth Age distinction is useful for developing this critique further (Gilleard and Higgs 2013), although the coherence of even these categories will later be questioned.

First, younger Third Age elderly in the Global North are increasingly economically active (Avramov and Maskova 2004; Palomba and Kotowska 2003), even as entrepreneurs (Kautonen et al. 2011). This may or may not be from choice but is becoming politically sanctioned through abolition of mandatory retirement ages, which are seen as ageist. For example, the UK's Employment Equality (Repeal of Retirement Age Provisions) Regulations 2011 started the phasing out of default retirement ages, employers now having to make an objectively justifiable case for calling on someone to retire, not just citing their age (BBC 2011). Thus, the key life course transition (2.2.1) of retirement is being undermined and with it associations it has displayed with demographic expressions such as lifestyle retirement migrations (8.4), for example.

Second, Third Age seniors are also often far from frail and immobile (Harris 2016). This is demonstrated by their wide involvement in the aforementioned lifestyle migrations and in continuing paid employment, and by their prominent presence within civil society generally-from volunteering and leading pressure groups (Hank and Erlinghagen 2010) to caring for grandchildren whose parents are in paid employment (Hank and Buber 2009). Not insignificant to these healthy and mobile seniors have been positive developments in biotechnology, including what Gilleard and Higgs (2013) termed "aspirational medicine," whereby "youthful desire for the body to remain a site of choice, autonomy, self-expression and pleasure has been extended to all portions of the adult life course" (p. 160). Reinforcing how "bodies are not predestined to get worse once a particular age is reached," aspirational medicine "seeks to establish a new set of possibilities that allow for a more embodied subjectivity in later life" (Gilleard and Higgs 2013: 166, 157).

Nonetheless, in spite of this implied positive non-dependent prognosis for the Third Age in 2050, an ageing population still presents complex and major global challenges (Dorling 2011: Chapter 7). Differentiation within the Third Age must be acknowledged from several angles. First, attention must be paid yet again to inequality and its numerous manifestations. Whilst many seniors within a specific society may wish to challenge ageism and carry on working well past 65 years of age, others may have little choice but to do this so as to have enough income to live a decent life. And all jobs are of course far from equal in the levels of remuneration, satisfaction and conditions they provide workers. Additionally, biotechnology and other means through which older people can carry on active, rewarding and healthy lives are also often expensive and thus unequally available. Aspirational medicine may thus the aspirational in more ways than just retaining bodily choice. The social class bias in older lifestyle retirement migrations (8.4) is further evidence for Third Age selectivity. In sum, the empowered Third Age is under present conditions not an equal option for seniors across the Global North let alone for those in the South, notwithstanding arguably more positive representations of older people found there (10.2.1.1).

Second, global population ageing is highly geographically uneven. This reflects the more general spatial unevenness of populations, as expressed through population pyramids and different DTM stages. Table 11.7 gives a sense of this variation by listing the top 10 countries in terms of actual and predicted percentages aged 80 years or over in 2015 and 2050, respectively. Globally, the percentage increase is three-fold, to 4.5 percent of the population, but this huge relative increase is expressed still more in global divergence, the top 10 for 2050 having predicted percentages well above this figure. Furthermore, note a greater mix of countries between Global North and South in

\section{ST PROOFS: NOT FOR DISTRIBUTION}


328 TWENTY-FIRST-CENTURY LIVES ACROSS SPACE

Table 11.7 Top 10 countries by percentage population aged $80+$ years, 2015 and 2050

\begin{tabular}{|c|c|c|c|}
\hline \multicolumn{2}{|c|}{2015 ranking } & \multicolumn{2}{|l|}{2050 ranking } \\
\hline Country & $\%$ Aged $80+$ & Country & $\%$ Aged 80 \\
\hline Japan & 7.8 & Martinique & 18.0 \\
\hline Italy & 6.8 & Italy & 15.6 \\
\hline Greece & 6.4 & Japan & 15.1 \\
\hline France & 6.1 & China & 15.0 \\
\hline Spain & 5.9 & Germany & 14.4 \\
\hline Portugal & 5.9 & Cuba & 14.3 \\
\hline Germany & 5.7 & Spain & 14.0 \\
\hline Belgium & 5.5 & Rep. of Korea & 13.9 \\
\hline Martinique & 5.4 & Portugal & 13.8 \\
\hline Lithuania & 5.3 & Singapore & 13.8 \\
\hline World & 1.7 & World & 4.5 \\
\hline
\end{tabular}

Source: derived from UN (2015a: Table S6).

2050 as compared to the dominance of Japan and Europe in 2015. Additionally, within countries there are now and will be in 2050 marked geographies of age. This means a concentration of seniors in certain locations, such as those favored by lifestyle retirement migrations (8.4) or in impoverished communities where younger adults have overwhelmingly out-migrated for employment, such as many rural areas (3.3.4). These geographies of senior concentration present highly spatial challenges for supply of Fourth Age support services in particular.

Third, whilst the Third Age concept presents a positive image of seniority, not least challenging ageist negative stereotypes, the body does still age (Box 10.1). One consequence is increased immobility and health problems generally with old age as a life course approaches its ultimate inevitable conclusion. No amount of biotechnological innovation can halt this and attendant manifestations of the "feared social imaginary" (Gilleard and Higgs 2013: vii; also Gilleard and Higgs 2010) of the Fourth Age. This reinforces how populations and areas with high percentages of older people are still important to identify and will increasingly require policy response. However, to identify them the conventional gerontic dependency ratio does not seem very helpful. Instead, Spijker and Maclnnes (2013) proposed an alternative indicator based not on age but on life expectancy (10.3.1) and number of people in employment. The dependent population is defined as the number of people with 15 years or less remaining according to life expectancy, while the independent population consists of the number of people in employment regardless of age. Spijker and Maclnnes's (2013) "real elderly dependency ratio" comprises the former divided by the latter. It is seen as declining for England and Wales from the late twentieth century to the present, then rising through the early twenty-first century but at a much slower rate and with much lower values than the conventional dependency rate. Even in 2050 the real dependency ratio is predicted to be far below its 1980 peak. Using this measure, Japan, notorious today for having high elderly dependency according to conventional measures (also Table 11.7), expresses much lower values, in line with much of Europe (Spijker and Maclnnes 2013).

Besides more appropriate statistical measurement of the "problem," greater imagination can also be given to "solutions." Accepting that Fourth Age populations do often require considerable care and attention-medical, everyday support, social (Gilleard and Higgs 2015)-and that in 2050 they will still be unevenly geographically concentrated at a variety of scales, the potential ameliorative role of international migration can be noted. On the one hand, the most ageing societies will increasingly experience population decline from natural decrease (3.2.2) through excess deaths over births. On the other hand, as the UN (2014a: 158) asserted in a context of much global anti-immigrant expression, there is need to recognize "the contributions [international] migration makes to the political, economic, social and cultural fabric of countries of origin and destination alike, as well as to the global community." Taken together, migration can potentially play a critical positive role in supporting the Fourth Age population (Harris 2016; Shutes 2011), with a further potential consequence that it may slow growth of the global population towards the symbolic 10 billion. Dorling (2013: 51) expressed this combined effect thus: 
people tend to rapidly adopt the fertility rates of the places they move to.... If we Europeans want to be well cared for in our old age, and we also want fewer future people in the world, the last thing we should be doing is trying to reduce migration.

\subsection{CONCLUSION: TWENTY-FIRST-CENTURY POPULATION GEOGRAPHY}

We are always on the move, and beneath us the rocks travel too, a long migration to a land they do not know. A new world.

(Sara Maitland 2014: 46)

To conclude both this chapter and the book one final consideration will be made, namely to reflect on the legacy, present and future prospects for Population Geography as an academic sub-discipline. Three questions will be asked: What has it achieved thus far? Is it still important? and Where will (and should) it be in 2050? These questions merit academic papers in their own right but are given just a few paragraphs here.

First, reiterating the conclusions of Chapter 1, Population Geography has evolved considerably as a sub-discipline since its mid-twentieth century foundations. One interpretation might see its profile peaking from near its emergence, notably in 1953 when Trewartha presented it alongside culture and the physical environment as one of three cornerstones of Geography, and on through the late 1960s'/early 1970s' rise of quantitative approaches and a Spatial Demography that impacted most notably on migration scholarship (1.3.3). Since then, as Chapter 1 showed, many Population Geographers have become concerned that their subject was being eclipsed by other subdisciplines, such as Geographic Information Systems (GIS) in one direction and Cultural Geography in another. Consequently, there has "been the repeated-some might say 'tired'-call for a (re)theorized population geography" (Tyner 2013: 702).

Regarding Population Geography today as relatively moribund would, however, be much too formal and nominal an interpretation of the sub-discipline. It partly relies too heavily on focusing just on what is included under the explicit "Population Geography" label. Instead, as the present book argued and sought to illustrate with evidence from its sources, Population Geography is not to be found only within the explicit sub-discipline-undertaken by equally explicitly defined "Population Geographers"-but is expressed widely across not only Human Geography but Social Science generally, and even beyond. In other words, population geography has spread and infiltrated widely, albeit often in academic disguise.

Even if attention is restricted to the demographic triumvirate of births, migrations and deaths-and this limited scope will be reflected on shortly-these elements of a life course are widely acknowledged as having important spatial dimensions and are widely researched and understood accordingly. Lives lived by life course cohorts (Chapter 2) have demonstrable geographical as well as widely taken-for-granted historical dimensions and this certainly includes their demographic expressions. Or, put differently, the relational construction of a life course, not least through Section 2.3's arenas of differential experience, does not take place on the head of a pin but is entangled through space and place: lives across space.

Such appreciation of the sheer but necessary ubiquity of recognizing the spatiality of lives lived sets up an immediate response to the second question. Yes, Population Geography is still of considerable importance today for understanding and explaining the demographic world. Indeed, popular, policy and political attention to the demographic triumvirate has probably never been stronger. Births and deaths may be near-unanimously accepted as inevitable but they are increasingly not left to the whims of nature. Their spatial expressions, likewise, are increasingly seen as very much more-than-natural and thus demanding of critical scholarly attention from social scientists. As for the intrinsically spatial practices of migration, never have they been so prominent on our TV screens and in political discourses, at least across the Global North. Many politicians and much popular opinion may seek to prevent many of migration's manifestations but the powerful political and economic forces that propel them clearly show the movement of humanity to be ultimately unstoppable. An interesting observation on the latter comes from British Pakistani writer Mohsin Hamid's (2014: 20) anti-sedentarist (5.2.1) imperative for the twenty-first century: 


\section{TWENTY-FIRST-CENTURY LIVES ACROSS SPACE}

I imagine that centuries hence, when people are finally free to move as they please around the planet Earth, they will look back at this moment and wonder ... how people ... could [cage] their fellow human beings ... merely for wanting to wander, as our species always have and always will.

Finally but staying with the future, to the third and most difficult of the questions raised, namely where will Population Geography be in 2050 ? The authors are particularly wary of predicting where it will go but a number of more normative observations will be made. Most represent continuation of the multi-dimensional expansion in scope of the subdiscipline (Bailey 2005) that Chapter 1 and the book overall has demonstrated.

First, the sheer significance of the twenty-first century challenges noted above will keep Population Geography prominent, whether or not badged thus, given the strong demographic aspects associated with these challenges. Inequality, global warming, resource depletion and biotechnology are all so uneven in spatial (and social) expressions and consequences that Geographers' attention must stay on them. However, the sheer complexity of the challenge they present also require Population Geographers to "up their game," adopting more multi-scalar approaches (for climate change: Barrett 2012; Birkenholtz 2011) and both inter- and multi-disciplinary investigations (Conway 2004).

Second, these twenty-first century challenges also suggest methodological innovation. Population Geography must continue embracing of novel perspectives to present, obtain and interrogate its ever more diverse material. In terms of presentation, technological developments not least within the internet can increasingly be used to express findings in imaginative terms. There is much scope to expand on Figure 10.8's Gapminder, for example, and as this chapter was being written Metrocosm (2016) came out with a most engaging computer visualization of the history of global urbanization (http://metrocosm.com/map-history-cities.html). In obtaining material, large-scale empirical data sets will remain indispensable. Some may become more challenging to compile with heightened ethical concerns about data gathering and surveillance, plus more prosaic costs factors, all reflected for example in the threatened loss of the UK Population Census (Box 3.2). Qualitative methods also need to evolve so as to be able to engage with the complexity of life course experiences. This can certainly learn from the novel methods being deployed in some mobilities research, for example (Büscher et al. 2010). Approaches to interrogate data need to and will evolve too, from GIS and spatial statistical software programs such as SpaceStat and GeoDa facilitating ever more complex statistical analyses, through expanding options within qualitative data analysis packages such as NVivo, to greater use of humanities-sourced techniques such as multi-voiced polyphonic presentations (5.5). Moreover, some of these new techniques will help build links between Population Geography and other demographic stakeholders, such as the interest often shown by policymakers for GIS (Hugo 2006).

Third, Population Geography will grow still further its understanding of what can and arguably should be covered under the triumvirate headings of births, migrations and deaths. For example, analysis of fertility can focus still more on the different family or household contexts in which births take place and on the biopolitics or socio-political negotiations involved in having children. Work on infertility, different forms of surrogacy and the diversity of non-nuclear family contexts seem especially fruitful. Migration studies will develop still further its productive engagement with mobilities, recognizing its place within a mobilities spectrum (5.2.2) rather than seeking to stand somewhat aloof as a very singular type of movement. Particularly fruitful should be further scholarship exploring the lifestyle mobilities (8.1.2) that blur the boundaries of the previously relatively fixed "intersections between travel, leisure and migration" (Cohen et al. 2015: 156). Blurring of differences and consequent challenging and expanding of categories-including those of home or usual/permanent address (5.1.1) and being a citizen all "in the mobile world of the twenty-first century" (Cresswell 2013: 82)-should be reflected widely across the domains of residential mobility, internal migration and international migration. For example, some forced movement is typically more residential mobility than (international) migration, such as women escaping domestic violence (Bowstead 2015), but this does not excuse the relative inattention it has received. Likewise, moving for love can be international as well as relatively local (D'Aoust 2013). For mortality, the established Population Geography connections with morbidity and ageing will be developed further, as in how Third Age seniors seek embodied ways to resist morbidity and death through lifestyle, medicine and cultural practices. This resistance may be unequally distributed but, as Gilleard and Higgs (2013: 166) observed, "desire to live better longer cannot be opposed on the grounds that not all will succeed or because such goals can never be permanently realised." Of death itself, whilst the relative rise of non-communicable disease mortality may be the dominant biomedical trend (10.4.1), Population Geographers are needed to maintain appreciation of how "likelihood to escape premature death is deeply, irrevocably spatial" (Tyner 2013: 703).

\section{ST PROOFS: NOT FOR DISTRIBUTION}


Fourth, other areas of growing interest to twenty-first century Population Geographers will take the subdiscipline still further from the conventional demographic triumvirate. This can be spurred on by the kind of life course framing the present book has encouraged. For example, not only are births, migrations and deaths intrinsically embodied but so too are all aspects of the life course. And this embodiment is linked to geography since, resisting any mindbody dualism, "it is by means of the body that space is perceived, lived-and produced" (Lefebvre 1991: 162). Thus, one can expect Population Geographers to demonstrate greater interest in the full range of embodied geographies (Teather 1999) that express a life. Embodiment is especially important for ageing and this is a further area that Population Geography can embrace still more fully. Moving away from a focus on ageing just in the context of morbidity and mortality, Skinner et al. (2015) have recognized much more to be done on geographies of ageing such as its, hidden, often very individual experiential geographies, ever-present questions of social justice and welfare in an ageing yet ageist world, and understanding better the geographies of relatively neglected groups such as indigenous people in the Global North or seniors in the South in general.

More generally, greater recognition of those who are somehow missing or excluded is also a critical dimension for Population Geography to develop further. Focus on the childless, whether or not from choice; non-movers, even when "objective" analysis suggests migration is the obvious thing to do; and those still alive who nonetheless experience extreme everyday life constraints due to considerable morbidity all important population categories hitherto relatively neglected. Taking this further, Tyner's (2013) surplus populations, diversely defined, merit much greater attention, not least from any concern with challenging inequalities.

Fifth, all this growth in scholarship, from enriching what is covered by and of interest within the demographic triumvirate to expanding beyond its shadow in terms of topics covered not only requires the aforementioned appropriate methodological expansion but also that Population Geography continues to embrace new developments within social theory. This should, of course, be undertaken critically and with sound scholastic purpose beyond simply jumping on the next bandwagon. Of interest, for example, are non-representational perspectives (Box 8.3) on demographic practices. This includes the potential involvement of affect in promoting either migration or staying put (e.g. Halfacree and Rivera 2012; Halfacree 2014a).

Sixth and finally, one can reflect briefly Population Geography may ultimately have become by 2050. As suggested in Section 2.4, its own expansionist ambitions may be ultimately self-destructive of a sub-discipline still generally recognizable today (even if much population geography is practiced under different sub-disciplinary headings). In particular, expansion beyond the demographic triumvirate to other more-or-less connected life course experiences and practices may herald the emergence of a more holistic sub-discipline from a life course perspective. This will focus not solely or even primarily on births, migrations and deaths but on all that a life encompasses: a Life Course Geography. However, a question to leave with the reader from this set of predictions is whether such a future would make too unwieldy a sub-discipline for academic and student alike? Instead, might we be better sticking with the core of births, migrations and deaths that the present book used to focus its material?

We are born, we live, and then we die (1.1); three seemingly simple facts of a life course with which this book began and then sought to illustrate using material from across the world. We hope that this has shown how such simple facts, not least as expressed through the relational playing out of lives across space, have been, are now and will in the future be of considerable interest and significance for scholars but also for students, policy makers, politicians, cultural arbiters, businesspeople, writers and artists... and, of course, each of us as individuals. "New worlds" of population are always emerging and there for discovery! 


\section{BIBLIOGRAPHY}

Abbasi-Shavazi, M. and McDonald, P. (2000) 'Fertility and multiculturalism: immigrant fertility in Australia, 1977-1991', International Migration Review 34(1): 215-242

Abu-Sahlieh, S. (1996) 'The Islamic conception of migration', International Migration Review 30: 37-57

Acton, T. (2010) Theorising mobility: migration, nomadism, and the social reconstruction of ethnicity', in Proceedings of Romani Mobilities in Europe: Multidisciplinary Perspectives International Conference, January, Refugee Studies Centre, University of Oxford

Adams, A., Freedland, M. and Prassl, J. (2015) 'The "zero-hours contract": regulating casual work, or legitimating precarity?', Oxford Legal Studies Research Paper No. 11/2015

Adams, W. (2005) 'Sustainability', in P. Cloke, P. Crang and M. Goodwin (eds) Introducing Human Geographies, 2nd edition, London: Arnold. pp. 125-132

Adepoju, A. (2005) 'Review of research and data on human trafficking in sub-Saharan Africa', International Migration 43(1-2): 75-98 Adey, P. (2010) Mobility, London: Sage

Adger, W. (2006) 'Vulnerability', Global Environmental Change 16: 268-281

Administration on Aging (2010) A Profile of Older Americans: 2010, U.S. Department of Health and Human Services, Administration on Aging

Ager, A. and Strang, A. (2008) 'Understanding integration: a conceptual framework', Journal of Refugee Studies 21(2): 166-191

Agnew, J. (1989) 'Sameness and difference: Hartshorne's The Nature of Geography and geography as areal variation', in N. Entrikin and S. Brunn (eds) Reflections on Richard Hartshorne's The Nature of Geography, Washington, DC: Association of American Geographers, pp. 121-140

Agnew, J. (2010) 'Slums, ghettos and urban marginality', Urban Geography 31(2): 144-147

Agrillo, C. and Nelini, C. (2008) 'Childfree by choice: a review', Journal of Cultural Geography 25(3): 347-363

Ahmad, A. (2008) 'Dead men working: time and space in London's ("illegal") migrant economy', Work, Employment and Society 22(2): $301-318$

Ahmed, N. (2013) Geographies of Gender and Generation: a Longitudinal, Intersectional Analysis of First Generation Bangladeshi Women in Tower Hamlets, London, Unpublished PhD thesis, Swansea University

Aitchison, C., Hopkins, P. and Kwan, M. (eds) (2007) Geographies of Muslim Identities: Diaspora, Gender and Belonging, London: Routledge

Al Jazeera (2015) 'Syrian refugees drown off coast of Turkey', 27 September, at: www.aljazeera.com/news/2015/09/syrianrefugees-drown-coast-turkey-150927104056192.html (accessed 08/16)

Al-Ali, N. and Koser, K. (eds) (2002) New Approaches to Migration?: Transnational Communities and the Transformation of Home, London: Routledge

Albrow, M. (1990) 'Introduction', in M. Albrow and E. King (eds) Globalization, Knowledge and Society, London: Sage, pp. 3-13

Alderman, H. and Behrman, J. (2004) 'Estimated economic benefits of reducing low birth weight in low-income countries', Washington DC, World Bank, HNP Discussion Paper

Allinson, J. (2006) 'Over-educated, over-exuberant and over here? The impact of students on cities', Planning, Practice and Research 21(1): 79-94

Allsopp, J., Sigona, N. and Phillimore, J. (2014) 'Poverty among refugees and asylum seekers in the UK: an evidence and policy review', University of Birmingham, Institute for Research into Superdiversity Working Paper

Alwin, D. and McCammon, R. (2003) 'Generations, cohorts, and social change', in J. Mortimer and M. Shanahan (eds) Handbook of the Life Course, New York: Springer, pp. 23-49 


\section{BIBLIOGRAPHY}

Ambrosini, J., Mayr, K., Peri, G. and Radu, D. (2011) 'The selection of migrants and returnees: evidence from Romania and implications', Cambridge, MA: National Bureau of Economic Research Working Paper No. 16912

Amin, A. and Thrift, N. (2002) Cities: Reimagining the Urban, Bristol: Polity Press

Amit, V. (2002) 'The moving "expert": a study of mobile professionals in the Cayman Islands and North America', in K. Olwig and N.

Sørensen (eds) Work and Migration: Life and Livelihood in a Globalizing World, London: Routledge, pp. 145-160

Ammerman, N. (ed.) (2007) Everyday Religion, Oxford: Oxford University Press

Anderson, B. (2010) 'Migration, immigration controls and the fashioning of precarious workers', Work, Employment and Society 24(2): 300-317

Anderson, B. (2014) Encountering Affect, Farnham: Ashgate

Anderson, B. and Harrison, P. (2010) 'The promise of non-representational theories', in B. Anderson and P. Harrison (eds) TakingPlace: Non-Representational Theories and Geography, Farnham: Ashgate, pp. 1-34

Anderson, B. and Rogaly, B. (2005) 'Forced labour and migration to the UK', Report Published by the TUC and COMPAS: Oxford Anderson, G. and Hussey, P. (2000) 'Population aging: a comparison among industrialized countries', Health Affairs 19(3): 191-203 Anderson, J. (2010) Understanding Cultural Geography, London: Routledge

Andreas, M. and Wagner, F. (eds) (2012) Realizing Utopia: Ecovillage Endeavors and Academic Approaches, Munich: Rachel Carson Centre

Andreas, P. (2009) Border Games: Policing the US-Mexico Divide, 2nd edition., London: Cornell University Press

Andrews, G., Cutchin, M., McCracken, K., Phillips, D. and Wiles, J. (2007) 'Geographical gerontology: the constitution of a discipline', Social Science and Medicine 65(1): 151-168

Andrews, M. (1999) 'The seductiveness of agelessness', Ageing and Society 19(03): 301-318

Andrijasevic, R. (2006) 'Lampedusa in focus: migrants caught between the Libyan desert and the deep sea', Feminist Review 82: $120-125$

Androff, D. (2011) 'The problem of contemporary slavery: an international human rights challenge for social work', International Social Work 54(2): 209-222

Angelini, V. and Laferrère, A. (2012) 'Residential mobility of the European elderly', CESifo Economic Studies 58(3): 544-569

Ansell, N. and Van Blerk, L. (2004) 'Children's migration as a household/family strategy: coping with AIDS in Lesotho and Malawi', Journal of Southern African Studies 30(3): 673-669

APA [American Psychological Association] (2011) 'Definition of terms: sex, gender, gender identity, sexual orientation', at: https://www.apa.org/pi/lgbt/resources/sexuality-definitions.pdf (accessed 07/16)

Apitzsch, U. and Siouti, I. (2007) 'Biographical analysis as an interdisciplinary research perspective in the field of migration studies', at: http://is.muni.cz/el/1423/podzim2014/SOC932/um/Apitzsch_Biographical_Analysis_April_2007.pdf (accessed 08/16)

Appadurai, A. (1996) Modernity At Large: Cultural Dimensions of Globalization, Minneapolis: University of Minnesota Press

Apparicio, P., Cloutier, M. and Shearmur, R. (2007) 'The case of Montreal's missing food deserts: evaluation of accessibility to food supermarkets', International Journal of Health Geographics 6(1), at: https://ij-healthgeographics.biomedcentral.com/ articles/10.1186/1476-072X-6-4 (accessed 08/16)

Arango, J. (2000) 'Explaining migration: a critical view', International Social Science Journal 52(165): 283-296

Archer, M. (2003) Structure, Agency and the Internal Conversation, Cambridge: Cambridge University Press

Arnett, J. (2000) 'Emerging adulthood: a theory of development from the late teens through the twenties', American Psychologist 55(5): 469-480

Arnot, C. (2009) 'No such thing as a classless society', Guardian 7 October

Arthur, M. and Rousseau, D. (1996) The Boundaryless Career, Oxford: Oxford University Press

Asia Society (n.d.) 'The slums of Asia', at: http://asiasociety.org/slums-asia (accessed 08/16)

Athwal, H. and Bourne, J. (2007) 'Driven to despair: asylum deaths in the UK', Race and Class 48(4): 106-114

Atkinson, R. and Blandy, S. (eds) (2013) Gated Communities: International Perspectives, London: Routledge

Atkinson, R. and Flint, J. (2004) 'Fortress UK? Gated communities, the spatial revolt of the elites and time-space trajectories of segregation', Housing Studies 19(6): 875-892

Attaran, A. (2005) 'An immeasurable crisis? A criticism of the Millennium Development Goals and why they cannot be measured', PLoS Medicine 2(10): e318

Austin Alchon, S. (2003) A Pest in the Land: New World Epidemics in a Global Perspective, Albuquerque: University of New Mexico Press

Avlund, K. (2010) 'Fatigue in older adults: an early indicator of the aging process?', Aging Clinical and Experimental Research 22(2): 100-115

Avramov, D. and Maskova, M. (2004) Active Ageing in Europe, Volume 2, Strasbourg: Council of Europe 
Azadi, H. and Ho, P. (2010) 'Genetically modified and organic crops in developing countries: a review of options for food security', Biotechnology Advances 28(1): 160-168

Bailey, A. (2005) Population Geography, London: Hodder Arnold

Bailey, A. (2009) 'Population geography: lifecourse matters', Progress in Human Geography 33(3): 407-418

Bailey, A. (2010) 'Population geographies, gender, and the migration-development nexus', Progress in Human Geography 34(3): $375-386$

Bailey, A. (2011) 'Population geographies and climate change', Progress in Human Geography 35(5): 686-695

Bailey, A. and Boyle, P. (2004) 'Untying and retying family migration in the New Europe', Journal of Ethnic and Migration Studies 30(2): 229-241

Bailey, l., Hopkins, R. and Wilson, G. (2010) 'Some things old, some things new: the spatial representations and politics of change of the peak oil relocalisation movement', Geoforum 41(4): 595-605

Bailey, N. (2012) 'How spatial segregation changes over time: sorting out the sorting processes', Environment and Planning A 44(3): $705-722$

Baker, B. and Rytina, N. (2013) Estimates of the Unauthorized Immigrant Population Residing in the United States: January 2012, Office of Immigration Statistics, Policy Directorate, US Department of Homeland Security

Bakewell, O. (2010) 'Some reflections on structure and agency in migration theory', Journal of Ethnic and Migration Studies 36(10): $1689-1708$

Bales, K. (2007) Ending Slavery: How we Free Today's Slaves, Berkeley, CA: University of California Press

Balkan Insight (2012) 'Romania leads Europe in abortion rate', 6 March, at: www.balkaninsight.com/en/article/romania-has-highestabortion-rate-in-europe (accessed 08/16)

Bandura, A. (1997) Self-efficacy: the Exercise of Control, New York: Freeman

Banks, S. (2004) 'Identity narratives by American and Canadian retirees in Mexico', Journal of Cross-Cultural Gerontology 19(4): $361-381$

Banks, S. (2009) 'Intergenerational ties across borders: grandparenting narratives by expatriate retirees in Mexico', Journal of Aging Studies 23(3): 178-187

Barcus, H. and Simmons, L. (2013) 'Ethnic restructuring in rural America: migration and the changing faces of rural communities in the Great Plains', Professional Geographer 65(1): 130-152

Barcus, H. and Werner, C. (2010) 'The Kazakhs of Western Mongolia: transnational migration from 1990-2008', Asian Ethnicity 11(2): 209-228

Barke, M. (2004) 'Rural tourism in Spain', International Journal of Tourism Research 6(3): 137-149

Barke, M. and Fuller, D. (2001) 'Race and ethnicity', in R. Pain, M. Barke, D. Fuller, J. Gough, R. MacFarlane and G. Mowl Introducing Social Geographies, London: Arnold, pp. 97-119

Barnes, C. and Mercer, G. (2003) Disability, Cambridge: Polity Press

Barrett, S. (2012) 'The necessity of a multiscalar analysis of climate justice', Progress in Human Geography 37(2): 215-233

Bartholomew, K. (1991) 'Women migrants in mind: leaving Wales in the nineteenth and twentieth centuries', in C. Pooley and I. Whyte (eds) Migrants, Emigrants and Immigrants: a Social History of Migration, London: Routledge, pp. 174-187

Bartram, D., Poros, M. and Monforte, P. (2014) Key Concepts in Migration, London: Sage

Basch, L., Glick-Schiller, N. and Szanton-Blanc, C. (1994) Nations Unbound: Transnational Projects, Postcolonial Predicaments, and Deterritorialized Nation-States, Langhorne, PA: Gordon and Breach

Bates, D. (2002) 'Environmental refugees? Classifying human migrations caused by environmental change', Population and Environment 23(5): 465-477

Baubock, R., Heller, A. and Zolberg, A. (eds) (1996) The Challenge of Diversity: Integration and Pluralism in Societies of Immigration, Avebury: Ashgate

Baudrillard, J. (1998) The Consumer Society, London: Sage

Baum-Snow, N. (2007) 'Did highways cause suburbanization?', Quarterly Journal of Economics 122(2): 775-805

BBC (2006) 'The rise of the "childfree"', at: http://news.bbc.co.uk/go/pr/fr/-/2/hi/europe/4813590.stm (accessed 05/10)

BBC (2011) 'Compulsory retirement age at 65 fully abolished', BBC News $1^{\text {st }}$ October, at: www.bbc.co.uk/news/business15127835 (accessed 08/16)

BBC (2012a) 'Trafficked: sex slaves seduced and sold', News 22 May

BBC (2012b) 'Human trafficking to the UK "rising"', News 18 October

BBC (2013a) 'Twelve arrested in US raid on Latin sex-trafficking ring', News 18 January

BBC (2013b) 'The girls stolen from the streets of India', News 8 January 


\section{BIBLIOGRAPHY}

Beale, C. (1975) 'The revival of population growth in non-metropolitan America', United States Department of Agriculture, Economic Research Service, ERS 605

Beaujeu-Garnier, J. (1966) Geography of Population, Harlow: Longman

Beaverstock, J. (1994) 'Re-thinking skilled international labour migration: world cities and banking organisations', Geoforum 25(3): 323-338

Beaverstock, J. (2002) 'Transnational elites in global cities: British expatriates in Singapore's financial district', Geoforum 33(4): 525-538

Beaverstock, J. (2005) 'Transnational elites in the city: British highly-skilled inter-company transferees in New York City's financial district', Journal of Ethnic and Migration Studies 31(2): 245-268

Beaverstock, J. and Boardwell, J. (2000) 'Negotiating globalization, transnational corporations and global city financial centres in transient migration studies', Applied Geography 20(3): 277-304

Beaverstock, J. and Smith, J. (1996) 'Lending jobs to global cities: skilled international labour migration, investment banking and the city of London', Urban Studies 33(8): 1377-1394

Becker, D. (2013) 'The past, present and future of transnational conflict in Jordan: a study of Syrian refugees in the Hashemite Kingdom', Masters Capstone Paper Project, Illinois State University

Beckett, K. (2005) 'Choosing Cesarean: feminism and the politics of childbirth in the United States', Feminist Theory 6(3): 251-275

Beck-Gernsheim, E. (2002) Reinventing the Family, Cambridge: Polity Press

Bedford, R. (1999) 'Mobility in Melanesia: bigman bilong circulation', Asia Pacific Viewpoint 40(1): 3-17

Bell, D. (2006) 'Variations on the rural idyll', in P. Cloke, T. Marsden and P. Mooney (eds) Handbook of Rural Studies, London: Sage, pp.149-160

Bell, D. and Jayne, M. (2010) 'The creative countryside: policy and practice in the UK rural cultural economy', Journal of Rural Studies 26(3): 209-218

Bell, D. and Valentine, G. (1995) 'Queer country: rural lesbian and gay lives', Journal of Rural Studies 11(2): 113-122

Bell, M. and Ward, G. (2000) 'Comparing permanent migration with temporary mobility', Tourism Geographies 2: 97-107

Bellwood, P. (2004) First Farmers: the Origins of Agricultural Societies, Oxford: Wiley-Blackwell

Bennett, J. (2006) History Matters: Patriarchy and the Challenge of Feminism, Manchester: Manchester University Press

Benson, M. (2011) The British in Rural France, Manchester: Manchester University Press

Benson, M. (2013b) 'Postcoloniality and privilege in new lifestyle flows: the case of North Americans in Panama', Mobilities 8(3): 313-330

Benson, M. (2014) 'Negotiating privilege in and through lifestyle migration', in M. Benson and N. Osbaldiston (eds) Understanding Lifestyle Migration, Basingstoke: Palgrave Macmillan, pp. 47-68

Benson, M. (2015) 'Lifestyle migration: from the state of the art to the future of the field', Two Homelands 42: 9-23

Benson, M. and O'Reilly, K. (2009a) 'Migration and the search for a better way of life: a critical exploration of lifestyle migration', Sociological Review 57: 608-625

Benson, M. and O'Reilly, K. (2009b) 'Lifestyle migration: escaping to the good life?', in M. Benson and K. O'Reilly (eds) Lifestyle Migration: Expectations, Aspirations and Experiences, Aldershot: Ashgate, pp. 1-14

Benson, M. and O'Reilly, K. (eds) (2009c) Lifestyle Migration: Expectations, Aspirations and Experiences, Farnham: Ashgate

Benson, M. and Osbaldiston, N. (eds) (2014a) Understanding Lifestyle Migration, Basingstoke: Palgrave Macmillan

Benson, M. and Osbaldiston, N. (2014b) 'New horizons in lifestyle migration research: theorising movement, settlement and the search for a better way of life, in M. Benson and N. Osbaldiston (eds) Understanding Lifestyle Migration, Basingstoke: Palgrave Macmillan, pp. 1-23

Berman, M. (1983) All that is Solid Melts into Air, London: Verso

Bernard, M., Liddle, J., Bartlam, B., Scharf, T. and Sim, J. (2012) 'Then and now: evolving community in the context of a retirement village', Ageing and Society 32(01): 103-129

Bernhardt, E. (1993) 'Fertility and employment', European Sociological Review 9(1): 25-42

Berry, B. (ed.) (1976) Urbanization and Counterurbanization, Beverly Hills: Sage Publications

Beyers, N., Gie, R., Zietsman, H., Kunneke, M., Hauman, J., Tatley, M. and Donald, P. (1996) 'The use of a geographical information system (GIS) to evaluate the distribution of tuberculosis in a high-incidence community', South African Medical Journal 86: 4-44

Bhabha, J. and Schmidt, S. (2008) 'Seeking asylum alone: unaccompanied and separated children and refugee protection in the US', Journal of the History of Childhood and Youth 1(1): 126-138

Bidner, C. and Eswaran, M. (2015) 'A gender-based theory of the origin of the caste system in India', Journal of Development Economics 114: 142-158

\section{ST PROOFS: NOT FOR DISTRIBUTION}


Bijker, R. (2013) 'Migration to less popular rural areas. The characteristics, motivations and search process of migrants', PhD Dissertation, University of Groningen

Binnie, J. and Valentine, G. (1999) 'Geographies of sexualities - a review of the progress', Progress in Human Geography 23(2): $175-187$

Binnie, J., Edensor, T., Holloway, J., Millington, S. and Young, C. (2007) 'Mundane mobilities, banal travels', Social and Cultural Geography 8(2): 165-174

Bio (2014) Homepage: www.bio.org (accessed 06/14)

Biraben, J.-N. (2003) 'The rising numbers of humankind', Population and Societies 394: 1-4

Birkenholtz, T. (2011) 'Network political ecology: method and theory in climate change vulnerability and adaptation research', Progress in Human Geography 36(3): 295-315

Bissell, D. and Fuller, G. (eds) (2011) Stillness in a Mobile World, London: Routledge

Bjerke, T., Kaltenborn, B. and Vitters $\emptyset$, J. (2006) 'Cabin life: restorative and affective aspects', in N. Mclntyre, D. Williams and K. McHugh (eds) Multiple Dwelling and Tourism. Negotiating Place, Home and Identity, Wallingford: CABI, pp. 87-102

Black, R. (1998) 'Putting refugees in camps', Forced Migration Review 2: 4-7

Black, R., Adger, W., Arnell, N., Dercon, S., Geddes, A. and Thomas, D. (2011) 'The effect of environmental change on human migration', Global Environmental Change 21: S3-S11

Blackman, L. and Venn, C. (2010) 'Affect', Body and Society 16: 7-28

Blakely, E. and Snyder, M. (1997) Fortress America: Gated Communities in the United States, Washington D.C.: Brookings Institution Press/Cambridge, MA: Lincoln Institute of Land Policy

Blakemore, K. and Boneham, M. (1994) Age, Race and Ethnicity: a Comparative Approach, Buckingham: Open University Press

Blanchflower, D. and Oswald, A. (2013) 'Does high home-ownership impair the labor market?', Forschungsinstitut zur Zukunft der Arbeit, IZA Discussion Paper No. 7640

Blinder, S. (2014) 'Briefing. Migration to the UK: asylum', University of Oxford: COMPAS

Blumstein, A., Rivara, F. and Rosenfeld, R. (2000) 'The rise and decline of homicide - and why', Annual Review of Public Health 21(1): 505-541

Blunt, A. and Dowling, R. (2006) Home, London: Routledge

Blunt, A. and Wills, J. (2000) Dissident Geographies, Harlow: Pearson Education

Boal, F. (2002) 'Belfast: walls within', Political Geography 21(5): 687-694

Bock, G. (1983) 'Racism and sexism in Nazi Germany: motherhood, compulsory sterilization, and the state', Signs 8(3): 400-421

Boeri, T. and Brücker, H. (2005) 'Why are Europeans so tough on migrants?', Economic Policy 20(44): 630-703

Bogue, D. (1969) Principles of Demography, New York: Wiley

Böheim, R. and Taylor, M. (2002) 'Tied down or room to move? Investigating the relationships between housing tenure, employment status and residential mobility in Britain', Scottish Journal of Political Economy 49(4): 369-392

Bollard, A., McKenzie, D., Morten, M. and Rapoport, H. (2011) 'Remittances and the brain drain revisited: the microdata show that more educated migrants remit more', World Bank Economic Review 25(1): 132-156

Bondi, L. (1991) 'Gender divisions and gentrification: a critique', Transactions of the Institute of British Geographers 16: 190-198

Bondi, L. (1999) 'Gender, class, and gentrification: enriching the debate', Environment and Planning D: Society and Space 17(3): 261-282

Bongaarts, J. (1978) 'A framework for analyzing the proximate determinants of fertility', Population and Development Review 4(1): $105-132$

Bongaarts, J. (2003) 'Completing the fertility transition in the developing world: the role of educational differences and fertility preferences', Population Studies 57(3): 321-335

Bonnet, C., Gobillon, L. and Laferrère, A. (2010) 'The effect of widowhood on housing and location choices', Journal of Housing Economics 19(2): 94-108

Boonyabancha, S. (2009) 'Land for housing the poor - by the poor: experiences from the Baan Mankong nationwide slum upgrading programme in Thailand', Environment and Urbanization 21(2): 309-329

Booth, R. (2013) 'Qatar World Cup construction "will leave 4,000 migrant workers dead"', Guardian 26 September

Borgegärd, L.-E. and Murdie, R. (1993) 'Socio-demographic impacts of economic restructuring on Stockholm's inner city', Tijdschrift voor Economische en Sociale Geografie 84: 269-280

Borjas, G. (1989) 'Economic theory and international migration', International Migration Review 23: 457-485

Borrell, C., Marí-Dell'Olmo, M., Serral, G., Martínez-Beneito, M. and Gotsens, M. (2010) 'Inequalities in mortality in small areas of eleven Spanish cities (the multicenter MEDEA project)', Health and Place 16(4): 703-711

\section{ST PROOFS: NOT FOR DISTRIBUTION}




\section{BIBLIOGRAPHY}

Borsdorf, A. and Hidalgo, R. (2009) 'Searching for fresh air, tranquillity and rural culture in the mountains: a new lifestyle for Chileans?', Die Erde 140(3): 275-292

Bose, M. (2007) 'Women's home-centred work in India: the gendered politics of space', International Development Planning Review 29(3): 271-298

Boseley, S. (2016) 'Precision medicine the theme at world's biggest cancer conference', Guardian 3 June

Boseley, S. and Davidson, H. (2015) 'Global obesity rise puts UN goals on diet-related diseases "beyond reach"', Guardian 9 October

Bosker, M., Brakman, S., Garretsen, H. and Schramm, M. (2012) 'Relaxing Hukou: increased labor mobility and China's economic geography', Journal of Urban Economics 72(2): 252-266

Bosworth, G. (2010) 'Commercial counterurbanisation: an emerging force in rural economic development', Environment and Planning A 42(4): 966-981

Bosworth, G. and Willett, J. (2011) 'Embeddedness or escapism? Rural perceptions and economic development in Cornwall and Northumberland', Sociologia Ruralis 51(2): 195-214

Bottomley, G. (1992) From Another Place: Migration and the Politics of Culture, Cambridge: Cambridge University Press

Boucher, D., Bennett, C., McFarlin, B. and Freeze, R. (2009) 'Staying home to give birth: why women in the United States choose home birth', Journal of Midwifery and Women's Health 54(2): 119-126

Bourdieu, P. (1977) Outline of a Theory of Practice, Cambridge: Cambridge University Press

Bourdieu, P. (1984) Distinction: a social critique of the judgement of taste, London: Routledge and Kegan Paul

Bowles, E. (1998) 'From village to camp: refugee camp life in transition on the Thailand-Burma border', Forced Migration Review 2: $11-14$

Bowstead, J. (2015) 'Forced migration in the United Kingdom: women's journeys to escape domestic violence', Transactions of the Institute of British Geographers 40(3): 307-320

Boyd, M. (1989) 'Family and personal networks in international migration: recent developments and new agendas', International Migration Review 23: 638-664

Boyle, P. (2002) 'Population geography: transnational women on the move', Progress in Human Geography 26: 531-543

Boyle, P. (2003) 'Population geography: does geography matter in fertility research?', Progress in Human Geography 27(5): 615-626

Boyle, P. (2004) 'Population geography: migration and inequalities in mortality and morbidity', Progress in Human Geography 28(6): 767-776

Boyle, P. and Halfacree, K. (1995) 'Service class migration in England and Wales, 1980-81: identifying gender-specific mobility patterns', Regional Studies 29: 43-57

Boyle, P. and Halfacree, K. (eds) (1998a) Migration into Rural Areas. Theories and Issues, Chichester: Wiley

Boyle, P. and Halfacree, K. (1998b) 'Migration into rural areas: a collective behaviour framework?', in P. Boyle and K. Halfacree (eds) Migration into Rural Areas: Theories and Issues, London: Wiley, pp. 303-316

Boyle, P. and Halfacree, K. (eds) (1999) Migration and Gender in the Developed World, London: Routledge

Boyle, P. and Norman, P. (2010) 'Migration and health', in T. Brown, S. McLafferty and G. Moon (eds) A Companion to Health and Medical Geography, Oxford: Blackwell, pp. 346-374

Boyle, P., Findlay, A. and Newbold, B. (2004) 'Editorial introduction to Population, Space and Place', Population, Space and Place 10(1): 1

Boyle, P., Halfacree, K. and Robinson, V. (1998) Exploring Contemporary Migration, Harlow: Addison, Wesley, Longman

Boyle, P., Norman, P. and Popham, F. (2009) 'Social mobility: evidence that it can widen health inequalities', Social Science and Medicine 68(10): 1835-1842

Boyle, P., Cooke, T., Halfacree, K. and Smith, D. (2001) 'A cross-national comparison of the impact of family migration on women's employment status', Demography 38: 201-213

Boyle, P., Cooke, T., Halfacree, K. and Smith, D. (2002) 'A cross-national study of the effects of family migration on women's labour market status: some difficulties from integrating microdata from two censuses', Journal of the Royal Statistical Society A 165: 465-480

Boyle, P., Cooke, T., Halfacree, K. and Smith, D. (2003) 'The effect of long-distance family migration and motherhood on partnered women's labour market activity rates in GB and the US', Environment and Planning A 35: 2097-2114

Boyle, P., Kulu, H., Cooke, T., Gayle, V. and Mulder, C. (2008) 'Moving and union dissolution', Demography, 45(1): 209-222

Bradley, D. (2011) 'Litwak and Longino's developmental model of later-life migration: evidence from the American community survey, 2005-2007', Journal of Applied Gerontology 30(2): 141-158

Bradley, H. (1989) Men's Work, Women's Work, Cambridge: Polity Press

Bradsher, J., Longino, C., Jackson, D. and Zimmerman, R. (1992) 'Health and geographic mobility among the recently widowed', Journal of Gerontology 47(5): S261-S268

\section{ST PROOFS: NOT FOR DISTRIBUTION}


Brah, A. and Phoenix, A. (2004) 'Ain't I a woman? Revisiting intersectionality', Journal of International Women's Studies 5: 75-86

Brama, A. (2008) 'Dynamics of ethnic residential segregation in Goteborg, Sweden, 1995-2000', Population, Space and Place 14: $101-117$

Bramwell, A. (1985) Blood and Soil: Walther Darré and Hitler's 'Green Party', Bourne End, Buckinghamshire: Kensal

Brandreth, G. (2013) Oxford Dictionary of Humorous Quotations, 5th edition, Oxford: Oxford University Press

Braun, L., Fausto-Sterling, A., Fullwiley, D, Hammonds, E., Nelson, A., Quivers, W., Reverby, S. and Shields, A. (2007) 'Racial categories in medical practice: how useful are they?', PLoS Medicine 4(9): 1423-1428

Breman, J. (1994) Wage Hunters and Gatherers, Delhi: Oxford University Press

Brenton, M. (2008) 'The cohousing approach to "living neighbourhoods"', Fact Sheet \#29, London: Housing Learning and Improvement Network

Brettell, C. (2008) 'Theorizing migration in Anthropology. The social construction of networks, identities, communities, and groups', in C. Brettell and J. Hollifield (eds) Migration Theory. Talking Across Disciplines, 2nd edition, London: Routledge, pp. 113-159

Brettell, C. (2013) 'Anthropology of migration', in I. Naess (ed.) The Encyclopedia of Global Human Migration, New Jersey: WileyBlackwell, at: http://onlinelibrary.wiley.com/doi/10.1002/9781444351071.wbeghm031/abstract (accessed 08/16)

Brettell, C. and Hollifield, J. (2008a) (eds) Migration Theory. Talking Across Disciplines, 2nd edition, London: Routledge

Brettell, C. and Hollifield, J. (2008b) 'Introduction. Migration theory. Talking across disciplines', in C. Brettell and J. Hollifield (eds) Migration Theory. Talking Across Disciplines, 2nd edition, London: Routledge, pp. 1-29

Bromley, R. and Mackie, P. (2008) 'Identifying the role of children in informal trade: evidence for urban policy', International Development Planning Review 30(2): 113-131

Bromley, R. and Mackie, P. (2009) 'Child experiences as street traders in Peru: contributing to a reappraisal for working children', Children's Geographies 7(2): 141-158

Bromley, R., Matthews, D. and Thomas, C. (2007) 'City centre accessibility for wheelchair users: the consumer perspective and the planning implications', Cities 24: 229-241

Bromley, R., Tallon, A. and Thomas, C. (2005) 'City centre regeneration through residential development: contributing to sustainability', Urban Studies 42(13): 2407-2429

Brookman, F. (2005) Understanding Homicide, London: Sage

Brooks, A. (2004) ' Under the knife and proud of it": an analysis of the normalization of cosmetic surgery', Critical Sociology 30(2): 207-239

Brooks, A. and Simpson, R. (2012) Emotions in Transmigration: Transformation, Movement and Identity, Basingstoke: Palgrave Macmillan

Brooks, R. and Waters, J. (2009) 'A second chance at "success" UK students and global circuits of higher education', Sociology 43(6): 1085-1102

Brossoie, N. (2008) 'Living options for adults needing assistance', Virginia Cooperative Extension, Publication 350-354

Brown, D. and Schafft, K. (2002) 'Population deconcentration in Hungary during the post-socialist transformation', Journal of Rural Studies 18(3): 233-244

Brown, D. and Swanson, L. (eds) (2003) Challenges for Rural America in the Twenty-First Century, University Park, PA: Penn State Press

Brown, D. and Wardwell, J. (eds) (1980) New Directions in Urban-Rural Migration: the Population Turnaround in Rural America, New York: Academic Press

Brown, D., Bolender, B., Kulcsar, L., Glasgow, N. and Sanders, S. (2011) 'Intercounty variability of net migration at older ages as a path-dependent process', Rural Sociology 76(1): 44-73

Brown, L. (2006) 'Global warming forcing U.S. coastal population to move inland: an estimated 250,000 Katrina evacuees are now climate refugees', Earth Policy Institute, Plan B Updates 16 August, at: www.earth-policy.org/plan_b_updates/2006/update57 (accessed 08/16)

Brown, R. (1982) 'Work histories, career strategies and class structure', in A. Giddens and G. Mackenzie (eds) Social Class and the Division of Labour, Cambridge: Cambridge University Press, pp. 119-136

Brown, T., McLafferty, S. and Moon, G. (eds) (2010) A Companion to Health and Medical Geography, Oxford: Blackwell

Browne, K. (2007) 'Drag queens and drag dykes: deploying and deploring feminities', in K. Browne, J. Lim and G. Brown (eds) Geographies of Sexualities, Farnham: Ashgate, pp. 113-124

Browne, K., Lim, J. and Brown, G. (eds) (2007) Geographies of Sexualities, Farnham: Ashgate

Brubaker, R. (1993) 'East European, Soviet, and post-Soviet nationalisms: a framework for analysis', Research on Democracy and Society 1: 353-378

Buck, T. (2014) 'Romanians in Spain plan to put down roots', Financial Times 16 January 


\section{BIBLIOGRAPHY}

Bukowski, C. (2009/1978) Factotum, London: Virgin Books

Buller H and Hoggart K. (1994) International Counterurbanization, Avebury: Aldershot

Bunce, M. (1994) The Countryside Ideal. Anglo-American Images of Landscape, London: Routledge

Bunce, M. (2003) 'Reproducing rural idylls', in P. Cloke (ed.) Country Visions, Harlow: Pearson, pp.14-30

Burdette, H. and Whitaker, R. (2005) 'A national study of neighborhood safety, outdoor play, television viewing, and obesity in preschool children', Pediatrics 116(3): 657-662

Burmeister, H. (2013) 'Rural revolution: documenting the lesbian land communities of Southern Oregon', MA Dissertation, Department of History, Portland State University

Burnley, I. and Murphy, P. (2004) Sea Change: Movement from Metropolitan to Arcadian Australia, Sydney: UNSW Press

Burton, I. (1963) 'The quantitative revolution and theoretical geography', Canadian Geographer 7: 151-162

Büscher, M., Urry, J. and Witchger, K. (eds) (2010) Mobile Methods, London: Routledge

Bush, R. (2010) 'Food riots: poverty, power and protest', Journal of Agrarian Change 10(1): 119-129

Bushin, N. (2005) 'For the Sake of the Children'?: Children's Experiences of Family Migration to the English Countryside, PhD thesis, Department of Geography, Swansea University

Bushin, N. (2009) 'Researching family migration decision-making: a children-in-families approach', Population, Space and Place 15(5): $429-443$

Butler, J. (1990) Gender Trouble: Feminism and the Subversion of Identity, London: Routledge

Butler, J. (1993) Bodies that Matter: the Discursive Limits of Sex, London: Routledge

Butler, R. and Parr, H. (eds) (1999) Mind and Body Spaces: Geographies of Illness, Impairment and Disability, London: Routledge

Butler, T. (1997) Gentrification and the Middle Classes. Aldershot: Ashgate

Butler, T. (2003) 'Living in the bubble: gentrification and its "others" in North London', Urban Studies 40(12): 2469-2486

Butler, T. (2007) 'For gentrification?', Environment and Planning A 39(1): 162-181

Butler, T. and Lees, L. (2006) 'Super-gentrification in Barnsbury, London: globalization and gentrifying global elites at the neighbourhood level', Transactions of the Institute of British Geographers 31(4): 467-487

Butler, T. and Robson, G. (2003) 'Negotiating their way in: the middle classes, gentrification and the deployment of capital in a globalising metropolis', Urban Studies 40(9): 1791-1809

Cadwallader, M. (1993) 'Commentary on Zelinsky's model', Progress in Human Geography 17: 215-217

Calasanti, T. (2005) 'Ageism, gravity, and gender: experiences of aging bodies', Generations 29(3): 8-12

Caldwell, J. (1982) Theory of Fertility Decline, London: Academic Press

Caldwell, J. (2001) 'Public health classics: population health in transition', Bulletin of the World Health Organization 79(2): 157-160

Caldwell, J. and Caldwell, P. (1987) 'The cultural context of high fertility in sub-Saharan Africa', Population and Development Review 13: 409-437

Caldwell, J. and Schindlmayr, T. (2003) 'Explanations of the fertility crisis in modern societies: a search for commonalities', Population Studies 57(3): 241-263

Caldwell, J., Caldwell, P. and Quiggin, P. (1989) 'The social context of AIDS in Sub-Saharan Africa', Population and Development Review 15(2): 185-234

Caldwell, J., Orubuloye, I. and Caldwell, P. (1992) 'Fertility decline in Africa: a new type of transition?', Population and Development Review 18: 211-242

Calero, C., Bedi, A. and Sparrow, R. (2008) 'Remittances, liquidity constraints and human capital investments in Ecuador', World Development 37(6): 1143-1154

Callard, F. (2003) 'The taming of psychoanalysis in geography', Social and Cultural Geography 4(3): 295-312

Callender, M. (2012) The Lives of Young Polish Migrants Residing in Northamptonshire, Unpublished PhD thesis, University of Northampton

Callinicos, L. (2005) Interview Segment, at: http://overcomingapartheid.msu.edu/video.php?id=65-24F-74 (accessed 08/16)

Callon, M. and Law, J. (2004) 'Introduction: absence-presence, circulation, and encountering in complex space', Environment and Planning D: Society and Space 22(1): 3-11

Camacho, A. (1999) 'Family, child labour and migration. Child domestic workers in metro Manila', Childhood 6(1): 57-73

Camarero, L., Cruz, F., González, M., del Pino, J., Oliva, J. and Sampedro, R. (2009) 'La población rural en España. De los desequilibrios a la sostenibilidad social', Barcelona: Fundación La Caixa, Serie Estudios, 27

Cameron, S., Lozier, J., Strange, J., Koch, J., Cordes, N., Solter, L. and Griswold, T. (2011) 'Patterns of widespread decline in North American bumble bees', Proceedings of the National Academy of Sciences 108(2): 662-667

Campanella, T. (2006) 'Urban resilience and the recovery of New Orleans', Journal of the American Planning Association 72(2): $141-146$

\section{ST PROOFS: NOT FOR DISTRIBUTION}


Cancian, S. (2011) 'Love in the time of migration: lovers' correspondence between Italy and Canada, 1948-1957', Diversite Urbaine 10(2): 91-109

Carolan, M. (2009) '“I do therefore there is": enlivening socio-environmental theory', Environmental Politics 18: 1-17

Carr, P., Lichter, D. and Kefalas, M. (2012) 'Can immigration save small-town America? Hispanic boomtowns and the uneasy path to renewal', Annals of the American Academy of Political and Social Science 641(1): 38-57

Carswell, G., and De Neve, G. (2013) 'From field to factory: tracing transformations in bonded labour in the Tiruppur region, Tamil Nadu', Economy and Society 42(3): 430-454

Casado-Díaz, M. (2006) 'Retiring to Spain: an analysis of differences among North European nationals', Journal of Ethnic and Migration Studies 32(8): 1321-1339

Casado-Díaz, M. (2009) 'Social capital in the sun: bonding and bridging social capital among British retirees', in M. Benson and K. O'Reilly (eds) Lifestyle Migration: Expectations, Aspirations and Experiences, Aldershot: Ashgate, pp. 87-102

Casado-Díaz, M., Kaiser, C. and Warnes, A. (2004) 'Northern European retired residents in nine Southern European areas: characteristics, motivations and adjustment', Ageing and Society 24(3): 353-381

Cass, N., Shove, E. and Urry, J. (2005) 'Social exclusion, mobility and access', Sociological Review 53(3): 539-555

Cassarino, J. (2004) 'Theorising return migration: the conceptual approach to return migrants revisited', International Journal on Multicultural Societies 6(2): 253-279

Cassidy, T. (2006) Birth: the Surprising History of How We Are Born, New York: Atlantic Monthly Press

Castells, M. (2000) 'Materials for an exploratory theory of the network society', British Journal of Sociology 51(1): 5-24

Castells, M. (2010) The Power of Identity, 2nd edition, Oxford: Blackwell

Castles, S. (2000) Ethnicity and Globalization: from Migrant Worker to Transnational Citizen, London: Sage

Castles, S. (2007) 'Twenty-first-century migration as a challenge to sociology', Journal of Ethnic and Migration Studies 33(3): 351-371

Castles, S. (2010) 'Understanding global migration: a social transformation perspective', Journal of Ethnic and Migration Studies 36(10): 1565-1586

Castles, S. and Miller, M. (1993) The Age of Migration, Basingstoke: Macmillan

Castles, S., de Haas, H. and Miller, M. (2014) The Age of Migration, 5th edition, Basingstoke: Palgrave Macmillan

Castree, N. (2014) 'The Anthropocene and geography I: the back story', Geography Compass 8(7): 436-449

Castrodale, M. and Crooks, V. (2010) 'The production of disability research in human geography: an introspective examination', Disability and Society 25: 89-102

Catholics Against Contraception (2012) Homepage: www.catholicsagainstcontraception.com/ (accessed 12/12)

CDC [Center for Disease Control] (1999) 'Achievements in public health, 1900-1999: family planning', MMWR Weekly 48(47): 1073-1080

CDC (2015) Homepage: www.cdc.gov (accessed 11/15)

CDC (2016) The NCHHSTP Atlas, at: www.cdc.gov/nchhstp/atlas/about-atlas.html (accessed 01/16)

Cerase, F. (1974) 'Expectations and reality: a case study of return migration from the United States to Southern Italy', International Migration Review 8(2): 245-262

Champion, A. (1992) 'Migration in Britain: research challenges and prospects', in A. Champion and A. Fielding (eds) Migration Processes and Patterns. Volume 1: Research Progress and Prospects, London: Belhaven Press, pp. 215-226

Champion, A. (1998) 'Studying counterurbanisation and the rural population turnaround', in P. Boyle and K. Halfacree (eds) Migration into Rural Areas. Theories and Issues, Chichester: Wiley, pp. 21-40

Champion, A. (ed.) (1989) Counterurbanization: the Changing Pace and Nature of Population Deconcentration, London: Edward Arnold

Champion, A. and Atkins, D. (1996) 'The counterurbanisation cascade: an analysis of the 1991 Census Special Migration Statistics for Great Britain', Seminar Paper 81, Department of Geography, University of Newcastle: Newcastle upon Tyne

Champion, T. (2001) 'Urbanization, suburbanization, counterurbanization and reurbanization', in R. Paddison (ed.) Handbook of Urban Studies, London: Sage, pp. 143-161

Champion, T. (2005) 'Population movement within the UK', in R.Chappell (ed.) Focus on People and Migration, Basingstoke: Palgrave Macmillan, pp. 91-113

Champion, T. and Hugo, G. (2004) 'Introduction: moving beyond the urban-rural dichotomy', in T. Champion and G. Hugo (eds) New Forms of Urbanization. Beyond the Urban-Rural Dichotomy, Aldershot: Ashgate, pp.3-24

Champion, T., Coombes, M. and Gordon, I. (2014) 'How far do England's second-order cities emulate London as human-capital "escalators"?', Population, Space and Place 20(5): 421-433 


\section{BIBLIOGRAPHY}

Chan, S. (2013) 'Internationalising higher education sectors: explaining the approaches in four Asian countries', Journal of Higher Education Policy and Management 35(3): 316-329

Chang, S., Sterne, J., Wheeler, B., Lu, T., Lin, J. and Gunnell, D. (2011) 'Geography of suicide in Taiwan: spatial patterning and socioeconomic correlates', Health and Place 17(2): 641-650

Chant, S. (1992) (ed.) Gender and Migration in Developing Countries, London: Belhaven Press

Chant, S. and Radcliffe, S. (1992) 'Migration and development: the importance of gender', in S. Chant (ed.) Gender and Migration in Developing Countries, London: Belhaven Press, pp. 1-29

Chapman, I. (2014) 'The end of Peak Oil? Why this topic is still relevant despite recent denials', Energy Policy 64: 93-101

Chapman, M. (1985) 'Me go walkabout: you too?', in M. Chapman and R. Prothero (eds) Circulation in Population Movement: Substance and Concepts from the Melanesian Case, London: Routledge and Kegan Paul, pp. 429-443

Chapman, M. (1987) 'Population movement studies at microscale: experience and extrapolation', GeoJournal 15(4): 347-365

Chapter 7 (2009) 'LID: getting the policy right', Chapter 7 News 6: 59-63

Chavez, L. (2001) Covering Immigration. Popular Images and the Politics of the Nation, Berkeley: University of California Press

Chavez, L. (2008) The Latino Threat: Constructing Immigrants, Stanford: Stanford University Press

Chen, Y., Wu, K., Yousuf, S. and Yip, P. (2012) 'Suicide in Asia: opportunities and challenges', Epidemiologic Reviews 34: 129-144

Cheng, H., Gurland, B. and Maurer, M. (2008) 'Self-reported lack of energy (anergia) among elders in a multiethnic community', Journal of Gerontology: Medical Sciences 63A: 707-714

Cheshire, L. (2010) 'A corporate responsibility? The constitution of fly-in, fly-out mining companies as governance partners in remote, mine-affected localities', Journal of Rural Studies 26(1): 12-20

Cheung, S. (2011) 'Migration control and the solutions impasse in South and Southeast Asia: implications from the Rohingya experience', Journal of Refugee Studies 25(1): 50-70

Chouinard, V. (1997) 'Making space for disabling difference: challenging ableist geographies', Environment and Planning D: Society and Space 15: 379-387

Chouinard, V., Hall, E. and Wilton, R. (eds) (2010) Towards Enabling Geographies, Farnham: Ashgate

Chu, J. (2006) 'To be 'emplaced': Fuzhounese migration and the politics of destination', Identities: Global Studies in Culture and Power 13: 395-425

Ciabarri, L. (2014) 'Dynamics and representations of migration corridors: the rise and fall of the LibyaLampedusa route and forms of mobility from the Horn of Africa (2000-2009)', ACME: An International E-Journal for Critical Geographies 13(2): 246-262

Clapson, M. (2003) Suburban Century: Social Change and Urban Growth in England and the USA, Oxford: Berg

Clark, S., Collinson, M., Kahn, K., Drullinger, K. and Tollman, S. (2007) 'Returning home to die: circular labour migration and mortality in South Africa', Scandinavian Journal of Public Health 35(69 supp.): 35-44

Clark, W. (1986) Human Migration, Beverly Hills, California: Sage

Clark, W. and Davies, S. (1990) 'Elderly mobility and mobility outcomes: households in the later stages of the life course', Research on Aging 12(4): 430-462

Clark, W. and Davies Withers, S. (2009) 'Fertility, mobility and labour force participation: a study of synchronicity', Population, Space and Place 15: 305-321

Clark, W. and Dieleman, F. (1996) Households and Housing: Choice and Outcomes in the Housing Market, New Jersey: Rutgers The State University of New Jersey

Clark, W. and Huang, Y. (2003) 'The life course and residential mobility in British housing markets', Environment and Planning A 35(2): 323-339

Clark, W., Deurloo, M. and Dieleman, F. (2003) 'Housing careers in the United States, 1968-93: modelling the sequencing of housing states', Urban Studies 40(1): 143-160

Clark, W., Deurloo, M. and Dieleman, F. (2006) 'Residential mobility and neighbourhood outcomes', Housing Studies 21(3): 323-342

Clausen, J. (1991a) 'Adolescent competence and the shaping of the life course', American Journal of Sociology 96: 805-842

Clausen, J. (1991b) 'Adolescent competence and the life course, or why one social psychologist needed a concept of personality', Social Psychology Quarterly 54: 4-14

Cleland, J. and Wilson, C. (1987) 'Demand theories of the fertility transition: an iconoclastic view', Population Studies 41(1): 5-30

Clifford, J. and Marcus, G. (eds) (1986) Writing Culture: the Poetics and Politics of Ethnography, Berkeley: University of California Press

Climo, J. (2000) 'Eldercare as "woman's work" in poor countries', Journal of Family Issues 21(6): 692-713

Cloke, P. (2003b) 'Knowing ruralities?', in P. Cloke (ed.) Country Visions, Harlow: Pearson, pp.1-13

Cloke, P. (2006) 'Conceptualizing rurality', in P. Cloke, T. Marsden and P. Mooney (eds) Handbook of Rural Studies, London: Sage, pp.18-28

\section{ST PROOFS: NOT FOR DISTRIBUTION}


Cloke, P., Crang, P. and Goodwin, M. (eds) (2005) Introducing Human Geographies, 2nd edition, London: Hodder

Coale, A. (1973) 'The demographic transition', International Population Conference: Liege, 1973, Vol. 1. Liege: IUSSP: 53-72

Coale, A. and Watkins, S. (eds) (1986) The Decline of Fertility in Europe, Princeton, NJ: Princeton University Press

Coates, C. (2001) Utopia Britannica. British Utopian Experiments: 1325-1945, London: Diggers and Dreamers Publications

Cockerham, W. (2005) 'Health lifestyle theory and the convergence of agency and structure', Journal of Health and Social Behavior 46(1): 51-67

Cohen, J. (2004) The Culture of Migration in Southern Mexico, Austin: University of Texas Press

Cohen, J. (1941) 'The geography of crime', Annals of the American Academy of Political and Social Science 217: 29-37

Cohen, R. (1987) The New Helots: Migrants in the International Division of Labour, Aldershot: Gower

Cohen, S., Duncan, T. and Thulemark, M. (2015) 'Lifestyle mobilities: the crossroads of travel, leisure and migration', Mobilities 10(1): 155-172

Colen, S. (1995) 'Like a mother to them: stratified reproduction and West Indian childcare workers and employers in New York', in F. Ginsburg and R. Rapp R. (eds) Conceiving the New World Order: the Global Politics of Reproduction, Berkeley: University of California Press, pp. 78-102

Collins, A. (2004) 'Sexual dissidence, enterprise and assimilation: bedfellows in urban regeneration', Urban Studies 41(9): 1789-1806

Collins-Kreiner, N. (2010) 'The geography of pilgrimage and tourism: transformations and implications for applied geography', Applied Geography 30(1): 153-164

Collinson, D. (1998) '"Shifting Lives": Work-Home Pressures in the North Sea Oil Industry', Canadian Review of Sociology/Revue Canadienne de Sociologie 35(3): 301-324

Collinson, M., Tollman, S., Kahn, K., Clark, S. and Garenne, M. (2006) 'Highly prevalent circular migration: households, mobility and economic status in rural South Africa', Paper prepared for Conference on African Migration in Comparative Perspective, Johannesburg, South Africa, June 2003, at: www.queensu.ca/samp/migrationresources/Documents/Collinson_highly.pdf (accessed 08/16)

Collyer, M. (2012) 'Deportation and the micropolitics of exclusion: the rise of removals from the UK to Sri Lanka', Geopolitics 17(2): 276-292

Collyer, M. and Haas, H. (2012) 'Developing dynamic categorisations of transit migration', Population, Space and Place 18(4): 468-481

Conlon, K. (2014) 'Countercultural communes: rejection or reflection of conventional mainstream gender norms?', PhD Dissertation, Rutgers University-Graduate School-Newark

Connell, J. (1992) 'Far beyond the Gulf: the implications of warfare for Asian labour migration', Australian Geographer 23: 44-50

Connell, J. and Gibson, C. (2003) Sound Tracks: Popular Music, Identity and Place, London: Routledge

Connelly, R., Roberts, K. and Zheng, Z. (2011) 'The settlement of rural migrants in urban China - some of China's migrants are not "floating" anymore', Journal of Chinese Economic and Business Studies 9(3): 283-300

Conradson, D. (2005) 'Landscape, care and the relational self: therapeutic encounters in rural England', Health and Place 11: 337-348

Conradson, D. (2011) 'The orchestration of feeling: stillness, spirituality and places of retreat', in D. Bissell and G. Fuller (eds) Stillness in a Mobile World, Abingdon: Routledge, pp. 71-86

Conradson, D. and Latham, A. (2005) 'Transnational urbanism: attending to everyday practices and mobilities', Journal of Ethnic and Migration Studies 31: 227-233

Conway, D. (2004) 'On being part of population geography's future: population-environment relationships and inter-science initiatives', Population, Space and Place 10(4): 295-302

Conway, K. and Houtenville, A. (2003) 'Out with the old, in with the old: a closer look at younger versus older elderly migration', Social Science Quarterly 84(2): 309-328

Cooke, L. and D'Emilio, F. (2015) 'EU free travel in danger as borders tighten, fences go up', Associated Press 12 November, at: www.usnews.com/news/world/articles/2015/11/12/eu-free-travel-zone-in-danger-as-nations-fence-out-migrants (accessed $08 / 16)$

Cooke, T. (2001) "'Trailing wife" or "trailing mother"? The effect of parental status on the relationship between family migration and the labor-market participation of married women', Environment and Planning A 33(3): 419-430

Cooke, T. (2008a) 'Migration in a family way', Population, Space and Place, 14(4): 255-265

Cooke, T. (2008b) 'Gender role beliefs and family migration', Population Space and Place 14(3): 163-175

Cooke, T. (2011) 'It is not just the economy: declining migration and the rise of secular rootedness', Population, Space and Place 17(3): 193-203

Cooper, R., Kaufman, J. and Ward, R. (2003) 'Race and genomics', New England Journal of Medicine 348(12): 1166-1170 


\section{BIBLIOGRAPHY}

Corbett, E., Watt, C., Walker, N., Maher, D., Williams, B., Raviglione, M. and Dye, C. (2003) 'The growing burden of tuberculosis: global trends and interactions with the HIV epidemic', Archives of Internal Medicine 163(9): 1009-1021

Corcoran, M. (2002) 'The process of reinvention of self: the experiences of returning Irish Emigrants', Éire-Ireland 37(1-2): 175-191

Corrao, G., Ferrari, P., Zambon, A., Torchio, P., Arico, S. and Decarli, A. (1997) 'Trends of liver cirrhosis mortality in Europe, 1970-1989: age-period-cohort analysis and changing alcohol consumption', International Journal of Epidemiology 26(1): 100-109

Corteen, K. (2002) 'Lesbian safety talk: problematizing definitions and experiences of violence, sexuality and space', Sexualities 5(3): 259-280

Cossman, R., Cossman, J., Jackson, R. and Cosby, A. (2003) 'Mapping high or low mortality places across time in the United States: a research note on a health visualization and analysis project', Health and Place 9(4): 361-369

Couch, D. and Liamputtong, P. (2008) 'Online dating and mating: the use of the internet to meet sexual partners', Qualitative Health Research 18(2): 268-279

Coulter, R. (2013) 'Wishful thinking and the abandonment of moving desires over the life course', Environment and Planning A 45(8): 1944-1962

Coulter, R., Van Ham, M. and Findlay, A. (2016) 'Re-thinking residential mobility. Linking lives through time and space', Progress in Human Geography 40(3): 352-374

Council of the European Union (2004) 'Council Regulation (EC) No 2007/2004 establishing a European Agency for the Management of Operational Co-operation at the External Borders of the Member States of the European Union', Official Journal of the European Union, L 349

Courgeau, D. (1989) 'Recent conceptual advances in the study of migration in France', in P. Ogden and P. White (eds) Migrants in Modern France, London: Unwin Hyman, pp. 60-73

Courtney, D. (2000) 'A quantification of Irish migration with a particular emphasis on the 1980s and 1990s', in A. Bielenberg (ed.) The Irish Diaspora, Harlow: Pearson, pp. 287-316

Courtney, K., Demeris, G., Rantz, M. and Skubic, M. (2008) 'Needing smart home technologies: the perspectives of older adults in continuing care retirement communities', Informatics in Primary Care 16: 195-201

Cowan, R. (2005) 'Immigrants tell of forced prostitution and slavery as trafficking gang is jailed', Guardian 2 November

Cox, K. and Golledge, R. (eds) (1969) Behavioral Problems in Geography: a Symposium, Evanston: Northwestern University

Cox, K. and Golledge, R. (eds) (1981) Behavioral Problems in Geography Revisited, London: Methuen

Coy, M. (2002) 'Gated communities in Latin American megacities: case studies in Brazil and Argentina', Environment and Planning B: Planning and Design 29(3): 355-370

Craddock, S. and Brown, T. (2010) 'Representing the un/healthy body', in T. Brown, S. McLafferty and G. Moon (eds) A Companion to Health and Medical Geography, Oxford: Blackwell, pp. 301-321

Craig, G. (2007) 'Slavery: alive and well and living in the UK', Regional Review, Summer: 20-21

Crane, A. (2013) 'Modern slavery as a management practice: exploring the conditions and capabilities for human exploitation', Academy of Management Review 38(1): 49-69

Crang, P. (1992) 'The politics of polyphony: reconfigurations in geographical authority', Environment and Planning D: Society and Space 10(5): 527-549

Crang, P. (2000) 'Cultural turn', in R. Johnston, D. Gregory, G. Pratt and M. Watts (eds) The Dictionary of Human Geography, Oxford, Blackwell, 4th edition, pp. 141-143

Cravey, A. (2003) 'Toque una ranchera, por favor', Antipode 35(3): 603-621

Crawley, H. (2006) Child First, Migrant Second: Ensuring that Every Child Matters, London: Immigration Law Practitioners' Association

Crawley, H. (2007) 'When is a child not a child? Asylum, age disputes and the process of age assessment', London: Immigration Law Practitioners' Association

Crawley, H. (2010) 'Moving beyond ethnicity: the socio-economic status and living conditions of immigrant children in the UK', Child Indicators Research 3(4): 547-570

Crawley, H., Hemmings, J. and Price, N. (2011) 'Coping with destitution: survival and livelihood strategies of refused asylum seekers living in the UK', Oxfam Research Report, at: http://policy-practice.oxfam.org.uk/publications/coping-with-destitution-survivaland-livelihood-strategies-of-refused-asylum-se-121667 (accessed 08/16)

Cresswell, T. (1996) In Place/Out of Place, Minneapolis: University of Minnesota Press

Cresswell, T. (2004) Place: a Short Introduction, Oxford: Blackwell

Cresswell, T. (2006) On the Move. Mobility in the Modern Western World, London: Routledge

Cresswell, T. (2010) 'Towards a politics of mobility', Environment and Planning D: Society and Space 28(1): 17-31

Cresswell, T. (2012) 'Mobilities II. Still', Progress in Human Geography 36(5): 645-653

Cresswell, T. (2013) 'Citizenship in worlds of mobility', in O. Söderström, D. Ruedin, S. Randeria, G. D'Amato and F. Panese (eds) Critical Mobilities, London: Routledge, pp. 81-100

\section{ST PROOFS: NOT FOR DISTRIBUTION}


Cresswell, T. and Merriman, P. (eds) (2011) Geographies of Mobilities, Farnham: Ashgate

Cribb, J., Hood, A., Joyce, R. and Phillips, D. (2013) Living Standards, Poverty and Inequality in the UK: 2013, London: Institute for Fiscal Studies

Cribier, F. (1987) 'Retiring to the seaside: a housing perspective', Housing Studies 2(1): 42-56

Crisp, J. (2004) 'New issues in refugee research: the local integration and local settlement of refugees: a conceptual and historical analysis', Geneva: Global Commission on International Migration

Crockett, L. (2002) 'Agency in the life course: concepts and processes', in R. Dienstbier and L. Crockett (eds) Agency, Motivation, and the Life Course, Lincoln: University of Nebraska Press, pp. 1-29

Cromartie, J. (2015) 'Development of ERS Rural-Urban classification systems', in National Academy of Sciences Workshop on Rationalizing Rural Area Classifications. Presentations. April 16-17, 2015, Washington, DC: National Academies

Croucher, K., Hicks, L. and Jackson, K. (2006) 'Housing with care for later life: a review of the literature', York: Joseph Rowntree Foundation.

Croucher, S. (2009) 'Migrants of privilege: the political transnationalism of Americans in Mexico', Identities: Global Studies in Culture and Power 16(4): 463-491

Croucher, S. (2012) 'Privileged mobility in an age of globality', Societies 2(1): 1-13

Crow, L. (1996) 'Including all of our lives: renewing the social model of disability', in B. Barnes and G. Mercer (eds) Exploring the Divide, Leeds: Disability Press, pp. 55-72

Crump, J. (2008) Final Report of the Foreclosure Data Committee, Housing Studies Program, University of Minnesota, at: www.house.leg.state.mn.us/dflpdf/DataGroupFinalReport.pdf (accessed 08/16)

Crush, J. (2000) 'Migrations past: an overview of cross-border migration in Southern Africa', in D. McDonald (ed.) On Borders: Perspectives on International Migration in Southern Africa, New York: SAMP/St Martin's Press, pp. 12-24

Crush, J. (2011) 'Complex movements, confused responses: labour migration in South Africa', Southern African Migration Programme, Policy Brief 25, at: www.queensu.ca/samp/sampresources/samppublications/policybriefs/brief25.pdf (accessed 08/16)

Crush, J., Jeeves, A. and Yudelman, D. (1991) South Africa's Labor Empire: a History of Black Migrancy to the Gold Mines, Boulder, CO: Westview Press

Crutzen, P. (2002) 'Geology of mankind', Nature 415(6867): 23

Crutzen, P. and Stoermer, E. (2000) 'The Anthropocene', International Geosphere-Biosphere Programme Newsletter 41: 12

Curtis, B. (2002) 'Foucault on governmentality and population: the impossible discovery', Canadian Journal of Sociology / Cahiers Canadiens De Sociologie 27(4): 505-33

Curtis, S. and Oven, K. (2012) 'Geographies of health and climate change', Progress in Human Geography 36: 654-666

Curtis, S., Riva, M. and Rosenberg, M. (2010) 'Health geography in public health', in T. Brown, S. McLafferty and G. Moon (eds) Companion to Health and Medical Geography, Oxford Blackwell, pp. 325-345

Cutchin, M. (2001) 'Deweyan integration: moving beyond place attachment in elderly migration theory', International Journal of Aging and Human Development 52(1): 29-44

Cuttitta, P. (2014) 'Borderizing the island setting and narratives of the Lampedusa border play', ACME: an International E-Journal for Critical Geographies 13(2): 196-219

D'Andrea, A. (2007) Global Nomads: Techno and New Age as Transnational Countercultures in Ibiza and Goa, London: Routledge

D'Aoust, A. (2013) 'In the name of love: marriage migration, governmentality, and technologies of love', International Political Sociology 7(3): 258-274

Dahlman, C. and Ó Tuathail, G. (2005) 'The legacy of ethnic cleansing: the international community and the returns process in postDayton Bosnia-Herzegovina', Political Geography 24(5): 569-599

Dai, D. (2010) 'Black residential segregation, disparities in spatial access to health care facilities, and late-stage breast cancer diagnosis in metropolitan Detroit', Health and Place 16(5): 1038-1052

Daily Mail (2011) 'An unrecognisable world: Global population of 9 billion will compete for food supplies in 2050 ', 22 February

Dalton, M., Longacre, M., Drake, K., Gibson, L., Adachi-Mejia, A., Swain, K., Xie, H. and Owens, P. (2011) 'Built environment predictors of active travel to school among rural adolescents', American Journal of Preventive Medicine 40(3): 312-319

Daniel, J. (2012) 'Making sense of MOOCs: musings in a maze of myth, paradox and possibility', Journal of Interactive Media in Education 2012(3): http://jime.open.ac.uk/articles/10.5334/2012-18/ (accessed 08/16)

Daniels, S. and Nash, C. (2004) 'Lifepaths: geography and biography', Journal of Historical Geography 30(3): 449-458

Darby, S., Hill, D., Auvinen, A., Barros-Dios, J., Baysson, H., Bochicchio, F., Deo, H., Falk, R., Forastiere, F., Hakama, M., Heid, I., Kreienbrock, L., Kreuzer, M., Lagarde, F., Mäkeläinen, I., Muirhead, C., Oberaigner, W., Pershagen, G., Ruano-Ravina, A., Ruosteenoja, E., Rosario, A., Tirmarche, M., Tomásek, L., Whitley, E., Wichmann, H. and Doll, R. (2005) 'Radon in homes and risk of lung cancer: collaborative analysis of individual data from 13 European case-control studies', British Medical Journal 330(7485): 223 


\section{BIBLIOGRAPHY}

Darlington, F., Norman, P. and Gould, M. (2015) 'Health and internal migration', in D. Smith, N. Finney, K. Halfacree and N. Walford (eds) Internal Migration, Farnham: Ashgate, pp. 113-128

Davidson, M. (2007) 'Gentrification as global habitat: a process of class formation or corporate creation?', Transactions of the Institute of British Geographers 32(4): 490-506

Davidson, M. and Lees, L. (2010) 'New-build gentrification: its histories, trajectories, and critical geographies', Population, Space and Place 16(5): 395-411

Davies, C. and Malik, S. (2014) 'Welcome to Luton: Romanian arrival greeted by two MPs and a media scrum', Guardian 1 January Davies, N. (1998) Dark Heart: The Shocking Truth about Hidden Britain, London: Vintage

Davis, K. and Blake, J. (1956) 'Social structure and fertility: an analytic framework', Economic Development and Cultural Change 4(3): 211-235

Davis, M. (1998) Ecology of Fear: Los Angeles and the Imagination of Disaster, London: Picador

Davis, M. (2004) 'Planet of slums', New Left Review 26, March-April: 5-34

Davis, M. (2006) Planet of Slums, London: Verso

Davison, K., Werder, J. and Lawson, C. (2008) 'Children's active commuting to school: current knowledge and future directions', Preventing Chronic Disease 5(3): 1-11

Dawson, J. (2006) Ecovillages, Schumacher Briefing 12, Totnes: Green Books

de Beauvoir, S. (1949) Le Deuxième Sexe, Paris: Gallimard

Dean, A. (2011) 'Will growing human population overwhelm our planet?', Asheville Citizen-Times, March 4

Dear, M. and Wolch, J. (1987) Landscapes of Despair: From Deinstitutionalization to Homelessness, Oxford: Polity Press

Dearling, A. (ed.) (1998) No Boundaries: New Travellers on the Road Outside of England, Lyme Regis: Enabler Publications

Defoort, C. (2008) 'Long-term trends in international migration: an analysis of the six main receiving countries', Population 63(2): 285-317

Del Casino, V. (2009) Social Geography, Oxford: Blackwell

Demeny, P. (2003) 'Population policy dilemmas in Europe at the dawn of the Twenty-First Century', Population and Development Review 29(1): 1-28

Den Besten, O. (2011) 'Negotiating children's outdoor spatial freedom: portraits of three Parisian families', in L. Holt (ed.) Geographies of Children, Youth and Families, Abingdon: Routledge, pp. 136-149

Deng, W., Long, L., Li, J., Zheng, D., Yu, J., Zhang, C., Li, K., Liu, H. and Huang, T. (2014) 'Mortality of major cancers in Guangxi, China: sex, age and geographical differences from 1971 and 2005', Asian Pacific Journal of Cancer Prevention 15(4): 1567-1574

Dennett, A. and Stillwell, J. (2008) 'Internal migration in Great Britain - a district level analysis using 2001 Census data', School of Geography, University of Leeds, Working Paper 01/08

Dewachi, O., Skelton, M., Nguyen, V., Fouad, F., Sitta, G., Maasri, Z. and Giacaman, R. (2014) 'Changing therapeutic geographies of the Iraqi and Syrian wars', The Lancet 383(9915): 449-457

Dickenson, D. (2004) 'Corporate South Africa's response to HIV/AIDS: why so slow?', Journal of Southern African Studies 30(3): 627-649

Diener, A. (2009) One Homeland or Two?: the Nationalization and Transnationalization of Mongolia's Kazakhs, Washington D.C.: Woodrow Wilson Center Press

Dienstbier, R. and Crockett, L. (eds) (2002) Agency, Motivation, and the Life Course, Lincoln, NE: University of Nebraska Press

Dirksmeier, P. (2008) 'Strife in the rural idyll? The relationship between autochthons and in-migrants in scenic regions of Bavaria', Erdkunde 62: 159-171

Dixon, D., Murray, J. and Gelatt, J. (2006) 'America's emigrants: US retirement migration to Mexico and Panama', Washington, DC: Migration Policy Institute, at http://www.migrationpolicy.org/research/americas-emigrants-us-retirement-migration-mexicoand-panama (accessed 03/17)

Dixon, L. and Ray, L. (2007) 'Current issues and developments in race hate crime', Probation Journal 54(2): 109-124

Doan, P. and Higgins, H. (2010) 'The demise of queer space? Resurgent gentrification and the assimilation of LGBT neighborhoods', Journal of Planning Education and Research, 6 January

Dobson, J. and Stillwell, J. (2000) 'Changing home, changing school: towards a research agenda on child migration', Area 32(4): 395-401

Docquier, F. and Rapoport, H. (2011) 'Globalization, brain drain, and development', Forschungsinstitut zur Zukunft der Arbeit, IZA Discussion Paper No. 5590

Docquier, F., Lowell, B. and Marfouk, A. (2009) 'A gendered assessment of highly skilled emigration', Population and Development Review 35(2): 297-321 
Doherty, B. and Hayes, G. (2012) 'Tactics, traditions and opportunities: British and French crop-trashing actions in comparative perspective', European Journal of Political Research 51(4): 540-562

Domosh, M. and Seager, J. (2001) Putting Women in Place, New York: Guilford Press

Donato, K., Gabaccia, D., Holdaway, J., Manalansan, M. and Pessar, P. (2006) 'A glass half full? Gender in migration studies', International Migration Review 40(1): 3-26

Dorling, D. (2011) So You Think You Know About Britain? The Surprising Truth about Modern Britain, London: Constable and Robinson

Dorling, D. (2012) The Visualization of Social Spatial Structure, Chichester: Wiley

Dorling, D. (2013) Population 10 Billion, London: Constable

Dorling, D. (2014) Inequality and the 1\%, London: Verso

Dorling, D., Mitchell, R., Shaw, M., Orford, S. and Smith, G. (2000) 'The ghost of Christmas past: health effects of poverty in London in 1896 and 1991', British Medical Journal 321(7276): 1547-1551

Dorman, S. and Chaisson, R. (2007) 'From magic bullets back to the Magic Mountain: the rise of extensively drug-resistant tuberculosis', Nature Medicine 13(3): 295-298

Dorn, M. and Laws, G. (1994) 'Social theory, body politics, and medical geography: extending Kearns's invitation', Professional Geographer 46(1): 106-110

Doward, J. (2014) ‘Qatar World Cup: 400 Nepalese die on nation's building sites since bid won', Guardian 16 February

Dozier, R. (2005) 'Beards, breasts, and bodies doing sex in a gendered world', Gender and Society 19(3): 297-316

Drain, P., Holmes, K., Hughes, J. and Koutsky, L. (2002) 'Determinants of cervical cancer rates in developing countries', International Journal of Cancer 100(2): 199-205

Drinkwater, S., Eade, S. And Garapich, M. (2006) 'Poles apart? EU enlargement and the labour market outcomes of immigrants in the UK', Forschungsinstitut zur Zukunft der Arbeit, IZA Discussion Paper No. 2410

Droogleever Fortuijn, J., van der Meer, M., Burholt, V., Ferring, D., Quattrini, S., Hallberg, I., Weber, G., and Wenger, G. (2006) 'The activity patterns of older adults: a cross-sectional study in six European countries', Population, Space and Place 12(5): 353-369

Dublin Airport (2015a) Flight timetables, at: www.dublinairport.com/gns/flight-information/flight-timetables.aspx (accessed 08/15)

Dublin Airport (2015b) 'Latest news. Almost 2.7 million passengers in July at Dublin Airport', at: www.dublinairport.com/gns/at-theairport/latest-news/15-08-14/Almost_2_7_Million_Passengers_In_July_At_Dublin_Airport.aspx (accessed 08/15)

Duckworth, V. (2002) 'Forget 2.4 children - now its down to 1.64', Independent 17 May

Duffy, P. (1995) 'Literary reflections on Irish migration in the Nineteenth and Twentieth Centuries', in R. King, J. Connell and P. White (eds) Writing Across Worlds.Literature and Migration, London: Routledge, pp. 20-38

Dufoix, S. (2013) 'Diasporas: historical and conceptual analysis', in I. Naess (ed.) The Encyclopedia of Global Human Migration, New Jersey: Wiley-Blackwell, at: http://onlinelibrary.wiley.com/doi/10.1002/9781444351071.wbeghm174/abstract (accessed $08 / 16)$

Dugmore, K., Furness, P., Leventhal, B. and Moy, C. (2011) 'Beyond the 2011 Census in the United Kingdom: with an international perspective', International Journal of Market Research 53(5): 619-650

Dun, O. and Gemenne, F. (2008) 'Defining "environmental migration"', Forced Migration Review 31:10

Duncan, S. and Phillips, M. (2011) 'People who live apart together (LATs): new family form or just a stage?', International Review of Sociology 21(3): 513-532.

Dunn, K. (2010) 'Embodied transnationalism: bodies in transnational spaces', Population, Space and Place 16: 1-9

Durkheim, E. (1897/1951) Suicide: a Study in Sociology, New York: Free Press

Duvander, A., Lappegård, T. and Andersson, G. (2010) 'Family policy and fertility: fathers' and mothers' use of parental leave and continued childbearing in Norway and Sweden', Journal of European Social Policy 20(1): 45-57

Dwyer, C. (2012) 'Performing migration: religious transnationalism and changing suburban landscapes', Paper presented at the Britain on the Move conference, London, 17-18 December

Dwyer, C., Gilbert, D. and Shah, B. (2013) 'Faith and suburbia: secularisation, modernity and the changing geographies of religion', Transactions of the Institute of British Geographers 38: 403-419

Dyck, I., Kontos, P., Angus, J. and McKeever, P. (2005) 'The home as a site for long-term care: meanings and management of bodies and spaces', Health and Place 11(2): 173-185

Dylan, B. (2004) Chronicles: Volume One, London: Simon \& Schuster

Dyson, T., Cassen, R. and Visaria, L. (2005) Twenty-First Century India: Population, Economy, Human Development and the Environment, Oxford: Oxford University Press

Eckstein, S. (1990) 'Urbanization revisited: inner-city slum of hope and squatter settlement of despair', World Development 18(2): $165-181$ 


\section{BIBLIOGRAPHY}

Edensor, T. (2008) 'Mundane hauntings: commuting through the phantasmagoric working-class spaces of Manchester, England', Cultural Geographies 15: 313-333

Edensor, T. (2011) 'Commuter: mobility, rhythm and commuting', in T. Cresswell and P. Merriman (eds) Geographies of Mobilities, Farnham: Ashgate, pp. 189-204

Edgington, R. (2008) '"Be receptive to the good earth": health, nature, and labor in countercultural back-to-the-land settlements', Agricultural History 82: 279-308

Ehrlich, P. (1968) The Population Bomb, New York: Sierra Club/Ballantine Books

Eimermann, M. (2015) 'Lifestyle migration beyond consumption - production binaries: Dutch migrants and multifunctional rural land use in Sweden', Two Homelands 42: 81-94

Ekerdt, D. (2010) 'Frontiers of research on work and retirement', Journals of Gerontology Series B: Psychological Sciences and Social Sciences 65(1): 69-80

El Nasser, H. (2008) 'Modern Suburbia not just in America anymore', USA Today, at: www.usatoday.com (accessed 07/10)

El-Bushra, J. (2000) 'Gender and forced migration: editorial', Forced Migration Review 9: 4-7

Elder, G. (1974) Children of the Great Depression, Chicago: University of Chicago Press

Elder, G. (1985) 'Perspectives on the life course', in G. Elder (ed.) Life Course Dynamics: Trajectories and Transitions, 1968-1980, Ithaca: Cornell University Press, pp. 23-49

Elder, G. (1994) 'Time, human agency, and social change: perspectives on the life course', Social Psychology Quarterly 57: 4-15

Elder, G., Kirkpatrick Johnson, M. and Crosnoe, R. (2003) 'The emergence and development of life course theory', in J. Mortimer and M. Shanahan (eds) Handbook of the Life Course, New York: Springer, pp. 3-19

El-Hinnawi, E. (1985) Environmental Refugees, Nairobi, Kenya: United Nations Environmental Programme

Elkington, J. (2004) 'Enter the triple bottom line', in A. Henriques and J. Richardson (eds) The Triple Bottom Line: Does it All Add Up, Abingdon: Earthscan, pp. 1-16

Ellermann, A. (2009) States against Migrants: Deportation in Germany and the United States, Cambridge: Cambridge University Press Ellingwood, K. (2009) Hard Line: Life and Death on the US-Mexico Border, New York: Vintage

Elrick, T. and Ciobanu, O. (2009) 'Migration networks and policy impacts: insights from Romanian-Spanish migrations', Global Networks 9(1): 100-116

Emple, H. (2010) 'The places of birth: navigating risk, control, and choice', Macalester College Honors Projects, Paper 24, at: http://digitalcommons.macalester.edu/geography_honors/24 (accessed 08/16)

Engelman, P. (2011) A History of the Birth Control Movement in America, Santa Barbara: Praeger

Engels, F. (1845/1987) The Condition of the Working Class in England, Harmondsworth: Penguin

England, K. (1991) 'Gender relations and the spatial structure of the city', Geoforum 22: 135-147

England, K. (1993) 'Changing suburbs, changing women: geographic perspectives on suburban women and suburbanization', Frontiers: A Journal of Women Studies 14: 24-43

Espenshade, T., Guzman, J. and Westoff, C. (2003) 'The surprising global variation in replacement fertility', Population Research and Policy Review 22(5-6): 575-583

Evans, B. (2008) 'Geographies of youth/young people', Geography Compass 2: 1659-1680

Evans, E. (2014) 'How green is my valley? The art of getting people in Wales to care about climate change', Journal of Critical Realism 13(3): 304-325

Evans, G., Gonnella, C., Marcynyszyn, L., Gentile, L. and Salpekar, N. (2005) 'The role of chaos in poverty and children's socioemotional adjustment', Psychological Science 16(7): 560-565

Evans, S. (2009) "That lot up there and us down here": social interaction and a sense of community in a mixed tenure UK retirement village', Ageing and Society 29(02): 199-216

Evans, Y., Herbert, J., Datta, K., May, J., Mcllwaine, C. and Wills, J. (2005) 'Making the city work: low paid employment in London', London: Department of Geography, Queen Mary, University of London

Faggian, A. and McCann, P. (2009) 'Universities, agglomerations and graduate human capital mobility', Tijdschrift voor Economische en Sociale Geografie 100(2): 210-223

Fairlie, S. (1996) Low Impact Development, Charlbury, Oxfordshire: Jon Carpenter

Fairlie, S. (2001) The Dowry. A Left Wing Defence of Rural England, South Petherton, Somerset: Chapter 7 Publications

Faist, T. (2008) 'Migrants as transnational development agents: an inquiry into the newest round of the migration-development nexus', Population, Space and Place 14(1): 21-42

Falkenberg, L., and Monachello, M. (1990) 'Dual-career and dual-income families: do they have different needs?', Journal of Business Ethics 9(4-5): 339-351

FAO [Food and Agriculture Organization] (2013) Homepage: www.fao.org/home/en/ (accessed 09/16)

\section{ST PROOFS: NOT FOR DISTRIBUTION}


Farstad, M. and Rye, J. (2013) 'Second home owners, locals and their perspectives on rural development', Journal of Rural Studies 30: $41-51$

Faustini, A., Hall, A. and Perucci, C. (2006) 'Risk factors for multidrug resistant tuberculosis in Europe: a systematic review', Thorax 61(2): 158-163

Fausto-Sterling, A. (2000) Sexing the Body: Gender Politics and the Construction of Sexuality, New York: Basic Books

Fausto-Sterling, A. (2005) 'The bare bones of sex: Part I, sex and gender', Signs 30: 1491-1527

Favell, A. (1998) 'The Europeanisation of immigration politics', European Integration online Papers (EloP) 2(10), at: http://ssrn.com/abstract=302731 (accessed 08/16)

Favell, A. (2003) 'Eurostars and Eurocities: towards a sociology of free moving professionals in Western Europe', University of California - San Diego, Center for Comparative Immigration Studies, Working Paper No. 71

Favell, A. (2008) 'Migration theory rebooted. Asymmetric challenges in a global agenda', in C. Brettell and J. Hollifield (eds) Migration Theory. Talking Across Disciplines, 2nd edition, London: Routledge, pp. 318-328

Fealy, G., McNamara, M., Treacy, M. and Lyons, I. (2012) 'Constructing ageing and age identities: a case study of newspaper discourses', Ageing and Society 32: 85-102

Fechter, A. (2007) 'Living in a bubble', in V. Amit (ed.) Going First Class. New Approaches to Privileged Travel and Movement, New York: Berghahn Books, pp. 33-52

Feijten, P. and Van Ham, M. (2007) 'Residential mobility and migration of the divorced and separated', Demographic Research 17: $623-653$

Feldman, I. (2012) 'The challenge of categories: UNRWA and the definition of a "Palestine Refugee"', Journal of Refugee Studies 25(3): 387-406

Fenagh Visitors Centre (2015) 'American Wake', at: www.fenagh.com/history/american-wake/ (accessed 12/15)

Fenton, J. (2004) '"A world where action is the sister of dream": surrealism and anti-capitalism in contemporary Paris', Antipode 36(5): 942-962

Ferreira, F., Gyourko, J. and Tracy, J. (2010) 'Housing busts and household mobility', Journal of Urban Economics 68(1): 34-45

Fesenmyer, L. (2014) 'Transnational families', in B. Anderson and M. Keith (eds) Migration: a COMPAS Anthology, Oxford: COMPAS, at: http://compasanthology.co.uk/wp-content/uploads/2014/02/Fesenmyer_COMPASMigrationAnthology.pdf (accessed 08/16)

Fielding, A. (1982) 'Counterurbanization in Western Europe', Progress in Planning 17: 1-52

Fielding, A. (1992a) 'Migration and culture', in A. Champion and A. Fielding (eds) Migration Processes and Patterns. Volume 1. Research Progress and Prospects, London: Belhaven Press, pp. 201-212

Fielding, A. (1992b) 'Migration and social mobility: South East England as an escalator region', Regional Studies 26: 1-15

Fielding, T. (1998) 'Counterurbanisation and social class', in: Boyle, P. and Halfacree, K. (eds), Migration into Rural Areas. Theories and Issues, Chichester: Wiley, pp. 41-60

Fielding, T. (2012) Migration in Britain, Cheltenham: Edward Elgar

Finch, J. (1983) Married to the Job: Wives' Incorporation in Men's Work, London: Allen and Unwin

Fincham, B., McGuinness, M. and Murray, L. (eds) (2009) Mobile Methodologies, Basingstoke: Palgrave Macmillan

Findlay, A. (1996) 'Skilled transients: the invisible phenomenon', in R. Cohen (ed.) Cambridge Survey of World Migration, Cambridge: Cambridge University Press, pp. 515-522

Findlay, A. and Graham, E. (1991) 'The challenge facing population geography', Progress in Human Geography 15: 149-162

Findlay, A., Short, D. and Stockdale, A. (2000) 'The labour-market impact of migration to rural areas', Applied Geography 20(4): 333-348

Findlay, A., Stockdale, A., Findlay, A. and Short, D. (2001) 'Mobility as a driver of change in rural Britain: an analysis of the links between migration, commuting and travel to shop patterns', International Journal of Population Geography 7(1): 1-15

Findlay, A., King, R., Smith, F., Geddes, A. and Skeldon, R. (2012) 'World class? An investigation of globalisation, difference and international student mobility', Transactions of the Institute of British Geographers 37(1): 118-131

Finke, P. (1999) 'The Kazaks of western Mongolia', in I. Svanberg (ed.) Contemporary Kazaks, New York: St. Martin's Press, pp. 103-139

Finney, N. and Robinson, V. (2008) 'Local press, dispersal and community in the construction of asylum debates', Social and Cultural Geography 9(4): 397-413

Finney, N. and Simpson, L. (2009) 'Sleepwalking to Segregation'?: Challenging Myths about Race and Migration, Bristol: Policy Press

Finney, N., Catney, G. and Phillips, D. (2015) 'Ethnicity and internal migration', in D. Smith, N. Finney, K. Halfacree and N. Walford (eds) Internal Migration, Farnham: Ashgate, pp. 31-45

Fischer, C. (1982) To Dwell Among Friends: Personal Networks in Town and City, Chicago: University of Chicago Press 


\section{BIBLIOGRAPHY}

Fischer, P. and Malmberg, G. (2001) 'Settled people don't move: on life course and (im-)mobility in Sweden', International Journal of Population Geography 7(5): 357-371

Fitzgibbon, K. (2003) 'Modern-day slavery? The scope of trafficking in persons in Africa', African Security Studies 12(1): 81-89

Flasher, J. (1978) 'Adultism', Adolescence, 13(51): 517

Fleshman, M. (2001) 'AIDS orphans: facing Africa's "silent crisis"', Africa Recovery 15(3): 1, 18-20

Flowerdew, R. (1991) 'Poisson regression models of migration', in J. Stillwell and P. Congdon (eds) Migration Models: Macro and Micro Approaches, London: Belhaven Press, pp. 92-112

Flowerdew, R. (1992) 'Labour market operation and geographical mobility', in A. Champion and A. Fielding (eds) Migration Processes and Patterns. Volume 1. Research Progress and Prospects, London: Belhaven Press, pp. 135-147

Flowerdew, R. and Aitkin, M. (1982) 'A method of fitting the gravity model based on the Poisson distribution', Journal of Regional Science 22: 191-202

Fontanella-Khan, J. (2014) 'Romanians in no rush to move to "racist" UK', Financial Times 12 January

Foresight (2011) Migration and Global Environmental Change: Future Challenges and Opportunities, London: Government Office for Science

Forrest, R. and Murie, A. (1991) 'Housing markets, labour markets and housing histories', in J. Allen and C. Hamnett (eds) Housing and Labour Markets. Building the Connections, London: Unwin Hyman, pp. 63-93

Fotheringham, A. and O'Kelly, M. (1989) Spatial Interaction Models: Formulations and Applications, Dordrecht: Kluwer

Fowler, H.W. and Fowler, F.G. (1964) Concise Oxford Dictionary of Current English, 5th edition, Oxford: Clarendon Press

Fox, J. (2002) 'Understanding a dynamic landscape: land use, land cover and resource tenure in Northeastern Cambodia', in S. Walsh and K. Crews-Meyer (eds) Linking People, Place and Policy: a GIScience Approach, Boston: Kluwer Academic Publishers, pp. $113-130$

Francisco, M. (2010) 'Re-inventing the rural through ecoimmigration - the Northeast Algarve', Paper presented at European Society for Rural Sociology Summer School, Córdoba, Spain, October

Fraser, M. (2010) 'Facts, ethics and event', in C. Jensen and K. Rödje (eds) Deleuzian Intersections. Science, Technology, Anthropology, Oxford: Berghahn, pp.57-82

Freeman, A. (2005) 'Moral geographies and women's freedom: rethinking freedom discourse in the Moroccan context', in G.-W. Falah and C. Nagel (eds) Geographies of Muslim Women: Gender, Religion, and Space, New York: Guilford, pp. 147-177

Freire-Medeiros, B. (2009) 'The favela and its touristic transits', Geoforum 40(4): 580-588

Freitas-Junior, R., Gonzaga, C., Freitas, N., Martins, E. and Dardes, R. (2012) 'Disparities in female breast cancer mortality rates in Brazil between 1980 and 2009', Clinics 67(7): 731-737

Frey, W. (1987) 'Migration and depopulation of the metropolis: regional restructuring or rural renaissance?', American Sociological Review 52(2): 240-257

Frey, W. (1990) 'America: beyond the transition', Population Bulletin 45(2): 1-51

Frey, W. (2002) 'Metro magnets for minorities and whites: melting pots, the new sunbelt, and the heartland', Population Studies Center Research Report No. 02-496, Institute for Social Research, University of Michigan

Frey, W. and Johnson, K. (1998) 'Concentrated immigration, restructuring and the "selective" deconcentration of the United States population', in P. Boyle and K. Halfacree (eds) Migration into Rural Areas. Theories and Issues. Chichester: Wiley, pp. 79-106

Frisvoll, S (2012) Power in the production of spaces transformed by rural tourism, Journal of Rural Studies 28(4): 447-457

Frontex (2014) Homepage: http://frontex.europa.eu/ (accessed 12/14)

Fry, C. (2000) 'Culture, age and subjective well-being: health, functionality and the infrastructure of eldercare in comparative perspective', Journal of Family Issues 20(6): 751-776

Fuguitt, G. (1985) 'The nonmetropolitan population turnaround', Annual Review of Sociology 11: 259-280

Fullilove, M. (2004) Root Shock, New York: Ballantine

Fullilove, M. (2014) '"The frayed knot." What happens to place attachment in the context of serial forced displacement?', in L. Manzo and P. Devine-Wright (eds) Place Attachment: Advances in Theory, Methods and Applications, London: Routledge, pp. $141-153$

Galanter, M. (1984) Competing Equalities: Law and the Backward Classes in India. Delhi: Oxford University Press

Gallagher, D. (1989) 'The evolution of the international refugee system', International Migration Review: Special Silver Anniversary Issue: International Migration and Assessment for the 90s 23(3): 579-598

Gallent, N. (2007) 'Second homes, community and a hierarchy of dwelling', Area 39: 97-106

Gallent, N., Mace, A. and Tewdwr-Jones, M. (2005) Second Homes. European Perspectives and UK Policies, Aldershot: Ashgate

Galvao, J. (2005) 'Brazil and access to HIV/AIDS drugs: a question of human rights and public health', American Journal of Public Health 95(7): 1110-1116

\section{ST PROOFS: NOT FOR DISTRIBUTION}


Gapminder (2014) Homepage: www.gapminder.org/ (accessed 08/16)

Gardner, A. (2010) 'Engulfed: Indian guest workers, Bahraini citizens and the structural violence of the Kafala system', in N. De Genova and N. Peutz (eds) The Deportation Regime, London: Duke University Press, pp. 196-223

Gardner, A., Pessoa, S., Diop, A., Al-Ghanim, K., Le Trung, K. and Harkness, L. (2013) 'A portrait of low-income migrants in contemporary Qatar', Journal of Arabian Studies 3(1): 1-17

Gardner, K. and Shukur, A. (1994) ' "I'm Bengali, I'm Asian and I'm living here!". The changing identity of British Bengalis', in R. Ballard (ed.) Desh-Pardesh: the South Asian Presence in Britain, London: Hurst and Company, pp. 142-164

Garside, J. (1993) 'Inner city gentrification in South Africa: the case of Woodstock, Cape Town', GeoJournal 30: 29-35

Gately, I. (2014) Rush Hour: How 500 Million Commuters Survive the Daily Journey to Work, London: Head of Zeus

Gatrell, A. and Elliott, S. (2015) Geographies of Health, 3rd edition, Chichester: Wiley

Gecas, V. (2003) 'Self-agency and the life course', in J. Mortimer and M. Shanahan (eds) Handbook of the Life Course, New York: Springer, pp. 369-388

Geest, S. (2002) 'From wisdom to witchcraft: ambivalence towards old age in rural Ghana', Africa 72: 437-463

Geist, C. and McManus, P. (2008) 'Geographical mobility over the life course: motivations and implications', Population, Space and Place 14(4): 283-303

Gesler, W. (1992) 'Therapeutic landscapes: medical issues in light of the new cultural geography', Social Science and Medicine 34: $735-746$

Gesler, W. (1996) 'Lourdes: healing in a place of pilgrimage', Health and Place 2(2): 95-105

Gesler, W. and Kearns, R. (2002) Culture/Place/Health, London: Routledge

Ghosh, P. (2016) 'Royal Society calls for review of European GM ban', BBC News, at: www.bbc.co.uk/news/science-environment36359682 (accessed 08/16)

Gibney, M. (2008) 'Asylum and the expansion of deportation in the United Kingdom', Government and Opposition 43(2): 146-167

Gibney, M. and Hansen, R. (2003) 'Deportation and the liberal state: the forcible return of asylum seekers and unlawful migrants in Canada, Germany and the United Kingdom', UNHCR New Issues in Refugee Research, Working Paper No. 77

Gibson, C. and Argent, N. (2008) 'Getting on, getting up and getting out? Broadening perspectives on rural youth migration', Geographical Research 46(2): 135-138

Gibson-Graham, J.K. (1996) The End of Capitalism (As We Knew It), Oxford: Blackwell

Gibson-Graham, J.K. (2006) A Postcapitalist Politics, London: University of Minnesota Press

Giddens, A. (1984) The Constitution of Society, Cambridge: Cambridge University Press

Giddens, A. (1991) Modernity and Self-Identity, Cambridge: Polity Press

Gielle, J. and Elder, G. (1998) Methods of Life-Course Research: Qualitative and Quantitative Approaches, Thousand Islands, CA: Sage

Gilbert, E. (1939) 'The growth of inland and seaside health resorts in England', Scottish Geographical Magazine 55: 21-27

Gill, N. (2010) 'Pathologies of migrant place-making: Polish migration to the UK', Environment and Planning A 42: 1157-1173

Gill, N. and Bialski, P. (2011) 'New friends in new places: network formation during the migration process among Poles in the UK', Geoforum 42: 241-249

Gilleard, C. and Higgs, P. (2010) 'Aging without agency: theorizing the fourth age', Aging and Mental Health 14(2): 121-128

Gilleard, C. and Higgs, P. (2013) Ageing, Corporeality and Embodiment, London: Anthem Press

Gilleard, C. and Higgs, P. (2015) 'Social death and the moral identity of the fourth age', Contemporary Social Science 10(3): 262-271

Gilmartin, M. (2008) 'Migration, identity and belonging', Geography Compass 2(6): 1837-1852

Gilroy, P. (1993) The Black Atlantic. Modernity and Double Consciousness, London: Verso

Gilroy, P. (1994) 'Diaspora', Paragraph 17(3): 207-212

Gilroy, P. (2000) Against Race: Imagining Political Culture Beyond the Color Line, Cambridge, Mass.: Harvard University Press

Gkartzios, M. (2013) '"Leaving Athens": Narratives of counterurbanisation in times of crisis', Journal of Rural Studies 32: 158-167

Glass, A. (2012) 'Elder co-housing in the United States: three case studies', Built Environment 38(3): 345-363

Glennie, P. and Thrift, N. (2009) Shaping the Day: a History of Timekeeping in England and Wales 1300-1800, Oxford: Oxford University Press

Glick, P. (1947) 'The family life cycle', American Sociological Review 12: 164-174

Goffman, K. and Joy, D. (2005) Counterculture through the Ages, New York: Villard

Golant, S. (1972) 'The residential location and spatial behaviour of the elderly', University of Chicago, Department of Geography, Research Paper 143, Chicago

Goldenberg, S. (2011) 'Planet Earth is home to 8.7 million species, scientists estimate', Guardian 23 August

\section{ST PROOFS: NOT FOR DISTRIBUTION}




\section{BIBLIOGRAPHY}

Golledge, R. (ed.) (1999) Wayfinding Behaviour: Cognitive Mapping and Other Spatial Processes, Baltimore: Johns Hopkins University Press

Goodall, C. (2011) 'Sanctuary and solidarity: urban community responses to refugees and asylum seekers on three continents', New Issues in Refugee Research: Research Paper No. 221, UNHCR

Gorman-Murray, A. (2009) 'Intimate mobilities: emotional embodiment and queer migration', Social and Cultural Geography 10(4): 441-460

Gosnell, H. and Abrams, J. (2011) 'Amenity migration: diverse conceptualizations of drivers, socioeconomic dimensions, and emerging challenges', GeoJournal 76(4): 302-322

Goss, J. and Lindquist, B. (1995) 'Conceptualizing international labor migration: a structuration perspective', International Migration Review 29: 317-351

Gough, J. (2001) 'Work, class and social life', in R. Pain, M. Barke, D. Fuller, J. Gough, R. MacFarlane and G. Mowl Introducing Social Geographies, London: Arnold, pp. 13-43

Gough, K. (2008) '"Moving around": the social and spatial mobility of youth in Lusaka', Geografiska Annaler Series B 90(3): 243-255

Gould, W. (2005) 'Vulnerability and HIV/AIDS in Africa: from demography to development', Population, Space and Place 11(6): 473-484

Gould, W. (2015) Population and Development, 2nd edition, London: Routledge

Gould, W. and Woods, R. (2003) 'Population geography and HIV/AIDS: the challenge of a "wholly exceptional disease"', Scottish Geographical Magazine 119(3): 265-281

Grabska, K. (2006) 'Marginalization in urban spaces of the Global South: urban refugees in Cairo', Journal of Refugee Studies 19(3): 287-307

Gráda, C. and O'Rourke, K. (1997) 'Migration as disaster relief: lessons from the Great Irish Famine', European Review of Economic History 1(1): 3-25

Graham, E. (1995) 'Population geography and post-modernism', Paper presented at the International Conference on Population Geography, September

Graham, E. (2000) 'What kind of theory for what kind of population geography?', International Journal of Population Geography 6: 257-272

Graham, E. (2004) 'The past, present and future of population geography: reflections on Glenn Trewartha's address fifty years on', Population, Space and Place 10(4): 289-294

Graham, E. and Boyle, P. (2001) 'Editorial introduction: (re)theorising population geography: mapping the unfamiliar', International Journal of Population Geography 7: 389-394

Granovetter, M. (1973) 'The strength of weak ties', American Journal of Sociology 78(6): 1360-1380

Green, A. (1997) 'A question of compromise? Case study evidence on the location and mobility strategies of dual-career households', Regional Studies 31: 641-657

Green, A. (2009) 'Older people and transitions from employment to nonemployment: international perspectives and policy issues', Professional Geographer 61(1): 46-58

Green, A. and Shuttleworth, I. (2015) 'Labour markets and internal migration', in D. Smith, N. Finney, K. Halfacree and N. Walford (eds) Internal Migration, Farnham: Ashgate, pp. 65-79

Green, A., Hogarth, T. and Shackleton, R. (1999) Long Distance Living. Dual Location Households, Bristol: Policy Press

Greene, M. and Kirby, D. (2012) 'The impact of tuition fees on access and student migration: lessons from Canada's Atlantic coast', Widening Participation and Lifelong Learning 14(1): 72-90

Greenfield, S. (2008) ID: The Quest for Identity in the 21st Century. London: Sceptre

Greenhalgh, S. (1996) 'The social construction of population science: an intellectual, institutional, and political history of twentiethcentury demography', Comparative Studies in Society and History 38(01): 26-66

Greenhalgh, S. (2008) Just One Child: Science and Policy in Deng's China, Berkeley: University of California Press

Greenhaus, J., Callanan, G. and Godshalk, V. (2010) Career Management, Thousand Oaks, CA: Sage

Greenpeace (2013) Bees in Decline, Amsterdam: Greenpeace International

Greenwood, M. (1975) 'Research on internal migration in the United States: a survey', Journal of Economic Literature 13: 397-433

Greenwood, M. (1985) 'Human migration: theory, models, and empirical studies', Journal of Regional Science 25: 521-544

Greer, G. (1970) The Female Eunuch, London: Harper Collins

Greer, G. (1984) Sex and Destiny: the Politics of Human Fertility, London: Harper Collins

Gregory, D. (2008) 'The Biopolitics of Baghdad: counterinsurgency and the counter-city', Human Geography 1: 6-27

Greil, A., Slauson-Blevins, K. and McQuillan, J. (2009) 'The experience of infertility: a review of recent literature', Sociology of Health and Illness 32(1): 140-162

\section{ST PROOFS: NOT FOR DISTRIBUTION}


Griffiths, D. and Maile, S. (2014) 'Britons in Berlin: imagined cityscapes, affective encounters and the cultivation of the self', in M. Benson and N. Osbaldiston (eds) Understanding Lifestyle Migration, Basingstoke: Palgrave Macmillan, pp. 139-159

Griffiths, J. (2000) Pip, Pip. A Sideways Look at Time. London: Flamingo

Griffiths, M. (2012) 'Anonymous aliens? Questions of identification in the detention and deportation of failed asylum seekers', Population, Space and Place 18(6): 715-727

Grigg, D. (1977) 'E.G. Ravenstein and the 'laws of migration”, Journal of Historical Geography 3: 41-54

Grimsrud, G. (2011) 'How well does the "counter-urbanisation story" travel to other countries? The case of Norway', Population, Space and Place 17(5): 642-655

Groen, J. and Polivka, A. (2010) 'Going home after Hurricane Katrina: determinants of return migration and changes in affected areas', Demography 47(4): 821-844

Grundy, E. (2006) 'Ageing and vulnerable elderly people: European perspectives', Ageing and Society 26: 105-134

Guardian (2013a) 'Nigeria's child brides suffer as tradition and sharia law combine to outweigh official ban', 3 September

Guardian (2013b) 'Death toll of African migrants rises after boat disaster near Lampedusa', 12 October

Guardian (2014a) 'London's soaring house prices make a long commute a little more attractive', 5 July

Guardian (2014b) 'Medical transplants. Where staff come from', 27 January

Guardian (2014c) Most refugees since second world war', 20 June

Guardian (2014d) 'The supermarket slave trail', 11 June

Guardian (2014e) 'I told my daughter her husband would kiss her and she should not shudder', 18 July

Guardian (2014f) 'World facing threat from old killers as antibiotics lose power, says WHO', Guardian 1 May

Guardian (2014g) 'Rise in Greek suicides linked to austerity', 22 April

Guardian (2014h) 'Anthropocene: is this the new epoch of humans?', 16 October

Gulati, L. (1993) In the Absence of their Men, New Delhi: Sage

Guldmann, J.M. (2004) 'Spatial interaction models of international telecommunication flows', in M. Goodchild and D. Janelle (eds) Spatially Integrated Social Science, Oxford: Oxford University Press, pp. 400-419

Gurran, N. and Blakely, E. (2007) 'Suffer a sea change? Contrasting perspectives towards urban policy and migration in coastal Australia', Australian Geographer 38(1): 113-131

Gururaja, S. (2000) 'Gender dimensions of displacement', Forced Migration Review 9: 13-16

Gustafson, P. (2001a) 'Roots and routes exploring the relationship between place attachment and mobility', Environment and Behavior 33(5): 667-686

Gustafson, P. (2001b) 'Retirement migration and transnational lifestyles', Ageing and Society 21(4): 371-394

Gustafson, P. (2008) 'Transnationalism in retirement migration: the case of North European retirees in Spain', Ethnic and Racial Studies 31(3): 451-475

Gustafson, P. (2009) 'Your home in Spain: residential strategies in international retirement migration', in M. Benson and K. O'Reilly (eds) Lifestyle Migration: Expectations, Aspirations and Experiences, Aldershot: Ashgate, pp. 69-86

Gustafson, P. (2014) 'Place attachment in an age of mobility, in L. Manzo and P. Devine-Wright (eds) Place Attachment: Advances in Theory, Methods and Applications, London: Routledge, pp. 37-48

Gutting, D. (1996) 'Narrative identity and residential history', Area 28: 482-490

Hagan, J. (1998) 'Social networks, gender, and immigrant incorporation: resources and constraints', American Sociological Review 63(1): 55-67

Hagan, J., Eschbach, K. and Rodriguez, N. (2008) 'US deportation policy, family separation, and circular migration', International Migration Review 42(1): 64-88

Hagga, J. (2000) 'High death rate among Russian men predates Soviet Union's demise', Population Reference Bureau, at: www.prb.org/Publications/Articles/2000/HighDeathRateAmongRussianMenPredatesSovietUnionsDemise.aspx (accessed $08 / 16)$

Hahn, H. (1986) 'Disability and the urban environment: a perspective on Los Angeles', Environment and Planning D: Society and Space 4: 273-288

Hakim, C. (2000) Work-Lifestyle Choices in the 21st Century: Preference Theory, Oxford: Oxford University Press

Hakim, C. (2003) 'A new approach to explaining fertility patterns: Preference Theory', Population and Development Review 29(3): 349-374

Hakim, C. (2004) Key Issues in Women's Work: Female Diversity and the Polarisation of Women's Employment, London: GlassHouse Press

Hakim, C. (2006) 'Childlessness in Europe', Research Report to the Economic and Social Research Council (ESRC). Research Grant RES-000-23-0074, at: http://178.79.155.39/wordpress/wp-content/uploads/2011/07/2006_Childlessness_inEurope1.pdf (accessed 04/12) 


\section{BIBLIOGRAPHY}

Halfacree, K. (1993) 'Locality and social representation: space, discourse and alternative definitions of the rural', Journal of Rural Studies 9: 23-37

Halfacree, K. (1994) 'The importance of "the rural" in the constitution of counterurbanization: evidence from England in the 1980s', Sociologia Ruralis 34: 164-189

Halfacree, K. (1995a) 'Household migration and the structuration of patriarchy: evidence from the U.S.A.', Progress in Human Geography 19: 159-182

Halfacree, K. (1995b) 'Talking about rurality: social representations of the rural as expressed by residents of six English parishes', Journal of Rural Studies 11: 1-20

Halfacree, K. (1996) 'Out of place in the country: travellers and the "rural idyll"', Antipode 28: 42-71

Halfacree, K. (2001) 'Constructing the object: taxonomic practices, "counterurbanisation" and positioning marginal rural settlement', International Journal of Population Geography 7: 395-411

Halfacree, K. (2004a) 'A utopian imagination in migration's terra incognita? Acknowledging the non-economic worlds of migration decision-making', Population, Space and Place 10: 239-253

Halfacree, K. (2004b) 'Untying migration completely: de-gendering or radical transformation?', Journal of Ethnic and Migration Studies 30: $397-413$

Halfacree, K. (2006) 'Rural space: constructing a three-fold architecture' in P. Cloke, T. Marsden and P. Mooney (eds) Handbook of Rural Studies, London: Sage, pp. 44-62

Halfacree, K. (2007a) 'Back-to-the-land in the Twenty-First Century - making connections with rurality', Tijdschrift voor Economische en Sociale Geografie 98: 3-8

Halfacree, K. (2007b) 'Trial by space for a "radical rural”: introducing alternative localities, representations and lives', Journal of Rural Studies 23: 125-141

Halfacree, K. (2008) 'To revitalise counterurbanisation research? Recognising an international and fuller picture', Population, Space and Place 14: 479-495

Halfacree, K. (2009) ' "Glow worms show the path we have to tread": the counterurbanisation of Vashti Bunyan', Social and Cultural Geography 10: 771-789

Halfacree, K. (2011a) '"A solid partner in a fluid world" and/or "line of flight"?: interpreting second homes in the era of mobilities', Norsk Geografisk Tidsskrift -Norwegian Journal of Geography 65: 144-153

Halfacree, K. (2011b) ' "Alternative" communities in rural Wales', in P. Milbourne (ed.) Rural Wales in the Twenty First Century: Society, Economy and Environment, Cardiff: University of Wales Press, pp. 65-88

Halfacree, K. (2012a) 'Heterolocal identities?: counter-urbanisation, second homes and rural consumption in the era of mobilities', Population, Space and Place 18: 209-224

Halfacree, K. (2012b) 'Interpreting rurality in the 21st century: from effacement to (re-)invention', in L. Kulcsár and K. Curtis (eds) International Handbook of Rural Demography, New York: Springer, pp. 387-400

Halfacree, K. (2013a) 'Running wild in the country?: mobilising rural in-migration', in L. Silva and E. Figueiredo, Elisabete (eds) Shaping Rural Areas in Europe, Springer, GeoJournal Libary 107: 11-23

Halfacree, K. (2013b) 'Utopian communities and migration', in I. Ness (eds) Encyclopedia of Global Human Migration, 5 Volumes, Chichester: Wiley-Blackwell, DOI: 10.1002/9781444351071.wbeghm570

Halfacree, K. (2014a) 'Jumping up from the armchair: beyond the idyll in counterurbanisation', in M. Benson and N. Osbaldiston (eds) Understanding Lifestyle Migration, Basingstoke, Palgrave Macmillan, pp. 92-115

Halfacree, K. (2014b) 'A critical response to the (non-) place of rural leisure users within the counterurban imagination', PASOS Journal of Tourism and Cultural Heritage 12(3): 515-523

Halfacree, K. (2016) 'Den Kopf auf dem Land freibekommen? Eine Relektüre künstlerischer Zurück zur Natur-Experimente der 70er Jahre' [Getting it together in the countryside?: revisiting artistic back-to-the-land experiments of the 1970s], in J. Springer and T. Dören (eds.) Draußen. Zum neuen Naturbezug in der Popkultur der Gegenwart, Bielefeld: Transcript, pp. 97-117

Halfacree, K. (forthcoming b) 'Countercultural back-to-the-land migration: evidence from the late 20th Century', manuscript available from author

Halfacree, K. and Boyle, P. (1993) 'The challenge facing migration research: the case for a biographical approach', Progress in Human Geography 17: 333-348

Halfacree, K. and Merriman, P. (2015) 'Performing internal migration', in D. Smith, N. Finney, K. Halfacree and N. Walford (eds) Internal Migration, Farnham: Ashgate, pp. 149-164

Halfacree, K. and Rivera, M.J. (2012) 'Moving to the countryside ... and staying: lives beyond representation', Sociologia Ruralis 52 : 92-114

Hall, C. and Müller, D. (eds) (2004) Tourism, Mobility and Second Homes, Clevedon: Channel View Publications 
Hall, D. (2002) Careers In and Out of Organizations, Thousand Oaks, CA: Sage

Hall, E. (2000) 'Blood, brain and bones": taking the body seriously in the geography of health and impairment', Area 32(1): 21-29

Hall, N. (2013) Hate Crime, Abingdon: Routledge

Hall, P. (1998) Cities in Civilization: Culture, Technology, and Urban Order, London: Weidenfeld and Nicolson

Hall, R. (1977) Passionate Crusader: the Life of Marie Stopes, New York: Harcourt Brace

Hall, R. (1995) 'Households, families and fertility', in R. Hall and P. White (eds) Europe's Population: Towards the Next Century, London: UCL Press, pp. 34-50

Hall, S (1990) 'Cultural identity and diaspora', in J Rutherford (ed.) Identity: Community, Culture, Difference, London: Lawrence and Wishart, pp. 222-237

Hall, S. (1999) 'Thinking the diaspora: home-thoughts from abroad', Small Axe 6:1-18

Halliday, J. and Coombes, M. (1995) 'In search of counterurbanisation: some evidence from Devon on the relationship between patterns of migration and motivation', Journal of Rural Studies 11: 433-446

Hamaaki, J., Hori, M., Maeda, S. and Murata, K. (2012) 'Changes in the Japanese employment system in the two lost decades', Industrial and Labor Relations Review 65(4): 810-846

Hamid, M. (2014) 'We are all migrants', Guardian, Review Section, 22 November, p. 20

Hammel, D. (2009) 'Gentrification', in R. Kitchin and N. Thrift (eds) International Encyclopedia of Human Geography, Volume 4, Oxford: Elsevier, pp. 360-367

Hamnett, C. (2010) 'On gentrification. "I am critical. You are mainstream": a response to Slater', City 14(1-2): 180-186

Hampton, J. (ed.) (1998) Internally Displaced People. A Global Survey, London: Earthscan

Handwerk, B. (2006) 'China's Three Gorges Dam, by the numbers', National Geographic News 9 June, at: www.Nationalgeographic. $\mathrm{com} /$ news (accessed 01/13)

Hank, K. and Buber, I. (2009) 'Grandparents caring for their grandchildren: findings from the 2004 survey of health, ageing, and retirement in Europe', Journal of Family Issues 30(1): 53-73

Hank, K. and Erlinghagen, M. (2010) 'Volunteering in "Old" Europe: patterns, potentials, limitations', Journal of Applied Gerontology 29(1): 3-20

Hannam, K., Sheller, M. and Urry, J. (2006) 'Editorial: mobilities, immobilities and moorings', Mobilities 1: 1-22

Hansen, K. (1995) 'Geographical mobility: March 1993 to March 1994', US Bureau of the Census, Current Population Reports, P20485, US Government Printing Office, Washington, D.C.

Hansen, N. and Philo, C. (2007) 'The normality of doing things differently: bodies, spaces and disability geography', Tijdschrift voor Economische en Sociale Geografie 98(4): 493-506

Happel, S. and Hogan, T. (2002) 'Counting snowbirds: the importance of and the problems with estimating seasonal populations', Population Research and Policy Review 21(3): 227-240

Hardill, I. (2009) 'Introduction: geographies of aging', Professional Geographer 61(1): 1-3

Hardill, I. and Baines, S. (2009) 'Active citizenship in later life: older volunteers in a deprived community in England', Professional Geographer 61(1): 36-45

Hardill, I., Spradbery, J., Arnold-Boakes, J. and Marrugat, M. (2005) 'Severe health and social care issues among British migrants who retire to Spain', Ageing and Society 25(05): 769-783

Hardin, G. (1968) 'The tragedy of the commons', Science 162: 1243-1248

Hardwick, S. (2008) 'Place, space, and pattern. Geographical theories in international migration', in C. Brettell and J. Hollifield (eds) Migration Theory. Talking Across Disciplines, 2nd edition, London: Routledge, pp. 161-182

Hardy, D. (2000) Utopian England. Community Experiments 1900-1945, London: E and FN Spon

Hareven, T. (2000) Families, History and Social Change, Oxford: Westview Press/Perseus Books

Harries, A., Hargreaves, N., Graham, S., Mwansambo, C., Kazembe, P., Broadhead, R., Maher, D. and Salaniponi, F. (2002) 'Childhood tuberculosis in Malawi: nationwide case-finding and treatment outcomes', International Journal of Tuberculosis and Lung Disease 6(5): 424-431

Harris, D. (2013) 'Death by injustice: Uganda's anti-homosexuality laws, Christian fundamentalism, and the politics of global power', The Catalyst 3(1): Article 4

Harris, J. and Todaro, M. (1970) 'Migration, unemployment and development: a two sector analysis', American Economic Review 60: 139-149

Harris, R. (2009) 'Slums', in R. Kitchin and N. Thrift (eds) International Encyclopedia of Human Geography, Oxford: Elsevier, pp. 157-162

Harris, S. and Throsby, D. (1998) 'The ESD Process: background, implementation and aftermath', in O. Hamilton and D. Throsby (eds), The ESD Process: Evaluating a Policy Experiment, Canberra: Australian Academy of Science, pp. 1-19 


\section{BIBLIOGRAPHY}

Harris, T. (2016) 'The real population time bomb isn't immigration - it's old age', Daily Telegraph 26 May

Hartmann, B. (1995) Reproductive Rights and Wrongs: the Global Politics of Population Control, 2nd edition., Boston, MA: South

End Press

Hartshorne, R. (1939) The Nature of Geography, Lancaster, PA: Association of American Geographers

Harvey, D. (1969) Explanation in Geography, London: Edward Arnold

Harvey, D. (1973) Social Justice and the City, London: Edward Arnold

Harvey, D. (1982) The Limits to Capital, Oxford: Blackwell

Harvey, D. (1984) 'On the history and present condition of geography: an historical materialist manifesto', Professional Geographer 36: $1-11$

Harvey, D. (1996) Justice, Nature and the Geography of Difference, Blackwell: Oxford

Harvey, D. (2001) Spaces of Capital, Edinburgh: Edinburgh University Press

Harvey, D. (2006) Spaces of Global Capitalism, London: Verso

Hassan, R., Zang, X. and McDonnell-Baum, S. (1996) 'Why families move: a study of residential mobility in Australia', Journal of Sociology 32(1): 72-85

Hattersley, L. and Creeser, R. (1995) Longitudinal Study 1971-1991: History, Organisation and Quality of Data, London: HM Stationery Office

Haug, B., Dann, G. and Mehmetoglu, M. (2007) 'Little Norway in Spain: from tourism to migration', Annals of Tourism Research 34(1): 202-222

Hayes, M. (2014) '"We gained a lot over what we would have had": the geographic arbitrage of North American lifestyle migrants to Cuenca, Ecuador', Journal of Ethnic and Migration Studies 40(12): 1953-1971

He, S. (2007) 'State-sponsored gentrification under market transition the case of Shanghai', Urban Affairs Review 43(2): 171-198

He, S. (2010) 'New-build gentrification in central Shanghai: demographic changes and socioeconomic implications', Population, Space and Place 16: 346-361

Healy, L. (2012) 'Unable to see the future: refugee youth in Malawi speak out', Forced Migration Review 40: 5-6

Hearn, J. (2012) 'A multi-faceted power analysis of men's violence to known women: from hegemonic masculinity to the hegemony of men', Sociological Review 60(4): 589-610

Heath, I. (2013) 'Overdiagnosis: when good intentions meet vested interests - an essay by lona Heath', British Medical Journal 347: $20-21$

Heath, S. (2004) 'Peer-shared households, quasi-communes and neo-tribes', Current Sociology 52(2): 161-179

Hedberg, C. and Do Carmo, R. (eds) (2012) Translocal Ruralism: Mobility and Connectivity in European Rural Spaces, Dordrecht: Springer

Hedberg, C. and Haandrikman, K. (2014) 'Repopulation of the Swedish countryside: globalisation by international migration', Journal of Rural Studies 34: 128-138

Hedman, E. (2008) 'Refuge, governmentality and citizenship: capturing "illegal migrants" in Malaysia and Thailand', Government and Opposition 43(2): 358-383

Heebels, B. and Van Aalst, I. (2010) 'Creative clusters in Berlin: entrepreneurship and the quality of place in Prenzlauer Berg and Kreuzberg', Geografiska Annaler: Series B, Human Geography 92(4): 347-363

Heidegger, M. (1971) 'Building dwelling thinking', in M. Heidegger Poetry, Language, Thought, Trans. A. Hofstadter, London: Harper and Row, pp. 145-161

Held, D., McGrew, A., Goldblatt, D. and Perraton, J. (1999) Global Transformations, Cambridge: Polity Press

Helderman, A., van Ham, M. and Mulder, C. (2006) 'Migration and home ownership', Tijdschrift voor Economische en Sociale Geografie 97(2): 111-125

Heleniak, T. (2002) 'Russia's demographic decline continues', Population Reference Bureau, at: www.prb.org/Publications/ Articles/2002/RussiasDemographicDeclineContinues.aspx (accessed 08/16)

Hemachudha, C. and Rosenfield, A. (1975) 'National Health Services and family planning: Thailand, a case study', American Journal of Public Health 65(8): 864-871

Hempstead, K. (2006) 'The geography of self-injury: spatial patterns in attempted and completed suicide', Social Science and Medicine 62(12): 3186-3196

Henry, K., Niu, X. and Boscoe, F. (2009) 'Geographic disparities in colorectal cancer survival', International Journal of Health Geographics 8(48), DOI: 10.1186/1476-072X-8-48

Herrnstein, R. and Murray, C. (1994) The Bell Curve, New York: Schuster and Schuster

Herslund, L. (2012) 'The rural creative class: counterurbanisation and entrepreneurship in the Danish countryside', Sociologia Ruralis 52(2): 235-255 
Hill, A. (2001) 'Lesbians the toast of the Two Ferrets', Observer 29 July

Hill, L. (2010) 'Gender and genre: situating Desperate Housewives', Journal of Popular Film and Television 38(4): 162-169

Hirsch, D., Sutton, L. and Beckhelling, J. (2012) The Cost of a Child in the Twenty-first Century, London: Child Poverty Action Group

Hirschman, C. (2005) 'Population and society: historical trends and future prospects', in C. Calhoun, C. Rojek and B. Turner (eds)

The Sage Handbook of Sociology, London: Sage, pp. 381-402

Hirt, S. and Stanilov, K. (2005) 'Twenty years of transition: the Evolution of urban planning in Eastern Europe and the former Soviet Union, 1989-2009', UN Habitat: Human Settlements Global Dialogue Series No. 5. HS/1147/09E

Hjälm, A. (2013) 'The "stayers": dynamics of lifelong sedentary behaviour in an urban context', Population, Space and Place 20(6): $569-580$

Hjorthol, R. (2008) 'Daily mobility of men and women: a barometer of gender equality?', in T. Uteng and T. Cresswell (eds) Gendered Mobilities, Farnham: Ashgate, pp. 193-212

Hjorthol, R. and Bjørnskau, T. (2005) 'Gentrification in Norway. Capital, culture or convenience?', European Urban and Regional Studies 12(4): 353-371

Hoare, A. and Corver, M. (2010) 'The regional geography of new young graduate labour in the UK', Regional Studies 44(4): 477-494

Hoare, P. (2006) England's Last Eden. Adventures in a Victorian Utopia, London: Harper Perennial

Hockey, J. and James, A. (2003) Social Identities Across the Life Course, Basingstoke: Palgrave Macmillan

Hoem, J., Kostova, D., Jasilioniene, A. and Muresan, C. (2009) 'Traces of the second demographic transition in four selected countries in Central and Eastern Europe: union formation as a demographic manifestation', European Journal of Population 25: 239-255

Hoey, B. (2005) 'From pi to pie: moral narratives of noneconomic migration and starting over in the postindustrial Midwest', Journal of Contemporary Ethnography 34: 586-624

Hoey, B. (2006) 'Grey suit or brown carhartt: narrative transition, relocation, and reorientation in the lives of corporate refugees', Journal of Anthropological Research 62: 347-371

Hoey, B. (2014) 'Theorising the "fifth Migration" in the United States: understanding lifestyle migration from an integrated approach', in M. Benson and N. Osbaldiston (eds) Understanding Lifestyle Migration, Basingstoke: Palgrave Macmillan, pp. 71-91

Hoffman, M. and Jamal, A. (2012) 'The youth and the Arab spring: cohort differences and similarities', Middle East Law and Governance 4(1): 168-188

Hofleitner, A., Chiraphadhanakul, T. and State, B. (2013) 'Coordinated migration', at: www.facebook.com/notes/facebook-datascience/coordinated-migration/10151930946453859/ (accessed 08/16)

Hogan, D. and Goldscheider, F. (2003) 'Success and challenge in demographic studies of the life course', in J. Mortimer and M. Shanahan (eds) Handbook of the Life Course, New York: Springer, pp. 681-691

Hohn, C. (1987) 'The family life cycle: needed extensions of the concept', in J. Bongrts, T. Burch and K. Wachter (eds) Family Demography: Methods and their Application, Oxford: Clarendon Press

Holdsworth, C., Finney, N., Marshall, A. and Norman, P. (2013) Population and Society, London: Sage

Holland, D., Fic, T., Rincon-Aznar, A., Stokes, L. and Paluchowski, P. (2011) 'Labour mobility within the EU. The impact of enlargement and the functioning of the transitional arrangements', London: National Institute of Economic and Social Research

Holloway, John (2002) Change the World Without Taking Power, London: Pluto Press

Holloway, John (2010) Crack Capitalism, London: Pluto Press

Holloway, Julian (2003a) 'Spiritual embodiment and sacred rural landscapes', in P. Cloke (ed.) Country Visions, Harlow: Pearson, pp. $158-177$

Holloway, Julian (2003b) 'Make-believe: spiritual practice, embodiment and sacred space', Environment and Planning A 35: $1961-1974$

Holloway, Julian (2006) 'Enchanted spaces: the séance, affect, and geographies of religion', Annals of the Association of American Geographers 96: 182-187

Holloway, L. and Halfacree, K. (2006) 'Rooted in the rural: seeking a back-to-the-land lifestyle in Britain', Paper presented at the Anglo-French Rural Geography Conference, Vichy, France, May

Holloway, S. and Jöns, H. (2012) 'Geographies of education and learning', Transactions of the Institute of British Geographers 37(4): $482-488$

Holmes, M. (2004) 'An equal distance? Individualisation, gender and intimacy in distance relationships', Sociological Review 52(2): 180-200

Hondagneu-Sotelo, P. and Cranford, C. (2006) 'Gender and migration', in J. Saltzman Chafetz (ed.) Handbook of the Sociology of Gender, New York: Springer pp. 105-126

Hopkins, P. and Pain, R. (2007) 'Geographies of age: thinking relationally', Area 39: 287-294

Hopkins, P., Kong, L. and Olson, E. (eds) (2012) Religion and Place, London: Springer Science and Business Media 


\section{BIBLIOGRAPHY}

Hörschelmann, K. (2011) 'Theorising life transitions: geographical perspectives', Area 43(4): 378-383

Howe, J. (2001) 'The headloading and footpath economy - walking in Sub-Saharan Africa', World Transport Policy and Practice 7(4): 8-12

Howitt, P. (1998) Sliding Doors. Dir. Peter Howitt. Paramount Pictures, 1998. Film.

Howkins, A. (2003) The Death of Rural England, London: Routledge

HPA [Health Protection Agency] (2013) Homepage: www.gov.uk/government/organisations/health-protection-agency (accessed 01/13)

Hu, F., Xu, Z. and Chen, Y. (2011) 'Circular migration, or permanent stay? Evidence from China's rural-urban migration', China Economic Review 22(1): 64-74

Huang, C., Barnett, A., Wang, X., Vaneckova, P., FitzGerald, G. and Tong, S. (2011) 'Projecting future heat-related mortality under climate change scenarios: a systematic review', Environmental Health Perspectives 119(12): 1681-1690

Hubbard, P. (2001) 'Sex zones: intimacy, citizenship and public space', Sexualities 4(1): 51-71

Hubbard, P. (2006) City, London: Routledge

Hubbard, P. (2008) 'Regulating the social impacts of studentification: a Loughborough case study', Environment and Planning A 40(2): 323-341

Huber, A. and O'Reilly, K. (2004) 'The construction of Heimat under conditions of individualised modernity: Swiss and British elderly migrants in Spain', Ageing and Society 24(3): 327-351

Huete, R., Mantecón, A. and Estévez, J. (2013) 'Challenges in lifestyle migration research: reflections and findings about the Spanish crisis', Mobilities 8(3): 331-348

Huff, J. and Clark, W. (1978) 'Cumulative stress and cumulative inertia: a behavioral model of the decision to move', Environment and Planning A 10: 1101-1119

Hughes, A. (1997) 'Rurality and "cultures of womanhood". Domestic identities and moral orders in village life', in P. Cloke and J. Little (eds) Contested Countryside Cultures, London: Routledge, pp.123-137

Hughes, B. and Paterson, K. (1997) 'The social model of disability and the disappearing body: towards a sociology of impairment', Disability and Society 12: 325-340

Hughes, D. (2000) 'The "Natasha" trade: the transnational shadow market of trafficking in women', Journal of International Affairs 53(2): 625-651

Hughes, G. and McCormick, B. (1981) 'Do council housing policies reduce migration between regions?', Economic Journal 91: 919-937

Hugo, G. (2006) 'Population geography', Progress in Human Geography 30(4): 513-523

Hugo, G. (2007) 'Population geography', Progress in Human Geography 31(1): 77-88

Huguet, J. (2012) 'Commentary: the demography of environmental migration', Asian Population Studies 8(2): 121-124

Hummer, R., Rogers, R., Nam, C. and Ellison, C. (1999) 'Religious involvement and U.S. adult mortality', Demography 36(2): 273-285

Humphreys, R. (2005) 'Yellow school bus - a US icon', BBC News 16 June, at: http://news.bbc.co.uk/1/hi/education/4098782.stm (accessed 08/16)

Hunt, S. (2005) The Life Course, Basingstoke: Palgrave

Huq, R. (2013) On the Edge: the Contested Cultures of Suburbia, London: Lawrence and Wishart

Hutchison, E. (2010) Dimensions of Human Behaviour: the Changing Life Course, London: Sage

Hutchison, E. (ed.) (2012) Essentials of Behavior. Integrating Person, Environment, and the Life Course, London: Sage

Huxley, A. (1932) Brave New World, London: Chatto and Windus

Hvistendahl, M. (2011) Unnatural Selection, New York: Public Affairs

Hyndman, J. and Mountz, A. (2008) 'Another brick in the wall? Neo-refoulement and the externalization of asylum by Australia and Europe', Government and Opposition 43(2): 249-269

Ide, N., Wyder, M., Kolves, K. and De Leo, D. (2010) 'Separation as an important risk factor for suicide: a systematic review', Journal of Family Issues 31(12): 1689-1716

IDMC [Internal Displacement Monitoring Centre] (2014) 'Sub-Saharan Africa', at: www.internal-displacement.org/sub-saharan-africa/ (accessed 11/14)

Imrie, R. (1996) Disability and the City, London: Paul Chapman

Imrie, R. and Edwards, C. (2007) 'The geographies of disability: reflections on the development of a sub-discipline', Geography Compass 1(3): 623-640

Inequality (2016) Homepage: http://inequality.org/ (accessed 09/16)

Ingle, M. (2013) 'Counterurbanisation and the emergence of a postproductivist economy in South Africa's arid Karoo region, 1994-2010', New Contree: A Journal of Historical and Human Sciences for Southern Africa 66: 55-69 
Ingold, T. (1995) 'Building, dwelling, living: how people and animals make themselves at home in the world', in M. Strathern (ed.) Shifting Contexts: Transformations in Anthropological Knowledge, London: Routledge, pp. 57-80

Ingold, T. (2008) 'Bindings against boundaries: entanglements of life in an open world', Environment and Planning A 40: 1796-1810 Inhorn, M. and Birenbaum-Carmeli, D. (2008) 'Assisted reproductive technologies and culture change', Annual Review of Anthropology 37(1): 177-196

International Rivers (2009) China's Three Gorges Dam: a Model of the Past, Berkeley, CA: International Rivers

Internet World Stats (nd) Homepage: www.internetworldstats.com/ (accessed 08/16)

IPCC [Intergovernmental Panel on Climate Change] (2007) Climate Change 2007: Impacts, Adaptation and Vulnerability. Working Group II Contribution to the Fourth Assessment Report of the Intergovernmental Panel on Climate Change, Cambridge: Cambridge University Presss

IPCC (2016) Homepage: www.ipcc.ch/ (accessed 09/16)

Iredale, R. (2000) 'Migration policies for the highly skilled in the Asia-Pacific region', International Migration Review 34(3): 882-906 Iredale, R. (2001) 'The migration of professionals: theories and typologies', International Migration 39(5): 7-26

Irons, M., Murphy, S. and Russell, J. (2014) 'History rolled in on a yellow school bus', Boston Globe 7 September

Jackiewicz, E. and Craine, J. (2010) 'Destination Panama: an examination of the migration-tourism-foreign investment nexus', Recreation and Society in Africa, Asia and Latin America 1(1): 5-29

Jackson, P. and Van der Wielen, J. (eds) (2002) Teleworking: International Perspectives, 2nd edition, London: Routledge

Jacob, J. (1997) New Pioneers, University Park, Pennsylvania: Pennsylvania University Press

Jaffe, R., Klaufus, C. and Colombijn, F. (2012) 'Mobilities and mobilizations of the urban poor', International Journal of Urban and Regional Research 36(4): 643-654

Jain, J. and Lyons, G. (2008) 'The gift of travel time', Journal of Transport Geography 16(2): 81-89

Janoschka, M. (2010) 'Between mobility and mobilization - lifestyle migration and the practice of European identity in political struggles', Sociological Review 58(s2): 270-290

Jasso, G. (2003) 'Migration, human development, and the life course', in J. Mortimer and M. Shanahan (eds) Handbook of the Life Course, New York: Kluwer Academic, pp. 331-364

Jefferies, J. (2005)' The UK population: past, present and future', at www.statistics.gov.uk/downloads/theme_compendia/ fom2005/01_fopm_population.pdf (accessed 12/11)

Jeffrey, C. (2010) 'Geographies of children and youth I: eroding maps of life', Progress in Human Geography 34: 496-505

Jennings, S. (2004) 'Coastal tourism and shoreline management', Annals of Tourism Research 31(4): 899-922

Jensen, O. (2009) 'Flows of meaning, cultures of movements - urban mobility as meaningful everyday life practice', Mobilities 4(1): $139-158$

Jian, M. (2013) 'A crime against the people', Guardian G2 6 May: 4-7

Jim, C., Yang, F. and Wang, L. (2010) 'Socio-ecological impacts of concurrent reservoir inundation and reforestation in the Three Gorges Region of China', Annals of the Association of American Geographers 100(2): 243-268

Jobes, P. (1984) 'Old timers and new mobile lifestyles', Annals of Tourism Research 11(2): 181-198

Jobes, P. (2000) Moving Nearer to Heaven: the Illusions and Disillusions of Migrants to Scenic Rural Places, Westport: Praeger

Johnson, K. and Daviss, B.A. (2005) 'Outcomes of planned home births with certified professional midwives: large prospective study in North America', British Medical Journal 330(7505): 1-7

Johnson, N. and Climo, J. (2000) 'Aging and eldercare in lesser developed countries', Journal of Family Issues 21(6): 683-691

Johnston, R. (1966) 'Central places and the settlement pattern', Annals of the Association of American Geographers 56(3): 541-549.

Johnston, R. (2009a) 'Geography and the social science tradition', in N. Clifford, S. Holloway, S. Rice and G. Valentine (eds) Key Concepts in Geography, 2nd edition, London: Sage, pp. 46-65

Johnston, R. (2009b) 'Census', in D. Gregory, R. Johnston, G. Pratt, M. Watts and S. Whatmore (eds) The Dictionary of Human Geography, 5th edition, Oxford: Wiley-Blackwell, pp. 74-75

Johnston, R. and Sidaway, J. (2004) Geography and Geographers, 6th edition, London: Arnold

Johnston, R., Poulsen, M. and Forrest, J. (2007) 'The geography of ethnic residential segregation: a comparative study of five countries', Annals of the Association of American Geographers 97(4): 713-738

Joint Committee on Human Rights (2007) 'The treatment of asylum seekers', UK Parliament, London, at: www.publications. parliament.uk/pa/jt200607/jtselect/jtrights/81/81 i.pdf (accessed 08/16)

Jones, A. (2012) Human Geography - the Basics, London: Routledge

Jones, A. (2013) 'Female infanticide in India and China', Sexual Health Exchange 2002 (3): 12-13

Jones, G. (1987) 'Leaving the parental home: an analysis of early housing careers', Journal of Social Policy 16(1): 49-74

Jones, H. (1990) Population Geography, 2nd edition, London: Paul Chapman Publishing 


\section{BIBLIOGRAPHY}

Jones, K., Patel, N., Levy, M., Storeygard, A., Balk, D., Gittleman, J. and Daszak, P. (2008) 'Global trends in emerging infectious diseases', Nature 251: 990-994

Jones, N. and Lake, I. (2013) 'The combined impact of rural residence and socio-economic status on premature mortality', Health and Place 24: 90-96

Jones, O. (2012) Chavs: the Demonization of the Working Class, London: Verso

Jones, O. (2014) The Establishment: and How They Get Away With It, London: Allen Lane

Jones, R. (1998) 'Remittances and inequality: a question of migration stage and geographic scale', Economic Geography 74(1): $8-25$

Jonsen, A. (2003) The Birth of Bioethics, Oxford: Oxford University Press

Joseph, A. and Hallman, B. (1998) 'Over the hill and far away: distance as a barrier to the provision of assistance to elderly relatives', Social Science and Medicine 46(6): 631-639

JSK [Jansankhya Sthirata Kosh] (2012) National Population Stabilisation Fund, at: www.jsk.gov.in/total_fertility_rate.asp (accessed 04/12)

Julca, A. (2013a) 'Remittances, motivation', in I. Naess (ed.) The Encyclopedia of Global Human Migration, New Jersey: WileyBlackwell, at: http://onlinelibrary.wiley.com/doi/10.1002/9781444351071.wbeghm447/abstract (accessed 08/16)

Julca, A. (2013b) 'Remittances and international migration', in I. Naess (ed.) The Encyclopedia of Global Human Migration, New Jersey: Wiley-Blackwell, at: http://onlinelibrary.wiley.com/doi/10.1002/9781444351071.wbeghm445/abstract (accessed 08/16)

Kabisch, N. and Haase, D. (2011) 'Evidence of urban population trends for the 21st Century', Population, Space and Place 17: 236-253

Kahanec, M. (2012) 'Labor mobility in an enlarged European Union', Forschungsinstitut zur Zukunft der Arbeit, IZA Discussion Paper No. 6485

Kalantaridis, C. and Bika, Z. (2006) 'In-migrant entrepreneurship in rural England: beyond local embeddedness', Entrepreneurship and Regional Development 18(2): 109-131

Kalipeni, E., Craddock, S., Oppong, J. and Ghosh, J. (2004) HIV and AIDS in Africa: Beyond Epidemiology, Oxford: Blackwell

Kaltenborn, B. (1998) 'The alternative home: motives of recreation home use', Norsk Geografisk Tidsskrift 52: 121-134

Kandel, W. and Parrado, E. (2005) 'Restructuring of the US meat processing industry and new Hispanic migrant destinations', Population and Development Review 31(3): 447-471

Kang, T. (2012) 'Gendered media, changing intimacy: internet-mediated transnational communication in the family sphere', Media, Culture and Society 34(2): 146-161

Kaplan, H. (1994) 'Evolutionary and wealth flows theories of fertility: empirical tests and new models', Population and Development Review 20(4): 753-791

Katz, S. and McHugh, K. (2010) 'Age, meaning, and place: cultural narratives and retirement communities', in T. Cole, R. Ray and R. Kastenbaum (eds) A Guide to Humanistic Studies of Ageing, Baltimore: Johns Hopkins University Press, pp. 271-292

Kaufmann, V. (2002) Re-Thinking Mobility. Contemporary Sociology, Aldershot: Ashgate

Kautonen, T., Tornikoski, E. and Kibler, E. (2011) 'Entrepreneurial intentions in the third age: the impact of perceived age norms', Small Business Economics 37(2): 219-234

Kay, J. (2001) 'Geography is still important', Financial Times, 10 January, p. 14

Kearns, R. (1993) 'Place and health: towards a reformed medical geography', Professional Geographer 45: 139-147

Kearns, R. and Gesler, W. (eds) (1998) Putting Health into Place: Landscape, Identity, and Well-Being, Syracuse: Syracuse University Press

Kearns, R. and Moon, G. (2002) 'From medical to health geography: novelty, place and theory after a decade of change', Progress in Human Geography 26(5): 605-625

Keddie, N. (1998) 'The new religious politics: where, when, and why do "fundamentalisms" appear?', Comparative Studies in Society and History 40: 696-723

Keddie, N. (2003) 'Secularism and its discontents', Daedalus 132(3): 14-30

Keller, G. (2013) 'Sectarian violence triggers Sunni-Alawi segregation in Syria', IRIN News 24 June, at: www.irinnews.org/analysis/ 2013/06/24/sectarian-violence-triggers-sunni-alawi-segregation-syria (accessed 08/16)

Kelly, B. (1993) Expanding the American Dream: Building and Rebuilding Levittown, New York: SUNY Press

Kelly, J. (2007) 'Children's living arrangements following separation and divorce: insights from empirical and clinical research', Family Process 46(1): 35-52

Kendig, H. (1990) 'A life course perspective on housing attainment', in D. Myers (ed.) Housing Demography: Linking Demographic Markets and Housing Structure, Madison, WI: University of Wisconsin Press, pp. 133-156

\section{ST PROOFS: NOT FOR DISTRIBUTION}


Kent, M. (2010) 'Smoking related deaths keep U.S. life expectancy below other wealthy countries', Population Reference Bureau, at: www.prb.org/Publications/Articles/2010/ussmoking.aspx (accessed 08/16)

Kerr, J., Rosenberg, D., Sallis, J., Saelens, B., Frank, L. and Conway, T. (2006) 'Active commuting to school: associations with environment and parental concerns', Medicine and Science in Sports and Exercise 38(4): 787-794

Khan, A. (2014) 'Why it's time to end kafala', Guardian 26 February, p. 17

Kim, T. and Locke, W. (2010) 'Transnational academic mobility and the academic profession', Centre for Higher Education Research and Information, The Open University, London

King, L. (2001) 'From pronatalism to social welfare? Extending family allowances to minority populations in France and Israel', European Journal of Population 17(4): 305-322

King, R. (ed.) (1991) Contemporary Irish Migration, Dublin: Geographical Society of Ireland

King, R. (2002) 'Towards a new map of European migration', International Journal of Population Geography 8: 89-106

King, R. and Ruiz-Gelices, E. (2003) 'International student migration and the European "year abroad": effects on European identity and subsequent migration behaviour', International Journal of Population Geography 9(3): 229-252

King, R., Connell, J. and White, P. (eds) (1995) Writing Across Worlds. Literature and Migration, London: Routledge

King, R., Warnes, A. and Williams, A. (1998) 'International retirement migration in Europe', International Journal of Population Geography 4: 91-111

King, R., Warnes, A. and Williams, A. (2000) Sunset Lives: British Retirement Migration to the Mediterranean, Oxford: Berg

King, R., Black, R., Collyer, M., Fielding, A. and Skeldon, R. (2010) The Atlas of Human Migration: Global Patterns of People on the Move, London: Earthscan

Kinnunen, T. (2010) '“A second youth": pursuing happiness and respectability through cosmetic surgery in Finland', Sociology of Health and IIIness 32(2): 258-271

Kinsella, K. and Phillips, D. (2005) Global Aging: the Challenge of Success, Washington DC: Population Reference Bureau

Kipgen, N. (2013) 'Addressing the Rohingya problem', Journal of Asian and African Studies 49(2): 234-247

Kiragu, E., Li Rosi, A, and Morris, T. (2011) 'States of denial. A review of UNHCR's reponse to the protracted situation of stateless Rohingya refugees in Bangladesh', UNHCR Policy Development and Evaluation Service, PDES/2011/13

Kirk, D. (1996) 'Demographic transition theory', Population Studies 50(3): 361-387

Kirschner, A., Berry, E. and Glasgow, N. (2006) 'The changing faces of rural America', in W. Kandel and D. Brown (eds) Population Change and Rural Society, Dordrecht: Springer, pp. 53-74

Kivivuori, J. and Lehti, M. (2006) 'The social composition of homicide in Finland, 1960-2000', Acta Sociologica 49(1): 67-82

Klein, A., Vaissiere, B., Cane, J., Steffan-Dewenter, I., Cunningham, S., Kremen, C. and Tscharntke, T. (2007) 'Importance of pollinators in changing landscapes for world crops', Proceedings of the Royal Society of London B: Biological Sciences 274(1608): 303-313

Klepp, S. (2010) 'A contested asylum system: the European Union between refugee protection and border control in the Mediterranean Sea', European Journal of Migration and Law 12(1): 1-21

Kley, S. (2011) 'Explaining the stages of migration within a life-course framework', European Sociological Review 27(4): 469-486 Kliot, N. (1987) 'The era of the homeless man', Geography 72: 109-121

Knodel, J., Havanon, N. and Pramualratana, A. (1984) 'Fertility transition in Thailand: a qualitative analysis', Population and Development Review 10(2): 297-328

Knopp, L. (1990) 'Some theoretical implications of gay involvement in an urban land market', Political Geography Quarterly 9: 337-352

Knox, E., Stewart, A., Gilman, E. and Kneale, G. (1988) 'Background radiation and childhood cancers', Journal of Radiological Protection 8(1): 9-18

Knox, P. and Pinch, S. (2009) Urban Social Geography. An Introduction, 6th edition, Harlow: Pearson

Koch, T. (2005) Cartographies of Disease: Maps, Mapping, and Medicine, Redlands, CA: ESRI Press

Köhler, B. and Wissen, M. (2003) 'Glocalizing protest: urban conflicts and the global social movements', International Journal of Urban and Regional Research 27(4): 942-951

Kong, L. (1990) 'Geography and religion: trends and prospects', Progress in Human Geography 14(3): 355-371

Kong, L. (2001) 'Mapping "new" geographies of religion: politics and poetics in modernity', Progress in Human Geography 25: 211-233

Kong, L. (2010) 'Global shifts, theoretical shifts: changing geographies of religion', Progress in Human Geography 34: 755-776

Koning, J. (2005) 'The impossible return? The post-migration narratives of young women in rural Java', Asian Journal of Social Science 33(2): 165-185

Korpela, M. (2009) 'When a trip to adulthood becomes a lifestyle: Western lifestyle migrants in Varanasi, India', in M. Benson and K. O'Reilly (eds) Lifestyle Migration: Expectations, Aspirations and Experiences, Aldershot: Ashgate, pp. 15-30 


\section{BIBLIOGRAPHY}

Korpela, M. (2014) 'Lifestyle of freedom? Individualism and lifestyle migration', in M. Benson and N. Osbaldiston (eds) Understanding Lifestyle Migration, Basingstoke: Palgrave Macmillan, pp. 27-46

Koser, K. and Salt, J. (1997) 'The geography of highly skilled international migration', International Journal of Population Geography 3: $285-303$

Kosiński, L. (1984) 'The roots of population geography', in J. Clarke (ed.) Geography and Population, Oxford: Pergamon, pp. 11-24

Koua, E. and Kraak, M.-J. (2004) 'Geovisualization to support the exploration of large health and demographic survey data', International Journal of Health Geographics 3(12): DOI: 10.1186/1476-072X-3-12

Krabel, S. and Flöther, C. (2014) 'Here today, gone tomorrow? Regional labour mobility of German university graduates', Regional Studies 48(10): 1609-1627

Krieger, N. (2003) 'Place, space, and health: GIS and epidemiology', Epidemiology 14(4): 384-385

Kritz, M., Lim, L. and Zlotnik, H. (eds) (1992) International Migration Systems. A Global Approach, Oxford: Clarendon Press

Kronenfeld, D. (2008) 'Afghan refugees in Pakistan: not all refugees, not always in Pakistan, not necessarily Afghan?', Journal of Refugee Studies 21(1): 43-63

Kuhlman, T. and Farrington, J. (2010) 'What is sustainability?', Sustainability 2(11): 3436-3448

Kulkarni, P. (2007) 'Estimation of missing girls at birth and juvenile ages in India', UNFPA India, at: http://india.unfpa.org/drive/ MissingGirlsatBirthpaper-August2007Kulkarni.pdf (accessed 04/12)

Kulu, H. (2008) 'Fertility and spatial mobility in the life course: evidence from Austria', Environment and Planning A 40(3): 632-652 Kulu, H. and Milewski, N. (2007) 'Family change and migration in the life course: an introduction', Demographic Research 17: 567-590 Kulu, H. and Steele, F. (2013) 'Interrelationships between childbearing and housing transitions in the family life course', Demography 50(5): 1687-1714

Kundera, M. (2002) Ignorance, London: Faber and Faber

Kunz, E. (1973) 'The refugee in flight: kinetic models and forms of displacement', International Migration Review 7: 125-146

Kunz, E. (1981) 'Exile and resettlement: refugee theory', International Migration Review 15: 42-51

Kunzig, R. (2011) 'Population 7 billion', National Geographic, January, at: http://ngm.nationalgeographic.com/2011/01/sevenbillion/kunzig-text (accessed 06/16)

Kurien, P. (2005) 'Being young, brown, and Hindu. The identity struggles of second-generation Indian Americans', Journal of Contemporary Ethnography 34: 434-469

Lalive, R. and Zweimüller, J. (2009) 'How does parental leave affect fertility and return to work? Evidence from two natural experiments', Quarterly Journal of Economics 124(3): 1363-1402

Lamptey, P., Wigley, M., Carr, D. and Collymore, Y. (2002) 'Facing the HIV/AIDS pandemic', Population Bulletin 57(3): 1-39

Larsen, J., Urry, J. and Axhausen, K. (2007) 'Networks and tourism: mobile social life', Annals of Tourism Research 34(1): 244-262

Laslett, P. (1994) 'The third age, the fourth age and the future', Ageing and Society 14(03): 436-447

Lauby, J. and Stark, O. (1988) 'Individual migration as a family strategy: young women in the Philippines', Population Studies 42(3): 473-486

Lauria, M. and Knopp, L. (1985) 'Toward an analysis of the role of gay communities in the urban renaissance', Urban Geography 6(2): 152-169

Law, C. and Warnes, A (1976) 'The changing geography of the elderly in England and Wales', Transactions of the Institute of British Geographers 1: 453-471

Lawson, H. and Leck, K. (2006) 'Dynamics of internet dating', Social Science Computer Review 24(2): 189-208

Lawson, V. (1999) 'Questions of migration and belonging: understandings of migration under neoliberalism in Ecuador', International Journal of Population Geography 5: 261-276

Le Nevez, C., Parker, M. and Whitfield, P. (2009) The Rough Guide to Wales, London: Rough Guides

Lea, J. (2008) 'Retreating to nature: rethinking 'therapeutic landscapes", Area 40: 90-98

Lee, E. (1966) 'A theory of migration', Demography 3: 47-57

Lee, J. (2005) 'Human trafficking in East Asia: current trends, data collection, and knowledge gaps', International Migration 43(12): 165-201

Lees, L. (2003) 'Super-gentrification: the case of Brooklyn Heights, New York City', Urban Studies 40(12): 2487-2509

Lees, L. (2014) 'The urban injustices of new Labour's "New Urban Renewal": the case of the Aylesbury Estate in London', Antipode 46(4): 921-947

Lefebvre, H. (1991/1974) The Production of Space, Oxford: Blackwell

Lefebvre, H. (2004) Rhythmanalysis: Space, Time and Everyday Life, London: Continuum

Legg, S. (2005) 'Foucault's population geographies: classifications, biopolitics and governmental spaces', Population, Space and Place 11(3): 137-156 
Leisch, H. (2002) 'Gated communities in Indonesia', Cities 19(5): 341-350

Leonard, S. (2008) 'Frontex: the EU External Borders Agency; exploring the nature of the beast', Paper presented at the 49th Annual International Studies Association Convention, San Francisco

Lerche, J. (2007) 'A global alliance against forced labour, unfree labour, neo-liberal globalization and the International Labour Organization', Journal of Agrarian Change 7(4): 425-452

Lesthaeghe, R. (1995) 'The second demographic transition in Western countries: an interpretation', in K. Mason and A. Jensen (eds) Gender and Family Change in Industrialized Countries, Oxford: Clarendon Press, pp. 17-62.

Lesthaeghe, R. (2010) The unfolding story of the second Demographic Transition. Report 10-696, research report. Population Studies Center, University of Michigan, Institute for Social Research, p. 5

Lesthaeghe, R. (2014) 'The second demographic transition: a concise overview of its development', Proceedings of the National Academy of Sciences 111(51): 18112-18115

Leung, M. (2013) "Read ten thousand books, walk ten thousand miles": geographical mobility and capital accumulation among Chinese scholars', Transactions of the Institute of British Geographers 38(2): 311-324

Levin, I. (2004) 'Living apart together: a new family form', Current Sociology 52(2): 223-240

Lewicka, M. (2011) 'Place attachment: how far have we come in the last 40 years?', Journal of Environmental Psychology 31(3): 207-230

Lewis, G. (1982) Human Migration: a Geographical Perspective, London: Croom Helm

Lewis, G. and Phoenix, A. (2004) '"Race", "ethnicity" and identity', in K. Woodward (ed.) Questioning Identity: Gender, Class, Ethnicity, London: Routledge, pp. 115-151

Lewis, O. (1969) 'The culture of poverty', in D. Moynihan (ed.) On Understanding Poverty: Perspectives from the Social Sciences, New York: Basic Books, pp. 187-220

Ley, D. (1994) 'Postmodernism', in R. Johnston, D. Gregory and D. Smith (eds) The Dictionary of Human Geography, Oxford, Blackwell, 3rd edition, pp. 66-68

Ley, D. and Samuels, M. (eds) (1978) Humanistic Geography: Prospects and Problems, Chicago: Maaroufa Press

Leyshon, A. and Thrift, N. (1995) 'Geographies of financial exclusion: financial abandonment in Britain and the United States', Transactions of the Institute of British Geographers 20(3): 312-341

Li, F. (1997) 'Representing difference: multiple voices of migrants from Hong Kong', Scottish Geographical Magazine 113: 74-81

Liefbroer, A. and Billari, F. (2010) 'Bringing norms back in: a theoretical and empirical discussion of their importance for understanding demographic behaviour', Population, Space and Place 16(4): 287-305

Lietaert, I., Derluyn, I. and Broekaert, E. (2014) 'Returnees' perspectives on their re-migration processes', International Migration 52(5): $144-158$

Lim, J. and Browne, K. (2009) 'Senses of gender', Sociological Research Online 14(1), www.socresonline.org.uk/14/1/6.html (accessed 10/14)

Lindstrom, D. and Berhanu, B. (1999) 'The impact of war, famine, and economic decline on marital fertility in Ethiopia', Demography 36(2): 247-261

Ling, R. and Horst, H. (2011) 'Mobile communication in the global south', New Media and Society 13(3): 363-374

Lingel, J., Naaman, M. and Boyd, D. (2014) 'City, self, network: transnational migrants and online identity work', in Proceedings of the 17th ACM conference on Computer Supported Cooperative Work, New York: ACM Press, pp. 1502-1510

Lino, M. (2013) 'Expenditures on children by families, 2012', U.S. Department of Agriculture, Center for Nutrition Policy and Promotion. Miscellaneous Publication No. 1528-2012, at: www.cnpp.usda.gov/sites/default/files/expenditures_on_children_by_ families/crc2012.pdf (accessed 08/16)

Lin-Yuan, Y. and Kosiński, L. (1994) 'The model of place utility revisited', International Migration 32: 49-70

Lipsicas, C., Mäkinen, I., Apter, A., De Leo, D., Kerkhof, A., Lönnqvist, J., Värnik, A., and Van Heeringen, C. (2012) 'Attempted suicide among immigrants in European countries: an international perspective', Social Psychiatry and Psychiatric Epidemiology 47(2): 241-251

Little, J. (2003) '"Riding the Rural Love Train": heterosexuality and the rural community', Sociologia Ruralis 43: 401-417

Little, J. and Austin, P. (1996) 'Women and the rural idyll', Journal of Rural Studies 12: 101-111

Litwak, E. and Longino, C. (1987) 'Migration patterns among the elderly: a developmental perspective', Gerontologist 27(3): 266-272

Lizárraga Morales, O. (2010) 'The US citizens retirement migration to Los Cabos, Mexico. Profile and social effects', Recreation and Society in Africa, Asia and Latin America 1(1): 75-92

Lloyd, C. (2010) Spatial Data Analysis: an Introduction for GIS Users, Oxford: Oxford University Press

Lloyd, C. (2011) Local Models for Spatial Analysis, Boca Raton: CRC Press 


\section{BIBLIOGRAPHY}

Lockwood, M. (1997) 'Sons of the soil? Population growth, environmental change and men's reproductive intentions in northern Nigeria', International Journal of Population Geography 3: 305-322

Loeffler, R. and Steinicke, E. (2007) 'Amenity migration in the US Sierra Nevada', Geographical Review 97(1): 67-88

Logan, J., Alba, R. and Zhang, W. (2002) 'Immigrant enclaves and ethnic communities in New York and Los Angeles', American Sociological Review 67(2): 299-322

Long, L. (1988) Migration and Residential Mobility in the United States, New York: Sage

Longhurst, N. (2013) 'The emergence of an alternative milieu: conceptualising the nature of alternative places', Environment and Planning A 45(9): 2100-2119

Longino, C. and Bradley, D. (2006) 'Internal and international migration', in R. Binstock and L. George (eds) Handbook of Aging and the Social Sciences, 6th edition, San Diego: Elsevier, pp. 76-93

Longino, C., Perzynski, A. and Stoller, E. (2002) 'Pandora's briefcase: unpacking the retirement migration decision', Research on Aging 24(1): 29-49

Loopstra, R., Reeves, A., Taylor-Robinson, D., Barr, B., McKee, M. and Stuckler, D. (2015) 'Austerity, sanctions, and the rise of food banks in the UK', British Medical Journal 350: h1775

Lorch, B. and Smith, M. (1993) 'Pedestrian movement and the downtown enclosed shopping center', Journal of the American Planning Association 59(1): 75-86

Lorenzen, J. (2012) 'Going green: the process of lifestyle change', Sociological Forum 27(1): 94-116

Lorimer, H. (2005) 'Cultural geography: the busyness of being "more-than-representational"', Progress in Human Geography 29: 83-94

Lubans, D., Boreham, C., Kelly, P. and Foster, C. (2011) 'The relationship between active travel to school and health-related fitness in children and adolescents: a systematic review', International Journal of Behavioral Nutrition and Physical Activity 8(5), DOI: 10.1186/1479-5868-8-5

Lucas, C. (2002) 'Relocalising the global food economy', BBC Lecture, Cardiff University, 1 May, at: www.carolinelucasmep.org.uk/ speeches/Relocalisingfood010502.htm (accessed 06/03)

Lynch, J., Smith, G., Kaplan, G. and House, J. (2000) 'Income inequality and mortality: importance to health of individual income, psychosocial environment, or material conditions', British Medical Journal 320(7243): 1200-1203

Mabey, R. (2005) Nature Cure, London: Chatto and Windus

Mace, A. (2009) 'Suburbanization', in R. Kitchin and N. Thrift (eds) International Encyclopedia of Human Geography, Oxford: Elsevier, pp. $77-81$

MacEachren, A., Gahegan, M., Pike, W., Brewer, I., Cai, G., Lengerich, E. and Hardisty, F. (2004) 'Geovisualization for knowledge construction and decision support', IEEE Computer Graphics Applications 24(1): 13-17

MacÉinrí, P. and White, A. (2008) 'Immigration into the Republic of Ireland: a bibliography of recent research', Irish Geography 41(2): $151-179$

Macfarlane, R. (2012) The Old Ways. A Journey on Foot, London: Hamish Hamilton

Macintyre, S., Ellaway, A. and Cummins, S. (2002) 'Place effects on health: how can we conceptualise, operationalise and measure them?', Social Science and Medicine 55(1): 125-139

Macintyre, T. (2010) 'Romashki; or, a life less ordinary. A narrative ethnography into an alternative rurality in Ukraine', MSc Dissertation, International Development Studies, Wageningen University

Madianou, M. and Miller, D. (2011) 'Mobile phone parenting: reconfiguring relationships between Filipina migrant mothers and their left-behind children', New Media and Society 13(3): 457-470

Maggio, G. and Cacciola, G. (2012) 'When will oil, natural gas, and coal peak?', Fuel 98: 111-123

Maguire, S. (2012) 'Putting adolescents and youth at the centre', Forced Migration Review 40: 4-5

Maheswaran, R., Strachan, D., Dodgeon, B. and Best, N. (2002) 'A population-based case-control study for examining early life influences on geographical variation in adult mortality in England and Wales using stomach cancer and stroke as examples', International Journal of Epidemiology 31(2): 375-382

Mahler, S. and Pessar, P. (2006) 'Gender matters: ethnographers bring gender from the periphery toward the core of migration studies', International Migration Review 40(1): 27-63

Mahroum, S. (2000) 'Highly skilled globetrotters: mapping the international migration of human capital', R\&D Management 30(1): 23-31

Maitland, S. (2009) A Book of Silence, London: Granta

Maitland, S. (2014) 'There. ., Archipelago 9: 36-46

Malakoff, D. (2011) 'Are more people necessarily a problem?', Science 333(6042): 544-546

\section{ST PROOFS: NOT FOR DISTRIBUTION}


Malecki, E. and Ewers, M. (2007) 'Labor migration to world cities: with a research agenda for the Arab Gulf', Progress in Human Geography 31(4): 467-484

Malkki, L. (1992) 'National Geographic: the rooting of peoples and the territorialization of national identity among scholars and refugees', Cultural Anthropology 7: 24-44

Malthus, T. (1798) An Essay of the Principle of Population, London: J. Johnson

Mann, M. (2005) The Dark Side of Democracy. Explaining Ethnic Cleansing, Cambridge: Cambridge University Press

Mann, S. and Seager, P. (2007) Upping Sticks: How to Move House and Stay Sane, Devon: White Ladder Press

Mannon, S., Petrzelka, P., Glass, C. and Radel, C. (2012) 'Keeping them in their place: migrant women workers in Spain's strawberry industry', International Journal of the Sociology of Agriculture and Food 19: 83-101

Marcuse, P. (1997) 'The enclave, the citadel, and the ghetto: what has changed in the post-Fordist US city', Urban Affairs Review 33(2): 228-264

Mares, P. (2001) Borderline: Australia's Treatment of Refugees and Asylum Seekers, Sydney: UNSW Press

Marinov, A. (2015) Inward Looking: the Impact of Migration on 'Romanipe' from the Romani Perspective, PhD thesis, Department of Geography, Swansea University

Mark, M. (2013) 'Nigeria's child brides: "I thought being in labour would never end"', Guardian 2 September

Markus, H. and Kitayama, S. (1991) 'Culture and the self: implications for cognition, emotion, and motivation', Psychological Review 98(2): 224-253

Marsden, M. (2005) Living Islam. Muslim Religious Experience in Pakistan's North-West Frontier, Cambridge: Cambridge University Press

Marshall, P. (2001) 'Digging for freedom', in C. Coates (2001) Utopia Britannica. British utopian experiments: 1325-1945, London: Diggers and Dreamers Publications, pp. 21-25

Marston, S. (2000) 'The social construction of scale', Progress in Human Geography 24: 219-242

Martin, P. and Midgley, E. (2003) 'Immigration: shaping and reshaping America', Population Bulletin 58(2)

Martínez, R. (2001) Crossing Over: a Mexican Family on the Migrant Trail, New York: Metropolitan Books

Martinson, J. (2014) 'Another year, another death of a gang rape victim in India', Guardian 2 January

Marx, K. (2010/1852) The Eighteenth Brumaire of Louis Bonaparte, cited at: www.marxists.org/archive/marx/works/1852/18thbrumaire/ch01.htm (accessed 08/16)

Mascarenhas, M., Flaxman, S., Boerma, T., Vanderpoel, S. and Stevens, G. (2012) 'National, regional, and global trends in infertility prevalence since 1990: a systematic analysis of 277 health surveys', PLoS Medicine 9(12), e1001356

Masquelier, A. (2006) 'Why Katrina's victims aren't refugees: musings on a "dirty" word', American Anthropologist 108(4): 735-743

Massey, D. (1984) Spatial Divisions of Labour, London: Methuen

Massey, D. (2005) For Space, London: Routledge

Massey, D.S. (1990) 'Social structure, household strategies, and the cumulative causation of migration', Population Index 56: 3-26

Massey, D.S. (1995a) 'The new immigration and ethnicity in the United States', Population and Development Review 21(3): 631-652

Massey, D.S. (1995b) 'Getting away with murder: segregation and violent crime in urban America', University of Pennsylvania Law Review 143(5): 1203-1232

Massey, D.S. and Capoferro, C. (2006) 'Sálvese quien pueda: structural adjustment and emigration from Lima', Annals of the American Academy of Political and Social Science 606(1): 116-127

Massey, D.S., Arango, J., Hugo, G., Kouaouci, A., Pellegrino, A. and Taylor, J. (1993) 'Theories of international migration: a review', Population and Development Review 19: 431-466

Massey, D.S., Arango, J., Hugo, G., Kouaouci, A., Pellegrino, A. and Taylor, J. (1998) Worlds in Motion: Understanding International Migration at the End of the Millennium, Oxford: Clarendon Press

Matthews, H. and Limb, M. (1999) 'Defining an agenda for the geography of children: review and prospect', Progress in Human Geography 23: 61-90

Matthews, H. and Vujakovic, P. (1995) 'Private worlds and public places: mapping the environmental values of wheelchair users', Environment and Planning A 27: 1069-1083

Matthews, M. (1987) 'Gender, home range and environmental cognition', Transactions of the Institute of British Geographers 12(1): 43-56

Maurer, M., Cuddihy, P., Weisenberg, J., Delisle, S., Strong, B., Gao, O., Kachnowski, S. and Howell, J. (2009) 'The prevalence and impact of anergia (lack of energy) in subjects with heart failure and its associations with actigraphy', Journal of Cardiac Failure 15(2): 145-151

Maxey, L. (2002) One Path Forward? Three Sustainable Communities in England and Wales. Swansea, Dept. of Geography, University of Wales, Swansea, unpublished Ph.D. thesis 


\section{BIBLIOGRAPHY}

May, J., Wills, J., Datta, K., Evans, Y., Herbert, J. and Mcllwaine, C. (2007) 'Keeping London working: global cities, the British state and London's new migrant division of labour', Transactions of the Institute of British Geographers 32(2): 151-167

Mayer, J. (2000) 'Geography, ecology and emerging infectious diseases', Social Science and Medicine 50: 937-952

Mayer, M. (2009) 'The "Right to the City" in the context of shifting mottos of urban social movements', City 13(2-3): 362-374

Mayo Clinic Staff (2013) 'HIV/AIDS', at: www.mayoclinic.com/health/hiv-aids/ (accessed 05/13)

McCoy, D., Montgomery, H., Arulkumaran, S. and Godlee, F. (2014) 'Climate change and human survival', British Medical Journal 348: g2351

McDonagh, J., Nienaber, B. and Woods, M. (eds) (2016) Globalization and Europe's Rural Regions, London: Routledge

McDorman, M., Declercq, E. and Menacker, F. (2011) 'Trends and characteristics of home births in the United States by race and ethnicity, 1990-2006', Birth 38: 17-23

McDowell, C. (2006) 'Displacement, return, and justice in the creation of Timor Leste', in N. Van Hear and C. McDowell (eds) Catching Fire: Containing Complex Displacement in a Volatile World, Lanham, MA: Lexington, pp. 181-212

McDowell, L., Batnitzky, A. and Dyer, S. (2009) 'Precarious work and economic migration: emerging immigrant divisions of labour in Greater London's service sector', International Journal of Urban and Regional Research 33(1): 3-25

McEwan, C. (2004) 'Transnationalism', in J. Duncan, N. Johnson and R. Schein (eds) A Companion to Cultural Geography, Malden, MA: Blackwell, pp. 499-512

McFalls, J. (2001) Population: A Lively Introduction, 4th edition, Population Bulletin 58(4), at: www.prb.org/pdf/populationlivelyintro.pdf (accessed 08/16)

McGuire, M. (2008) Lived Religion: Faith and Practice in Everyday Life, Oxford: Oxford University Press

McHugh, K. (2000) 'Inside, outside, upside down, backward, forward, round and round: a case for ethnographic studies in migration', Progress in Human Geography 24: 71-89

McHugh, K. (2003) 'Three faces of ageism: society, image and place', Ageing and Society 23(2): 165-185

McHugh, K. (2007) 'Generational consciousness and retirement communities', Population, Space and Place 13(4): 293-306

McHugh, K. and Mings, R. (1991) 'On the road again: seasonal migration to a sunbelt metropolis', Urban Geography 12: 1-18

McIntyre, N. (2006) 'Introduction', in N. Mclntyre, D. Williams and K. McHugh (eds) Multiple Dwelling and Tourism. Negotiating Place, Home and Identity, Wallingford: $\mathrm{CABI}, \mathrm{pp} .3-14$

McIntyre, N. (2009) 'Re-thinking amenity migration: integrating mobility, lifestyle and social-ecological systems', Die Erde140 (3): 229-250

McIntyre, N., Williams, D. and McHugh, K. (eds) (2006) Multiple Dwelling and Tourism. Negotiating Place, Home and Identity, Wallingford: $\mathrm{CABI}$

McKay, G. (1996) Senseless Acts of Beauty, London: Verso

McKendrick, J. (1999) 'Multi-method research: an introduction to its application in Population Geography', Professional Geographer 51(1): 40-50

McKenzie, F. (2010) 'Fly-in fly-out: the challenges of transient populations in rural landscapes', in G. Luck, D. Race and R. Black (eds) Demographic Change in Australia's Rural Landscapes, Dordrecht: Springer, pp. 353-374

McKeown, R. (2009) 'The Epidemiologic Transition: changing patterns of mortality and population dynamics', American Journal of Lifestyle Medicine 3(1): 19S-26S

McLeod, K. (2000) 'Our sense of Snow: the myth of John Snow in medical geography', Social Science and Medicine 50(7): 923-935

McLuhan, M. (1962) The Gutenberg Galaxy, Toronto: University of Toronto Press

McNabb, R. (1979) 'A socio-economic model of migration', Regional Studies 13: 297-304

McNeilly, A. (2002) 'Lactational endocrinology: the biology of LAM', in M. Davis, C. Isaacs, L. Hanson and A. Wright (eds) Integrating Population Outcomes, Biological Mechanisms and Research Methods in the Study of Human Milk and Lactation, Dordrecht: Springer, pp. 199-205

McNevin, A. (2006) 'Political belonging in a neoliberal era: the struggle of the sans-papiers', Citizenship Studies 10(2): 135-151

McPherson, K., Steel, C. and Dixon, J. (1994) 'ABC of breast diseases. Breast cancer - epidemiology, risk factors and genetics', British Medical Journal 309(6960): 1003-1006

McRae, S. (2003) 'Constraints and choices in mothers' employment careers: a consideration of Hakim's Preference Theory', British Journal of Sociology 54(3): 317-338

McWilliams, J. (2000) The 1960s Cultural Revolution, Westport, Conn.: Greenwood Press

Meadows, D., Meadows, D., Jorgens, R. and Behrens, W. (1974) The Limits to Growth: a Report for the Club of Rome's Project on the Predicament of Mankind, New York: Universe Books

Meek, J. (2014) Private Island: Why Britain Now Belongs to Someone Else, London: Verso 
Meijering, L. (2006) Making a Place of their Own. Rural Intentional Communities in Northwest Europe, Utrecht/Groningen: Netherlands Geographical Studies 34

Meijering, L., Huigen, P. and van Hoven, B. (2007) 'Intentional communities in rural space', Tijdschrift voor Economische en Sociale Geografie 98: 42-52

Melzer, S. (2011) 'Does migration make you happy? The influence of migration on subjective well-being', Journal of Social Research and Policy 2(2): 73-92

Mendoza, C. and Morén-Alegret, R. (2013) 'Exploring methods and techniques for the analysis of senses of place and migration', Progress in Human Geography 37(6): 762-785

Meng, X. and Zhang, J. (2001) 'The two-tier labor market in urban China: occupational segregation and wage differentials between urban residents and rural migrants in Shanghai', Journal of Comparative Economics 29(3): 485-504

Menjívar, C. (2013) 'Domestic violence, abuse, and migration', in I. Naess (ed.) The Encyclopedia of Global Human Migration, New Jersey: Wiley-Blackwell, at: http://onlinelibrary.wiley.com/doi/10.1002/9781444351071.wbeghm182/abstract (accessed $08 / 16)$

Merriman, P. (2012) Mobility, Space and Culture, Abingdon: Routledge

MetCouncil [Metropolitan Council] (2012) Population Estimates data download for 2010, at: http://stats.metc.state.mn.us/ data_download/(accessed 02/12)

Metrocosm (2016) 'The history of urbanization', at: http://metrocosm.com/map-history-cities.html (accessed 09/16)

Metykova, M. (2010) 'Only a mouse click away from home: transnational practices of Eastern European migrants in the United Kingdom', Social Identities 16(3): 325-338

Meyer, E. and Post, L. (2006) 'Alone at night. A feminist ecological model of community violence', Feminist Criminology 1(3): 207-227

Mezirow, J. (2000) 'Learning to think like an adult', in J. Mezirow and Associates Learning as Transformation, San Francisco: JesseyBass, pp. 3-33

Michelson, W. (1977) Environmental Choice, Human Behavior, and Residential Satisfaction, New York: Oxford University Press

Michielin, F. and Mulder, C. (2008) 'Family events and the residential mobility of couples', Environment and Planning A 40(11): 2770-2790

Middleton, J. (2011) 'Walking in the city: the geographies of everyday pedestrian practices', Geography Compass 5(2): 90-105

Middleton, N., Gunnell, D., Frankel, S., Whitley, E. and Dorling, D. (2003) 'Urban-rural differences in suicide trends in young adults: England and Wales, 1981-1998', Social Science and Medicine 57(7): 1183-1194

Middleton, N., Sterne, J. and Gunnell, D. (2008) 'An atlas of suicide mortality: England and Wales, 1988-1994', Health and Place 14(3): 492-506

Miles, M. and Crush, J. (1993) 'Personal narratives as interactive texts: collecting and interpreting migrant life-histories', Professional Geographer 45: 84-94

Milligan, C. (2009) There's No Place like Home: Place and Care in an Ageing Society, Farnham: Ashgate

Milligan, C. and Wiles, J. (2010) 'Landscapes of care', Progress in Human Geography 34(6): 736-754

Mills, M. (1997) 'Contesting the margins of modernity: women, migration, and consumption in Thailand', American Ethnologist 24(1): 37-61

Milne, W. (1991) 'The human capital model and its econometric estimation', in J. Stillwell and P. Congdon (eds) Migration Models. Macro and Micro Approaches, London: Belhaven Press, pp. 137-151

Milo, R. and Kahana, E. (2010) 'Multiple sclerosis: geoepidemiology, genetics and the environment', Autoimmunity Reviews 9: A387-A394

Mincer, J. (1978) 'Family migration decisions', Journal of Political Economy 86: 749-773

Mingay, G. (ed.) (1989) The Rural Idyll, London: Routledge

Mings, R. (1997) 'Tracking "snowbirds" in Australia: winter sun seekers in far north Queensland', Australian Geographical Studies 35(2): 168-182

Mirowsky, J. and Ross, C. (2003) Education, Social Status, and Health, New York: de Gruyter

Mitchell, C. (2004) 'Making sense of counterurbanization', Journal of Rural Studies 20: 15-34

Mitchell, R., Shaw, M. and Dorling, D. (2000) Inequalities in Life and Death, Bristol: Polity Press

Moen, P. (2003) 'Midcourse: navigating retirement and a new life stage', in J. Mortimer and M. Shanahan (eds) Handbook of the Life Course, New York: Kluwer Academic/Plenum, pp. 267-291

Mojumdar, A. (2012) 'I became pregnant again and again', Guardian 12 July: 13

Molho, I. (1986) 'Theories of migration: a review', Scottish Journal of Political Economy 33: 396-419

Möller-Leimkühler, A. (2003) 'The gender gap in suicide and premature death or: why are men so vulnerable?', European Archives of Psychiatry and Clinical Neuroscience 253(1): 1-8 


\section{BIBLIOGRAPHY}

Monbiot, G. (2006) Heat: How to Stop the Planet Burning, London: Allen Lane

Montepare, J. and Lachman, M. (1989) "'You're only as old as you feel": self-perceptions of age, fears of aging, and life satisfaction from adolescence to old age', Psychology and Aging 4(1): 73-78

Montgomery, J. (1962) Abodes of Love, London: Putnam

Montgomery, J. (1996) 'Components of refugee adaptation', International Migration Review 30(3): 679-702

Moon, B. (1995) 'Paradigms in migration research: exploring "moorings" as a schema', Progress in Human Geography 19: 504-524

Moore, S. (2016) Commentary, in Guardian, Second Section, p.5

Moorehead, C. (2005) Human Cargo: a Journey Among Refugees, London: Chatto and Windus

Moraes, H. (1995) Henrietta, London: Penguin

Moran, L., Skeggs, B., Corteen, K. and Tyrer, P. (2003) 'The formation of fear in gay space: the "straight" story', Capital and Class 80: 173-199

Morawska, E. (2001) 'Structuring migration: the case of Polish income-seeking travellers to the West', Theory and Society 30(1): $47-80$

Morgan, S. (2003) 'Is low fertility a twenty-first-century demographic crisis?', Demography 40(4): 589-603

Morley, D. (2000) Home Territories: Media, Mobility and Identity, London: Routledge

Mormont, M. (1987) 'Rural nature and urban natures', Sociologia Ruralis 27: 3-20

Morokvasic, M. (2004) 'Settled in mobility: engendering post-wall migration in Europe', Feminist Review 77(1): 7-25

Morris, J. (1986) The Matter of Wales, London: Penguin

Morrison, P. and Wheeler, J. (1976) 'Rural renaissance in America?', Population Bulletin 31(3): 1-27

Morrow-Jones, H. (1988) 'The housing life-cycle and the transition from renting to owning a home in the United States: a multistate analysis', Environment and Planning A 20:1165-1184

Mort, F. (1995) 'Archaeologies of city life: commercial culture, masculinity, and spatial relations in 1980s London', Environment and Planning D: Society and Space 13(5): 573-590

Mortimer, J. and Shanahan, M. (eds) (2003) Handbook of the Life Course, New York: Springer

Moscardo, G., Pearce, P., Morrison, A., Green, D. and O'Leary, J. (2000) 'Developing a typology for understanding visiting friends and relatives markets', Journal of Travel Research 38(3): 251-259

Moser, S. (2008) 'Personality: a new positionality?', Area 40: 383-392

Moss, L. (ed.) (2006) The Amenity Migrants: Seeking and Sustaining Mountains and Their Cultures, Wallingford: CAB International

Motlagh, J. (2014) 'These aren't refugee camps, they're concentration camps, and people are dying in them', Politzer Center on Crisis Reporting, at: http://pulitzercenter.org/reporting/asia-myanmar-burma-concentration-camps (accessed 08/16)

Mountford, A. (1997) 'Can a brain drain be good for growth in the source economy?', Journal of Development Economics 53(2): 287-303

Mountz, A. (2011) 'The enforcement archipelago: detention, haunting, and asylum on islands', Political Geography 30(3): 118-128

Mowl, G., Pain, R. and Talbot, C. (2000) 'The ageing body and the homespace', Area 32: 189-197

Mulder, C. (1993) Migration Dynamics: a Life Course Approach, Amsterdam: Thesis Publishers

Mulder, C. (2007) 'The family context and residential choice: a challenge for new research', Population, Space and Place 13, 265-278

Mulder, C. (2013) 'Family dynamics and housing: conceptual issues and empirical findings', Demographic Research 29: 355-378

Mulder, C. and Cooke, T. (2009) 'Family ties and residential locations', Population, Space and Place 15(4): 299-304

Mulder, C. and Wagner, M. (2001) 'The connections between family formation and first-time home ownership in the context of West Germany and the Netherlands', European Journal of Population/Revue Européenne de Demographie 17(2): 137-164

Murdoch, J. and Marsden, T. (1994) Reconstituting Rurality, London: UCL Press

Murphy, D. and Hall, C. (2011) 'Energy return on investment, peak oil, and the end of economic growth', Annals of the New York Academy of Sciences 1219(1): 52-72

Murphy, M. (1987) 'Measuring the family life cycle: concepts, data and methods', in A. Bryman, B. Bytheway, P. Allatt, and T. Keil (eds) Rethinking the Life Cycle, Basingstoke: Macmillan, pp. 30-50

Murphy, P. (2002) 'Sea Change: re-inventing rural and regional Australia', Transformations 2: 1-12

Murray, H. (2012) 'Hope for reform springs eternal: how the sponsorship system, domestic laws and traditional customs fail to protect migrant domestic workers in GCC Countries', Cornell International Law Journal 45: 461-485

Murshidi, M., Hijjawi, M., Jeriesat, S. and Eltom, A. (2013) 'Syrian refugees and Jordan's health sector', The Lancet 382(9888): 206-207

Murtagh, E. and Murphy, M. (2011) 'Active travel to school and physical activity of Irish primary schoolchildren', Pediatric Exercise Science 23: 230-236 
Myers, K. and Farquhar, D. (1998) 'Improving the accuracy of death certification', Canadian Medical Association Journal 158(10): $1317-1323$

Myklebost, H. (1989) 'Migration of elderly Norwegians', Norsk Geografisk Tidsskrift - Norwegian Journal of Geography 43(4): $191-213$

Naimark, N. (2001) Fires of Hatred: Ethnic Cleansing in Twentieth-Century Europe, Cambridge, MA: Harvard University Press Nam, C. (1994) Understanding Population Change, Illinois: F.E. Peacock, Inc.

NARM [North American Registry of Midwives] (2006) Annual Report, at: http://narm.org/pdffiles/2006AnnualReport.pdf (accessed $08 / 16)$

Nasser, N. (2003) 'South Asian ethnoscapes: the changing cultural landscapes of British cities', Global Built Environment Review 3: $26-39$

National Academy of Sciences (2015) Workshop on Rationalizing Rural Area Classifications. Presentations, April 16th-17th, Washington, D.C.: National Academies

Neal, T. (2013) A Labour of Leisure: an Ethnographic Account of a Village in Rural France, Unpublished PhD Thesis, Department of Town and Regional Planning, University of Sheffield

Nelson, L. and Wells, C. (2004) 'Global epidemiology of childhood tuberculosis', International Journal of Tuberculosis and Lung Disease 8(5): 636-647

Nelson, Z. (2014) 'Lampedusa boat tragedy: a survivor's story', Guardian 22nd March

Newbold, B. (1999) 'Evolutionary immigrant settlement patterns: concepts and evidence', in K. Pandit and S. Davies Withers (eds) Migration and Restructuring in the United States: a Geographic Perspective, New York: Rowman and Littlefield Publishers, pp. 250-272

Newbold, B. (2010) Population Geography, Lanham: Rowman and Littlefield Publishers

New Zealand Statistics (n.d.) 'Statistical Standard for Ethnicity 2005', at: www2.stats.govt.nz (accessed 06/15)

Ní Laoire, C. (2000) 'Conceptualising Irish rural youth migration: a biographical approach', International Journal of Population Geography 6: 229-243

Ní Laoire, C. (2007) 'The "green green grass of home"? Return migration to rural Ireland', Journal of Rural Studies 23: 332-344

Ní Laoire, C. (2008) '"Settling back"? A biographical and life-course perspective on Ireland's recent return migration', Irish Geography 41(2): 195-210

Niamir-Fuller, M. and Turner, M. (1999) 'A review of recent literature on pastoralism and transhumance in Africa', in M. Niamir-Fuller (ed.) Managing Mobility in African Rangelands: the Legitimization of Transhumance, London: Intermediate Technology Publictions, pp. 18-46

Nicholl, D. (2002) An Introduction to Genetic Engineering, Cambridge: Cambridge University Press

Nicholls, N. (2009) 'Estimating changes in mortality due to climate change', Climatic Change 97: 313-320

Niimi, Y., Ozden, C. and Schiff, M. (2010) 'Remittances and the brain drain: skilled migrants do remit less', Annals of Economics and Statistics/Annales d'Économie et de Statistique 97/98: 123-141

Nijhuis, M. (2014) 'The WWF's report on the shockingly rapid decline in wildlife should surely move us to action', Observer 5 October No Frontex Days Catania (2016) Homepage: http://nofrontex.blogspot.co.uk/p/blog-page_1.html (accessed 08/16)

No One is Illegal (2016) Homepage: www.noii.org.uk/ (accessed 08/16)

Norman, P., Boyle, P. and Rees, P. (2005) 'Selective migration, health and deprivation: a longitudinal analysis', Social Science and Medicine 60(12): 2755-2771

Norström, T. and Ramstedt, M. (2005) 'Mortality and population drinking: a review of the literature', Drug and Alcohol Review 24(6): $537-547$

North, P. (2010) 'Eco-localisation as a progressive response to peak oil and climate change - a sympathetic critique', Geoforum 41(4): 585-594

Northup, S. (1853) Twelve Years a Slave, Auburn, NY: Derby and Miller

Noulas, A., Scellato, S., Lambiotte, R., Pontil, M. and Mascolo, C. (2012) 'A tale of many cities: universal patterns in human urban mobility', PloS One 7(5): e37027, DOl:10.1371/journal.pone.0037027

Nowok, B., Van Ham, M., Findlay, A. and Gayle, V. (2013) 'Does migration make you happy? A longitudinal study of internal migration and subjective well-being', Environment and Planning A 45(4): 986-1002

NRDC [Natural Resources Defense Council] (2014) 'An introduction to climate change', at: www.nrdc.org/globalwarming/ climatebasics.asp (accessed 06/14)

Nurchayati (2011) 'Bringing agency back in: Indonesian migrant domestic workers in Saudi Arabia', Asian and Pacific Migration Journal 20(3-4): 479-502

O'Connell Davidson, J. (2006) 'Will the real sex slave please stand up?', Feminist Review 83(1): 4-22 


\section{BIBLIOGRAPHY}

O'Connell Davidson, J. (2010) 'New slavery, old binaries: human trafficking and the borders of "freedom"', Global Networks 10(2): 244-261

O'Connell Davidson, J. (2011) 'Moving children? Child trafficking, child migration, and child rights', Critical Social Policy 31(3): 454-477

O'Connor, J. (1991/2008) True Believers, London: Vintage

O'Connor, J. (1993) 'Introduction', in D. Bolger (ed.) Irish in Exile: Irish Writers Abroad, Dublin: New Island Books, pp. 11-18

O'Dell, T. (2009) 'My soul for a seat. Commuting and the routines of mobility', in E. Shove, F. Trentmann and W. Wilk (eds) Time, Consumption and Everyday Life, Oxford: Berg, pp. 85-98

O'Reilly, K. (2014) 'The role of the social imaginary in lifestyle migration: employing the ontology of practice theory', in M. Benson and N. Osbaldiston (eds) Understanding Lifestyle Migration, Basingstoke: Palgrave Macmillan, pp. 211-234

O'Reilly, K. and Benson, M. (2009) 'Lifestyle migration: escaping to the good life?', in M. Benson and K. O'Reilly (eds) Lifestyle Migration: Expectations, Aspirations and Experiences, Farnham: Ashgate, pp.1-13

OECD [Organisation for Economic Co-operation and Development] (2012) Education at a Glance 2012: Highlights, at: www.oecd.org/edu/EAG\%202012_e-book_EN_200912.pdf (accessed 08/16)

OECD (2016) 'Income inequality', at: https://data.oecd.org/inequality/income-inequality.htm (accessed 08/16)

Oels, A. (2013) 'Rendering climate change governable by risk: from probability to contingency', Geoforum 45: 17-29

Ogden, P. (2000) 'Weaving demography into society, economy and culture: progress and prospect in population geography', Progress in Human Geography 24: 627-640

Ohnmacht, T., Götz, K. and Schad, H. (2009) 'Leisure mobility styles in Swiss conurbations: construction and empirical analysis', Transportation 36(2): 243-265

Oliver, M. (1998) 'Theories of disability in health practice and research', British Medical Journal 317: 1446-1449

Olshansky, S. and Ault, B. (1986) 'The age of delayed degenerative diseases', Milbank Quarterly 64(3): 355-391

Olshansky, S., Carnes, B., Rogers, R. and Smith, L. (1997) 'Infectious diseases - new and ancient threats to world health', Population Bulletin 52(2): 1-52

Olwig, K. and Sørensen, N. (2002) 'Mobile livelihoods', in N. Sørensen and K. Olwig (eds) Work and Migration. Life and Livelihoods in a Globalizing World, London: Routledge, pp. 1-20

Ombelet, W., Cooke, I., Dyer, S., Serour, G. and Devroey, P. (2008) 'Infertility and the provision of infertility medical services in developing countries', Human Reproduction Update 14(6): 605-621

Omran, A. (1971) 'The Epidemiologic Transition: a theory of the epidemiology of population change', Milbank Memorial Fund Quarterly 49(4): 509-538

O'Neill, M. and Hubbard, P. (2010) 'Walking, sensing, belonging: ethno-mimesis as performative praxis', Visual Studies 25(1): 46-58

O'Neill, T. (2001) '"Selling girls in Kuwait": domestic labour migration and trafficking discourse in Nepal', Anthropologica 43(2): 153-164

O'Toole, C. (2002) 'Sex, disability and motherhood: access to sexuality for disabled mothers', Disability Studies Quarterly 22(4): $81-101$

Ono, M. (2015) 'Commoditization of lifestyle migration: Japanese retirees in Malaysia', Mobilities 10(4): 609-627

ONS [Office for National Statistics] (2013) Homepage: www.ons.gov.uk/ (accessed 11/13)

Onyx, J. and Leonard, R. (2005) 'Australian grey nomads and American snowbirds: similarities and differences', Journal of Tourism Studies 16(1): 61-68

Orbach, S. (1988) Fat is a Feminist Issue, 2nd edition, London: Arrow Books

Orubuloye, l., Caldwell, P. and Caldwell, J. (1993) 'The role of high-risk occupations in the spread of AIDS: truck drivers and itinerant market women in Nigeria', International Family Planning Perspectives 19(2): 43-48+71

Osbaldiston, N. (2010a) 'The authentic place in the amenity migration discourse', Space and Culture 20(1): 1-13

Osbaldiston, N. (2010b) 'Elementary forms of place in Seachange', Journal of Sociology 46(3): 239-256

Osbaldiston, N. (2012) Seeking Authenticity in Place, Culture, and the Self. The Great Urban Escape, New York: Palgrave Macmillan

Osbaldiston, N. (2014) 'Beyond ahistoricity and mobilities in lifestyle migration research', in M. Benson and N. Osbaldiston (eds) Understanding Lifestyle Migration, Basingstoke: Palgrave Macmillan, pp. 163-187

Osbaldiston, N. (2015) 'A cultural sociological reading of lifestyle migration', Two Homelands 42: 25-36

Osborne, H. (2014) 'London's soaring house prices make a long commute a little more attractive', Guardian 5 July

Osili, U. and Long, B. (2008) 'Does female schooling reduce fertility? Evidence from Nigeria', Journal of Development Economics 87(1): 57-75

Oswin, N. (2013) 'Geographies of sexualities: the cultural turn and after', in N. Johnson, R. Schein and J. Winders (eds) The WileyBlackwell Companion to Cultural Geography, Chichester: Wiley, pp. 105-117

\section{ST PROOFS: NOT FOR DISTRIBUTION}


Ouyang, H. (2013) 'Syrian refugees and sexual violence', The Lancet 381(9884): 2165-2166

Over the Hill (2014) Homepage: www.ruralmedia.co.uk/Over-The-Hill.aspx (accessed 11/14)

Overvåg, K. (2009) Second Homes in Eastern Norway, Trondheim: Norwegian University of Science and Technology, Doctoral Theses at NTNU 238

Owusu, G., Agyei-Mensah, S. and Lund, R. (2008) 'Slums of hope and slums of despair: mobility and livelihoods in Nima, Accra', Norsk Geografisk Tidsskrift 62: 180-190

Pachucki, M. and Breiger, R. (2010) 'Cultural holes: beyond relationality in social networks and culture', Annual Review of Sociology 36: 205-224

Pain, R. (1991) 'Space, sexual violence and social control: integrating geographical and feminist analyses of women's fear of crime', Progress in Human Geography 15(4): 415-431

Pain, R. (1997) 'Social geographies of women's fear of crime', Transactions of the Institute of British Geographers 22(2): 231-244

Pain, R. (2000) 'Place, social relations and the fear of crime: a review', Progress in Human Geography 24(3): 365-387

Pain, R. (2001) 'Age, generation and life course', in R. Pain, M. Barke, D. Fuller, J. Gough, R. MacFarlane and G. Mowl Introducing Social Geographies, London: Arnold, pp. 141-163

Pain, R. and Smith, S. (eds) (2008) Fear: Critical Geopolitics and Everyday Life, Farnham: Ashgate

Pallarès-Blanch, M., Prados Velasco, M. and Tulla Pujol, A. (2014) 'Naturbanization and urban-rural dynamics in Spain: case study of new rural landscapes in Andalusia and Catalonia', European Countryside 6(2): 118-160

Palmary, I., Burman, E., Chantler, K. and Kiguwa, P. (eds) (2010) Gender and Migration: Feminist Interventions, London: Zed Books

Palmer, C. (2013) 'We really needed a guidebook', Guardian 14 September, Family Section: 3

Palomba, R. and Kotowska, I. (2003) The Economically Active Population in Europe, Population Studies 40, Strasbourg: Council of Europe

Pandit, C. (2011) 'The impact of foreclosures on crime in American cities', Sociological Insight 3: 82-109

Paniagua, A. (2008) 'The environmental dimension in the constitution of new social groups in an extremely depopulated rural area of Spain (Soria)', Land Use Policy 25(1): 17-29

Paris, C. (2011) Affluence, Mobility and Second Home Owenership, London: Routledge

Parr, H. (2002) 'Medical geography: diagnosing the body in medical and health geography, 1999-2000', Progress in Human Geography 26: 240-251

Parr, H. and Butler, R. (1999) 'New geographies of illness, impairment and disability', in R. Butler and H. Parr (eds) Mind and Body Spaces: Geographies of Illness, Impairment and Disability, London: Routledge, pp. 1-24

Parreñas, R. (2001) Servants of Globalization: Women, Migration and Domestic Work, Stanford, CA: Stanford University Press

Parutis, V. (2011) 'Economic migrants" or "middling transnationals"? East European migrants' experiences of work in the UK', International Migration 52(1): 36-55

Pascual-de-Sans, À. (2004) 'Sense of place and migration histories. Idiotopy and idiotope' Area 36(4): 348-357

Passel, J. (2006) 'The size and characteristics of the unauthorized migrant population in the US', Pew Hispanic Center Research Report, March 7, at: http://citeseerx.ist.psu.edu/viewdoc/download?doi=10.1.1.693.412\&rep=rep1\&type=pdf (accessed 08/16)

Pattisson, P. (2013) 'Revealed: Qatar's World Cup "slaves"', Guardian 25 September

Pavlish, C. (2005) 'Action responses of Congolese refugee women', Journal of Nursing Scholarship 37(1): 10-17

Paxson, C. and Rouse, C. (2008) 'Returning to New Orleans after Hurricane Katrina', American Economic Review 98(2): 38-42

Peach, C. (2009) 'Slippery segregation: discovering or manufacturing ghettos?', Journal of Ethnic and Migration Studies 35(9): 1381-1395

Peake, L. (2010) 'Gender, race, sexuality', in S. Smith, R. Pain, S. Marston and JP Jones (eds) Handbook of Social Geographies, London: Sage, pp. 55-76

Pearce, J., Richardson, E., Mitchell, R. and Shortt, N. (2010) 'Environmental justice and health: the implications of the socio-spatial distribution of multiple environmental deprivation for health inequalities in the United Kingdom', Transactions of the Institute of British Geographers 35(4): 522-539

Pearce, J., Richardson, E., Mitchell, R. and Shortt, N. (2011) 'Environmental justice and health: a study of multiple environmental deprivation and geographical inequalities in health in New Zealand', Social Science and Medicine 73(3): 410-420

Peet, R. (1998) Modern Geographical Thought, Oxford: Blackwell

Pena, A. (2013) 'Remittances and undocumented migration', in I. Naess (ed.) The Encyclopedia of Global Human Migration, New Jersey: Wiley-Blackwell, at: http://onlinelibrary.wiley.com/doi/10.1002/9781444351071.wbeghm443/abstract (accessed 08/16) 


\section{BIBLIOGRAPHY}

Penz, G. (1992) 'Development refugees and distributive justice: indigenous peoples, land, and the developmentalist state', Public Affairs Quarterly 6(1): 105-131

Perec, G. (1968/2011) The Art and Craft of Approaching Your Head of Department to Submit a Request for a Raise, Translated by D. Bellos, London: Vintage

Perez, T. (2010) 'Climate refugees are a growing problem', in M. Haerens (ed.) Refugees, Detroit, Ml.: Greenhaven Press, pp. 31-35 Perry, G. (2014) Playing to the Gallery, London: Particular Books

Pessar, P. and Mahler, S. (2003) 'Transnational migration: bringing gender in', International Migration Review 37(3): 812-846

Peterson, G. (2013) 'Return migration', in I. Naess (ed.) The Encyclopedia of Global Human Migration, New Jersey: Wiley-Blackwell, at: http://onlinelibrary.wiley.com/doi/10.1002/9781444351071.wbeghm453/abstract (accessed 08/16)

Peterson, R. and Krivo, L. (1993) 'Racial segregation and black urban homicide', Social Forces 71(4): 1001-1026

Petroskas, D. (2011). Homelessness, Housing, and Health Geography: the Impact of Housing on the Health of Chronically III Homeless Adults, PhD dissertation, University of Minnesota, Minneapolis

Peutz, N. (2006) 'Embarking on an anthropology of removal', Current Anthropology 47(2): 217-231

Pherali, T. (2012) 'Academic mobility, language, and cultural capital: the experience of transnational academics in British Higher Education Institutions', Journal of Studies in International Education 16(4): 313-333

Phillimore, J. and Goodson, L. (2006) 'Problem or opportunity? Asylum seekers, refugees, employment and social exclusion in deprived urban areas', Urban Studies 43(10): 1715-1736

Phillips, D. (1994) 'The changing geography of disease distributions', Geografiska Annaler, Series B Human Geography 76(2): 71-89

Phillips, M. (1993) 'Rural gentrification and the processes of class colonisation', Journal of Rural Studies 9: 123-140

Phillips, T. (2015) 'China ends one child policy after 35 years', Guardian 29 October

Philo, C. (1992) 'Neglected rural geographies: a review', Journal of Rural Studies 8: 193-207

Philo, C. (1997) 'Of other rurals?', in P. Cloke and J. Little (eds) Contested Countryside Cultures, London: Routledge, pp. 19-50

Philo, C. (2000) 'More words, more worlds: reflections of the "cultural turn" and human geography', in I. Cook, D. Crouch, S. Naylor and J. Ryna (eds) Cultural Turns / Geographical Turns, Harlow: Prentice Hall, pp. 26-53

Philo, C. (2005) 'Sex, life, death, geography: fragmentary remarks inspired by "Foucault's population geographies"', Population, Space and Place 11(4): 325-333

Pickerill, J. and Krinsky, J. (2012) 'Why does Occupy matter?', Social Movement Studies 11(3-4): 279-287

Pickerill, J. and Maxey, L. (eds) (2009a) Low Impact Development. The Future in our Hands, Wordpress online, at www.jennypickerill.info/wp-content/uploads/Low-Impact-Development-Book.pdf (accessed 03/17)

Pickerill, J. and Maxey, L. (2009b) 'Geographies of sustainability: low impact developments and radical spaces of innovation', Geography Compass 3(4): 1515-1539

Pickett, K. and Pearl, M. (2001) 'Multilevel analyses of neighbourhood socioeconomic context and health outcomes: a critical review', Journal of Epidemiology and Community Health 55(2): 111-122

Piketty, T. (2014) Capital in the Twenty-First Century, Cambridge, MA: Harvard University Press

Pile, S. (1996) The Body and the City: Psychoanalysis, Space and Subjectivity, London: Routledge

Pile, S. (2010) 'Emotions and affect in recent human geography', Transactions of the Institute of British Geographers 35: 5-20

Pillay, N. (2013) 'Maternal mortality and morbidity: a human rights imperative', The Lancet 381: 1159-1160

Piore, M. (1979) Birds of Passage: Migrant Labour in Industrial Societies, Cambridge: Cambridge University Press

Plane, D. and Jurjevich, J. (2009) 'Ties that no longer bind? The patterns and repercussions of age-articulated migration', Professional Geographer 61(1): 4-20

Planned Parenthood (2012) Homepage: www.plannedparenthood.org/ (accessed 12/12)

Plumer, B. (2013) 'These maps show how Asia is taking over the oil markets', Washington Post Blog 26 August, at www.washingtonpost.com/news/wonk/wp/2013/08/26/these-maps-show-how-asia-is-taking-over-the-oil-markets/ (accessed 09/16)

Pogge, T. (2004) 'The first United Nations millennium development goal: a cause for celebration?', Journal of Human Development 5(3): 377-397

Polivka, L. (2011) 'Neoliberalism and postmodern cultures of aging', Journal of Applied Gerontology 30(2): 173-184

Pollak, J. and Slominski, P. (2009) 'Experimentalist but not accountable governance? The role of Frontex in managing the EU's external borders', West European Politics 32(5): 904-924

Pollard, S. (2013) 'Overcrowded UK cannot take another tide of immigrants', Daily Express 8 February

Pooley, C. (2013) 'Mobility', in I. Naess (ed.) The Encyclopedia of Global Human Migration, New Jersey: Wiley-Blackwell, at: http://onlinelibrary.wiley.com/doi/10.1002/9781444351071.wbeghm376/abstract (accessed 08/16)

Pooley, C. and Turnbull, J. (1998) Migration and Mobility in Britain since the Eighteenth Century, London: UCL Press

\section{ST PROOFS: NOT FOR DISTRIBUTION}


Pooley, C., Turnbull, J. and Adams, M. (2005) A Mobile Century?: Changes in Everyday Mobility in Britain in the Twentieth Century, Farnham: Ashgate

Porter, G. (2011) 'I think a woman who travels a lot is befriending other men and that's why she travels": mobility constraints and their implications for rural women and girls in sub-Saharan Africa', Gender, Place and Culture 18(1): 65-81

Porter, G., Hampshire, K., Abane, A., Robson, E., Munthali, A., Mashiri, M. and Tanle, A. (2010) 'Moving young lives: mobility, immobility and inter-generational tensions in urban Africa', Geoforum 41(5): 796-804

Porter, R. (1994) London. A Social History, London: Penguin

Portes, A. and Rumbaut, R. (2006) Immigrant America: a Portrait, 3rd edition, Berkeley: University of California Press

Portes, A., Fernández-Kelly, P. and Haller, W. (2009) 'The adaptation of the immigrant second generation in America: a theoretical overview and recent evidence', Journal of Ethnic and Migration Studies 35(7): 1077-1104

Portes, A., Guarnizo, L. and Landolt, P. (1999) 'The study of transnationalism: pitfalls and promises of an emergent research field', Ethnic and Racial Studies 22(2): 217-237

Posel, D. (2004) 'Have migration patterns in post-apartheid South Africa changed?', Journal of Interdisciplinary Economics 15(34): $277-292$

Poston, D. and Bouvier, L. (2010) Population and Society. An Introduction to Demography, Cambridge: Cambridge University Press

Poston, D., Davis, M. and Deng, D. (2012) 'Demographic structure and process in rural China', in L. Kulcsár and K. Curtis (eds) International Handbook of Rural Demography, New York: Springer, pp. 137-154

Potts, D. (2005) 'Counter-urbanisation on the Zambian copperbelt? Interpretations and implications', Urban Studies 42(4): 583-609

Pourrut, X., Kumulungui, B., Wittmann, T., Moussavou, G., Délicat, A., Yaba, P., Nkoghe, D., Gonzalez, J.-P. and Leroy, E. (2005) 'The natural history of Ebola virus in Africa', Microbes and Infection 7(7): 1005-1014

Pratt, G. (2000) 'Class', in R. Johnston, D. Gregory, G. Pratt and M. Watts (eds) The Dictionary of Human Geography, Oxford, Blackwell, 4th edition, pp. 85-88

Pray, L. (2008) 'Embryo screening and the ethics of human genetic engineering', Nature Education 1(1): 207

PRB [Population Reference Bureau] (2001) 'Understanding and using population projections', Washington D.C.: PRB, at: www.prb.org/Publications/Reports/2001/UnderstandingandUsingPopulationProjections.aspx (accessed 09/16)

PRB (2002) Family Planning Worldwide, 2002 Data Sheet, Washington D.C.: PRB

PRB (2003) Maternal Mortality a Leading Cause of Death in Cambodia, Washington, D.C.: PRB

PRB (2007) World Population Highlights 2007: Overview of World Population, at: www.prb.org/Publications/Articles/ 2007/623WorldPop.aspx (accessed 08/16)

PRB (2008) Family Planning Worldwide, 2008 Data Sheet, Washington D.C.: PRB

PRB (2009) 2009 World Population Data Sheet, Washington, D.C.: PRB

PRB (2010) 2010 World Population Data Sheet, Washington D.C.: PRB

PRB (2011) 'Educational differences in adult mortality in the United States', at: www.prb.org/Multimedia/Video/2011/us-educationmortality-policy-seminar.aspx (accessed 08/16)

PRB (2012) Population and Economic Development: 2012 Data Sheet, Washington, D.C.: PRB

PRB (2014) 2014 World Population Data Sheet, Washington D.C.: PRB

PRB (2015a) World Population Clock, at: www.prb.org/ (accessed 09/15)

PRB (2015b) The Urban-Rural Divide in Health and Development. Data Sheet, Washington, DC: PRB, at www.prb.org/pdf15/urbanrural-datasheet.pdf (accessed 08/16)

PRB (2015c) 'Infant Mortality Rate (infant deaths per 1,000 live births), 1970 and 2014', at: www.prb.org/DataFinder/Topic/ Rankings.aspx?ind=5 (accessed 01/16)

PRB Staff (2004) Transitions in World Population. Population Reference Bureau: Population Bulletin 59(1), Washington, D.C.: PRB

Price, M. and Benton-Short, L. (eds) (2008) Migrants to the Metropolis: the Rise of Immigrant Gateway Cities, Syracuse: Syracuse University Press

Prieto-Rodríguez, J. and Rodríguez-Gutiérrez, C. (2000) 'The added worker effect in the Spanish case', Applied Economics 32(15): 1917-1925

Pritchett, L. (1994) 'Desired fertility and the impact of population policies', Population and Development Review 20(1): 1-55

Proctor, J. (2006) 'Introduction: theorizing and studying religion', Annals of the Association of American Geographers 96: 165-168

Putnam, R. (2000) Bowling Alone: the Collapse and Revival of American Community, New York: Simon and Schuster

Querol V. (2010) 'Rural events, participation and mobilities among youth in Castelló Province', Paper presented at European Society for Rural Sociology Summer School, Córdoba, Spain, October

Rabe, B. and Taylor, M. (2010) 'Differences in opportunities? Wage, unemployment and house-price effects on migration', Institute for Social and Economic Research, University of Essex, Working Paper No. 2010-05 


\section{BIBLIOGRAPHY}

Rabušic, L. and Manea, B. (2008) 'Hakim s preference theory in the Czech context', Czech Demography 2: 46-55

Radl, J. (2012) 'Too old to work, or too young to retire? The pervasiveness of age norms in Western Europe', Work, Employment and Society 26(5): 755-771

Raghuram, P. (2013) 'Theorising the spaces of student migration', Population, Space and Place 19(2): 138-154

Raijnan, R., Schammah-Gesser, S. and Kemp, A. (2003) 'International migration, domestic work, and care work: undocumented Latina Migrants in Israel', Gender and Society 17(5): 727-749

Ralph, D. and Staeheli, L. (2011) 'Home and migration: mobilities, belongings and identities', Geography Compass 5(7): 517-530

Ramstedt, M. (2003) 'Alcohol consumption and liver cirrhosis mortality with and without mention of alcohol - the case of Canada', Addiction 98(9): 1267-1276

Ratha, D. (2013) 'Remittances and poverty alleviation in poor countries', in I. Naess (ed.) The Encyclopedia of Global Human Migration, New Jersey: Wiley-Blackwell, at: http://onlinelibrary.wiley.com/doi/10.1002/9781444351071.wbeghm441/abstract (accessed 08/16)

Ratha, D. and Silwal, A. (2012) 'Remittance flows in 2011: an update', World Bank Migration and Development Brief 18, at: https://openknowledge.worldbank.org/handle/10986/16176 (accessed 08/16)

Raymer, J., Abel, G. and Smith, P. (2007) 'Combining census and registration data to estimate detailed elderly migration flows in England and Wales', Journal of the Royal Statistical Society: Series A 170(4): 891-908

Rees, P. and Hardill, I. (2015) 'Later-life and internal migration', in D. Smith, N. Finney, K. Halfacree and N. Walford (eds) Internal Migration, Farnham: Ashgate, pp. 129-148

Rees, P., Wohland, P., Norman, P. and Boden, P. (2011) 'A local analysis of ethnic group population trends and projections for the UK', Journal of Population Research 28(2-3): 149-183

Refugees International (2003) 'Iraq: Focus on women's needs', 30 April

Reher, D. (2004) 'The demographic transition revisited as a global process', Population, Space and Place 10(1): 19-41

Rehkopf, D. and Buka, S. (2006) 'The association between suicide and the socio-economic characteristics of geographical areas: a systematic review', Psychological Medicine 36(2): 145-157

Rehm, J., Taylor, B., Mohapatra, S., Irving, H., Baliunas, D., Patra, J. and Roerecke, M. (2010) 'Alcohol as a risk factor for liver cirrhosis: a systematic review and meta-analysis', Drug and Alcohol Review 29(4): 437-445

Reisig, V. And Hobbiss, A. (2000) 'Food deserts and how to tackle them: a study of one city's approach', Health Education Journal 59(2): 137-149

Relph, E. (1976) Place and Placelessness, London: Pion

Rérat, P., Söderström, O. and Piguet, E. (2010) 'New forms of gentrification: issues and debates', Population, Space and Place 16: $335-343$

Reuveny. R. (2008) 'Eco-migration and violent conflict: case studies and public policy implications', Human Ecology 36: 1-13

Rican, S. and Salem, G. (2010) 'Mapping disease', in T. Brown, S. McLafferty and G. Moon (eds) A Companion to Health and Medical Geography, Oxford: Blackwell, pp. 96-110

Rich, A. (1980) 'Compulsory heterosexuality and lesbian experience', Signs 5: 631-661

Richards, P. (1999) 'Homicide statistics', UK House of Commons Research Paper No. 99/56

Riera, J. (2010) 'The boundaries between the refugee and migrant problems are often blurred' in M. Haerens (ed.) Refugees, Detroit, Ml.: Greenhaven Press, pp. 25-30

Rigby, A. (1974) Alternative Realities, London: Routledge and Kegan Paul

Rigg, J. (1997) Southeast Asia: the Human Landscape of Modernization and Development, London: Routledge

Rigg, J. (2006) 'Land, farming, livelihoods, and poverty: rethinking the links in the rural South', World Development 34: 180-202

Rigg, J. (2007) An Everyday Geography of the Global South, London: Routledge

Riley, J. (2001) Rising Life Expectancy: a Global History, Cambridge: Cambridge University Press

Riley, J. (2005) 'Estimates of regional and global life expectancy, 1800-2001', Population and Development Review 31(3): 537-543

Rindfuss, R., Entwisle, B., Walsh, S., Prasartku, P. Sawangdee, Y., Crawford, T. and Reade, J. (2002) 'Continuous and discrete: where they have met in Nang Rong, Thailand', in S. Walsh and K. Crews-Meyer (eds) Linking People, Place and Policy: a GIScience Approach, Boston: Kluwer Academic Publishers, pp.7-38

Ritchey, P. (1976) 'Explanations of migration', Annual Review of Sociology 2: 363-404

Rivera, M.J. (2004) 'Las representaciones sociales del habitar en la sociedad metropolitana', PhD thesis, Department of Sociology, Universidad Pública de Navarra

Rivera, M.J. (2007) 'Migration to rural Navarre: questioning the experience of counterurbanisation', Tijdschrift voor Economische en Sociale Geografie 98: 32-41

Rivza, B. and Teichler, U. (2007) 'The changing role of student mobility', Higher Education Policy 20(4): 457-475

\section{ST PROOFS: NOT FOR DISTRIBUTION}


Rizzo, A. (2014) 'Rapid urban development and national master planning in Arab Gulf countries. Qatar as a case study', Cities 39: 50-57

Robards, J., Evandrou, M., Falkingham, J. and Vlachantoni, A. (2014) 'Mortality at older ages and moves in residential and sheltered housing: evidence from the UK', Journal of Epidemiology and Community Health 68: 524-529

Robbins, P. (2004) Political Ecology: a Critical Introduction, Oxford: Blackwell

Robehmed, S. (2012) 'Why is Hebden Bridge the lesbian capital?', BBC New Magazine online, 9 February, at: www.bbc.co.uk/ news/magazine-16962898 (accessed 10/15)

Roberts, A. (2008) Albion Dreaming. A Popular History of LSD in Britain, London: Marshall Cavendish Limited

Roberts, M. (2005) 'Male infertility "is increasing"', BBC News 23 June, at: http://news.bbc.co.uk/1/hi/health/4118976.stm (accessed 08/16)

Robinson, V. (1998) 'Defining and measuring successful refugee integration', in Proceedings of ECRE International Conference on Integration of Refugees in Europe, Antwerp, November

Robinson, V. and Coleman, C. (2000) 'Lessons learned?: a critical review of the Government programme to resettle Bosnian quota refugees in the United Kingdom', International Migration Review 34(4): 1217-1244

Rockström, J. and 28 colleagues (2009) 'A safe operating space for humanity', Nature 461(7263): 472-475

Rodríguez, V., Fernández-Mayoralas, G. and Rojo, F. (2004) 'International retirement migration: retired Europeans living on the Costa del Sol, Spain', Population Review 43(1): 1-36

Rodríguez González, C., Bustillo Mesanza, R. and Mariel, P. (2011) 'The determinants of international student mobility flows: an empirical study on the Erasmus programme', Higher Education 62(4): 413-430

Rodríguez-Planas, N. and Farré, L. (2014) 'Migration, crisis and adjustment in an enlarged EU: the Spanish perspective', Forschungsinstitut zur Zukunft der Arbeit, IZA Discussion Paper No. 8091

Rofe, M. (2006) 'New landscapes of gated communities: Australia's Sovereign Islands', Landscape Research 31(3): 309-317

Rogers, A. (1967) 'A regression analysis of interregional migration in California', Review of Economics and Statistics 49: 262-267

Rogers, A. (1989) 'The elderly mobility transition: growth, concentration, and tempo', Research on Ageing 11: 3-32

Rogers, A. (1992a) 'Introduction', in A. Rogers (ed.) Elderly Migration and Population Redistribution, London: Belhaven Press, pp. $1-17$

Rogers, A. (1992b) 'Elderly migration and population redistribution in the United States', in A. Rogers (ed.) Elderly Migration and Population Redistribution, London: Belhaven Press, pp. 226-249

Rogers, A. and Castro, L. (1986) 'Migration', in A. Rogers and F. Willekens (eds) Migration and Settlement, Dordrecht: Reidel, pp. 157-209

Rogers, A., Castro, L. and Lea, M. (2005) 'Model migration schedules: three alternative linear parameter estimation methods', Mathematical Population Studies 12: 17-38

Rogers, A., Raquillet, R. and Castro, L. (1978) 'Model migration schedules and their applications', Environment and Planning A 10: 475-502

Rogers, R., Hummer, R., Nam, C. and Peters, K. (1996) 'Demographic, socioeconomic, and behavioral factors affecting ethnic mortality by cause', Social Forces 74(4): 1419-1438

Rogge, J. and Akol, J. (1989) 'Repatriation: its role in resolving Africa's refugee dilemma', International Migration Review 23(2): 184-200

Rolshoven, J. (2007) 'The temptations of the provisional. Multilocality as a way of life', Ethnologia Europaea 37(1/2): 17-25

Romano, D. (2005) 'Whose house is this anyway? IDP and refugee return in post-Saddam Iraq', Journal of Refugee Studies 18(4): $430-453$

Roper, J., Ganesh, S. and Inkson, K. (2010) 'Neoliberalism and knowledge interests in boundaryless careers discourse', Work, Employment and Society 24(4): 661-679

Rose, A., Sinka, K., Watson, J., Mortimer, J. and Charlett, A. (2002) 'An estimate of the contribution of HIV infection to the recent rise in tuberculosis in England and Wales', Thorax 57(5): 442-445

Rose, D. (1984) 'Rethinking gentrification: beyond the uneven development of Marxist urban theory', Environment and Planning D: Society and Space 2(1): 47-74

Rose, D. (1989) 'A feminist perspective of employment restructuring and gentrification: the case of Montréal', in J. Wolch and M. Dear (eds) The Power of Geography, Boston: Unwin Hyman, pp. 118-138

Rose, D. and Le Bourdais, C. (1986) 'The changing conditions of female single parenthood in Montréal's inner city and suburban neighborhoods', Urban Resources 3: 45-52

Rose, G. (1997) 'Situated knowledges: positionalities, reflexivities and other tactics', Progress in Human Geography 21: 305-320

\section{ST PROOFS: NOT FOR DISTRIBUTION}




\section{BIBLIOGRAPHY}

Rose, M. (2006) 'Gathering "dreams of presence": a project for the cultural landscape', Environment and Planning D: Society and Space 24: 537-554

Roseman, C. (1971) 'Migration as a spatial and temporal process', Annals of the Association of American Geographers 61: 589-598

Rosen, G. and Grant, J. (2011) 'Reproducing difference: gated communities in Canada and Israel', International Journal of Urban and Regional Research 35(4): 778-793

Rosenberg, M. (2011) 'New spaces of the geographies of health and health care', Geojournal 76: 109

Ross, N. (2007) '"My journey to school ... ": foregrounding the meaning of school journeys and children's engagements and interactions in their everyday localities', Children's Geographies 5(4): 373-391

Rossi, P. (1955) Why Families Move, Glencoe, Illinois: Free Press

Roudi-Fahimi, F., El Feki, S. and Tsai, T. (2011) Youth Revolt in Egypt, a Country at the Turning Point, Washington D.C.: Population Reference Bureau

Rovin, K., Hardee, K. and Aklilu, K. (2013) 'Linking population, fertility, and family planning with adaptation to climate change: perspectives from Ethiopia', African Journal of Reproductive Health 17(3): 15-29

Rowles, G. (1978) Prisoners of Space? Exploring the Geographical Experience of Older People, Boulder, CO: Westview Press

Rowles, G. (1983) 'Between worlds: a relocation dilemma for the Appalachian elderly', International Journal of Aging and Human Development 17(4): 301-314

Rubin, H. (2003) Self-made Men: Identity and Embodiment Among Transsexual Men, Nashville: Vanderbilt University Press

Ruddick, S. (2003) 'The politics of aging: globalization and the restructuring of youth and childhood', Antipode 35: 335-364

Rudrappa, S. (2010) 'Making India the "mother destination": outsourcing labor to Indian surrogates', Research in the Sociology of Work 20: 253-285.

Ruíz-Godoy, L., Rios, P., Cervantes, F., Osornio-Vargas, A., García-Cuellar, C. and García, A. (2007) 'Mortality due to lung cancer in Mexico', Lung Cancer 58(2): 184-190

Ryan, D., Cooley, B. and Benson, C. (2008) 'Theoretical perspectives on post-migration adaptation and psychological well-being: towards a resource-based model', Journal of Refugee Studies 21(1): 1-18

Ryan, L. and Sales, R. (2013) 'Family migration: the role of children and education in family decision-making strategies of Polish migrants in London', International Migration 51(2): 90-103

Ryan, L., Sales, R., Tilki, M. and Siara, B. (2009) 'Family strategies and transnational migration: recent Polish migrants in London', Journal of Ethnic and Migration Studies 35(1): 61-77

Sabel, C., Pringle, D. and Schoerström, A. (2010) 'Infectious disease diffusion', in T. Brown, S. McLafferty and G. Moon (eds) A Companion to Health and Medical Geography, Maryland: Blackwell, pp. 111-132

Sabotka, T., Zeman, K. and Kantorova, V. (2003) 'Demographic shifts in the Czech Republic after 1989: a second demographic transition view', European Journal of Population 19(3): 249-277

Sachs, J. (2007) 'Bursting at the seams', BBC Reith Lecture, at: www.bbc.co.uk/radio4/reith2007/lecture1.shtml (accessed 09/16)

Sachs, J. (2012) 'From millennium development goals to sustainable development goals', The Lancet 379(9832): 2206-2211

Saenz, R. (2007) 'The growing color divide in U.S. infant mortality', Population Reference Bureau, at: www.prb.org/Publications/ Articles/2007/ColorDivideinInfantMortality.aspx (accessed 08/16)

Sage, J., Evandrou, M. and Falkingham, J. (2013) 'Onwards or homewards? Complex graduate migration pathways, well-being, and the "parental safety net"', Population, Space and Place 19(6): 738-755

Sager, T. (2006) 'Freedom as mobility: implications of the distinction between actual and potential travelling', Mobilities 1(3): 465-488

Saith, A. (2006) 'From universal values to Millennium Development Goals: lost in translation', Development and Change 37(6): $1167-1199$

Salazar, N. (2010) 'Tourism and cosmopolitanism: a view from below', International Journal of Tourism Anthropology 1(1): 55-69

Salazar, N. (2014) 'Migrating imaginaries of a better life ... until paradise finds you', in M. Benson and N. Osbaldiston (eds) Understanding Lifestyle Migration, Basingstoke: Palgrave Macmillan, pp. 119-138

Samers, M. (2008) 'At the heart of "migration management": immigration and labour markets in the European Union', in C. Gabriel and H. Pellerin (eds) Governing International Labour Migration: Current Issues, Challenges and Dilemmas, London: Routledge, pp. $128-144$

Samers, M. (2010) Migration, London: Routledge

Sami, S., Williams, H., Krause, S., Onyango, M., Burton, A. and Tomczyk, B. (2014) 'Responding to the Syrian crisis: the needs of women and girls', The Lancet 383(9923): 1179-1181

Sanchez, L. and Pancheco, E. (2012) 'Rural population trends in Mexico: demographic and labor changes', in L. Kulcsár and K. Curtis (eds) International Handbook of Rural Demography, New York: Springer, pp. 155-168 
Sankar, P., Cho, M., Condit, C., Hunt, L., Koenig, B., Marshall, P., Lee, S. and Spicer, P. (2004) 'Genetic research and health disparities', Journal of the American Medical Association 291(24): 2985-2989

Santana, P. (2000) 'Ageing in Portugal: regional iniquities in health and health care', Social Science and Medicine 50(7): 1025-1036

Santos, A., McGuckin, N., Nakamoto, H., Gray, D. and Liss, S. (2011) Summary of Travel Trends. 2009 National Household Travel Survey, US Department of Transportation, Federal Highway Administration, Report No. FHWA-PL-II-022

Sargisson, L. (2001) 'Politicising the quotidian', Environmental Politics 10: 68-89

Sargisson, L. (2007) 'Imperfect utopias: green intentional communities', Ecopolitics Online 1(1): 1-24

Sassen, S. (1991) The Global City: New York, London, Tokyo, Princeton, NJ: Princeton University Press

Sassen, S. (1998) Globalization and its Discontents. Essays on the New Mobility of People and Money, New York: New Press

Sassoon, J. (2008) Iraqi Refugees: the New Crisis in the Middle East, London: IB Tauris

Savage, M. (2010) 'The politics of elective belonging', Housing, Theory and Society 27(2): 115-161

Savage, M., Bagnall, G. and Longhurst, B. (2005) Globalization and Belonging, London: Sage

Scarpa, S. (2008) Trafficking in Human Beings: Modern Slavery, Oxford: Oxford University Press

Schatzki, T. (2009) 'Timespace and the organization of social life', in E. Shove, F. Trentmann and W. Wilk (eds) Time, Consumption and Everyday Life, Oxford: Berg, pp. 35-48

Schewel, K. (2015) 'Understanding the aspiration to stay: a case study of young adults in Senegal', University of Oxford, Centre on Migration, Policy and Society, Working Paper No. 119

Schillmeier, M. (2011) 'Unbuttoning normalcy - on cosmopolitical events', Sociological Review 59: 514-534

Schlich, R., Schönfelder, S., Hanson, S. and Axhausen, K. (2004) 'Structures of leisure travel: temporal and spatial variability', Transport Reviews 24(2): 219-237

Schootman, M., Lian, M., Deshpande, A., McQueen, A., Pruitt, S. and Jeffe, D. (2011) 'Temporal trends in geographic disparities in small-area-level colorectal cancer incidence and mortality in the United States', Cancer Causes Control 22(8): 1173-1181

Schultz, C. (2014) 'Nearly 6,000 migrants have died along the Mexico-U.S. border since 2000', Smithsonian.com October 1, at: www.smithsonianmag.com/smart-news/nearly-6000-migrants-have-died-along-mexico-us-border-2000-180952904/?no-ist (accessed 03/17)

Schuster, L. (2005) 'A sledgehammer to crack a nut: deportation, detention and dispersal in Europe', Social Policy and Administration 39(6): 606-621

Schuster, L. (2011) 'Turning refugees into "illegal migrants": Afghan asylum seekers in Europe', Ethnic and Racial Studies 34(8): 1392-1407

Schuster, L. and Majidi, N. (2013) 'What happens post-deportation? The experience of deported Afghans', Migration Studies 1(2): $221-240$

Scott, G. (2000) "What goes around comes around": informal activities and reciprocity networks in Westport, New Zealand', New Zealand Geographer 56(1): 39-45

Scott, J. (1988) Gender and the Politics of History, New York: Columbia University Press

Scott, S. (2006) 'The social morphology of skilled migration: the case of the British middle class in Paris', Journal of Ethnic and Migration Studies 32(7): 1105-1129

Scudder, T. (2005) The Future of Large Dams: Dealing with Social, Environmental, Institutional and Political Costs, London: Earthscan

Selman, P. (2009) 'The rise and fall of intercountry adoption in the 21st century', International Social Work 52(5): 575-594

Senior, M. (1979) 'From gravity modelling to entropy maximising: a pedagogic guide', Progress in Human Geography 3: 175-210

Şerban, M. and Voicu, B. (2010) 'Romanian migrants to Spain: in- or outside the migration networks. A matter of time?', Revue D'études Comparatives Est-Ouest 41(04): 97-124

Serbanescu, F., Morris, L., Stupp, P. and Stanescu, A. (1995) 'The impact of recent policy changes on fertility, abortion, and contraceptive use in Romania', Studies in Family Planning 26(2): 76-87

Settersten, R. (2003) 'Age structuring and the rhythm of the life course', in J. Mortimer and M. Shanahan (eds) Handbook of the Life Course, New York: Springer, pp. 81-98

Shaffer, M., Kraimer, M., Chen, Y. and Bolino, M. (2012) 'Choices, challenges, and career consequences of global work experiences: a review and future agenda', Journal of Management 38(4): 1282-1327

Shah, N. (1995) 'Structural changes in the receiving country and future labor migration - the case of Kuwait', International Migration Review 29(4): 1000-1022

Shakespeare, T. (1993) 'Disabled people's self-organisation: a new social movement?', Disability, Handicap and Society 8: 249-264

Shakespeare, T. (2006) Disability Rights and Wrongs, London: Routledge

Shanahan, M. (2000) 'Pathways to adulthood in changing societies: variability and mechanisms in life course perspective', Annual Review of Sociology 26: 667-692 


\section{BIBLIOGRAPHY}

Shanahan, M. and Elder, G. (2002) 'History, agency, and the life course', in R. Dienstbier and L. Crockett (eds) Agency, Motivation, and the Life Course, Lincoln, NE: University of Nebraska Press, pp. 145-186

Shanahan, M. and Macmillan, R. (2008) Biography and the Sociological Imagination: Contexts and Contingencies, New York: WW Norton and Company

Sharkey, J. (2009) 'Measuring potential access to food stores and food-service places in rural areas in the US', American Journal of Preventive Medicine 36(4): S151-S155

Sharlet, J. (2010) 'Straight man's burden. The American roots of Uganda's anti-gay persecutions', Harper's Magazine September: $36-48$

Shaw, M., Mitchell, R. and Dorling, D. (2000) 'Time for a smoke? One cigarette reduces your life by 11 minutes', British Medical Journal 320, 1 January: 53

Shaw, M., Tunstall, H. and Dorling, D. (2005) 'Increasing inequalities in risk of murder in Britain: trends in the demographic and spatial distribution of murder, 1981-2000', Health and Place 11(1): 45-54

Shaw, M., Davey Smith, G., Thomas, B. and Dorling, D. (2008) The Grim Reaper's Road Map: an Atlas of Mortality in Britain, Bristol: Policy Press

Sheller, M. and Urry, J. (2006) 'The new mobilities paradigm', Environment and Planning A 38: 207-226

Shelton, N., Birkin, M. and Dorling, D. (2006) 'Where not to live: a geo-demographic classification of mortality for England and Wales, 1981-2000', Health and Place 12(4): 557-569

Shen, J. (1998) 'China's future population and development challenges', Geographical Journal 164(4): 32-40

Shen, Q. (2000) 'Spatial and social dimensions of commuting', Journal of the American Planning Association, 66(1): 68-82

Shinozaki, K. (2004) Negotiating Citizenship in Transnational Migration: the Case of Filipina and Filipino Migrant Domestic Workers in Germany, Ph.D. dissertation, Ochanomizu University, Japan

Shkolnikov, V., McKee, M. and Leon, D. (2001) 'Changes in life expectancy in Russia in the mid-1990s', The Lancet 357(9260): 917-921

Short, B. (2006) 'Idyllic ruralities', in P. Cloke, T. Marsden and P. Mooney (eds) Handbook of Rural Studies, London: Sage, pp.133-148

Short, J. (1991) Imagined Country, London: Routledge

Shortt, N., Richardson, E., Pearce, J. and Mitchell, R. (2012) 'Mortality inequalities by environment type in New Zealand', Health and Place 18(5): 1132-1136

Shove, E., Trentmann, F. and Wilk, W. (eds) (2009) Time, Consumption and Everyday Life, Oxford: Berg

Shrestha, L. (2006) 'The changing demographic profile of the United States', Congressional Research Service Report for Congress, Washington D.C., at: www.dtic.mil/dtic/tr/fulltext/u2/a462164.pdf (accessed 08/16)

Shryock, H. (1964) Population Mobility within the United States, Chicago: Community and Family Study Center, University of Chicago Shubin, S. (2012) 'Living on the move: mobility, religion and exclusion of Eastern European migrants in rural Scotland', Population, Space and Place 18(5): 615-627

Shubin, S. (2015) 'Migration timespaces: a Heideggerian approach to understanding the mobile being of Eastern Europeans in Scotland', Transactions of the Institute of British Geographers 40(3): 350-361

Shucksmith, M. (1983) 'Second homes - a framework for policy', Town Planning Review 54: 174-193

Shutes, I. (2011) 'Social care for older people and demand for migrant workers', Migration Observatory, University of Oxford, at: www.migrationobservatory.ox.ac.uk/policy-primers/social-care-older-people-and-demand-migrant-workers (accessed 09/16)

Silvers, A. (1977) 'Probabilistic income-maximising behaviour in regional migration' International Regional Science Review 2: 29-40

Silvey, R. (2001) 'Migration under crisis: household safety nets in Indonesia's economic collapse', Geoforum 32(1): 33-45

Silvey, R. (2004) 'Power, difference and mobility: feminist advances in migration studies', Progress in Human Geography 28(4): 490-506

Silvey, R. (2006a) 'Geographies of gender and migration: spatializing social difference', International Migration Review 40(1): 64-81

Silvey, R. (2006b) 'Consuming the transnational family: Indonesian migrant domestic workers to Saudi Arabia', Global Networks 6(1): $23-40$

Silvey, R. and Lawson, V. (1999) 'Placing the migrant', Annals of the Association of American Geographers 89: 121-132

Simini, F., González, M., Maritan, A. and Barabási, A. (2012) 'A universal model for mobility and migration patterns', Nature 484(7392): 96-100

Šimon, M. (2014) 'Exploring counterurbanisation in a post-socialist context: case of the Czech Republic', Sociologia Ruralis 54(2): $117-142$

Simpson, D. (2008) 'Nomadic urbanism: the senior full-time recreational vehicle community', Interstices: Journal of Architecture and Related Arts, at: http://interstices.aut.ac.nz/ijara/index.php/ijara/article/download/143/217 (accessed 08/16) 
Sinding, S. (2007) 'Overview and perspective', in W. Robinson and J. Ross (eds) The Global Family Planning Revolution: Three Decades of Population Policies and Programs, Washington D.C.: World Bank, pp. 1-12

Singh, M. and Shandilya, S. (2012) 'Internal migration in India', Journal of Business Management \& Social Sciences Research 1(3): 66-69

Singh, P. and Velásquez, S. (2013) 'Remittances and poverty alleviation, Ecuador and Bangladesh', in I. Naess (ed.) The Encyclopedia of Global Human Migration, New Jersey: Wiley-Blackwell, at: http://onlinelibrary.wiley.com/doi/10.1002/9781444351071. wbeghm442/abstract (accessed 08/16)

Singh, S., Casterline, J. and Cleland, J. (1985) 'The proximate determinants of fertility: sub-national variations', Population Studies 39(1): 113-135

Singhal, A. and Rogers, E. (1989) India's Information Revolution, London: Sage

Singhal, A., Cody, M., Rogers, E. and Sabido, M. (2003) Entertainment-Education and Social Change: History, Research and Practice, Mahway, NJ: Lawrence Erlbaum Associates

Sjaastad, L. (1962) 'The costs and returns of migration', Journal of Political Economy 70: 80-93

Skeldon, R. (1995) 'The challenge facing migration research: the case for greater awareness', Progress in Human Geography 19 : 91-96

Skeldon, R. (2008) 'International migration as a tool in development policy: a passing phase?', Population and Development Review 34(1): $1-18$

Skelton, T. (2002) 'Research on youth transitions: some critical interventions', in M. Cieslik and G. Pollock (eds) Young People in Risk Society, Aldershot: Ashgate, pp. 100-116

Skelton, T. and Hamed, N. (2011) 'Adult anxieties versus young people's resistance: negotiating access to public space in Singapore', in L. Holt (ed.) Geographies of Children, Youth and Families, Abingdon: Routledge, pp. 203-220

Skinner, M., Cloutier, D. and Andrews, G. (2015) 'Geographies of ageing. Progress and possibilities after two decades of change', Progress in Human Geography 39(6): 776-799

Slater, T. (2006) 'The eviction of critical perspectives from gentrification research', International Journal of Urban and Regional Research 30(4): 737-757

Slater, T. (2011) 'Gentrification of the city', in G. Bridge and S. Watson (eds) The New Blackwell Companion of the City, Oxford: Blackwell, pp. 571-585

Slater, T. (2013) 'Expulsions from public housing: the hidden context of concentrated affluence', Cities 35: 384-390

Sloan, M. (2013) 'The post-move satisfaction of individuals moving within New Zealand', PhD Thesis, Victoria University of Wellington

Smailes, P. (1997) 'Socio-economic change and rural morale in South Australia, 1982-1993', Journal of Rural Studies 13(1): 19-42

Smaje, C. (2013) '"Decreasing the wealth of the kingdom": poverty statistics and statistical poverty', 30 July, at: http://stats. cwslive.wiley.com/details/feature/5063831/Decreasing-the-wealth-of-the-kingdom-poverty-statistics-and-statisticalpoverty.html (accessed 08/16)

Small, M., Harding, D. and Lamont, M. (2010) 'Introduction: reconsidering culture and poverty', Annals of the American Academy of Political and Social Science 629: 6-27

Smith, D. (1984) 'Recollections of a random variable', in M. Billinge, D. Gregory and R. Martin (eds) Recollections of a Revolution, London: Macmillan, pp. 117-133

Smith, D. (2002) 'Patterns and processes of studentification in Leeds', Regional Review April: 6-7

Smith, D. (2009) ' "Student geographies", urban restructuring, and the expansion of higher education', Environment and Planning A 41(8): 1795-1804

Smith, D. (2011) 'Geographies of long-distance family migration: moving to a "spatial turn"', Progress in Human Geography 35(5): 652-668

Smith, D. and Higley, R. (2012) 'Circuits of education, rural gentrification, and family migration from the global city', Journal of Rural Studies 28(1): 49-55

Smith, D. and Holt, L. (2005) '"Lesbian migrants in the gentrified valley" and "other" geographies of rural gentrification', Journal of Rural Studies 21: 313-322

Smith, D. and Holt, L. (2007) 'Studentification and "apprentice" gentrifiers within Britain's provincial towns and cities: extending the meaning of gentrification', Environment and Planning A 39(1): 142-161

Smith, D. and Jons, H. (2015) 'Education and internal migration', in D. Smith, N. Finney, K. Halfacree and N. Walford (eds) Internal Migration, Farnham: Ashgate, pp. 47-63

Smith, D. and King, R. (2012) 'Editorial Introduction: re-making migration theory', Population, Space and Place 18(2): 127-133

Smith, D. and Phillips, D. (2001) 'Socio-cultural representations of greentrified Pennine rurality', Journal of Rural Studies 17: 457-469

\section{ST PROOFS: NOT FOR DISTRIBUTION}




\section{BIBLIOGRAPHY}

Smith, J. and Jehlička, P. (2007) 'Stories around food, politics and change in Poland and the Czech Republic', Transactions of the Institute of British Geographers 32(3): 395-410

Smith, M. (2001) Transnational Urbanism: Locating Globalization, Oxford: Blackwell

Smith, M. and Guarnizo, L. (1998) (eds) Transnationalism from Below, New Brunswick, NJ: Transaction Publishers

Smith, N. (1979) 'Towards a theory of gentrification: aback to the city movement by capital not people', American Planning Association Journal 45: 538-548

Smith, N. (1984) Uneven Development, Oxford: Blackwell

Smith, N. (1996) The New Urban Frontier, London: Routledge

Smith, N. (2002) 'New globalism, new urbanism: gentrification as global urban strategy', Antipode 34(3): 427-450

Smith, N. and Williams, P. (eds) (1986) Gentrification of the City, London: Routledge

Smith, P. (1999) 'The elementary forms of place and their transformations: a Durkheimian model', Qualitative Sociology 22(1): 13-36

Sobotka, T., Skirbekk, V. and Philipov, D. (2011) 'Economic recession and fertility in the developed world', Population and Development Review 37(2): 267-306

Solana-Solana, M. (2010) 'Rural gentrification in Catalonia, Spain: a case study of migration, social changes and conflicts in the Empordanet area', Geoforum 41: 508-517

Sorkin, M. (1992) Variations on a Theme Park: The New American City and the End of Public Space, New York: Hill and Wang

Southall, H. (1991) 'The tramping artisan revisits: labour mobility and economic distress in early Victorian England', Economic History Review 44(2): 272-296

Southerton, D. (2009) 'Re-ordering temporal rhythms: coordinating daily practices in the UK in 1937 and 2000', in E. Shove, F. Trentmann and W. Wilk (eds) Time, Consumption and Everyday Life, Oxford: Berg, pp. 49-63

Spar, D. (2006) The Baby Business: How Money, Science, and Politics Drive the Commerce of Conception, Cambridge, Mass.: Harvard Business School Press

Sparke, M. (2009) 'Triangulating globalization', Journal of Historical Geography 35(2): 376-381

Sparks, P. (2012) 'Rural health disparities', in L. Kulcsár and K. Curtis (eds) International Handbook of Rural Demography, New York: Springer, pp. 255-271

Speare A. and Goldscheider, F. (1987) 'Effects of marital status change on residential mobility', Journal of Marriage and the Family 49(2): 455-464

Sperry, S. (2008) 'The politics of adoption', National Geographic Magazine 11 February

Spicer, N. (2008) 'Places of exclusion and inclusion: asylum-seeker and refugee experiences of neighbourhoods in the UK', Journal of Ethnic and Migration Studies 34(3): 491-510

Spijker, J. and Maclnnes, J. (2013) 'Population ageing: the timebomb that isn't', British Medical Journal 347(6598): 1-5

Spinney, J. (2011) 'A chance to catch a breath: using mobile video ethnography in cycling research', Mobilities 6(2): 161-182

Sporton, D. (1999) 'Mixing methods in fertility research', Professional Geographer 51(1): 68-76

Srivastava, R. (2005) 'Bonded labor in India: its incidence and pattern', Working Paper, Cornell University ILR School, at: http://digitalcommons.ilr.cornell.edu/forcedlabor/18/ (accessed 08/16)

Stack, S. (2000) 'Suicide: a 15-year review of the sociological literature part I: cultural and economic factors', Suicide and LifeThreatening Behavior 30(2): 145-162

Stafford, R. (2009) Elderburbia: Aging with a Sense of Place in America, Santa Barbara, CA: Praeger

Stansell, C. (2010) 'Global feminism in a conservative age: possibilities and pieties since 1980', Dissent 57(2): 49-53

Stark, O. (1991) The Migration of Labor, Oxford: Blackwell

Stark, O. and Bloom, D. (1985) 'The new economics of labor migration', American Economic Review 75: 173-178

Statewatch (2012) Homepage: www.statewatch.org (accessed 12/12)

Statistics South Africa (2014) Homepage: http://cs2016.statssa.gov.za/ (accessed 10/14)

Stavropoulou, M. (2008) 'Drowned in definitions?', Forced Migration Review 31: 11-12

Stein, B. (1983) 'The commitment to refugee resettlement', Annals of the American Academy of Political and Social Science 467: 187-201.

Stein, B. (1986) 'Durable solutions for Developing Country refugees', International Migration Review 20(2): 264-282

Stern, N. (2006) Stern Review on the Economics of Climate Change, Cambridge: Cambridge University Press

Stevenson, D. (2003) Cities and Urban Cultures, Maidenhead: Open University Press

Stiglitz, J. (2012) The Price of Inequality, New York: WW Norton and Company

Stockdale, A. (2006) 'Migration: pre-requisite for rural economic regeneration?', Journal of Rural Studies 22(3): 354-366

Stockdale, A. (2014) 'Unravelling the migration decision-making process: English early retirees moving to rural mid-Wales', Journal of Rural Studies 34: 161-171 
Stockdale, A. and MacLeod, M. (2013) 'Pre-retirement age migration to remote rural areas', Journal of Rural Studies 32: 80-92

Stockdale, A., MacLeod, M. and Philip, L. (2013) 'Connected life courses: influences on and experiences of "midlife" in-migration to rural areas', Population, Space and Place 19(3): 239-257

Storey, D. (2012) Territories, 2nd edition, Abingdon: Routledge

Storey, K. (2001) 'Fly-in/fly-out and fly-over: mining and regional development in Western Australia', Australian Geographer 32(2): $133-148$

Storey, K. (2010) 'Fly-in/fly-out: implications for community sustainability', Sustainability 2(5): 1161-1181

Stouffer, S. (1940) 'Intervening opportunities: a theory relating mobility and distance', American Sociological Review 5: 845-867

Stoyanov, P. and Frantz, K. (2006) 'Gated communities in Bulgaria: interpreting a new trend in post-communist urban development', GeoJournal 66(1-2): 57-63

Streib, G. (2002) 'An introduction to retirement communities', Research on Aging 24(1): 3-9

Stutzer, A. and Frey, B. (2008) 'Stress that doesn't pay: the commuting paradox', Scandinavian Journal of Economics 110(2): 339-366

Subramanian, S., Nandy, S., Irving, M., Gordon, D., Lambert, H. and Davey Smith, G. (2006) 'The mortality divide in India: the differential contributions of gender, caste, and standard of living across the life course', American Journal of Public Health 96(5): 818-825

Sule-Odu, A., Fakoya, T., Oluwole, F., Ogundahunsi, O., Olowu, A., Olanrewaju, D. and Sofekun, E. (2008) 'Postpartum sexual abstinence and breastfeeding pattern in Sagamu, Nigeria', African Journal of Reproductive Health 12(1): 96-100

Sunil, T., Rojas, V. and Bradley, D. (2007) 'United States' international retirement migration: the reasons for retiring to the environs of Lake Chapala, Mexico', Ageing and Society 27(04): 489-510

Suri, J. (2009) 'The rise and fall of an international counterculture, 1960-1975', American Historical Review 114: 45-68

Sustain/Elm Farm Research Centre (2001) Eating Oil. Food Supply in a Changing Climate, at: http://library.uniteddiversity.coop/ Food/Eating_Oil-Food_Supply_in_a_Changing_Climate.pdf (accessed 09/16)

Sutton, A. (2004) 'The Three Gorges Project on the Yangtze River in China', Geography 89(2): 111-126

Swyngedouw, E. (1997) 'Excluding the other: the contested production of a new "Gestalt of scale" and the politics of marginalization', in R. Lee and J. Wills (eds) Geographies of Economies, London: Arnold, pp. 167-177

Szewczyk, A. (2013) 'Stepping-Stone Migration': Polish Graduates in England, Unpublished PhD thesis, Loughborough University

Tait, S. (2007) 'Television and the domestication of cosmetic surgery', Feminist Media Studies 7(2): 119-135

Tammaru, T. and Kontuly, T. (2011) 'Selectivity and destinations of ethnic minorities leaving the main gateway cities of Estonia', Population, Space and Place 17: 674-688

Tannock, S. (2015) 'Bad attitude? Migrant workers, meat processing work and the local unemployed in a peripheral region of the UK', European Urban and Regional Studies 22(4): 416-430

Tanser, F., Sharp, B. and Le Sueur, D. (2003) 'Potential effect of climate change on malaria transmission in Africa', The Lancet 362(9398): 1792-1798

Taylor, L. (2011) 'No boundaries: exurbia and the study of contemporary urban dispersion', GeoJournal 76(4): 323-339

Taylor, P. (1989) Political Geography, 3rd edition, Harlow: Longman

Taylor, P. (1993) Political Geography, Harlow: Longman

Taylor, P., Hoyler, M., Walker, D. and Szegner, M. (2001) 'A new mapping of the world for the new millennium', Geographical Journal 167(3): 213-222

Teather, E.K. (1999) Embodied Geographies, London: Routledge

Teferra, D. and Altbach, P. (2004) 'African higher education: challenges for the 21st century', Higher Education 47(1): 21-50

Teichler, U. (2004) 'The changing debate on internationalisation of higher education', Higher Education 48(1): 5-26

Telegraph (2015) 'Syrian refugees rescued from sinking boat off Greek island of Lesbos, in pictures', 14 November

Tholen, B. (2010) 'The changing border: developments and risks in border control management of Western countries', International Review of Administrative Sciences 76(2): 259-278

Thompson, E.P. (1967) 'Time, work-discipline, and industrial capitalism', Past and Present 38: 56-97

Thompson, F. (1982) The Rise of Suburbia, Basingstoke: Palgrave Macmillan

Thrift, N. (1986) 'Little games and big stories: accounting for the practice of personality and politics in the 1945 general election', in K. Hoggart and E. Kofman (eds) Politics, Geography and Social Stratification, London: Croom Helm, pp. 86-143

Thrift, N. (1987) 'Introduction: the geography of late twentieth century class formation', in N. Thrift, N. and P. Williams (eds) Class and Space, London: Routledge and Kegan Paul, pp. 207-253

Thrift, N. (2004a) 'Thick time'. Organization 11(6): 873-880

Thrift, N. (2004b) 'Intensities of feeling: towards a spatial politics of affect', Geografiska Annaler: Series B 86: 57-78

Thrift, N. (2007) Non-Representational Theory: Space, Politics, Affect, London: Routledge 


\section{BIBLIOGRAPHY}

Thrift, N. (2009) 'Space: the fundamental stuff of geography', in N., Clifford, S. Holloway, S. Rice and G. Valentine (eds) Key Concepts in Geography, 2nd edition, London: Sage, pp. 85-96

Tian, N., Wilson, J. and Zhan, F. (2010) 'Female breast cancer mortality clusters within racial groups in the United States', Health and Place 16(2): 209-218

Tibaijuka, A. (2010) 'Adapting to urban displacement', Forced Migration Review 34: 4

Tilly, C. (2011) 'The impact of the economic crisis on international migration: a review', Work, Employment and Society 25(4): 675-692

Timperio, A., Ball, K., Salmon, J., Roberts, R., Giles-Corti, B., Simmons, D., Baur, L. and Crawford, D. (2006) 'Personal, family, social, and environmental correlates of active commuting to school', American Journal of Preventive Medicine 30(1): 45-51

Todaro, M. (1969) 'A model of labor migration and urban unemployment in less developed countries', American Economic Review 59: $138-148$

Todaro, M. (1976) Internal Migration in Developing Countries, Geneva: International Labour Office

Topping, A. (1995) 'Ecological roulette: damming the Yangtze', Foreign Affairs September/October: 132-146

Torkington, A., Larkins, S. and Gupta, T. (2011) 'The psychosocial impacts of fly-in fly-out and drive-in drive-out mining on mining employees: a qualitative study', Australian Journal of Rural Health 19(3): 135-141

Tovar-Guzmán, V., Hernández-Girón, C., Barquera, S., Rodríguez-Salgado, N. and López-Carrillo, L. (2001) 'Epidemiologic panorama of stomach cancer mortality in Mexico', Archives of Medical Research 32(4): 312-317

Trevena, P., McGhee, D. and Heath, S. (2013) 'Location, location? A critical examination of patterns and determinants of internal mobility among post-accession Polish migrants in the UK', Population, Space and Place 19(6): 671-687

Trewartha, G. (1953) 'A case for Population Geography', Annals of the Association of American Geographers 43(2):71-97

Tsenkova, S. (2010) 'Informal settlements in post-communist cities: diversity factors and patterns', Urbani Izziv 21 (2): 73-84

Tunstall, R., Lupton, R., Green, A., Watmough, S. and Bates, K. (2012) 'Disadvantaged young people looking for work: a job in itself?', York: Joseph Rowntree Foundation

Turner, A. (2012) Review of Shaping the Day: A History of Timekeeping in England and Wales, 1300-1800, by Paul Glennie and Nigel Thrift, English Historical Review, 127(528), 1217-1219

Turner, T. and Niemeier, D. (1997) 'Travel to work and household responsibility: new evidence', Transportation 24(4): 397-419

Tyldum, G. and Brunovskis. A. (2005) 'Describing the unobserved: methodological challenges in empirical studies on human trafficking', International Migration 43(1/2): 17-34

Tykwer, T. (1998) Run, Lola Run. Dir. Tom Tykwer. Sony Pictures Classics, 1998. Film.

Tyler, I., Gill, N., Conlon, D. and Oeppen, C. (2014) 'The business of child detention: charitable co-option, migrant advocacy and activist outrage', Race and Class 56(1): 3-21

Tyner, J. (2013) 'Population geography I: surplus populations', Progress in Human Geography 37(5): 701-711

Tyner, J. (2015a) 'Population geography II: mortality, premature death, and the ordering of life', Progress in Human Geography 39(3): 360-373

Tyner, J. (2015b) 'Population geography III: precarity, dead peasants, and truncated life', Progress in Human Geography 40(2): 275-289

Tyrrell, N. and Kraftl, P. (2015) 'Lifecourse and internal migration', in D. Smith, N. Finney, K. Halfacree and N. Walford (eds) Internal Migration, Farnham: Ashgate, pp. 15-29

Uhlenberg, P. (1974) 'Cohort variations in family life cycle experiences of US females', Journal of Marriage and the Family 36: 284-292

Ullman, E. (1980) 'Geography as spatial interaction', reprinted in R. Boyce (ed.) Geography as Spatial Interaction, Seattle: University of Washington Press, pp. 13-27

UN [United Nations] (1995) Report of the International Conference on Population and Development. Cairo 5-13, September 1994, at: www.unfpa.org/public/site/global/publications/pid/1973 (accessed 04/12)

UN (2000) 'Protocol to Prevent, Suppress and Punish Trafficking in Persons, Especially Women and Children, Supplementing the United Nations Convention against Transnational Organized Crime', at: www.refworld.org/docid/4720706c0.html (accessed $08 / 16)$

UN (2009) 'Five "mega-trends" - including population growth, urbanization, climate change - make contemporary displacement increasingly complex, Third Committee told', at: www.un.org/press/en/2009/gashc3964.doc.htm (accessed 09/16)

UN (2010) World Urbanization Prospects: the 2009 Revision, New York: UN

UN (2011) 'As world passes 7 billion milestone, UN urges action to meet key challenges', UN News Centre at: www.un.org/ apps/news/story.asp?NewsID=40257\#.V6HJbjV39Vc (accessed 08/16)

UN (2012) 'Changing levels and trends in mortality: the role of patterns of death by cause', UN, Department of Economic and Social Affairs, Population Division ST/ESA/SER.A/318

\section{ST PROOFS: NOT FOR DISTRIBUTION}


UN (2014a) Framework of Actions for the Follow-up to the Programme of Action of the International Conference on Population and Development Beyond 2014, New York: UN

UN (2014b) The Millennium Development Goals Report 2014, New York: UN

UN (2015a) World Population Prospects. The 2015 Revision, New York: UN Department of Economic and Social Affairs, Population Division, at https://esa.un.org/unpd/wpp/publications/files/key_findings_wpp_2015.pdf (accessed 08/16)

UN (2015b) The Millennium Development Goals Report 2015, New York: UN

UN (2016) UN Habitat - 'Youth', at: http://unhabitat.org/urban-themes/youth/ (accessed 08/16)

UN (2016b) Population Division, at: https://esa.un.org/unpd/wup/ (accessed 09/16)

UN Demographic Yearbook (2006) at: http://unstats.un.org/unsd/demographic/sconcerns/mortality/mort2.htm\#DYB

UN Population Division (2011) World Population Prospects: the 2010 Revision, at: www.un.org/en/development/desa/population/ publications/pdf/trends/WPP2010/WPP2010_Volume-I_Comprehensive-Tables.pdf (accessed 06/16)

UNAIDS [Joint United Nations Programme on HIV/AIDS] (2010) UN AIDS Report on the Global AIDS Epidemic, 2010, at: www.unaids.org/globalreport/Global_report.htm (accessed 08/16)

UNAIDS (2015) AIDS by the Numbers 2015, Geneva: UNAIDS

UNDP [United Nations Development Programme] (2009) Human Development Report 2009. Overcoming Barriers: Human Mobility and Development, New York: UNDP, at: http://hdr.undp.org/sites/default/files/reports/269/hdr_2009_en_complete.pdf (accessed 08/16)

UNFCCC [United Nations Framework on Climate Change] (2016) Homepage: http://newsroom.unfccc.int/ (accessed 09/16)

UNFPA [United Nations Population Fund] (2007a) State of World Population 2007, New York: UNFPA

UNFPA (2007b) 'Potential contributions to the MDG agenda from the perspective of ICPD', New York: UNFPA

UNFPA (2012a) By Choice, Not by Chance. Family Planning, Human Rights and Development, New York: UNFPA, at: www.unfpa.org/sites/default/files/pub-pdf/EN_SWOP2012_Report.pdf (accessed 08/16)

UNFPA (2012b) Ageing in the Twenty-First Century: a Celebration and a Challenge, New York: UNFPA

UNHCR [United Nations High Commissioner for Refugees] (1995) The State of the World's Refugees: in Search of Solutions, Oxford: Oxford University Press

UNHCR (2006) The State of the World's Refugees 2006: Human Displacement in the New Millennium, Oxford: Oxford University Press

UNHCR (2010) 2009 Global Trends. Refugees, Asylum-seekers, Returnees, Internally Displaced and Stateless Persons, Geneva: UNHCR

UNHCR (2011) 'States Parties to the 1951 Convention relating to the Status of Refugees and the 1967 Protocol. 1 April', UNHCR, at: www.unhcr.org/protect/PROTECTION/3b73b0d63.pdf (accessed 08/16)

UNHCR (2014) Monthly Information Kit No. 5. Syrian Refugee Response/Iraq, Reporting Period April 2014, UNHCR, at: http://reliefweb.int/report/iraq/monthly-information-kit-no5-syrian-refugee-response-iraq-may-2014-reporting-period-april (accessed 08/16)

UNHCR (2015a) UNHCR Global Trends. Forced Displacement in 2014, UNHCR, at: www.unhcr.org/uk/statistics/country/ 556725e69/unhcr-global-trends-2014.html (accessed 08/16)

UNHCR (2015b) Syria Regional Refugee Response. Inter-agency Information Sharing Portal, at: http://data.unhcr.org/ syrianrefugees/regional.php\#_ga=1.20947768.1482554276.1446993082 (accessed 11/15)

UNHSP [United Nations Human Settlement Programme] (2003) The Challenge of Slums, London: Earthscan

UNODC [United Nations Office on Drugs and Crime] (2012) Global Report on Trafficking in Persons 2012, New York: UN, at: www.unodc.org/documents/data-and-analysis/glotip/Trafficking_in_Persons_2012_web.pdf (accessed 08/16)

UNODC (2014) Global Study on Homicide 2013, Vienna: UNODC

UNStats [United Nations Statistics Division] (2015) Homepage: http://unstats.un.org/unsd/default.htm (accessed 12/15)

Urdal, H. (2008) 'Demographic aspects of climate change, environmental degradation and armed conflict', United Nations Expert Group Meeting on Population Distribution, Urbanization, Internal Migration and Development, UN/POP/EGM-URB/2008/18

Urdal, H. (2012) 'A clash of generations? Youth bulges and political violence', United Nations Population Division, Expert Paper No. $2012 / 1$

Urry, J. (2000) Sociology Beyond Societies, London: Routledge

Urry, J. (2007) Mobilities, Cambridge: Polity Press

US Census Bureau (2000) Annual Geographical Mobility Rates, By Type of Movement: 1947-1999, at: www.census.gov/ population/socdemo/migration/tab-a-1.txt (accessed 05/13)

US Census Bureau (2010) FactFinder QuickFacts, at www.census.gov (accessed 02/12)

\section{ST PROOFS: NOT FOR DISTRIBUTION}




\section{BIBLIOGRAPHY}

US Census Bureau (2010) International Data Base, at http://www.census.gov/population/international/data/idb/country.php (accessed April 2017)

USCB [United States Census Bureau] (2013) Homepage: www.census.gov/en.html (accessed 08/16)

USDOS [United States Department of State] (2008) Trafficking in Persons Report: 2008, Washington, DC: USDOS

Valentine, G. (1989) 'The geography of women's fear', Area 21(4): 385-390

Valentine, G. (1997) 'A safe place to grow up? Parenting, perceptions of children's safety and the rural idyll', Journal of Rural Studies 13: $137-148$

Valentine, G. (2001) Stranger Danger, London: Continuum

Valentine, G. (2003) 'Boundary crossings: transitions from childhood to adulthood', Children's Geographies 1: 37-52

Valentine, G. and Harris, C. (2014) 'Strivers vs skivers: class prejudice and the demonisation of dependency in everyday life', Geoforum 53: 84-92

Van Blerk, L. (2008) 'Poverty, migration and sex work: youth transitions in Ethiopia', Area 40(2): 245-253

Van Blerk, L. and Ansell, N. (2006) 'Children's experiences of migration: moving in the wake of AIDS in southern Africa', Environment and Planning D: Society and Space 24(3): 449-471

Van Dam, F., Heins, S. and Elbersen, B. (2002) 'Lay discourses of the rural and stated and revealed preferences for rural living. Some evidence of the existence of a rural idyll in the Netherlands', Journal of Rural Studies 18: 461-476

Van de Kaa, D. (1987) 'Europe's second demographic transition', Population Bulletin 42(1): 1-59

Van der Klis, M. and Mulder, C. (2008) 'Beyond the trailing spouse: the commuter partnership as an alternative to family migration', Journal of Housing and the Built Environment 23(1): 1-19

Van Ham, M. and Feijten, P. (2008) 'Who wants to leave the neighbourhood? The effect of being different from the neighbourhood population on wishes to move', Environment and Planning A 40(5): 1151-1170

Van Hear, N. (with Brubaker, R. and Thais, B.) (2009) 'Managing mobility for human development: the growing salience of mixed migration', Human Development Research Paper (HDRP) Series 20, UNDP

Van Hear, N. and McDowell, C. (eds) (2006) Catching Fire: Containing Forced Migration in a Volatile World, Lanham, MD: Lexington Books

Van Kempen, R. and Şule Özüekren, A. (1998) 'Ethnic segregation in cities: new forms and explanations in a dynamic world', Urban Studies 35(10): 1631-1656

Van Rie, A., Beyers, N., Gie, R., Kunneke, M., Zietsman, L. and Donald, P. (1999) 'Childhood tuberculosis in an urban population in South Africa: burden and risk factor', Archives of Disease in Childhood 80(5): 433-437

Van Til, W. (1972) 'The great America cop-out', at: www.indstate.edu/education/sites/education.indstate.edu/files/TL/Van\%20Til/ 19\%20The\%20Great\%20American\%20Cop-Out.pdf (accessed 08/16)

Vaneigem, R. (1967/1994) The Revolution of Everyday Life, London: Rebel Press

Vannini, P. and Taggart, J. (2013) 'Making sense of domestic warmth: affect, involvement, and thermoception in off-grid homes', Body and Society20(1): 61-84

Vannini, P. and Taggart, J. (2013) 'Voluntary simplicity, involuntary complexities, and the pull of remove: the radical ruralities of offgrid lifestyles', Environment and Planning A 45(2): 295-311

Vannini, P. and Taggart, J. (2014) 'Do-it-yourself or do-it-with? The regenerative life skills of off-grid home builders', Cultural Geographies 21(2): 267-285

Vannini, P. and Taggart, J. (2014a) 'No man can be an island: lifestyle migration, stillness, and the new quietism', in M. Benson and N. Osbaldiston (eds) Understanding Lifestyle Migration, Basingstoke: Palgrave Macmillan, pp. 188-208

Vassilev, R. (2004) 'The Roma of Bulgaria: a pariah minority', Global Review of Ethnopolitics, 3(2): 40-51

Vassin, S. (1996) 'The determinants and implications of an aging population in Russia', RAND Corporation Conference Proceedings, at: www.rand.org/pubs/conf_proceedings/CF124/CF124.chap6.html (accessed 08/16)

Vejdovsky, B. and Hemingway, M. (2011) Hemingway: a Life in Pictures, Richmond Hill, Ontario: Firefly Books

Venhorst, V., Van Dijk, J. and Van Wissen, L. (2010) 'Do the best graduates leave the peripheral areas of the Netherlands?', Tijdschrift voor Economische en Sociale Geografie 101(5): 521-537

Vepsäläinen, M. and Pitkänen, K. (2010) 'Second home countryside. Representations of the rural in Finnish popular discourses', Journal of Rural Studies 26: 194-204

Vertovec, S. (1999) 'Conceiving and researching transnationalism', Ethnic and Racial Studies 22: 447-462

Vertovec, S. (2004) 'Migrant transnationalism and modes of transformation', International Migration Review 38: 970-1001

Vertovec, S. (2007) 'Introduction: new directions in the anthropology of migration and multiculturalism', Ethnic and Racial Studies 30(6): 961-978

Vertovec, S. and Cohen, R. (eds) (1999) Migration and Transnationalism, Aldershot: Edward Elgar 
Viallon, P. (2012) 'Retired snowbirds', Annals of Tourism Research 39(4): 2073-2091

Vias, A. (2012) 'Micropolitan areas and urbanization processes in the US', Cities 29: S24-S28

Vidal, J. (2016) 'Pollution has risen 8\% in last five years, WHO data shows', Guardian 12 May

Visser, G. (2002) 'Gentrification and South African cities: towards a research agenda', Cities 19(6): 419-423

Visser, G. and Kotze, N. (2008) 'The state and new-build gentrification in central Cape Town, South Africa', Urban Studies 45(12): 2565-2593

Viswanath, K. and Mehrotra, S. (2007) '"Shall we go out?" Women's safety in public spaces in Delhi', Economic and Political Weekly 42(17): 1542-1548

Vitali, A., Billari, F., Prskawetz, A. and Testa, M. (2009) 'Preference theory and low fertility: a comparative perspective', European Journal of Population/Revue européenne de Démographie, 25(4): 413-438

Wagner, F. (2012) 'Ecovillage research review', in M. Andreas and F. Wagner (eds) Realizing Utopia: Ecovillage Endeavors and Academic Approaches, Munich: Rachel Carson Centre, pp. 81-94

Walby, S. (1986) Patriarchy at Work, Cambridge: Polity Press

Walby, S. (1989) 'Theorising patriarchy', Sociology 23: 213-234

Walby, S. (1990) Theorizing Patriarchy, Oxford: Blackwell

Walby, S. (2003) 'The myth of the nation-state: theorizing society and polities in a global era', Sociology 37(3): 529-546.

Walby, S., Armstrong, J. and Strid, S. (2012) 'Intersectionality: multiple inequalities in social theory', Sociology 46: 224-240

Walker, K. (2013) 'Commuting', in I. Naess (ed.) The Encyclopedia of Global Human Migration, New Jersey: Wiley-Blackwell, at: http://onlinelibrary.wiley.com/doi/10.1002/9781444351071.wbeghm145/abstract (accessed 08/16)

Wallerstein, I. (1974) The Modern World-System, New York: Academic Press

Wallerstein, I. (1979) The Capitalist World Economy, Cambridge: Cambridge University Press

Wallerstein, I. (1983) Historical Capitalism, London: Verso

Walters, M. (2003) Six Modern Plagues and How We are Causing Them, Washington, DC: Island Press/Shearwater Books

Walters, W. (2002) 'Place characteristics and later-life migration', Research on Aging 24(2): 243-277

Wang, H., Dwyer-Lindgren, L., Lofgren, K., Rajaratnam, J., Marcus, J., Levin-Rector, A., Levitz, C., Lopez, A. and Murray, C.J. (2012) 'Age-specific and sex-specific mortality in 187 countries, 1970-2010: a systematic analysis for the Global Burden of Disease Study 2010', The Lancet 380(9859): 2071-2094

Warde, A. (1991) 'Gentrification as consumption: issues of class and gender', Environment and Planning D. Society and Space 9: 223-232

Warnes, A. (ed.). (1982) Geographical Perspectives on the Elderly, Chichester: Wiley

Warnes, A. (1992) 'Migration and the life course', in A. Champion and A. Fielding (eds) Migration Processes and Patterns. Volume 1. Research Progress and Prospects, London: Belhaven Press, pp. 175-187

Warnes, A. and Williams, A. (2006) 'Older migrants in Europe: a new focus for migration studies', Journal of Ethnic and Migration Studies 32: 1257-1281

Warrington, M. (2001) "I must get out”: the geographies of domestic violence', Transactions of the Institute of British Geographers 26(3): 365-382

Washington, H. (2015) Demystifying Sustainability: Towards Real Solutions, London: Routledge

Waters, J. (2006) 'Geographies of cultural capital: education, international migration and family strategies between Hong Kong and Canada', Transactions of the Institute of British Geographers 31(2): 179-192

Waters, J. (2012) 'Geographies of international education: mobilities and the reproduction of social (dis)advantage', Geography Compass 6(3): 123-136

Waters, J. and Leung, M. (2012) 'Young people and the reproduction of disadvantage through transnational higher education in Hong Kong', Sociological Research Online 17(3) at: http://www.socresonline.org.uk/17/3/6.html (accessed 03/17)

Watkins, F. and Jacoby, A. (2007) 'Is the rural idyll bad for your health? Stigma and exclusion in the English countryside', Health and Place 13(4): 851-864

Watson, W. (1964) 'Social mobility and social class in industrial societies', in M. Glucksmann and E. Devons (eds) Closed Systems and Open Minds, Edinburgh: Oliver and Boyd, pp. 129-157

Watts, M. (2001) '1968 and all that. .,', Progress in Human Geography 25(2): 157-188

Webber, M. (1964) 'Order in diversity: community without propinquity', in L. Wingo (ed.) Cities in Space: the Future Use of Urban Land, Baltimore: Johns Hopkins University Press, pp. 23-54

Webster, C. (2003) 'The nature of the neighbourhood', Urban Studies 40(13): 2591-2612

Weeks, J. (2005) Population, 9th edition, Belmont, CA: Wadsworth 


\section{BIBLIOGRAPHY}

Weidinger, T. and Kordel, S. (2015) 'German spa towns as retirement destinations: how (pre) retirees negotiate relocation and locals assess in-migration', Two Homelands 42: 37-53

Weissbrodt, D. and Anti-Slavery International (2002) 'Abolishing slavery and its contemporary forms', New York and Geneva: UN, at: www.ohchr.org/Documents/Publications/slaveryen.pdf (accessed 08/16)

Welch, M. and Schuster, L. (2005) 'Detention of asylum seekers in the US, UK, France, Germany, and Italy A critical view of the globalizing culture of control', Criminal Justice 5(4): 331-355

Weller, S. (2006) 'Situating (young) teenagers in geographeis of children and youth', Children's Geographies 4: 97-108

Werner, C. and Barcus, H. (2009) 'Mobility and immobility in a transnational context: changing views of migration among the Kazakh diaspora in Mongolia', Migration Letters 6(1): 49-62

Weston, K. (1995) 'Get thee to a big city: sexual imaginary and the great gay migration', GLQ: A Journal of Lesbian and Gay Studies 2(3): 253-277

Wharton, E. (1934) A Backward Glance, New York: D. Appleton-Century Incorporated

Whatmore, S. (1993) 'On doing rural research (or breaking the boundaries)', Environment and Planning A 25: 605-607

Whatmore, S. (2002) Hybrid Geographies: Natures, Cultures, Spaces, London: Sage

White, P. and Jackson, P. (1995) '(Re)theorising population geography', International Journal of Population Geography 1: 111-123

White, S. (1980) 'A philosophical dichotomy in migration research', Professional Geographer 32: 6-13

Whitehead, J. and More, N. (2007) 'Revanchism in Mumbai? Political economy of rent gaps and urban restructuring in a global city', Economic and Political Weekly 42(25): 2428-2434

Whitehead, M. (2007) Spaces of Sustainability: Geographic Perspectives on the Sustainable Society, London: Routledge

Whitley, E., Gunnell, D., Dorling, D. and Davey Smith, G. (1999) 'Ecological study of social fragmentation, poverty and suicide', British Medical Journal 319: 1034-1037

Whitman, S., Ansell, D., Orsi, J. and Francois, T. (2011) 'The racial disparity in breast cancer mortality', Journal of Community Health 36(4): 588-596

Whittaker, D. (2006) Asylum Seekers and Refugees in the Contemporary World, London: Routledge

Whitzman, C. (2007) 'Stuck at the front door: gender, fear of crime and the challenge of creating safer space', Environment and Planning A 39(11): 2715-2732

WHO [World Health Organization] (2004) 'Key facts from the World Health Report', Geneva: WHO

WHO (2012) 'Trends in maternal mortality: 1990-2010. WHO, UNICEF, UNFPA and The World Bank estimates', Geneva: WHO WHO (2013a) 'Infectious diseases', at: www.who.int/topics/infectious_diseases/en/ (accessed 05/13)

WHO (2013b) 'Global tuberculosis report 2013', at: www.who.int/tb/publications/global_report/en/ (accessed 08/14)

WHO (2013c) 'Noncommunicable diseases', at www.who.int/mediacentre/factsheets/fs355/en/ (accessed 08/14)

WHO (2014a) 'WHO methods and data sources for country-level causes of death 2000-2012', Geneva: WHO

WHO (2014b) 'Estimates for 2000-2012. Cause-specific mortality', at: www.who.int/healthinfo/global_burden_disease/ estimates/en/index1.html (accessed 11/14)

WHO (2015a) 'Gender', at: www.who.int/gender-equity-rights/understanding/gender-definition/en/ (accessed 06/15)

WHO (2015b) 'Life expectancy', at: http://apps.who.int/gho/data/node.main.688 (accessed 08/16)

WHO (2016) 'Causes of child mortality', at: www.who.int/gho/child_health/mortality/causes/en/ (accessed 08/16)

Whyte, W. (1956) The Organisation Man, New York: Touchstone

Wibberley, G. (1978) 'Mobility in the countryside', in R. Cresswell (ed.) Rural Transport and Country Planning, London: Leonard Hill, pp. $3-16$

Wilbur, A. (2013) 'Growing a radical ruralism: back-to-the-land as practice and ideal', Geography Compass 7(2): 149-160

Wilbur, A. (2014) 'Back-to-the-house? Gender, domesticity and (dis)empowerment among back-to-the-land migrants in Northern Italy', Journal of Rural Studies 35: 1-10

Wiles, J. and Allen, R. (2010) 'Embodied ageing in place: what does it mean to grow old?', in V. Chouinard, E. Hall and R. Wilton (eds) Towards Enabling Geographies, Farnham: Ashgate, pp. 217-235

Wilkinson, R. (1996) Unhealthy Societies: the Afflictions of Inequality, London: Routledge

Wilkinson, R. and Pickett, K. (2009) The Spirit Level: Why Equality is Better for Everyone, London: Penguin

Williams, A. (2010) 'Spiritual therapeutic landscapes and healing: a case study of St. Anne de Beaupre, Quebec, Canada', Social Science and Medicine 70(10): 1633-1640

Williams, A. and Patterson, G. (1998) '"An empire lost but a province gained": a cohort analysis of British international retirement in the Algarve', International Journal of Population Geography 4: 135-156

Williams, A., King, R. and Warnes, A. (2004) 'British second homes in Southern Europe: shifting nodes in the scapes and flows of migration and tourism', in C. Hall and D. Müller (eds) Tourism, Mobility and Second Homes, Clevedon: Channel View Publications, pp. 97-112

\section{ST PROOFS: NOT FOR DISTRIBUTION}


Williams, C. and Millington, A. (2004) 'The diverse and contested meanings of sustainable development', Geographical Journal 170(2): 99-104

Williams, D. and Kaltenborn, B. (1999) 'Leisure places and modernity: the use and meaning of recreational cottages in Norway and the USA', in D. Crouch (ed.) Leisure/Tourism Geographies. Practices and Geographical Knowledge, London: Routledge, pp. 214-230

Williams, G., Meth, P. and Willis, K. (2009) Geographies of Developing Areas, Abingdon: Routledge

Williams, R. (1973) The Country and the City, London: Chatto and Windus

Williams, Z. (2012) 'All thanks to a condom called Panther', Guardian 7 July: 34-35

Williams, Z. (2015) 'Poverty goals? No, it's extreme wealth we should target', Guardian 19 October

Williamson, J. (2002) Coping with City Growth During the British Industrial Revolution, Cambridge: Cambridge University Press

Williamson, K. (2011) 'Between a rock and a hard place: unaccompanied children seeking asylum in Ukraine', UNHCR New Issues in Refugee Research: Paper 223

Willis, K. and Yeoh, B. (2000) 'Gender and transnational household strategies: Singaporean migration to China', Regional Studies 34(3): 253-264

Wills, J., May, J., Datta, K., Evans, Y., Herbert, J. and Mcllwaine, C. (2009) 'London's migrant division of labour', European Urban and Regional Studies 16(3): 257-271

Wilson, A. (1967) 'A statistical theory of spatial distribution models', Transportation Research 1: 253-269

Wilson, A. (1970) Entropy in Urban and Regional Modelling, London: Pion

Wilson, E. (2000) Bohemians. The Glamorous Outcasts, London: IB Tauris

Wilson, K. and Martin, W. (1982) 'Ethnic enclaves: a comparison of the Cuban and Black economies in Miami', American Journal of Sociology 88: 135-160

Wilson, T. (2010) 'Model migration schedules incorporating student migration peaks', Demographic Research 23(8): 191-222

Wilson, W. (1987) The Truly Disadvantaged, Chicago: University of Chicago Press

Wilson, W. (1996) When Work Disappears: the World of the New Urban Poor, New York: Alfred P. Knopf

Wiltshire, R. (1990) 'Employee movement in large Japanese organisations', in J. Johnson and J. Salt (eds) Labour Migration: the Internal Geographical Mobility of Labour in the Developed World, London: David Fulton, pp. 32-52

Winders, J. (2005) 'Changing politics of race and region: Latino migration to the US South', Progress in Human Geography 29(6): 683-699

Winders, J. (2007) 'Bringing back the (b)order: post-9/11 politics of immigration, borders, and belonging in the contemporary US South', Antipode 39(5): 920-942

Wolff, S. (2008) 'Border management in the Mediterranean: internal, external and ethical challenges', Cambridge Review of International Affairs 21(2): 253-271

Wolpert, J. (1964) 'The decision process in a spatial context', Annals of the Association of American Geographers 54: 537-558

Wolpert, J. (1965) 'Behavioural aspects of the decision to migrate', Papers of the Regional Science Association 15: 159-169

Wolpert, J. (1966) 'Migration as an adjustment to environmental stress', Journal of Social Issues 22: 92-102

Wood, E. (1995) Democracy against Capitalism, Cambridge: Cambridge University Press

Wood, W. (1994) 'Forced migration: local conflicts and international dilemmas', Annals of the Association of American Geographers 84(4): 607-634

Woods, M. (2005) Rural Geography, London: Sage

Woods, M. (2009) 'Rural geography: blurring boundaries and making connections', Progress in Human Geography 33: 849-858

Woods, M. (2011) Rural, London: Routledge

Woods, R. (1979) Population Analysis in Geography, Harlow: Longman

Woods, R. (1982) Theoretical Population Geography, Harlow: Longman

Woods, R. (1993) 'Commentary on Zelinsky's model', Progress in Human Geography 17: 213-215

World Bank (1994) Averting the Old-age Crisis: Policies to Protect the Old and Promote Growth, Oxford: Oxford University Press

World Bank (2014) 'Age dependency ratio, old (\% of working-age population)', at: http://data.worldbank.org/indicator/ SP.POP.DPND.OL/countries?display=default (accessed 11/14)

Worth, N. (2008) 'The significance of the personal within disability geography', Area 40: 306-314

Worth, N. (2009) 'Understanding youth transition as "Becoming": identity, time and futurity', Geoforum 40: 1050-1060

Worth, N. (2011) 'Evaluating life maps as a versatile method for lifecourse geographies', Area 43: 405-412

Wright, E.O. (2009) 'Understanding class. Towards an integrated analytical approach', New Left Review 60: 101-116

Wrigley, N. (2002) "Food deserts" in British cities: policy context and research priorities', Urban Studies 39(11): 2029-2040

Wu, F. (2004) 'Residential relocation under market-oriented redevelopment: the processes and outcomes in urban China', Geoforum 35: $453-470$ 


\section{BIBLIOGRAPHY}

Wu, F. (2010) 'Gated and packaged suburbia: packaging and branding Chinese suburban residential development', Cities 27(5): 385-396

Wu, J., Huang, J., Han, X., Gao, X., He, F., Jiang, M., Jiang, Z., Primack, R. and Shen, Z. (2004) 'The three gorges dam: an ecological perspective', Frontiers in Ecology and the Environment 2(5): 241-248

Wulff, M., Champion, A. and Lobo, M. (2010) 'Household diversity and migration in mid-life: understanding residential mobility among 45-64 year olds in Melbourne, Australia', Population, Space and Place 16(4): 307-321

Würfel, M. (2012) 'The ecovillage: a model for a more sustainable, future-oriented lifestyle?', in M. Andreas and F. Wagner (eds) Realizing Utopia: Ecovillage Endeavors and Academic Approaches, Munich: Rachel Carson Centre, pp. 11-17

WWF [World Wide Fund for Nature] (2014) Living Planet Report 2014, Gland, Switzerland: WWF International

Wyman, M. (2005) 'Emigrants returning: the evolution of a tradition', in M. Harper (ed.) Emigrant Homecomings: the Return Movement of Emigrants 1600-2000, Manchester: Manchester University Press, pp. 16-31

Yang, T., Noah, A. and Shoff, C. (2015) 'Exploring geographic variation in US mortality rates using a spatial Durbin approach', Population, Space and Place 21(1): 18-37

Yang, Y. and Morgan, S. (2003) 'How big are educational and racial fertility differentials in the U.S.?', Social Biology 50(3-4): 167-187 Yardley, J. (2007) 'Chinese dam projects criticized for their human costs', New York Times 19 November

Yeoh, B., Huang, S. and Gonzalez, J. (1999) 'Migrant female domestic workers: debating the economic, social and political impacts in Singapore', International Migration Review 33(1): 114-136

Yeung, H. (2003) 'Globalization', in A. Rogers and H. Viles (eds) The Student's Companion to Geography, 2nd edition, Oxford: Blackwell, pp. 103-107

Yeung, Y. (2009) 'Mega-cities', in R. Kitchin and N. Thrift (eds) International Encyclopedia of Human Geography, Oxford: Elsevier, pp. $40-47$

Yin, S. (2007) 'Gender disparities in health and mortality', Population Reference Bureau, at: www.prb.org/Publications/Articles/ 2007/genderdisparities.aspx (accessed 08/16)

Yorgason, E. and della Dora, V. (2009) 'Geography, religion, and emerging paradigms: problematizing the dialogue', Social and Cultural Geography 10(6): 629-637

Young, A., Morales, M., McCabe, S., Boyd, C. and d'Arcy, H. (2005) 'Drinking like a guy: frequent binge drinking among undergraduate women', Substance Use and Misuse 40(2): 241-267

Young, J. (1973) 'The Hippie solution: an essay in the politics of leisure', in I. Taylor and L. Taylor (eds) Politics and Deviance, Harmondsworth: Penguin, pp. 182-208

Young, L. and Ansell, N. (2003) 'Fluid households, complex families: the impacts of children's migration as a response to HIV/AIDS in Southern Africa', Professional Geographer 55(4): 464-476

Young, R., Marshall, S., Domene, J., Graham, M., Logan, C., Zaidman-Zait, A., Mart, A. and Lee, C. (2008) 'Transition to adulthood as a parent-youth project: transfer, career promotion, and relational processes', Journal of Counseling Psychology 55: 297-307

Zaiceva, A. and Zimmermann, K. (2008) 'Scale, diversity, and determinants of labour migration in Europe', Oxford Review of Economic Policy 24(3): 427-451

Zasada, I., Alves, S., Müller, F., Piorr, A., Berges, R. and Bell, S. (2010) 'International retirement migration in the Alicante region, Spain: process, spatial pattern and environmental impacts', Journal of Environmental Planning and Management 53(1): 125-141

Zelinsky, W. (1966) A Prologue to Population Geography, Englewood Cliffs, NJ: Prentice-Hall

Zelinsky, W. (1971) 'The hypothesis of the mobility transition', Geographical Review 61: 219-249

Zelinsky, W. and Lee, B. (1998) 'Heterolocalism: an alternative model of the sociospatial behaviour of immigrant ethnic communities', International Journal of Population Geography 4: 281-298

Zetter, R. (1991) 'Labelling refugees: forming and transforming a bureaucratic identity', Journal of Refugee Studies 4: 39-62

Zetter, R. (2007) 'More labels, fewer refugees: remaking the refugee label in an era of globalization', Journal of Refugee Studies 20(2): 172-192

Zetter, R. and Deikun, G. (2010) 'Meeting humanitarian challenges in urban areas', Forced Migration Review 34: 5-7

Zhao, Y. (1999) 'Labor migration and earnings differences: the case of rural China', Economic Development and Cultural Change 47(4): 767-782

Zhao, Y. (2003) 'The role of migrant networks in labor migration: the case of China', Contemporary Economic Policy 21(4): 500-511

Zheng, H., Yan, S., Qin, Z. and Jin, L. (2012) 'MtDNA analysis of global populations support that major population expansions began before Neolithic Time', Scientific Reports 2: 745

Zhou, Y. and Ma, L. (2000) 'Economic restructuring and suburbanization in China', Urban Geography 21(3): 205-236

Zolberg, A., Suhrke, A. and Aguayo, S. (1989) Escape from Violence, New York: Oxford University Press

\section{ST PROOFS: NOT FOR DISTRIBUTION}


Zsembik, B. and Fennell, D. (2005) 'Ethnic variation in health and the determinants of health among Latinos', Social Science and Medicine 61(1): 53-63

Zuehlke, E. (2009) 'Child mortality decreases globally and immunization coverage increases, despite unequal access', Population Reference Bureau, at: www.prb.org/Publications/Articles/2009/childmortality.aspx (accessed 08/16)

Zuiches, J. (1980) 'Residential preferences in migration theory', in D. Brown and J. Wardwell (eds) New Directions in Urban-Rural Migration, New York: Academic Press, pp. 163-188

Zukin, S. (1996) The Cultures of Cities, Chichester: Wiley-Blackwell

Zukin, S. (2008) 'Consuming authenticity: from outposts of difference to means of exclusion', Cultural Studies 22(5): 724-748

Zweiniger-Bargielowska, I. (2010) 'Building a British superman: physical culture in interwar Britain', Journal of Contemporary History 41: 595-610 
